

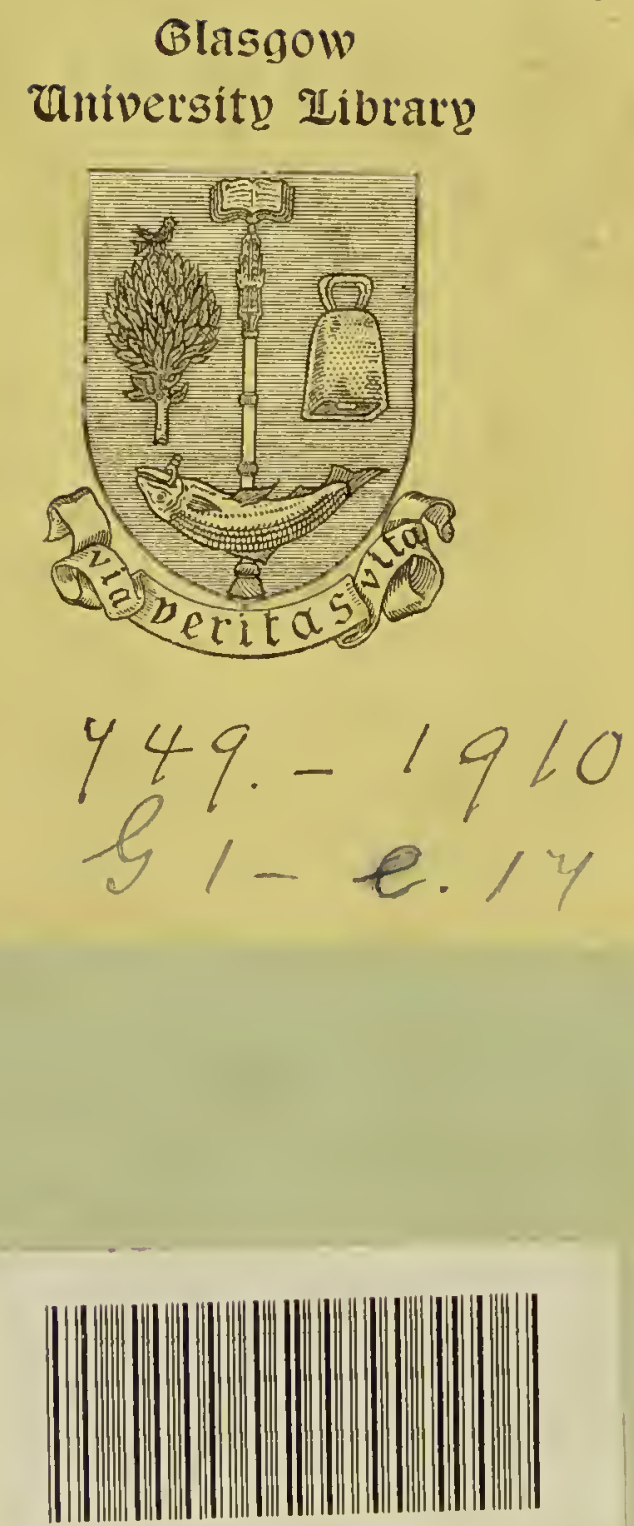

30114005578243 
Glasgow University Library

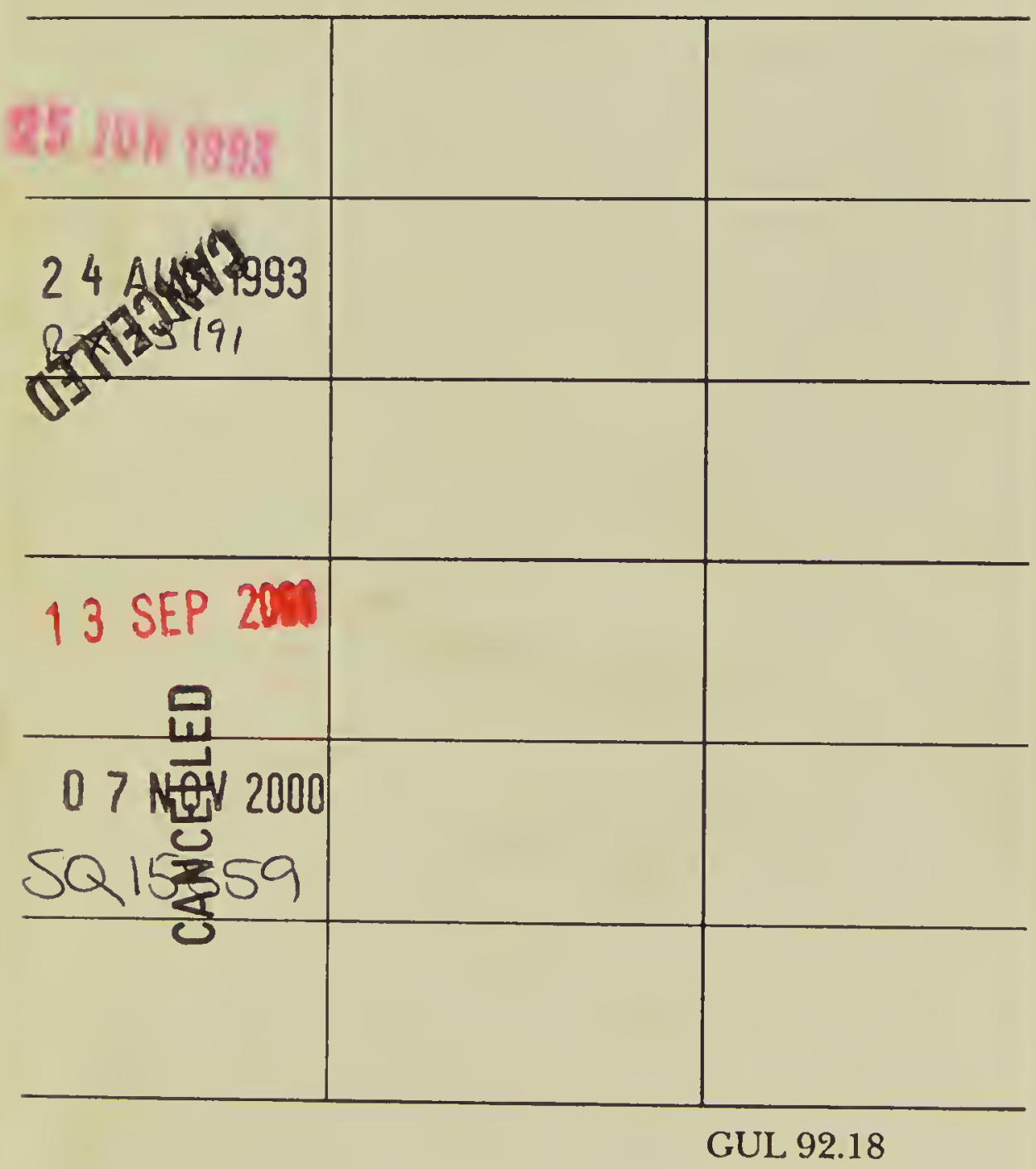







\title{
HISTORY
}

\author{
$\mathrm{OF}$
}

\section{THE HUMAN BODY}

\author{
BY
}

HARRIS HAWTHORNE WILDER

Professor of Zoology in Smith College

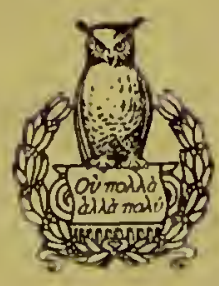

This Volume is sold in England by GEORGE BELL AND SONS PORTugal, St. LinCOLn's In N, LONDON 
COPYRIGHT, I9O9,

$\mathrm{BY}$

HENRY HOLT AND COMPANY 
Seinem Lehrer und Freunde

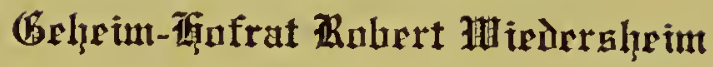

$$
\text { wird dieses Buch }
$$

in Liebe und Dankbarkeit gewidmet.

Der Verfasser. 



\section{PREFACE}

THIs book has a twofold purpose: first, to present the results of modern anatomical and embryological research relative to the human structure in a form accessible to the general student, and, secondly, to furnish students of technical human anatomy with a basis upon which to rest their knowledge of details.

Regarding the first of these purposes, it may be said that, while many of the phases of the doctrine of evolution have been thoroughly exploited, and their general teaching has become the property of the general scholar, the contribution to thought furnished by anatomy has been considered of too technical a character for popular presentation. It is true that this science necessarily rests upon a material basis, and involves a mass of extremely intricate details, and it is also true that a more or less complete knowledge of these is absolutely necessary before the contribution of this science to evolutionary thought can be appreciated; but in these respects anatomy does not cliffer from other branches of natural science, the essential teachings of which are already a matter of general knowledge. If, then, the technical difficulties have been surmounted in the case of Geology, Astronomy and general Zoölogy, it is not too much to hope that in the course of the next few years the mission of Anatomy may also become generally known, especially since its results touch human interests more closely than do those of any of the kindred sciences.

Concerning the second purpose, that of assisting in the technical study of human anatomy, it is hardly necessary to present an argument, since the great advantages of studying human anatomy in connection with both comparative anatony and embryology are patent to all who have employed this method. While there are still a few human anatomists who present the old argument that the science is too full of detail already to allow the assumption of additional facts, the ex- 
perience of everyone who has learned the parts of some complicated organ like the brain, by the old method, and has had it later eluciclated by the new, is a sufficient refutation of such a position. It takes but a little experience with anatomy, as taught by the modern comparative method, to see that this latter furnishes a rational basis for an absolute knowledge of the fundamental relationships, while the old method is largely an intricate system of mnemonics. A student of the older anatomy must needs remember arbitrarily that two given parts are related in a certain way and not in the reverse way, and if his memory is inadequate to the task he has nothing to save him, while a student, furnished with a morphological basis for his knowledge and able to refer the parts back to a time in which they were in a much simpler condition, will know that they must be related in a certain definite way, and cannot be otherwise arranged.

The present work has especially the needs of the medical student in mind, since it is not a general comparative anatomy, but, as its title signifies, a "history of the human body," in which the structure of the lower vertebrates is expounded only so far as is needed to throw light upon the relations found in Man. Thus the lines that do not lead in this direction, but represent specialized side-branches, like those of birds or snakes, are barely touched upon, other than as illustrations of principles similar to those under consideration, although certain exceptional modes of development or eccentric specializations are often mentioned on account of their general interest.

The technical terms of human anatomy employed in this work conform in general to the list prepared by the Basle Anatomical Nomenclature (BNA), but in cases where these terms differ widely from those in common use in America the latter are placed in brackets after the BNA term. In cases where the BNA nomenclature is not in accord with morphological principles, these terms are rejected, but are indicated in brackets or otherwise. Of these, the most important are the following: 
I. In the case of the bones of the carpus and tarsus. For these the BNA nomenclature employs the terms used on the Continent, and especially Germany (e.g., triquetrum, multangulum majus, etc.), instead of those to which the Americans and English are accustomed. The synonomy of these terms is presented in the form of a table, but as both sets are purely arbitrary and describe the shapes and relative sizes as found in Man alone, there seems no reason why one should be preferred to the other, or, indeed, why either should be longer perpetuated, in preference to the simple system employed by comparative morphologists.

2. In several cases in which terms of orientation are still employed with reference to Man in a standing position (e.g., superior and inferior instead of anterior and posterior; anterior and posterior instead of ventral and dorsal). Thus, in the case of the columns of the spinal cord, it is thought best to reject the BNA terms posterior and anterior in favor of the more natural dorsal and ventral, as employed in the case of all other animals. In the same way the two vence cave are referred to as anterior and posterior instead of superior and inferior.

3. In the case of the pads of the palm and sole. Here the principle involved is one of use rather than position, and the point at issue depends upon the true function of these parts. The two views held at present are (I) that their function is tactile, and (2) that it is mechanical, preventing the tendency to slip by presenting a surface covered by ridges. [cf. Chapter IV.] The BNA term for these pads is toruli tactiles, a term which does not accord with the view expressed here.

In a few cases the adoption of the new nomenclature involves changes in well-established terms; for example, ductus [vas] deferens, stratum germinativum [mucosum], and renal [Malpighian] corpuscles; and in some there is a slight change in spelling, as thyrcoid and chorioid, but as these are all in the interest of exactness and do not violate morphological principles, they are employed here.

In the case of a work which, like the present one, attempts 
to cover a large field, in each and every point of which there are opposing views, both as to the facts themselves and to their interpretation, errors and misinterpretations are inevitable, and the writer craves the indulgence of those who have directed their special attention to any one of the subjects touched upon here. The book is primarily intended as an interpretation of the work of the specialists in anatomy, especially during the last half-century, and its mission will be accomplished if it serves to render the facts obtained more accessible to the general reader.

Dryads' Green, Northampton,

May, 1909 


\section{CONTENTS}

PAGE

Preface • • • • • • • • • • • • • •

CHAPTER

I. The Continutity of Life . . . . . . . . I

II. The Phylogenesis of Vertebrates . . . . . 26

III. The Ontogenesis of Vertebrates . . . . . 48

IV. The Integument and the Exoskeleton . . . 76

V. The Endosieleton . • . . . . . . . . 122

VI. The Muscular System . . . . . . . . I89

VII. The Digestive and Respiratiory Systems • • . 257

ViII. The Vascular System . . . . . . . . 3I7

IX. The Uro-genital System . . . . . . . . 365

X. The Nervous System . . . . . . . . 406

XI. The Sense-Organs . . . . . . . . . . 465

XII. The Ancestry of the Vertebrates . . . . . 506

ApPEndix • . . . . . . . . . . . 539 



\section{PLATES}

PAGE

Plate I. Diagrams showing Vertebrate development; stages I and II. Based upon a stereogram by KingsLEY. 62

Plate II. Diagrams showing Vertebrate development; stages III and IV. Based upon a stereogram by KingSLEY. $6_{3}$

Plate III. Development of uro-genital system in Amniotes from stage of sexual indifference (a) to male, b), and to female (c). In part after GEGEnBAur. . 386

PLATE IV. Longitudinal median sections of Vertebrate brains corresponding to the first half of the series in Fig. I 7 in the text. [(b) and (c) after EDINGER]. $4 \mathrm{I} 4$

Plate V. Longitudinal median sections of Vertebrate brains corresponding to the seccond lualf of the series in Fig. II 7. [After EDINGER]. . . . 4I 5

Plate VI. Diagram of cranial nerves in Anamnia. [After Wiedersheim]. . . . . . . 448

Plate VII. Diagram of cranial nerves in Ammiota. [After WiEDERSHEIM]. $\quad \cdot \quad \cdot \quad \cdot \quad \cdot \quad 449$

Plate VIII. Inter-relation of Trigeminus, Facialis, Glossopharyngeus, and Vagus, together with the sympathetic ganglia in man. Based upon diagrams by several anatomists (Arnold, Gray, Gegenbaur). . 456 
"Man still bears in his bodily frane the indelible stamp of his locely origin."

Charles Darwin: "Descent of Man" (closing sentence) 


\section{CHAPTER I \\ THE CONTINUITY OF LIFE}

"Ich sage immer und wiederhole es, die Welt könntt nicht bestehen, wenn sie nicht so einfach wäre."

JoHANN Wolfgang Goethe, in Eckermann, Gespräche mit Goethe. II Apr., I827.

ONE of the grandest generalizations formulated by modern biological science is that of the continuity of life; that the protoplasmic activity within the body of each living being now on earth has continued without cessation from the remote beginnings of life upon our planet, and that from that period until the present no single organism has ever arisen save in the form of a bit of living protoplasm detached from a preexisting portion; that the eternal flame of life, once kindled upon this earth, has passed from organism to organism, and is still going on, existing and propagating, incarnated within the myriad animal and plant forms of the present day. Built up of carbon, hydrogen, oxygen, nitrogen, together with traces of a few other elements, yet of a complexity of structure that has hitherto resisted all attempts at complete analysis, protoplasm is at once the most enduring and the most easily destroyed of substances; its molecules are constantly breaking down to furnish the power for the manifestations of vital phenomena, and yet, through its remarkable property of assimilation, a power possessed by nothing else upon earth, it as constantly builds up its substance anew from the surrounding medium, usually in excess of that lost by disintegration, and possessed of qualities identical with those of the parent mass. The continuity, then, is not one of material, but of qualities, and it is this that makes an organism the same from birth till death. An acorn, a sapling, an oak 
- all are the same organism, although the bulk of the acorn is but the hundrecth part of the sapling, and that the thousandth part of the oak, and although every particle that constituted the organism in an early stage may have been eliminated long before the next stage is reached. Upon the attainment of a certain size-limit, the most or the whole of the constantly accumulating excess is freed from the parent organism, in the form of germinal particles, each of which, still continuing the process of assimilation, wrests building material from its surroundings, from other organisms as well as from inorganic substances, and, if successful, develops into a new organism, which often to the minutest details reproduces the parent from which it arose.

Through this power of assimilation there is a constant encroachment of the organic upon the inorganic, a constant attempt to convert all available material into living substance, and to indefinitely multiply the total number of individual organisms. This tendency receives a check, however, from two sources: from the forces of the inorganic world, since each organism is particularly sensitive to surrounding conditions, and, secondly, from other organisms. It has been to offset these that all variations in organisms have taken place, changes which have furnished a great power of adaptation to various conditions and have resulted in the invasion and occupancy of all environments in which the conditions do not absolutely prohibit protoplasmic activity.

Thus have developed all the plant and animal forms which have ever appeared on the earth, and since no one of these can have arisen spontaneously, but depends for its development upon a bit of living protoplasm thrown off from a previously existing organism, it follows that all living beings may be traced back through continuous though converging lines of life to the first beginning of all life-the primordial protoplasm. Difficult as this may be to follow in the case of the more complex organisms, those which, through constant modification, have departed most widely from the original condition, this continuity of life is easily seen in the one- 
celled organisms, or Protozoa, which are the simplest in structure of all living things. The essential body substance consists of a minute mass of semi-fluid protoplasm, in the interior of which lies a denser portion which constitutes its most important organ, the mucleus. This latter is the physiological center for the control of all the vital functions of the animal, and is undoubtedly extremely complex in structure, even in the simplest members of the group. In some protozoans the protoplasm is enclosed by a thin but fine cell-membrane, which preserves for the animal a more or less definite shape; in other cases there is no such membrane, and the protoplasm is free to assume an irregular and constantly changing outline, each species, however, still preserving a certain characteristic range of form.

Through the intaking of other organisms, either alive or in a state of disintegration, the protoplasm of all Protozoa has the power of adding to its bulk, through assimilation; a process perhaps more than all others characteristic of life and not imitated in any way by lifeless matter. For this process a nucleus is absolutely essential, for it has been experimentally proven that non-nucleated fragments of the simpler Protozoa are capable of continuing their existence for some time, and can even receive foreign materials, yet have no power of assimilation. A fragment containing a nucleus, on the other hand, will continue to grow and will ultimately completely restore the lost part.

This process of growth is limited, however, not by any failure in the vital process, but by the mathematical law of the ratio of surface to mass.* The intaking of both food and oxygen, and also the expulsion of all waste products, take place on the external surface, or, in the case of those covered by a cell-membrane, over a restricted portion of that area, but on

* This law is that the surfaces of homologous solids are to each other as the squares, and their masses as the cubes, of their homologous dimensions. A protozoan which has increased to twice its normal size, i. $\varepsilon$., twice its original diameter, has increased its surface four times and its mass eiglit times. It has therefore reduced its proportionate surface by one half, and its supply of food and oxygen in the same degree. 
account of the law just mentioned, the mass of a growing animal increases faster than its external surface, and the time is soon reached at which it is in danger both of starving and of suffocation. To offset this, recourse is had to a process called fission, which effects at the same time a relief from the physiological difficulty and a multiplication of the individual.

In its simplest form this reproduction by fission, as it is termed, is inaugurated by (I) a lengthening of the nucleus; (2) a contraction of its middle portion, producing a form
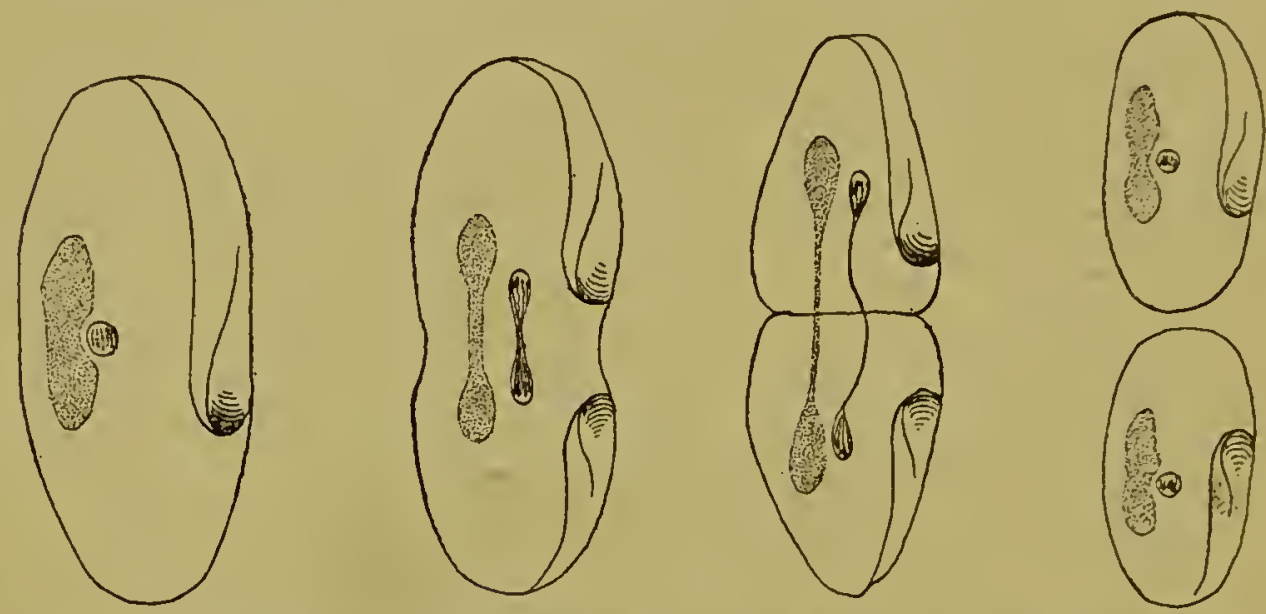

FIG, I. Simple fission. Diagrams based on the infusorian Paramacium. In all the figures the macronucleus is on the left, the micronucleus on the right. The division of the micronucleus is effected by mitosis, that of the macronucleus is direct.

like an hour-glass, and (3) a separation of the two halves, forming two independent nuclei, each half of the original size. A similar subdivision of the body of the cell follows, the arrangement being such that each piece becomes supplied with one of the two nuclei, and is capable of beginning an independent existence. In certain other cases the proceeding is more complicated. The organism surrounds itself with a shell or cyst, secreted by the protoplasm, and after a quiescent period, breaks up, not into two, but a larger number, usually four, eight or sixteen, which become released by the bursting of the cyst and swim out into the water, each in its turn to assimilate foreign matter until of the size for another encystment.

It is but natural to refer to the undivided organism as the 
"parent," and to the resultant organisms, whether two or one, as the "offspring," yet it is here plain that we do not have to do with either parent or children in the usual sense. The "parent," as such, ceases to exist the moment it becomes divided; yet no death has ensued, for there is no dead body. The vital activity of protoplasm has been perpetuated, without an interruption, from the undivided mass to each piece resulting from the fission, or in other words, the life is continuous.

In a restricted sense, then, a protozoan is immortal: its
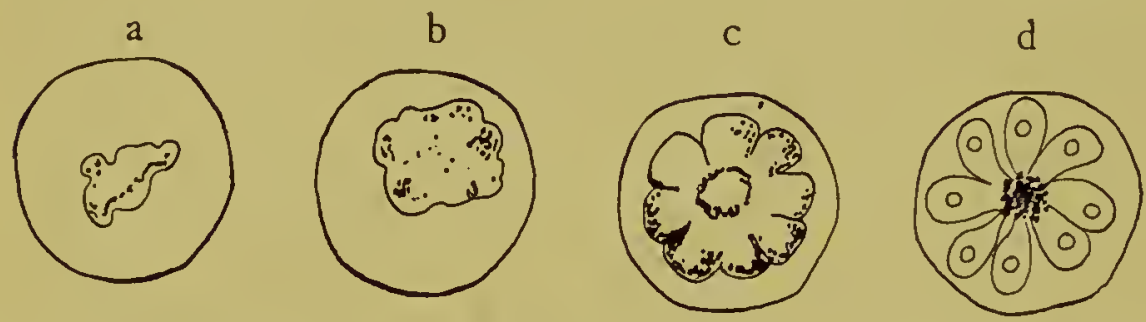

FIG. 2. Multiple fission as shown by the parasite of malaria, Hamamaba malaria. [After Ross and Fielding-Ould.]

The enclosing outline represents a human blood corpuscle, within which the transformation takes place.

(a) Young amoboid stage formed from a sporozoid. (b) Older amoboid stage, showing growth. (c) Beginning of multiple fission. (d) Division of the mass into eight sporozoids. At this stage the sporozoids become liberated through the distintegration of the remains of the corpuscle, and invade the plasma. From this they enter new corpuscles, and assume the amœboid form as at $a$, thus completing the cycle.

vital activities have been continuous, without interruption from the beginning of life upon the planet. It is not meant, of course, that a protozoan is indestructible, for countless numbers of them are continually succumbing to mechanical or chemical injury; but each accident of this sort extinguishes a life which has existed without cessation from the first life of all. The actual material particles are constantly changing, even while a protozoan is retaining its identity as an individual, yet that which is continuous from moment to moment in such an individual, is equally so during and after each fission, and is perpetuated without interruption, in each piece, so long as it does not meet with conditions which destroy it.

Aside from the phenomena of reproduction by fission, there is another procedure which has been observed in many forms 
of Protozoa, and while in the present state of knowledge it cannot be asserted that it is a universal procedure, existing in all species, it is very likely that this or a similar process is oc-
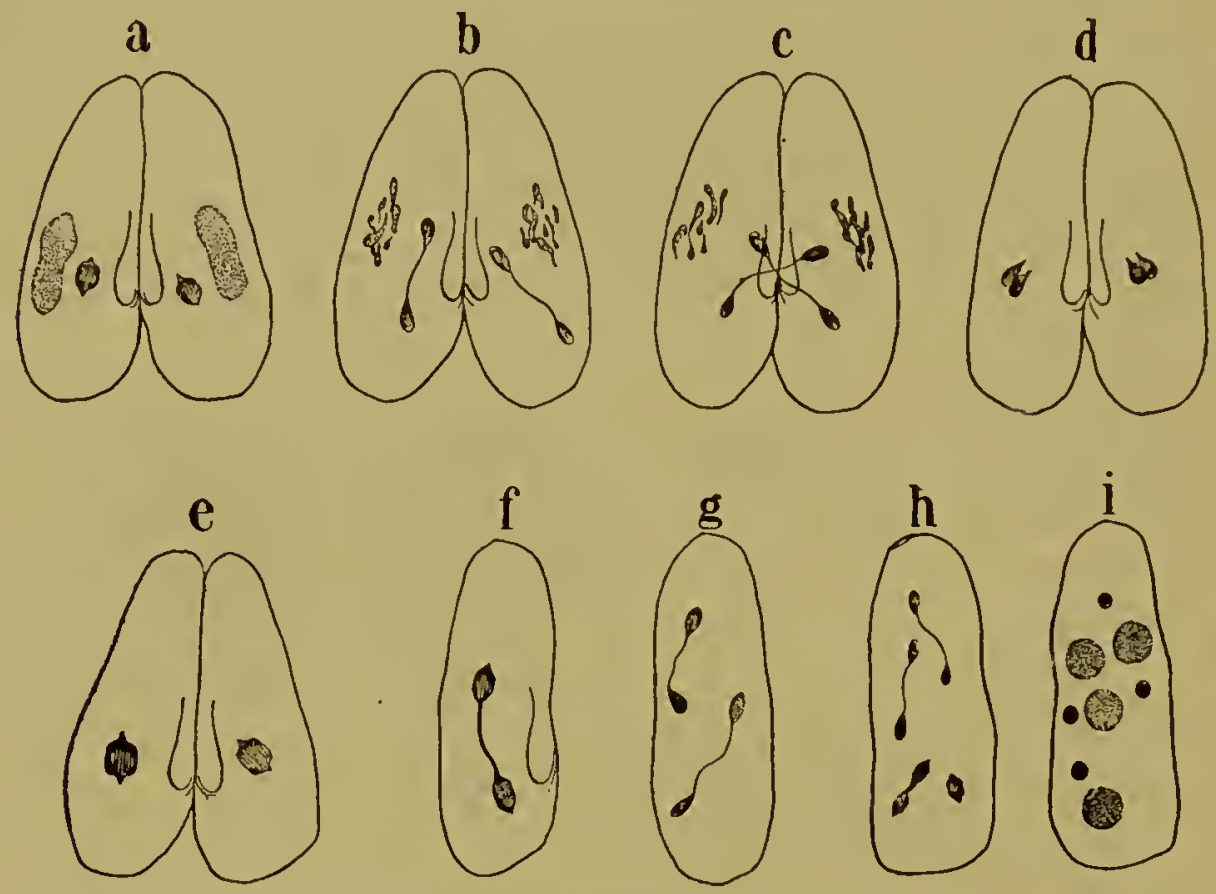

FIG. 3. Conjugation. Diagrams based on the infusorian Paramacium. Here the two gametes are of the same size and the fusion is temporary with similar results in the case of each.

(a) The two micronuclei are forming mitotic figures preparatory to division. (b) The two micronuclei have elongated; the macronuclei are disintegrating. (c) One-half of each micronucleus passes into the other individual through the mouth. (d) Fusion occurs in each individual between the half nucleus that originally belonged to it and the half nucleus that has come from the other. This forms a fusion-nucleus. (e) The fusion-nuclei form mitotic figures preparatory to division. At about this time the two individuals separate. (f), (g), (h) The fusion-nucleus divides three times in succession, eventually iorming eight nuclei. (i) Four of the eight nuclei enlarge and form macronuclei, and four remain small and become micronuclei. These become associated in pairs, one microand one macro-nucleus, and are distributed to four individuals that result from two successive divisions. Each of these, evidently as the result of the conjugation, has a renewed power of fission, and multiplication continues in this way [cf. Fig. I] until the power becomes diminished, when it is renewed by a new conjugation [cf. Fig. 5 (a)].

casionally undergone in all cases. This is the process of conjugation [Fig. 3] which, in the cases best studied, seems to bear a definite relation to the process of reproduction by fission. In these cases the number of fissions which can occur in succession appears to be limited, for after a series of these 
it seems that the reproductive force becomes lessened, causing longer pauses between successive fissions, and ultimately the death of the organisms. It is at this time, when the fissions are farther between and carried on with less activity, that conjugation appears. This consists typically of the temporary fusion of two individuals, during which there is a mutual interchange of certain of the elements of the nuclei. When this is accomplished the two individuals, or gametes, as they are here termed, separate, and begin anew a fresh series of fissions as at first.

The purpose of the process thus seems to be something like a rejuvenescence, by means of which the reproductive activity may be renewed; yet, that the action is chemical rather than physiological is suggested by experiments in which a similar increase of activity, taking the place of conjugation, may be induced by the addition of food-substances like beef broth to the water containing the species under investigation.

In many cases the process of conjugation is rendered more complicated by the introduction of two sorts of individuals, macro-and micro-gametes, which are evidently produced for this especial purpose by a variation in the usual course of the fission process. In this case the two usually unite permanently and form a zygote, which becomes thus endowed with special reproductive activity. [Fig: 4.]

In multicellular organisms the matter becomes still more complicated, but is essentially the same so far as concerns protoplasmic continuity. Here only certain cells, which are called germ-cells, act as gametes and conjugate, producing the new organisms by their repeated divisions, while the remainder, often vastly preponderating over the former in actual bulk, build up a body or soma, which forms a shelter and protection for the germ cells. Somata possess a high degree of adaptability to external conditions, and become modified to fit them, so that in this way they and the germ-cells contained within them may come to be developed in places and under circumstances where otherwise they could not possibly exist.

In this way all animal and plant forms have been produced, 


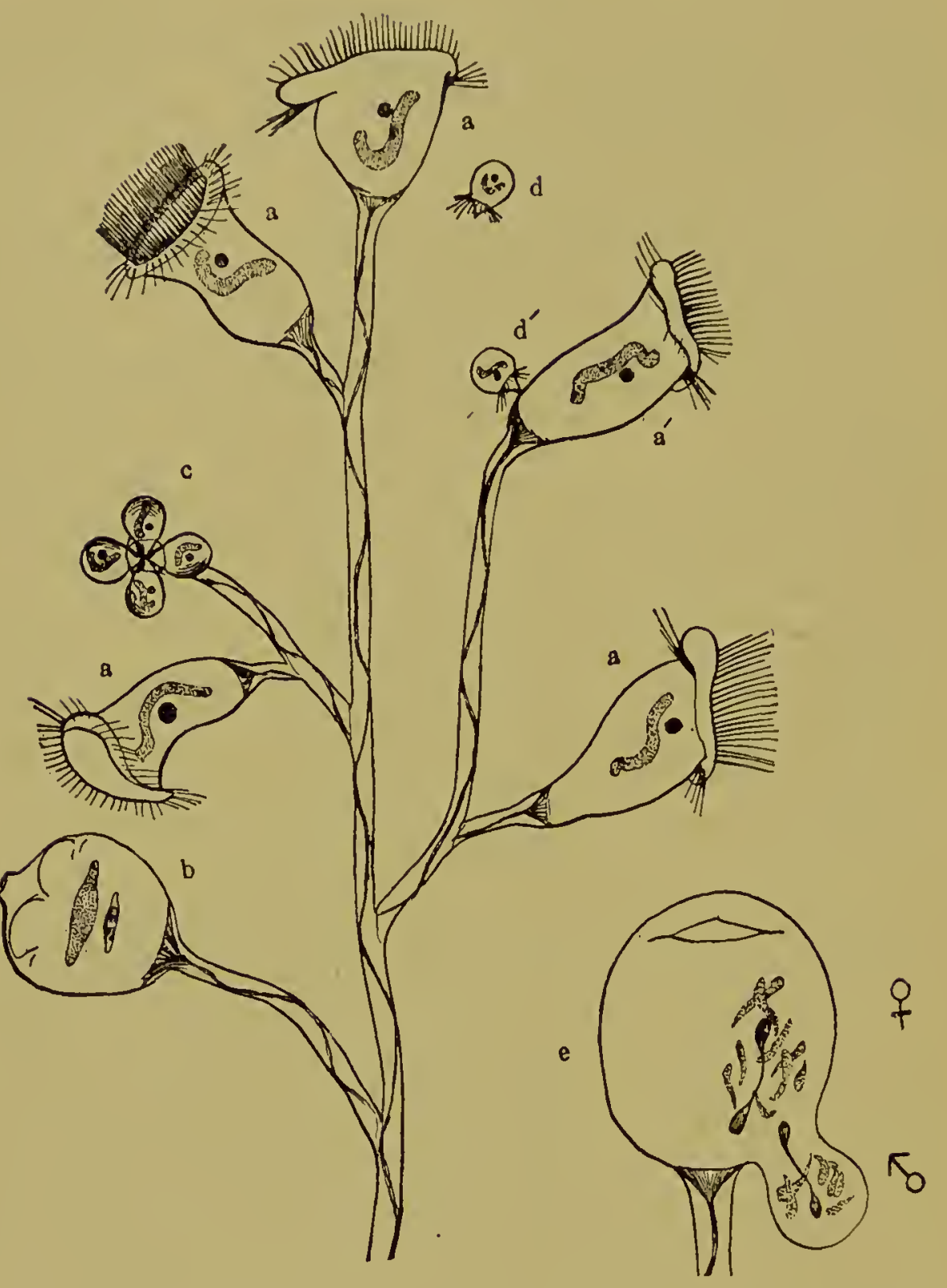

FIG. 4. Carchesium, a sessile protozoan colony, showing conjugation. [Diagram in part after Bütschli and SchewiakofF.]

a a Macrozooids, which, by their division produce either $b$, other macrozooids, or $c$ microzooids, which eventually become free. $d$ Frce-swimming microzooids, perhaps from another colony. d microzooid (here a microgametc) in conjugation with a macrozooid (macrogamete). In each of the above individuals may be sccil a a macrozm marcronucleus and a spherical micronucleus. $e$ Detail of conjugation. The macronucleus of each component is shown broken into fragments previous to dissolution; the two micronuclei are dividing mitotically into two halvcs, onc-half of cach destined to pass into the other component. The micro- and macro-gametcs are designated, rcspectively, as male and female. [Subsequent stages sinilar to those shown in Fig. 3.] 
cach being but the temporary dress of a proliferating mass of protoplasm; a detacled mass of tissue, which feeds, breathes, and often moves and perceives, for the better support and protection of the continuous living protoplasm. The soma is mortal, and after a longer or shorter period loses its vitality and goes to dissolution; the germ, in the restricted sense of being coëval with life upon the earth, is immortal; and yet, in spite of the far greater value of the latter, the two are very closely associated. As the soma becomes modified, the germ becomes equally so, since each germ, as it develops, repro-
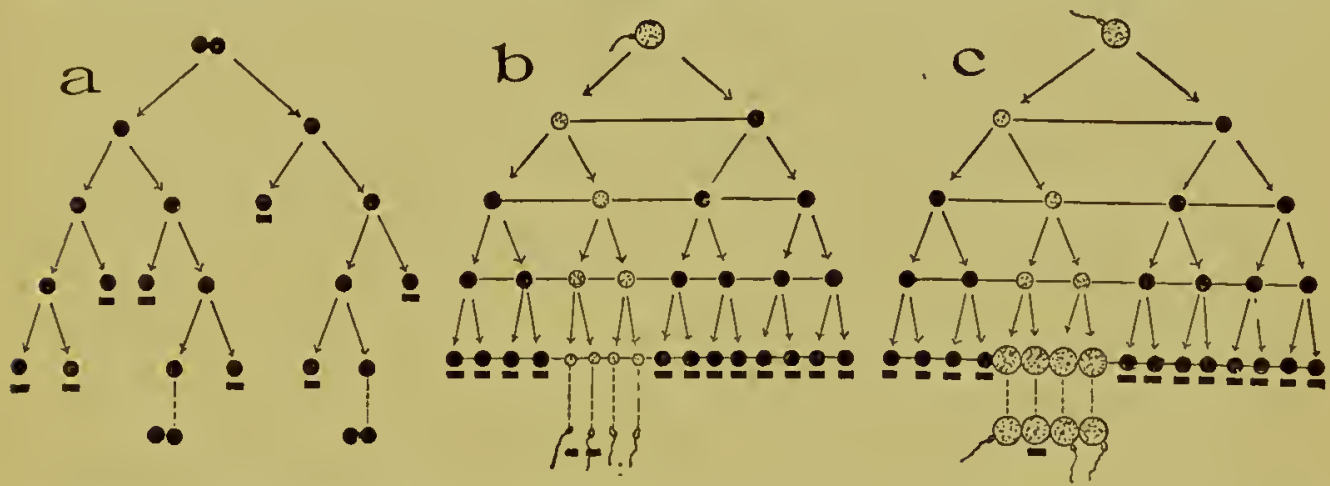

FIG. 5. Diagrams illustrating the life cycle in unicellular and multicellular organisms.

The round dots represent cells. In (b) and (c) the germ cells are gray, the somatic cells black. In all cases the destruction of a cell is indicated by a heavy black bar placed beneath it.

(a) Protozoan type, with equivalent gametes. The series begins with a con. jugation, after which the gametes separate and a series of simple fissions follows in the case of each gamete. After several generations of these, in which many of the individuals produced are destroyed, conjugation again appears, completing the cycle.

(b) Life cycle in a male Metazoan. The cycle begins with a conjugation between a macro- and a micro-gamete (ovum and spermatozoön), after which thcre follows a series of simple fissions, which differ from those of $(a)$ in the perpetual union of the components thus formed, represented here by connecting lines. There is thus built up an interdependent cell-colony, the soma, shown in the fifth row from the top. Certain of the somatic cells become microgametes (=spermatozoa), destined rest conjugation, and caple of independent existence when separated from the rest. The remaining somatic cells perish simultaneously.

(c) Same as (b), but the gametes produced are macrogametes (=ova), and the soma is consequently female. The conjugation of thesc cells with microgametes is the completed lower row, thus completing the life cycle. The bars placed beneath destruction.

duces a soma almost identical with that from which it came; a result which can be explained only by supposing that each germ contains a controlling mechanism, directing and determining the development of every individual part in the future soma. 
The differences between unicellular and multicellular organisms in these respects may be graphically expressed in the accompanying diagrams. [Fig. 5.]

In the first of these (a), which represents the condition in the simpler unicellular animals, a cycle of cell generations begins with a conjugation, a procedure during which a part of the nuclear material of the two conjugating individuals is mutually exchanged, the result seeming to be an increased activity of division for some time. The resulting cell generations are followed in the diagram in the case of but one of the two conjugating individuals, that of the other being similar. Several generations are indicated, as also the chance mortality of individuals, the result of this last being to keep the total number of individuals in each generation approximately the same in spite of the geometrical ratio in which the individual cells tend to increase.

The two other diagrams (b) and (c) represent a similar cycle of cell generations in two multicellular organisms, male and female, respectively. In these, the cycle begins with the union of a male and female germ-cell, that is, a permanent conjugation between a micro- and a macro-gamete, forming a fertilized ovum. Because of the cellular differentiation due to a necessary adaptation, the male cell is small and active and equipped with a locomotive organ in the form of a vibratile flagellum, while the female cell is more or less immobile and furnished with a large amount of yolk, the food supply for the embryo during its early development, when it cannot obtain its own nourishment. After the conjugation there ensues a series of cell generations, as in the other case, with the essential difference that here they remain in organic continuity with one another and form, not independent individuals, but the component parts of a multicellular organism. The number of such generations is often very great, certainly much greater than here represented, and the cells early begin a differentiation of form and function which leads eventually to the formation of all the tissues necessary to build up the adult body or soma. Among those early cells are the 
primordial germ-cells, differently marked in the diagrams, which seem to retain the general qualities of the first egg-cell and to resist the tendency to specialization seen in the others. From these the final germ-cells develop, small and mobile in the case of the male, large and provided with yolk in the case of the female. These, liberating themselves from the soma, unite in pairs to form another cycle like the first, while all the generations of the somatic cells are sooner or later brought to an end simultaneously, the death of the individual.

This organic connection between the cells, which constitutes the essential difference between unicellular and multicellular organisms, has its advantages as well as its disadvantages. The chief among the first is the great power of differentiation among individual cells or cell-groups, with the resultant division of labor; a great disadvantage lies in the fact that through this very specialization of function, any vital accident occurring in one part drags down to death all the other cells of the organism. The germ-cells alone are the immortal parts, the continuous principle which survives the destruction of the soma, and each contains within itself, expressed in the form of an ultra-complex mechanism, the ability to reproduce in its cell descendants every detail of the soma from which it originated.

In this is seen the primary value of the soma, which becomes clear when taken in connection with the struggle on the part of nature to develop as much protoplasm as possible. The soma is a mass of protective cells, capable of a high degree of specialization, and thus able to adapt itself in accordance with the needs of every environment in which it is possible for organic beings to exist. Even its death is an adaptation, for by this means new and perfect somata are constantly taking the place of those whose usefulness as guardians of the germ-cells has become impaired by the inevitable injury to which organisms are constantly exposed. Life is continuous in the germ-cells from generation to generation and has been carried into all enviromments and protected and multiplied through a constant succession of perishable somata. 
The adaptations of the soma are extremely gradual, and thus, if all forms that have ever existed could be arranged in order, they would form a continuous series, not in the form of a straight line, but in that of a profusely branching tree, since from one parent form two or more varieties are constantly arising, capable of inhabiting a slightly different environment, and, if successful, continuing along separate lines of development. As a matter of fact, however, the fauna and fiora of the world at present represent, for the most part, but isolated units in the great system, and while a careful study of the structure of every known form has led to the restoration of many portions of this tree, there are in other places great gaps filled thus far only by inferences, and therefore matters of continual controversy.

This continuity of all life and the recognition of animals and plants as no more than the countless adaptive forms of the plastic soma, enable the zoölogist to trace out with considerable accuracy the history of those series of which the records are the best preserved, a history which, while lying in the past, is represented in the present by forms which arose in earlier periods, the complete adaptation of which has allowed them to successfully struggle with their competitors and thus to survive with but little change to the present day. It is in this sense, then, that there can be a history of the human body, the history of the struggles and successes and failures of our remote ancestors, as they successively encountered the various environments wherein this history has been enacted. The ocean, the marsh, the prairie, the forest, each has formed the complex stage-setting of an historic period and has contributed to the formation of the human soma. Man's body was, like all others, not made new, but adapted, and this not once, but repeatedly. Old organs have been readapted to new uses or are retained as merely functionless rudiments, new organs have arisen through the change of function of some preexisting part, the body has in all its details been molded and shaped with each new change to the end of producing the highest degree of physiological efficiency, and this always with sole 
reference to the problem in hand and with no regard to the future inconveniences which may arise from a certain form or arrangement.

To learn this history we must turn to the comparative anatomy of vertebrates. Some of them are still so similar to the early stages of our own development that we may almost look upon them as our former selves; others represent development along other lines to which their environment and its necessities have brought them, and they show us what we might have been, had chance led us in their direction.

The first period of vertebrate history was an aquatic one, in which the environment was represented, not merely by the water, which developed a certain kind of respiration, and allowed a style of locomotive organs inadmissible on land, but by the vast hordes of carnivorous enemies generated in the depths of the ocean; yet, through these struggles was gained an exoskeletal armor with which to ward off the attacks of the powerful molluscs and crustaceans of the Silurian seas; and of the armor plates thus obtained the relics are still retained in the cranial region, forming the dermal bones of the skull (frontals, parietals, squamosals, etc.).

Profound changes became necessary when our ancestors left the ocean and sought refuge in the marshes and upon land; changes not merely in the mode of respiration, but in the entire skeletal and muscular system, owing to the great difference in specific gravity between water and air. Differences in food caused modifications in the digestive system, and all surfaces exposed to the air developed glands in profusion to resist the drying effect of sun and wind. During this period were acquired pentadactylous extremities, lungs and larynx, and the salivary and lacrimal glands. The organism became modified in countless ways, as the attempts to inhabit dry lands, apart from the marshes, ushered in the next great period, that of the rocks and plains.

Here began a complete aërial respiration, the development of the permanent kidneys, which replaced the Wolffian bodies of amphibians, and the formation of a cornified epidermis, with 
its proliferations in the form of scales, horns and claws. The great increase in the size and strength of the limbs, begun in the previous period, reached here a high degree of perfection, and towards the end of this period vertebrates were for the first time enabled by the help of these to lift their bodies completely from the ground and exchange the crawling movements for a definite walk.

But the most important of all the changes produced by a land environment has been the rapid increase in the size and efficiency of the central nervous system, which became developed in part through the need of controlling the larger limb muscles, and in part in response to the far more varied environment afforded by the land surfaces and the consequent necessity of recording a larger number of sensory impressions. By a curious and indirect method this development, especially that of the perceptive centers of the brain, has been still more encouraged in a certain group of rather generalized mammals through the occupation of an arboreal environment. The direct result of this was, that in these animals, which were, in the main, large enough to grasp the boughs in climbing, a prehensile paw with an opposable first digit was developed on both anterior and posterior limbs, and this new tool, especially the anterior set, which became hands, from now on allowed the animals to grasp all sorts of objects, and expose them to a more careful scrutiny, thus causing a continually greater development of the recording centers of the brain.

As this arboreal environment has been the latest in the line of human history previous to the assumption of a strictly terrestrial life, there are still in man's body more evidences of this than of the earlier stages, but these, because they are the latest, are also the most superficial, and consist of such characters as the flattened nails, the pectoral position of the mammæ, and the opposable thumbs.

The latest change of all, the assumption of an erect position and the emancipation of the anterior limbs from all locomotive functions, has necessitated a few modifications, especially changes in the pelvic girdle and in the relative size and 
strength of the muscles of the legs, but has effected little in the way of actual change of structure, so that anatomically man still stands very near his arboreal kinsmen that represent the immediate past in the history of human development.

This study of the succession of forms upon the earth is termed race history or phylogenesis, and forms one of the two sources from which the past history of animal development may be obtained. The other is the sequence of stages recorded during the embryonic development of each individual, and is termed the developmental history or ontogenesis. By what is at once the most natural and the most mysterious law of nature each individual animal inherits, not only the structure of its immediate parents, the attainment of which means the end of its development, but also that of its entire line of ancestors, which appear in approximately the natural order of succession and constitute the stages of its ontogenetic development.

As a result of this it follows that the two records, phylogenetic and ontogenetic, run closely parallel, and each serves in many places to bridge a gap or explain an obscure period in the other. This parallelism of the two records lies at the basis of all morphological speculation, and forms what is often termed the law of biogenesis.* It must not be expected, however, that the correspondence in the two records is complete, since numerous disturbing causes must be taken into consideration which tend to modify each record quite independently of the other. In the race history there are many gaps caused by extinction, and the forms that have come down to us from earlier periods have become much changed from their former condition and represent their ancestors in a qualified sense only; while in the individual development there are many characters that are in no sense historic, and have to do with such immediate environmental problems as nutrition or protection. These latter characteristics, which

* The "Biogenetisches Grundgesetz" of Haeckel; formulated by him as follows: "Die Ontogenie (Keimesgeschichte) ist eine kurze Wiederholung der Phylogenie (Stammesgeschichte)." 
are called canogenetic, or modern, are clearly of no importance in such inquiries as the present, and must be carefully distinguished from those that are palingenetic, that is, actual repetitions of past history.

It is essential, then, in order to interpret correctly the two records, phylogenetic and ontogenetic, and from them to reproduce the past history of our race, with its solutions of the details of man's structure, that the nature of each form of record be thoroughly understood. The phylogenetic or racehistory is the plainer and more direct of the two, and presents fewer technical difficulties to the student, but it contains at present extensive gaps, not yet filled in by the discovery of fossil remains; the manuscript is plain and clear, but has suffered much from the ravages of time and is fraginentary at best : the ontogenetic, on the other hand, presents a more continuous story, but the difficulties in the way of investigation are very great; here the manuscript is written in a microscopic hand, and is, moreover, a palimpsest, scribbled over with extraneous material, added at late dates and connected with the exigencies of development.

The characteristics of the phylogenetic record may be made clear by the aid of the accompanying diagram [Fig. 6], which represents a purely hypothetical case, and the conditions involved may be presented in the form of laws, as follows:

I. Development has not been in a single direction, but in many, since the constant rivalry between allied forms causes them to continually push their way into new environments, the gradual adaptation to which causes a greater and greater divergence between the descendants of those that entered the new environment and those that remained in the old.

To illustrate this by the diagram, suppose 29 to represent a terrestrial carnivorous animal, living on the border of the ocean and preying upon the forms of life found upon the shore, or within shallow water. Pressed by the struggle for existence, in this instance represented by the scarcity of this sort of food, certain individuals venture farther out into deeper water and attempt to capture fish. Thus begins the 
establishment of a group which becomes more and more aquatic, as represented by the divergent line leading to 34 , until finally a completely aquatic fish-eating animal or group of animals is the result, the form at the end of the line, 34, representing the highest point of specialization attained.

The remaining descendants of 29 , continuing to live in precisely the same habitat as their ancestors, as is here indicated

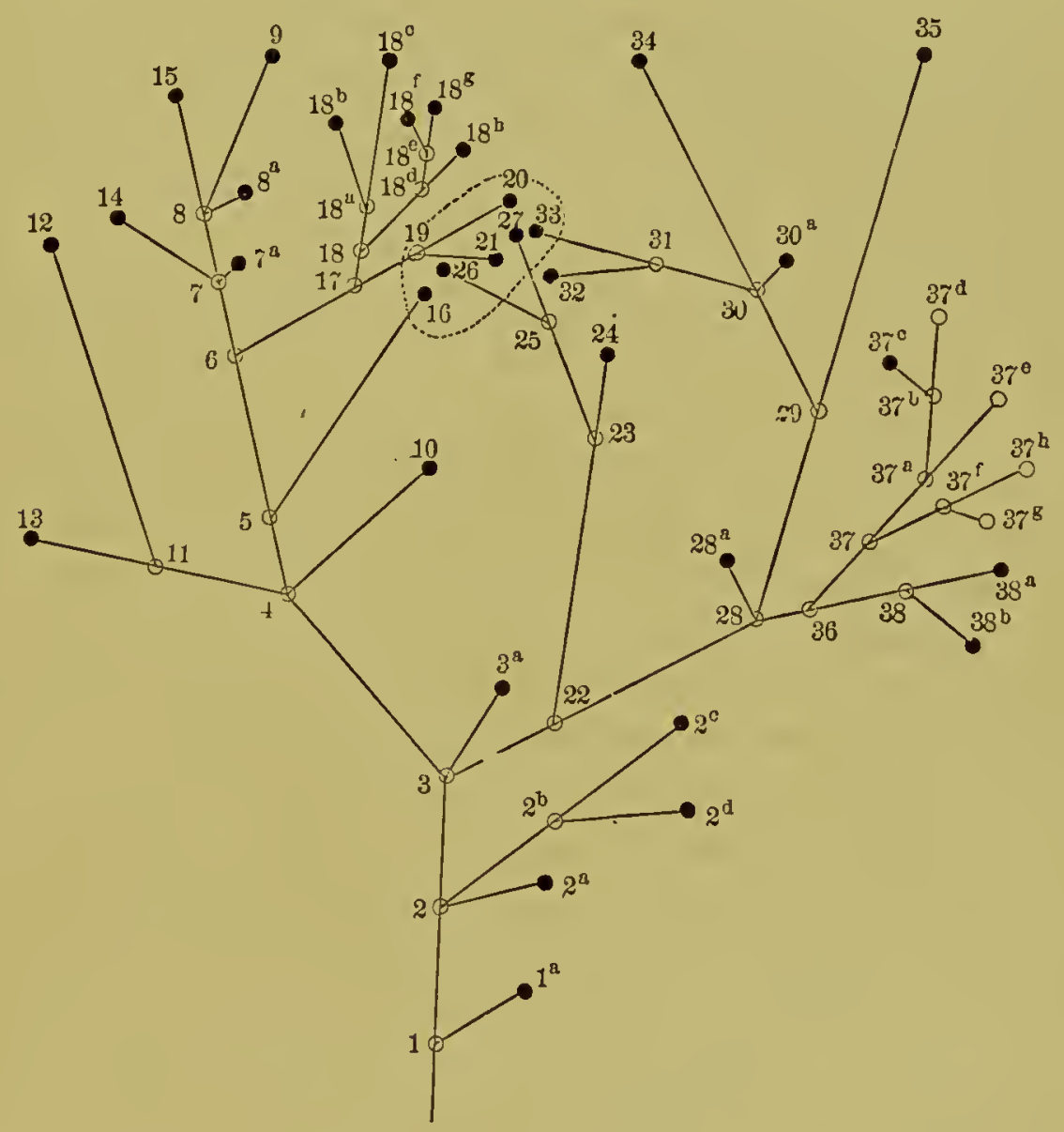

Fig. 6. Hypothetical tree illustrating the interrelations of organisms.

Extinct forms are represented by open circles, living forms by solid black ones. The same number distinguished by exponent letters significs a close relationship. The dotted areas suggest some special environment, the inhabitants of which show "adaptive resemblance" although representing several unrelated lines.

by the continuance of the line 29-35, in the same direction as 28-29, either remain exactly as their ancestors, or probably become more highly specialized in the same direction. Con- 
tinual divergencies on the part of animals, as they seek new environments in this way, produce the numerous divergent branches, the relative time of the divergence being expressed by the position of the intersection and the amount of the modification by the length of the line.

II. Although animal forms are not related to one another as members of a single linear series, they yet form a continuum, and any two living forms, however great the structural difference between them, are connected to one another by a continuous chain of animals, a connection which will become apparent by tracing the lines backwarls along the ancestral course of each until they meet at their earliest common ancestor.

Thus, in tracing the relationships of 9 and $\mathrm{I} 4$, neither form is ancestral to the other, but both arose from the common ancestor 7 , back of which their history is identical. As the ancestral forms are now wholly extinct, they are no longer available for study save when found in the fossil state, but their place may often be supplied by modern forms which are but little modified from the condition of the actual ancestors. Thus the recent forms $8 \mathrm{a}$ and $7 \mathrm{a}$ are almost as useful in reproducing this part of the phylogenetic history as 8 and 7 would be, and through them the inter-relationship of 9 and I4 may be readily traced. This may be stated as a third law:

III. Although the actual ancestral forms lying at the forking of the branches no longer exist and have seldom been found in a fossil state, many clerws of their structure may be obtained by the study of those of their descendants which have retained most completely the ancestral environment, and which have, therefore, kept many or most of the ancestral characteristics.

Thus in studying the relationships and comparing the structure of two such divergent forms as 32 and 34 , the living form $30 a$ would be of the greatest assistance, as it would enable the investigator to see what was the common structural heritage from which, through two lines of modification, the two forms in question have developed.

IV. Among the fossil remains of extinct forms rohich geo- 
logical investigation has unearthed, many forms have been found which are the actual ancestors of groups now distinct, and they have thus been of the greatest value in tracing out phylogenetic relationships. Others, however, represent a series of forms which developed, culminated and became extinct before modern times, thus presenting a group of great value to the student, but having no bearing upon the present discussion.

Perhaps the most famous of the ancestral forms found in a fossil state is the Archoopteryx, a definite transition between reptiles and birds. Of this, two specimens were discovered in the lithographic slate quarry at Solenhofen, Germany. Others, of almost equal importance, have assisted greatly in suggesting the relationship between amphibians and reptiles, and have furnished clews to the proper arrangement of the orders of living mammals. As illustrations of large groups of animals whose history lies wholly in the past may be mentioned the trilobites, a group of crustacean-like articulates, which became wholly extinct at the end of the Palæozoic Age, and the ammonites, a group of cephalopod molluscs.

$\mathrm{V}$. The relative amount of structural difference between any two divergent forms is proportionate to the amount of contrast between their environments, and not necessarily to the amount of time that has elapsed since their divergence from the common ancestor.

That time has in itself no power to modify an animal species is shown by the slight differences that exist in some cases between certain living forms and their fossil allies. Perhaps the most conspicuous example of this is the brachiopod, Lingula, a worm enclosed in a bivalve shell. This form has existed from the earliest Silurian times to the present day, and yet there are hardly sufficient differences between the earliest fossil Lingulce and those now alive to allow them to be treated as distinct species. As a rule, however, successive geological periods show almost a complete change in their fauna and flora, and most of the modern forms are quite recent in origin.

The persistence of ancestral types in a slightly modified 
condition is indicated in the diagram by such forms as $3 a$ or $30 a$ where the shortness of the line connecting the living form with its ancestor indicates but little change from the earlier condition.

VI. As a given environment tends to exert a similar infuence upon all of its occupants, members of quite distantly related groups which become associated in the same environment often become so similarly infuenced as to bear, superficially, at least, a great resemblance to one another. This is called "analogical resemblance" and has been productive of many mistakes in the attempt to clear up phylogenetic relationships.

Many striking examples of this are found among vertebrates. Thus a pelagic environment, as seen among the extinct ichthyosaurs and the modern Cetacea, has changed the fore-limbs into fin-like paddles, reduced the hind-limbs to functionless rudiments, shortened the neck, and given head and body a piscine form; limbless, attenuated forms occur among fishes, amphibians and several groups of reptiles other than snakes; and a grazing habit produced in the herbivorous reptilian group of the dinosaurs a close resemblance to the large ungulate mammals of a later day.

This law is illustrated in the diagram by the forms included by the dotted line, which represents a given environment, invaded by members of several groups. Here the descendants, not only of related forms like 5 and 6 , but those of quite distant ancestors, as 6 and 30 , have become similarly modified, until they may resemble one another so closely as to deceive the casual observer. Forms 20 and 33, representing totally distinct stocks, may thus bear so close a superficial resemblance as to be popularly classed together under the same general term.*

* Thus whales and porpoises are vulgarly supposed to be fishes; shrewmoles, mice; and bats, birds. Salamanders are usually confused with lizards; and certain blind and limbless lizards (Rhineura) which occur in Florida and burrow in the earth, so closely resemble earth-worms as to deceive at first glance a professional naturalist. 
In the above exposition of phylogenesis there can be seen at once both its advantages and its disadvantages as an historical record.

In cases in which a line of descent is well represented by a series of adult animals, the advantage of being able to study large forms with functional parts is obvious; but where the extinction of intermediate forms has obliterated the record at some important point, the phylogenetic data fail completely and must be supplied by the parallel history found in the individual development of the nearest allied forms. The greatest assistance has often been furnished by palæontology, but as the hard parts alone leave their imprint in the rocks, they are of little or no assistance in the history of many of the systems. Again, through the metamorphosis of the earlier geological formations and the consequent obliteration of all organic remains occurring in them, the palæontological record has lost beyond hope of recall all of its early stages, and at the period of the first fossiliferous strata, the main classes of animals as we have them at present, had already become established.

It is here that the study of comparative embryology lends its assistance, since in the embryological record the earliest stages are preserved, although often overlaid with secondary modifications. By its aid may be traced, not only the lines connecting any two forms (Rule II. above), but it furnishes faint though definite clews to the early history of animal development previous to the beginning of the palæontological record. Its defects, though many, are not the same as those of the phylogenetic record, and the two thus reinforce one another to a remarkable degree, each completing the gaps left in the other, and corresponding closely in those places in which both records are preserved.

The exposition of developmental history, or ontogenesis, may be given in the form of laws as in the former case.

I. The developmental history of an animal includes all stages from that of the fertilized egg (ovum) to that of the sexually mature adult, and is not in any way interrupted by 
the act of birth or hatching. These latter are purcly external phenomena and mark no important stage in the development of the animal save in the line of certain necessary adaptations. The birth period often varies considerably in allied forms.

These external phenomena are wholly adaptive and are regulated by the conditions imposed by the struggle for existence. Thus aquatic salamanders lay eggs which pass through all the stages from the beginning outside of the body of the parent, but in the more terrestrial species, although closely allied to the foregoing, the eggs are detained in the oviducts of the mother, where development continues throughout the larval period and the young are produced in a practically adult condition.

It is advantageous to some species to produce a large number of immature offspring, relying upon chance for the survival of a few of them; under other circumstances it has been proven the better course to produce a small number of welldeveloped-young, furnished with a better equipment for fighting the battle of life.

II. In developmental history a given species reproduces in miniature its ozm ancestral history, and thus passes through those stages only through which its actual ancestors have also passed.

Thus, in the diagram, form $I 8 b$ has passed through the stages I $8 a$, I $8, I 7,6,5,4,3,2$, and I as well as the innumerable stages between these points as represented by the lines connecting them, but would not reproduce any stage in the history of some allied form through which the latter has passed since the divergence, such as 7 or I9.

The only stages common to any two recent forms, allied or not, are those below the point represented by their latest common ancestor. This may be formulated as follows:

III. In any two given forms only those developmental stages which represent common ancestors are the same in both. From the point at which their ancestors diverged their developmental histories are distinct and differcht. It follow's from this that the more closcly allied the two forms, the mor' 
completely will their cmbryonic development coincide, and converscly, in forms widely apart the divergence bcgins very early and only the first of the two developmental histories will be coincident.

To illustrate those points: if the development of 16 and 34 be compared, only the early stages $\mathrm{I}, 2$ and 3 will be seen to coincide; if, however, the developmental histories of $2 \mathrm{I}$ and I 6 be taken, they will be found coincident as far as their last common ancestor, 5. In closely allied forms, such as $37 f$ and $37 \mathrm{~g}$, almost the entire embryological history in the two animals will closely correspond, differences being noted only at the last.

IV. The more highly specialized the animal, the more changes its ancestors have passed through; and therefore so much the more is to be recapitulated ontogenetically. This is effected in part by lcngthening the embryonic period and in part by sliding over or dropping out some of the stages.

In the fish, for example, after the development of a simple circulation designed for a water-breathing vertebrate, there is nothing farther to do than to perfect and to mature it as it is ; in the mammal, however, the circulatory system, which is at first like that of the embryonic fish, must become successively modified as amphibian, reptilian, and finally mammalian; a much longer history, which involves numerous changes and adaptations.

$V$. The different historic stages are not given the same time value, but the earlier the stage, the more it is accelerated. The earlier stages also lose in distinctness and detail and are more often lost than the later ones. It follows from this that the early part of the history is best learned from the lower forms, in arhich the stages sought are not very remote from
the adult condition.

The approximate time values of the developmental stages are seen in the development of the hen's egg; the segmentation stages, and the formation of blastula and gastrula, which represent all the earlier invertebrate portion of the history, are passed through in a few hours; the establishment of the mesodermic somites (myomeres), which makes it a vertebrate, is 
well marked by the end of the second day; at the age of four days the embryo is sauropsidan, at five or six definitely avian, and the remaining fifteen days are spent in perfecting the details first of a gallinaceous bird, and lastly of the particular species to which it belongs. Furthermore, the remainder of the history, until the adult stage is reached, that is, the latest historical period, requires many months. The value of the study of the more primitive forms is well seen by the formation of the mesoderm, and especially that part of it which give rise to the myomeres or primitive muscle segments. In Amphioxus, a form considerably below the fishes, the mesoderm arises from the primordial intestine in the form of paired diverticula, from the dorsal part of which the myomeres arise; in fishes and amphibians these elements are not distinct diverticula, but still possess cavities or the rudiments of them; and in birds and mammals the myomeres arise as solid cubes cut from an indifferent cell mass, and give absolutely no clew to their early history.

VI. In studying an embryological record one must constantly distinguish between palingenetic characters, or those which are true repetitions of the past history, and cenogenetic characters, or those which have been more recently acquired as the result of some special adaptation. One of the most universal among these latter is the presence of yolk, a food supply for the embryo, which lies between or within the cells and, when excessive, causes misleading distortions in the proportion of parts and effects the obliteration of many important features.

In general the actual size of an egg is due to the amount of yolk it contains, and thus the historic records are reproduced with greater faithfulness in very small ones. This is well shown by the comparison of the almost yolkless egg of $\mathrm{Am}$ phioxus with that of the bircl, which represents the other extreme. In the one the cylindrical form of the primitive vertebrate is well preserved and appears almost at the beginning; in the other the dorsal portion of the future body lies for a time almost flat on the surface of an enormous sphere of yolk, and is enabled later to assume the cylindrical form 
only through a secondary adaptation by which the embryonic and vitelline (yolk) portions of the egg become nearly separated from one another, the connection being retained through a narrow stalk.

It will be seen by the above exposition of the two historical records, phylogenetic and ontogenetic, that they are by no means complete and that the fragments that exist are often difficult to interpret. This has necessarily occasioned a large amount of controversy among morphologists, not alone in the interpretation of the facts, but even in some cases in the recognition of the facts themselves, owing to the great mechanical difficulties in the way of their examination. As in all earnest investigation, however, the differences grow less as the work progresses, and at the present time there is a practical agreement upon the main features of vertebrate history, the differences being confined mainly to details. In some cases in the following chapters attempts have been made to set forth divergent views, but, for the most part, both for the sake of clearness and in order to present the matter within suitable limits, the selection has been made of that theory which, in the judgment of the writer, possesses the greatest probability.

The significance of an anatomical fact depends upon the phylogenetic position of the animal studied, yet at the same time it must be remembered that the only criterion we possess for making the phylogenetic arrangement is that of the anatomical structure, so that the two lines of investigation are mutually dependent and are likely to become equally modified by the presentation of each new fact. As a basis for this history of the human body, which is at the same time a history of vertebrates, especially of those that lie in the direct line of human ancestry, it is thus necessary to consider the various vertebrate'groups, both living and extinct, so far as we know them, and study their mutual relationships as deduced from their structure and development. This is, in fact, a bricf study of vertebrate phylogenesis, and will be considered in the next chapter. 


\title{
CHAPTER II
}

\section{THE PHYLOGENESIS OF VERTEBRATES*}

\begin{abstract}
"The Epicureans, according to whom animals had no creation, doe suppose that by mutation of one into another, they were first made; for they are the substantial part of the world; like as Anaxagoras and Euripides affirme in these tearmes: nothing dieth, but in changing as they doe one for another they show sundry formes."

Plutarch's Morals; transl. by Philemon Holland, 1603, p. 846.
\end{abstract}

Although no great subdivision of animals, with the possible exception of the echinoderms (star-fish, sea-urchins, etc.), possesses a more isolated position than do the vertebrates, this latter group is connected in an obscure way with the invertebrate world through a series of animal forms of uncertain position themselves and usually grouped together under the name of Prevertebrata or Protochordata. These comprise a worm-like form, Balanoglossus, that burrows in the mud along the sea-coasts, the sac-like tunicates, and the small and slender Amphioxus. Formerly classed at great distances from one another among molluscs, worms and even plants ( $e$. g., sessile tunicates), they are now united, owing to the common possession of pharyngeal gill-slits, a dorsal nervous system, and an internal skeletal rod, the notochord, although in some cases these two latter characteristics are transitory structures that appear only during the early steps of development.

The highest of these animals, and consequently the one nearest the true vertebrates, is Amphioxus, a small marine creature something like a headless fish, which is found in the

* For a detailed classification of vertebrates, to accompany this chapter, the reader is referred to the Appendix 
shore water of the warmer seas, usually buried in the sand in a perpendicular position, with the anterior end projecting into the water, expanded into a sort of hood for the collection of its food. When fully grown it is about two inches in length and is in the form of a cylinder, flattened laterally; and pointed at either end. It is divided into a succession of body segments, somites, by $\mathrm{V}$-shaped lines, which represent the edges of the partitions of connective tissue, the myocommata. These run through the masses of body muscles, and divide them into segmental portions, the myomeres. The internal skeletal axis, which forms one of the chief characteristics of the group of vertebrates, is here represented by a flexible cylindrical rod of a substance resembling cartilage, running through the body from tip to tip. This rod, the notochord, shows no trace of segmentation, and it is thus seen, as is also the case in all vertebrate embryos, that the segmentation so fundamentally characteristic of vertebrates, and so well marked in their internal skeleton (vertebræ, ribs, etc.), was acquired first by the muscular system, perhaps as an adaptation to facilitate the flexibility of the body, and that it was secondarily carried over to the skeleton.

In arranging a phylogenetic tree of the vertebrates, Amphioxus should be placed at the bottom, although, if absolute accuracy is demanded, neither Amphioxus nor any modern animal, with its later modifications, should be placed at any point along the main stems of the phylogenetic tree, but all should be placed at the termini of branches; proximity to the ancestral line being indicated by the shortness of the branch.

If, however, later modifications, since they have undoubtedly affected all modern forms to a greater or less extent, may be left out of account, and if the successive animal forms may be placed in the positions occupied by their direct ancestors, we may thus form a phylogenetic tree like the one given here, which expresses the relationships of modern forms to one another in a simple and essentially correct manner.

Above Amphioxus ensues a great gap, the greatest in the entire series, bridged over by no forms, either living or fos- 
sil, with which we are acquainted, and only suggested in part by the members of the next higher group, the cyclostomes. This group comprises eel-like forms, to be carefully distinguished, however, from true eels or from any of the true vertebrates,

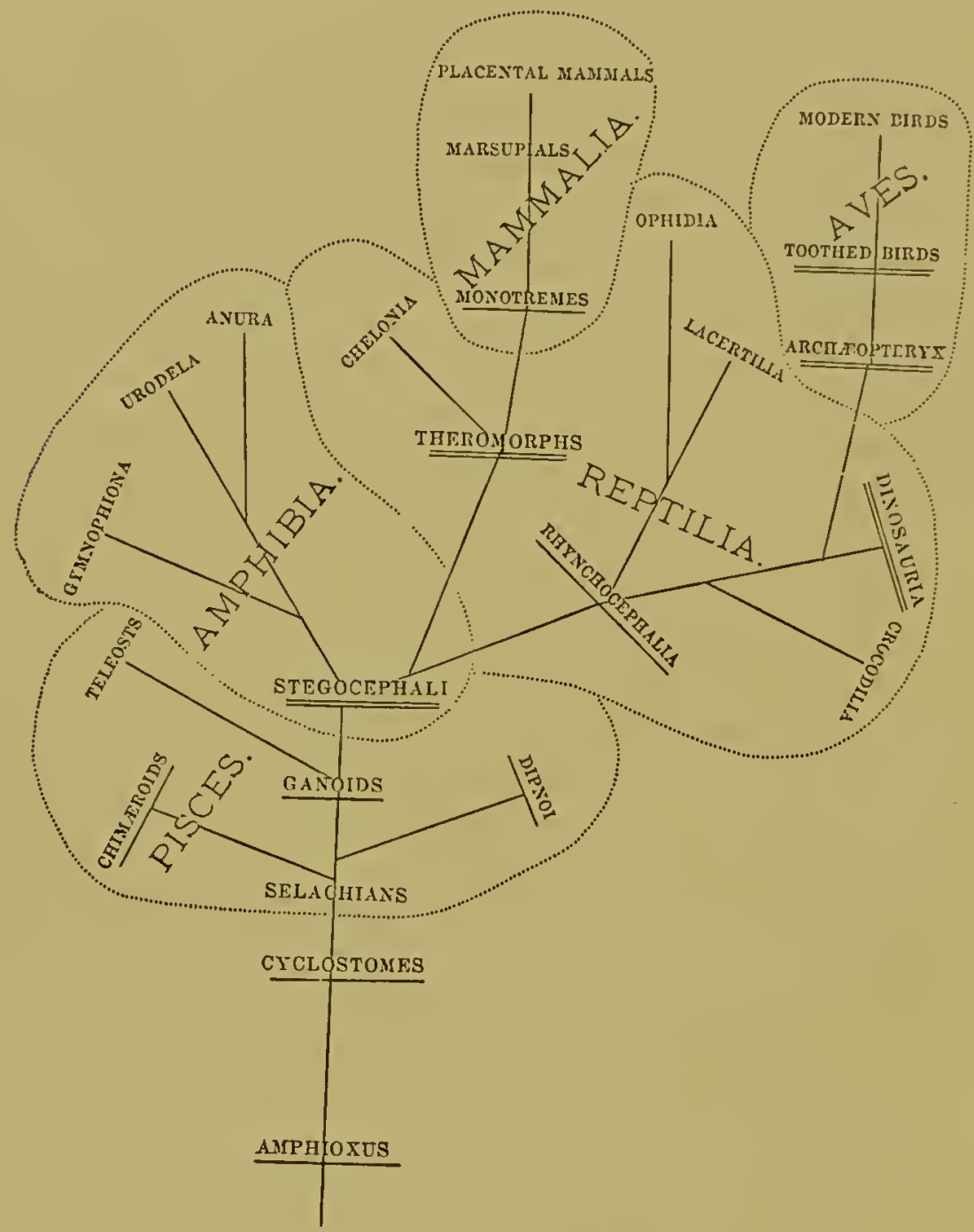

FIG. 7. Phylogenetic tree of vertebrates.

Double underscoring indicates an extinct group; single underscoring one that has but a few living representatives. The boundaries of the Classes are represented by dotted lines.

since they possess neither jaws nor teeth in the sense of those of the higher vertebrates, but have the mouth surrounded by a circular lip which is capable of being extended so as to remind one of the hood possessed by Amphioxus. Within this mouth there are variously shaped spines or plates which serve 
as teeth. A most important distinction between Amphioxus and the cyclostomes, however, lies in the fact that the latter possess a definite head, with brain and sense organs, parts which exist only in a rudimentary or potential sense in $\mathrm{Am}$ phiorus.

In distinction from the cyclostomes, or "round-mouths," are the true vertebrates, which are termed gnathostomes, or "jaw-mouths," the possession of jaws being a constant characteristic of the entire group. The lowest class of gnathostomes is that of the fishes (Pisces), but these are in turn subdivided into several groups, some of which represent lateral branches, that is, specializations along definite directions, and thus not in the direct line of human history. The most primitive group is that of selachians, which comprises the sharks and dog-fish, and the skates or rays. This group of animals is absolutely fundamental for the morphologist and represents the first great stage in the main line of vertebrate history. Selachians have a wholly cartilaginous skeleton, the mouth upon the lower side of the head and not at the anterior end, as in other fish, and five gill-slits which open separately and free, not covered by an operculum (gill-flap). Their position in the tree is clearly in the main line above the cyclostomes.

The ganoid fishes are also of great importance to us. They represent a few remnants of what was the dominant group during the Devonian epoch and are the direct descendants of the selachians. As in the case of all such remnants, they are extremely diverse in structure among themselves and are placed in a single group rather more for convenience than because of a very close relationship to one another. They are characterized by the tendency of the scales to fuse into bony plates, a tendency which in the past resulted in the development of a special group, the placoderms, which were entirely covered by a suit of mail formed in this way. Similar plates cover the head in all modern ganoids and they occur in rows along the body in a few forms (sturgeons). The skeleton is mainly cartilaginous in 
the lower representatives of this group, but becomes more or less bony in the higher. The gill-slits no longer open directly and separately to the outside, as in their selachian ancestors, but are grouped together and covered by a gill-flap or opercullum.

The two remaining groups of fishes, teleosts and dipnoans, represent independent lateral branches that have specialized in accordance with certain definite lines and are consequently not in the direct line of man's ancestry. Such groups often form collateral testimony of considerable morphological value and are thus not without importance even in the present line of speculation. The teleosts have an almost completely ossified skeleton and are the descendants of the bony ganoids, with which they are so closely connected through intermediate forms that the separation between them is mainly an artificial one.* They are essentially a modern group and constitute the great majority of the fishes in the world to-day, thus taking the place of the ganoids of earlier times. The dipnoi are represented by but three forms, one found in Africa, one in Australia and one in South America. They are fresh-water fishes and are remarkable for their power of sustaining long periods of drought by digging into the mud, and breathing air through a modified air-bladder. They were thus formerly considered the link between fishes and amphibians, but later researches into their structure do not confirm this view.

As a matter of fact the amphibians seem to have come from the ganoids, although by means of forms now lost, and to have developed first into the Stegocephali, a group wholly extinct but well represented by fossil remains occurring in and about the coal deposits. These had many of the characteristics of our modern amphibians, but possessed scales arranged in definite rows, organs which are entirely lacking in all living representatives of this Class, with the exception of the

* Although the employment of the two terms "ganoid" and "teleost" is a convenient one in comparative anatomy, modern ichthyologists tend strongly to the rejection of both terms and the fusion of the two groups into a single one, the Teleostomi. Cf. Appendix. 
Gymnophiona, which still possess scale rudiments, not visible externally. The Stegocephali are of extreme importance, since they were the ancestors both of the present-day amphibians and of the two main reptilian lines, and the survival of a single representative would have been of priceless value to morphologists. As it is, however, we are in possession of a large number of fossil remains, many of them extremely well preserved, and representing four distinct orders; and further discovery along this line may well be expected at any time. Of the soft parts the fossil imprints furnish but little evidence, a lack which must be supplied by the study of the urodeles, undoubtedly their nearest living allies and presumably not very different in the essential internal features.

These latter animals, though not quite in the direct line of human ancestry, are thus of the greatest importance as the best representatives of what may be called the amphibian stage. The urodeles comprise the tailed amphibians, their most typical representatives being the forms known as salamanders and newts, also in many sections, unfortunately, "lizards," owing to their superficial resemblance to these latter animals. The more primitive members of this group are often large (IO-40 cm.), and the giant Cryptobranchus of Japan, the largest of all living amphibians, attains the length of a meter.

The Anura, or tailless amphibians, include frogs, toads and tree-toads, and attain their tailless condition in part by a retrogressive development of the caudal region and in part through the excessive development of the ilia and the thigh muscles, a feature connected with their jumping habits. The Gymnophiona are blind subterranean forms, burrowing in the earth like earth-worms, to which they bear considerable resemblance. They are mitch attenuated, are without external limbs, and have their bodies clearly marked off into annular segments. They occur only in the warmer parts of the world and consist of but few forms.

Arising also from the Stegocephali come the reptiles, which have apparently developed along two lines, the one leading to 
the birds, the other to the mammals. Of the first of these, the oldest group is that of the Rhynchocephalia, mainly fossils, but with a single living species, which fate has preserved in New Zealand, the Sphenodon (Hatteria). This represents the ancestor of lizards and snakes, Lacertilia and Ophidia respectively, and also a group of extinct reptilian giants, the dinosaurs, whose nearest living allies are the crocodiles. Here this line would have ended, so far as human knowledge is concerned, had it not been for the chance discovery, about the middle of the nineteenth century, of two specimens of one of the most remarkable "missing links" ever found, the Archcopteryx, a form midway between reptiles and birds, and of undoubted affinity to the stem of the dinosaurs. This creature was bird-like, possessed wings and a certain number of contour feathers, but had a long vertebrated tail, several free digits in the hand, furnished with curving claws, and a heavy jaw containing conical teeth, reptilian in character. This discovery, followed by that of the toothed birds, completed the chain of evidence, and supplied one of the most isolated groups of vertebrates with a definite line of ancestry.

The other line of reptiles, which may have arisen from the Stegocephali more or less independently of the first, was that beginning with the theromorphs, an extinct group, many of which attained a gigantic size. Some members of this group are so near the mammals in many particulars that it has been only with the greatest care, and through the consideration of all the available parts, that their reptilian nature has been determined. In studying the remains of these forms, especially those of the sub-group of theriodonts, the most of which were small animals, like the earliest mammals, it seems impossible not to assign them a close relationship to the latter, probably that of actual ancestry. Indeed, there is at present but one other claimant for that position, and that is the group of Stegocephali, and as these were contemporary with the theromorphs, and at one time probably graded into them by imperceptible transitions, the two views are not very wide apart. All things considered, it seems that the gap between 
Stegocephali and the mammals requires some intermediate link, and thus the addition of the theromorphs in this place seems rather a completion than an opposition to the theory of Stegocephalan ancestry.

The only living reptiles associated with the same branch as the theromorphs are the turtles (Chelonia), which, although highly specialized in the matter of trunk skeleton, are of the greatest value in regard to their soft parts, which are undoubtedly similar to those of the extinct members of the branch, and are thus the best living representatives of the important stage between amphibians and the early mammals.

The earliest mammalian remains are contemporary with those of the theromorphs, and are those of small forms, like the most mammalian of the reptilian remains. These are apparently nearly related to the monotremes, the lowest living mammals, which are represented by two forms occurring in Australia and New Zealand, the Duck-bill Platypus (Ornithorhynchus) and the spiny ant-eater (Echidna). The latter has no connection with the true ant-eaters (Myrmecophagida) of South America, which are placental mammals. The monotremes are strongly reptilian in certain skeletal features; like true reptiles and unlike all other mammals, they possess a single terminal orifice, that of a common cloaca, into which open the alimentary canal, the ureters and the genital ducts; and they actually lay eggs, that is, very immature embryos, surrounded by a thin, cornified shell. The mammary glands, one of the essential characteristics of the class of mammals, are seen here in a very simple condition. They consist of two lateral groups of integumental glands, apparently of the tubular type, which open separately in the bottom of an oval depression, the mammary pocket. There are no teats, and the young obtain the secretion either directly from the depressions or by sucking at the hair in this region.

The next group above the monotremes are the marsupials, with the exception of the opossum also confined to the Australian region. As in the previous group, the young are born 
in an immature state, but are unprotected by an egg-shell, and are matured in an external abdominal pouch (marsupium) until able to care for themselves." The relation between the monotremes and the modern marsupials is hardly close enough to justify an immediate succession, but suggests that each group, as we now know it, has descended from more primitive ancestors that were thus related; that is, that the ancestor of modern marsupials was a direct descendant of the ancestor of the monotremes.

Beyond the marsupials all the mammals are placental, that is, the embryos are retained for a longer time within the uterus of the parent and are nourished by means of an organ formed in part from the mucous membrane of the uterus and in part from tissue furnished by the embryo but not included within its body. This organ is termed the placenta and is connected with the body of the embryo through an umbilical cord. This cord contains fetal blood vessels which connect proximally with the main circulatory system of the embryo and develop distally into a system of capillaries that lie in villi in the embryonal portion of the placenta, obtaining their nourishment and effecting the interchange of respiratory gases through osmotic transmission. There is thus no direct organic continuity between mother and offspring, and neither nerves nor blood vessels are continuous from one to the other. Indeed, in the lower placental mammals the connection between the maternal and embryonal portions of the placenta is very loose and the two easily separate at birth, although in the higher forms the connection becomes more intimate and the separation takes place between the muscular and mucous coat of the uterus, thus involving an actual loss of maternal tissue.

The placental mammals, although their appearance was comparatively recent, geologically speaking, have specialized in all directions, and now occupy almost every available environment, not only of the land, but of the water. Some are fitted to pursue and drag down large herbivorous animals, while others feast upon dead bodies or suck the blood of the living after 
the manner of parasites. Many are specially adapted to the capture of insects, either on or beneath the surface of the ground, or on trees, and some have even developed the power of flight by which they may follow their prey through the air. The hosts of the vegetable feeders are as highly differentiated and become specially adapted to feed either upon low herbage or the leaves of trees, roots, bark or fruits, and have even developed one group of oceanic forms, fitted to browse upon the sea-weeds and other submerged vegetation.

These various lines of specialization, together with the usual extinction of intermediate forms, have produced a series of more or less isolated groups, or Orders, the interrelationships of which have been deciphered in part by the labors of anatomists, in part by those of palæontologists, but are still more or less uncertain. A suggestion of this is shown in the accompanying phylogenetic tree of mammals (Fig. 8.), which takes into consideration both living and extinct groups, so far as known.

The earliest mammalian forms, of which we possess only fragmentary remains, were more like the reptiles, and especially the theromorphs, than any now extant, but possessed many of the characters of the monotremes, which may be considered their somewhat highly specialized descendants. To this group has been given the name Pantotheria, and as the ancestors of all the rest they may form the main trunk of the phylogenetic tree. The monotremes are the nearest living descendants, and they have been derived from them through an ancient and closely related group, the Multituberculata, All three of these groups were reptilian in structure, and may be classed together and in contrast to all the other mammals, as the Sub-class Prototheria.

While still primitive, however, the Pantotheria began to differentiate along two lines, the one somewhat resembling the marsupials, the other the insectivores, and thus early these two lines of development became inaugurated. Eventually the reptilian characters were dropped, and the animals, passing over into the Sub-class Eutheria, or typical mammals, be- 
came respectively the Didelphia, or marsupials, and the Monodelphia, or placentals. The first of these lines then differ-

PROTOTHERIA

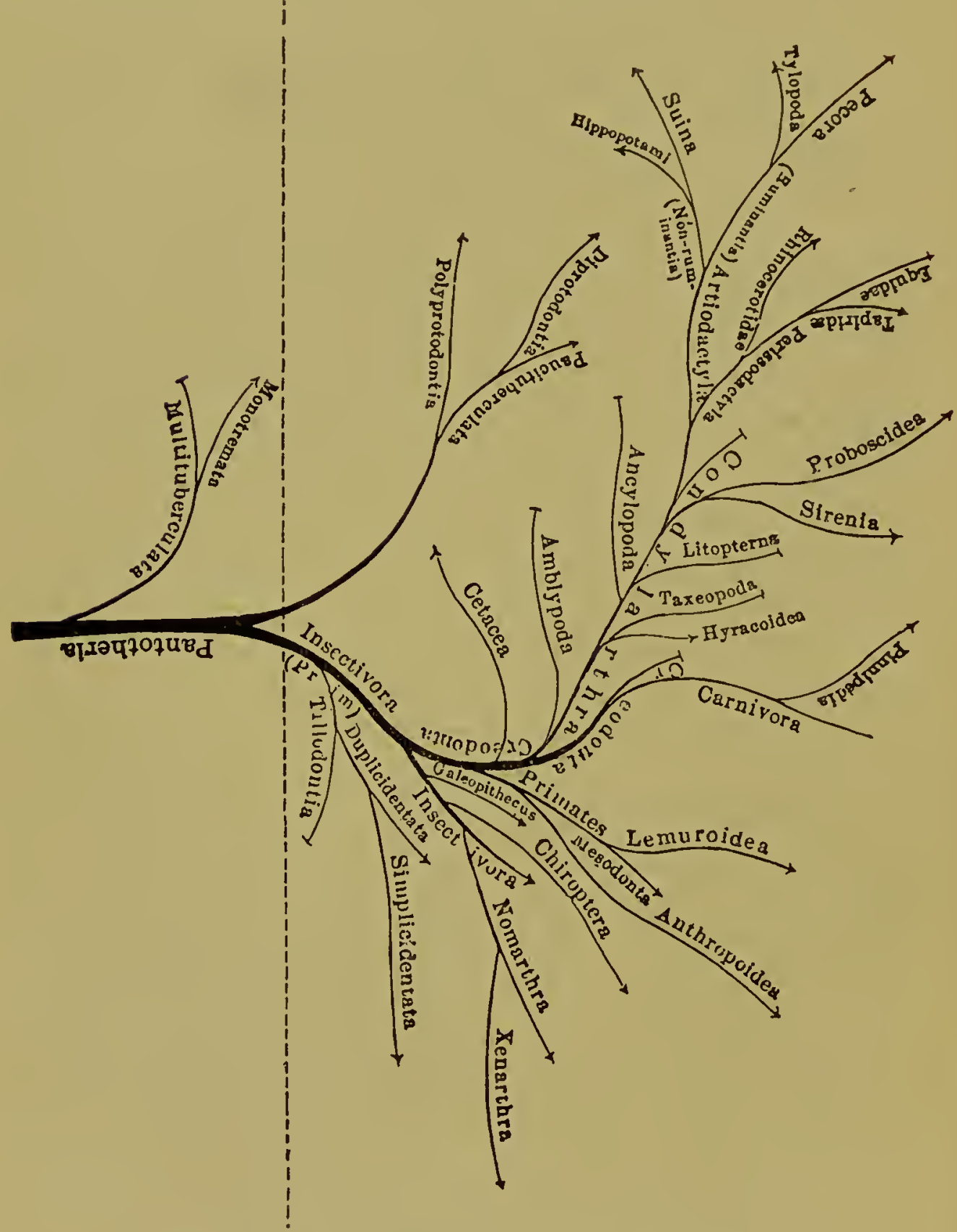

FIG. 8. Phylogenetic tree of mammals.

A branch that terminates in an arrow point still possesses living representatives, one that ends in a short cross bar is extinct.

entiated into the marsupialian Orders of the present time, distinguished mainly by variation in the dentition, and the second, 
which resembled the present-day Insectivora, passed over into that Order.

This insectivorous stem, in addition to perfecting its own type along the narrow lines first laid down, developed several lines of differentiation, and it was from these that all the higher placental mammals have arisen. A very primitive stem is that of the Rodentia, of which the extinct group of Tillodontia may have been the first; succeeded by the $D u$ plicidentata or gnawing animals, like the rabbits, in which, back of the two sharp upper incisors, there is a second reduced pair, and later by the Simplicidentata, like squirrels, rats, mice, and beavers, in which the upper incisors consist of a single pair.

The branch represented here as immediately above the last, suggesting a little less primitive character, is that leading to the group usually called the Edentata, and consisting of the sloths, armadilloes, ant-eaters, besides several extinct forms, such as the Megatherium, Megalonyx, and Glyptodon, the first two like the sloths, the last like an armadillo. In the more specialized of these there is a peculiar joint between two of the vertebræ of the back, and they are called the Xenarthra in contrast to those in which this joint is normal, the Nomarthra. This group has always been exclusively American, the living forms mainly South American.

From this same generalized group, the Insectivora, there have developed two distinct lines of flying or soaring forms, the Chiroptera or bats, and the Galeopithecus, a single species found in Madagascar, but not nearly related to any of the other stems.

By far the most prolific of the stems proceeding from the Insectivora is that which started with the extinct group of Creodonta: These animals were at first small, generalized mammais, scarcely distinguishable from the parent insectivores, but they gradually took on special characters which suggest the modern Carnivora, which are considered their direct descendants. Before specializing along this line, however, some of them began to differentiate in several other di- 
rections and thus gave origin to the Primates, the Condylarthra, a generalized form of ungulate, and probably a line of aquatic carnivorous forms, destined to become the most erratic and singular of all mammals, the Cetacea, or whales and porpoises. These earliest ancestors of divergent lines were very much alike, and the early primate, carnivorous, and hoofed forms, were all very generalized, and without the differential characteristics that their descendants later developed. The most primitive of the Primates were a group called the Mesodonta, of which the modern lemurs are the most direct descendants. Very early, however, forms like the modern monkeys, Anthropoidea, began to make their appearance, forms in which the orbit was entirely separated from the temporal fossa, and in which the dentition was the same as in the monkeys of the Old World and in Man; and in these we find the direct human ancestors.

The creodont stem developed, as stated above, the modern Carnivora, including the cats, dogs, bears, and weasels, and from this, at an early date, there probably arose a carnivorous line that adapted itself to the sea. This is the Pimmipedia, or those with fin-like feet, the seals, the walrus, sea-lion, etc.

The remaining stem, that of the Condylarthra, was perhaps the most prolific of all in respect to the amount of variation, and the extent of modification, for it has produced the Sirenia, aquatic forms, nearly as highly specialized as the whale; the Proboscidia, or elephants, with an excessive modification of the nose; and an enormous variety of animals with a reduction of toes, the series reaching its absolute limit in the horse, which has lost all the digits but one, this becoming greatly strengthened to serve the purpose of an entire foot.

The original Condylarthra have long been extinct, as well as the earlier derivatives, the Amblypoda, Ancylopoda, Taxeopoda, and Litopterna; but, fortunately, of all these there is left a single solitary Genus, Procavia or Hyrax, for which the Order Hyracoidea has been made. This is a little animal of about the size of a rabbit; the one referred to in the King 
James Bible as the "coney." It frequents Syria and the adjoining countries, and a related species is found in South Africa; the sole survivors of the early ungulates.

The modern ungulate forms, aside from Hyrax, may be represented by two stems, the one leading to the Proboscidea and Sirenia, the other branching immediately into the Perissodactyla, with an odd number of functional digits, and the Artiodactyla, with an even number. The Proboscidea include the two species of living elephants, besides several extinct ones, like the mammuth, the mastodon, and the dinotherium, and the Sirenia consist of two living genera of unwieldly aquatic herbivores, the manatee or sea-cow, and the dugong, which subsist on sea-weeds and consequently do not wander far from the coasts. The Perissodactyla include the three lines represented by the tapir, the rhinoceros, and the horse; and the Artiodactyla embrace the non-ruminant pigs and hippopotami, and the almost numberless species of ruminants, such as cattle, sheep, antelopes, and deer. Of these perhaps the most distinct are the giraffes, and the Tylopoda, or camels.

In reviewing the two phylogenetic trees as given in Figs. 7 and 8, it will be seen that it is precisely those forms that are the most needed to show the interrelationships of groups that have suffered the most from the extinction of their species, which is but another way of expressing the fact that generalized and transition forms are not as well fitted for the struggle for existence as are their more specialized and better adapted descendants, and are hence often exterminated by the very races which have developed from them. This extermination tends to isolate the terminal groups and thus to disguise the plan of development, as may be seen by reference to Fig. 7, in which the distinction is shown between living and extinct groups. The effect of extinction will here be shown if the reader imagines the extinct groups completely blotted out, which will leave the modern orders entirely cut off from one another.

The same principle may be seen also in the second diagram, the phylogenetic tree of mammals (Fig 8). Here the groups 
of the greatest importance in showing relationships are the primitive Insectivora, the Mesodonta, the Condylarthra, and the Creodonta, and although the existence of the first could be surmised from their modern descendants, the discovery of the fossil remains of the others were absolutely essential to the reconstruction of the original relations between the three great groups of primates, carnivores, and ungulates. It is thus not surprising that the various orders of mammals have, until recently, been treated like isolated groups, and that, even yet, any scheme that may be offered must be looked upon as provisional and liable to be modified by the bringing to light of new evidence, especially that from palæontological sources.

It will be noticed that the branch leading to the Primates, the order to which Man belongs, is represented in the diagram as one of the shortest and least specialized, a presentation which, although opposed to the prevailing opinion, is in strict accord with the facts; since in anatomical structure these animals show comparatively little deviation from the primitive mammalian type and do not exhibit the extreme specialization displayed by the groups representing most of the other terminal branches. Such aberrant orders as those of the bats, whales, and horses, which have departed farthest from the original mammalian environment, show in consequence the greatest modifications and are thus the most specialized; certain other groups, the peculiarities of which are not so striking, are still greatly modified in comparison with the Primates. Thus the majority of the ungulates show a reduction in the original number of digits, the extremes resulting in either two, as in the camels and deer, or one, as in the horse; but the Primates, together with the rodents and modern insectivores, preserve the original number of five, inherited directly from the amphibians and reptiles. The teeth of ungulates are characterized by a great complexity in the folding of the enamel layer, and in the number and arrangement of the cusps; those of rodents are specialized for the purpose of gnawing, and in the Cetacea they are either secondarily reduced to the form of simple cusps, all alike, or are lost altogether; the Primates, 
however, are very simply constructed in these particulars and remain close to the lower type as shown in the marsupials. Primates are also primitive in their muscular system, possessing in many instances a single undifferentiated musclemass where the members of other Orders show a complex group of muscular units.

Aside from the adaptation of their extremities to an arboreal life, the one line of development by which the Primates have become differentiated is in that of their central nervous system, and especially that of the cerebrum, which has given them a far greater capacity for recording their sensory impressions, and thus of profiting by experience, the basis for the development of reason. It is chiefly in this respect that the human species has developed so far beyond the condition of the other Primates that the world has long, and perhaps willingly, been deceived in regard to their true relationship. In spite of all prejudice, however, man is, anatomically speaking, a typical primate, closely related, even in many of the smaller details, to the rest, and the only way in which he has proved superior, through the excessive development of the cerebral hemispheres, is not a modification calculated to produce important correlated changes in the other parts. Of the two living Sub-orders, the Lemuroidea and the Anthropoidea, the former are the more primitive and more nearly represent the generalized Mesodonta from which the race sprung. In the completeness of the partition which separates the orbital and temporal fossa, Man is seen to be an Anthropoid; and in important characters, such as the reduction of the premolars from three to two, he agrees with the Catarrhine division of this Sub-order. If we employ the usual schedule: of values to be attached to points of structural difference, as. used for the purpose of classification, we cannot fairly place: him in a Family apart from the large tailless apes of the Old. World, and aside from this we have several intermediate links, which the researches of the past few years have brought to light, and which reduce even the slight gap formerly considered to be between them. 
The date of Man's appearance on the earth has been pushed back many thousands of years beyond what was formerly believed to be possible, and this has been absolutely proven by the most indisputable facts. Crania of the present human type have been discovered in Europe in association with the remains of such extinct forms as the cave-bear and the hairy mammuth, and numerous carvings and incised drawings have been discovered in which the latter animal has been portrayed by an eye-witness and with much artistic ability. This brings the present species, Homo sapiens, with proportions like that of the modern European, back to the end of the last glacial epoch, or, as some think, to a time contemporary with it.

Aside from this, there have also been found, dating from about the same period, remains of men, or man-like creatures, of proportions unknown at the present time and constituting a distinct species, Homo primigenius ( $H$. neanderthalensis). Such remains have been found at Spy in Belgium, in the Neanderthal near Düsseldorf, at Cannstadt in Prussia, in the bed of the river Liane near Boulogne-sur-mer, and in other localities, the specimens all closely corresponding to one another and equally unlike the present living species. The forehead and cranium of the "Neanderthal man," as seen from these specimens, was extremely low and flat, and the superciliary ridges above the eyes were so heavy and prominent that they formed together a pair of projecting arches hung over the deep-set eyes. There was almost no chin. The height was that of a rather small man; the arms were not excessively long, but the thigh-bones were permanently curved and the tibix were short, so that an absolutely erect position was impossible. In spite of the general ape-like appearance and the low character of the cranium, the actual capacity of the latter was about that of a modern Australian, and the presence of flint implements in association with the remains show that this species could lay claim to being termed a man although of a distinct type from the one that has survived.

The fossil remains of an animal, in many respects pre- 
cisely intermediate between Homo primigenius and the anthropoid apes, were discovered in Java in I89 I in deposits of the late Tertiary period, and were named Pithecanthropus erectus, the generic name, "ape-man," having been proposed some years before for the then hypothetical transition form, the " missing link" of popular fancy. These remains consist of a cranium, a femur and three molar teeth, and although not found in contact with one another, their relation to their surroundings was such as to declare them the fragments of a single skeleton.

In the cranium the ape-like characters seen in Homo primigenius are here still more pronounced; the cranial vault is still lower, the superciliary ridges are still more prominent, closely approximating those of a chimpanzee or gibbon. The proportions of the teeth suggest a dental arcade intermediate between the fiattened form seen in man and the elongated arch of the living anthropoids; the probable shape of the tongue and hard palate, as deduced from this, would seem to have allowed the production of many of the more elementary sounds occurring in human speech. An independent fact that corroborates this conclusion was determined later when the configuration of the brain surface was obtained by means of a cast of the interior of the cranium, for here the center of articulate speech (the left lower frontal convolution) was found to have been more developed than in the highest apes but considerably less so than in man.

The femur does not exhibit the transitional characters which one would be led to expect from the nature of the cranium, for it is essentially human in form and shows a higher type than that of the European Homo primigenius. Pithecanthropus must thus represent a parallel or collateral form in which the development in the direction of an erect position had reached a high plane while the cranium and brain remained at a stage intermediate between the highest apes and the Neanderthal man.

Concerning the ancestry of Pithecanthropus and its relationships to the apes the widest opinions still prevail, but the 
trend of opinion leads to the rejection of the four living anthropoids (gorilla, chimpanzee, orang and gibbon) as direct ancestral forms. Owing to the modifications time is apt to produce in animal species it seems more logical to expect to find the connection in some extinct type, as, for example, the European Dryopithecus of the middle Miocene. As the case stands at present, however, there are few animal species concerning which so many of the intermediate links have bee11 preserved as in the case of man, and to the scientist the " missing-links," the discovery of which would be of the greatest importance, are not those representing intermediate anthropoidal forms, but those lying in the far greater gaps lower down, as, for example, between lemurs and primitive insectivores, or between the Pantotheria and the theromorphs, which would throw further light upon the reptilio-amphibian ancestry of the Mammalia.

Naturally the phylogenetic stages which lie in the direct line of human ancestry are of the most value as historical records, and as such form the main subject of study for the morphologist, but collateral lines furnish many helpful suggestions, and in cases where a group of animals which represents an ancestral line has become wholly extinct, dependence must be placed upon the nearest related group, although not directly in the line of descent. With this in mind it will be seen from the foregoing that the phylogenetic stages of the greatest value in the present discussion are the following:

I. Amphioxus.

2. Cylostomes.

3. Selachians.

4. Ganoids.

5. Urodeles (as a substitute for the Stegocephali).

6. Reptiles (preferably the chelonians, as the nearest living allies of the theromorphs).

7. Monotremes (the nearest living allies of the Pantotheria).

8. Marsupials (probably not very near the direct line, but suggestive of the conditions in the primitive Insectivora). 
9. Inscctivora (of the modern type, still quite primitive. The rodents are valuable here also as collateral lines, descended from the primitive Insectivora).

Io. Lemurs (practically modern Mesodonta, and hence representing fairly well the immediate ancestors of the anthropoids).

I1. Cercopithecidoe (tailed monkeys of the Old World).

12. The large tailless apes of the Old World (Gorilla, Chimpanzee, Orang, Gibbon).

I3. Pithecanthropus (extinct).

14. Homo primigenius (extinct).

15. Homo sapiens.

In this list an attempt has been made to enumerate only living forms, specimens that are still available to the anatomist for dissection and full comparison. In two cases, however, I3 and I4, this resolution was broken, owing to the vital importance of these forms. It must also be remembered that we possess at least a partial skeletal record of some of the extinct groups that lie in the direct line of ancestry, and that these records, although extremely fragmentary, are of the utmost value. It will also be seen that these stages are not those of coördinate groups, but that they grade from Classes to Orders, then to Families, and finally to Genera and Species; this is, however, the natural manner of considering an ancestry, for the early stages are the less detailed and are expressed equally well by all the members of a large group, while the finishing touches, which separate genera from genera and species from species, consist of slight differences, more recent and superficial in character.

A sinilar gradation is seen in the developmental history, as studied in comparative embryology, in which the earliest features laid down are those of the main subdivisions; then come in succession those of the Class, the Order, the Family, and so on until the distinguishing characters of the Species make their appearance, the latter usually not fully expressed until maturity.

The truth of this actual recapitulation of the history became 
apparent to the early morphologists, one of the greatest of whom thus expressed his feelings while gradually tracing back from the adult condition the developmental history of the skull of the common fowl: "Whilst at work I seemed to myself to have been endeavoring to decipher a palimpsest, and not one erased and written upon again just once, but five or six times over. Having erased, as it were, the characters of the culminating type,- that of the gaudy Indian bird,-I seemed to be among the sombre Grouse; and then, towards the end of incubation, the characters of the Sand-grouse and Hemipod stood out before me. Rubbing these away, in my downward work, the form of the Tinamou looked me in the face; then the aberrant Ostrich seemed to be described in large archaic characters; a little while, and these faded into what could just be read off as pertaining to the sea-turtle; whilst, underlying the whole, the Fish in its simplest Myxinoid form could be traced in morphological hieroglyphics." *

In following out the historical development of the different systems, as outlined in the ensuing chapters, both embryonic and phylogenetic records have been drawn upon as the primary sources from which this history may be deduced, and the conclusions which have the corroboration of both may be naturally considered the most trustworthy ones. Each of these two records has its advantages and its disadvantages; in the former the stages are continuous, although the early ones are obscure, and all parts of the record are apt to be overlaid and mystified by cænogenetic changes; in the latter the record is far more fragmentary and its stages are discontinuous, but the facts are usually plainer and more easily read. An adult lower animal which represents a phylogenetic stage in the history of a higher shows the parts in full physiological efficiency, while in an embryonic stage the organs are at the best not wholly functional, and often render it difficult to imagine an adult animal with the same relationship of parts; on the other hand, in places where a long historic period has no known living or

* W. K. Parker, in Trans. Roy. Philos. Sor, I869, pp. 803-804. 
fossil representative among adult animals, the only clew is that furnished by embryology. It will be seen that in a few cases, notably in the history of the transition from fins to walking limbs between fishes and amphibians, both records are unsatisfactory, and in such cases the only hope of a definite solution lies in the future discovery of some extinct form which may bridge the gap and thus furnish a clew by which the two discontinuous threads may be united. 


\title{
CHAPTER III
}

\section{THE ONTOGENESIS OF VERTEBRATES}

\begin{abstract}
". . the embryological record, as it is usually presented to us, is both imperfect and misleading. It may be compared to an ancient manuscript, with many of the sheets lost, others displaced, and with spurious passages interpolated by a later hand. . . L Like the scholar with his manuscript, the embryologist has by a process of careful and critical examination to determine where the gaps are present, to detect the later insertions, and to place in order what has been misplaced."
\end{abstract}

Francis Balfour, Comparative Embryology. Vol. I, p. 3 .

WITH the exception of a few cases of asexual reproduction, that is, cases in which an individual arises from a single parent. every multicellular organism results from a conjugation between a macro- and a micro-gamete. These are called the ovum and spermatozoön, respectively, and are the product of two distinct parent individuals. Precisely the same phenomenon occurs frequently among colonial unicellular organisms, where an entire colony produces gametes of only one sort, and in this case the distinction between such a colony and the mass of cells which constitute the body of a simple Metazoan is extremely slight and depends solely upon the amount of differentiation between the individual cells and the consequent degree of mutual interdependence attained. In both cases the cell mass, aside from the gametes, constitutes a soma, composed in the one case of homogenous, in the other of heterogenous, cells. The soma, or cell colony, is perishable and restricted to a definite time of existence; the gametes by their conjugation produce zygotes, each of which, by its repeated division, may form a new soma, that is, the colony, or the individual, of the succeeding generation. 
Among multicellular organisms, the gametes are produced in definite organs, the gonads, or germ-glands, which produce but a single sort of gamete, either macro-gametes (ova) or micro-gametes (spermatozoa). Those glands are termed respectively ovaries and testes, and may occur in the same or in different individuals. In the latter case the individuals are said to be of separate sexes and are termed male and female, the former secreting the micro-, the latter the macrogametes. Individuals possessing both sorts of gonads are termed hermaphroditic or bisexual, but owing to the fact that usually the two sorts of organs are functionally active at different times, the organisms are seldom functionally bisexual, but alternately male and female. Such hermaphroditic forms are frequent among invertebrates, and occur regularly in certain classes, but in vertebrates they are found only among the Cyclostomes (Myxinoids), although in all cases the curious homology between the parts in the two sexes (Cf. Chap. VIII) suggests that the phenomenon may have been widespread or even universal among the ancestors of modern vertebrates.

In most aquatic animals the gametes are liberated in the water and conjugation takes place without any act on the part of the parents, through the motor action of the microgametes themselves, exactly as in Protozoa; in terrestrial forms, however, since the gametes need a liquid medium, this latter is supplied by glands, and the seminal fluid of the male, in which the micro-gametes swim actively, is conveyed to the female by some form of copulation.

Since the superficial phenomena are so obvious that they are universally recognized without technical study, while the essential details require for their detection the care and patience of an experienced microscopist, and since especially the parallel phenomena occurring among the Protozoa have remained unknown until within comparatively recent years, it may be easily comprehended that the terms in common use relative to these phenomena fail to express the underlying biological principles and are not of universal applicability. Thus 
the micro-gametes, first discovered in the seminal fluid of mammals, * were termed spermato-zoa, or sperm animals, a term expressing the view held at that time that they were parasitic or adventitious organisms occurring in a fertilizing or quickening fluid, and from this the act of mixing the spermatic fluid with the ova was termed fertilization. This term is now applied technically to the entrance of the spermatozoön
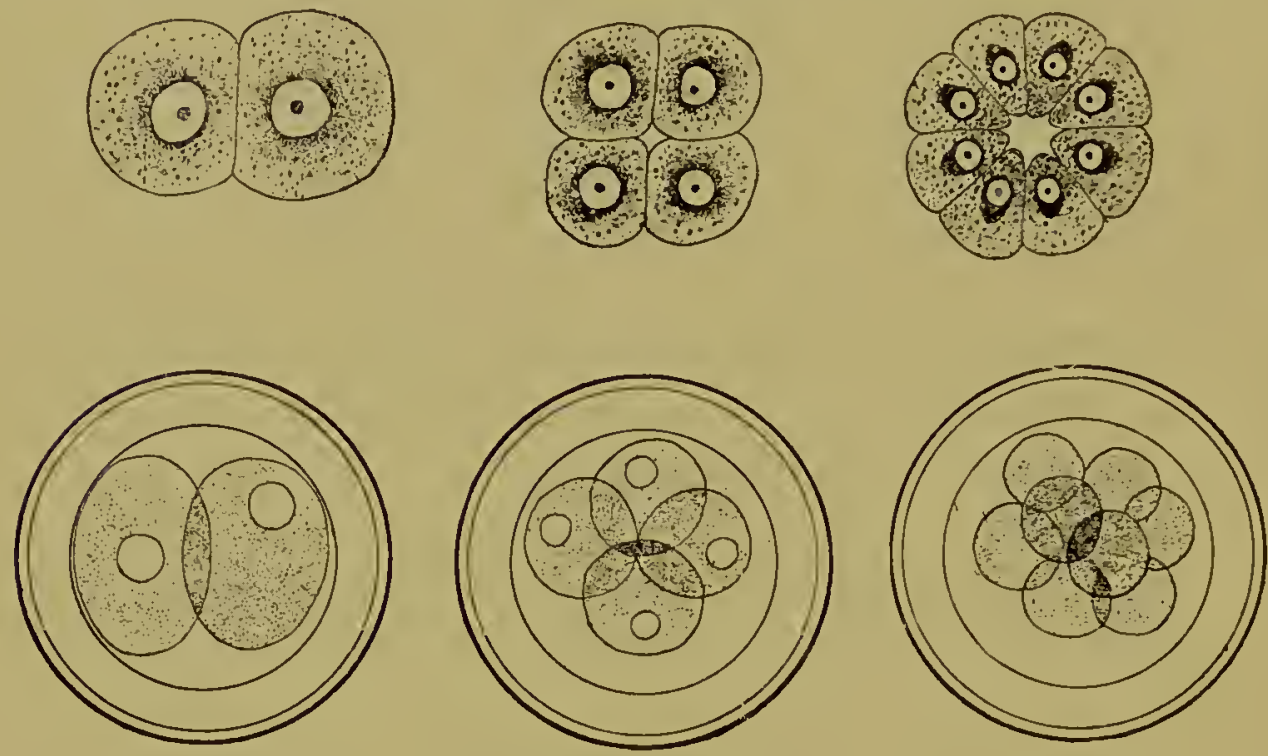

FIG. 9. Earliest stages of Metazoan development.

The upper row represents the egg of Sycandra, a calcareous sponge [after F. E. Scrulze]; the lower row represents that of the rabbit [after Bischoff]. In the rabbit the egg is surrounded by a thick capsule, the zona pellucida. The egg of the sponge is without this and floats freely in the water.

into the ovum, $i$. $e$., to the union of the two gametes, and is thus synonymous with conjugation, when applied to Metazoa. Furthermore, since, in the majority of cases, the bulk of the ovum so far exceeds that of the spermatozoön that the latter appears to be lost in the process, the term ovum, or egg, is commonly used to designate not only the macro-gamete (the unfertilized egg), but also the double cell resulting from the conjugation (the fertilized egg), a use of terms which necessitates constant watchfulness in order to guard against confusion. Ovum and spermatozoön, the macro- and micro-ga-

* Discovered in 1677 by Ludwig Hamm, a pupil of Leeuwenhock. 
metes respectively of a conjugation, are essentially Protozoa, and thus the first stage in the development of multicellular animals is an historic repetition representing the first and simplest of organisms. The spermatozoön with its motor organ still retains its protozoan character even in the highest of the vertebrates, but the ovum, loaded down with yolk, bears for the most part little resemblance to an active organism. Even here, however, in certain sponges and hydroid polyps, a more primitive form of ovum is still preserved, for it is here amœboid in form and possesses functional pseudopodia, being often impossible to distinguish from genuine Amœbæ, the simplest of Protozoa. This is a good illustration of Rule $V$ of ontogenesis as given in the previous chapter, since it is to be expected that here, among the lowest and simplest of the Metazoa, the early stages would receive the fullest attention in the ontogenetic recapitulation.

In size ova vary greatly, but the difference is due mainly to the actual amount of food stuff, or yolk, which is required in each case: this in turn is proportional, not to the size of the adult animal, but to the degree of maturity at which it is most advantageous for the young animal to begin its free existence. Some animals produce a few very large eggs and thus use up their reproductive energy in developing yolk; others produce large quantities of tiny eggs which will develop into innumerable minute larvæ. Both extremes and all intermediate grades are the result of adaptation to the various conditions that surround the different organisms and thus regulate the size of the egg, as well as the size and shape of the parts in the adult. Thus, for example, the ova of jellyfish, earth-worms, many molluscs, star-fish, and most mammals, are very small, almost microscopic; those of insects, crustaceans and fishes are usually of an appreciable size, those of frogs and of certain fish are still larger, while the eggs of reptiles and birds are enormous, those of the latter having reached the extreme limit relative to the size of the parent.

In the eggs of placental mammals, which are practically yolkless, there is no great difference in actual size between 
such extremes as those of the elephant and the mouse; in the birds, on the other hand, the true egg, $i$. e., the yellow sphere usually termed the "yolk," is approximately proportionate to the size of the parent. This difference is due to the fact that in mammals the egg is little more than the first cell of the new individual, since the food supply comes entirely from outside sources, while in birds the food is placed wholly within the egg and is the only source available to the young bird.

The spermatozoön, never having yolk to give it bulk, is always small, usually far beyond the limits of the unaided eye. Its form is typically that of an oval cell-body or "head" to which is attached a locomotive flagellum, which may attain an appreciable dimension in respect to length, but is always extremely delicate.

When the seminal fluid and the ova are brought together there is always a vast excess of spermatozoa, and in cases in which direct observation has been possible, as in aquatic forms, in which the mingling of the elements occurs freely in the water, the eggs are seen to be assailed by dozens of active spermatozoa, each endeavoring to effect an entrance. To permit the entrance of one and only one of the entire number, several devices are made use of by the eggs of various species; one of these is the encasement of the entire ovum in a shell, in which there is a single minute opening, the micropyle, through which a single spermatozoön enters and in so doing effectually blocks the way for all successors. In other cases the entrance of a spermatozoön seems to cause some chemical or physical change which renders the egg substance impervious to the other male cells or incompatible with their continued existence. In the eggs of echinoderms (star-fish, sea-urchins, etc.) the stimulus of an entering spermatozoön causes the in:mediate formation of an external membrane which effectually prevents any farther entrance. In mammals it is probable that the zona radiata proves an impassable barrier to all spermatozoa except those that approach it in a direction perpendicular to its surface, thus greatly reducing the number that 
are in condition to enter the egg. It is also likely that here, as in many other cases, several spermatozoa may actually enter the egg substance, but that all except one are simply added to the yolk and serve as food.*

The spermatozoön, after the entrance into the egg is once effected, drops its locomotor apparatus and becomes merely a nucleus, which fuses with the one belonging to the egg, a procedure similar to conjugation in the Protozoa. The egg cell thus becomes furnished with a fusion-nucleus, and may be considered from now on the first cell of a new organism. From it arise all the cells of the developing animal through the process of fission, and, since a division of the nucleus always precedes the division of the cell, it follows that this fusion-nucleus is in the same way the primary one from which all later nuclei are to be derived. Since now, as has been shown by direct observation, this fusion-nucleus becomes divided in such, a way as to effect an exactly equal division of both maternal and paternal components, and since the process has been found to continue as far as the investigators have been able to follow it, it is extremely probable that the nucleus of each and every cell of the adult organism contains an element derived from each of its parents.

Herein lies a material basis for the phenomena of heredity, and it thus becomes evident that all hereditary traits and characters are perpetuated through the direct transmission and growth of a bit of material furnished by each parent and handed down to each cell of the organism. Although this is still the mystery of mysteries to the biologist, the careful study of the past twenty or thirty years, directed upon this very point, has revealed much, but in so doing has added more

* Until 1875 it was generally supposed that more than one spermatozoön took part in the fertilization of an egg. The true facts in the case were first determined by observation, and later proven by direct experiment. Polyspermy, or the introduction into the egg of more than one spermatozoön, has been experimentally brought about in the eggs of various marine animals by such methods as the application of heat and been abnormal. 
that is still unknown. It has shown the nucleus to be a microcosm of extraordinary complexity, and has opened up a new world, the very existence of which has until lately remained unsuspected.

What seems to be the essential element of all nuclei, found alike in plants and animals, is a substance which, from its extreme susceptibility to staining fluids when artifically treated for purposes of microscopic examination, has been designated by the non-committal term of chromatin. During functional

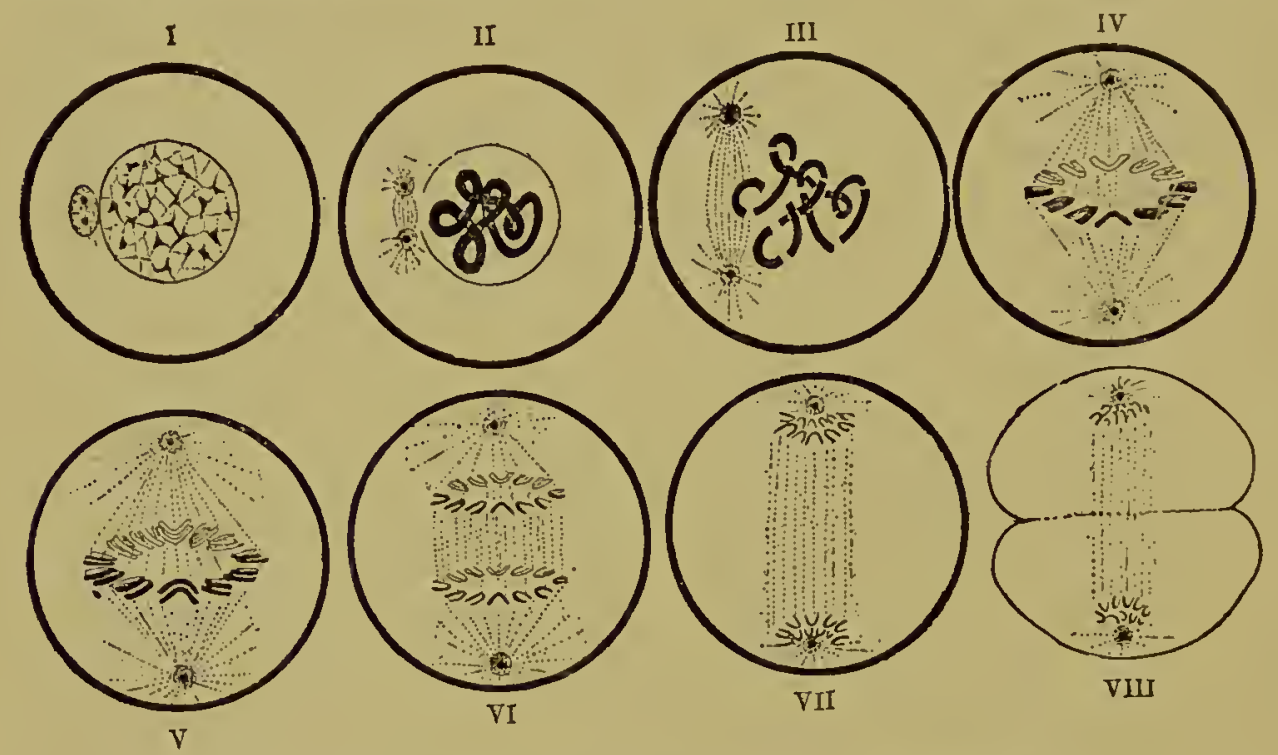

FIG. 10. Diagrams representing normal mitosis.

In I the nucleus is "resting"; the centrosome is seen by its side. In II the spireme appears, which in III becomes separated into chromosomes. In IV the centrosomes have become placed at opposite poles, while the chromosomes form an equatorial plate midway between them. Each chromosome divides longitudinally an equatorial plate midway becomes drawn to the two opposite poles. In VIII the cell divides into two.

activity this substance is diffused throughout the nucleus in little, irregular masses, but assumes the form of a continuous thread or chain preparatory to a cell division, and eventually becomes separated into a definite number of equal bodies, the chromosomes. The number of these found in any somatic cell of a given species of animal is always the same for that species, but may be different in an allied species, and the number seems to bear no reference to the size or the degree of complexity of the animal. For instance, the number four occurs in Ascaris, the pin-worm, eight in certain nematode 
worms, twelve in the mole-cricket, and sixteen in a water beetle, the rat and Man, as well as in the pine and the onion. Cyclops, a minute crustacean, possesses twenty-four, as do also the frog, mouse, snail, lily and a fern (Osmunda). The earth-worm has thirty-two chromosomes, the torpedo thirty-six, and Artemia, a small shrimp, the unusual number of I68. Whenever a cell divides in a growing or proliferating tissue, the maintenance of the same number of chromosomes in each of the two resulting cells is effected by means of a complex mechanism of minute threads, radiating from two opposite centers, which resilts in the separation of each individual chromosome into equal halves, thus assuring for each daughter cell, not merely the same number of chromosomes, but halves of the same ones. This process is known as mitosis or karyokinesis.

To the general rule concerning the constancy in the number of the chromosomes, there is, however, one very important exception, and that is, in the germ cells, that become the gametes in a conjugation, the starting point of a new organism. Here, owing to a difference in their mode of formation, the number of chromosomes in a given species is exactly onehalf of that characteristic of the somatic cells of the same species, and it is only by the fusion of the two gametes, ovum and spermatozoön, that the normal somatic number is restored. This reduction of the number of chromosomes is brought about through an extremely complex process, the essentials of which are: first, the formation of certain germ-cells, spermatogonium or oögonium, which develop twice the normal number of chromosomes, and, secondly, two successive divisions of the cells, and of the number of chromosomes also, by means of which four cells are produced, each with one-half the normal number. In the case of the male cells each of the four is effective, and, through a metamorphosis in its form, becomes a functional spermatozoön; but in the case of the female, owing to the disadvantages which would arise from the division of the yolk into four ova of equal size, one of them retains it all and becomes a functional ovum while the 
others become yolkless, abortive, eggs, attached to the ovum and called polar globules. Owing to the enormous disparity in size between the abortive and the functional ova the divisions of the oögonium by which they are formed were for a long time not recognized as true cell divisions, but the polar globules were spoken of as extruded or cast off from the
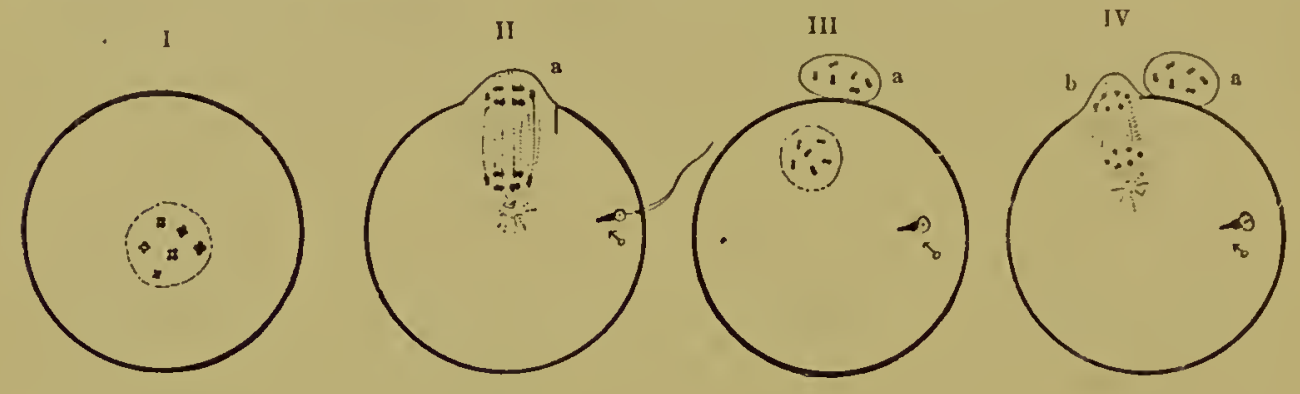

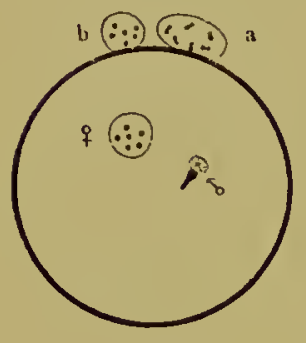

V

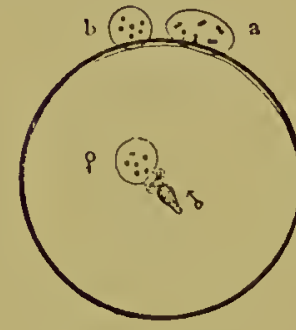

VI

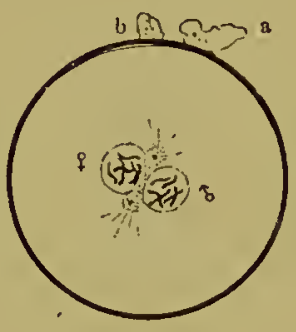

VII

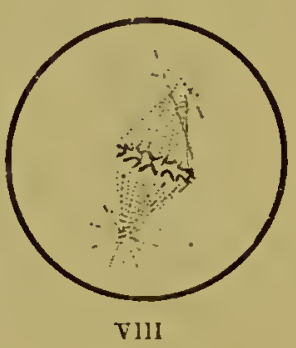

V111

FIG. II. Diagram of fertilization.

Stage I represents the egg just previous to maturation. The chromosomes, arranged in tetrads, are twice the number found in somatic cells, which, in this dia. gram is assumed to be I2. At II a mitotic figure is formed, which, in III, results in the formation of two cells; a little one, the first polar globule, $a$, and the egg, each with a reduced number of chromosomes, in this case 12 . In $I V$ and $V$ a second mitotic figure is formed, which results in the expulsion of a second polar globule, $b$, and the reduction of the chromosomes of the egg nucleus to six, onehalf the normal number. Meanwhile a spermatozoon head has entered the egg, composed mainly of chromatin, the equivalent of the six chromosomes of the reduced egg nucleus, and a new centrosome, to replace that of the egg which was destroyed during the expulsion of the second polar globule. The spermatozoon head rotates through $180^{\circ}$, thus bringing the centrosome between the male and female germ nuclei, as in VI. The first cleavage spindle is seen forming in VII and VIII, after which the cell divides into two and development begins.

"egg," terms which express the phenomena as observed, but mask their true biological significance.

Usually, owing probably to the rudimentary condition of the polar globules and their lack of function, the globule formed by the first of the two reductive divisions, and hence the equivalent of the definite ovum plus one abortive egg, does not carry through its division into two, but remains as 
a single mass, and is spoken of as the "first polar globule" in distinction from that resulting from the second division, which is termed the "second." Strictly speaking, the first and second polar globules are not equivalent, but the first is the equivalent of two abortive eggs and the second of but one; and corresponding to this the first polar globule possesses twice the number of chromosomes exhibited by either the second globule or the functional egg. Furthermore, the two polar globules are frequently not extruded until after the entrance of the spermatozoön, the presence of which seems to act as a stimulus for these cell divisions. In these cases, the unfertilized "egg" is, strictly speaking, not the ovum, but the oögonium, which requires the two reductive divisions to become the equivalent of the spermatozoön.

To illustrate this by an actual example, let us suppose an animal that possesses in each somatic cell sixteen chromosomes. The spermatogonium would thus possess thirty-two which, by the reductive divisions, would result in the formation of four spermatozoa, each with eight. Similarly the oögonium would possess thirty-two chromosomes, a number which would be reduced to sixteen by the expulsion of the first polar globule, the latter body having the like number. The second reductive division would result in the formation of a second polar body with eight chromosomes, and would leave eight in the egg. This number, when added to the same amount introduced by the spermatozoön, restores the normal number, sixteen, and thus forms the first cell of the new organism, equipped with the regular somatic number, one-half from either parent.

In this is seen a provision for avoiding that enormous increase in the number of chromosomes that otherwise must be the inevitable result of each conjugation. Furthermore, when taken in connection with the fundamental law of heredity that in the long run the two parents are equally potent in transmitting their characteristics to their offspring and that neither sex has the preponderance of influence in this direction, it is seen that the hereditary substance must lie in the chromosomes alone, since these are the only elements in athich both parcnts 
are cqually represented. Neither the preponderating bulk of the orum (macro-gamete) nor the flagellum and other locomotor apparatus of the spermatozoön (micro-gamete) are of any significance in hereditary transmission, but are mere adaptive characters, of provisional functional importance, and without influence in directing the development of the new organism; achile the chromosomes, to effect the union and equal division of which the other parts have been developea, form the true germ-plasm, transmitted in direct continuity from both parents and entering every cell as it develops, directing both the architectural plan which these cells assume and also their gradual differentiation into the tissues which form the adult soma of the succeeding generation.

In this "Continuity of the germ-plasm" is found the material basis also for the recapitulation theory, the law of biogenesis explained in the first chapter; for the contimuously living chromatin, which pervades each cell of an organism, has in its own existence actually experienced all the somatic modifications of its entire past history, traces of which it must retain in some form of structural expression, enabling it to control the development of the soma during every stage of its existence. How this is effected is far beyond our present means of observation, and perhaps of experiment, but the results presuppose an inconceivably complex structure in the chromatin in order to render such results possible.

The first stage in the development of all Metazoa, that of the fertilized ovum or zygote, is followed, in most cases immediately after fertilization, by a succession of cell-divisions, or cleavages, as they are here termed, which, in typical cases, follow a general geometrical plan and result in the formation of a mass of cells that shape themselves into a definite embryological stage, that of the blastula. As the various geometrical forms assumed by the cells during the cleavage stages are all represented among colonial one-celled organisms, so there are also a few such that, in the arrangement of their cells, closely resemble the blastula. In this stage the cells form a hollow sphere, one cell in thickness, and in cases in which the blastula 
floats freely in the water, as in that of many of the invertebrates, each cell is provided with long vibratile flagella, by which the colony is moved. This larval form is closely imitated by such an organism as Volvox, which is usually reckoned as a plant, but serves to show a physiologically functional adult organism in the corresponding stage. The folding in, or collapse of one portion of the blastula, as in the diagram,
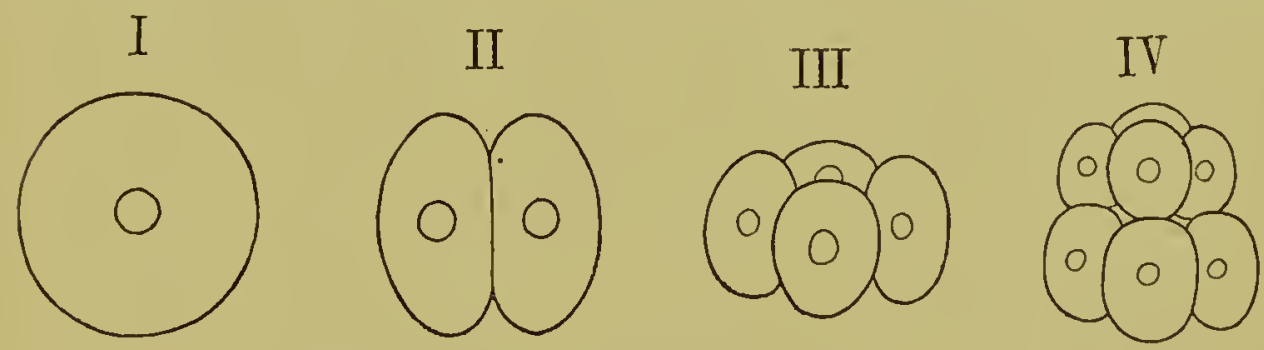

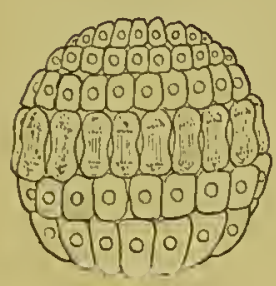

V
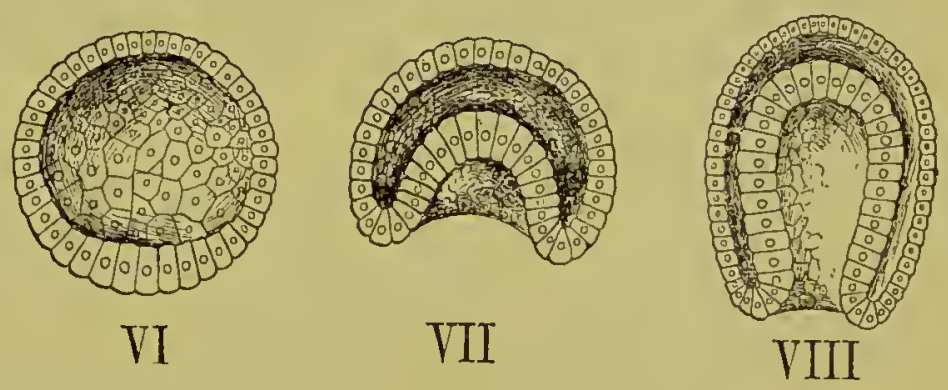

FIG. 12. Early Metazoan development; typical. [After models of Amphioxus by HATSCHEK.]

I, the egg. II, III, and IV, cleavage stages. V and VI, blastula: in VI, which represents a somewhat older stage than V; one-half has been removed. VII represents the beginning of the gastrular invagation, and VIII is the completed gastrula,
both sectioned as in VI. produces a two-layered cup which forms the next important ontogenetic stage, the gastrula, and in attaining this the embryo passes beyond the Protozoa in its imitative repetition and assumes the essential form of the simplest of the Metazoa, the Colenterata. A typical gastrula is radiate in structure, and possesses a central axis with two poles, oral and apical, the former characterized by the presence of the gastrula mouth or protostome. This latter leads into the large central cavity, the gastrocole, which has developed from the exterior at the expense of the cavity of the blastula, the blastocalc. In some 
gastrulae this latter cavity is completely obliterated by the completion of the process of invagination, but often remains as a space between the two layers, the ectoderm and endoderm.

When this type is completed and becomes an adult animal it often assumes a considerable complexity of structure but never gets far away from the original plan and does not develop more than the two primary layers. The fresh-water hydra is an example of one of the simplest cœlenterates or gastrula-animals, and the coral polyps and medusæ represent the more complex ones. In none of these does a blastocole appear, in the simpler forms ectoderm and endoderm are everywhere in contact, and in the more complex medusæ the space between them is filled by a gelatinous tissue developed from the other layers, and termed mesenchyme.*

Up to this point the course of development is the same for all Metazoa, allowing for the adaptive modifications always met with in the application of a general plan to a group of organisms.

From this point on, however, there is a divergence in the course of development, and the various branches of the higher Metazoa proceed along different paths, yet all develop, although through different means, the three following attributes, which differentiate them from the lower Metazoa, the Colenterata:

I. The formation of a third germ element, the mesoderm, situated between ectoderm and endoderm.

2. The formation of a new cavity or system of cavities, the metaccle, lined wholly by the mesoderm.

3. The attainment of a new body axis, and a bilateral, instead of a radiate, symmetry.

Onitting all further reference to the other branches, it appears that in the branch leading to the vertebrates the gas-

* This is carefully to be distinguished from the mesoderm, or middle layer, which appears first in animals above the colenterates and is always in the form of a definite layer. The mesenchyme never appears as a layer, but its cells serve to fill in the spaces between the true germ layers, and the structures formed from this source are thus determined by the form of the surrounding tissues. 
trula assumes the form and position shown in Fig. I $3, b$, in which it becomes placed horizontally, with the apical pole for-
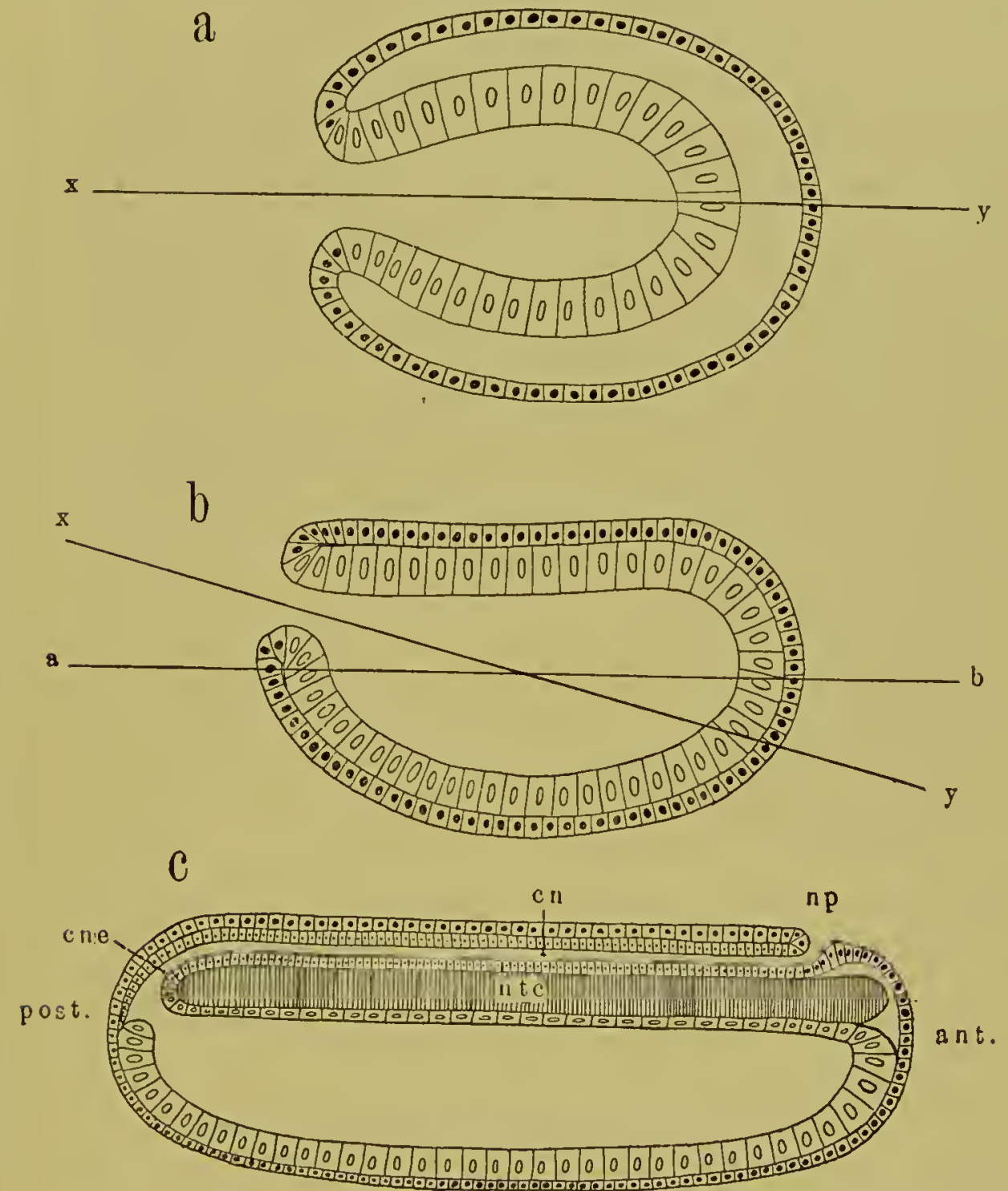

FIg. 13. Diagrams of gastrulæ. [Based on models of Amphioxus by HATSCHEK.]

(a) Typical gastrula, as in Fig. I2, VIII, but differently placed, for comparison with the others. (b) Early gastrula of Amphioxus, a probable ancestor of the
vertebrates.
(c) Later embryo of the samc.

$x y$, primary axis, $i$. $e$., that of the gastrula; $a b$, secondary axis, that of the adult Amphioxus; $c n$, neural canal; cne, neurenteric canal; $n p$, neuropore; $n t c$, notochord.

ward and the protostome posterior and dorsal and in the median line. The plan of structure is a bilateral one, with 
dorsal, ventral and two lateral aspects. If this metamorphosis has any biogenetic value, that is, if it is indicative of a genuine historic stage in the phylogeny of vertebrates, it suggests an ancestral gastrula that sank to the bottom, lay upon its side and exchanged a free swimming for a crawling mode of locomotion, apical pole forward. Such an hypothetical form as this corresponds, however, to nothing known at the present time, but may well have disappeared without trace, since a similar fate has happened to the transition forms linking the vertebrates to the other Metazoa, leaving the group unusually isolated. [See Chapter XII.]

There now occur several simultaneous changes which inaugurate the essential vertebrate structure and are best explained by the help of the accompanying diagrams. [Plates I. and II.] The gastrula has now become considerably elongated in the direction of the newly acquired secondary axis and is represented as cut transversely across, the diagram representing the posterior portion and showing the cross-section as well as a portion of the length. The most superficial of these changes involves a longitudinal mid-dorsal stripe, which becomes gradually turned in, forming a trough. Through the fusion in the median line of the edges of the trough, the turned-in portion becomes a tube, which ultimately frees itself from its attachment to the rest of the ectoderm, and forms the neural tube, the anlage* of the nervous system. The walls of this tube, by an excessive thickening of certain definite portions, become the brain and spinal cord, and the lumen is perpetuated as the ventricles of the brain and the canalis centralis of the cord, the embryonal communication between these cavities being retained throughout life.

A somewhat similar structure, also median, arises from the dorsal wall of the endoderm. This appears at first as an inverted trough, and possesses a narrow lumen, but it eventually

* The word Anlage is borrowed from the German to express a conception for which there is no English equivalent. It signifies the first visible indication of a part that appears in the embryo, and may thus signify either a definite cell-mass or a slight change in the arrangement of cells. 


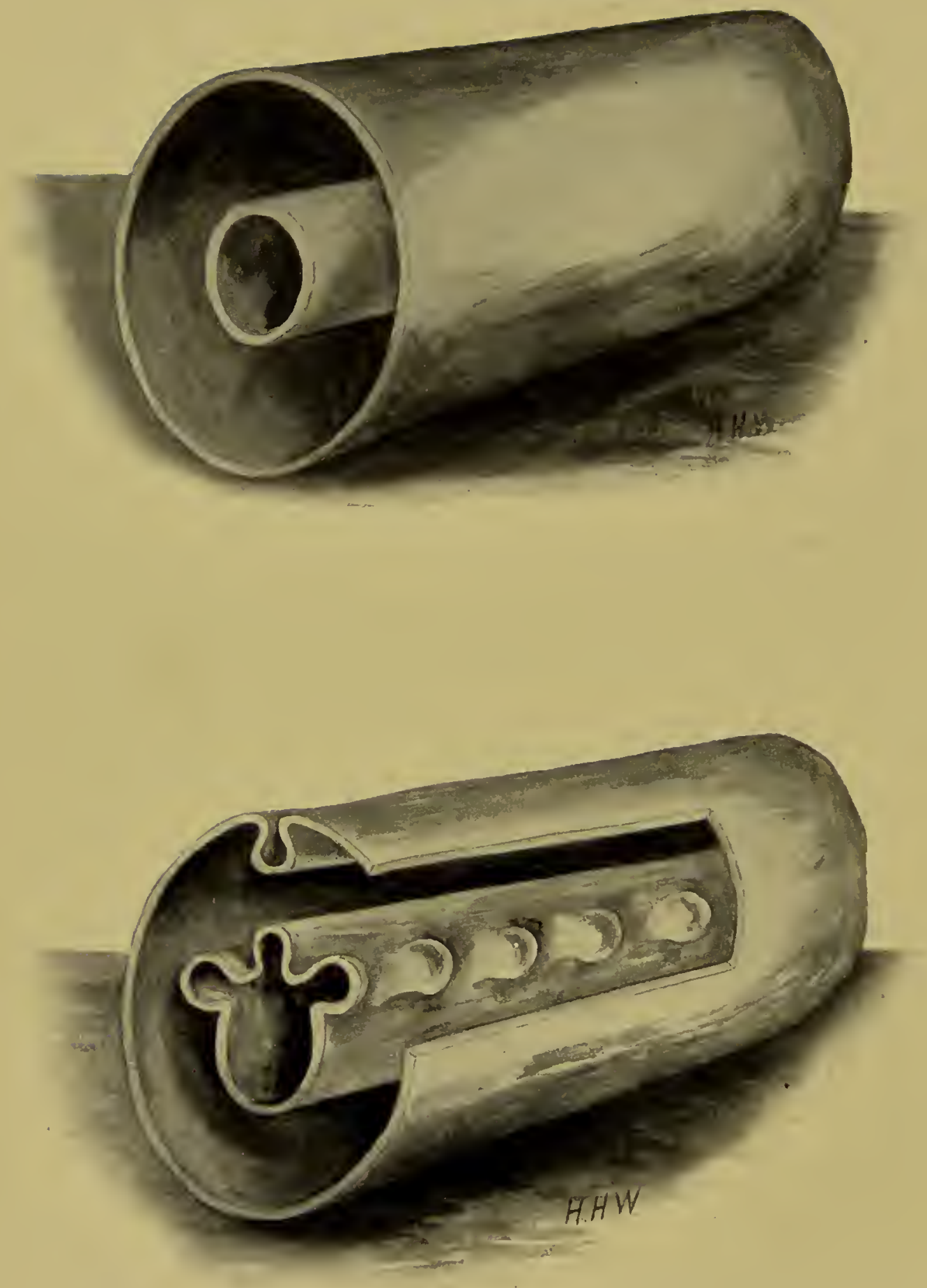

Plate I. Diagrams showing Vertebrate development, explained in the text; stages I and II. Based upon a stereogram by Kingsley. 

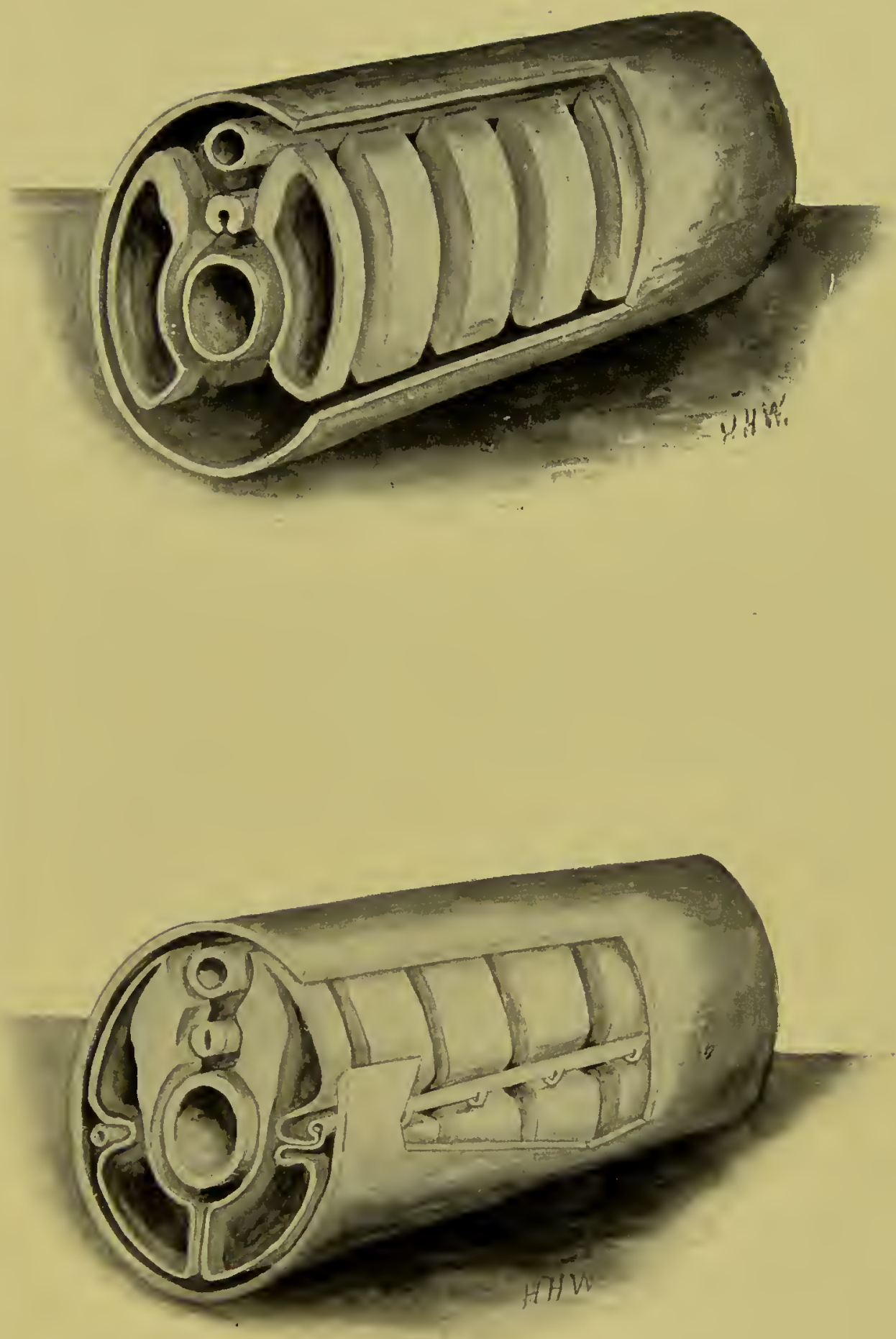

Plate II. Diagrams showing Vertebrate development; stages III and IV. Based upon a stereogram by KingSLEY. 

becomes pinched off from its place of origin, not as a tube, but as a solid rod of cells, the notochord, which forms the precursor of the vertebral column.

A third procedure, more complicated than the other two, is that involved in the formation of the mesoderm. Like the notochord, this arises also from the endoderm, and appears typically in the form of paired, lateral pockets, the mesodermic diverticula. There is reason to suppose that originally, that is, in certain of the lost forms between the creeping gastrula and Amplioxus, these diverticula were used as gonads, or sac-like cavities, in the lining of which the germ cells were developed, but in the vertebrates this function is retained by but a very small portion of their surface, as will be shown later. These diverticula soon separate themselves from the intestine and expand until they fill practically the entire space between ectoderm and endoderm and lie in close contact to one another. They thus form a series of paired cavities, the metacoles, those of each side separated by transverse partitions composed of the walls of adjacent diverticula, and those of the two lateral series similarly separated by longitudinal partitions which lic in the median line above and below the intestine. The early loss of the transverse partitions converts the segmental series of lateral cavities into a single pair, one for each side, while a similar reduction of the greater portion of the ventral longitudinal partition throws the two cavities together and forms eventually a single large metacnele or body cavity, lined by the mesoderm. One layer of this invests the outer body wall, the other the intestine, the parietal and visceral layers respectively. The longitudinal partitions, both dorsal and ventral, serve as suspensory ligaments in. the intestine and are termed mesenteries. The dorsal one is retained throughout its cntire extent; the ventral one disappears posterior to the liver. It will be noticed that the mesodermic diverticula during their development have expanded at the expense of the protocœle, the original cavity included between cctoderm and endoderm, and thus at the completion of the process the protocole has become reduced to a com- 
plicated system of narrow spaces lying everywhere between the other layers. The protocœle is thus called the primary and the metacole the secondary body-cavity, and it is this latter, the one lined by the mesoderm and included between its two layers, that forms the permanent body-cavity of verte-
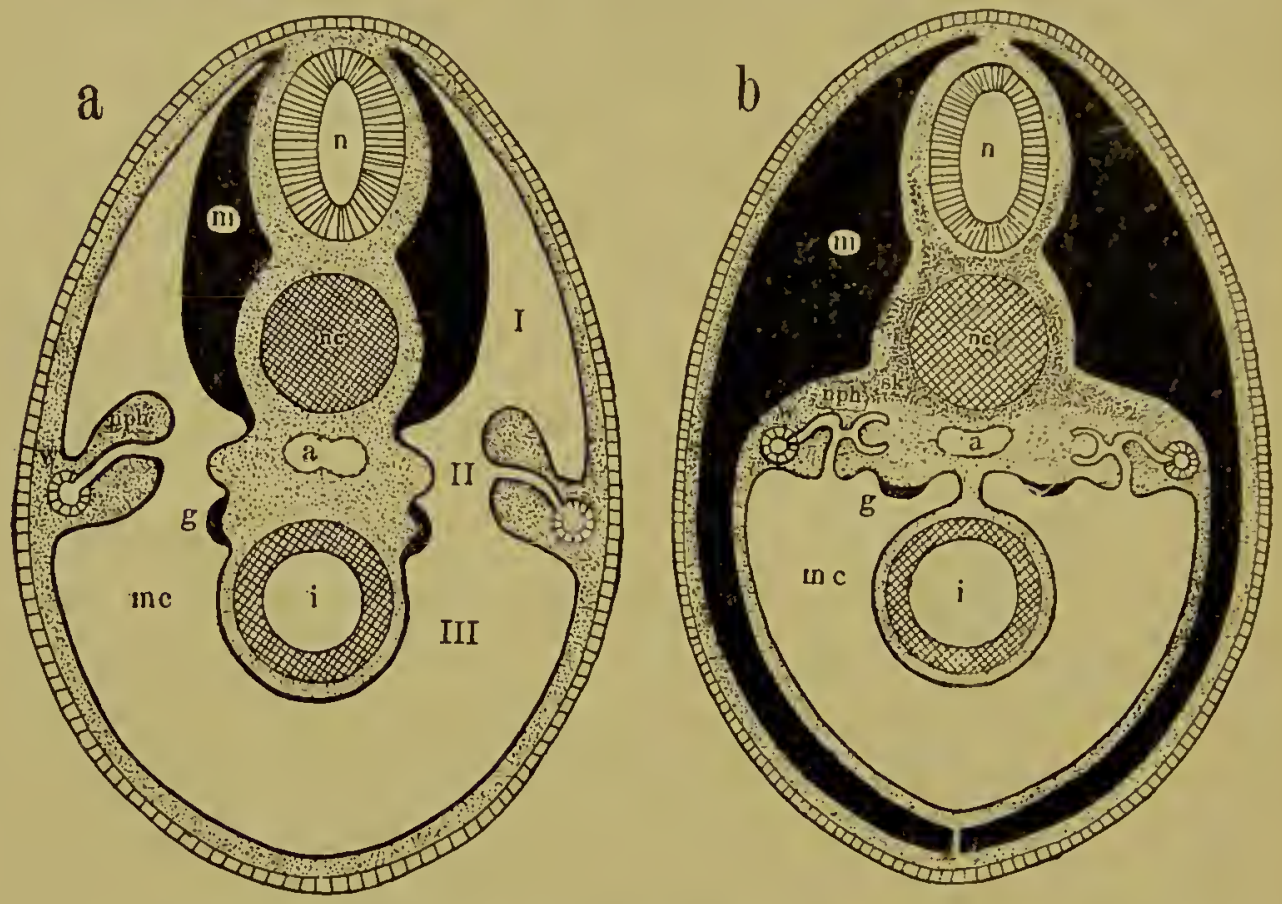

Frg. I4. Diagrammatic cross sections through vertebrate embryos, based upon the conditions found in selachians. [Modified, after vaN WIJHE.]

(a) Earlier stage, in which the three parts of the mesodermic diverticula are still continuous. (b) Later stage, in which the epimeres of the mesodermic diverticula have separated from the meso-hypomeres and form a continuous layer around the body, interrupted only at the mid-dorsal and the mid-ventral lines.

In all the figures the ectoderm is represented by square cells, the endoderm by rossing diagonal lines, the mesoderm by solid black, the mesenchyme by dots. $I$, epimere; II, mesomere; III, hypomere; $a$, aorta; $g$, gonad; $i$, intestine; $m$, myotome of epimere; $m c$, metacole (the definite colons); $n$, nerve cord; $n c$, notochord: nph, nephridium; sk, sklerotome, the anlage of the axial skeleton; $w$, protonephrotic duct (Wolffian duct).

brates, the so-called colom or pleuro-peritoneal cavity. The narrowed spaces of the protocœle become filled with embryonal connective tissue cells, the mesenchyme, which never assume the form of a definite layer, and are produced by proliferation from the mesoderm, and perhaps from the other two as well. Canals are left here and there which in time are built up into a continuous system of vessels, with walls of 
connective tissue, and form the vascular system (blood-vessels and lymphatics).

The arrangement of the various embryonic elements at this point is shown in the accompanying diagrams based upon selachian embryos, and exhibiting the actual proportions as they exist in a rather primitive vertebrate. [Fig. I4.] The general arrangement of parts in an adult dog-fish is not materially different from the last of these. Through the formation of a restricted middle area, the mesodermic diverticula become divided into dorsal, middle and ventral portions, the epimere, mesomere and hypomere respectively, each with a distinct, separate history.

The epimere, the inner wall of which becomes greatly thickened, eventually cuts itself off from the remaining meso-hypomere, and expands both dorsally and ventrally between the latter and the ectoderm until it meets the opposite one in the mid-dorsal and mid-ventral lines, separated only by thin strips of connective tissue. From the thickened inner wall of this develop the voluntary muscles of the body, the segmentation of which is retained among the fishes throughout the greater part of the body, and still appears in unmistakable traces among the highest forms. The mid-ventral connective tissue partition separating the muscle masses of the two sides becomes the linea alba, a conspicuous white line, which persists in all vertebrates. The cavity of the epimere becomes suppressed by the growth of the inner wall and thus comes to nothing.

The consecutive meso-hypomeres soon lose their independence through the breaking down of the transverse partitions, as described above, but the metameric repetition found among the parts derived from them continues to suggest their origin as separate diverticula. From the narrowed mesomere there arise the essential organs of the urogenital system, many parts of which retain throughout life the indications of a segmental origin. The cavities of the mesomere become those of the . systems derived from it.

The hypomeres, fused into a single bag or sac, form the 
definite cœlom, or pleuro-peritoneal cavity, of which they furnish the lining nembrane, the peritoneum. The outer layer (parietal mesoderm) lines the body wall; the inner (visceral mesoderm) invests the primary intestine and, later on, its derivative organs, as lungs, pancreas and liver. In all except mammals the membrane is a continuous one, but here, through the formation of the diaphragm and the consequent setting apart of a separate thoracic cavity, the portion thus cut off is treated as a distinct membrane and called the pleura.

Although the above sketch represents the underlying plan upon which the development of all vertebrates is based, it is not found in an unmodified condition save in the lowest classes. It is most typically represented in the development of $\mathrm{Am}$ phioxus, for which the foregoing description, save in a few points, might well be used; in the selachians, also, the modifications are not very great and the plan may be easily traced. In the amphibians, however, the plan is so much obscured, especially in its earlier stages, that for a long time, during the early history of the science of embryology, the homologies were not recognized. These modifications become still greater in the Sauropsida and Mammalia, in which, without the help of the amphibians, which here, as elsewhere, form a valuable connecting link, the recognition of the early stages would be hardly possible. The principal disturbing factor, at least in amphibians and the sauropsida, is the presence of increasingly greater quantities of yolk, which presents numerous mechanical problems, and its influence is felt with equal emphasis in the case of placental Mammals, where the egg, although yolkless, has evidently become so through a secondary reduction and still follows in its development that of the yolk-filled eggs of the Sauropsidan type.

One of the most important modifications in the developmental history of the higher classes concerns the appearance and subsequent development of the mesoderm and the formation of the definite colon. In Amphioxus the pairs of diverticula arise in quite typical fashion from the sides of the primitive intestine, and this procedure is almost as easily 


\section{THE ONTOGENESIS OF VERTEBRATES}

recognized in the case of the selachians. The amphibians show considerable modification, and these are the last in the ascending scale in which the diverticula are provided from the first
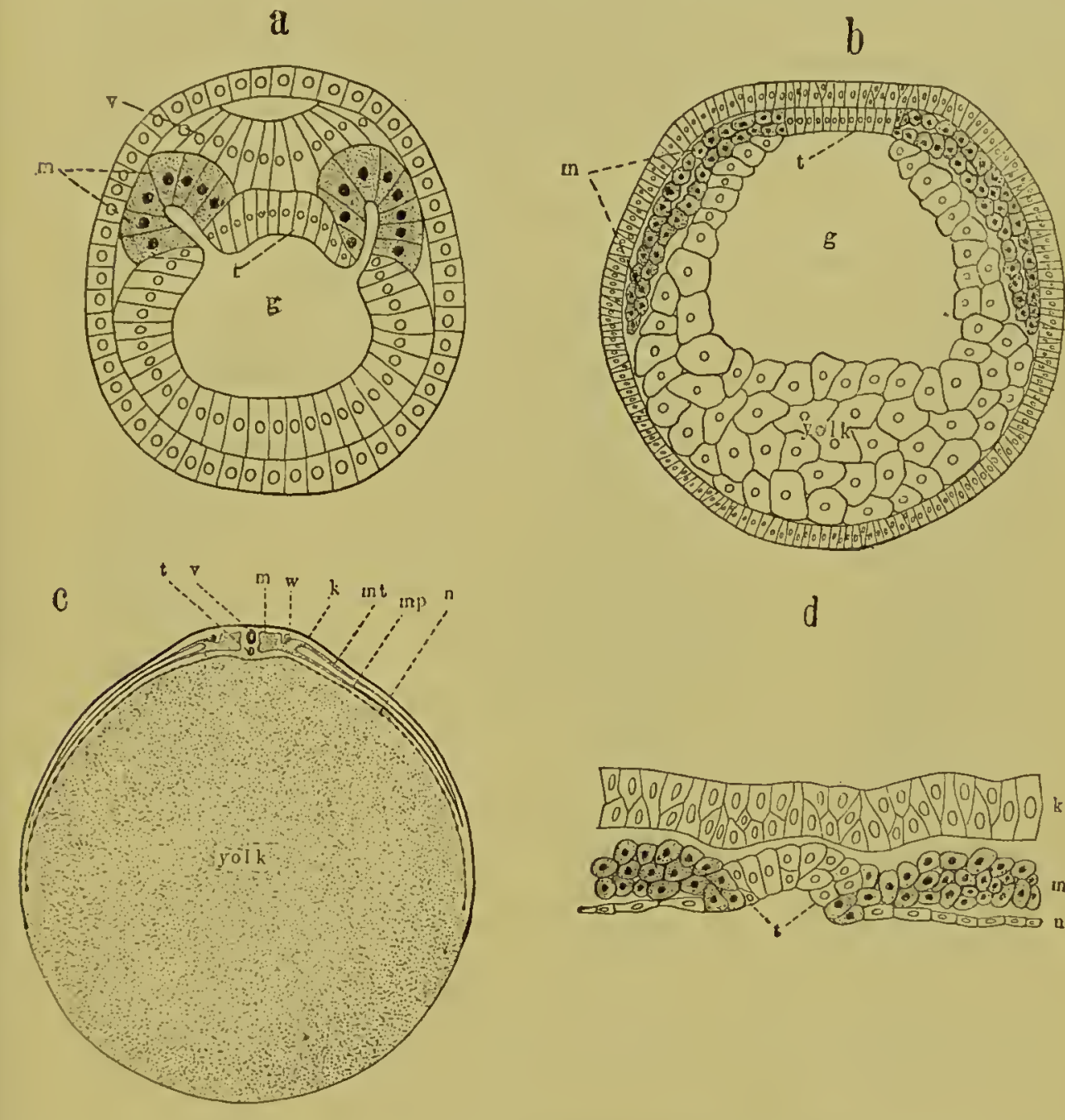

$d$

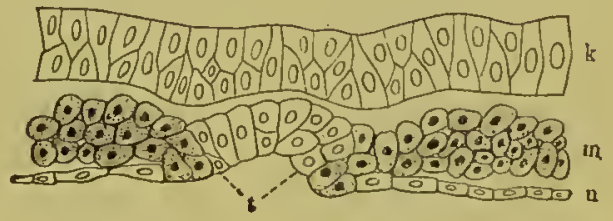

FIG. I5. Four cross-sections of vertebrate embryos showing development of the mesoderm.

(a), Amphioxus [after Hatschek]; (b) Triton (a salamander) [after Hert. WIG]; (c) bird, diagrammatic; $(d)$ mole [after HEAPE].

$m p$, visceral layer of the mesoderm; $w$, nerv; $m$, parietal layer of the mesoderm; g, gastrocolc.

with a definite lumen, which is here in the form of an irregular crack between the outer and inner cell layers.

Above this class the mesoderm appears first in the form of an irregular cell layer which starts at the sides of the notochord and invades the space between ectoderm and endoderm. 
In it the region of the epimeres becomes easily distinguished by a great increase in the thickness of the layer, and an indication of the separate diverticula appears through a series of transverse fissures, which divide the mass into separate square blocks, the so-called mesodermic somites. These first appear at about the middle of the body, and are added to progres-
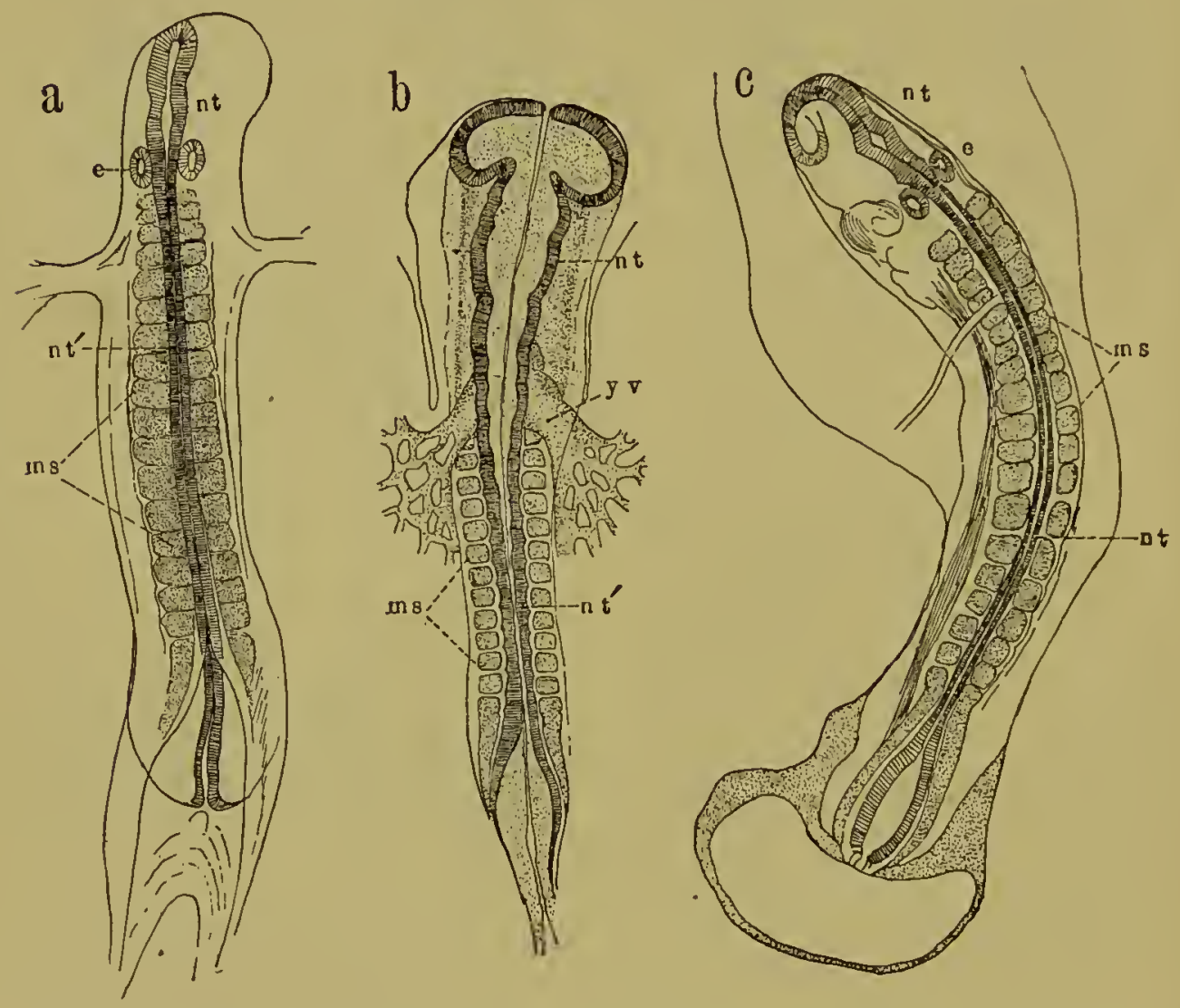

FIG. I6. Three early vertebrate embryos, showing mesodermic somites.

(a) turtle [after Mrtsukuri]. (b) chick [after Duval]. (c) pig [after Keibel]. $n t$, nerve cord (brain); $n t^{\prime}$, nerve cord (spinal cord); ms, mesodermic sorites; $e$, ear; yz, yolk veins.

sively both anteriorly and posteriorly until the full number is reached. The meso-hypomeric region remains for a time as a single undivided layer, but ultimately splits into two, outer and inner, containing between them a single undivided space, the future cœlom. This latter is here called a schizocole, in respect to its mode of origin.

There is thus attained in the higher vertebrates a much shortened and greatly modified method of producing the ele- 
ments for the later developments, hardly recognizable on comparing it with the more expanded and simple form found among the lower types. Aside from such modifications as those mentioned, which are explained through mechanical exigencies, there appear to be differences in the origin of the first mesoderm cells themselves, differences which tend to shake our faith in the absolute homology of the germ layers. Since, however, in spite of such variation in the early history, the same embryonic elements eventually appear in all cases, so that the anlagen of the principal organs are the same for all, it is hardly possible that the early modifications, however profound, have any deeper significance than that of cænogenetic adaptations to the various changed conditions of development.

The presence of yolk has a great modifying influence, both on the general shape of the early embryo and upon the definiteness of its stages. Yolk is an inert substance, the presence of which in large quantities within the cells interferes with their normal division and with their assumption of normal positions. Beyond a certain proportion, in fact, no cell division is possible, and the egg comes to consist of two portions, (I) the protoplasmic area, in which all cell divisions take place, and which ultimately becomes developed into the embryo, and (2) the yolk-sac. These two ạreas are indicated in some eggs, as in those of the frog, by a difference in color, the protoplasmic area being deeply pigmented and the yolk area not. The extreme of disproportion is seen in the bird's egg, where the protoplasmic area is represented by the light yellow embryonal disc, about 4-5 $\mathrm{mm}$. in diameter, which floats on the upper surface of the huge, non-cellular yolk mass. In such cases, the embryo, when passing through the early stages, or until after the establishment of the mesodermic somites and the formation of head and tail, is spread out on the surface of the spherical yolk, in proportion to which it is so small as to be almost flat, but later on becomes nearly separated from it, retaining its connection by a narrow yolk-stalk attached in the umbilical region. The embryo grows at the expense of the yolk-sac, and as the former increases in size, the latter dimin- 

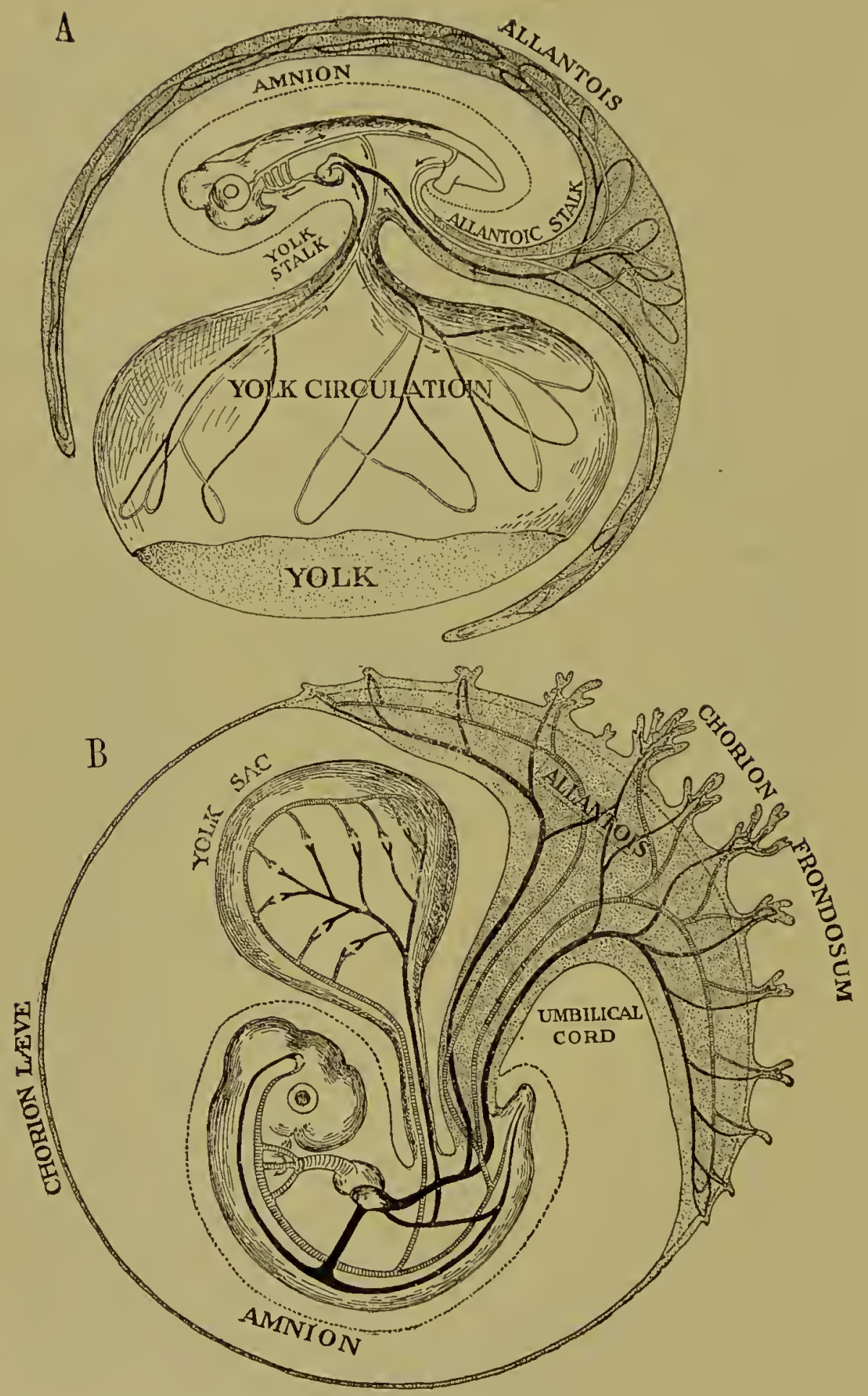

FIG. 1\%. Diagrams of Amniotes, showing the relation of the extraembryonal membranes.

(A) Sauropsidan, with functional yolk-sac and respiratory allantois. (B) Mam. mal, with functionless yolk-sac and with the allantois converted into an umbilical cord and placenta. 
ishes, so that by the time the animal assumes a free life the yolk-sac has nearly or wholly disappeared.

The embryos of the higher vertebrates differ from those of the lower in one very conspicuous feature, and that is, in the possession of fetal membranes, external to the embryo and designed in part for protection and in part for the obtaining of nourishment. The two membranes of the most extensive occurrence are the amnion and the allantois, which are present in reptiles, birds and mammals and absent in fishes and amphibians, a difference which is expressed in the two terms Amniota and Anamnia (with and without amnion), applied respectively to the two divisions in question.

The amnion appears to be solely for the protection of the embryo. It is a thin transparent membrane, composed of parietal mesoderm and ectoderm, and is formed by the growth of folds about the embryo. It invests the latter on all sides and forms about it an enclosed space, the amniotic cavity, in which the embryo lies, immersed in a colorless amniotic Aluid, of about the same specific gravity as the embryo itself. The allantois is in the form of an empty sac, composed of two layers, visceral mesoderm and endoderm, and develops from the umbilical region of the embryo. In reptiles and birds it pushes its folded edges between yolk-sac and amnion on the inner, and the shell on the outer, side, and thus comes to completely invest the former and line the latter with a double membrane. In this there develop two large allantoic (umbilical) arteries and two allantoic veins, and the organ thus serves as an excellent respiratory organ, affecting the interchange of gases through the porous shell. In placentai mammals the egg-shell is replaced by a membranous chorion, and the allantois effects a close union with this, either involving the entire surface or more generally a restricted area, and this surface, entering into a more or less intimate relationship with the mucous membrane of the maternal uterus, forms the essential organ of nutrition, the placenta. That portion of the chorion which is involved in the formation of a placenta is covered by branching processes, the chorionic villi, forming a surface 
known as the chorion frondosum in distinction from the smooth area, the chorion lceve. A diffuse placenta, where the villi cover the entire external surface of the chorion, is the most primitive type, and is found in pigs, horses, whales and porpoises; if a small portion of the chorion is left smooth, the placenta is bell-shaped, as in some edentates and lemurs. By a continuation of this process, that is, by a farther extension of the smooth area, the placenta becomes discoidal, which is the form characteristic of Man and the higher anthropoids, insectivores, bats and rodents; in the lower monkeys there are two such discs, placed at opposite poles, the placenta discoidea duplex. It is the single discoidal type, as found in man, that gave the name "placenta" to this organ, as the word signifies a round, flat cake.

If there are two smooth areas at opposite poles, with placental villi between them, the zonary placenta is formed, the type characteristic of all carnivores, elephants, Hyrax and the Sirenia. A very distinct type of placentation is the cotyledonal, characteristic of ruminants. Here the placental structure is confined to small nodules or cotyledons scattered over the entire surface of the chorion, and varying in number from three to five in the deer to more than a hundred in the sheep and cow.

All of the above forms of placentation are easily derived from the primitive diffuse type, and as a rule actually pass through the changes during early development, the form finally assumed being attained through the growth of smooth areas (chorion lave).

The methods of placentation may be again divided with reference to the relationship to the uterine mucous membrane; in one type the villi at birth are simply drawn out of the maternal portion, leaving pits, and in the other type the union between fetal and maternal elements is more intimate and the separation occurs between the mucous and muscular walls of the uterus itself, thus involving the loss of maternal mucous membrane, called in this connection, the decidua. The latter of these types, in which the placenta becomes a far more spe- 
cialized organ, is termed deciduate, the former indeciduate. In ungulates and in many of the edentates the placenta is indeciduate, in most others it is deciduate.

When the fertilized egg of a placental mammal first enters the uterus it does not at once become fixed, and development proceeds for some time before there is any attempt at the formation of a placenta. Meanwhile the egg passes through a series of typical cleavage stages and attains the condition of a hollow sphere, similar to the blastula of more typical. ontogenesis. This, however, is not a blastula, but the blastodermic vesicle, upon one side of which there develops an embryonal area similar to that of the bird, that is, spread out in the form of a flattened disc, and not cylindrical as in the case of other yolkless eggs. This apparently useless circumlocution can be understood only on the ground, supported also by the early development in the marsupials and monotremes, that mammals have been derived from ancestors having large, yolk-filled eggs and that the secondary reduction of this substance has been too recent to effect a corresponding modification in the course of development. Adhesion to the walls of the uterus occurs through the formation of chorionic villi over the surface of the blastodermic vesicle, in which the form of placentation characteristic of the species soon becomes manifest.

The later developmental history of vertebrates subsequent to the formation of the germ layers and the establishment of the anlagen of the various systems, belongs to that division of the subject known as organogeny, or the development of the various organs, and cannot be followed further in this place; it receives a fuller treatment, however, in the ensuing chapters, where the systems are considered separately and where embryological facts are made use of in so far as they are needed to explain the history of the several organs. Most of the systems arise from a single germ layer, often, indeed, from a definite restricted locality in one of them, the anlage of which appears at an early period, and there is thus a time at which an organ, however complex and difficult to understand as it exists in the adult, is exceedingly simple. This primitive 
condition furnishes the best possible starting point from which to follow its gradual modifications step by step until the adult form is reacled. The derivation and original anlage of most of the systems have been given above and are expressed graphically in several of the diagrams [Plates I and II; Fig. I4], but it may be also useful to introduce the chapters on the several systems by a table which shows the derivation of each. In studying this it must be borne in mind that the mesenchyme, which is everywhere distributed and forms all of the connective tissues of the body, enters into the final structure of every other part, and hence is not taken into consideration here.

Embryonal Element.

Derivative

I. Ectoderm........ Epidermis; including that of the entire external surface, as well as the more external parts of mouth cavity, rectuin, and other cavities opening to the exterior.

Epidermic structures; including all glands of the integument, nails and claws, hair and feathers, horny scales, the enamel of the teeth and the crystalline lens.

Nervous System; including brain and cord; peripheral nerves and sympathetic system with the ganglia associated with each; the epithelium of the sense organs, and the tapetum of the eye.

II. Endoderm........Alimentary canal, that is, its essential layer, the mucous membrane; also all organs derived from this, as thymus and thyroid glands, larynx, trachea and lungs, liver and pancreas.

Notochord; the anlage about which the vertebræ (mesenchymatous structures) are formed.

III. Mesoderm.

a. Epimeres... Voluntary muscles, except those of jaw, hyoid and branchial arches.

b. Mesomeres. Urogenital system, including the germ glands.

c. Hypomeres. Peritoneum; including pleura of mammals; germ-glands; voluntary muscles of jaw, 


\section{THE ONTOGENESIS OF VERTEBRATES}

hyoid and branchial arches, including the muscles of the larynx.

IV. Mesenchyme...... Connective tissues; including those in the strict sense, also cartilage and bone, and the corium. Involuntary muscles of the viscera and of the skin.

Vascular system; including heart, blood-vessels and blood; lymphatics; and the septum of the diaphragm. 


\title{
THE INTEGUMENT AND THE EXOSKELETON
}

\begin{abstract}
"Seit Huxley seine Schrift 'Zeugnisse für die Stellung des Menschen in der Natur' veröffentlicht hat, sind $3 \mathrm{r}$ Jahre vergangen, und wenn man erwägt, was in diesem Zeitraum auf dem Gebiet der physischen Anthropologie, der Embryologie und Morphologie überhaupt gearbeitet und erreicht worden ist, so ist es, meine ich, an der Zeit, den Blick wieder einmal rückwärts zu richten, das zu einem einheitlichen Ganzen zusammenzufassen, was an vielen Orten zerstreut liegt, un daraus endlich zu ersehen, was der Mensch war, was er ist, und was er sein wird."

Robert Wredersheim, Der Bau des Menschen, r893, p. 3 .
\end{abstract}

THE usual invertebrate form of integument is composed of a single layer of epidermic cells, the external surface of which is covered by a non-cellular structure formed from the cell walls. This outer element is often a transparent cuticula,* or in other cases may consist of vibratile cilia. Beneath the integument, and separated from it by a thin layer of connective tissue, lie the muscles.

The integument of Amphioxus conforms to this general type, but in all true vertebrates important changes take place, rendering it quite different in structure and of far greater complexity. The epidermis becomes many-layered and loses the external cuticula, although cilia persist in a few early larval forms, and the underlying connective tissue becomes thick, often much exceeding the epidermis in this respect. As this latter layer, the corium [cutis], is almost indissolubly as-

* The flattened outer cells of the epidermis, which form the stratum corneum, are, under certain circumstances, easily separated from the next, and form a thin layer often termed the "cuticle." The use of the word in this connection is questionable, on account of its liability of being confused with the non-cellular cuticula of invertebrates 
sociated with the epidermis, while very loosely attached on its under side to the parts which it covers, the two form together an easily detachable part, known as the skin or hide, similar in general function to the integument of invertebrates, but far more complex in structure. The vertebrate integument is further characterized by a great variety of secondary structures, involving one or both layers and either remaining beneath the surface, as is the case with glands and pigment, or projecting conspicuously beyond, as in hairs, feathers and scales.

Concerning the integument itself, in so far as it can be treated apart from its accessory organs, it may be noted that the epidermis is always several cells deep and is in constant growth, being renewed from the innermost layer in about the same proportion as it is worn off at the surface. This inner layer is a fairly definite one and is termed the stratum germinationm [str. mucosum or Malpighii]. Its cells are constantly proliferating and the older cell generations are gradually pushed toward the surface, becoming flattened and more cornified as they progress. They thus form a protective covering for the more delicate cells that lie beneath them, and compose a layer, which, in distinction to the stratum germinativum, is called stratum corneum. Some authorities distinguish for convenience a stratum lucidum, lying between the two, although the exact limits of none except the stratum germinativum are definitely fixed.

It is evident that, in order to avoid an excessive growth of these upper layers, there must be some way by which they may be continually removed. This is accomplished in reptiles and amphibians by periodic moults or ecdyses, through which the entire surface layer is cast off by a single process, and quite often in one continuous piece, after the formation of a new layer beneath it. In many forms with a cornified skin, like snakes and lizards, these cast-off "skins," the exuvic, are matters of common observation, and are seen to reproduce most faithfully every scale, horn or other protuberance characteristic of the animal; in certain other cases the cast-off skin 
is eaten by the owner. In birds and mammals there is no periodic moult, so far as the skin is concerned, and no continuous layer cast off, but the dead and dried cells are constantly being worn from the surface and pass away unnoticed. In these animals, however, there is usually a definite period for the renewal of the accessory parts, the feathers and hairs, a form of moult to be carefully distinguished from the foregoing.

The corium, in common with other connective tissues and in contrast to the epidermis, is not composed wholly of cells, but consists in great measure of fibers, which run in all directions between the cells and are produced through their agency. These fibers, which, though not the formative element of the corium, are the most important structural ones, form a rather loose and often very elastic felting, which, in many vertebrates, notably mammals, forms the main bulk of the skin. In fact, it is this layer alone, which, artificially thickened by the action of tannin, is used for leather, the epidermis being first removed by maceration. The corium is the thickest in mammals, but is also fairly thick in amphibians and in many fishes. In reptiles and birds it is thin, the amount of protection thus lost being compensated for by the dense and firm covering afforded by the accessory epidermic structure, scales and feathers respectively. Birds have the thinnest corium of all vertebrates, a condition undoubtedly correlated with the development of the feather coat, which renders the protection of a thick corium superfluous.

In the formation of the accessory organs each of the two layers furnishes materials characteristic of itself, and, although in later growth a structure that originates in one layer can, and generally does, invade the province of the other, there is a definite place of origin for each element involved. Thus from the epidermis come integumental glands of all sorts, although they usually dip down into the corium from which they receive a fibrous investment. Pigment may be derived from either layer, but more usually from the corium, and when found in the epidermis, as it commonly is, it is more likely to 
have wandered in from the corium than to have originated in place. Blood-vesscls, with the single exception of the pharyngeal mucous membrane of lungless salamanders,* are entirely confined to the corium. Sensory nerve endings of the simplest type are distributed freely through the epidermis, but the more specialized forms remain in the corium, although they may be located in papillæ pushed up into the epidermic zone. The epidermis thus forms a bloodless covering with but slight sensitiveness, the main function of which is to protect the more delicate structures included in the lower layer. Aside from this general protection afforded by the unmodified epidermis, both layers of the skin have the power of originating hard parts, which enter into the formation of certain accessory external structures that form a more or less complete exoskeleton. Thus the corium produces true bone, with the haversian canals and other osseous characters, while the epidermis forms horn and enamel, the latter superficially resembling bone, but harder and with a different structure. The structures formed from these may be composed of one substance and involve but a single layer in their formation, although the other usually coöperates in some other way, or again may be composites formed from material furnished by both layers

Thus exclusively horny structures, such as hairs or feathers, are formed from the epidermis alone, but, through the necessity of nourishment, they dip down into the richly vascular corium, which forms special organs to further this result. The dermal scutes of ganoids, and the dermal bones of higher forms arise wholly within the corium, while a tooth is a composite structure composed of dentine, a hard sort of bone, from the corium, overlaid with enamel from the epidermis.

As exoskeletal structures are universal among vertebrates, and often form their most obvious characteristics, and especially as they have interesting morphological histories of their own, they deserve special treatment, and will be taken up in the order of their appearance.

* Sce Chapter VII, under Respiration. 
The indifferent or generalized condition that serves as the starting point for all exoskeletal elements is found in the body covering of the dog-fish, which consists of imbricated rows of pointed scales, that is, rows arranged in such a way that the scales of one row cover the intervals of the one behind it. This typical arrangement is seen also in the scales of other fishes and reptiles and in the feather papillæ on the skin of a plucked
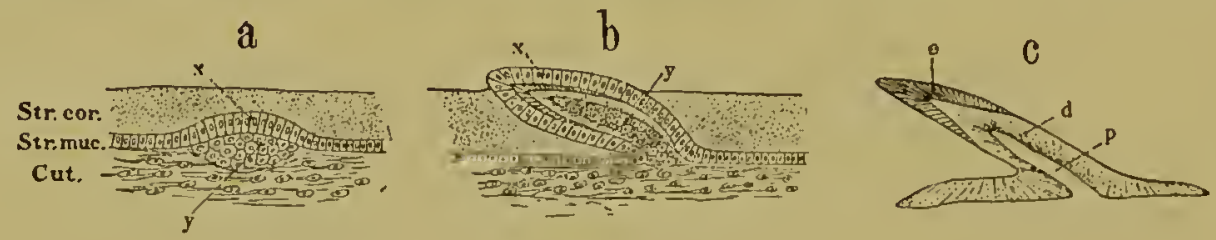

d

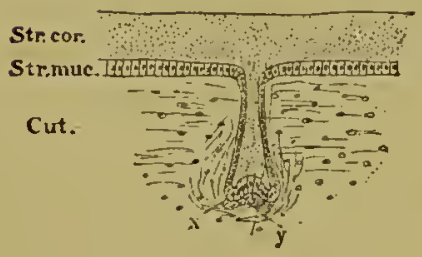

e

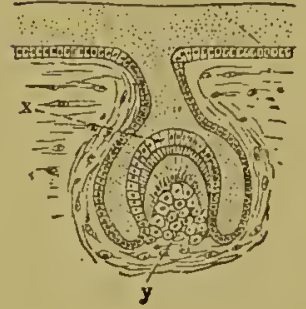

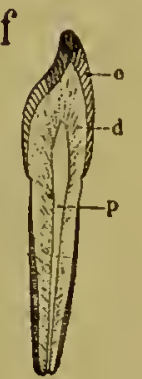

FIG. I8. Comparison in development and structure between a placoid scale and a tooth.

(a), (b), and (c) represent the scale; (d), (e), and (f) the tooth. In all the figures the stratum corneum is dotted, the stratum germinativum is represented by a layer of large cells with nuclei; and the cutis is presented as composed of fibers with scattered cells.

$x$, enamel membrane; $y$, cutis papilla; $e$, enamel; $d$, Jentine; $p$, pulp cavity.

bird. A similar, though less obvious, plan underlies the arrangement of the hair in mammals, as will be shown later.

The scales in the dog-fish are of the form known as placoid, each consisting of an approximately flat base from which rises a sharp-pointed cusp, inclined in the direction of the free edge of the scale, or posteriorly when the scale is in place. This scale is somewhat complex in structure and consists of a basis or core of dentine overlaid by a layer of enamel, especially thick over the cusp, which is almost wholly composed of it. The scale is hollow beneath and a nutrient papilla formed from the corium finds its way into the interior. It arises in 
the skin of the embryo from a fold which involves about equally both layers, and the scale develops between them, the dentine being formed from the corium and the enamel from the epidermis.

In selachians the jaws are equipped with several rows of pointed teeth, usually arranged like the scales which cover the surface, and as the former have exactly the same embryonic history as the latter and are composed of the same two layers, it must be concluded that they were once simple placoid scales like the rest, and that their later modifications have been due to the difference of the function to which they have become subjected, an inference sufficient to account for their slight changes in form as well as for their increased size and hardness, which is correlated with the greater amount of work to be accomplished. These teeth, seen here almost at their point of departure from generalized placoid scales, are inherited by all higher vertebrates, although in some cases, like turtles and birds, they have become secondarily lost. Aside from the correspondence in form, arrangement and structure, the homology is clearly shown by the development, which proceeds in all cases from a fold, involving both corium and epidermis, in which the tooth subsequently appears. These organs, when once acquired, are subjected to great variations as an accommodation for the prehension and mastication of the innumerable kinds of food; they develop as pointed needles, fangs for inoculating poison, sharp-edged chisels, flat surfaces for grinding, and ornamental tusks, in all retaining the general structure characteristic of placoid scales. The morphology of the teeth will be taken up somewhat more at length in the chapter on the digestive system, with which those parts become so early associated.

In ganoids, to which, as the lineal descendants of selachians, one should look for the next phase of this history, the scales develop from the corium alone, the epidermis remaining passive. There is thus formed a type of scale that is composed entirely of dentine, and lacks all trace of enamel. This dentine, however, is very fine and hard in character and usu- 
ally presents an extremely smooth and polished surface which has been often referred to as genuine enamel. Scales of this
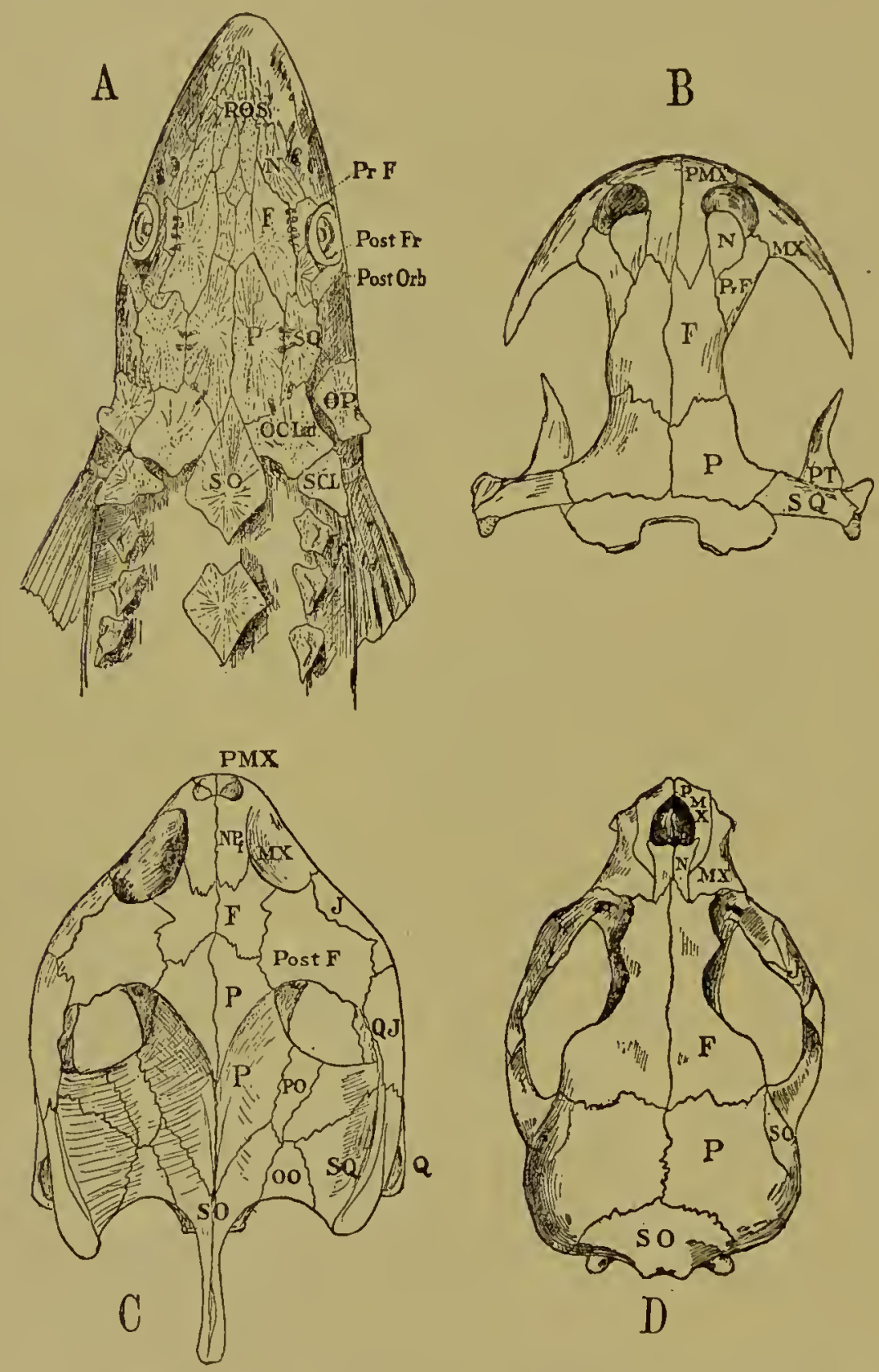

FIG. I9. Dorsal views of various skulls, showing the dermal bones.

(A) sturgeon (Acipenser). (B) salamander (Amolysloma). (C) turtic. (D) sea-lion (Otaria).

ROS, rostral plates; $N$, nasal; $F$, frontal; Pr. $F$, pre-frontal Post. Fr, post-frontal ; $P M X$, pre-maxillary; $M X$, maxillary; $J$, jugal; $Q J$, quadrato-jugal; $P$, parictal; $S Q$, squamosal; $P T$, pterygoid; $P O$, pro-otic; $O O$, opisth-otic; $S O$, supra-occipital; Oc. Lat., lateral occipital; $O P$, opcrcular; S. Cl., supra-claviclc.

type, termed ganoid ' $i$. c., shining; from which the Order receives its name), are usually rhomboid in shape and lack the 
cusp or point of the placoid type. In the sturgeon the scales consolidate into large, bony shields or scutes, and this principle was carried to the extreme in the long extinct and nearly related groups of placodcrms, where the entire fish was covered with a heavy suit of mail, probably as a protection from the huge molluscan forms which then thronged the seas. In the sturgeon, however, these plates are not continuous, but are arranged in longitudinal rows along the back and sides, leaving large areas unprotected. In all ganoids similar scutes corer the entire head, and fit together by their edges, forming sutures, but leaving no appreciable intervals. These are fairly definite in number and arrangement in the different species and form the so-called dermal bones of the skull. [Cf. Chap. V.] Certain of these, as the frontals, parietals, maxillaries and squamosals, persist in the highest groups; others, like the opercular and rostral series, disappear completely, while of an extensive orbital series one alone persists as the lacrimal. The dermal bones that line the mouth cavity, such as the vomers, palatines and parabasal, retain the indications of their origin longer than do the others, since, in many cases among both fishes and amphibians, they are covered with teeth which are arranged in imbricated series over a considerable area; occasionally, even, as in the vomers of the frog larva, these elements begin as separate conical teeth which fuse secondarily to form the plate, thus repeating ontogenetically their mode of origin. Nearly always, however, the history is curtailed, and the dermal bones first appear as thin, lace-like structures, lying in the sub-cutaneous connective tissue, and enlarge from definitely located "centers of ossification" by marginal additions.

The scales of teleosts are developmentally and structurally like those of ganoids, the ancestors of the group, but in form, although often rhomboid in the young, they become approximately circular, and are hence termed cycloid. Ctcnoid scales are a variety of this in which the inner margin attached to the skin is extended into numerous small processes like the teeth of a comb.

Modern amphibians have a soft, slimy skin, without exoskeletal structures of any kind, save in the rare order of cocil- 
ians (Gymnophiona), in which scale rudiments lie in pits sunk beneath the surface. In the extinct group of Stegocepha!i, which possessed many amphibian characters, the body was covered ventrally with well-cleveloped, imbricated scales. These facts together furnish sufficient proof that amphibians were originally scaly and that the present naked condition is due to a secondary reduction. These scales were probably bony, like those of ganoids and teleosts.

It is an abrupt transition from the scales of fishes to those of reptiles, since, in this latter class, the scales are purely epidermic in origin and are composed of horn (keratin), a substance allied to enamel, without trace of bone. The corium, it is true, nourishes the scales by means of richly vascular papilla placed beneath each, but furnishes none of the hard parts. There is no doubt that in some way these scales must be related to the bony ones of ganoids and teleosts, but the relation appears to be an indirect one. They may have had a common origin in scales which, like those of the placoid type, possess both elements, the one emphasizing the epidermic portion, the other that of the corium. This would seem to conflict with the direct derivation of reptiles from the ganoids as we know them, and shows the incompleteness of our records at this point.

Aside from scales the reptilian integument possesses a great variety of other exoskeletal forms, such as spines, combs, and claws, all made of keratin, and equally unlike anything in ganoids or amphibians. In this wealth of horny exoskeletal elements the reptiles are closely followed by their lineal descendants, the birds, where the scales are represented by the far more elaborate, but strictly homologous, feathers, and where beak and feet are encased in horny coverings. The covering for the beak has evidently replaced teeth, as in turtles, and is undoubtedly a recently acquired character, since fossil birds occur in the Cretaceous formation, in all respects like modern birds save in the presence of conical teeth set in sockets; furthermore, tooth germs have been found in the jaws of the embryos of several species of modern birds, transitory 
in character and never developing far enough to break through the gums.

The scales of mammals are commonly little emphasized, owing to the conspicuous nature of the hairy coat, principally associated with them, but they are, nevertheless, of great morphological value. They occur. in definite regions and only in certain forms, but are so widely distributed that, were all other reasons absent, their former more extensive distribution would be strongly suggested. In most cases they are found only on tails and paws, but in the Manidce, an edentate group, the entire dorsal surface of the body and limbs is covered with large, imbricate scales, and in the closely related armadillos, similar scales fuse to form a dorsal carapace, as well as shields for the head, tail and limbs. Scale formation on paws and tail occurs mainly in marsupials, rodents and insectivores, and may be seen particularly well on the dorsal surface of the paws of moles and shrews, or on the flat tails of the beaver and muskrat, in which the scales are usually rounded and regularly imbricated. Where the tail is cylindrical, as in the rats and mice, the scales are arranged in rings, those of one row standing in imbricated relation to the one which it overlaps.

In structure the scales are epidermic, like those of reptiles, underlaid by corium papillæ. They usually remain more or less embryonic, and the epidermis, though cornified, does not develop definite hard parts, but in the Manida distinct horn scales are produced, as thick and heavy as those of reptiles, the main difference being that there are here no periodic ecdyses, and the scales are shed and renewed singly, as occasion requires. In young armadillos the scales that form the carapace and shields are like those of Manis, but they become soon reinforced by ossifications of the corium, one for each scale, which enlarge and finally fuse to form an osseous substructure. These corium elements are plainly secondary structures and are not to be considered as primary elements of the mammalian scale, which, as stated above, is entirely epidermic. Aside from the sporadic occurrence of scaled areas in 
various mammals, as previously noticed, a more definite proof of the former completeness of the scaly coat is found in the relationship between scales and hairs in scaled areas, and in

a

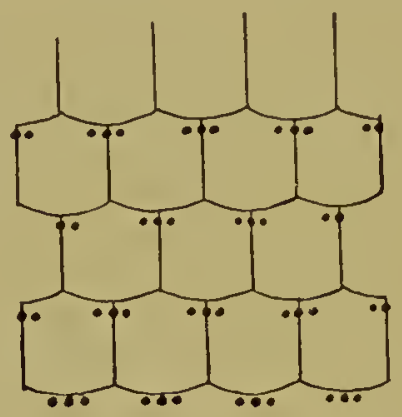

b
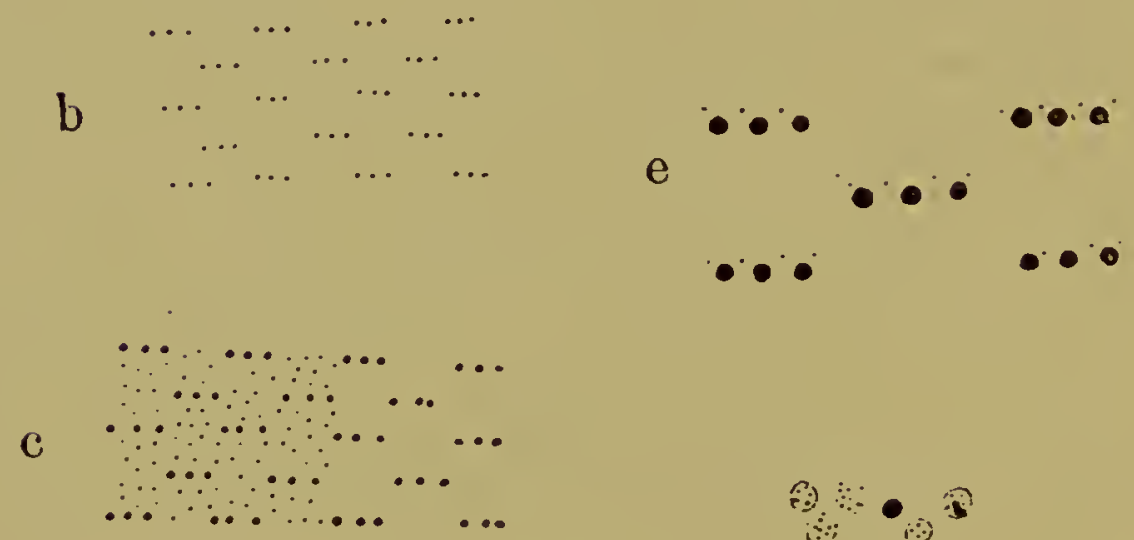

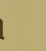


will be noticed that scattered hairs appear among the scales in a definite relationship, and that a group of three hairs, one median and two lateral, projects from beneath the margin of each scale, the median hair being somewhat longer and stouter than the others. It further appears that there is a similar arrangement of hairs, usually in groups of three, upon hair arecis not associated with scales, the hair groups being arranged in imbricated series, and that this arrangement is general, even in nuammals without trace of scales. There are some modifica-

a
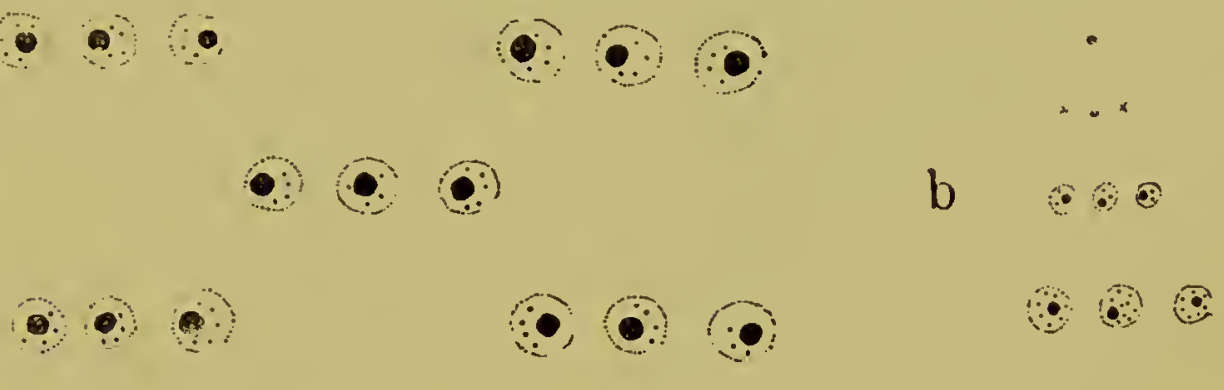

FIG. 2I. Hair arrangement

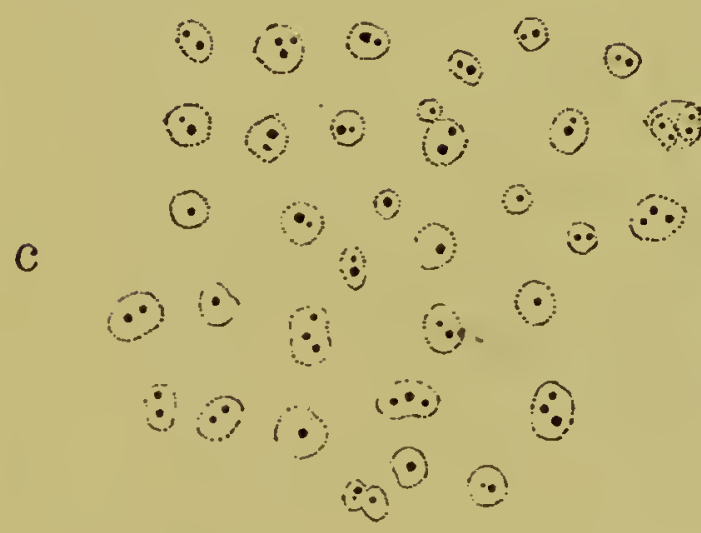
in various maminals.

(a) Ursus arctos (brown bear). Front of chest. Diagrammatic. [After DE MEIJERE.] (b) Canis familiaris (dog). Four developmental stages. The adult arrange. ment is like that of (a). [Nfter DE MEIJERE.] (c) Homo. Scalp of negro. Camera drawing from the actual object.

tions of this, due to secondary changes, such as the need of a thick fur, but even in these modifications the original plan is still apparent. Thus, in the pig, there are two sets of bristles, a coarser set arranged in imbricated groups of three, and a finer set, filling the intervening areas without definite arrangement; to obtain a thick fur, as in the rabbit, each hair in the group may become a bundle of hairs, the bundles being arranged in groups of three as in the typical case; even the number three is not always kept, and groups of five occur, 
with two lateral hairs on each side, or groups of seven with three. Occasionally, as in the dog and cat, the plan becomes partly obscured in the adult, but is evident during development,
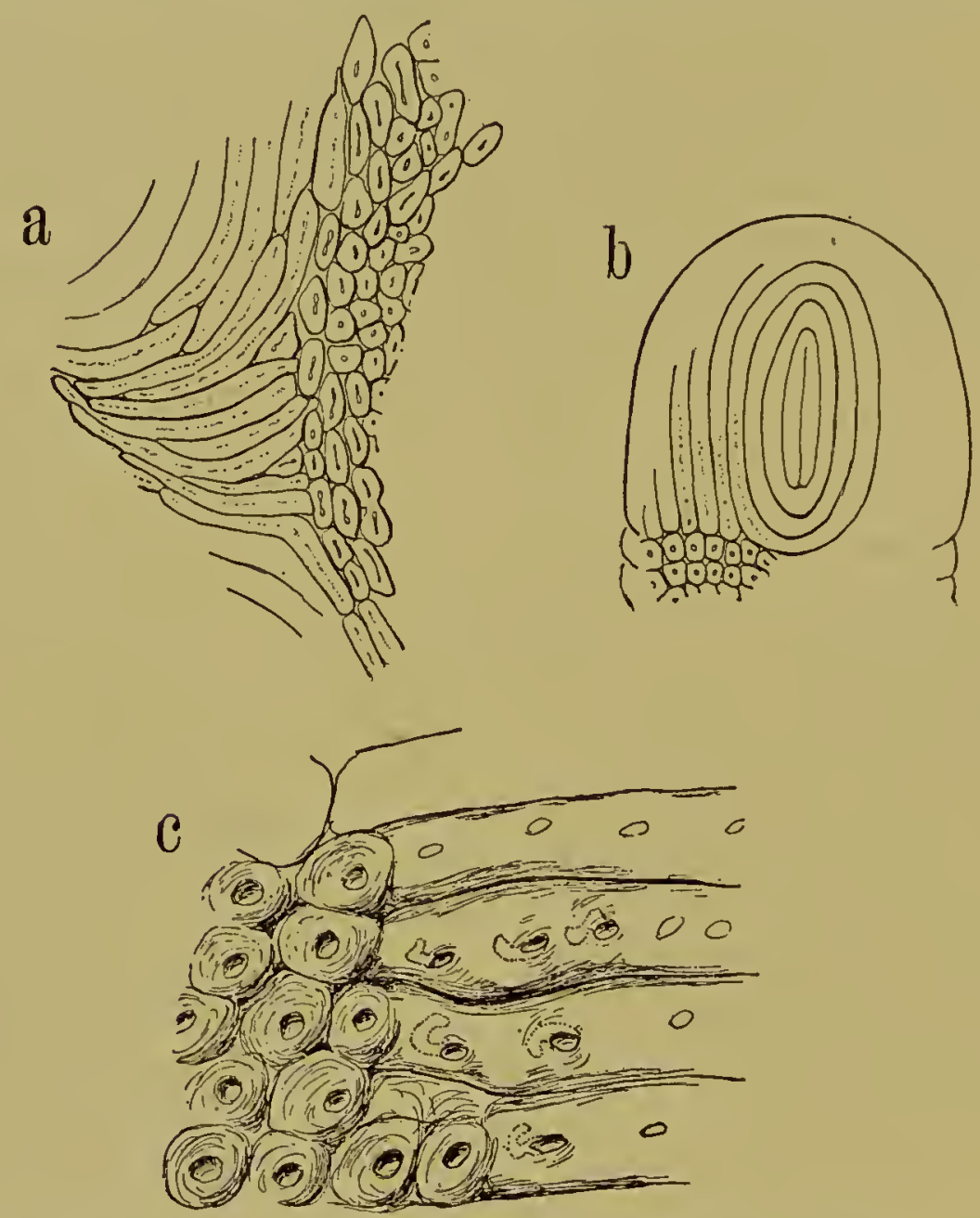

FIG. 22. Formation of friction ridges from single rows of epidermic warts. [After Miss Whipple.]

(a) Midas rosalia. (Brazilian monkey). Proximal portion of hypothenar pad. (b) Midas rosalia. Apical pad. (c) Homo. Advanced fetus. Side of finger in tran. sition region. The dotted lines indicate the position of sweat-glands.

the three-hair group being definitely marked in the advanced fetus.

A still further corroboration of the former presence of scales in mammals may be obtained from the study of the lower surfaces of the paws, where, except in such extreme modifications as the ungulates, scales either still exist or have left a permanent record in a peculiar configuration of the epidermis, 

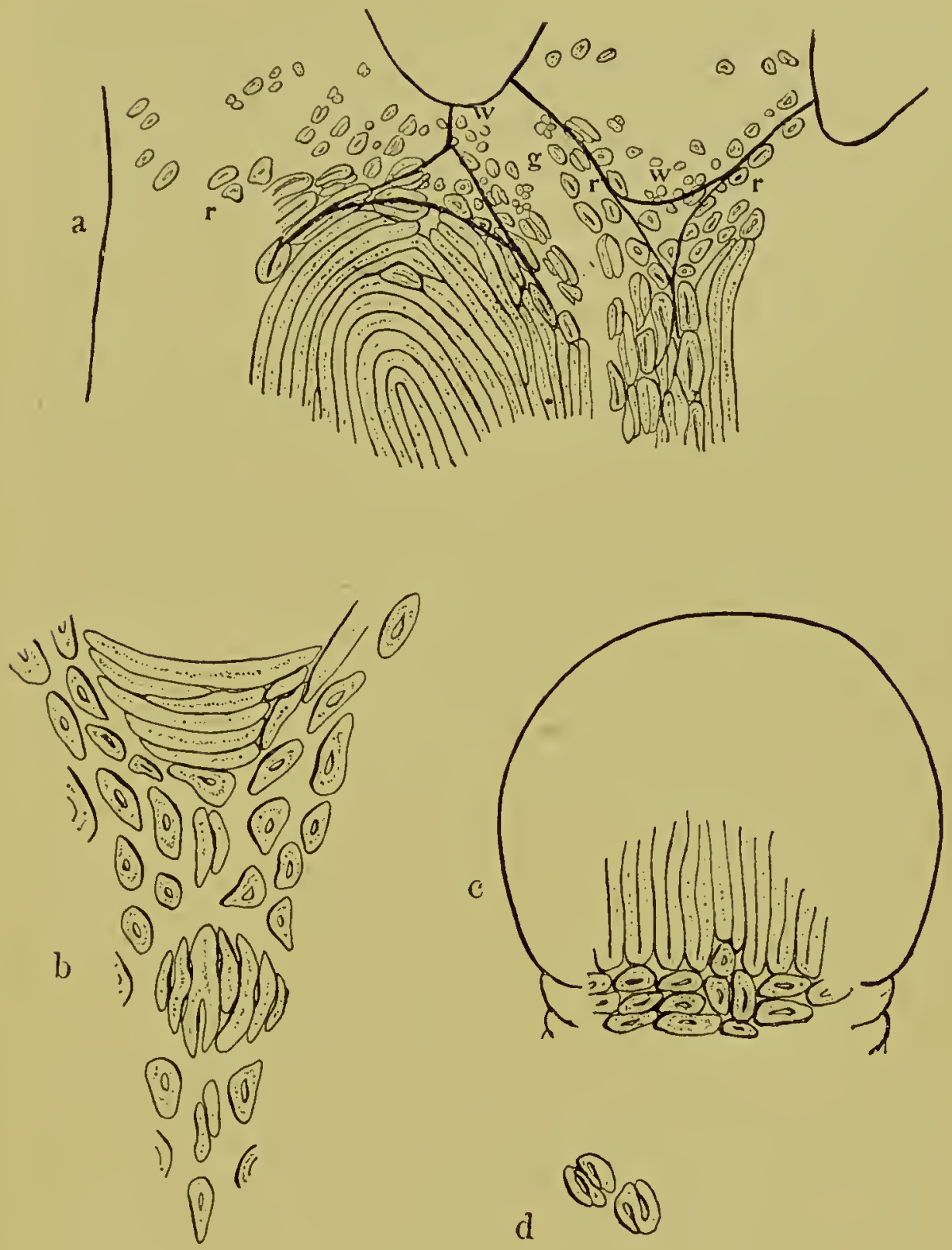

FIG. 23. Formation of friction ridges in pairs from rings formed by the confluence of epidermic warts. [After Miss Whipule.]

All the figures are taken from Lemur macaco (semi-ape from Madagasear).

(a) Detail of area below the interspace between index and medius. Here are seen individual isolated warts w; groups of these forming rings $g$; fully formed rings $r$; also the formation of ridges in pairs by the lengthening of rings in one of the pattern is also suggestive. pad. (d) Detail showing two Apieal perthods of formation of three ridges from the rings. 
directly traceable to them. In their simplest form the scales or scale rudiments are in the form of rounded, wart-like epidermic organs, which cover the entire surface in Ormithorhynchus and large portions of it in marsupials, insectivores and lemurs. They possess a more or less imbricated arrangement, and their identity with scales is shown, not merely by their structure and development, but by a comparison with the scaled dorsal surface of the paw in such cases as that of the shrew or the star-nosed mole, where the transitions from one form to the other may be seen along the edges of the paw. This primitive condition is modified in most cases by the presence of characteristic mammalian organs, the pads, which are used as contact surfaces, and are typically eleven in number, five for the tips of the digits, four for the distal margin of palm or sole, below the interdigital intervals, and two near the wrist or ankl.. Upon these the scale rudiments become arranged in rowes, and by their fusion form friction ridges, so called from their use, which is to prevent slipping, like the parallel ridges seen on the handles of certain steel instruments. These friction ridges are always arranged at right angles to the direction in which there is the greatest tendency to slip, that is, directly across the pads in walking forms, but arranged in concentric circles about the highest part of the pad in the arboreal lemurs and monkeys where slipping in all directions is equally to be expected. Owing to the general principles that the separate scale rudiments form friction ridges on the actual contact surfaces only, it follows that when the pads remain high their surfaces alone are ridged, while the depressed areas are covered with separate units, but when, as in lemurs and monkeys, there is a progressive tendency to utilize the entire surface for contact, the ridged areas spread in exact correspondence with the acquirement of contact surface, until, in the higher primates, the entire ventral surface of the pazes becomes covered with ridges, leaving separate scale rudiments only along the boundaries, where this modified skin meets that of the dorsal surface.

Had the friction ridges, which completely cover the palmar 
and plantar surfaces in Man and the other higher primates developed primarily in forms in which the entire surface was used for contact, it may be assumed that they would have taken some simple form, designed with reference to the area as a whole; since, however, they have passed through the longer and more complex history caused by the introduction and secondary reduction of various pads, they have preserved the indications of the former relief by an arrangement otherwise without cause or meaning. This may be seen by a comparison of the lower surface of the paw in some animal in which the pad system is in full function with that of one in which the inequalities of the surface have become secondarily reduced. (Fig. 24.) The one is an actual relief, the other a flat sketch; the one possesses raised pads surrounded by folds of skin which diverge in three directions from points known as triradii, the other indicates the former location of the pads by whorls and other patterns, and that of the folds by the arms of embracing triradii. Thus in the field-mouse (Fig. 24, a) there are present four interdigital pads, the first situated immediately below the interval between thumb and index, the second below the interval between the latter and digit III, and so on. Each of these is inclosed by folds of skin which diverge in three directions from points known as triradii, and there are three triradii about each pad except the third, which possesses a fourth one, located between digits III and IV. Below these lie the thenar and hypothenar pads, the folds of which are often well marked, though not especially so in this case. The apical pads at the ends of the digits also possess folds, not well shown in the figure, with two triradii, one upon each side. Turning now to the paws of Macacus, a small monkey (Fig. 24, b), in which the relief has been reduced to a flat surface, each of the above features (except the thenar in this especial case) is expressed by the configuration of the ridges, as indicated in the figure. The ridges essential in marking the paim are represented by solid lines, although in reality not different from the rest. Each pad is represented by a figure or pattern, of which the four interdigital are the 
most typical, and are in the form of concentric circles, the center coincident with the summit of the pad. The spiral form
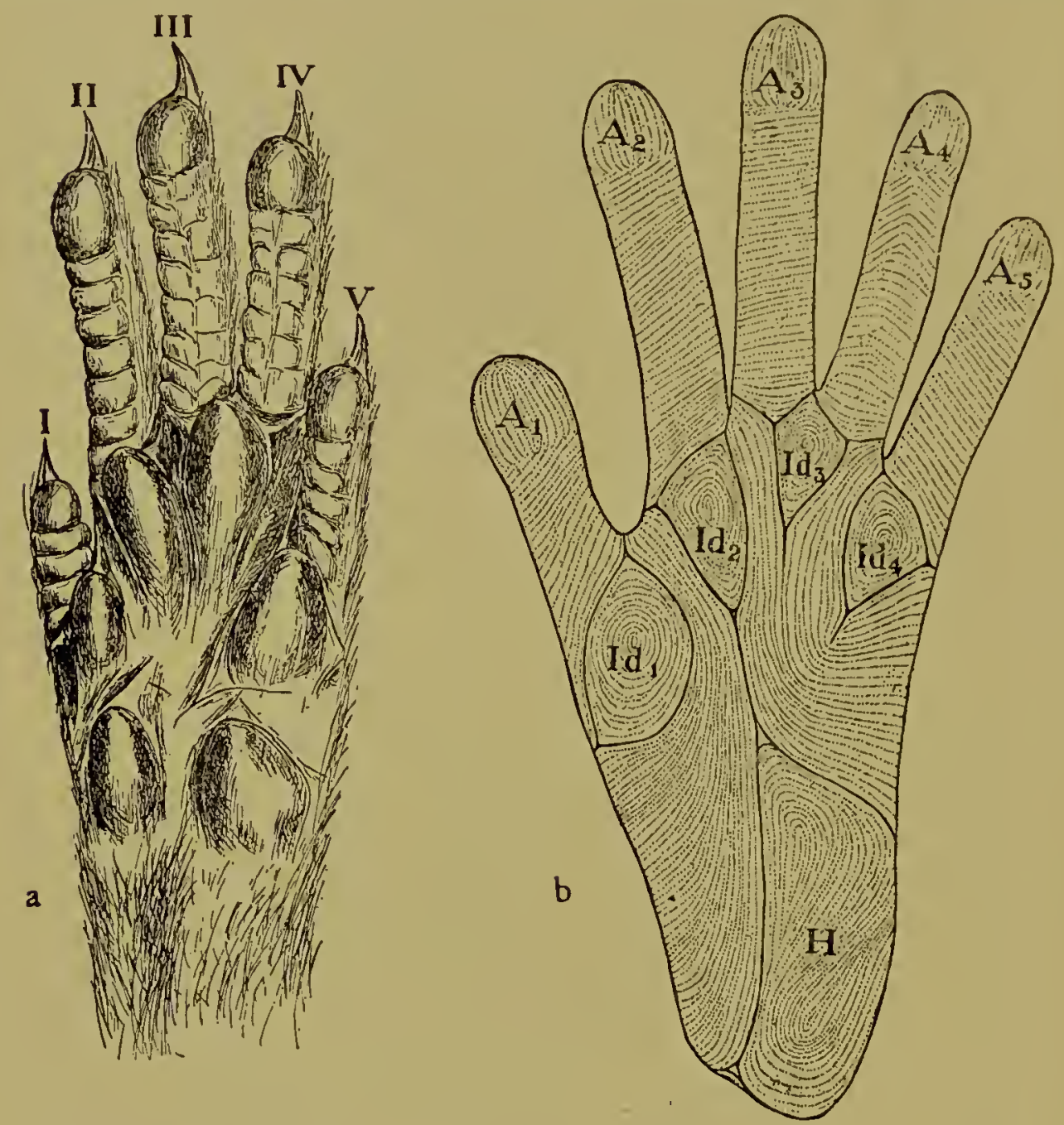

Fig. 24. Ventral surface of anterior chiridium of an insectivore and of a primate showing correspondence between relief and arrangement of friction ridges. [After Miss WhipPLE.]

(a) Crocidura corulea (shrew-mouse). Fore paw showing walking-pads enclosed by triangular folds of skin. (b) Macacus sp? (Old World monkey). Hand, covered by friction ridges, the arrangement of which corresponds to the relief of (a). The pads are represented by concentric circles, and the triangular folds by triradii. These latter features are here designated by heavy lines, although in the real object they are not more conspicuous than the others.

of the hypothenar is a degeneration from the primitive type, to which it is connected by the existence of transitional forms, either in other individuals of the same species or in different 
species. The thenar pattern has here become entirely reduced, but is often present. The apical patterns are also modified, but in a lateral view would show the triradii.

Inasmuch, however, as in the Primate hand and foot the ridges are still of considerable functional importance, they are apt to become modified at each point of the surface in accordance with the use of that point, and it thus happens that in different species and in different parts of the surface there are varying degrees of faithfulness to the ancient records. Thus in Macacus the use of the hand is such that thenar, hypothenar and apical pads tend to degenerate, while the interdigitals are preserved in their typical relations, while in the human hand the reverse is the case, and the apical patterns are nearly always well marked, and often in the form of typical whorls with two lateral triradii; while the interdigital patterns are usually lost or obscurely indicated. A hypothenar pattern is frequent, especially in the white race, and occasionally occurs as a whorl with three triradii; but the thenar is of rare occurrence, and then usually associated with the first interdigital. These changes are in part explained by the tendency of the ridges to assume an approximately transverse direction, a tendency in which the right hand has surpassed the left, owing to the long preferential use of the former.

In the human foot the apical patterns are about as well marked as in the hand, but with a smaller percentage of the primitive whorl type; the four interdigital pads are fairly well indicated and often appear in infants as rounded elevations. Of these the most constant is the first, placed on the ball of the foot below the great toe, and is frequently of the whorl type, occasionally with three triradii; the primitive condition again corresponding to the functional importance of the region which here bears the main force of the body during a portion of each step. The hypothenar is occasionally indicated by a loop on the outer edge, but the thenar is practically lost. An additional loop, of uncertain morphological significance, occasionally occurs on the heel. 


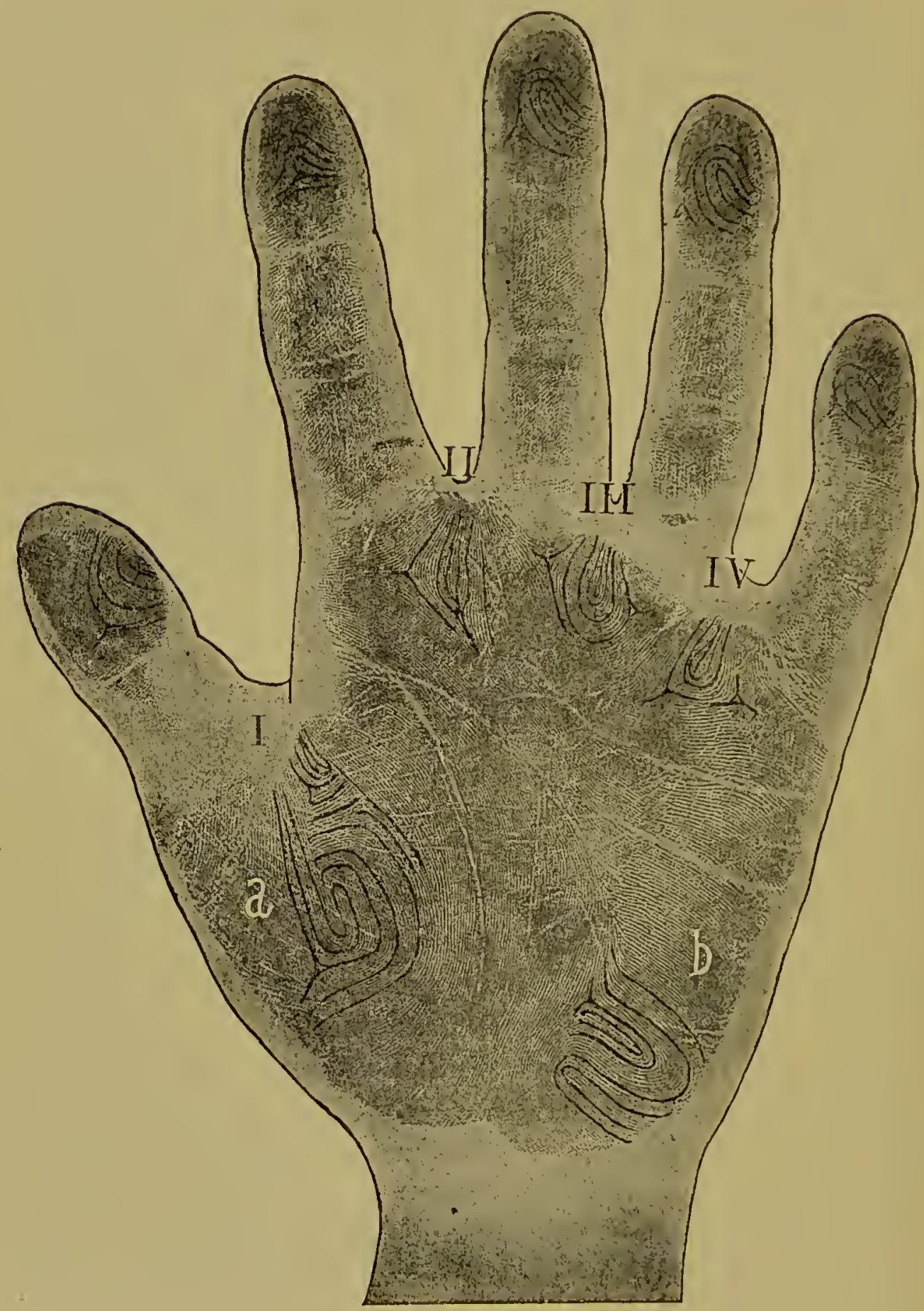

FIG. 25. Print of right hand of boy (Anglo-American), showing a complete set of friction-skin patterns.

I, II, III, IV, the four interdigitals; $a$, the thenar; $b$, the hypothenar; the five apical patterns (not lettered) are seen on the finger-tips. 
It thus appears that, aside from the sporadic distribution of scales in various mammals, the palmar and plantar surfaces, save in the most modified cases (Ungulates, Cetacea), are covered with scale elements, either distinct or united in rows to form ridges; and, furthermore, that in other parts of the body the hair follicles occur in definite groups, arranged in alternate series; facts that can be interpreted only as indicative of the former presence of a scaly coat.

That this stage is actually passed through in embryo mammals has not as yet been definitely determined, but some circumstances seem to indicate that the vestiges of this covering may be looked for in the epitrichium, which is a superficial epidermic formation without definite structure so far as is known. This at one time covers the surface, but save in the palmar and plantar regions disintegrates and disappears; contributing in man to the formation of the vernix caseosa, found upon the surface of the new-born infant. Upon the palms and soles, however, at least in man, where it has been mainly studied, it appears to persist and take part in the formation of the friction ridges.

This brings with it the suggestion that the epitrichium represents the primitive scaly coat of ancestral mammals, the greater part of which becomes lost by an embryonal ecdysis. How this epitrichium appears and what its fate is on surfaces where scales persist, other than the palms and soles, or in the few scaled mammals, is not known; but in the sloths and ant-eaters, nearly related to the last, it is especially firm and remains until birth as a definite covering. In many mammals the similarity to a moulting external layer is increased by the presence of a thick layer over the nails or claws, continuous with the epitrichium, and cast off with it, the eponychium.

The hair, which forms the characteristic coat of present-day mammals, may be safely considered as once accessory to a covering of scales, which it has secondarily replaced, as explained in the foregoing, but this does not account for its origin, or suggest its primary function. An attempt has been made to homologize hairs with the integumental sense organs 
of amphibians, owing to a similarity in the early stages of development; but although this view may receive some little extra support from the considerable degree of sensitiveness

a

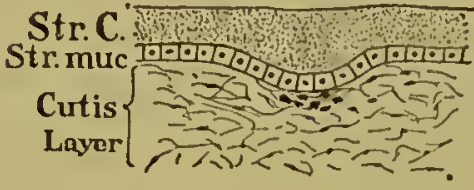

b

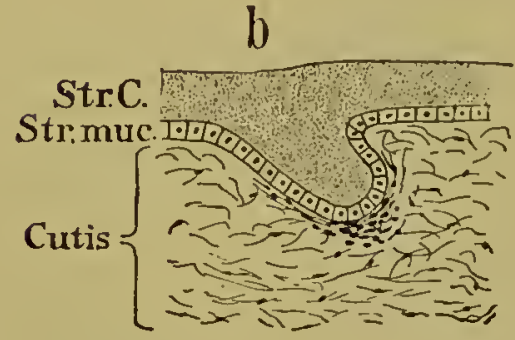

C

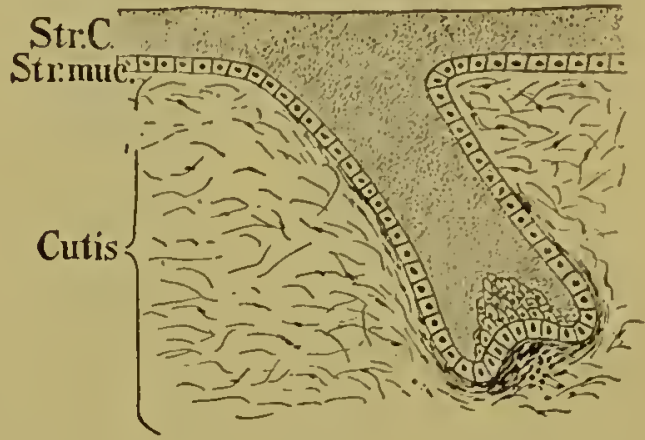

d

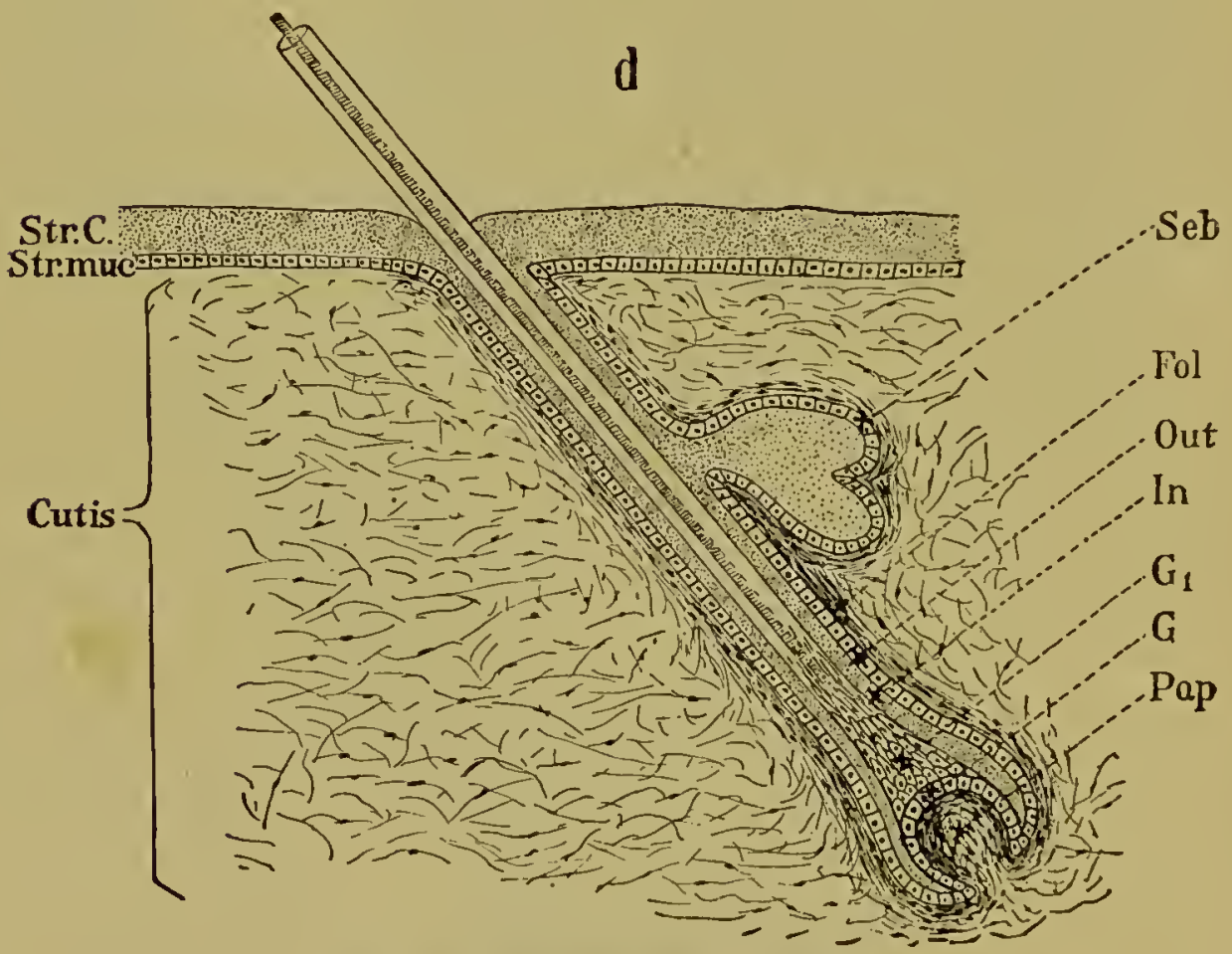

FIG. 26. Development of hair.

sir. $c$, stratum corneum; str. muc., stratum germinativum; seb, sebaceous gland; fol, follicle; out, outer root sheath; in, inner root shcath; $G$, hair germ; $G_{1}$, beginning hair; pap, corium papilla.

which some hairs attain, the evidence in favor of it is slight, and the idea does not receive general credence. It seems likely that the hair developed subsequently to the scales and 
not before them, and may have exercised some function of protection, possibly that of a fringe upon or near the free edges to prevent the accumulation of dirt in the folds where they overlap, a purpose for which organs of similar appearance but of different origin are frequently employed in insects and crustaceans.

In development the hair is wholly epidermic, formed by the stratum germinativum, but dips down into the corium in the form of a solid column of rapidly proliferating cells, the outer layer of which soon differentiates into a sheath or follicle, while the inner cells become horny and form a shaft which projects beyond the surface and becomes the hair. Growth is constantly kept up at the bottom of the follicle, and proceeds from a small area of actively proliferating cells which are nourished by a corium papilla and form the true root, or matrix, of the hair. From an inspection of the following figure (Fig. 26), it becomes evident that this matrix is merely a specialized portion of the stratum germinativum and that the hair consists of the upper layers derived from it, and renewed from beneath as in the superficial skin. When a hair is pulled out, the break usually occurs immediately above the matrix, and the lost portion involves the hair, the epidermic sheath, and quite often the follicular sheath as well, parts that are easily regenerated so long as the matrix remains. Associated with this structure are typically two sorts of glands, tubular and acinous, which are formed as outpushings from the sides of the follicle and grow down into the corium. These develop in various mammals to subserve many different purposes, often becoming dissociated from the original connection with the hair. To these two types all forms of integumental glands occurring in mammals may be referred. Their modifications and transformations may be considered later.

The occurrence and distribution of the hair are in strict accordance with the needs of the animal, and show great differences, corresponding to the various environments to which mammals have become adapted. The hair may differ in length, in caliber, in thickness (i.e., the number of groups in 
a given area), in texture, or in form; it may be increased to a thick, matted wool, or may show every degree of reduction down to a total loss. It may develop into bristles, as in the hog, or even form spines, as in the hedgehog and porcupine, although this latter result is usually brought about by the confluence of numerous individual hairs. The "horn" of the rhinoceros is such a structure, and not a true horn. Many variations in thickness are brought about by modifications in the hair groups, or by the interpolation of supernumerary hairs independent of the group system. In the former case the number of single hairs in each group may be increased, or each primary hair may be represented by a bundle; or again, each primary hair may be accompanied by a series of accessory hairs, arranged as satellites about the former. In the latter case there is usually a marked difference between the hairs that are included in the primary system and those that are not, as is seen in the case of the hog, in which there are two sizes of bristles, coarse ones in groups, and finer ones interpolated without system (Fig. 20, c).

It may be said in general that arctic forms and those living at high altitudes are the most plentifully supplied with hair, while tropical and sub-tropical forms are sparsely covered. An aquatic life tends to reduce the hair coat; if the animal is but semi-aquatic, as seals and otters, the hair is reduced to the form of a fine plush, but in the Cetacea and Sirenia, which are wholly aquatic, the reduction is almost a complete one. Many apes are but scantily supplied with hair, the ventral side of body and limbs being but sparsely covered, while the upper part of the face and ears are nearly bare. The same tendency is continued farther in Man, who shows considerable racial variation, ranging from the hairy Ainus and certain hairy individuals in the white race to the smooth and beardless Malays. That Man was formerly supplied with a thick coat of hair, however, is shown by the fetal condition, at one stage of which the entire body, not excepting the face, is covered by a coat of fine down, the lanugo. This mainly disappears before birth, and becomes eventually re- 
placed by the permanent coat, which usually shows but slight development save in certain definite localities. The lanugo persists in a reduced condition on the face, especially in females, forming the down which gives to the cheeks their characteristic bloon. Abnormal hairiness in man, or hypertrichosis, is fortunately rare, and is of two kinds; the one, hypertrichosis vera, is due to an excessive growth of the permanent coat which replaces the lanugo; the other, pseudohypertrichosis, is the result of the persistence of the lanugo.

Localized hypertrophy in various mammals in the form of manes, crests or tufts of hair, is of frequent occurrence and is used for various purposes, such as defense from flies or other noxious insects, attraction of the other sex, or as a protection from the teeth of rivals. Under this general head come also the beard of man, which corresponds in position and direction to that found in other primates, and the long hair of the head. The other locations in Man in which long hair occurs, the axillary and pubic regions, do not seem to belong here, and probably represent portions that escaped reduction rather than hypertrophy. The obvious function of the cranial hair is a protection from the sun, and its location suggests that it is developed with reference to the erect and not the quadrupedal position, in which latter case it would have extended farther down the back. The axillary and pubic tufts may be for lessening the friction between the limbs during motion; it has been also suggested that they possessed a use in transitional forms in furnishing places to which the infant might cling, thus leaving the arms of the parent free for climbing. In support of this latter view it may be noticed that the distances between these locations correspond approximately to the proportions of a normal infant, and that an infant thus attached is also in the right position for nursing.

Aside from differences in caliber and length, the hair of various mammals differs markedly in structure, in color, in the shape of its cross-sections in various places and in the shape assumed by each hair. In structure a hair consists of a firmer cortex of varying thickness enclosing a softer medulla; a 
single layer of epidermic cells covers the cortex externally. Differences in color and luster are due to the amount of pigment in the cortex, the sculpture of the epidermic covering and the presence or absence of air in the medulla.

The cells of the epidermic covering may fit smoothly upon one another or may project like scales. A typical illustration of this latter case is that found in wool, and by virtue of this peculiarity the separate hairs may be made to cling together by causing the minute teeth to interlock, a result effected throught the act of spinning. To this peculiarity the possibility of wool as a textile fabric is due.

In Man there is much racial variation in the hair of the head, a character of considerable value in ethnology. The degree of waviness or curliness is due to the shape of the single hairs; if they are cylindrical, that is, circular in crosssection, they are perfectly straight, as in the typical Mongolian; a slight degree of flatness with an elliptical cross-section, allows the hairs to become wavy, as in many Europeans; if more flat, they are curly, and if very flat, the hairs are woolly, as in the Negroes. In this last class there are two subdivisions, the Eriocomi, where the hair is evenly distributed, making a solid mat, and the Lophocomi, the "cheveux en grains de poivre," in which the hair is collected into little tufts with partings between them. This latter peculiarity is seen in adult Bushmen and Hottentots and in the children of most other negro races.

The degree of flatness of the cross-section is expressed by an index in which the longer diameter is considered unity and the shorter is compared with it in the form of a decimal fraction. Thus, in a perfectly cylindrical hair, the index would be Ioo, in one in which the breadth of the oval is half the length the index would be 50 . As a matter of fact there is no index so high as 100 , but it ranges between 85 , that of the Japanese, and 40-50, that of the Hottentots. In Europeans it varies between 62 and 72 . In length the hair varies greatly, straight hair being the longest and woolly hair the shortest. In races with either extreme (straight or woolly) 
the hair of the two sexes is of equal length, but in those with way or curly hair that of the female considerably exceeds in length that of the male.

The hair exhibits a definite slant or direction of grozeth, which varies in different parts of the body, so that one may speak of hair-streans or hair-currents. This direction is the one shown by the follicle and by the hair immediately after its emergence from the skin, and is entirely unrelated to the various directions which the free masses may temporarily assume under the influences of gravitation, wind, or other external forces. It is thus best seen in animals with a coat of short, appressed hair, like horses or short-haired dogs, and is often quite obscured in those with long hair, or in those with soft, plush-like fur, like seals and moles. In these latter, however, it may be accurately ascertained by shaving or clipping the hair.

In general it may be said that a given area. shows a definite direction, the lines of which may be parallel or somewhat divergent, two adjacent areas being separated either by a parting, where the streams diverge from one another, or by a raised crest or seam where they converge. At certain points special features may be noticed, the most important of which are the vortex or whorl, the rhomboid and the feathcring. In the vortex variously directed hair currents unite to form a spiral figure, which either converges to form a central tuft, convergent vortex, or starts at the center and diverges; divergent vortex. The first type of vortex often marks a point at which some projecting organ is later to appear, as at the corners of the forehead in the calf before the appearance of the horns; or else one where a former projecting organ has disappeared, as at the umbilicus; but, on the other hand, there are numerous instances where such a relationship cannot be established. The significance of the second type is unknown. Either type may form either a right- or a left-handed spiral (clockwise or contra-clockwise). A rhomboid is an open space of the shape designated by the name, and appears where the corners of four areas meet. It is thus 


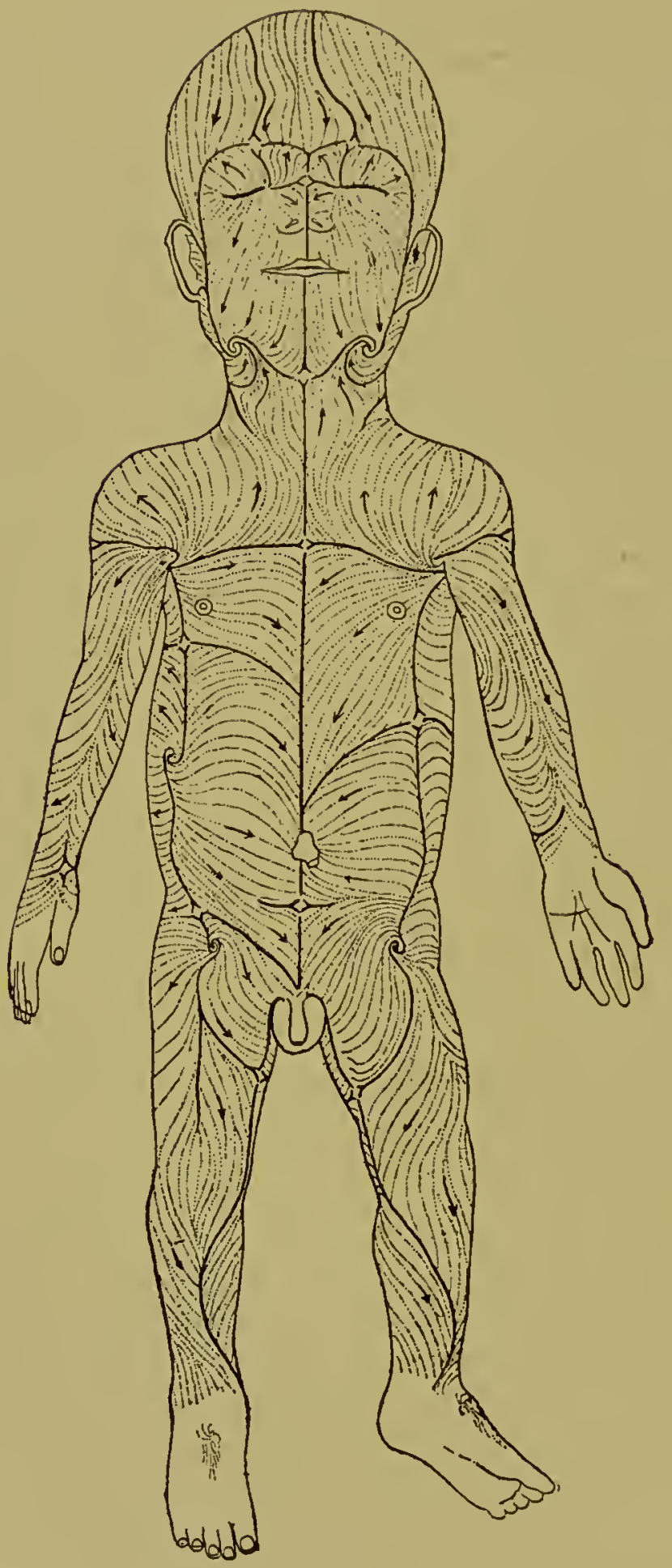

FIg. 27. Hair direction in human fetus. [After Vorgr.]

The black lines designate the lines of parting, the arrows show the direction of the hair currents. Rhomboids and vortices are also shown. 
always so arranged that the hairs converge at two opposite corners and diverge at the other two. A feathering is a special form of area, usually more extensive than the two last, occurring only in association with a vortex, of which it forms a continuation in one direction. It is in the form of a long and narrow ellipse, and the hair currents run along a central axis and diverge to the margin.

All of the above forms may be readily seen upon our domestic animals, and are often well marked in man, especially in individuals whose skin is covered with very short appressed hairs. An especially good object is the broad, square chest of the bull-dog, on which are usually three vortices and three rhomboids; a vortex above and a rhomboid below in the median line, a lateral rhomboid on each side of the vortex, and a lateral vortex on each side of the rhomboid. Aside from these there occurs a vortex on each elborv, usually one on each side of the neck, and upon the hinder parts a pair of especially conspicuous vortices, above which, at the base of the tail, are two rhomboids. Individual variation may show departures from this description.

In Man the various features are present and often well marked, but as they require for their expression a certain grade of pilosity, they are usually overlooked. Here, also, as in other animals, there is considerable individual variation, and a feature marked on one person may be absent on another; the two sides, also, are not necessarily symmetrical. The most conspicuous vortex is the one at the crown of the head, easily observed in boys with short hair. This may be either clockwise or contra-clockwise, and seems to follow no rule in this respect. Other vortices occur above the angle of the jaw and in front of the axilla. Rhomboids occur along the mid-ventral line; one of them is situated at the angle between the throat and the chin, immediately above the thyreoid protuberance, a second at the anterior end of the sternum, and a third on the abdomen, midway between the umbilicus and the pubic eminence. A rhomboid is found constantly upon the lower part of the ulna, a little above the wrist. 
The study of hair direction has excited an occasional interest among morphologists, and a number of theories have been advanced to explain the origin of the various features, but there has been as yet too little morphological work in this field to allow much theorizing or to serve as a basis for definite conclusions. The general tendency of the hair to slope backwards from the point of the nose to the end of the tail suggests the influence of the air-currents upon a rapidly moving body, or at least an adaptation to them, the same phenomenon being strikingly exhibited by the direction of feathers in birds, and that of scales in reptiles; in the same way the general downward slope of the hair along the sides of quadrupeds suggests the influence of gravitation, especially when taken in connection with the apparent hair direction in the sloth, which shows a parting along the mid-ventral line and is directed ventrodorsally, as if in correlation with the customary inverted position of the animal. In opposition to this, however, it may be pointed out that in certain areas the direction is the reverse of that which either of the above forces would produce, and as for the case of the sloth, the direction observed may be that assumed by the long hair after emerging from the surface, since the direction of the follicles seems never to have been investigated. Darwin's well-known attempt to attribute the hair direction on the human arms to the direct influence of tropical rains upon the arms of simians, when held above the head for protection, is at variance with the facts, and hence must be dismissed from the discussion.

Recently a new line of explanation has been sought in the influence of underlying parts, especially that of the sub-cutaneous muscles, the constant traction of which influences the hair follicles over definite areas, but this idea cannot as yet be considered to have passed the stage of a vague hypothesis, especially since many of the observations are fallacious, and hence have no weight in establishing the conclusions.

It seems likely, since the hairs originated in association with a complete coat of scales and at a time which must be designated as premammalian, and since the original hair direc- 
tion must have been the same as that of the scales which preceded them, that this original direction would have been retained after the loss of the scales, and that the hereditary transmission of this may account for at least a general plan underlying the variations occurring in the mammals of the present day. The existence of individual variations, known to be considerable in man and certain domestic animals, points to a diminution of the original functional importance, which has become no longer sufficient to retain the various features at a definite standard.

Aside from the formation of horny scales, feathers, and hair, the epidermis produces numerous other organs composed of keratin, and fitted for various uses. The most of these appear as isolated instances to subserve a particular purpose in a small group of animals, but in one case, that of claws or ndils, the organs are possessed by both Sauropsida and Mammalia and form a strictly homologous series throughout, which presents some interesting modifications.

The first employment of this substance in this locality appears to be in the dog-fish, where the fins are lengthened beyond the limits of the fish skeleton by numerous horn threads, set close together and forming two series, overlapping the cartilaginous rays on each side. Otherwise there is little use of keratin among fishes and almost none at all among amphibians, unless there be included a certain form of wart found in toads and due to the local thickening of the stratum corneum. One species of salamander also (Siren) possesses horny plates in the mouth, serving the purpose of teeth. In turtles, a dorsal carapace and a ventral plastron are formed from parts of the endoskeleton, with the addition of dermal elements, and these are covered by large plates of keratin, the so-called "tortoise-shell." The jaws of the same animal are also covered with horny plates equipped with a sharp cutting edge, and a precisely similar formation produces the characteristic beak of birds, although it is hardly to be supposed that the two structures are genetically connected. Aside from the coat of imbricated scales, many reptiles possess horns, 
crests and other cornified structures, many of which are undoubtedly scale modifications; and in birds there are occasionally horny structures, often with a core of bone, like tire spurs of the game cock, of doubtful morphological value. The lower legs and feet of birds are encased by a horny epidermis, a part of which is covered by definite scales, while other parts of it are divided by grooves into square or polygonal areas. The skin of crocodiles is marked in much the same way and does not form overlapping scales, yet it is highly probable that in both cases the areas separated are the equivalent of scales, since overlapping is not a necessary characteristic of these organs.

In mammals there are many special organs composed of keratin. The "whalebone" of whales is derived from the epidermis of the hard palate and forms a thick fringe which hangs from the upper jaw and is employed as a strainer. There are three types of horns: that of the rhinoceros, formed by a coalescence of numerous keratin fibers, probably the morphological equivalent of hairs; the hollow type found in some ruminants, in which a hollow keratin structure is fitted over a core of bone; and, thirdly, the solid horn of deer and antelopes, where the final structure is composed of the bony core alone, the epidermis being represented by the "velvet," an external covering which atrophies after the horn is completed and is rubbed off by the animal. Thus this last, in its final condition, cannot be counted among epidermic structures.

In reptiles, birds and mammals the ends of the digits are armed by horny structures, strictly homologous throughout, although variously denominated as clares, nails or hoofs, in accordance with their shape. In the typical claw (Fig. 28, a) the parts to be noted are the convex dorsal plate (Krallenplatte), the concave ventral plate (Sohlenhorn) and the apical pads of the digit (Zehenballen). In the sauropsidan claw (a) the two plates are of about equal importance and the terminal pad is represented by an unmodified scale or by several scales. In the typical mammalian claw (b) the ventral plate is somewhat reduced and the terminal pad is well developed 
and covered with friction-ridges. In monkeys (d) the dorsal plate is flatter and broader as an adaptation to the prehensile hand or foot and does not project much beyond the end of the digit; the ventral plate is much reduced in extent and is not very horny, and the terminal pad has decreased in volume and is indicated mainly by the friction-ridges, which are in the form of a loop or whorl. The extreme of this line of develop-
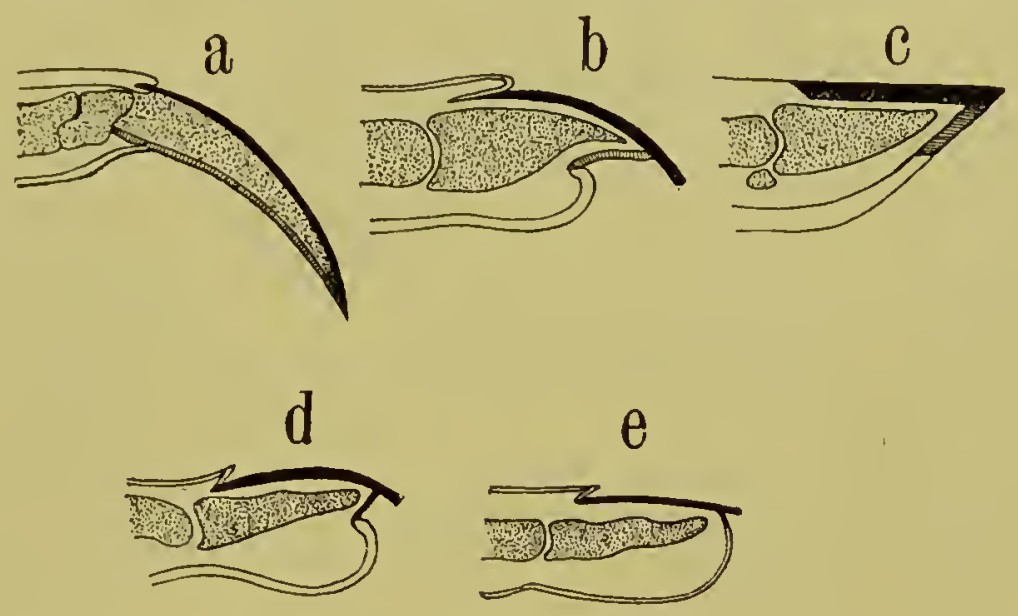

FIG. 28. Diagrammatic longitudinal sections through digits of various mammals, to illustrate the morphology of claws, hoofs, and nails. [(a), after Gegenbaur; (b)-(e), after Boas.]

(a) Echidna. (b) Typical unguiculate. (c) Horse. (d) Monkey. (e) Man.

The dorsal plate is represented by solid black; the ventral plate is striped. The bones are dotted.

ment is reached by man (e) in which the last remnant of the ventral plate appears in the narrow strip of skin between the inner surface of the nail ( $i$ e., the dorsal plate) and a terminal fold where the friction-ridges commence. The terminal pad is much as in monkeys. In the hoofed quadruped another line of development is shown (c) in which the ventral plate forms a horny, though rather soft, surface for contact with the ground. There is no pad, and the soft integument representing that area lies behind the hoof, continuous with the ventra! plate.

Glands occur in the integument of all vertebrates, profusely. in fishes, amphibians and mammals, rarely and strictly localized in the sauropsida. They are always derived from the stratum germinativum of the epidermis and vary greatly in 
complexity of development and in the nature of their secretion. The principles underlying gland formation are very simple, and may be briefly considered in this place before taking up in detail their occurrence and distribution in verte-

a b $\quad c \quad d \quad e$

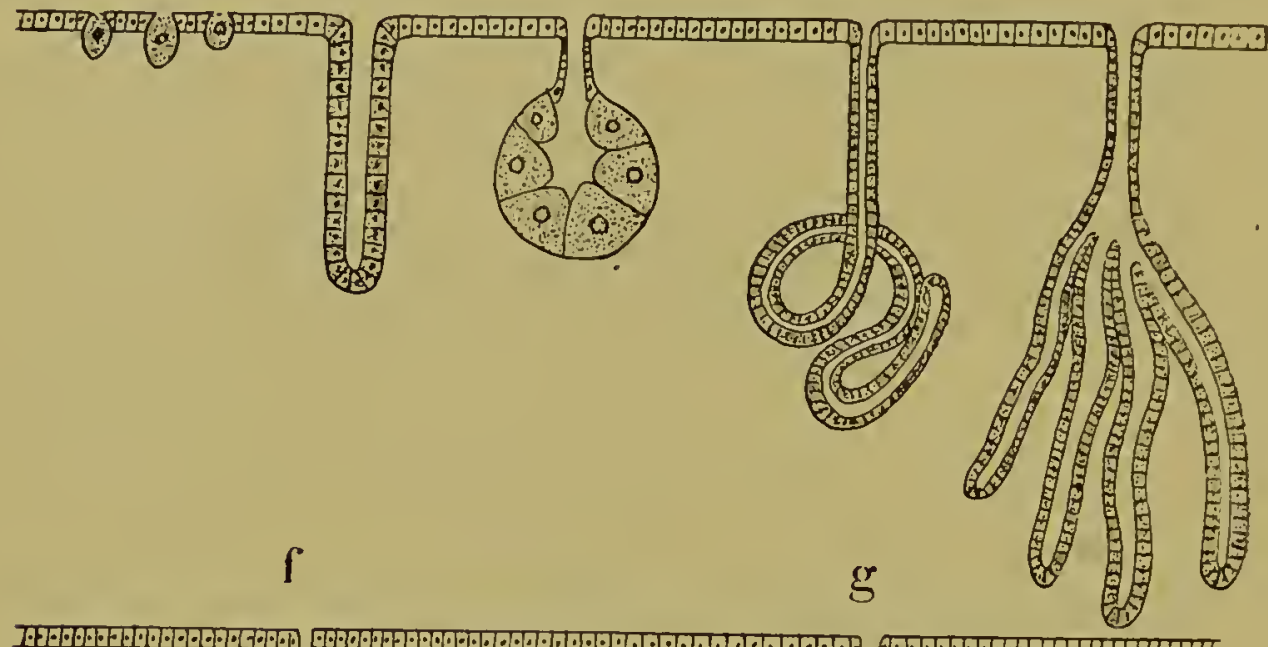

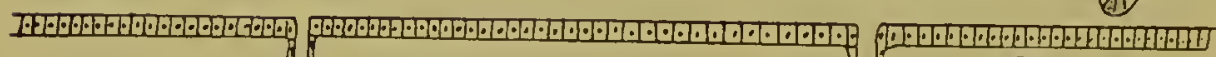
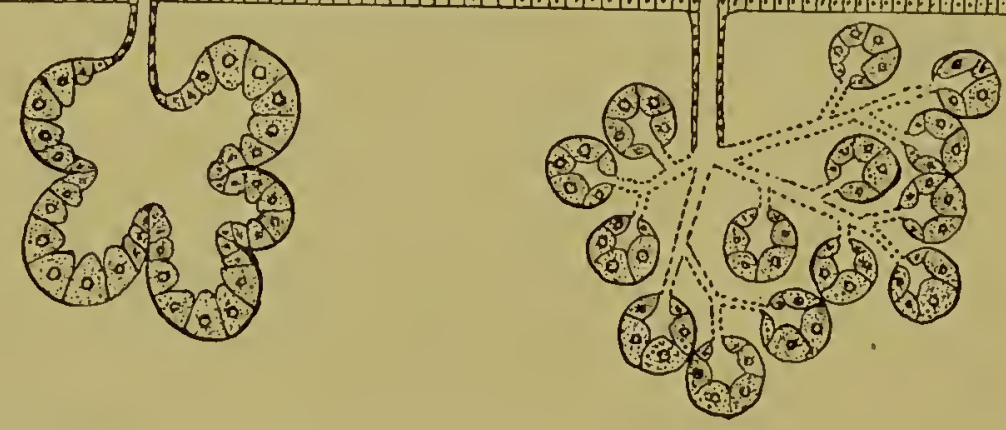

FIG. 29. Diagrams of various types of glands, shown as invaginations from a layer of indifferent epithelium.

(a) represents a region in which certain of the surface cells are differentiated as unicellular glands. (b) is a simple tubular gland and (c) a simple acinous gland, each formed from a complex of gland cells. Tubular glands may become coiled (d), or branched (e). Acinous glands may consist of a single acinus, as in (c), or of several, as in (f). A still greater complexity is seen in (g), where each acinus possesses its own excurrent duct, all being collected into a common duct which leads to a single outlet.

brate integument. The protoplasm of all cells has the power of storing up some form of secondary material, metaplasm, extracted from the materials supplied to it, and a gland cell differs from another mainly in the fact that its metaplasm is of use to some other part of the organism and that its chief value to the organism lies in the material which it produces. 
Usually also a gland cell, specialized as it were in this direction, secretes these products in greater abundance than in the case of other cells. As a single cell may thus have all the attributes of a gland, the simplest glands are composed of but one such elementary unit and are unicellular. Such simple glands are of extensive occurrence among animals and are generally used where a surface is to be kept uniformly moistened with some secretion, as a protection against water or air, and where there is no special auxiliary structure, like the eyelids of land vertebrates, to insure an even distribution. The majority of glands, however, are multicellular and represent various solutions of the problems of how to increase the physiological efficiency within a definite space, $i$. $e$, , how to increase the effective secreting surface without increasing the mass. The diagrams in Fig. 29 represent various solutions of this problem, as well as varying degrees of physiological efficiency, the most complex form being in general the most successful. Beginning with single cells opening upon a free surface it is evident that the efficiency increases with the number of gland cells in a given space, the limit of this type being reached when all the cells have become thus employed. If, however, the problem allows the utilization of a certain amount of depth, the efficiency may become much increased by folding or invaginating portions of the original surface below the general level, either in the form of tubules (b) or flask-shaped globules, acini $(\mathrm{c})$. Each of these primary types may become still further complicated in several ways. The tubular form may become convoluted $(\mathrm{d})$ or branched $(\mathrm{e})$, and the acinous form may develop secondary acini (f). Through a slight cellular differentiation the cells nearest the outlet of the gland may become flattened and form a non-secreting duct through which may pass the fluid manufactured in the secretory portion. This principle may be extended to the secondary acini, and when these latter become profusely multiplied, the result is a definitely localized and very effective organ, as in $(g)$. These varied forms are not sharply defined, and even the fundamental types of tubular and acinous glands may grade 
into one another in such a way as to make the classification indeterminate.

Glands may be also divided according to their method of furnishing the secretion, since some cells, when surcharged, liberate their fluid by bursting, and thus become destroyed, while others allow their secretion to pass through their walls, retaining their physiological life for an indefinite period. In the former case the supply of cells is kept up by a constant proliferation from a zone of growth; in the other case the community of cells retains its identity. The glands in the former case are termed necrobiotic, in the other they are vitally secretory. This physiological distinction is often of use in determining homologies at times when the structure is non-committal or misleading.

Unlike most other structures, the integumental glands of vertebrates do not appear to have a continuous history in the various Classes, but are developed in each Class, or even in specific cases, to suit the needs of particular environment or habits. In fishes and amphibians the main function of integumental glands is to secrete a protective slime. to defend the surface from the action of the water, to which, as a secondary function, probably accidental at first, there is often added to the secretion some acrid or even actively poisonous quality, for defense against predaceous animals. The glands supplying this function are often of the unicellular type, with a narrowed neck at the surface, and called beaker cells from their shape; the simple acinous type, in the form of flaskshaped glands, is widely distributed among amphibians, where the glands often occur in clusters, causing a conspicuous protuberance. The integument of the Sauropsida is characterized by an almost complete absence of glands, certain special ones appearing in definite localities and employed for some special purpose. Such are the cloacal glands of snakes, which secrete for defensive purposes a milky fluid having a nauseating odor, and the musk glands of certain turtles, which may be defensive or used as a sexual allurement. In the males of certain lizards a single line of glands opens along a definite row of scales on 
the inner aspect of the femora; at the time of pairing these secrete a gummy fluid which hardens into short spines or teeth, employed during copulation. In birds the sole integumental

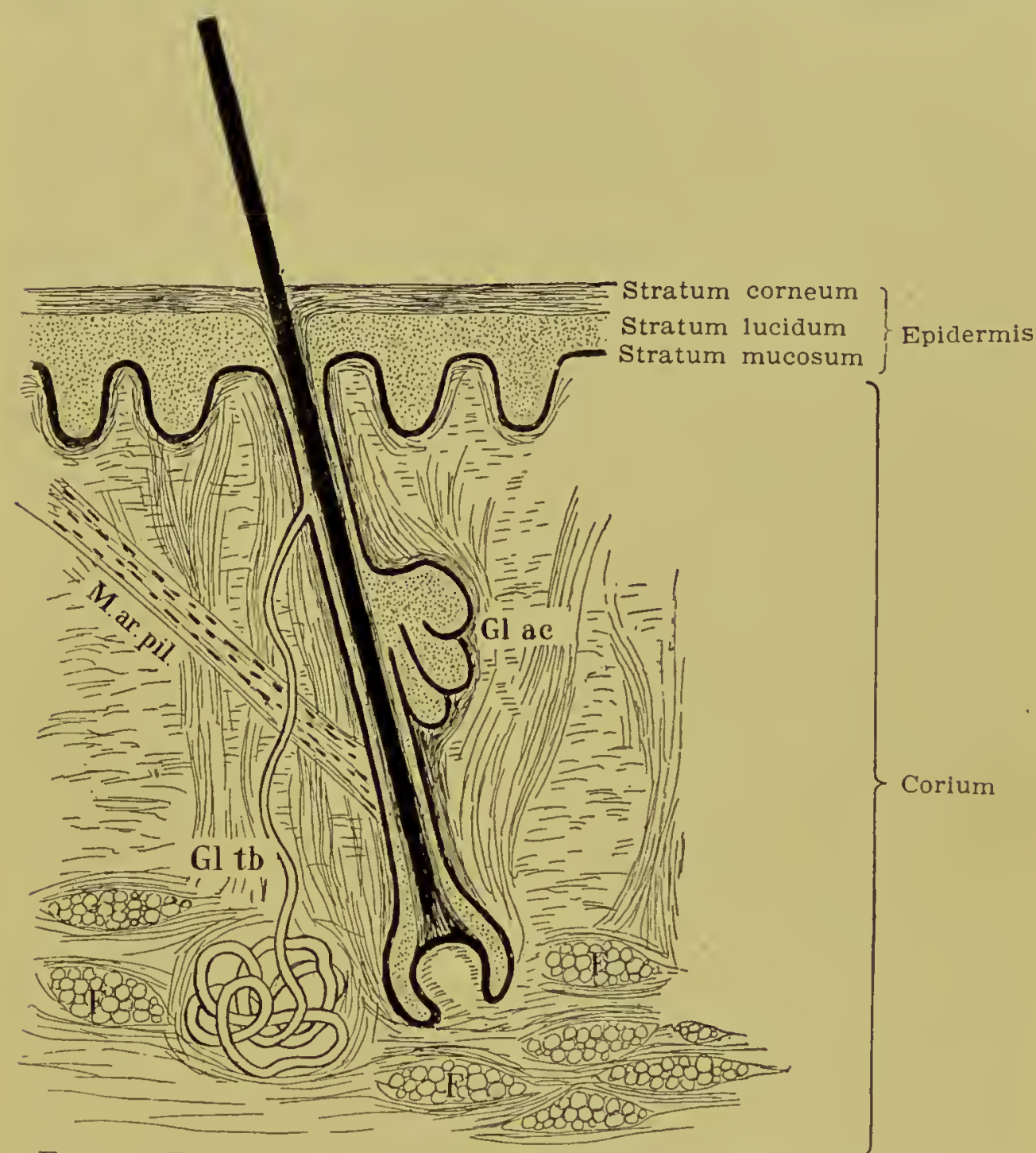

FIG. 30. Typical mammalian hair with its accessory parts. [After WEBER.]

Gl. ac., acinous gland, usually sebaceous; $G l$. $t$ b., tubular gland, usually perspiratory in function; $M$, ar. pil,, arrector pilarum muscle.

gland is the uropygial, a compound mass situated on the dorsal aspect of the tail rudiment, and secreting an oily fluid used for anointing the feathers.

In mammals, the skin is, as a rule, profusely glandular, and the glands possess the highest degree of physiological differentiation. In spite of their diversity, however, they may 
all be referred to two primary forms, each originally developed in association with a hair. This primitive condition is still common, though often with some slight modifications. and is shown in diagrammatic form in Fig. 30. The glands arise as outpushings from the wall of the follicle, into which they empty. One of these types is a long, slender tube, often convoluted at its free end, a tubular gland; the other short and somewhat lobed, an acinous gland. Aside from this morphological distinction the tubular gland is vitally secretory, the acinous necrobiotic. As a secondary modification either type may exist without a hair, but such cases are exceptional. It seems likely that such a complex as that represented in the figure was originally associated, as are the hairs, with the primary scales, one for each, the glands being associated with the median hair only, but this cannot as yet be definitely asserted.

Each of the two glandular elements is capable of great modifications, both morphologically and physiologically. The tubular type is not always convoluted, but may be straight and simple, or branched. Its characteristic secretion is a thin, colorless, watery fluid, the perspiration or sweat, but it is viscous and reddish in the hippopotamus and albuminous and of a blue color in Cephalophus, a South African antelope. A much modified form of these glands furnishes the thick and oily ear-wax. In distribution these glands are often found over the entire body (hippopotamus, bear), but may be strictly localized, as in most rodents, where they are found mostly on the ventral surface of the paws. They fail entirely in, the two aquatic orders of Sirenia and Cetacea, also in Manis, in a sloth (Cholopus), and an insectivore (Chrysochloris). They are usually found on the palmar and plantar surfaces, where, in man and the monkeys, their openings are readily seen at regular intervals along the middle line of the friction ridges. In this location, by moistening the ridges, they undoubtedly assist the firmness of the grasp, often a factor of vital importance in an arboreal animal. When distributed over the general surface and yielding the customary colorless watery 
fluid, these glands are usually called sweat glands (glandulce sudoriparc), but enough has been said to show that this term is too limited to be employed in general. In Man, where they fulfill this function, they do not appear to be evenly distributed, and there seem to be individual differences in the extent and copiousness of the secretion. As it has been found that these glands rarely occur in the integument of the Fuegians, there are undoubtedly marked racial differences, but there is, at present, little knowledge upon this point.

The primary use of the second, or acinous, type of integumental gland seems to be to furnish an oily secretion for the lubrication of the hair, forming the sebaceous glands; and corresponding to this use, they appear less inclined than does the other type to become disassociated from a hair follicle. Modified forms do occur, however, often unconnected with hair follicles, and modified in their secretion to subserve some special use. Such are the tarsal [meibomian] glands of the eyelid, which are properly the hypertrophied sebaceous glands of the eyelashes, whose purpose it is to supply a line of oil for the edges of the lids and thus prevent the overflow of tears. Other modified forms of this type are found at the orifices of the body, as the lips, the anus, and upon the external genitals (Tyson's glands, preputial glands, etc.). Corresponding to their primary function as sebaceous glands, they are wanting in Cetacea and in adult Sirenia; the scale-covered Manis retains only the modified orificial glands. They are, however, wholly wanting in some sloths (Cholapus) and in an African insectivore (Chrysochloris), the same animals in which the glands of the tubular type are also wanting.

Aside from these small, generally distributed, glands, the integument of mammals is especially characterized by the occurrence of localized masses of glands, often voluminous in size and furnishing a secretion intended for a special purpose. The elements of which these masses are composed are sometimes of the tubular and sometimes of the acinous type, or of both sorts together. Of these the anal sacs are widely distributed, composed mainly of tubular glands, and forming 
from two to five sacs which open into the rectum immediately within the antus, from which in some cases they may be protrucled by being turned inside out. The best known of these are the two lateral ones of the skunk, weasel, and allied forms, which are covered with a muscular layer derived from the levator ani muscle, and secrete an ill-smelling fluid as defense. Other similar glands secrete odoriferous fluids employed as a mode of sexual attraction, some of which are agreeable to man and are used in the manufacture of perfumes (musk, civet).

In their simplest form such glands open separately, but near together, the surface covered by the opening being designated as a glandular area, usually free from hair or nearly so; but in many cases this glandular area becomes depressed and forms a sac or bursa sunken beneath the surface and serving at times as a reservoir for the secretion. It is as such a structure that the mammary or milk glands, so characteristic of the Class of Mammalia, have arisen, and their appearance in the momotreme, Echidna, exhibits nearly their original condition. The female of this animal possesses upon the ventral side an integumental pouch, the marsupium, in which the eggs are placed, and in which the young are nurtured when hatched. Opening into the sides of this pouch is a pair of glandular sacs or pouches, supplied with glands which are probably of the tubular týpe, although long supposed to be acinous. These sacs are the mammary pockets, at the bottom of which lies the glandular area with its numerous openings. The secretion, which is a form of milk, pours out into the pockets, where it is taken up by the young. There are no traces of nipples, but the lips of the sac, the corium wall, fit around the nose of the young and prevent loss (Fig. 3I, a). A slight advance is seen in the young Halmaturus, a marsupial, where the mammary pocket is deeper, and a rudimentary nipple is formed by the elevation of the middle of the glandular area at the bottom (d). This structure is still further developed in the young opossum (c), and in this latter animal the functional activity of these organs causes the complete extrusion of the nipples, and the mammary pocket is lost (f). 
This type of nipple, in reality a mammary pocket turned inside out, is the most usual among the higher animals. In it the glandular area forms the nipple itself, while the corium wall, the rampart-like lip of the pocket, becomes the areola, a circular

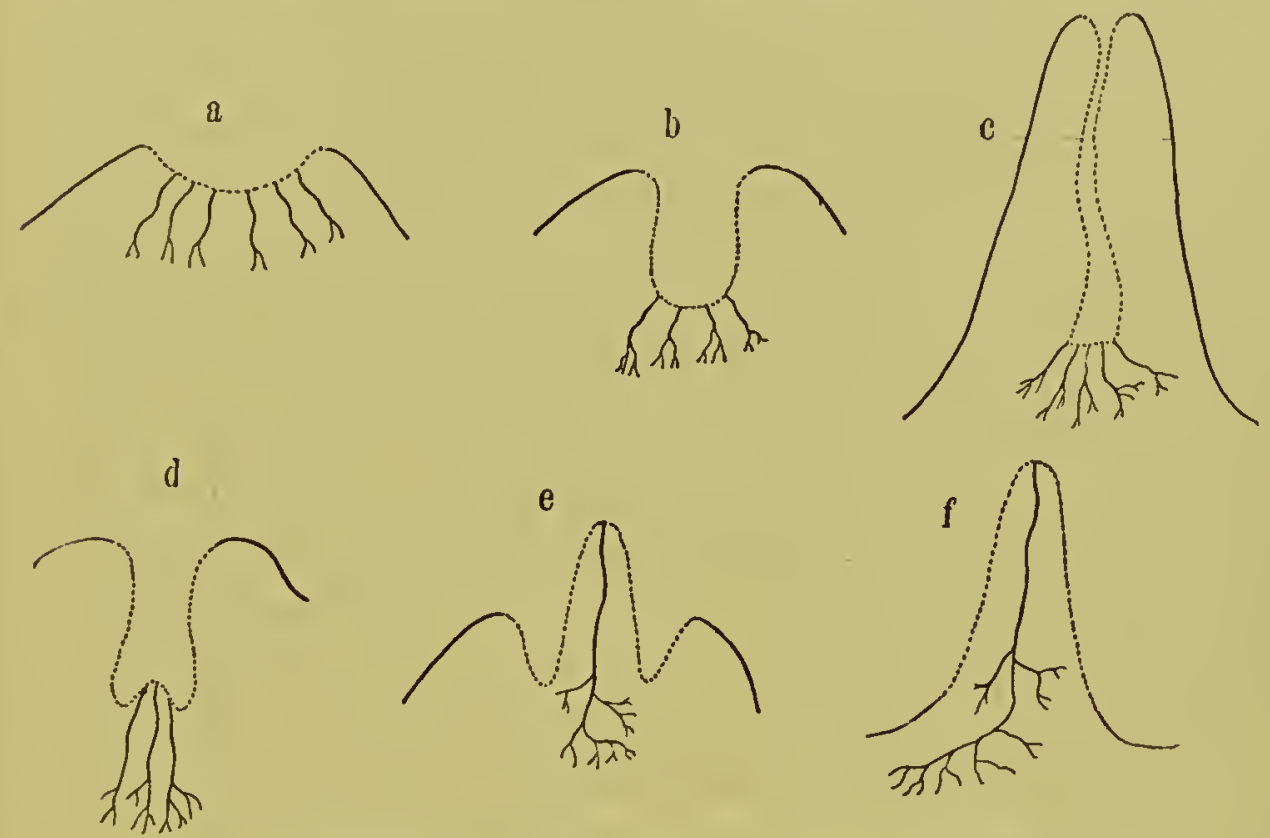

FIG. 3I. Morphology of nipples. [After WEBER.]

(a) Primary condition, as in Echidna. (b) Embryo calf, comparable with the condition seen in (a). (c) Cow (adult) showing "false" nipple produced by the prolongation of the cutis wall. (d) Halmaturus (a marsupial) previous to lactation. (e) Didelphys (a marsupial) previous to lactation. (f) Didelphys during lactation, showing "true" nipple, produced by the eversion of the mammary pocket.

In all the figures the area glandularis, $i$. e., the surface of the mammary pocket, is represented by a dotted line; the cutis wall by a full line. The branching lines opening either at the bottom of the depression or at the summit of the elevation, are
the milk glande.

area of modified skin surrounding the nipple. Quite another type of nipple, though derived equally with the former from the mammary pocket of the Echidna, is that occurring in ruminants. In this the glandular area remains at the bottom of the pocket, while the surrounding corium wall becomes elevated more and more until a long pendulous nipple is formed from that (Fig. $3 \mathrm{I}, \mathrm{b}$ and $\mathrm{c}$ ). The mammary pocket is here retained as a long lactiferous duct running through the nipple. In many placental mammals the earliest embryonic indication of the mammary glands consists of a lateral ridge, 
extending from axilla to groin, occupying the position of a future row of nipples. By the suppression of this mammary ridge at regular intervals there arises a series of elevaticns which at first sight appear to be the nipples, but which become secondarily reduced and eventually come to form actual depressions. These are evidently the ontogenetic repetitions of the mammary pockets, since from these, after the manner detailed above, the true nipples arise, faithfully repeating the stages shown in Fig. 3I. The mammary ridge perhaps represents the wall of the marsupium or pouch, thus suggesting that the placental mammals have been derived from ancestors which possessed a marsupium. The conclusion is not necessary, however, that these ancestors were the Didelphia of the present day, but that the common ancestors of both modern types of mammals, marsupial and placental, possessed this organ, and that the Didelphia have retained it as a functional organ, while in the Monodelphia but few traces remain. The occurrence of a marsupium among the monotremes, the only living representatives of the Prototheria, points to the same thing. (See Fig. 8, and the accompanying text.)

The number and position of the nipples vary much in the different groups of monodelphic mammals, and furnish a series of illustrations of adaptation, both to the habits of life and the number of the young. In that type which appears to be the most primitive, there is a series of nipples arranged in a lateral row upon either side and extending from axilla to groin. In this case, as in pigs, most carnivora, and many rodents, the animal lies upon one side while nursing. By the suppression of the anterior end of this series inguinal mamme are produced, as in ungulates, which nurse their young while standing erect. In the Cetacea the single pair of inguinal nipples lies in the bottom of a pocket, not the prinnitive one of the Echidna, but one secondarily developed to solve the problem of nursing under water. The lips of this pocket fit tightly about the snout of the young, which can suckle beneath the surface, being at the same time able to breathe through the nostrils, which in these animals have migrated backward from 
their primary position. By a suppression of the posterior portion of this series, pectoral mamma are produced, either two pairs, as in some lemurs, or a single pair, as in the majority of primates, and in bats. In the aquatic Sirenia, also, the mamma are pectoral. As they bear but a single young at a time and nurse it by clasping it in the flippers while standing upright in the water, these animals, as suggested by the name, are probably the real origin of the well-nigh universal mermaid myth. The pectoral position is the most convenient for arboreal animals like the Anthropoidea and enables them to carry the offspring in one arm and leave the other free for climbing.

In many animals with a restricted number of mamma there have been frequently observed cases of supernumerary nipples or supernumerary mammæ. These are termed respectively hyperthelism and hypermastism, and are looked upon as atavistic and indicative of the former development of a complete series, of which those normally developed form a part. They are often noted in the adult human subject, and the anlagen of numerous pairs of nipples occur regularly in the embryo. The occurrence of six-nippled sheep that have a tendency to cast two young at a birth has been recently made the subject of experiment with a view to perpetuating the latter peculiarities, and thus form a race adapted to countries with a short summer, like Canada. Whether there is a definite correlation between these two characters, or whether in Man there is any correspondence between hypermastism and a tendency to produce twins, has never been determined.

The occasional occurrence of mammæ in unusual positions, as on the thigh or the back, as has been noted in the human subject, is a displacement, and not a reversion, and hence has no normal morphological meaning.

The occurrence of rudimentary nipples in the male is the rule among placental mammals, but seems not to be the case in monotremes and marsupials. If this be true, this is a definite instance of the hereditary transmission to the male sex of parts that developed first in the female, and formed for a long 
time an exclusive characteristic. Since neither natural selection nor sexual selection could have a part in this transmission, it has been brought forward as a case of the direct transmission of an acquired characteristic. It may, however, be a phenomenon similar to the strange series of homologies of the various parts of the reproductive organs, treated in full in Chapter IX., where such an explanation is inadmissable, although at the present time no satisfactory one can be offered. Although usually the only parts of the mammary apparatus to occur in the male are the rudimentary nipples, yet cases of so-called gynecomastism are known, in which well-defined and even functional mammæe occur in persons of the male sex, unaccompanied by any sexual abnormality.

Pigment is a coloring matter, occurring in the form of granules, and existing in certain cells as a form of metaplasm secreted by them and retained within their substance. These pigment cells are found in both epithelium and connective tissue. Pigment often occurs in the interior of the body, notably in the peritoneum of amphibians, where it lines the cœlom and invests the organs with a brown or even black covering. It occurs in the integument or the integumental structures of all vertebrates except certain white animals, and in albinos, the peculiarity of which consists of a total absence of pigment from all parts of the body. These two cases may be readily distinguished by observing the iris of the eye, which retains its pigment in normally white animals, but lacks it in albinos, giving the eyes a pinkish cast. As a general rule the integument of vertebrates is pigmented when without accessory structures, or when these form an insufficient covering, but in those birds and mammals in which the feathers or hair are respectively sufficient to entirely conceal the shin, these accessory parts receive the color and the integument is unpigmented. This rule is further emphasized by the fact that bare places, like the head and neck of vultures and the ischial callosities of monkeys, or scantily covered places like the entire integument of elephants and rhinoceroses, are pigmented, and occasionally highly colored. 
The pigment of vertebrate integument is usually contained in branching connective tissue cells, produced in the corium, but capable of wandering into the epidermis. In some cases, as in Man and monkeys, the stratum germinativum of the epidermis is pigmented, and varying degrees of this are responsible for the great variety of skin color in Man. Although the connection is hard to prove, it is a general truth that human races that live in the tropics are the darkest and that the skin grows gradually paler in races nearing the poles, there being a correlation in this respect between skin color and hair color. As instances of this, there may be recalled the sooty blackness of the Sudanese, and the dark color of the aborigines of India, which may be compared with the lighter color of Europeans and northern Asiatics. More convincing cases of this are seen in representatives of the same race; such as is shown by the contrast between the Italians and Spaniards on the one hand and the Scandinavians on the other, or between the American Indians in Canada and those in Mexico. A similar reduction in pigment is found in people living at high altitudes in comparison with the same race living in the bordering lowlands. The importance of these correlations has been repeatedly denied, and there are numerous instances of exceptions, often conspicuous ones like the dark skin and hair of the Eskimo, and at the present state of our knowledge too much cannot be urged on this point.

Another point of interest lies in the regions of the body in which the pigmentation is the densest. As a general rule it may be observed, not in vertebrates alone but in invertebrates as well, that the darkest and most deeply colored parts are those that lie uppermost, exposed to the light, while the under parts are lacking in pigment. That this bears no relation to the architecture of the body may be seen in the case of the flounder, a fish that is much compressed laterally and has the habit of lying upon one side at the bottom in rather sliallow water. This habitual lower side, which is sometimes the left, sometimes the right, half of the body, is entirely colorless, while the upper side is marked with a complicated pattern 
resembling the sandy or muddy bottom on winich the fish lies.

This principle is a general one and applies to the distribution of pigment, whether in the integument itself or in its accessory structures, a fact that may be readily seen by comparing a form with a naked skin, like a frog or a porpoise, with one covered with hair. In terrestrial mammals the difference is not always a marked one and is apt to be greater in short-legged forms that creep close to the ground; in birds, which in the majority of cases expose nearly the entire surface to the light, the body may apparently be of uniform color, but here the deeper pigmentation is confined to the exposed surfaces of the feathers, while the portions which are shielded from the sun are less deeply colored or even without pigment. The white ventral surface of aquatic birds like snipe and gulls is a protective coloring and comes under another principle. In man the distribution of pigment is also unequal, the darker areas being, in all races, the back and the dorsal aspect of arms and legs, while the chest and abdomen, the ventral aspect of the limbs, and especially the palms and soles, are lighter in color. This distribution, it will be noticed, corresponds to the influence of the light, when man assumes the quadrupedal, and not the usual human, position. Aside from these general areas, a deep local pigmentation occurs in the axilla and groin, about the anus and the external genitals, and upon the nipples and areola, where it is evident that the pigmentation is for some other purpose and can bear no relation to the distribution of light. In this general connection between a darker color and the increase of the sun's light and heat, there must be some physiological advantage which a dense pigmentation gives its possessor, an advantage which seems to be a real one whenever there is a chance for comparison between a black man and a white man in the tropics in regard to their relative power of enduring the heat of the sun. Although the subject is still an obscure one, it seems probable that the presence of a dense layer of pigment in the stratum germinativum effectually prevents the direct action of the light upon the surface capil- 
laries, thus allowing them to expand and retain the blood at the surface where the excess of heat can be constantly thrown off. Thus repeated observations have been made in Samoa* when whites and natives have been together and doing the same work, that the skin of the latter would be dry and glowing as in a fever, while that of the former was cold and damp. Under these circumstances the Samoans would be constantly giving off heat while the whites were compelled to retain theirs.

In the presentation of the above facts in connection with one another, the conclusion seems almost unavoidable that the various conditions are directly due to the solar action upon each individual, and to the propagation of the conditions thus acquired until the physiological advantages become inborn in the race. Although this may seem at first the simpler explanation, there are numerous biological facts associated with heredity that seem to render impossible so direct a transmission of somatic peculiarities, and point to a more indirect method of attaining the same end through individual variation and the selection for survival in each generation of those in which the desired peculiarity is the most marked. This explanation, however, is in many points as unsatisfactory as the other when applied to this case, since we know that the struggle for existence in man has never been severe enough to compel the extinction of individuals differing from others by a shade of color; neither is sexual selection operative here, since among a primitive people all who are not physically unfit become the propagators of the race. The matter must be left at present as one in which the facts are evident but the explanation of them obscure; the problem is to be solved sometime, and when solved will offer an explanation of the relation of structure to environment everywhere.

* According to Dr. A. Krämer.-Die Samoa Inseln, Stuttgart, I903. 


\title{
CHAPTER V
}

\section{THE ENDOSKELETON}

\begin{abstract}
“. . . our 'physic' and 'anatomy' have embraced "such infinite varieties of being, have laid open such new worlds in time and space, have grappled, not unsuccessfully, with such complex problems, that the eyes of Vesalius and of Harvey might be dazzled by the sight of the tree that has grown out of their grain of mustard seed."

Thomas Henry Huxley, in nis essay: On the advisableness of improving natural knowledge.
\end{abstract}

An endoskeleton or internal framework for the support of the muscles and the protection of the viscera is one of the distinguishing characteristics of vertebrates, for with the exception of a few sporadic cases in which internal skeletal parts occur, invertebrates are without such a system. The vertebrate endoskeleton is a part of the connective tissue system of the body and, in its usual sense, includes only bone and cartilage, although both developmentally and physiologically the associated ligaments and other connective tissues belong with the former.

Primarily the endoskeleton consists of three systems. originally distinct from one another, the axial, the visccral, and the appendicular. The axial includes the vertebral column and a large part of the skull; the visceral includes the lower jaw, certain elements in and about the upper jaw, the hyoid apparatus, and the branchial or gill arches; and the appendicular includes the shoulder and hip girdles and the skeleton of the free limbs.

Of these, the axial is the oldest, and is represented in its simplest form by the notochord, although this organ soon yields in functional importance to the connective tissue sheath which enwraps it, from which, in higher forms, the main ele- 
ments of the vertebræ are derived. The notochord, which is endodermic and arises from the primitive alimentary canal in the manner related above, is the only portion of the endoskeleton not formed from the mesenchyme, and is hence not a

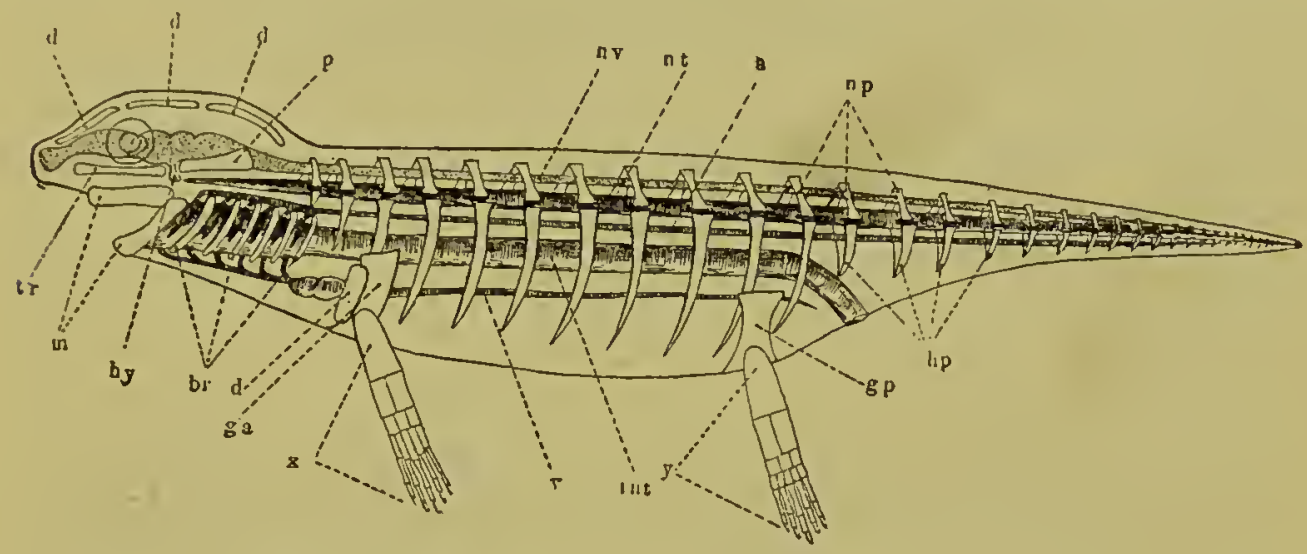

Fig. 32. Diagram of vertebrate, showing relation of skeleton to soft parts.

Axial SKeleton: $t r$, trabecula; $p$, parachordal; $d$, dermal bones of skull; $n t$, notochord; $n p$, neuropophyses; $h p$, hamapophyses.

Visceral SKEleton: $m$, mandible; $b r$, branchial arches.

APpendicular SKeleton: $g a$, anterior; $d$, its dermal element; $g p$, posterior girdle; $x$, anterior free limb; $y$, posterior free limb.

SofT PARTs: $n v$, nervous system; $h y$, hypophysis; $a$, aorta; $v$, sub-intestinal vein (an embryonic organ); int, intestine.

connective tissue; but this becomes gradually replaced by skeletal elements formed from true mesenchymatous tissue, so that in the higher forms the adult skeleton is wholly from this latter source. The notochord seems to have been a very ancient form of endoskeleton, antedating that formed of connective tissue and functioning in the immediate ancestors of the present-day vertebrates. It is present in what may be nearly its original condition in Amphioxus, where it appears as a cylindrical rod, extending through the longitudinal axis of the body from end to end. This rod furnishes a certain degree of rigidity and allows the animal to maintain a fixed length, while permitting a considerable amount of flexibility through its elasticity. In about the same condition it appears as a constant organ during the early embryonic life of every vertebrate; and, although it is usually replaced in great measure by mesenchymatous elements, yet in some fishes, even in those 
as high as ganoids, it retains much of its original appearance and function. Both in Amphioxus and in these forms, as well as in all embryos, it is formed of a semi-gelatinous tissue, often called pre-cartilage, and is surrounded by a firm sheath of connective tissue, which, in those adult forms with a persistent notochord, supplies the necessary firmness and rigidity in which particular the notochord alone would be inadequate. This sheath becomes, in fact, of far greater importance than the notochord, and the cartilaginous and osseous tissues formed from it come to encroach more and more upon the yielding tissue within, and eventually supply the main elements used in the construction of the vertebræ.

The first stage in this advance is seen in the lamprey and other cyclostomes, where there appear pairs of little cartilages, lying upon the side of the notochord sheath and projecting upwards to protect the nerve cord, which lies along the dorsal side of the notochord. These little cartilages are of two kinds. The primary ones develop from the edges of the intermuscular septa, and are hence intersegmental, a point which is important to remember in connection with the relative position of the vertebræ in higher forms. A secondary set alternate with these, and form intercalary pieces, protecting the intervals between the first set.

A second advance over the condition found in Amphioxus is seen in the formation of a head, which, since here the notochord no longer extends to the tip of the snout but ends a little behind the plane of the eyes, has been supposed to be in part a transformation of the anterior end, and in part a new formation added anterior to this. Whether this may be safely assumed or not, the anterior termination of the notochord forms in the embryo an important topographical point, the portion of the head along the sides of the notochord being referred to as parachordal, and that anterior to it as prachordal. The hypophysis, an organ lying in the median line and depending from the lower surface of the brain, lies at the anterior point of the notochord, and will thus serve to mark the boundary between these two portions of the head. 
The next few stages in the history of these parts, lying between the condition above described and definite vertebræ, are still somewhat a matter of controversy, since, in the various

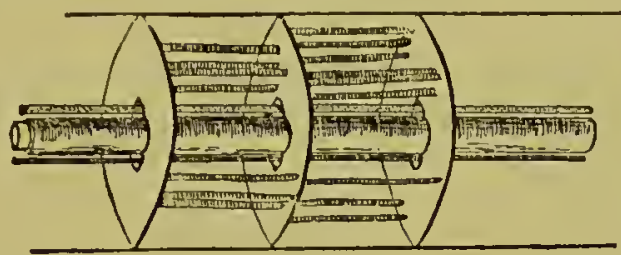

a

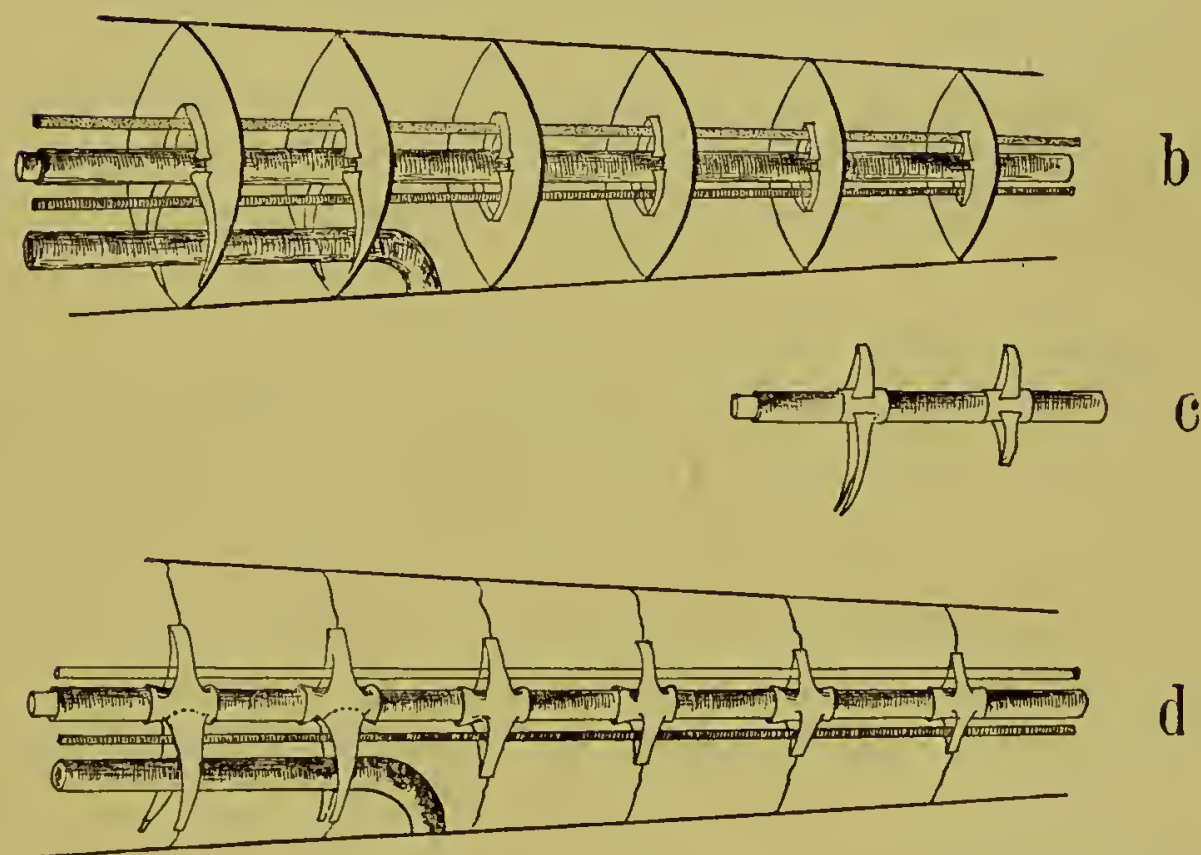

FIG. 33. Diagrams illustrating a theory of the development of the vertebrate.

(a) Condition previous to the formation of vertebral anlagen (caudal region). The body is divided into segments by transverse myocommata through which run the notochord, the nerve cord, and the aorta. In the region of the colom the myocommata open ventrally and allow the alimentary canal to pass. There is no trace of bone or cartilage. (b) Later stage, in which skeletal bridges have formed along the edges of the myocommata, both dorsally and ventrally. (c) Detail of still later stage, in which the sheath of the notochord has chrondrified (or ossified) at the points whcre the bridges come in contact with it. (d) Completed vertebra, formed by the fusion of the elements shown in (c).

types of fish, where these stages should be sought, numerous modifications have taken place which are to be explained as 
special adaptations, admirably fitted to the habits and environment of the various species, but covering up the original racehistory which we are secking to interpret. While, then, one cannot be dogmatic about this portion of the history, the following seems to be the most likely course of development, and its various stages are to be found, with some little modification, in living species.

It would seem, then, that the primary pairs of neural processes, for the purpose of better fulfilling their mission of protecting the nerve cord, became more elongated until they finally met in the middle line above the nerve cord, thus forming a series of intersegmental neural arches, shaped like inverted $\mathrm{Vs}$; and since the aorta, lying immediately beneath the notochord, needed a similar protection, other arches became developed for this purpose, situated immediately below the former, and with their points directed downwards.

When these two systems of arches were well established, they would naturally seek to secure a firmer attachment to the notochordal sheath by spreading out their bases, and as the two sets of arches, neural and hamal, were opposite each other, each neural arch being associated with its corresponding hæmal arch, the enlarged bases would grow together. To support the increasing weight of these parts, the notochordal sheath would then chondrify or ossify beneath these bases in the form of rings, and the fusion of three elements, a neural and a hæmal arch and a notochordal ring, would form a vertebra of the type found in the ordinary bony fish. The condition just described, with neural and hæmal arches alike, is that found in the tail region, posterior to the visceral cavity, while in the trunk the hæmal arches are open and their halves widely divergent, forming the ribs, which embrace the visceral cavity. The rings, which are formed from the notochordal sheath, begin in the center of the future vertebræ, and as they grow, expand along both edges until they come in contact with the preceding and succeeding ones. At the same time, however, the ossification has proceeded inwards as well. restricting the notochord, and as the central portion of the ring 
is the oldest, that part becomes the most restricted, often completely severing the notochord at this point, or intra-vertebrally; while the notochord is retained at practically its original caliber at the newest edges of the ring, or inter-vertebrally. The completed ring, which forms the body or centrum of the vertebra, is cylindrical, with concave ends like the interior of conical cups. Such vertebræ are called amphicolous (=both ends hollow), and the hollows of the adjacent vertebræ enclose masses of notochord in the form of two cones, placed base to base.

Although the above sketch is, in a way, hypothetical, many of the stages described actually occur as the adult condition in various fishes, especially ganoids, and the final condition is exactly shown by the teleosts, as one may have frequent occasion to observe. That the evolution of the vertebral column up to this point has been somewhat after the plan here given, may be generally conceded, and it is hoped that important links in the history may be discovered in the field of palæontology, which has already furnished us so many valuable records and bridged so many gaps.

Above the fish the development of the vertebral column has been, not so much in the acquirement of new elements, as in the regional modification of those already possessed. This is strikingly shown by the comparison of the vertebral column of a fish with that of a reptile or mammal; in the first of these the vertebræ are all very much alike, while in the second they are differentiated into successive groups, and in cases in which this differentiation has reached its extreme each vertebra may be sufficiently unlike the rest to be identified by the anatomist when isolated, a feat impossible in the former case.

This regional differentiation is due chiefly though indirectly to the change of environment from water to land, a change which necessitates the replacement of soft and weak fins by two pairs of firm limbs, and substitutes for the evenly distributed buoyancy of the water a fixed support at two points, the shoulder and hip girdles. In the first land animals the limbs were small and weak, and progress was attained through 
a sinuous motion of the body. Even here, however, the infuence of the limbs would be felt, since they would cling to the surface and thus furnish definite fixed points where the sinuous motion of the vertebræ would be lessened. By a gradual increase in the size and strength of the limbs, the animal would attain the power of crawling, that is, of dragging the body over the ground through the action of the limb muscles as well as those of the back, and finally the limbs would become strong enough to bear the entire weight and the body would be lifted wholly above the surface of the ground, thus changing the crawling motion into a true walk. This gradual development of the free limbs is accompanied by important correlated changes in the vertebral column, due in the main to two causes. The first of these is the increased size and functional importance of the limb girdles, or those parts of the limb skeleton enclosed within the body, to which the free part is movably attached, usually by a ball-and-socket joint; and the second is the increase in size of the proximal limb muscles. As the limbs become larger and stronger, their girdles, $i$. e., the proximal portion of their skeleton, feel the need of a stronger support and a more intimate association with the axial skeleton, a need especially felt by the hip-girdle, since here the greater weight is sustained. This girdle, seeking the necessary support, grows dorsally around the body, until it meets the ends of a pair of ribs with which it articulates. In the lower forms the ribs are very short and are borne upon the end of short transverse processes, and the girdle with its dorsally developing process, known as the ilium, the rib, the transverse process, and the vertebra, form a complete chain around the body.

At first this association involves but a single vertebra, the location of which is apt to vary. Thus in Necturus, the most primitive salamander now in existence, the hip-girdle is usually attached to the Igth vertebra, counting from the head, but the 2oth is occasionally employed instead, and two cases have been reported in which the attachment was to the $r 8$ th. Cases are also known in which the attachment is oblique, either to the 
Igth on one side and the 20 th on the other, or to the 18 th on one side and the Igth on the other.

In these low forms this sacral vertebra shows no special modification save that it may be slightly stouter than its fellows, and have longer transverse processes and stouter ribs, but as the posterior limbs increase in size and functional power their girdle increases with them and may form similar attachment to two or more adjacent vertebræ, which may become more and more modified and form a more or less complete
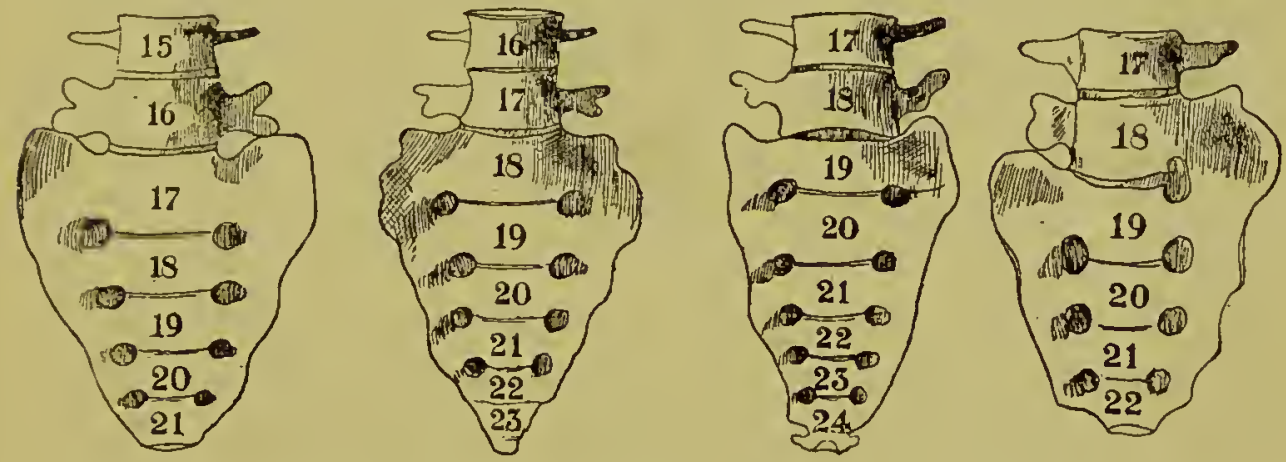

FIG. 34. Variations in the composition of the human sacrum. [After GeGenbaur.]

fusion into a single piece, the sacrum. This anchylosis of adjacent sacral vertebræ is the most complete in birds and in Man, and for the same reason, namely, the employment of the hind limbs alone for the support of the body; although in the two cases the number and arrangement of the associated parts differs very considerably.

Variation in the sacral region is not confined to the lower forms, although it is more frequent in these latter (e. g., Necturus) and becomes relatively stable in the higher and more specialized classes. As shown in Fig. 34, there is variation both in the point of attachment of the hip bones and in the number of vertebræ involved in the composition of the human sacrum, and similar variations have been noted in other mammals. These, like the variation in the number of ribs and in the groups of vertebræ, not infrequent in the human subject, should serve to dispel the idea that the body of man or any 
other animal is formed in accordance with a definite pattern or is constructed upon any other principle save those of heredity and environment.

The anterior, or pectoral, girdle never becomes directly attached to the vertebral column, and consequently the latter receives no direct modification through the development of the former, but the use of this region as a secondary center of support causes a division of function between the vertebræ that lie anterior and posterior to it, the first forming the neck. By the establishment of this point and the sacrum, the two centers of support, the vertebral column becomes divided into regions, the differentiation of which depends upon the degree of development of the limbs and the amount of difference in the function to which these parts are subjected. Beginning anteriorly the vertebræ anterior to the first center of support are the cervical or neck vertebræ, the first one or two of which are especially modified to bear the head and allow of its special motions. The vertebræ between the shoulder-girdle and the sacrum are spoken of in general as the trunk vertebra, and in birds and mammals allow a further subdivision into thoracic and lumbar, the former being provided with free ribs, and the latter being without them. Then follow the sacral vertebra, usually more than one in forms above the amphibia, followed by the caudal vertebrce or tail. The correlation between the regional differentiation and the development of the limbs is especially emphasized by such forms as the whales, which have secondarily lost the hind limbs, and snakes, which have lost both pairs, since in the former the deprived region, and in the latter the entire vertebral column, have lost all trace of such differentiation.

The second cause of modification of the vertebral column is correlated with the first and is directly due to the increase in size of the limb muscles and their consequent need of broader and stronger points of origin. The limb muscles, in the case of animals with well-developed limbs, are usually broad, fanshaped sheets, like the trapezius and latissimus dorsi, attached wholly or in part to certain processes of the vertebral column, 
and cause much local differentiation in the varying degrees of development of these processes. Although topographically related to the trunk, and classed with trunk muscles in works on anatomy, they belong morphologically to the limbs, and when these latter are small, as in salamanders, the muscles are small also, seldom extending to the vertebral column, and thus exercise little or no modifying influence upon it.

Other modifications of the vertebral column are due to the movement of the body as a whole and to the separate and more or less specialized motions of the head and tail. Thus, to perform the crawling movement of salamanders and most reptiles, where a sinuous motion of the body axis forms the principal mode of locomotion, there must be a large amount of flexibility, especially in regard to lateral movements, between the separate vertebræ; and thus the amplicœlous form of intervertebral articulation, the restricted motion of which proves sufficient for fishes, becomes converted into true balland-socket joints by the ossification (or chondrification) of the ball of notochord contained in the cavities between each pair of adjacent cups, and by its anchylosis to one of the contiguous vertebræ. This forms the ball; the unmodified cupshaped end of the other vertebra serves as a socket. The anchylosis of the notochordal balls may take place with either the preceding or the succeeding vertebra; in the former case each vertebra of the series will have the cup at its anterior, and the ball at its posterior end, forming the type known as procolous, while in the latter case the reverse condition is the result, such vertebræ being designated as opisthocolous.

Both of these conditions are common among amphibians and reptiles, but with the attainment of limbs sufficiently stout to entirely sustain the weight of the body such a flexibility of the vertebral column is not only unnecessary but becomes a positive detriment, and thus in mammals the vertebra become acclous, that is, the articulations are reduced to mere flat contact surfaces, and the notochordal balls are transformed into the intervertebral cartilages that serve as cushions. In the cervical vertebræ of many mammals the opisthocœlous 
type of articulation is retained. In birds even this restricted motion is undesirable except in neck and tail, owing to the use of the entire body as an air-ship which must be held in a rigid position, and the trunk-vertebræ ossify into two completely anchylosed pieces, the first including the thoracic, and the second the lumbar, sacral, and a part of the caudal, vertebræ.

The head is responsible for many modifications of the vertebral column, developed in part in response to the necessity of turning it in all directions, and in part to the need of lifting it from the ground, or even sustaining it above the level of the rest of the body. Like many others, these problems are associated with a terrestrial environment and are not experienced by fishes, in which the main encleavor is to retain the head in a rigid state as the direct anterior extension of the body axis, since a pliant head would render a change of direction while swimming of almost momentary occurrence and would entirely forbid those quick, arrow-like propulsions upon which most fishes depend for safety and for the successful pursuit of their prey. In the first experience of a terrestrial life, however, all this becomes changed. The turning of the head not only gives an increased power of observation, but is necessary in attack and defense, and thus the vertebræ lying between the skull and the place of support for the anterior limbs become differentiated to form a cervical or neck region, the main endeavor of which is to gain flexibility and increase the mobility of the head.

Although in some of the higher terrestrial vertebrates this power is but little used, in others it develops to an extraordinary extent, notably among the birds, in which this is the only part of the vertebral column, except the tail, to which motion is allowed. Here, in some instances, the neck not only becomes extremcly flexible, but greatly elongated, accompanied by extraordinary modifications of the trachea and the bloodvessels, in order to accommorlate themselves to the rapid changes of shape and position of which the neck becomes capable.

On the other hand, certain mammals, like the whales and 
porpoises, and the dugongs or sea-cows, which, having descended from terrestrial ancestors, have become secondarily adapted to an aquatic life, form a remarkable corroboration of the statement that a movable neck is incompatible with a natatory habit, since in these the seven cervical vertebræ, typical of the Mammalia, have become greatly flattened anteroposteriorly, and are either fitted so closely together that but little motion is possible between them, or are even anchylosed into a single piece, thus not only reducing the length of the neck region and approximating the head to the shoulders, but depriving it of motion, two important piscine characteristics.

Not only are the intervertebral articulations in the cervical region extremely flexible in general, but that of the first with the skull and the first with the second become especially modified, changes which often profoundly affect the shape of these vertebræ. The first of these articulations is a double modified ball-and-socket joint, the protuberances, or occipital condyles, occurring upon the occipital region of the skull along the lateral edges of the foramen magnum, and fitting into saucershaped depressions on the anterior face of the first vertebra, or atlas. In Amphibia and Mammalia these condyles are wide apart and distant from one another, while in reptiles and birds. they coalesce in the mid-ventral line and form what appears to be a single median condyle, the two components being usually indicated by a median groove. In all cases the motion between the skull and the atlas is in one plane only and imparts to the head the bowing motion.

The turning from side to side is effected by the articulation of the first vertebra with the second, and is due to a curious modification by which the body of the first vertebra remains disconnected from its own neural arch and anchyloses with that of the second vertebra, the axis, forming its pivot-shaped odontoid process, around which the ring-shaped atlas may rotate. This typical relation of the first two vertebræ, occurring in reptiles, birds and mammals, is modified in amphibians through a secondary inclusion of the elements of the atlas 
within the skull, leaving the axis with its pivot to serve as the first free vertebra. This bone secondarily acquires articular surfaces to articulate with the lateral conclyles.

In animals whose limbs are strong enough to sustain the body above the ground the. weight of the head and the necessity of holding it in place beyond the anterior center of support causes considerable modification of the vertebræ, the influences sometimes reaching beyond the middle of the body. In these cases the head is held up in part by muscles, but in mammals there is also an important auxiliary apparatus in the form of a strong ligament, the ligamentum muche, which extends between the occipital region of the skull and the spinous processes of the cervical and dorsal vertebrx on a principle similiar to that of a check rein.

In mammals with large and heavy heads, especially when the weight is augmented by voluminous horns or large tusks, the weight sustained by this ligament becomes enormous, and not only is the ligament developed in proportion, but so, also, are the occipital crests and the spinal processes of the vertebræ, which serve it as points of attachment, the processes especially involved being those of the anterior dorsal region opposite the shoulders. This correlation between a heavy head and exaggerated spinous processes is such that from a slight indication of the one in a fossil the other may be assumed. In the Cetacea, which have the buoyancy of the water to assist them, and in Man, through the erect position of whom the head becomes almost perfectly balanced upon the summit of the vertebral column, this entire apparatus, including the ligament and its points of attachment, becomes much reduced, but from a totally different cause in the two instances.

The tail, or post-sacral region of the vertebral column, is developed strictly in correlation with the needs of the animal and varies in development from a voluminous portion of the body, containing a large number of vertebre and furnished with metameric muscles, to a mere rudiment, invisible ex- 
ternally. Examples of the former may be seen in salamanders and in many snakes, in which the caudal region, that posterior to the cloacal orifice, may be even more extensive than the remainder of the body; the opposite condition appears in the frog, where the long caudal notochord of the tadpole becomes in the adult consolidated into an unsegmented urostyle, situated between the two elongated ilia and entirely enclosed by the soft parts. Similar reductions are found in birds, in which the tail skeleton consists of six free and six anchylosed vertebræ, and in the higher anthropoids, in which the 3-5 embryonic vertebra become in the adult consolidated into a single piece $(\operatorname{cocc} y x)$.

There are two distinct sets of ribs developed among Vertebrates, having a slightly different history, but subserving the same general purpose, that of protecting the viscera, and of furnishing attachments for the muscles. Since one set is sufficient for use in the same animal, both do not occur simultaneously save in a single instance, but the one set is, with some exceptions, characteristic of fishes, the other of higher forms. The origin of the first of these sets has been already explained in the discussion of vertebræ, where they were described in teleost fishes as expansions of the lower or hæmal arches. The other ribs have in their origin no direct connection with the vertebra, that is, they are not derived from portions of them, but develop from the free edges of the intermuscular septa, the myocommata, where they border upon the visceral cavity. This process of rib formation does not necessarily involve the entire free edge of the septa, but is confined in lower forms to the extreme dorsal region, bordering on the vertebræ, with which they articulate. Thus in selachians, almost the only fish that possess this sort of rib, and in amphibians, they are very short, being functionally scarcely more than movable tips for the transverse processes and of no value for the protection of the viscera. They make no attempt to reach around the body, and thus never come into relation with a sternum. This latter, to us the typical relation for ribs to assume, appears first in reptiles, which thus form the prototype 
of the later development in birds and mammals. In these latter a typical rib possesses two well-marked segments, a dorsal and a ventral, often bent at an angle to each other; both
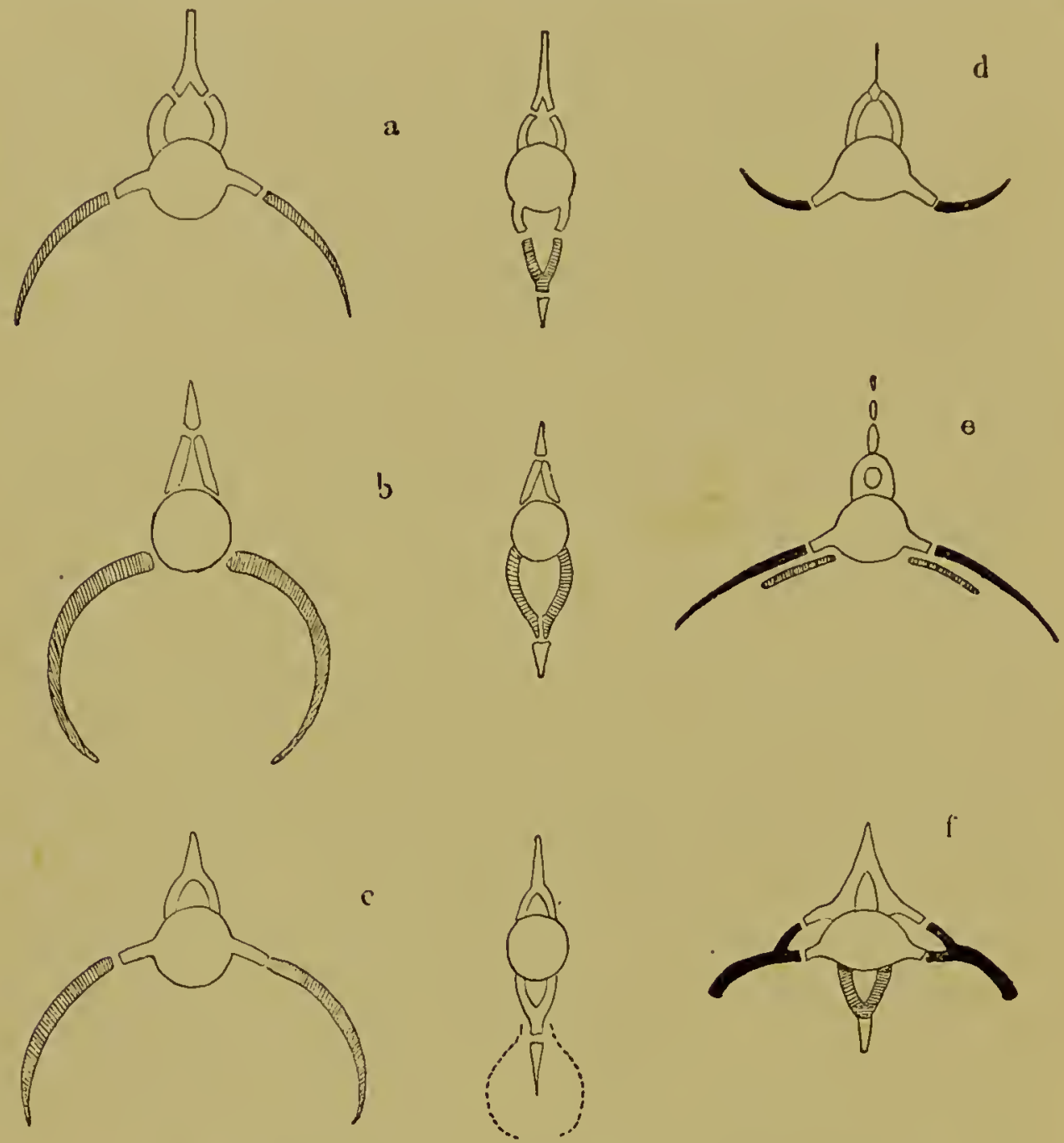

Fig. 35. Morphology of ribs. [After WiedersheIm.]

(a) Ganoid. (b) Dipnoan. (c) Teleost. (d) Selachian. (e) Polypterus (a special case among ganoids). (f) Urodele.

In the three first the condition in both trunk and tail is given. In all the figures the "fish rib" is striped, the myocommatous rib is black, and the basal stumps are outlined.

may be fully ossified, as in birds, or the ventral segments may remain cartilaginous, forming the so-called "costal cartilages," characteristic of mammals. In birds the dorsal segments possess flat uncinate processes, which extend backwards from their posterior edges and overlap the succeeding rib, thus 
effecting here the rigidity necessary in all parts of the body in adaptation to flight while allowing for play of the respiratory motions.

The distribution of the two types of ribs among vertebrates is a little unusual, since the second or myocommatous type appears, not only in amphibians and amniotes, the higher groups, but also in the selachians, one of the most primitive. This is one of the many indications of kinship between these and the higher forms, and suggests the direct descent of the amphibians from selachian-like ancestors, thus disposing of the remaining fishes as collateral lines, in which the piscine type attains its special line of development, without relationship to the higher classes, save through a common ancestor. The hæmal arch ribs, or true "fish-ribs," are characteristic of teleosts, dipnoi, and most ganoids; in one of the latter, Polypterus, both sets appear simultaneously, the myocommatous set being functional, while the hæmal arch set is rudimentary, not attached to the vertebræ, and hence of little use to the fish, but of great significance to the anatomical historian.

In a strict sense it cannot be said that the fish-rib or hæmal arch ribs are in all cases exactly homologous with one another, or even that they are in all cases formed mainly from the hæmal arches, since recent investigation has demonstrated the existence of other elements, derived directly from the bodies of the vertebræ, and normally supporting the hæmal arches, which are often concerned in the formation of the ribs; but not only would an exposition of this lead us too far into details, but would take us away from the main inquiry, since the phenomena do not occur on the direct road traced in our present history. The conception of these ribs as expanded hæmal arches is not in any case far from the truth, since the other elements concerned are themselves functionally if not morphologically parts of the hæmal arches.

As shown by amphibians and reptiles every vertebra between the second (axis) and the sacrum is typically furnished with a pair of ribs, which in these Classes are usually free. In birds and mammals certain of these become anchy- 
losed to the vertebra which bear them, leaving a set of thoracic vertebræ (the "dorsal "vertebræ of the older terminology) interpolated between two groups, cervical and lumbar, in which the rib elements are fused. In the cervical vertebra these fused ribs form the ventral element of the plainly double transverse processes, and enclose between themselves and the original transverse process (diapophysis) the vertebral foramina. In the lumbar vertebræ the rib elements form the large wing-like transverse processes (pleurapophyses), and are thus seen to be not equivalent to the processes of the same name in other regions.

The number, both of free ribs and of vertebræ forming each group, differs considerably, not only in different mammals, but even in different individuals of the same species. Thus in Man, although twelve pairs of free ribs is the rule, the rib element of the last ( 7 th) cervical vertebra is occasionally free, "cervical rib," and, more commonly, a free rib appears on the first lumbar vertebra. As this is perhaps the rule rather than the exception in the gorilla, one of Man's nearest living allies, this anomaly is often called the "gorilla rib." Variation in the sacral vertebræ has already been noticed [Cf. Fig. 34 and accompanying text].

The origin of the sternum is still a matter of controversy, and it seems likely that there may have been two sternums, of different origin, the one succeeding the other during historical development, the archisternum and the neosternum. Fishes lack the part entirely, but it is present in some form in all other vertebrates save in a few aberrant cases, for example, the snakes, where it is incompatible both with their mode of locomotion by means of the ends of the very numerous ribs, and with their habit of swallowing huge mouthfuls, far too large to pass through the ring formed by the vertebrx, ribs, and sternum, as is the usual arrangement. What is apparently the first indication of a sternum is seen in the salamander Necturus, perhaps the lowest amphibian, in which from three to five of the thoracic myocommata chondrify in the ventral region, forming small V-shaped elements, indefi- 
nite in shape and irregular in occurrence, as is usually found in an organ at its beginning; before the type has become fixed.
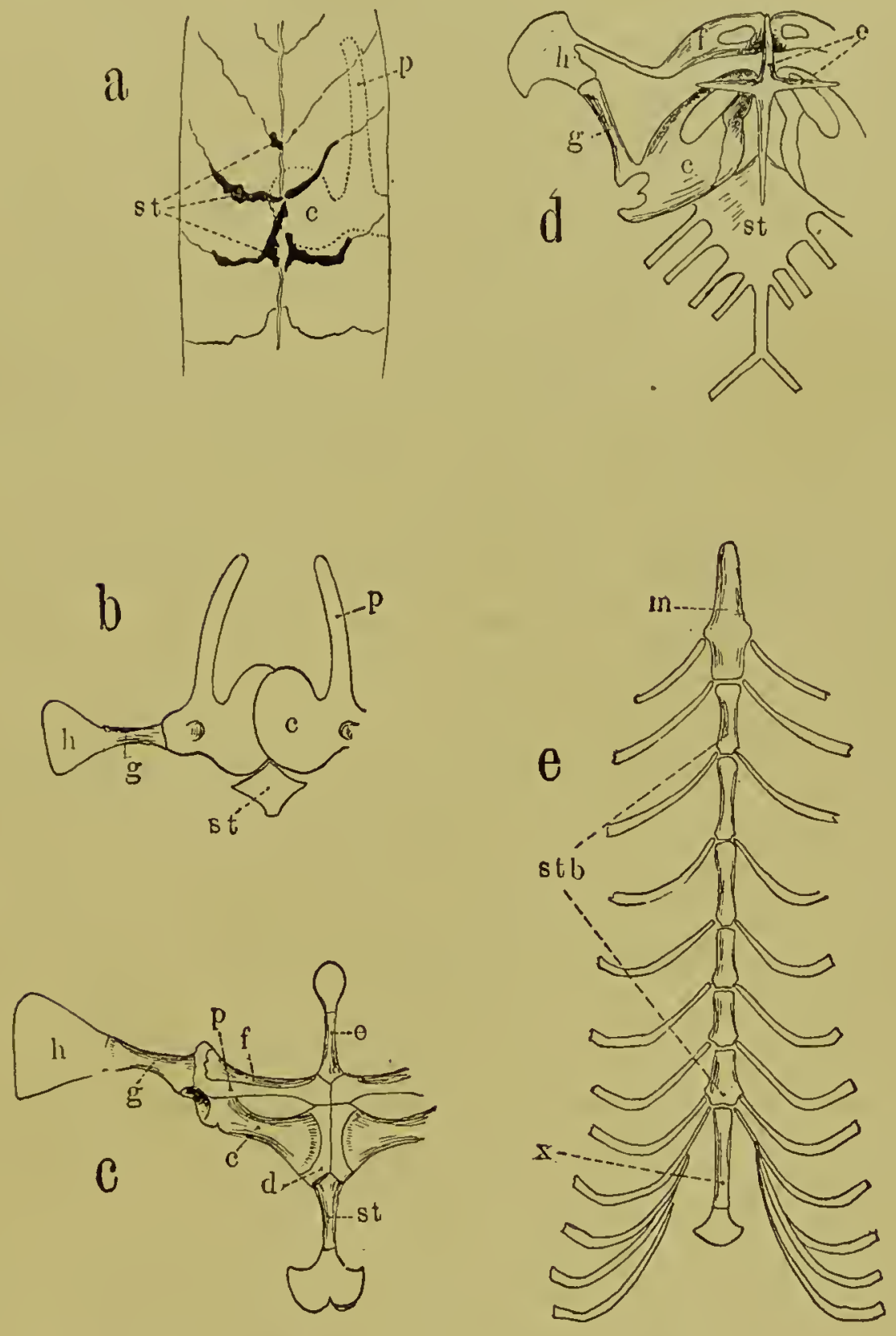

Fig. 36. Morphology of the sternum.

(a) Necturus (a primitive salamander). (b) A higher salamander. (c) Frog. (d) Laceria (Europcan lizard). (e) Cat.

$c$, coracoid; $d$, epicoracoid; $e$, cpistcrnum; $f$, clavicle; $g$, scapula; $h$, suprascapula, $p$, procoracoid; st, sternum; $m$, manubrium; $s t b$, sternebra; $x$, xiphistcrnum.

If we seek the reason for their appearance we shall probably find it in an attempt to lessen the pressure upon an important 
point. One of the sternal elements, usually the largest, lies in the fourth myocomma, in close connection with the overlapping coracoids, and as at the same point in higher salamanders there is a definite sternal plate of a rhomboid shape, this latter has evidently developed from the element in question, while the others have been lost. This must also be the same piece found in frogs and other tailless amphibians, again in the same relationship to the coracoids, and entering into a more or less complete connection with the two halves of the shoulder-girdle in forming the skeletal armature that covers the pectoral region. As the ribs of all amphibia are very short and rudimentary, and do not reach even half way around the body, there is never the slightest attempt at a connection between them and the sternal piece, a characteristic that definitely distinguishes this archisternum from the neosternum of the Amniota. This last organ, the second form of sternum, is characteristic of reptiles, birds, and mammals, and is not only always connected with several pairs of thoracic ribs, but undoubtedly owes its origin to them, being probably due to the fusion of the ribs in the mid-ventral line. This fusion forms in reptiles and birds a flat plate, especially extensive in the latter, where it serves as a place of origin for the enormously developed muscles of flight, but in the mammals the sternum, continuous with the ribs while in the cartilaginous state, ossifies in the form of a series of separate elements, the sternebre, one for each pair of ribs involved. The original number of these elements may be retained throughout life, as in most mammals, or may become reduced by a secondary fusion to a smaller number.

The confinement of the sternum to the thoracic region leaves the ventral abdominal surface unprotected, an affair of no great moment so long as an animal remains small, or not very much elongated, but when, as in the Crocodilia, the elongation of the body greatly increases the extent of the unprotected surface, while at the same time the increase in size renders the body ponderous, the pressure exerted on the abdominal viscera by the weight of the body as the animal 
crawls, or even lies passively on the surface of the ground, must be very great. It is evidently to overcome this in part and furnish some protection for the soft parts that there develops in this region a series of skeletal elements precisely similar in origin to the primitive sternal pieces of Necturus, formed by the ossification of the ventral portion of the abdominal myocommata. Developing along the mid-ventral line also, many of the pieces become connected together and form a system of "abdominal ribs," as they have been called, better known as the parasternum. As these do not appear to be represented in any other Order, they are of no phylogenetic value, but serve to explain the reason for the origin of the archisternum in the salamanders by furnishing an exact physiological parallel. Associated with the sternal region, both in Amphibia and in the Amniota, there is a rather problematic element, known as the episternum, of which no continuous history is yet known, so that it is not even certain that the various elements in different animals called by that name are homologous. The typical episternum is a skeletal piece occurring in lizards and consisting of a thin cross-shaped or $\mathrm{T}$ shaped piece lying, as its name denotes, upon (i.e., on the ventral side of) the sternum, and a little anterior to it.

This part is not clearly present in other vertebrates, but similar pieces occur in several cases, and are often designated by the same name. Thus, in the shoulder-girdle-sternum complex of the frog there is a piece extending anteriorly along the mid-ventral line, between the clavicles, and closely resembling the true sternum (archisternum) that extends posteriorly. This has been often called the episternum, but is more likely a portion of the archisternum, formed like the other, from myocommata. Again, the well-known "wish-bone" of birds is formed by a fusion of the two clavicles with a middle piece, the interclavicle, which forms the "head" and is es-" pecially well developed in the common fowl. This element also has been identified with the episternum by some investigators, as have also certain parts of the keel of the sternum, which develop from separate centers of ossification. 
Among the Mammalia the lowest Order, the Monotremata, possess in this region a large $T$-shaped bone, the stem of which, very broad and flat, articulates with the true sternum, forming its anterior extension, while the lateral arms are applied along the sides of the clavicles. This piece has been called by some an episternum and by others an interclavicle, but its precise homologies are not definitely determined. In all other.mammals the clavicles apparently articulate directly with the most anterior of the sternal pieces, the marubrium;

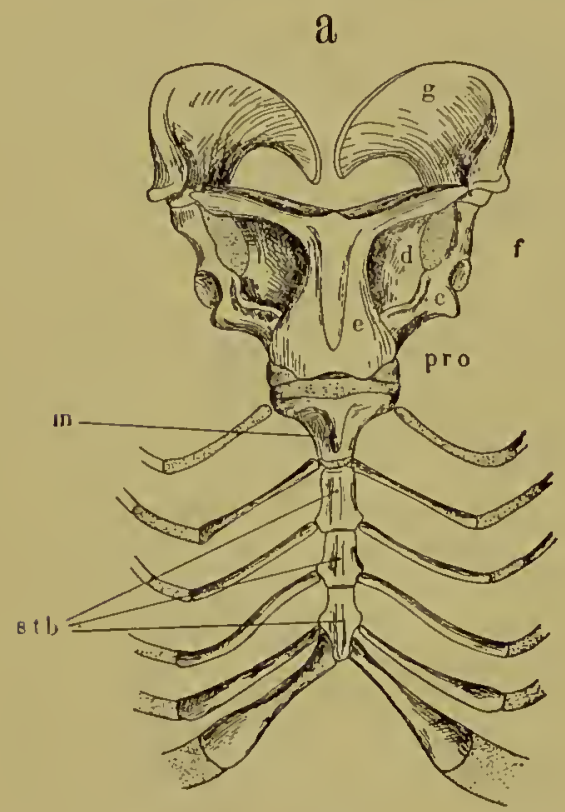

b

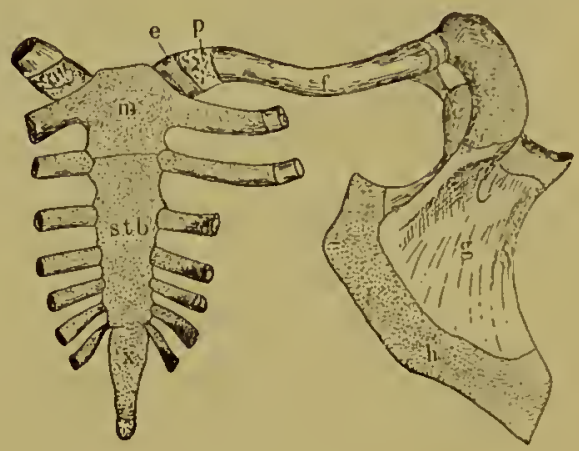

FIG. 37. Sternum and shoulder-girdle of mammals. [After W. K. PARKER.]

(a) Ornithorhynchus. (b) Human embryo.

$c$, coracoid; $d$, epicoracoid; $e$, episternum; $f$, clavicle; $g$, scapula; $h$, suprascapula; $m$, nanubrium; stb, sternebræ; $x$, xiphisternum.

but in the embryo there are found definite disc-shaped skeletal elements, interposed between the two, which develop later into thin, interarticular discs. These, usually designated omosternum, have been likened to the lateral arms of the T-shaped bone of the Monotremata.

The ontogenetic history of the skull, a complex of skeletal elements developed at the anterior end of the notochord, is singularly constant in all classes, and we may thus feel confident that we have in this a repetition of stages once passed 
through by the adult ancestors of the present-day vertebrates. It is true that the early stages thus indicated do not correspond with the adult condition of any form now living, but of the two types in which we might expect to find a correspondence with this period of the history, Amphioxus has no head, and, of course, no skull, and the cyclostomes with their parasitic habit are too much modified to be reliable; there is, moreover, an enormous gap between the two and a second, almost as great, between the latter and the selachians, so that it may well be conceded that adult animals representing the stages indicated by the embryonic history once existed in the places now left vacant. Nothing could fit better into this ontogenetic history at a later period than the selachian skull, as will be shown further on, thus verifying the record at an important point, and rendering it more probable that the earlier embryonic stages, so constant in appearance in all vertebrates, are equally accurate in reproducing the conditions once found in forms now lost to us. To outline the history, then, with the help of embryology, it appears that the ancestral vertebrate, after the acquirement of the prochordal addition to its head, developed several pairs of external sense organs in the cephalic region, three of which, the nasal sacs, the eyes, and the (inner) ears, have persisted. Of others there are indications in early embryonic life, such as the one placed between the eye and ear and supplied by the seventh nerve, and there are reasons to believe that the original sense organ of the second pair was not the eye as we have it now, but the lens alone, in the form of a simple capsule; but these matters hardly belong in this place and are suggested merely as indications of the elaborate past history of the head, entirely gone from the world of adult life, but now restored in part by the labors of a generation of embryologists. At this tirne the notochord, terminating at the hypophysis, a downgrowth of the brain just anterior to the ear capsules, was the only skeletal element in the head, and could have had little value as an organ of support, and none whatever as an organ of protection. This condition of affairs is represented in Fig. 38 , A, which, it 


\section{I44 HISTORY OF THE HUMAN BODY}

must be noted, represents the head as seen, not from above, as it is more usually drawn, but from below, a view that enables one to see the notochord and its termination behind the hypophysis. To this condition there are added, but no one yet knows how or from what source, two pairs of laterally placed cartilages (Fig. 38, B), the one alongside the noto-
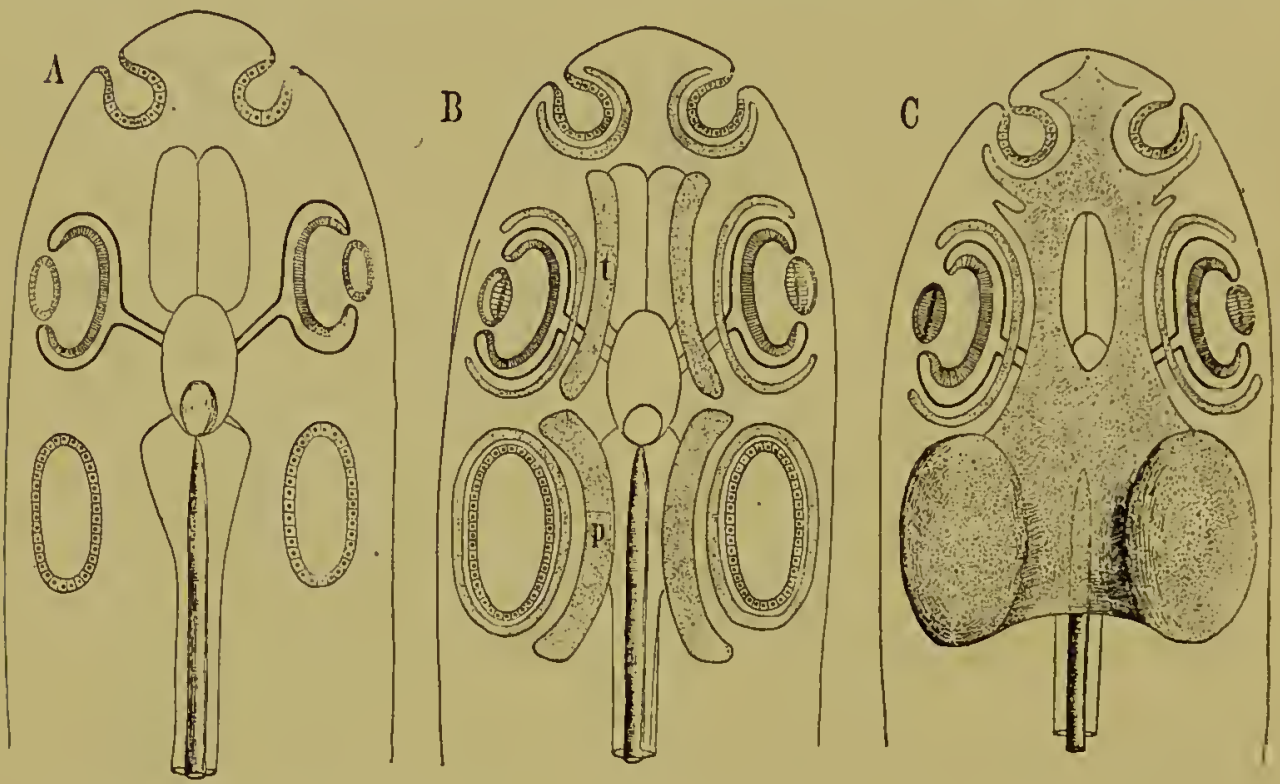

FIG. 38. Diagrams showing the development of the primordial skull. Since this organ develops primarily beneath the brain as a support the figures represent the ventral aspect.

(A) Early stage, before the appearance of cartilage. The notochord is seen lying along the nerve cord as far forward as the hypophysis. The three sense-organs, nose, eye, and ear, have already appeared. (B) This stage shows the trabecula [t], the parachordals [p], and the capsules around the sense-organs. (C) In this the trabeculx, the parachordals, and the nasal and otic capsules have fused into a single mass, the primordial skull, or chondrocranium. The anterior end of the notochord is imbedded in this. The cartilaginous capsule of the eye remains free to allow the necessary movements of the eyeball.

chord and the other anterior to it, the parachordal and prachordal elements respectively. The former are rather flat, of an elongated crescentic shape, filling in the space between the notochord and the ear capsules; the latter are elongated and rod-like or beam-like, hence often termed the trabeculc (diminutive of trabs, trabis, a beam), and lie a little beneath the eyes and nearer the median line.

At the same time the three persisting pairs of sense organs 
become enclosed by cartilaginous capsules, differing somewhat in their development, according to the needs of the organ. Thus the nasal capsules remain open anteriorly for the free admission of the fluids to be tested, the eye-capsules involve the sclera alone, while, the otic capsules usually become entirely closed and develop fairly thick walls, since sound vibrations can pass easily through solids and do not need a special opening. As these several cartilaginous elements, the para- and præchordals and the sense capsules, increase in size, they fuse together in about the following manner. The two trabecula expand anteriorly and fuse with each other across the middle line and with the nasal capsules as well; extending backwards, they fuse with the parachordals. 'These latter, growing in width, fuse both with the otic capsules and with each other, including in this fusion the anterior end of the notochord, which becomes lost in the general mass.

There is thus formed a single, curiously shaped piece of continuous cartilage, composed of all the elementary pieces, with the natural exception of the otic capsules, which must remain free to allow the turning of the eyeball (Fig. $38, \mathrm{C}$ ). These pieces fuse so completely that all boundaries are lost, and we can speak only of a parachordal or a trabecular region, and so on, without assigning definite boundaries. This consolidated piece is termed the primordial skull or chondrocranium, and remains at this stage in selachians, where it is characteristic of the entire Order, being throughout life without trace of ossification and with such slight modifications only as are necessary for the adaptations of the various adult forms. It is a natural supposition drawn from common experience that a skull is intended for the protection of the brain, but in this case the function is rather that of support, since it lies laterally to and in part beneath the brain, leaving practically the entire dorsal and the anterior part of the ventral aspects without protection. In the adult selachians, indeed, these deficiencies are made up in part by the formation of firm membranes, continuous with the cartilage and closing in the open fontanelles, but they are plainly secondary modifi- 
cations, for use during active adult life, and are not emphasized in the embryonic history of higher forms.

This stage of the chondrocranium, or the selachian stage, as it may be called, is passed through with during the development of all the higher vertebrates, and although in the various forms the shape and proportion of the parts often differ widely in anticipation of the various needs of the adult, they all possess in common the origin in the same way, from the same elemental parts, and the characteristic regions may in all cases be readily identified.

For the next stage in this history it will not be necessary to have recourse to embryology save to verify the conclisions, since it is represented with almost diagrammatic clearness among the ganoids, a very few of which have been, by a fortunate chance, saved from the general destruction of the Order during an earlier geological period. This siage may be thus conveniently denominated the ganoid stage, for the type of which we may select the sturgeon. Although similar to the selachians in many respects, this animal differs markedly from them in its external covering, for while the former is evenly and uniformly covered by small placoid scales arranged in a regularly imbricated pattern, the sturgeon possesses $a$ series of large, bony plates, or scutes, as they are called, which may be considered as having been formed originally from the fusion of the basal pieces of many scales. These scutes are arranged on the body in longitudinal rows, leaving the intervening regions bare, but are continued over the head as somewhat modified scutes, the edges of which are in contact, thus forming an external armor, with sutures between the different scutes (Fig. I9, A). Immediately beneath this lies a cartilaginous skull, very similar to that of selachians, and the dermal armor encases it like an external skull, which it really is. These dermal plates are quite definite in their arrangement, and the same general plan may be followed throughout the Order of ganoids. The snout, or rostrum, is covered by a series of small rostral plates, which extend back as far as the nostrils; back of these openings may be found a 
pair of nasals; behind these again, and between the eyes, is a pair of frontals, often accompanied by prec-and post-frontals. Behind these is a pair of parietals, and one or more supraoccipitals. On the sides of the head, at about the level of the parietals, are the squamosals, and around the eye are several orbitals, distinguished as pre-, supra-, post-orbitals, etc. The operculum, or gill-flap, which is present in these fishes, is covered and augmented by supra-, sub-, and pre-operculars.

In short, to anticipate the history a little at this point, we see in the dermal scutes the first appearance of the so-called dermal bones of the skull which in later forms are to sink in beneath the surface and become internal, thus coming into close connection with the primordial skull and the osseous elements derived from it. They are not all inherited by higher forms exactly as they occur in the ganoid, the question of their retention being based in each case upon their functional importance. Thus, the opercular series, retained in the fish, becomes lost with the reduction of the part which they cover; the orbital series is retained in part by reptiles, but becomes lost in birds and mammals, with the single exception of one of the præ-orbitals, which becomes the lacrimal; and the supraoccipital series becomes reduced to a single piece. On the other hand, certain ones are retained in all higher vertebrates, and are recognizable throughout, although by secondary fusions and divisions they are not always strictly homologous. Thus, the frontals may or may not include the originally separate præ- and post-frontals, and in a given case the absence of one of these latter elements as a distinct piece may mean either that it has fused with one of the others or has been gradually reduced in size until it has become lost. The frontals in some form, however, are among the most constant of dermal elements, and the same may be said of the parietals, squamosals and nasals, which can be traced in all the vertebrate classes (Fig. I9). The ventral side of the cranium becomes also encased in a similar manner by dermal bones that develop in the roof of the mouth, among which are the vomers, the palatines, the pterygoids, and the extensive parabasal 
(parasphenoid), which forms almost the entire base of the cranium in fishes and amphibians. Certain of these last named do not develop, strictly speaking, in association with the cranitum, but are formed about certain elements of the visceral skeleton, as will be explained below, but as these latter clements early lose their physiological independence and become closely incorporated with the original chondrocranium, the statement is in no way misleading.

These dermal plates thus form an almost complete case of bone, surrounding and protecting the internal cartilaginous skull, and, by supplying the deficiencies of the latter, effect the complete enclosure of the brain within the skeletal parts. There are thus formed two skulls, one within the other, and in the ganoids, where the relation between the two is not as yet a very intimate one, the outer or bony skull may be easily removed from the other.

The next step in advance is one shown also among the ganoids, and consists of the strengthening of the chondrocranium directly by the development of centers of ossification within the cartilage itself (endochondral ossification) forming definite osseous elements, called from their mode of origin cartilage bones, in distinction from the other, the dermal bones. Among these centers may be enumerated the exoccipitals, the pro-otics, epiotics, and opisthotics, which together form the petrosals, the ali-sphenoids, the orbito-sphenoids, and the ethmoids, well-known elements in the skulls of higher vertebrates, but here found at their inception, arising as isolated areas of the chondrocranium and developing at the expense of the cartilage, clearly differing from the dermal bones in origin.

We thus find in the skull of the ganoids the elements of the vertebrate skull almost at their beginning, and can trace the origin of parts familiar to us as they appear in the specialized skulls of mammals, where, under the cloak of an exactly similar external appearance, their diverse origin has become lost. The ganoids seem thus a vital link in the story of the skull, yet even had they become entirely extinct, as they 
came very near being, this portion of the history might have been deciphered from the embryological records, since even in the inammals the primordial skull develops from its primitive elements, the cartilage bones appear as centers of ossification within it, and the dermal bones, never preformed in cartilage, appear as subcutaneous ossifications in the connective tissue.
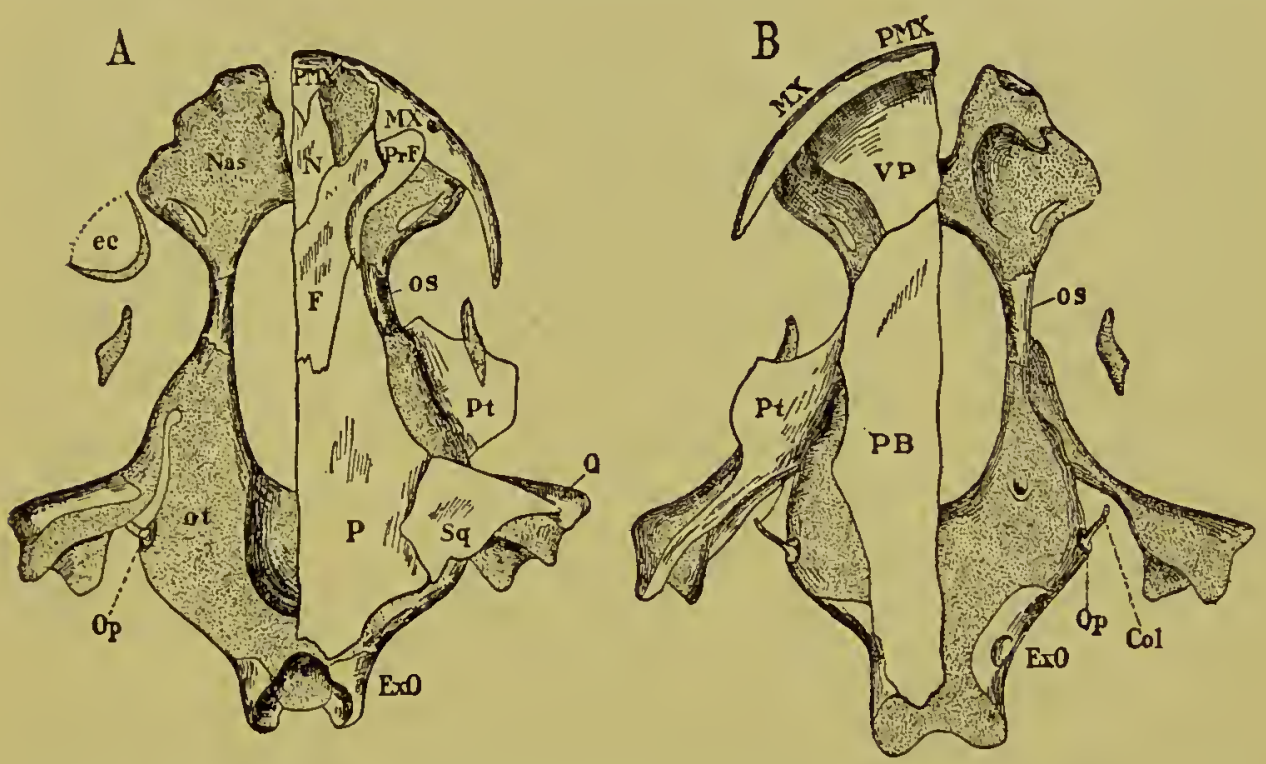

FIG. 39. Two views of the skull of Cryptobranchus allegheniensis, a primitive salamander, a little higher than Necturus.

(A) Dorsal. (B) Ventral.

Dermal Bones: $p m$, premaxillary; $m x$, maxillary; $n$, nasal; $f$, frontal; pr. $f$, pre-frontal; $p$, parietal; $s q$, squamosal; $p t$, pterygoid; $v p$, vomero-palatine; $p b$, para. basal.

Cartilage Bones: os, orbitosphenoid; $q$, quadrate; ex. 0 , exoccipital op, operculum, sule.

OTHER Parts: col, columella; nas, nasal capsule; $e c$, eye capsule; ot, otic cap-

In both figures the dermal bones have been retained on the right side of the skull and removed on the left.

In that case, however, we could hardly have obtained an idea of the appearance of the adult ganoids, since embryology, with its distortion of the facts through an early assumption of the proportions of the perfected animal, is an unsafe guide upon which to base more than very general conclusions. Had the ganoids been lost we would have believed in a general way in the former existence of fish-like forms in which the dermal bones were still in the form of an exoskeletal armature, but their exact appearance and relationship would have given rise 
to endless controversy, such as always occurs with regard to places where the records are incomplete, and this vital period in the history of the skull would have lost much of its reality.

As to the necessity which caused the appearance of these endochondral ossifications in the primordial skull, there has been pointed out a curious relationship between them and the principal cranial nerves, namely, that the ossifications develop about their places of exit from the brain cavity as though to protect them. Thus we have the olfactory nerve surrounded by the ethmoid, the optic nerve perforating the orbito-sphenoid, and similar relations existing between the trigeminus and the alisphenoid, the facial nerve and the proötic, and the ninth and tenth and the exoccipital. These are certainly the topographical conditions, but whether a causal relation really exists between them is not known.

In completing the history of the skull, it remains to notice the amphibian stage, best exhibited by urodeles, and the amniote stage, typically represented by reptiles and mammals. In the first of these the dermal bones are no longer external at any stage of their development and have become definitely incorporated with the skull as physiological parts of the internal skeleton. Aside from this the characteristically piscine elements, like the rostrals, the orbitals and those associated with the operculum, have become lost, and the bones assume more the number and relationships of the higher terrestrial forms (Fig. I9, B, and Fig. 39).

In the Amniota one of the fundamental changes is the loss of the parabasal as the main element of the cranial floor, and its functional replacement by a series of median cartilage bones, the basi-occipital, basi-sphenoid and pra-sphenoid. The parabasal may be entirely lost, but in the light of recent investigation it seems probable that it is continued as the median vomer, which is thus not the same as the paired vomers, which form a portion of the roof of the mouth of fishes and amphibians, and which, if this view is the correct one, probably disappear in amniotes.

Another characteristic is the secondary fusion of elements, 
and as the purpose of this procedure is wholly physiological, the object being to insure local strength or gain muscular attachments, the process does not respect origin, but oftentimes involves both dermal and cartilage bones, and may even include, as well, elements of the visceral skeleton. The results are thus bone-complexes, each of which, in the adult, is a morphological puzzle, to the history of which we have in this stage no clew. Among mammals these consolidations are very extensive, especially among the primates, but even this condition is eclipsed by that in birds, where the fusion reaches its extreme and nearly all of the bones of the cranium proper are fused in the adult into a single piece, so that, in order to properly study the skull, observation must be made upon a newly-hatched fledgeling or even upon an advanced embryo extracted from the egg.

In the skulls of both amphibians and amniotes, even in the most completely ossified ones, there remain certain portions of the unossified chondrocranium, especially about the nose and internal ears. In mammals there develop from this the cartilages of the external nose which, although often highly specialized, are to be considered in respect to origin the most ancient parts of the skull.

In giving the account of the earlier stages in the development of the chondrocranium, nothing was said concerning the subsequent history of the cartilage of the eye-ball, which could not unite in the formation of the skull. In many fish the outer coating of the eye-ball (sclera) remains cartilaginous and occasionally becomes very thick and heavy (c.g., swordfish). There are also traces of cartilage in the sclera of many salamanders. Both cartilage and bone occur in the sclera of certain birds, notably hawks and owls, in the latter of which a series of long, palisade-like ossicles forms an elongated tube, shaped something like the tubes of an opera-glass. Whether these are the direct descendants of the primitive capsule seems very doubtful, and it is more probable that these developments have been called out de novo in response to functional necessity. 
The visceral skcleton is associated with the anterior portion of the alimentary canal and the parts derived from it, and seems to have developed primarily along the sides of the pharynx for the regulation of those most primitive of vertebrate respiratory organs, the gill-slits. Although Amphioxus possesses a very regular and quite complicated system of skeletal bars to support its eighty or ninety pairs of gill-slits, these cannot as yet be brought into definite relationship with the visceral skeleton of the higher vertebrates; the same may be said of the visceral skeleton of the cyclostomes, which is in the form of a complicated pharyngeal basket, bearing little apparent resemblance either to the skeletal bars of Amphioxus or to the succession of simple arches characteristic of the fishes. It is in the selachians that we first meet with a visceral skeleton to which that of the higher forms can be certainly referred, and it is here, therefore, that the morphological history of the vertebrate visceral skeleton must start.

It consists of a series of pairs of cartilages, more or less modified from the form of simple rods, and alternating with gill-slits that open from the exterior into the pharyngeal cavity. In most selachians there are seven well-developed pairs of these, besides a few cartilages that may represent rudiments of others, but as in two very primitive genera there are, respectively, eight and nine regular pairs, besides the rudiments, eleven or twelve original pairs can be accounted for, thus suggesting a former condition with a large number of gill-slits, a supposition that compares well with the testimony furnished by Amphioxus. The selachian condition, showing all the pieces that may be accredited to the visceral skeleton, together with the chondrocranium, is given in Fig. 40 , somewhat diagrammatized from an actual preparation. It may be safely assumed that at one time these visceral arches were all similar to one another, associated with similar gillslits and all gill-bearing, although important modifications have now taken place in certain ones of them. These modifications are of the highest importance in this history and may now be considered, with constant reference to the figure. 
The first, or mandibular pair, which is the most modified of all, becomes folded about the mouth in such a way as to make a serviceable pair of jaws, both upper and lower, with
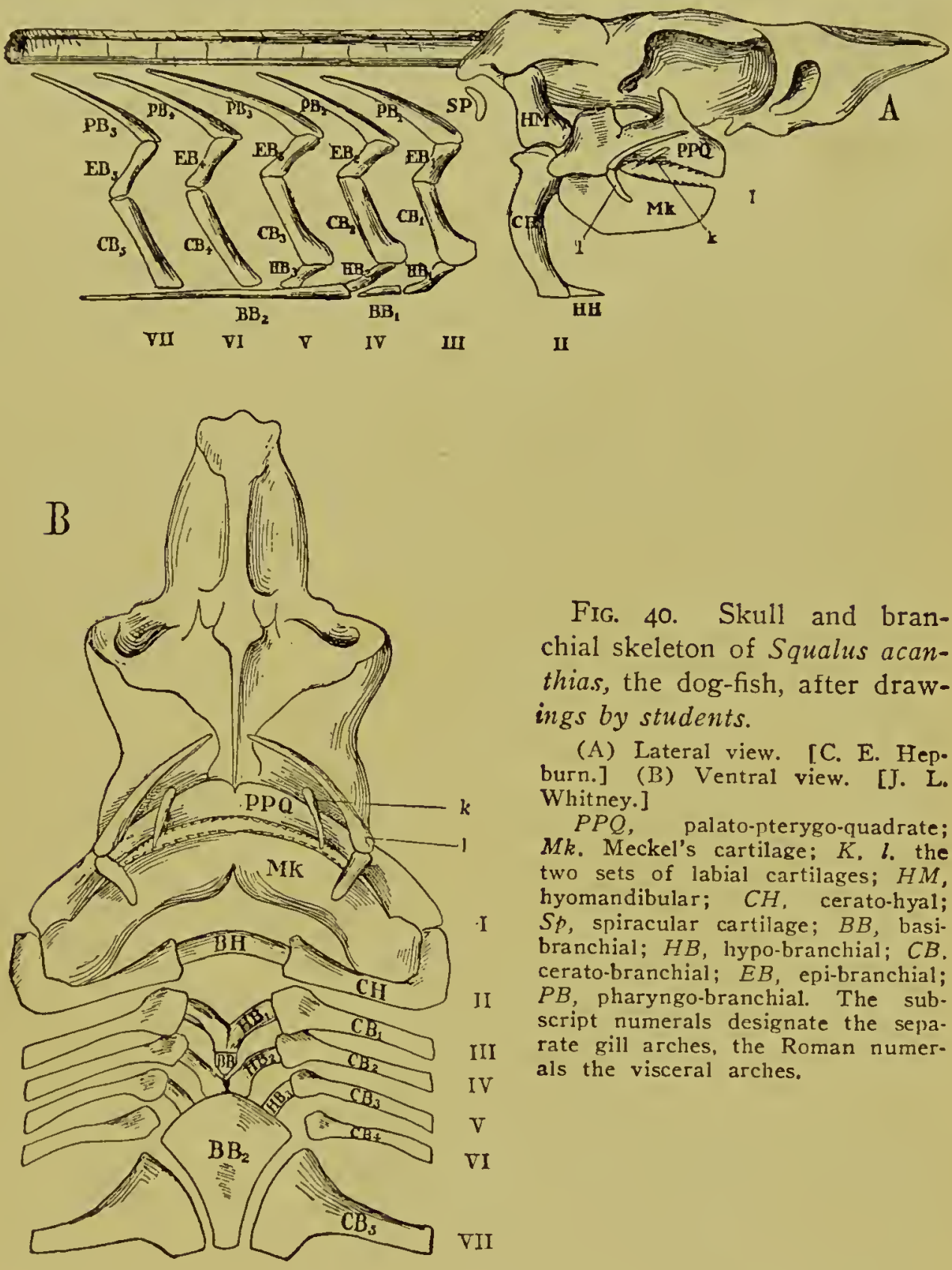

Fig. 40. Skull and branchial skeleton of Squalus acanthias, the dog-fish, after drawings by students.

(A) Lateral view. [C. E. Hep. burn.] (B) Ventral view. [J. L. Whitney.] $P P Q$,

paiato-pterygo-quadrate; $M k$. Meckel's cartilage; $K, l$. the two sets of labial cartilages; $H M$, hyomandibular; $C H$, cerato-hyal; $S p$, spiracular cartilage; $B B$, basibranchial; $H B$, hypo-branchial; $C B$. cerato-branchial; $E B$, epi-branchial; $P B$, pharyngo-branchial. The subscript numerals designate the separate gill arches, the Roman numerals the visceral arches.

each of which several rows of pointed placoid scales are associated to serve as teeth. In short, one hardly knows whether to describe these parts as gill-arches covered with placoid 
scales, or as jaws equipped with teeth, since their origin as the first, and their present and future function as the second, are both so clearly indicated.

We have thus revealed in these organs a valuable bit of history, since we can here observe the jazus and teeth of vertebrates almost at their birth, and can learn the source from which the material was derived and how it was first transformed. It cannot be said, however, that the jaws as used here have developed directly into those of the higher vertebrates, since in the intermediate history there is much addition of new material and replacement of old, with the one object in view of increased physiological effectiveness.

The assumption of an upper and lower jaw marks an epoch in early vertebrate history, since these organs, once acquired, replace forever the circular jawless mouth, and hood-like lip, characteristic of both Amphioxus and cyclostomes. So radical must have been the change that some think that even the mouth opening is a different one, that the one associated with the new jaws was once merely a gill-slit like the rest, through which some ancestor acquired the habit of admitting food, and that its manifest advantage over the other mouth in its convenient skeletal equipment, caused the disappearance of the old one and the perfection of the new; there are, however, certain indications that point to the former possession of a still older mouth, the palcostoma, homologons with that of the tunicate, and with this both the hood-like mouth of the cyclostomes and the slit form of other vertebrates may be contrasted as the neostoma, or secondary mouth. This last expression applies simply to the opening, which is probably homologous in all vertebrates, but becomes completely metamorphosed by the addition of jaws. [Cf. Chapter $\mathrm{X}$., sub Hypophysis.]

The use of placoid scales as teeth is also a new idea, and they are certainly a great advance over the horny epidermic excrescences that arm the circular lip of the cyclostomes. Placoid scales are composite structures, composed of dentine covered over by enamel, and they are so perfectly fitted for the 
purpose that they have had no rivals for the office; thus, although the changes in form and arrangement have been numberless, the teeth of even the highest vertebrates, composed of dentine overlaid by enamel, attest their origin from placoid scales. Aside from the testimony of comparative anatomy, the embryonic history of the teeth, even in the highest form, is a direct corroborative testimony to this mode of origin.

Associated with the first pair of visceral arches, the primitive jaws, are the cartilages of the second pair, the hyoid, also emancipated from the function of bearing gills and modified in part to assist in the action of the jaws. Like the first, these arches also consist of two pieces, a dorsal and a ventral one. The first, called the hyomandibular, is more or less detached from the other and forms an intermediate piece, technically called a suspensorium, between the cranium and the true jaws. To this is also attached the ventral piece, or hyoid proper.

The remaining five arches, the genuine branchial arches, are all much alike, and are gill-bearing, associated with gillslits. Each one consists of four pieces, two dorsal and two ventral, the two sets bent at an angle with each other. Along the mid-ventral lines the pairs are united and held to one another by median pieces, the basi-branchials, of which there is typically one for each pair, but in living selachians the full number is seldom represented. The additional gill arches in the two primitive forms have been referred to above and are represented in the diagram, Fig. 4I, A, by dotted lines.

Aside from these definite and well-developed visceral arches, as they may be called collectively, there are the rudiments mentioned above, functionally of little importance, and held by some to be the reduced remnants of still other arches. Such are the labial cartilages, lying at the angle of the mouth and used to strengthen the integumental folds of the lips; another of these is the spiracular cartilage, crescentic in shape and surrounding the spiraculum or blow-hole, perhaps a modified gill-slit, anterior to the others. These, if admitted to the 
series, will considerably increase the number of original visceral arches and render more probable their descent from a form like Amphioxus.

In the other Orders of fishes the visceral skeleton becomes somewhat modified, but the seven pairs of visceral arches are recognizable in all cases. The most marked changes are those affecting the jaws, and are primarily due to the extensive development of dermal bones, which reinforce the cartilaginous bars, as they do in the case of the chondrocranium. The lower jaw becomes almost entirely encased by them, the principal dermal elements being the dentary, which covers the outside and bears the most or all of the teeth, and the angulare, which covers the inner side.

Lying within these, as if bound in splints, is the cartilaginous lower jaw, the original visceral arch, which is destined from now on to lose its functional importance save at its posterior end, which here emerges from the splints and presents a rounded articular surface. This piece is called the articulare, and sometimes ossifies, forming a cartilage bone. The entire cartilaginous arch, thus subordinated, the mandibular cartilage, is also called Meckel's cartilage, the name commemorating the distinguished German anatomist, Johann Friedrich Meckel [I $781-1833$ ], who first saw it in the embryo human jaw, lying encased in the dermal bones, much as in the adult ganoid or teleost.

The original upper jaw, however, loses still more prestige, since its function as a jaw is entirely usurped by a set of dermal bones, the premaxillary and maxillary, placed parallel to, but outside of it. In spite of this, however, it becomes directly encased by other dermal bones, the palatine and the pterygoid, and is retained as an accessory upper jaw in some fishes and a few salamanders. Its posterior end, like that of the lower jaw, remains free and, after the reduction of the anterior portion, fits in between the hyomandibular and the articulare of the lower jaw as an extra suspensory piece. In later development it ossifies as a cartilage bone, under the name of quadrate. As for the ultimate fate of the anterior 
portion, the dermal palatine and pterygoid enlarge at its expense, and it is retained as an unimportant bit of cartilage in some amphibians, but beyond these it is not seen. The two dermal pieces, which originally encased it, hovever, retain considerable importance and usually appear in the skulls of amniotes along a curve approximately parallel to that of the maxillaries, but interior to it, thus marking the former position of the lost cartilage. These bones are often toothbearing in fishes and amphibians, retaining this much of their former function.

Of the five pairs of true branchial arches, the first four are retained in ganoids and teleosts as gill-bearers, while the fifth lies in the floor of the pharynx and is often covered with sharp teeth arranged in several rows.

A notable modification, which, however, is mainly an external one, is found in the development of the operculum or gill-flap, which appears as a fold of skin and becomes reinforced by special dermal bone. This ultimately develops posteriorly so as to cover all the gill-slits in such a way that they seem to be internal, and are not visible from the exterior, as in the case of most selachians.

The subsequent history of the visceral skeleton and its fate in amphibians, reptiles, birds, and mammals may be quickly outlined. These Classes are fundamentally terrestrial, and never possess genuine.internal gills, and thus the main changes are due to a loss of function, which, by throwing the branchial arches out of employment, would have caused them to disappear, were it not that they could be in part employed to subserve some other necessary function. It will be seen that in most cases this has been their fate, but this very change of function, although it saves them from complete destruction, of necessity causes considerable modification, oftentimes a profound one.

Beginning with the most anterior arches, the mandibular and hyoid, the hyomandibular seems to disappear entirely, although doubtfully identified by some morphologists with certain other elements in the otic region, and leaves to the quad- 

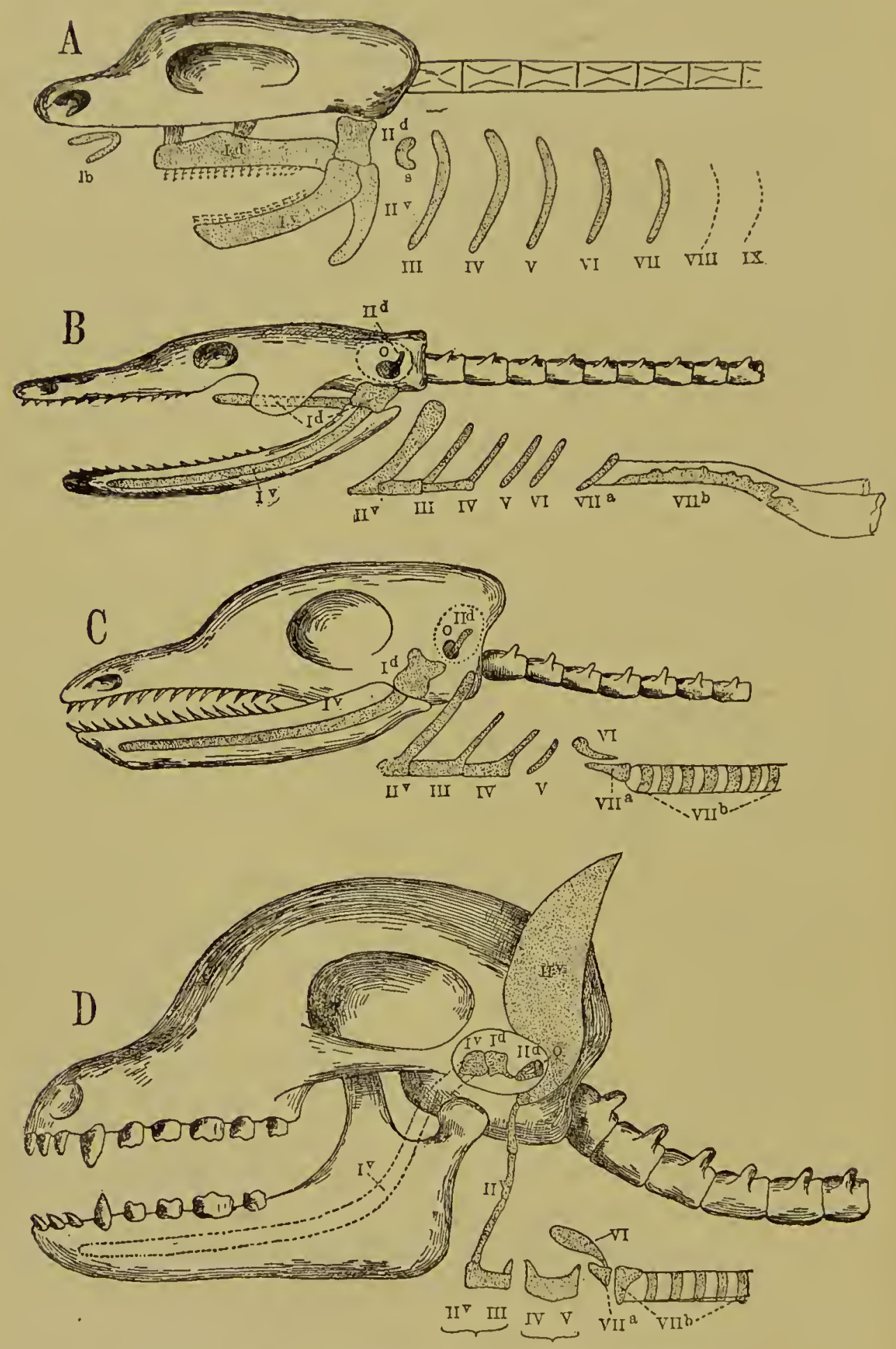

FIG. 4I. Morphology of the visceral skeleton.

(A) Selachian. (B) Amphibian. (C) Sauropsidan. (D) Mammal.

In all the figures the visceral arches are designated by Roman numerals; in the case of the first two the dorsal and ventral segments are further designated by ex. ponent letters ( $d$ and $v$ ). Other designations are, $l b$, labial cartilage; $s$, spiracular cartilage; $o$, operculum. In arch VII the arytrenoid and tracheal cartilages are designated by the exponents $a$ and $b$, respectively. The designations for the stapes represent an interpretation at variance with the one given in the text, but formerly widely accepted. 
rate the responsible rôle of being the only suspensory piece for the mandible. As a cartilage bone it persists in amphibians, reptiles, and birds. The several elements of the mandible remain distinct in amphibians and reptiles, but consolidate in birds, the proximal end, which articulates with the quadrate, being in all cases the free posterior end of Meckel's cartilage (=articulare). In mammals a great change takes place in these parts, the history of which is repeated in the developing embryo, through which the facts first came to light. Here both quadrate and articulare, external at first, as in amphibians and reptiles, become drazen into the tympanic cavity (middle ear) where, still retaining approximately their original shape, though proportionately reduced in size, they become the incus and malleus, respectively, while the mandible, each half consolidated into a single bone, forms a new articulation directly zith the skull in the petrosal region. The old articulation of the mandible, that between quadrate and articulare, now incus and malleus, after having served so long and well in the mastication of food, emancipated from this coarse work and remaining almost embryonic in point of size, becomes attuned to sound waves and assists in their transmission! The third and innermost bone of the tympanic cavity, the stapes, has been for a long time a true bone of contention, in spite of its small size. Some authorities have attempted to identify it with the missing hyomandibular, the dorsal half of the second visceral arch, which disappears above the fish. It appears, however, to have had a double origin, one for the loop, the other for the base. The first seems to have been originally derived in amphibians from the cartilaginous wall of the otic capsule, and to be thus a part of the chondrocranium, and not. an element of the visceral system. The oval base is an ossified membrane, secondarily fused with the other piece. The foramen in this minute bone, to which it owes its stirrup-like shape in man and in some other mammals, transmits an artery which in man disappears in the embryo, but in Insectivora and rodents is retained throughout life, the arteria stapedialis.

The remaining arches subserve in part the original function of gill-bearers so long as there is opportunity, which occurs 
only in a few amphibians, and then mainly during larval life, and are otherwise modified to assist in the functions performed by two organs that develop in the region, the tongue and the larynx. The latter makes its appearance in a very simple condition, associated with two bag-like lungs, in the most primitive of the salamanders, and utilizes as the first laryngeal cartilage, cartilago lateralis, the last of the gill-arches ( $5^{\text {th }}$ branchial or 7 th visceral arch). This element, which, by subdivision and metamorphosis, develops into a pair of arytcnoid cartilages and a series of lateral tracheal pieces, shows itself capable of becoming a complicated mechanism, sufficient for the needs of amphibians; but in the reptiles the 4th gill-arch (6th visceral) becomes associated with it as the epiglottis, which here appears for the first time. The reptilian larynx, with but little modification, is employed by the birds, but in mammals the next two gill-arches, counting anteriorly, the 2nd and 3 rd ( 4 th and $5^{\text {th }}$ visceral) become associated together in front of the larynx and form the protecting shield-like piece, the thyreoid, which in the lowest Order (Monotremata) still appears like two pairs, of arches covering the larynx ventrally.

It may be said in general that in all the Classes, from the amphibians on, those visceral arches not employed as jaws or as laryngeal cartilages form a hyoid or hyo-branchial complex and furnish a skeletal equipment for the tongue, an organ which often develops voluminously, fitted for very special work. Thus, in the amphibians in general this complex consists of the 2nd to the 6th visceral arches, inclusive; in reptiles and birds of the 2 nd to the 5 th, and in mammals of the 2 nd and 3 rd only. In the latter these two remaining arches form a complex consisting of a median piece, the basi-hyal, and two pairs of cornua, the anterior pair representing the 2nd visceral arch, the true hyoid of fishes and the posterior pair the 3 rd visceral or ist gill-arch. In most mammals the anterior cornua of this hyoid complex consist of a chain of small bones, which, enumerated from the basi-hyal ("body of the hyoid"), are: cerato-hyal, epi-hyal, 
stylo-hyal, and tympano-hyal, the last closely associated with the external opening of the ear. In man and the higher apes the two latter are fused with the skull to form the "styloidprocess of the temporal bone," and this is connected with the cerato-hyal (="lesser or anterior cornua ") by the stylohyoid ligament, which replaces the missing epi-hyal. Occasionally a rudiment of this latter bone is found in the middle of the ligament.

A recently described and very singular metamorphosis of a portion of a visceral arch is that by which in mammals the outer end of the 2 nd or hyoid arch, naturally located near the external opening of the ear, segments off and becomes transformed into the cartilage of the external ear-flap, the auricula or pinna, which in the various Orders responds readily to the varied environments of different mammals and exhibits a great range of variation in shape and size.

Of what a series of changes and unexpected metamorphoses has the visceral skeleton shown itself capable, and what vicissitudes have the several elements experienced! Beginning as a set of similar arches, regulating the opening and closing of gill-slits, they become jaws, vocal organs, supports for the tongue, suspensory pieces for the mandible, tympanic ossicles and flapping external ears. Indeed, a well-recognized, though not generally accepted, theory derives from them also the skeleton of the free limbs, including the shoulder and hip girdles, and even the long bones of the limbs and the numerous smaller pieces in carpus and tarsus and in the digits. But even without this latter theory, which appears untenable, the subject presents a remarkable history of repeated change of function, of the adaptation of old material to new purposes, of the dethronement of the old systems and the employment of their organs for the development of the new; we see here Nature constantly exploring new environments, and then adapting function to environment, and material to function, constantly making over old organs in obedience to mechanical laws and never originating or creating new ones de novo. The results are thus often imperfect and 
incomplete, and are frequently obtained by a very indirect and circuitous route, and although many an animal form and many myriads of individuals have succumbed as the result of some mechanical disadvantage which the skill of a simple human mechanic could have remedied, there is never the least sign or indication of such an interference. Everything. develops as an inevitable result of natural law, as a part of the general plan which is broad enough to include the entire universe and which is willing to sacrifice countless hecatombs of lives rather than submit to a single exception to its laws.

In about the same degree as the visceral skeleton of the true vertebrates is suggested by the gill armature of Amphioxus, so do its fin-folds and the rows of spines enclosed by them suggest a simple condition from which may be derived the appendicular or limb skeleton. In Amphioxus a continuous though very low fin-fold begins near the anterior end, runs the entire length of the animal, and is continuous around the tail with a median ventral one as far forward as the atriopore, where it divides into two, which, as the metapleural folds, continute almost to the mouth.

This fold system is supported throughout its entire length by a skeleton of gelatinous fin-spines, thus forming an apparatus, which, if developed slightly more than in Amphioxus, would be very serviceable in retaining the equilibrium while swimming, serving as dorsal and ventral keels. The skeletal fin-rays would become developed as well as the external folds, and the general appearance would be not unlike that suggested by Fig. 42, a. It will be noticed that in Amphio.rus certain parts of the caudal fin are wider than the rest, showing how responsive this fold is to a localized increase of function, and this allows one to draw the hypothetical ancestor with certain areas of the fin-fold marked by a greater width, corresponding to the places where the greatest stress would be apt to come in an actively swimming animal. As a farther departure from Amphioxus, the division of the median ventral fin takes place at the antus, and not at an atriopore, since this latter is probably a special organ developed to supply the 
needs of Amphioxus, and not transmitted to any of its descendants.

Judging from the final results as we see them in the great Class of fishes, it may be supposed that the inequalities in the development of this fin-fold increased through localized func-

a

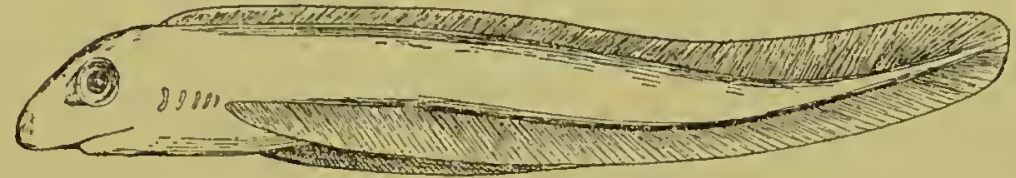

b

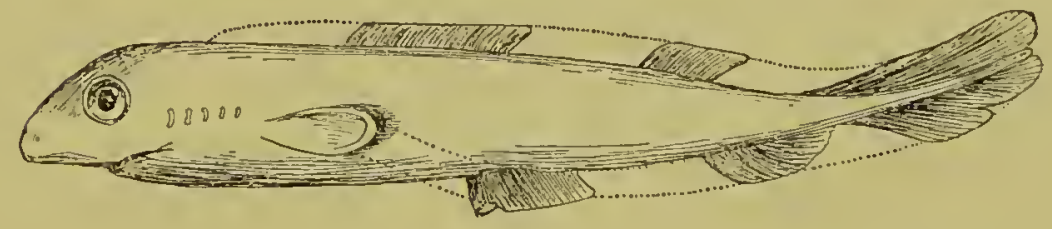

C

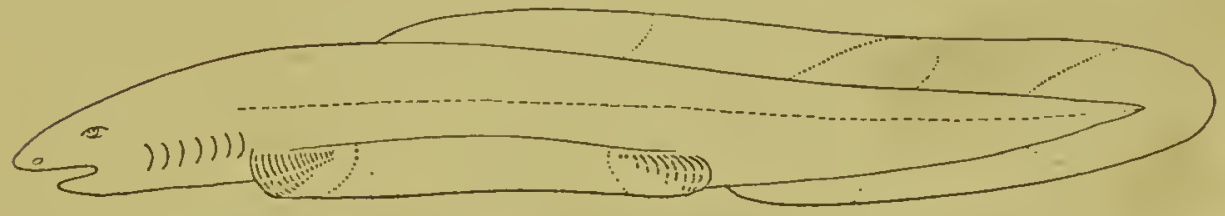

FIG. 42. Diagrams illustrating the fin-fold theory.

(a) and (b) [after WIEDERSheIM] represent the unmodified theory. A continuous fin-fold, stiffened by skeletal rays, extends along the median dorsal line, around the tail, and along the mid-ventral line as far as the cloaca, where it divides into two lateral folds that extend along the sides of the trunk. The retention of portions of this fold and the loss of the intermediate portions results in the formation of both median and paired fins. In (c) [after RABL] is shown RABL's modification of this theory. The two lateral folds are from the beginning distinct from the median one, and are hence subjected to external influcnces, especially at their free anterior and posterior ends, thus modifying the first and last rays the most, and the others in a progressively decreasing series towards the middle of the fold. When, later, the first and the last portions become set off (along the dotted lines) and the intermediate portion suppressed, they form fins, of which the anterior one shows greater modifications along its anterior, the other along its posterior border, precisely as is the actual case among fishes.

tional activity, until certain portions became especially zuell developed, while the intermediate portions were entirely lost. (Fig. 42, b). The significance of this is seen if this figure be now compared with any good, typical fish, which shows the perfected type resulting from this process. Here the fins are all alike in structure, proving their derivation from a common origin, but are divided into two groups in accordance with their position, median and paired. Of the median fins the 
dorsal (one or more) and the anal function as keels to retain the equilibrium and prevent the body from rolling sideways, and the caudal fin is the main organ of propulsion, but may act in part also as a rudder to regulate the direction in which the animal moves. The paired fins, which bear the inappropriate names of pectorals and ventrals, act as subsidiary oars or paddles and seem mainly to guide and maintain the course.

A recent suggestion, which serves as an addition to the finfold theory, is given in Fig. 42, c, in which it is supposed that the lateral folds are primarily distinct from the median one, and that the paired fins develop from their free extremities, where the stress of motion naturally comes. This furnishes a reason other than chance for the formation of two, and only two, pairs of fins, and also explains a sort of symmetry shown in the two sets of fins of many fishes, since the free edge, and hence, the strongest development, is anterior in the forward fin and posterior in the back one.

The median fins, being of use only in the water, disappear above the fish, although the necessity of similar organs for aquatic life is well shown by the fact that in secondarily aquatic higher vertebrates which have returned to the water although derived from a terrestrial ancestry, some new form of median fins becomes developed. Such animals have lost the serviceable median fins of fishes, with their strong skeletal spines, and, as they cannot recall them, are forced to develop some makeshift arrangement to serve the purpose. Thus, aquatic amphibians develop a tail fin of integument without skeletal support, the tail of the crocodile is supplied with keels formed of projecting scales, and the whale has manufactured perfect dorsal and caudal fins out of whole cloth, as it were, since they are made from thick, though hairless, mammalian integument, reinforced by connective tissue. The dorsal fin of this latter, as is the case with other external details, is wonderfully fishlike, but the caudal fin is flattened the other way, and extends laterally, instead of up and down, as in fishes.

To summarize, then, the original median fins of fishes have 
developed to subserve the needs of an aquatic life, and disappear forever with the assumption of a terrestrial environment, although, in secondarily aquatic forms, similar organs are developed from other sources. The paired fins, on the other hand, useful in fishes, assume their highest importance on land, and become the two pairs of free limbs. To these more than to any other organ, the higher vertebrates owe their extraordinary development, and their high degree of success in occupying so many sorts of environment, since by this means they have been able to possess the surface of the earth, to occupy the trees, to burrow in the ground, to return to the sea and even to conquer the problem of aërial navigation. It is, moreover, probable that the emancipation of the fore limbs from the function of locomotion and the acquirement by them of prehensible powers, which enable them to seize objects and bring them to the immediate attention of the sense organs, have been the chief causes of the excessive brain development which has achieved for the Primates the greatest success thus far attained in the domination of the world.

Corresponding to the great variety of functions of which the paired limbs are capable, there is an equally vast series of modifications of structure, presenting an army of forms which include various sorts of ambulatory limbs, paddles, grasping organs, tools for excavating the earth, and wings of several sorts. Among the modifications there must be included also the numerous cases of limb reduction, which may affect the fore or hind limbs or both, and exhibit every stage of reduction down to total loss. Thus the amphibian Siren has lost its hind legs totally, while retaining its fore legs, and similarly in the case of the whale the fore legs become developed into enormous flippers or paddles, while the hind legs are reduced to useless rudiments entirely concealed beneath the skin. The opposite tendency is seen in the ostrich, where the legs are very short and heavy, while the wings are much reduced and almost without function, and is still better exhibited in the kiwi-kiwi of New Zealand, in which the wings have totally clisappeared. In several groups the complete or nearly 
complete reduction of both pairs is correlated with an excessive lengthening of the body, locomotion being effected by an undulatory movement of the entire animal. In snakes, which progress mainly through the action of their very numerous ribs, the loss of both pairs of limbs is usually a total one, but in the boas the posterior limbs appear as spur-like rudiments, situated upon either side of the cloacal orifice, and are of considerable use in climbing trees. Aside from the snakes a similar form is assumed by several groups of reptiles and amphibians, the adaptation fitting them in some cases for a life similar to that of snakes, and in others for a subterranean existence. These latter, which include at least one group of lizards and one of amphibians, burrow in the earth like earth-worms, and as in the process of this adaptation they have lost their eyes, reduced their head and arranged their scales in the form of annular segments, they resemble these latter animals almost to the point of deception.

Through all their vicissitudes, however, the number of free limbs is constant in all vertebrates, except when secondarily reduced, and consists of two pairs, corresponding, as we suppose, to the number of original points at which the primitive fin-fold became hypertrophied. Although this number may be reduced as a special adaptation, it can never be increased, and the favorite mythological conceptions of human and other vertebrate forms with supernumerary limbs are far more impossible and absurd than is usually recognized, since they are generally held to be merely contrary to experience, but are here seen to violate the fundamental principles of development.* It might, indeed, have been possible in the first place for the lateral fin-folds to have hypertrophied in three or more places instead of two, a result which some slight change of environment or habit would have then easily effected, but the

* Cases of monstrosities with extra limbs, in which the total number exceeds four, are not violations of this principle, but are anomalies due to a multiplication of certain of the anlagen, like monsters with two heads or two bodies. The cause of such redundancy is as yet imperfectly understood, but enough has been already proven to show that it lies in the germ, in which the abnormality probably exists in the form of redundant germinal elements. 
time for accomplishing this is now long past and, the number of limb-anlagen once established, no subsequent change is possible. Here again the principle is clearly enunciated that there is never any anticipation for the future in the history of development, and that each step is taken with sole reference to the needs of the animal that takes it. Although a given form is destined to be the ancestor of a great group of higher animals, yet there is no prescience exhibited in the details of its structure other than the needs of its own economy, and the task of laying down the lines in accordance with which its numberless descendants are to be constructed is left to the chance of the necessary adaptations. These condicioning characteristics may or may not be the best for the future, but in either case they are transmitted to posterity, to grant them success or failure, as the case may be.

The form assumed by the free paired limbs throughout the Class of fishes is that of a fin or ichthyopterygium, a type consisting of a thin double membrane supported by a variable number of fin-rays; a single type also underlies the countless modifications exhibited by the higher vertebrates, the handform or chiropterygium, a type consisting of three main divisions, proximal, medial, and distal, the last terminating in five digits. Simple as it is to refer all modifications existing in higher vertebrates to the latter, and in the fishes to the former type, no satisfactory explanation has thus far been forthcoming to bridge the wide gap existing between the two. That the two possess an independent origin would involve the total suppression of the appendicular apparatus formed from the fin-fold and the development de novo of two pairs of appendages in the same place, and for the same or a similar purpose, a supposition which involves too much improbability to be considered for a moment. The free limbs of the one type must be strictly homologous with those of the other, and the fact of the present distinctness of the two types is undoubtedly due to the extinction of transition forms. This transition must have taken place at the epoch at which the vertebrates first attained the land, a transition which must have been a com- 
paratively abrupt one, characterized by a rapid adjustment to the needs of a terrestrial life. It thus follows that the transition forms themselves must have been put at a disadvantage when in competition both with their immediate descendants, which were better fitted for the land, and with their immediate ancestors, which had never left the water, and their rapid extinction was a necessary consequence. The deficiency in the historical record at this place, however, has not prevented speculation on this subject; on the contrary, it has proved an especially at-
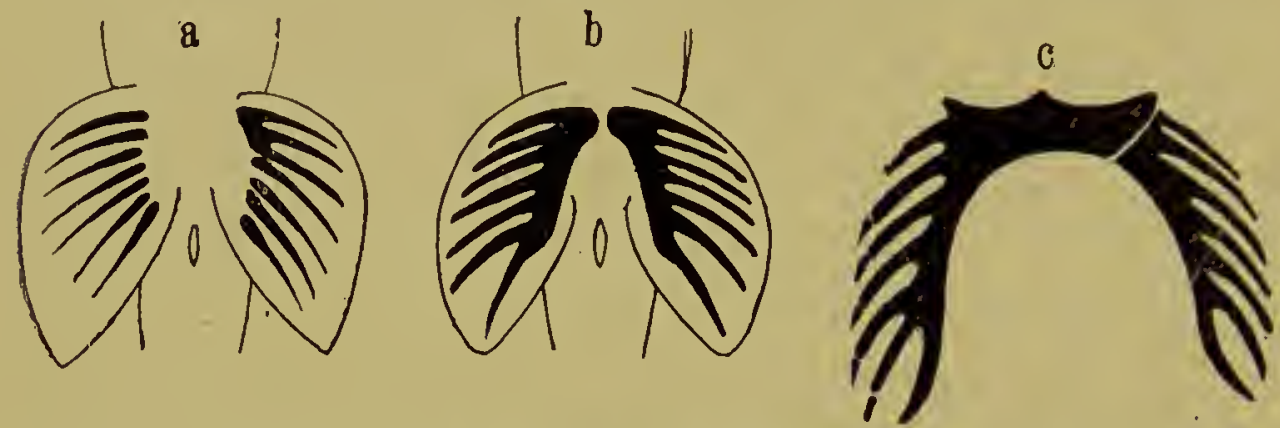

FIG. 43. Diagrams illustrating the development of the fin skeleton; based on that of selachians. [After WiEDERSHEIM.] In (a) and (c) the right side shows a slightly older stage than the left.

tractive field for the anatomical philosopher, and the discussion of some of the leading theories of this question will be considered farther on in the present chapter.

Aside from this problem, however, the history of the development of the limbs is by no means clear in other respects, and although the faith in their complete homology throughout is universal, the manner of their development and the relation of the various forms to one another cannot be agreed upon. Embryology, which is usually so suggestive, is practically silent here, since the record seems in all cases to be much abbreviated. The best that can be done, therefore, is to arrange a sequence of adult forms which seem to show transitions from one type to another, paying as much regard as possible to the lines of descent as indicated by the other parts. In this way have been sketched the histories which follow, and in reading this it must be remembered that a history founded merely on a succession of adult forms, and re- 
maining unsubstantiated by a parallel series of embryonic stages, rests upon an insecure foundation and is liable to receive considerable modification through the discovery of additional facts. In tracing these histories the anterior and posterior limbs must be treated separately, since, although the free limbs are plainly serially homologous, and often correspond quite closely, part for part, the girdles, although equally a portion of the appendicular skeleton, differ fundamentally from one another and must have had a somewhat different early history.

Beginning with the posterior limb, which is more conservative than the anterior, and probably retains more primitive characteristics, it may safely be supposed that at its origin as a localized flap of a once continuous fin-fold, its skeleton consisted of a series of spines or rays, independent of one another, and somewhat longer at their bases, tapering to their free extremities (Fig. 43, a). To insure strength and to gain a concerted action, a very natural step would be to widen these at their bases still farther until they fuse, forming a piece something like a comb, with long teeth far apart (Fig. 43, b). As these organs become of still greater importance and need a firmer support, the basal portions, corresponding to the backs of the combs, would be likely to grow invards until they meet and fuse across the mid-ventral line, thus forming a very primitive girdle, with which the free part would become movably articulated (Fig. 43, c). This last case, is, however, not a hypothetical one, but drawn directly from the posterior girdle and free limb skeleton of the dog-fish, a typical selachian, in which the free limb consists of a basipterygium, bearing a series of rays, the whole being movably attached to a girdle in the form of a ventral band. Although there is at present an impassable space between this free limb and that of even the simplest amphibian, the girdles of the two forms are not so far apart, since a broadening of the middle piece into a plate, and the extension of its ends dorsally until they come in contact with a pair of ribs, would convert the one into the other. (Cf. Fig. 44, d.) 
The fault in the above theory is that, while offering a direct transition from the selachian to the amphibian form, it leaves
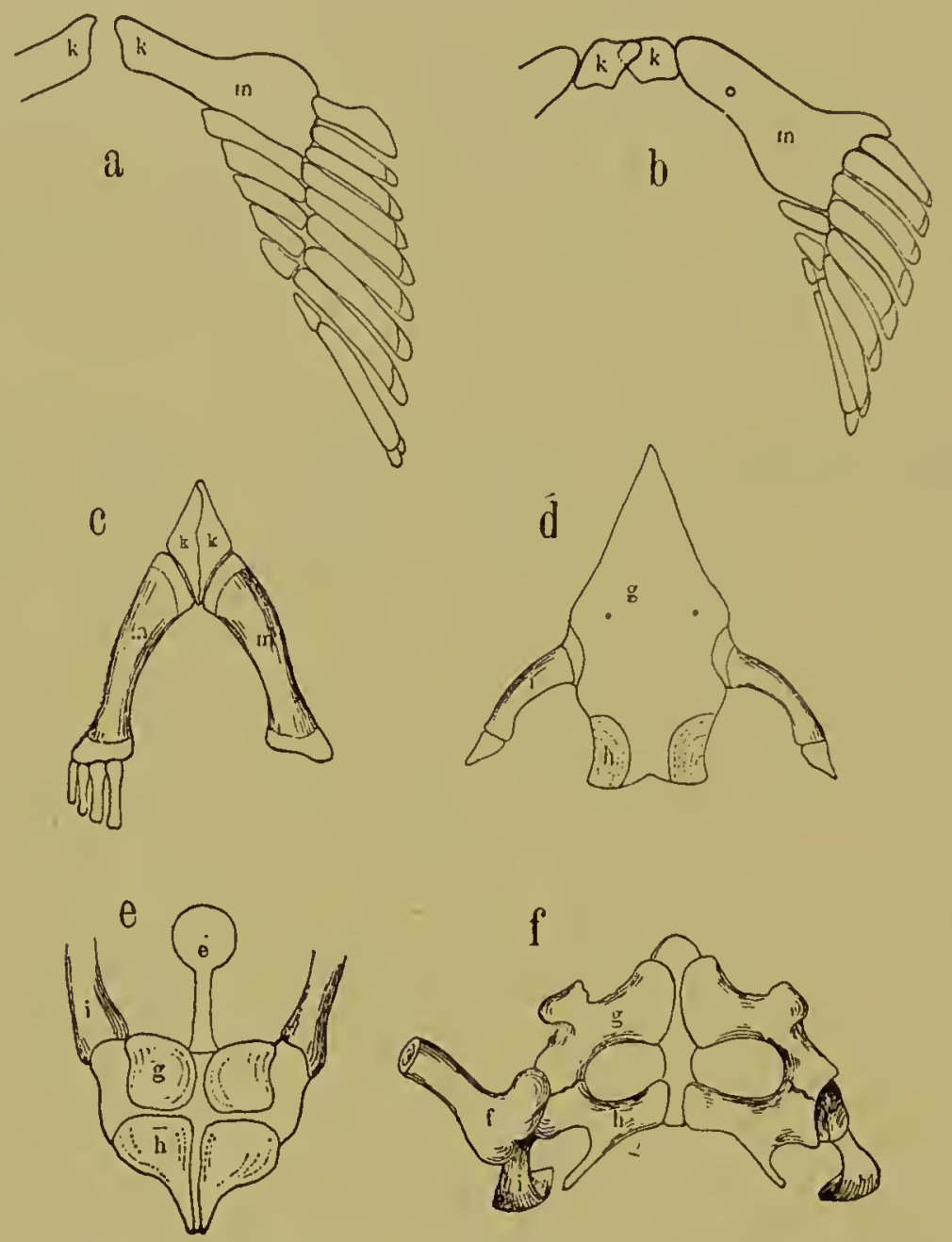

FIG. 44. Series illustrating a theory of the plyylogenetic development of the pelvic girdle. [Mainly after WiEDERSHEIM.]

(a) Acipenser (sturgeon). (b) Scaphyrhynchus (shovel-nosed ganoid). (c) Polypterus (ganoid). (c) Necturus (primitive salamander). (e) Dactylethra (South African frog). (f) Turtle.

In (a) the part $m$ is formed by a fusion of the anterior rays. The pieces $k k$, segmented off from $m$ in (b), form in (c) a rhomboidal plate. In (d) this plate has grown large and bears a pair of ossified ilia, $i$, and a pair of centers of ossification, the ischia, $h$. In (g) appear two more ossific centers, the pubes, $g$. (f) is a typical pelvic girdle, with all its parts. The epipubis, $c$ in (e), is incidental and unimportant in this connection.

no solution for the various conditions that occur in ganoids, some of which, at least, ought to be included in the line of descent. For these a plausible solution is offered in Fig. 44, 
although here the fault lies in the derivation of ganoid conditions directly from the primitive form without accounting for that of the selachians. The first of these figures is that of Acipenser, the sturgeon (Fig. 44, a), and still represents the primitive condition of parallel fin-rays, save that several of the anterior ones have fused in order to meet the greater strain imposed upon them. This tendency has progressed still farther in a related ganoid, Scaphyrhynchus (Fig. 44, b), where the proximal portions of nearly all the rays are included in the heavy basal piece, which bears both the distal portions of the rays of which it is composed as well as the few original rays which have not entered into its formation. In this an important step is the formation of a pair of little pieces, which are segmented off from the proximal ends of the two basal pieces, and which serve to interpret the condition found in Polypterus, in many ways the highest of the living ganoids and the one nearest the amphibians (Fig. 44, c). On this the basal piece of each fin has partly ossified and becomes a long limb-bone highly suggestive of a femur, and the two are attached to a small mid-ventral piece, a rudimentary girdle, which is divided by a suture into two portions, plainly the same as the two small inner pieces of Scaphyrhynchus, here united to form a rhomboid plate.

The derivation from this of the condition found in the urodele Necturus becomes at once evident in a comparison of the two (Fig. 44, c and d), in the latter of which the steps in advance consist of the enlargement of the plate, its connection with the vertebral column through two small processes, the ilia, that extend dorsally, and the appearance of two centers of ossification posteriorly. In this the real transition from the fish type to that characteristic of the higher vertebrates consists of the direct connection between the hipgirdle and the vertebral column, a condition never found in fishes. That this attachment has been newly acquired at the stage represented by Necturus is evidenced by the lack of difference between the sacral vertebra, to which the attachment is made, and the adjacent ones, as well as by the frequency of 
variation in the vertebra selected for this attachment, as explained above.

Within the plate itself are two ossifications, which represent the first appearance of the ischiadic bones, destined to become an important element in the hip-gircles of higher forms; and in some of the higher amphibians, another pair of osseous elements, the pubic bones, also appear in much the same condition (Fig. 44, e).

From the latter form of girdle to that of a reptile (Fig. $44, f$ ), the step is a smaller one, the main. difference being in the formation of a large obturator foramen between the ventral elements, pubis and ischium, a foramen present, though insignificant in Necturus and other amphibia (Fig. 44, d and e). In some reptiles the obturator foramina become confluent, forming a heart-shaped foramen cordiforme.

Allowing for considerable variation in form and proportion, the pelvic girdle of mammals is similar to that of reptiles, and consists of the three elements, ilium, ischium, and pubis, the first dorsal, the other two ventral. The ilium is attached to the sacrum, which varies somewhat in the number of vertebræ involved in its formation, and the ischium and pubis of the two sides usually unite in the mid-ventral line to form a symphysis, although in man the symphysis involves the pubic bones alone, the ischia being wide apart. This is doubtless in correlation with the enormous size of the head of the human infant, for the passage of which through the pelvic outlet provision must be made. A similar case is found in birds, where, with the single exception of the African ostrich, not the ischia alone, but the pubes also are wide apart to allow for the passage of the enormous eggs, characteristic of the Class, and out of all proportion to anything that exists elsewhere in nature.

The history of the shoulder-girdle differs considerably from the foregoing in its later development as it becomes complicated by the addition of membrane bones from without, as in the case of the skull. Although in its first appearance, in selachians, it differs somewhat in form from the hip-girdle, 
it is probable that its early history is similar, and that it was formed by a coalescence of basal portions of the fin-rays, as in the other case, its size and shape being modified in accordance with differences in function. In the adult selachians it extends dorsal to the insertion of the fin into long points, and the two halves become distinct, forming a symphysis at the mid-ventral line, thus somewhat resembling a pair of mandibles. Each lateral piece is termed scapulo-coracoid, as a suggestion of the two elements to which it is to give rise,
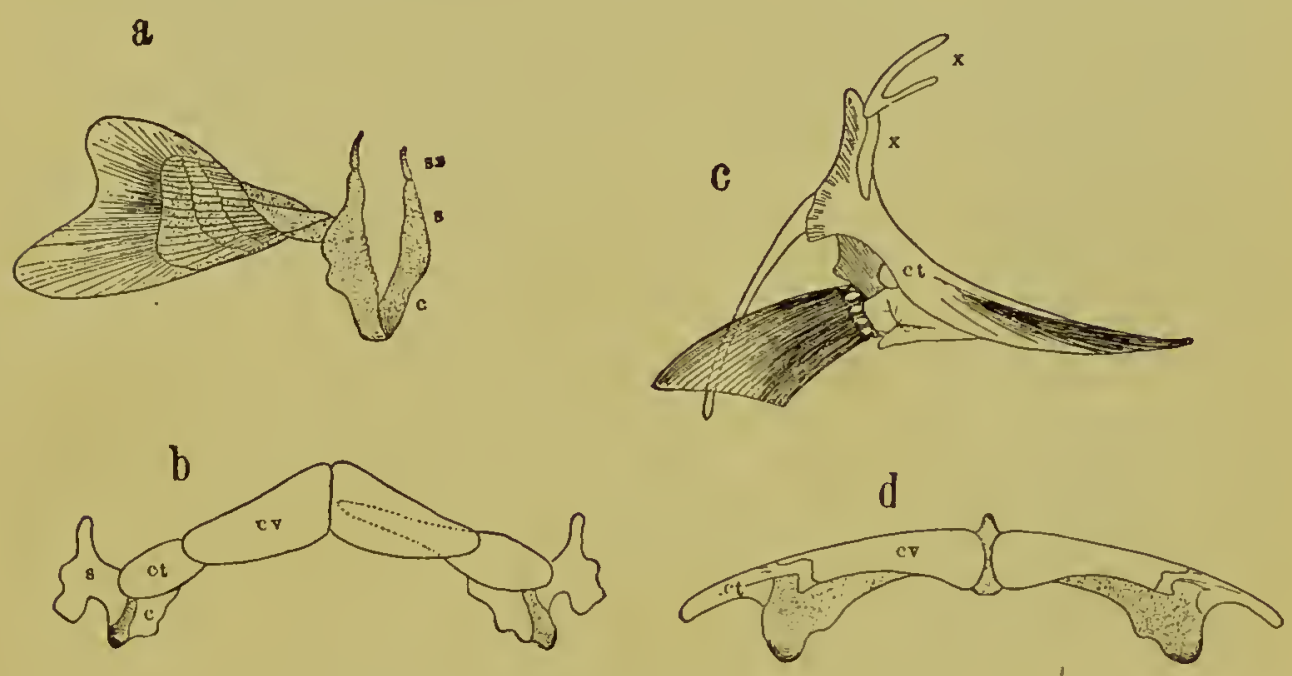

FIG. 45. Shoulder-girdle of fishes.

(a) Dog-fish (selachian) Ceratodus (dipnoan).

(b) Polypterus (ganoid).

(c) Cod (teleost).

(d)

$s$, scapula; ss, suprascapula; $c$, coracoid; $c v$, clavicle; $c t$, cleithrum; $x x$, supracleithra,

but, as in the case of the primordial skull, there is no suggestion of boundaries between the two portions, although it may be vaguely indicated by the point of insertion of the fin, the portion dorsal to it being the scapular portion, and the other the coracoid.

In ganoids there becomes associated with this cartilaginous girdle a series of dermal bones, formed, as elsewhere, by the fusion of scales. There are two of these bones investing each lateral piece, the clavicles being ventral and the cleitlura lateral to these. Of these the former are a little larger, and form the symphysis in the mid-ventral line, excluding the 
cartilage. In teleosts the cleithra become excessively developed and function as the entire girdle, while the clavicles are wanting and the cartilaginous element is much reduced. There are also other small clorsal pieces, the supra-cleithra (post-temporal and supra-clavicle of many authors), which connect the girdle with the skull.

As the teleosts represent the perfection of the fish type, but are not in the direct line of descent, their condition represents a specialization not closely related to higher forms, and the direct history is carried over, with a small interval, from the ganoids to the amphibians. In these latter the dernıal element is but little in evidence, while the cartilaginous part is voluminous and shows centers of ossification. In urodeles each half-girdle is in the form of a thin plate, wrapped abolit the side of the body and incompletely divided into three portions, a dorsally extended scapula, containing an ossified area, and two ventral extensions separated by a notch, the coracoid and procoracoid, both cartilaginous. There are here no dermal elements. In the tailless amphibians the cartilaginous foundation is much the same as in urodeles, but there is also an ossified area in the coracoid, and a dermal clavicle, in the form of an inverted trough, which fits closely over the procoracoid, forming a compound piece. The two lateral halves become also more or less closely associated with median sternal (and episternal) elements, and in the more specialized frogs the whole forms a complicated skeletal armature protecting the vital organs and forming a functional thorax, something like that of higher forms, although without rib components and not involved in the respiratory process.

In the Amniota there appear three parts to the girdle, scapula, coracoid, and clavicle, corresponding in the main to those of Amphibia. The two first are preformed in cartilage and ossify later on in development; but the clavicle of reptiles and birds has no cartilaginous stage, and seems thus to represent the dermal element alone. In birds the two clavicles fuse with a median interclavicle (possibly an episternum), to form the furcula or "wish-bone." In mammals the scapula receives the most emphasis, while the coracoid is never present as a 
distinct bone save in the monotremes. In others it ossifies from a separate center, but soon fuses with the scapula to form the coracoid process. The clavicle is large and well developed in those forms in which strength of shoulder is especially required, as in most cases among the Rodentia, Insectivora and

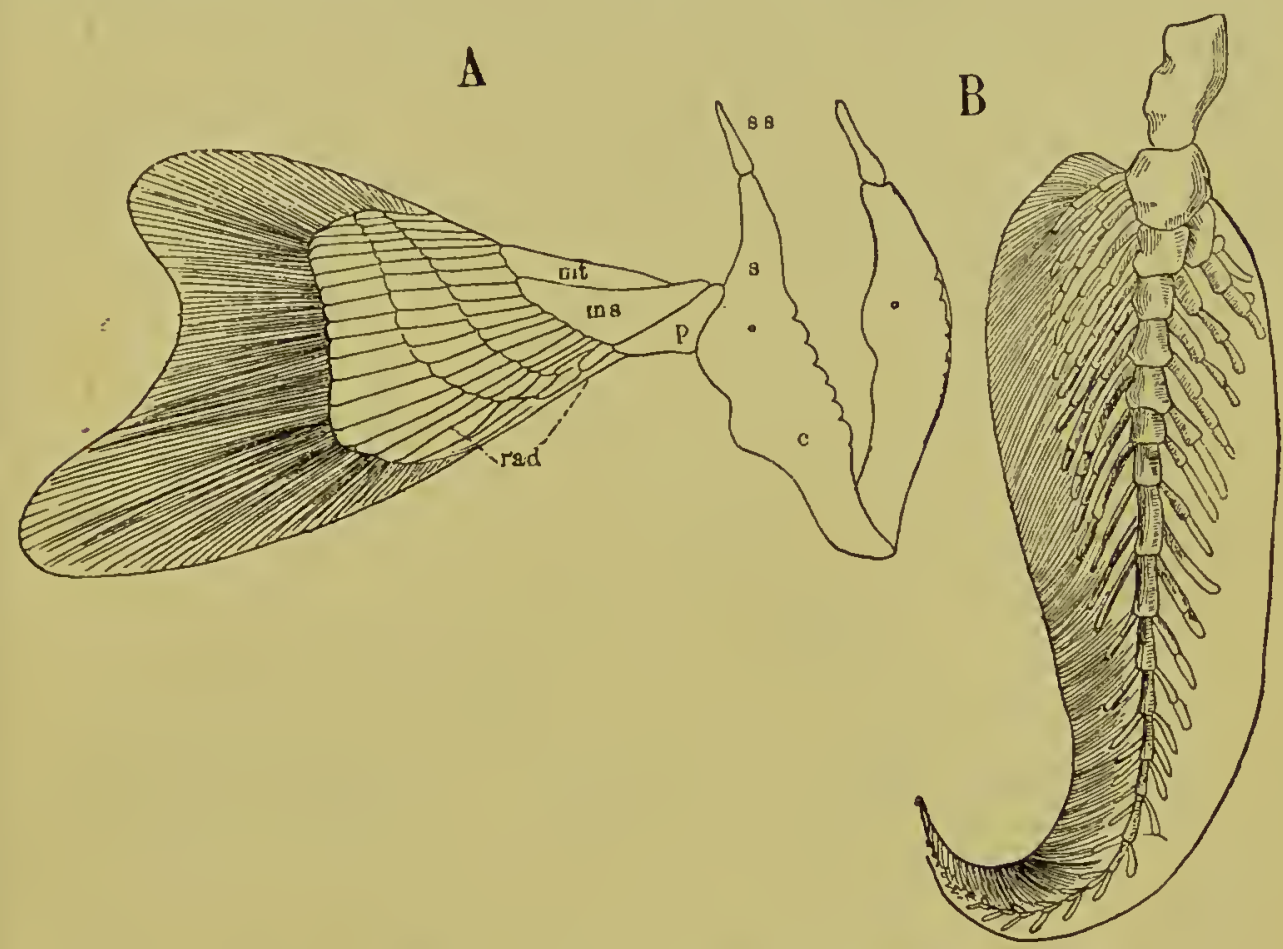

FTc. 46. Anterior fins of fishes.

(A) Dog-fish (selachian). (B) Ceratodus (dipnoan).

$s$, scapula; ss, suprascapula; $c$, coracoid; $p$, propterygium; $m s$, mesopterygium; mt, metapterygium; rad, radials. In $B$ there are radials on both sides of a central axis.

Primates, but is rudimentary in Carnivora, and is entirely wanting in hoofed mammals and in Cetacea. It is stated by some investigators that the mammalian clavicle is in development a compound piece, formed, as in amphibians, of a cartilaginous core, overlaid by a dermal element. If this be so, the former may be the procoracoid, and the latter the true clavicle, but there is some doubt concerning the actual conditions of development, and the whole matter needs further investigation.

The early history of the free limbs has not been wholly deciphered. The fins of fishes exhibit a great variety of form, based upon a series of fin-rays, either distinct or united to basal pieces. Of these latter the posterior limb of selachians shows one, the basi-pteryginm, and the anterior limb three, 
named in order, beginning at the front, pro-, meso-, and metapterygium (Fig. 46, A). These latter pieces have often been considered as primitive, and various attempts have been made to derive from them the long bones of the limbs in terrestrial forms; but they are not always found in ganoids, which ought to show here, as elsewhere, transitions to the higher verte-
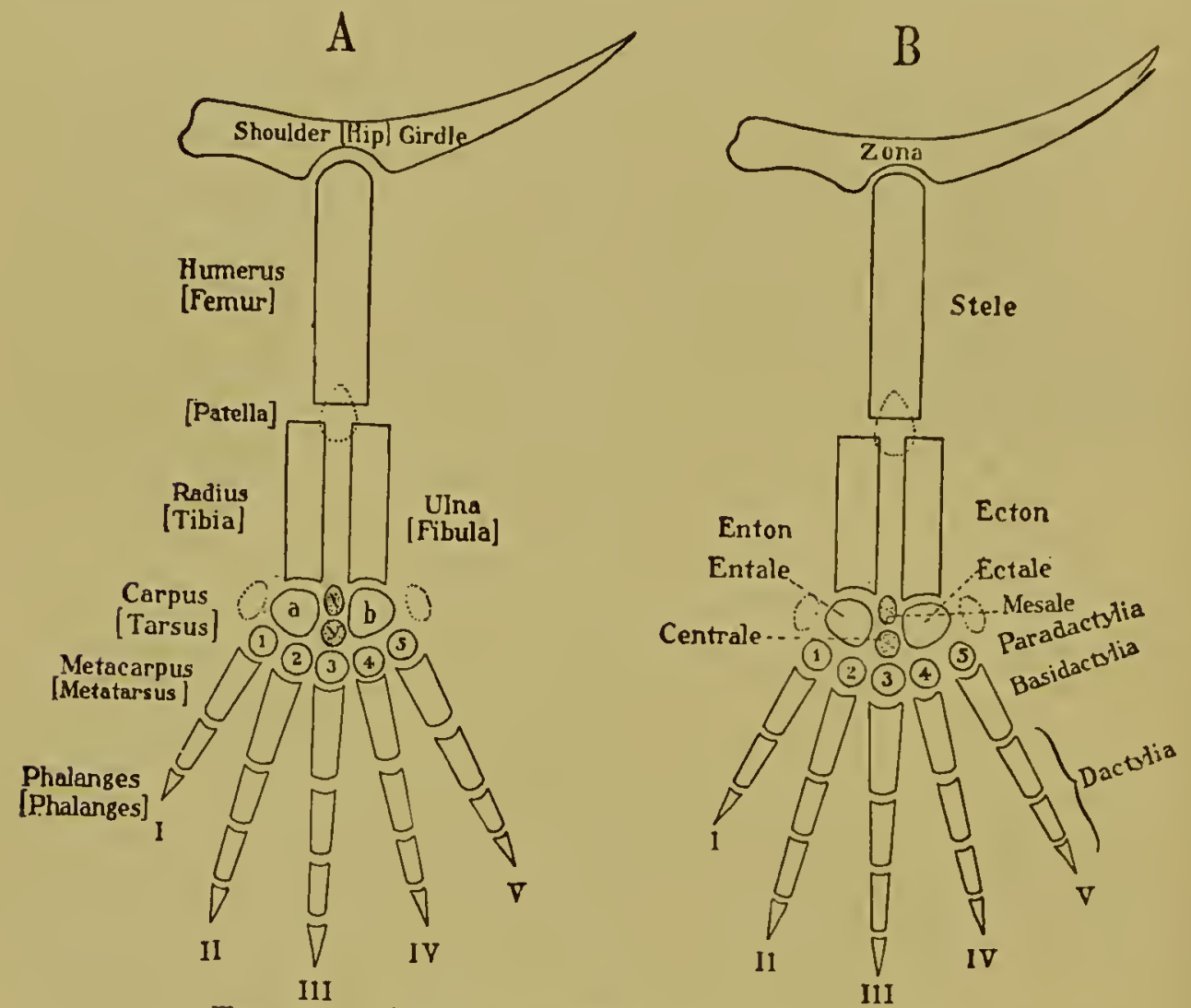

FIG. 47. Diagrams of typical free limb skeleton.

(A) According to the usual nomenclature; names belonging to the posterior limb are bracketed. (B) Suggestion for a common nomenclature for both limbs.

In (A) the separate carpal and tarsal pieces are as follows: $a$, radiale [tibiale]; $b$, ulnare [fibulare]; $x$, intermedium; $y$, centrale; $1-5$, carpalia [tarsalia].

In both diagrams the more constant sesamoids are indicatef by dotted lines.

brates. The Dipnoi show a beautifully symmetrical type oi fin-skeleton, which consists of a jointed central axis with rays upon either side, a type which many have regarder as the primitive form from which all the other cases have been derived, and have named it accordingly the archipterygium. (Fig. 46, B). The highly specialized Dipnoi, however, are not the proper animals to which to look for primitive condi- 
tions, and it is also true that as a rule it is unsymmetrical and not symmetrical forms that show the early conditions of a part.

Inmediately above the fishes the chiropterygium or handform appears, a type so definite and fixed in character that all limbs from the amphibians on may be directly referred to it, while it appears in almost its typical condition in animals widely separated. (Fig. 47.) It consists of a proximal joint formed of a single bone, a second joint of two, followed by a series of several small pieces from which extend five digits, each composed of several bones. Unfortunately the parts were originally named without much reference to the striking correspondence (serial homology) between the anterior and posterior limbs, and thus some of the corresponding parts have received very different names, leading to a redundancy of terms. The nomenclature and correspondence are indicated in the following table:

Anterior Limb.

Posterior Limb.

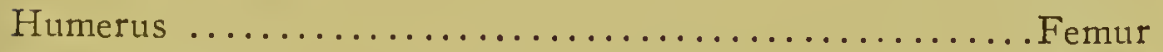

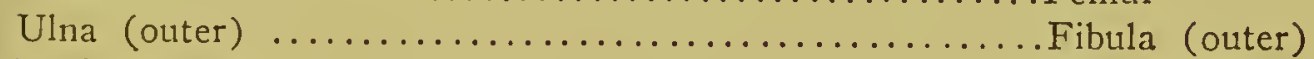

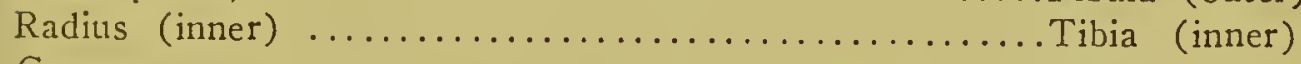

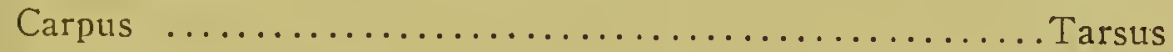

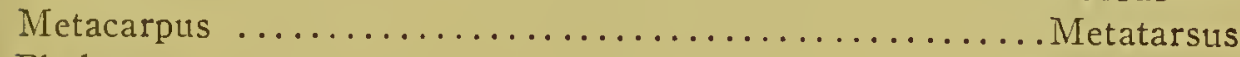

Phalanges $\ldots \ldots \ldots \ldots \ldots \ldots \ldots \ldots \ldots \ldots \ldots \ldots \ldots \ldots$ Phalanges

The digits are designated either by number, I-V, or else the Latin names for the fingers are used, pollex, index, medius, annularis, minimus. These names are applied equally to both members, except that for digit I of the posterior limb the term hallux is employed instead of pollex.

The nomenclature of the bones of carpus and tarsus has caused by far the most difficulty, as they are extremely variable and liable to fuse with one another or to disappear. Still, in spite of much irregularity, they are reducible to a type or pattern, as given in the diagram, to which the individual cases may be referred. In this typical form (Fig. 47, A), there is a piece at the distal end of each of the two limb bones of the second joint, and five at the proximal ends of the five meta- 
carpals [or metatarsals]. These are called radiale and ulnare [tibiale and fibulare], and the five carpalia [or tarsalia] respectively. Of the latter set the individual bones are designated by a number, as tarsale $I V$, carpale $I I$, carpale $V$, etc. Aside from these there are two median pieces: the intermedium, lying between the two proximal elements, and the centrale, distal to it and enclosed by both rows.

The close similarity that exists between the anterior and posterior limb skeleton, and the frequency with which the two need to be compared, piece by piece, leads one frequently to wish that the nomenclature of the two should be unified throughout, as has already been done in part in the distal portion. Probably the chief objection to this lies in the diverse ideas which still exist concerning the serial homology of the parts (treated here in Chapter VI), yet the practical advantage that has already resulted from a partial uniform nomenclature in carpus and tarsus shows the possibility of completing such a scheme as a working hypothesis, without raising the question of serial homology. Such a scheme is shown in Fig. 47, B, which may be compared with $A$ of the same figure, that shows the nomenclature now in use.

In addition to the definite carpal and tarsal bones, which belong to the primary limb skeleton, there are certain other elements of sporadic occurrence, that are situated in or about the tendons of muscles and serve some mechanical purpose in connection with the action of those parts. These are sesamoid bones, and are suggested in the diagran by dotted lines. Of these the most ustial are a radial and an ulnar one [tibial and fibular] placed upon the free edges of the carpus [or tarsus]. Sesamoids also occur in other pertions of the limb skeleton; thus the patella, constantly found ir birds and mammals, occurs in the posterior limb between the first and seconrl long joints, and forms the protuberance at the knee. Small sesamoids, often associated in pairs, are found on the flexor side of the digits between the phalanges.

Althougin the above scheme for a typical carpus or tarsus and its nomenclature seems to serve the purpose of naming the parts in all cases (Fig. 48), there are many 
facts which forbid us from imagining that it is really a primitive condition. Thus the animal in which it comes to its most perfect realization is the turtle, the carpus of which is almost diagrammatic, while the salamanders, where a more primitive type is to be expected, depart almost as widely from the diagrams as do the mammals. From certain indications it seems probable that in the early carpus and tarsus there were two centralia (Fig. 48, a), and that the separate bones were ar-
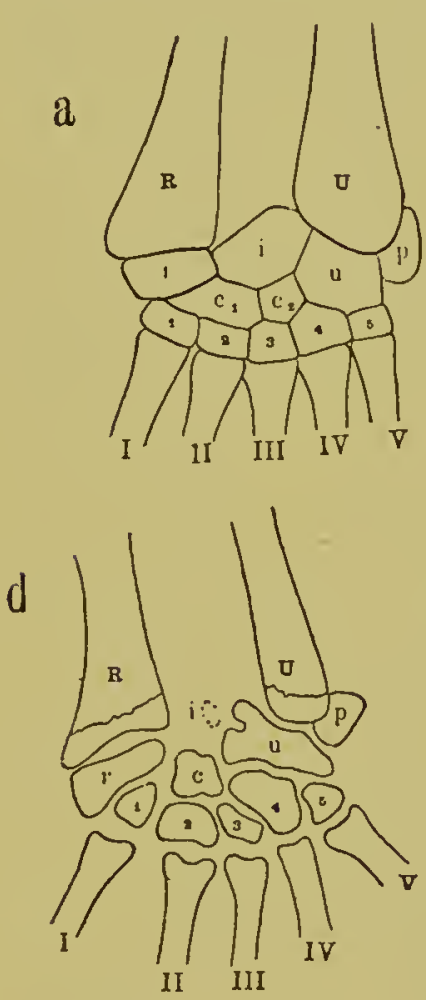

b
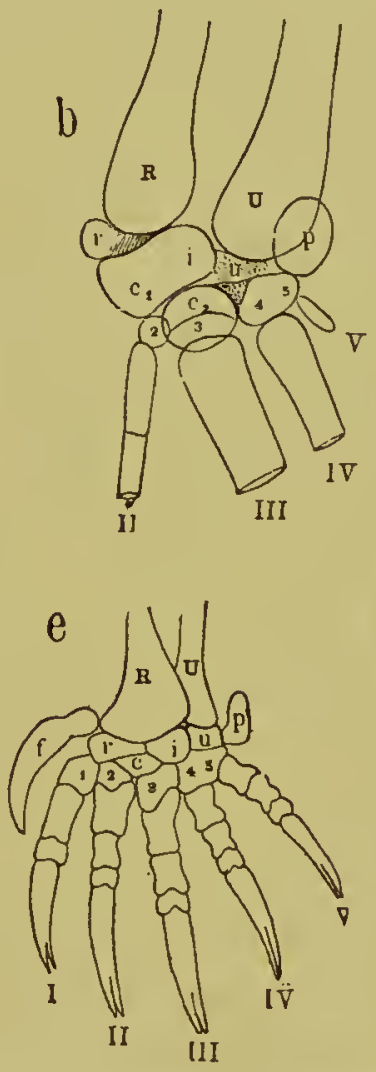

Flix. 48. Various forms of carpus. Figures NorSA; figure (e), after Flower.

(a) Sphenodon (Hatteria), a New Zealand lizard. (b) Chick embryo, early stage. (c) Chick embryo, later stage. (d) Lacerta, a European lizard. (e) Talpa, European mole. (f) Pig.

$R$, radius; $U$, ulnar; $r$, radiale; $u$, ulnare; $i$ intermedium; $c$, centrale; $I-5$, car. palia; $p$, pisiforme; $f$ : os falciforme; I-V digits.

ranged, not symmetrically, but in oblique rows continued more or less directly to the digits, and suggesting the derivation of both carpus [and tarsus] and digits from long fin-rays, divided into numerous joints.

In the nomenclature of the carpal and tarsal bones employed in human anatomy we have an unusually good illustra- 
tion of parts named with reference merely to a single animal form, for the names given them, c.g., cuneiform, trapezium, multangulum majus, os magmum, etc., are wholly relative and might not apply even to closely allied animals. Since, however, these older names are still more or less used, it may be well to compare them with the more unusual nomenclature given above, a comparison which may be best shown in the form of a table as follows:

\begin{tabular}{|c|c|c|c|}
\hline Older Nomenclature & \multicolumn{2}{|c|}{$\begin{array}{c}\text { MORPHOLOGICAL } \\
\text { NAMES }\end{array}$} & \multirow{2}{*}{$\frac{\text { OLDER NOMENCLATURE }}{\text { Foot }}$} \\
\hline Hand & Hand & Foot & \\
\hline scaphoides (naviculare)* & radiale & tibiale & \\
\hline lunare (lunatum) & \multicolumn{2}{|c|}{ intermedium } & $\{$ astragalus (talus) \\
\hline cuboides (triquetrum) & ulnare & fibulare & (ter \\
\hline $\begin{array}{l}\text { anlage in embryo, } \\
\text { with occasional } \\
\text { persistence }\end{array}$ & \multicolumn{2}{|c|}{ centrale } & naviculare \\
\hline $\begin{array}{l}\text { trapezium (mult- } \\
\text { angulum majus) }\end{array}$ & carpale I & tarsale I & entocuneiforme \\
\hline $\begin{array}{l}\text { trapezoides (mult- } \\
\text { angulum minus) }\end{array}$ & 'carpale II & tarsale II & mesocuneiforme \\
\hline $\begin{array}{l}\text { os magnum (capi- } \\
\text { tatum) }\end{array}$ & carpale III & tarsale III & ectocuneiforme \\
\hline unciforme (hamatum) & $\begin{array}{l}\text { carpale IV } \\
\text { carpale } \mathrm{V}\end{array}$ & $\begin{array}{l}\text { tarsale } I V \\
\text { tarsale } V\end{array}$ & os cuboideum \\
\hline & $\begin{array}{l}\text { radial } \\
\text { sesamoid }\end{array}$ & $\begin{array}{l}\text { tibial } \\
\text { sesamoid }\end{array}$ & \\
\hline pisiforme & $\begin{array}{l}\text { ulnar } \\
\text { sesamoid }\end{array}$ & $\left(\begin{array}{c}\text { fibular } \\
\text { sesamoid }\end{array} \mid\right.$ & \\
\hline
\end{tabular}

* Synonyms used more frequently by European anatomists, are giren in parentheses. These latter have been adopted by the BNA, but the substitution of them in America for the more familiar terms placed first in the above list, will be difficult to accomplish, and it is a question if it be desirable, since neither set of terms rests upon a morphological basis. 
Aside from the normal bones in carpus and tarsus there occur occasionally supernumerary elements of greater or less frequency. Thus in Man, in which the subject has been naturally investigated the most thoroughly, there have been recorded for the carpus fifteen or sixteen such elements, aside from the occasional occurrence of the division of a normal element into two (bipartite). The summary of such elements, so far as known, resting upon the investigation of several thousand human carpi, is shown in Fig. 49. Similar supernumerary elements occur in the tarsus; the most important of

at

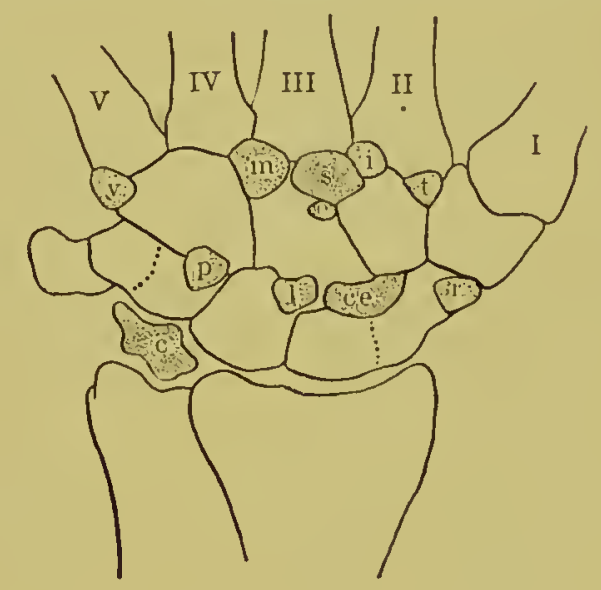

$b$

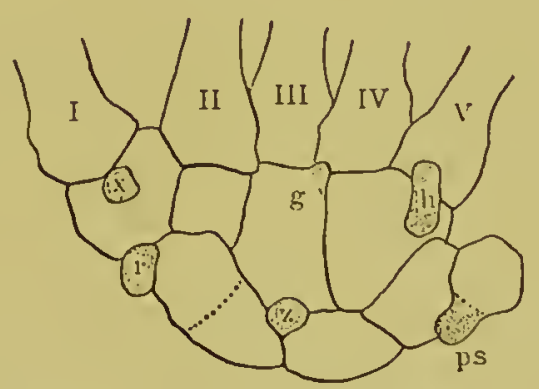

Fig. 49. Diagrams of the supernumerary carpal bones. [After Pfitrzer.]

$r$, radiale externum (constant in apes); $c e$, centrale; $l$, (epilunatum); $z$, hypolunatum; $c$, triquetrum secundarium; $p$, epipyramis; $y$, prætrapezium; $s$, styloideum; $i$, parastyloideum; $e$, metastyloideum; $m$, capitatum sccondarium; $g$, ossiculum Gru. Of the normal proprius; $v$, os Vesalianum; $p s$, pisiforme secundarium. bipartite. This peculiarity has been also observed in the lunare and the trapezoides.

which are the trigomum, associated with the astragulus, and present in $8 \%$ of the cases studied; the tibiale extcrmum (I II $2 \%$ ); the peroneum (8-9\%); and an intermetatarseum (8- 
$9 \%$ ), a derivative, sometimes of the first, sometimes of the second, metatarsal.

The typical number of digits is five, but this number is frequently recluced by the loss of digits at either end of the series. Instances of recluction, usually with vestiges of the missing digits, are of frequent occurrence and range from cases with the loss of the first alone, as in certain salamanders, or of the last alone, as in the feet of birds, to the extreme case exhibited by the horse, in which the middle digit is alone developed, accompanied by vestiges of II and IV.

In the pig and the ox two digits, II and $V$, considerably reduced in size, are set behind the two well developed ones, III and IV, and terminate in horny spurs that do not touch the ground. Digit I is entirely wanting.

The occasional occurrence of hyperdactylism, or cases with supernumerary digits, in all groups of tetrapod vertebrates, together with the presence, in numerous cases, of extra bones, sesamoid and otherwise, beyond and at the sides of the true digits (e.g., os falciforme in Fig. 48, e), have often been interpreted as pointing to a previous condition with more than five digits, a condition that would well accord with the derivation of the hand from a fin, since in most cases the latter structures possess more than five fin-rays. These phenomena are not, however, so interpreted by all morphologists, and the subject is a controversial one at present. Such an hypothetical digit placed before the thumb or great toe is called a precpollex or pre-hallux respectively; the one continuing the series beyond the fifth is a post-minimus.

The chiridial appendage just described, although it forms the universal plan upon which is based that of all tetrapod vertebrates, is yet capable of great modification and has enabled its various possessors to adapt it to a great variety of uses. The detailed consideration of this belongs to the field of special comparative anatomy and does not come within the scope of this work, yet as an illustration of the general principle of adaptation a few cases may be mentioned which show certain of the modifications acquired in the successful adap- 
tation of the free limb to locomotion in the two difficult elements of water and air (Fig. 50). The chiricial appendage (cheiropterygium) is primarily a walking leg (Fig. 50, a), designed to aid in pushing the body along the ground or, when extremely developed, to support the body above the surface and assume the entire function of locomotion. In becoming
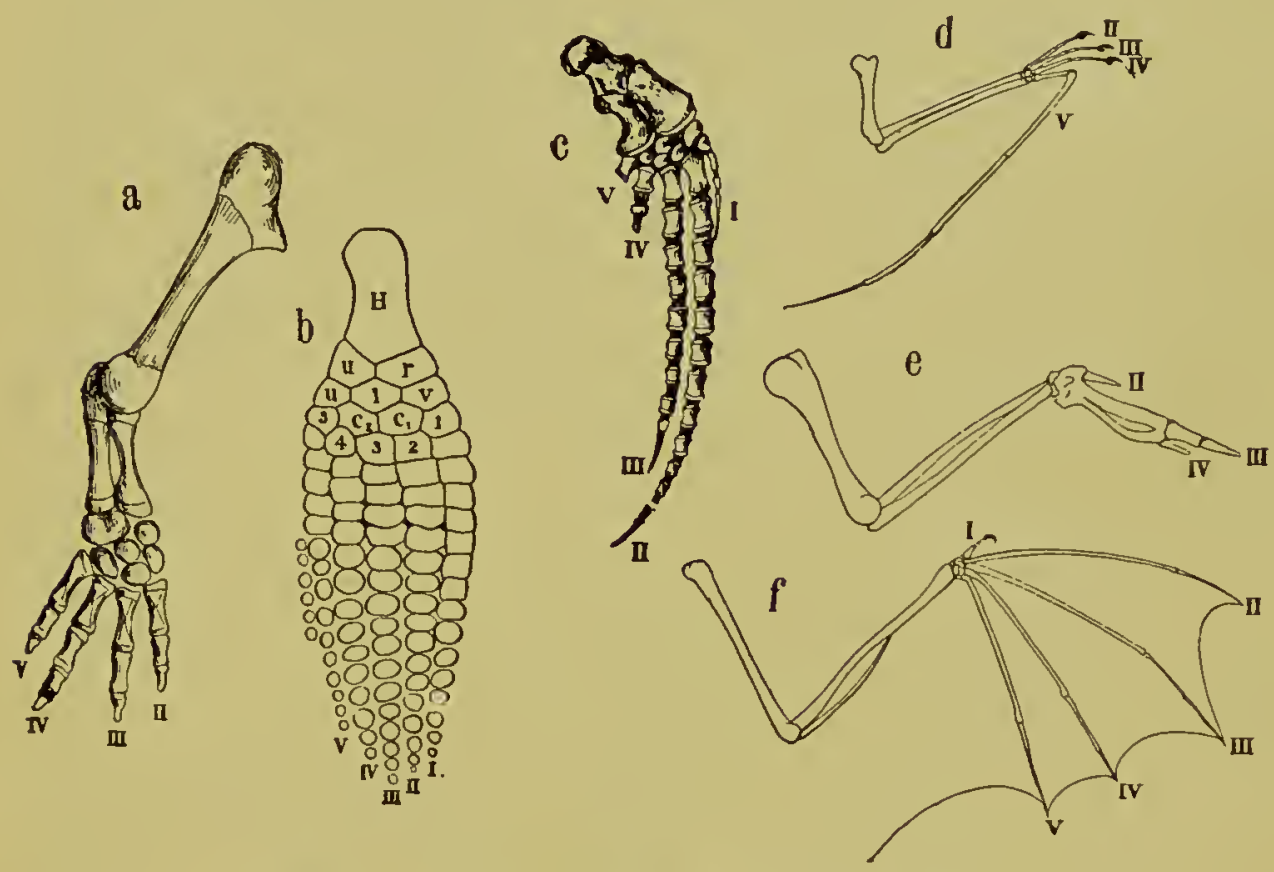

FIG. 50. Modifications of the fore limb.

(d) Necturus, a primitive salamander. (b) Ichthyosaurus, an extinct marine lizard. (c) Globicephalus, a cetacean (dolphin). (d) Ptcrodactyl, an extinct flying reptile. (e) Bird. (f) Bat.

Figure (a) represents an unmodified limb skeleton; (b) and (c), limbs modified for swimming; (d), (e), and $(\hat{r})$, those modified for flight. Designations as in the previous figure.

modified to subserve the needs of an aquatic life it needs to change into a paddle, which it does by elongating the digits, pressing them close together, and surrounding them by a web of integument. The digits also often become hyperphalangeal, that is, they develop an unusual number of phalanges, as is seen both in the marine lizard Ichthyosaurus and in the entirely unrelated branch represented by the Cetacea. [Fig. 50: compare (b) and (c).]

In Ichthyosaurus the five regular digits are retained, and there is also a row of small bones along the outer edge, con- 
sidered by some to be a sixth digit, a "post-minimus." It is probably a row of sesamoids employed here to widen the paddle and thus increase its effectiveness. In the cetaceans the external digits suffer some reduction while the two middle ones, II and III, are lengthened.

In adaptation to flight the chiridial appendage forms a framework for a thin surface, without appreciable weight, and formed either of integument or of integumental structures of some sort. There have been at least three independent and perfectly successful attempts to solve this most difficult mechanical problem, each one involving profound changes in the limb skeleton. In the extinct ptcrodactyls the principal modification consisted of an extreme lengthening of the little finger to form a framework for the wing membrane; in the bats a similar result has been attained by lengthening all the digits except the pollex; and in the birds, where the development of feathers necessitates the formation of a firm framework without motion between the parts, there is formed a bone complex composed of carpal and metacarpal elements and several phalanges. [Fig. 50; (d) to (f).]

At the conclusion of this subject it may not be without interest to review briefly the subject of the transition between the two types of free limb, the ichthyopterygium and the cheiropterygium, in which, although as yet no theory has gained general credence, or has even passed beyond the stage of an ingenious speculation, many interesting suggestions have been advanced, some of which may be near the truth, as may at any time be shown by the discovery of the fossil remains of transition animals, unknown at present.

In general the similarity between fin-rays and digits is seen by everyone, and this probable homology is rendered more likely by the fact that the bones of the digits in animals with the hand-form of limb are preformed in cartilage, thus suggesting the fin-rays of the selachians and ganoids. Both are divided by joints into movable segments; both are supplied with flexor and extensor muscles; and in some fishes the rays are prolonged by the addition of horn threads, in structure 
and position recalling the claws or nails of the hand-form, although placed on both sides instead of one. Even the usually excessive number of the fin-rays is no real objection, since in some fishes this number is a very limited one, and the theories of pre-pollex and post-minimus point to a previous larger number. Thus in a way the derivation of hand or foot from the fish-fin may be accounted for; but an insuperable difficulty lies in the presence of the two lengths of limb bones interposed between the girdle and the distal complex, which seem to correspond with nothing found in the fin. It is to be remembered, however, that these are not especially long in the more primitive forms, like salamanders, so that the main obstacle lies not so much in their length as in their very existence, which has never received a satisfactory explanation.

An early suggestion along this line was based upon the anterior fin of selachians with its three basal pieces (Fig. 5I, a). The mesopterygium is usually much the largest and shows a tendency to form the sole connection with the shoulder-girdle. If this becomes established, the mesopteryginm becomes a humerus, and the pro- and metapterygium, slipping away from the girdle, and bearing the free rays, would become respectively radius and ulna (Fig. $5 \mathrm{I}, \mathrm{b}$ and $\mathrm{c}$ ). This theory seemed for a time to receive especial corroboration from the structure of the paddle of the extinct sea-lizards, Ichthyosaurus and Pleisiosaurus, but the time is now passed for drawing such broad conclusions from a chance resemblance in some highly specialized form, aside from which it must be noted that the theory is based upon the anterior fin alone, leaving the more primitive posterior one out of the question.

When, a little after this, the biserial dipnoid fin was heralded as the primitive type, and named in consequence the "archipterygium," it turned thought in a new direction, and an effort was made to seek in the more primitive cases of the hand-form a central axis with lateral rays proceeding from it. In one such attempt the central axis was formed by humerus, ulna, ulnare, carpale $\mathrm{V}$ and the fifth digit, to which the remaining bones served as lateral rays upon the inner or radial side, 
thus forming a uniserial form, as in the selachians; in another the hind limb of the salamander Ranodon was used as a basis,
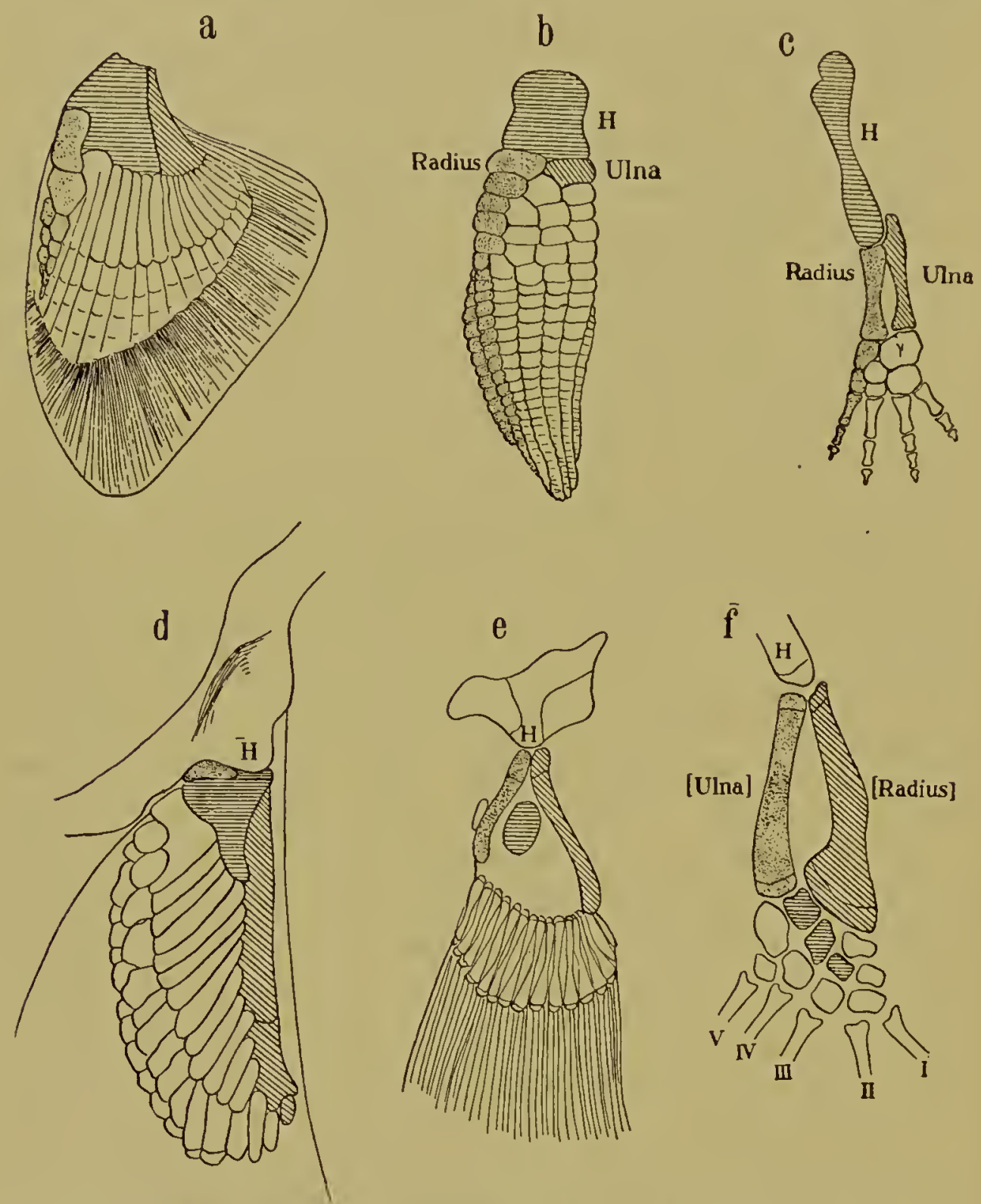

FIG. 5I. Two theories of derivation of the chiridium (cheiropterygium) from the fin (ichthyopterygium). The figures represent the anterior limb in all cases. [a-c, after Huxley; d-f, after Pollard.]

(a) Cestracion, a primitive selachian. (b) Ichthyosaurus, an extinct lizard. (c) Necturus, a primitive salamander. (d) Chlamydoselachus, a primitive selachian. (e) Polypterus, a ganoid. (f) Ranodon, a slamander.

In the three upper figures the ulna is dotted, the radius designated by a diagonal striping; in the three lower the reverse is the case.

and femur, fibula, intermedium, the two centralia, carpale II and the second digit were taken as the central axis, leaving one 
digit upon the inner, and three upon the outer side to serve as lateral rays. Aside from the unsafe basis, however, upon which all such theories are founded, their use as an argument is nullified by the ease with which, in turn, each digit, with its associated bones, may serve either as a hypothetical central axis or as a lateral ray.

As opposed to the theories thus far recorded, which seek to derive the entire free limb from the fin, is a more recent one, based upon the anterior fin of the ganoid Polypterus, in which ulna and radius are derived from the fin, while the humerus represents an element to be detached later from the shoulder-girdle. In this fin (Fig. 5I, c), the pro- and metapterygium, already in the form of long bones, articulate with a projecting point of the girdle, while the meso-pterygium, reduced to a small, disc-shaped element, lies between them but detached from both. If now the long pro-and meta-pterygia are taken as radius and ulna respectively, ignoring the meso-pterygium for the present, the projecting process of the shoulder-girdle, which articulates with both, and which might easily become detached from the main mass if of functional advantage, would become a humerus. The mesopterygium would thus become intermedium or intermedium and centrale, leaving the remaining parts of the carpus, the metacarpus and phalanges, to be formed from fin-rays. This is in many ways the most satisfactory solution thus far, and the fact that it rests upon the condition found in a single species is no real objection, since it is altogether probable that terrestrial vertebrates were originally derived from a single form, perhaps a single species, and that Polypterus, with its close anatomical correspondence with the lowest urodelous amphibia, is nearly allied to that form. A more serious objection lies in the fact that the posterior fin cannot be as easily developed into a cheiropterygium as can the anterior one; still the anterior and posterior limbs may not have had exactly the same origin or early history, since a later similarity of use would cause a convergence in anatomical structure. Moreover, as a matter of fact, the pelvic fin of Polypterus exhibits proximally 
a single long bone, showing at least a tendency in a similar direction. Here the matter must rest for the present from lack of further evidence, but the solution may be found at any time, as has happened in so many other cases, through the discovery of the fossil remains of some transition form. The pick of the geologist may unearth the key to this problem, a true palæontological Rosetta stone, by the aid of which the discrepant records written on fin and foot may be deciphered and brought into harmony.*

* Within the past few years a number of fossil Polypteri have been discovered in the Triassic beds along the shores of Lake Tanganyika, in the waters of which their living descendants are still found. This would seem a. favorable locality from which to expect results. 


\section{CHAPTER VI}

\section{THE MUSCULAR SYSTEM}

"When we no longer look at an organic being as a savage looks at a ship, as something wholly beyond his comprehension; when we regard every production of nature as one which has had a long history; when we contemplate every complex structure and instinct as the summing up of many contrivances, each useful to the possessor, in the same way as any great mechanical invention is the summing up of the labor, the experience, the reason, and even the blunders of numerous workmen; when we thus view each organic being, how far more interesting . . . does the study of natural history become!"

CH. Darwin, Origin of Species. Chapter XV.

VERTEBRATES possess two sorts of muscular tissue, unstriated, or involuntary, in the form of cells, and striated or voluntary, in the form of long fibers usually formed from cell-complexes. The cells of the involuntary type are mesenchymatous in origin and are usually associated together to form layers that supply the walls of certain internal organs and the larger blood vessels with the power of expansion and contraction. All voluntary muscles, on the other hand, are derived directly from the mesoderm, and the ultimate contractile organs are here not the cells themselves, but long fibrils of contractile substance built up by the cells, or often by long rows of cells, and organically connected with them. The musculature of the heart, in some characteristics seemingly intermediate between the two classes of muscular tissue, is a modification of the first or involuntary type, the cells of which possess a peculiar shape and become marked with striæ. This derivation is rendered clear through the embryological development of the heart, which is seen to be originally an expanded blood vessel with an hypertrophied muscular coat. 
The muscles of the second type are in general under the control of the animal's will, and are thus termed voluntary, although there are regions, as along the pharynx and œsophagus, where genuine striated muscle may become involuntary and depend for its action upon external stimuli.

The striated muscles, which form the subject of this chapter, fall into three anatomical divisions corresponding to those of the skeleton, the axial muscles, the appendicular muscles, and the visceral muscles. In the embryo the appendicular muscles are derived directly from the axial, both arising from the dorsal portion of the mesodermic somites, the epimeres; while the visceral muscles, limited to the anterior part of the body, arise from the ventral portion, the hypomeres, the element which in the remainder of the body develops into the pleuro-peritoneum, and furnishes no voluntary muscles. This difference in origin sharply divides the voluntary musculature into two fundamental groups, (I) the parietal, arising from the epimeres and including the axial and appendicular musculatures, and (2) the visceral, arising from the hypomeres, and limited to the anterior part of the body.

Aside from these three primary divisions of striated muscles, there occur in almost all vertebrates certain superficial muscular elements, usually in the form of subcutaneous layers and intimately associated with the corium. These have often been treated as a distinct group of muscles, but recent investigation has placed it beyond doubt that we have here to do with muscular elements which have developed independently in different animals in response to certain physiological needs and have been derived from the most convenient subjacent skeletal muscles, whether parietal or visceral. However, in spite of their secondary origin from other muscular groups, they have differentiated so far structurally that it is far more convenient to treat them as a distinct group, the integumental muscles, rather than to consider them with the various muscles from which they were originally derived. The order of treatment, therefore, of the muscles, as described in this chapter, will follow the plan just outlined: the first to be considered will 

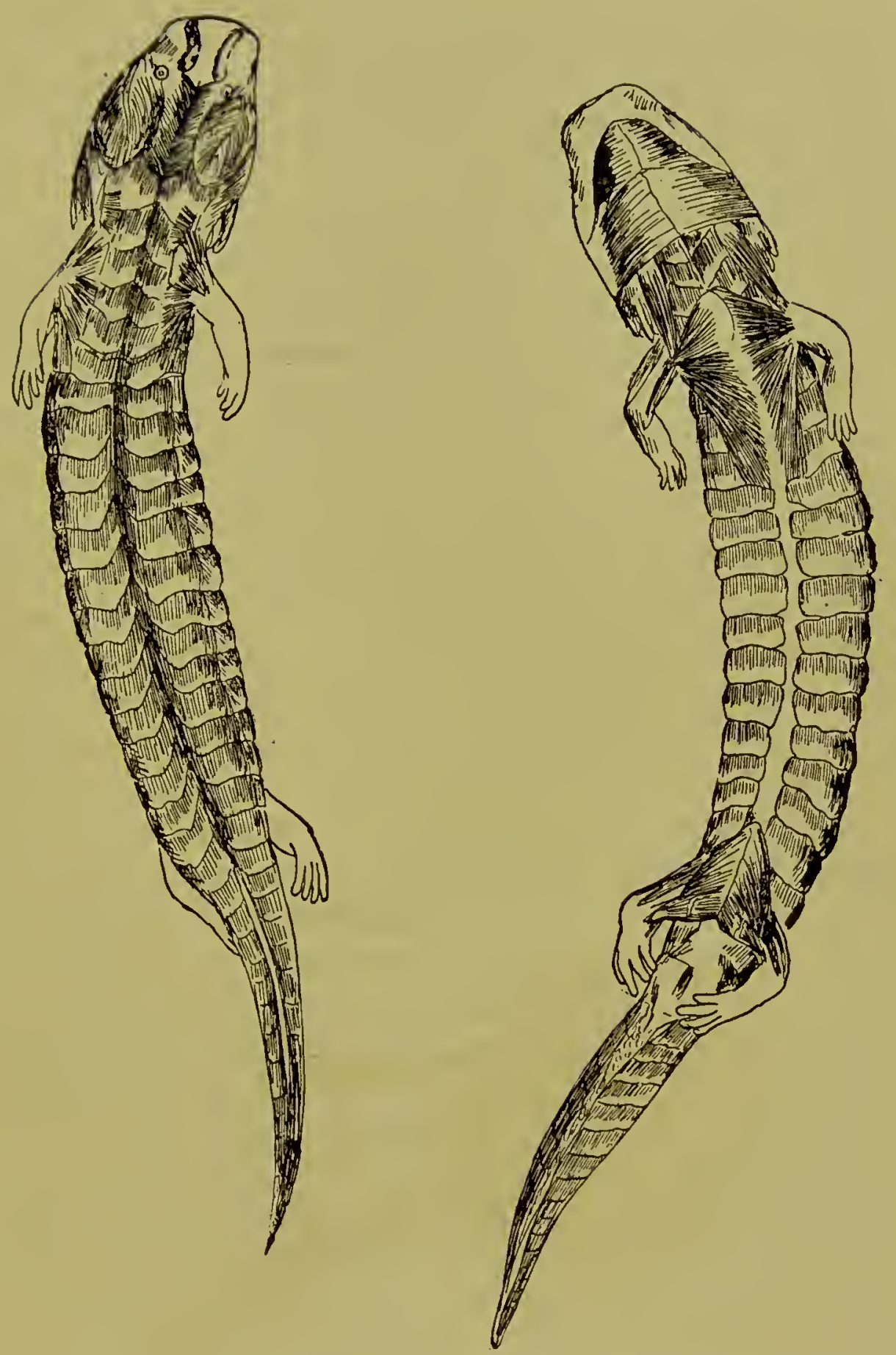

FIG. 52. Necturus, with the integument removed, showing the primitive myotomic muscles and the differentiation in the regions of the limbs and the visceral skeleton. 
be the parietal or epimeric muscles, consisting of (I) the arial, and (2) the appendicular musculature. This will be followed by a short sketch of the visceral or hypomeric muscles; and, lastly, the integumental muscles, a secondary system, will be considered.

The primary groups of skeletal muscles, with the exception of the integumental, can be well shown in a condition approaching the primitive one by carefully removing the skin from a salamander and inspecting the muscles as they lie in their natural position (Fig. 52). In this preparation the axial muscles form the bulk of the muscular system, and are seen to consist of a series of muscle somites, or myotomes, separated by thin perpendicular planes of connective tissue, the myocommata. These axial muscles are divided by a horizontal furrow, which runs along each side, into dorsal and ventral masses, a distinction which is of fundamental importance, as these masses are innerved respectively from the dorsal and ventral branches of the spinal nerves. The groove dividing them marks the place of the lateral line of fishes, in which are located a row of specialized sense organs. The axial muscles begin at the base of the skull and continue to the end of the tail, but show some interruption of their course in two places, corresponding to the attachments of the two pairs of limbs. That of the anterior limbs, however, is seen to be more superficial in character, and when the appendicular muscles, that spread out in thin fan-like sheets over the trunk nyotomes, are removed, the myotomic muscles are displayed in an almost uninterrupted sequence. In the case of the posterior limbs, however, the skeletal girdle comes to lie imbedded within the body muscles, and this, together with the cloaca, causes a considerable hiatus in the sequence of the myotomes on the ventral side, although dorsally the sequence is unbroken. The muscles attached to the free limbs and their girdles form the appendicular group, of little proportional importance here, but destined in the higher vertebrates, with the development of larger and more powerful limbs, to assume a far greater bulk, and in some cases to even surpass that of the axial 
muscles. There will be noticed this difference in the proximal muscles of the anterior and posterior limbs, that while those of the latter arise almost exclusively from the girdle itself, those of the anterior limbs are spread out fan-like over the axial myotomes, and arise either from the myocommata or from the integument. In the higher forms this difference receives much greater emphasis, and in both birds and mammals the appendicular muscles of the fore limbs completely enwrap the body both dorsally and ventrally, thus concealing the axial muscles entirely, from the hips to the neck, except in the vontral abdominal region.

Corresponding to the extensive development of the visceral skeleton in the animal under consideration, the visceral musculature is also large and well shown. This occupies the ventral and lateral regions of the head and neck, and includes the massive muscles of the mandible, which extend over the top and sides of the skull. In the higher forms these muscles, with the exception of those of the mandible, lose in bulk, but perhaps gain in complexity, supplying tongue, pharynx, and the laryngeal region.

Although thus far the facts presented rest upon a secure morphological basis, and although it is comparatively easy to follow the fate of the primary muscle masses as a whole in the separate vertebrate Classes, the further emphasis of the separate units which differentiate from the primary masses, that is to say, the homology of the individual muscles, is a subject fraught with especial difficulties, and is one in which the ground is still uncertain, owing to the lack of fixed principles to direct the investigation. To begin with the axial muscles in their undifferentiated condition as a series of similar myotomes and follow out the various fate of their derivatives throughout vertebrates as the parts become modified to subserve countless special uses; to start with the limb muscles in the form of myotomic buds and follow the transformation outwardly expressed by the varied shape of fin, wing or leg; and finally to discover a fundamental plan in the complicated visceral muscular system of selachians, and carry out the 
history of these elements as they gradually assume control of such different parts as the jaws, the auditory ossicles and the laryngeal cartilages; such would be the history of the muscular system as it may sometime be written, a history even the outlines of which are in many places still waiting to be established.

A great barrier in the way of morphological study of the muscles lies in the fact that there is no definite criterion of homology, no simple way of absolutely proving that a given muscle in a certain animal is morphologically the same as a similarly related one in another species. This can be proven in a fairly satisfactory way by tracing the race history through a series of forms, provided that no extensive hiatus occurs in the series; but more often the animals to be considered are isolated forms, separated by wide gaps from their nearest living allies. With other organs the embryological record furmishes valuable clews and often traces a continuous history, by the aid of which the condition in isolated adult forms may be interpreted; but here not only are the embryological conditions extremely difficult to interpret, but in the majority of cases the historic stages are not there, and the final condition is seen to arise suddenly from a mass of apparently undifferentiated cells.

In attempting to establish a basis for homology it is to be remembered that a muscle is not always a definite organ like a bone or blood vessel, but is rather a mass of muscular fibers set apart for a more or less distinct purpose and differentiated from the surrounding muscle masses in proportion to the definiteness and precision of its action. One muscle may be entirely isolated from the adjacent fibers by a firm cover of connective tissue and provided with a special tendon attached to a definite skeletal process; another may be a bundle of fibers but partially separated from a larger mass and acting only in connection with it. Often, too, the action produced by a muscle is not precise enough to prevent numerous variations, and thus may vary considerably in different individuals of a single species or even in the two sides of the same individual. It is 
thus necessary to study all possible individual as well as specific variations of a muscle or a muscle group before laying down the outlines of its history.

When the study of comparative myology was in its infancy, muscles were homologized and named from their general location, appearance and use, without regard to their developmental history, a method which, while fairly safe when applied to closely allied forms, was apt to be very misleading when applied to animals as different, for example, as members of the different vertebrate Classes. Thus, if the starting point were the human subject, as was the former universal custom, it would be quite easy to recognize such a muscle as the deltoid in the apes and monkeys, which possess prehensile arms of a similar shape and used in a similar way, but it would be much more difficult to determine the same muscle in the ox, which uses its fore legs so differently, and the difficulty might become insurmountable in a form as different as a turtle or a frog.

Jomewhat more reliable as criterions for homology than position or use are the origin and insertion, the points at which the muscle fibers are attached, although these are altered by increase or decrease in volume or by a slight change in use; and, if the change is marked, may attain quite different relationships to the skeletal parts. There is, however, some difference in the relative value of these two points, the origin being the more constant in certain regions, the insertion in others. It has long been considered an axiom of comparative myology to give the credit for greater constancy to the origin in all cases, but in the muscles of the appendicular skeleton the reverse seems to be the case, since here the insertions are at the distal end and are effected through narrow tendons, the precise attachment of which is a matter of great importance in the action of the muscle, while the origins occupy large and rather indefinite areas, the extent of which is relative to the degree of development in each case.

Undoubtedly the most reliable criterion for muscular homology is that based upon the constant relation between a given 
muscle and the nerve which supplies it, since the nerve which originally supplies a given prinitive element, such as a myotome or a limb-element, never forsakes it, but follows it through all its vicissitudes and continues to supply its derivatives, of whatever complexity or form, through all the changes of relation, which are often very great. A conspicuous example of this is the facial nerve (VIIth cranial), which, in spite of its name, is not, so far as it is a motor nerve, originally associated with the face but with the hyoid region of the neck. In the lower mammals this nerve supplies an integumental muscle covering the side of the neck, of which the human platysma is a remnant, and as it happens that in higher forms this sheet becomes extended over the face and differentiates into the various slips that form the mimetic musculature, its nerve follows it, multiplying its branches in strict accordance with the growth and differentiation of the muscle, until it covers the entire face with its ramification and earns the name of facialis. Another branch of the same nerve supplies the digastric muscle of the mandible in the lower vertebrates (the equivalent of the posterior belly of the like-named muscle of mammals), and when in reptiles a small slip detaches itself from this muscle and wanders into the middle ear to become the stapedius, a minute branch of the nerve in question follows it to its ultimate location and furnishes it with its nerve supply.

Were it possible to follow each motor nerve fiber from its origin to its connection with its muscle, it would probably serve as an absolute criterion for muscular homology, but there is much chance of error in the fact that an anatomical nerve is not a single fiber, but a bundle of them, and while each fiber is presumably constant in its supply, there is some variation in the way in which they are put into bundles, so that no one can be sure that a given nerve is always quite homologous with one in a like location in another animal. This is especially true of the innervation of the limbs, where the nerve supply, after proceeding from a certain fairly definite number of nerve roots, passes into a plexus, in which the 
fibers become divided up and reunited in new combinations; and although in two allied forms the final nerves that emanate from the plexus may be constant in number and position, no one can be quite sure that their make-up in individual fibers is the same, and indeed it is more than likely that they are not. Furthermore, in cases in which a single muscular element has differentiated into a group of well-separated muscles, each with a specific action, all will be supplied by branches of the same nerve, and the innervation will furnish no clew to homology, beyond that of identifying them as members of the same limited group.

Thus the criterion of nerve supply, although in theory an accurate and definite method, often fails in its application through variation in the make-up of the separate nerve bundles, and while undoubtedly the best criterion we possess, it cannot be employed in all cases. It is the most reliable in its application to the axial muscles, where there has been the least amount of differentiation, and where the primitive segmentation is still evident or but slightly disguised; it has also proven of value in the muscles of the visceral system, especially in the case of fishes and amphibians, but in such cases as the highly differentiated linı muscles, this method is difficult of application, and cannot be followed beyond the homologizing of the larger groups. Here the character most to be depended on is the insertion, since these are in most cases by narrow tendons and hence very definite, while the origins spread over a greater or less area in proportion to the size of the muscular "belly," or fleshy mass, and are thus somewhat inconstant in individuals of the same species, or probably in the same individual at different periods of its life.

Although, in the study of the morphology of the muscles, through the incompleteness of the embryological record and the technical difficulties in the way of examining it, reliance has hitherto been placed mainly on the comparison of adult forms, much may undoubtedly be learned concerning the history of individual muscles and muscle groups from their ontogeny, especially in those cases in which the animal passes 
through an active larval period, and hence exhibits the earlier stages in functional activity and consequently in greater completeness.

From both this source and from the study of adult comparative anatomy certain principles may be deduced concerning the formation of muscles, some of which may be noted here.

I. Separation of an indifferent mass into several elements. This may be done in several ways:

(a) By the growth of process from the surrounding skeletal parts, thus furnishing separate points of origin for different bundles of fibers.

(b) By the growth of a skeletal process across the fibers of a long, band-like muscle, thus cutting it in two; a secondary segmentation.

(c) By the higher specialization of the parts of insertion, thus causing a splitting up of the muscle bundle.

Illustrations of the first of these may be seen in the development of the processes on the vertebræ, which results in the breaking up of the indifferent myotomes into the extremely complex slips seen in the muscles of the vertebral column of higher forms. The second method is rare, and occurs in the case of one of the hyoid muscles of the frog (the fourth petro-hyoideus), and in two of the occipito-cervical muscles in the mammals (obliqui capitis, cf. Fig. 55). The third case finds a complete illustration in the differentiation of the digits in mammals and the splitting up, both of the tendons and of the bellies of the common flexors and extensors, in exact accordance with the degree of independent action of the separate digits.

\section{Fusion of separate elements.}

This may occur in the case of the degeneration of a function, but it is seen in a progressive instance in the case of long muscles derived from elements taken from separate myotomes. Thus the external oblique muscle of the abdomen is a metameric muscle formed by contributions from a series of successive myotomes. In salamanders this is barely differentiated 


\section{THE MUSCULAR SYSTEM}

and is still crossed by the myocommata, that mark the original elements, but as the higher forms are reached the myocommata disappear, and the primary segmental nature of the muscular sheet is shown only by the distribution of nerves and blood vessels. In the rectus abdominis the same thing has taken place, but here the effacement of the myocommata is not complete, and a few of them are still present, even in mammals, forming the "tendinous interscriptions" of human anatomy (3-4 in man).

III. Extension over a much larger area of an element originally belonging to one or two myotomes.

This is seen especially well in the case of certain of the muscles of the anterior limb, notably the pectoralis and the latissimus dorsi. Small and not very extensive in the earliest land animals, these muscles increase with the importance of the limbs to which they belong, and may eventually extend over a large number of myotomes. In their final condition such muscles closely resemble such a sheet as that of the external oblique of the abdomen, but their composition is very different, the one being the result of the fusion of elements derived from several myotomes, the other the extension of an element derived from one or a few.

IV. Further cxtension of a pair of muscles that have come to meet in the median line through the formation of a median skcletal crest or keel.

The two most conspicuous examples of this are the pectoralis muscle on the ventral side of the thorax and the temporalis of the mandible. Of these, one is originally an appendicular, the other a visceral muscle, each probably a derivative of a single myotome. Both extend their area of origin with their increase of function and size, the one dorsally over the sides and top of the skull, the other ventrally over the chest. Further progress being stopped by the meeting of the two muscles in the median line, they obtain an extended point of attachment through the formation of a median ridge. The most excessive instance of this is seen in the enormous keel of the sternum in certain birds, notably the humming-bird. 
Both phylogenetically and ontogenetically the arial muscles are the first to appear, and forcibly suggest some lost ancestor in the form of a segmented worm, the muscles of which were mainly longitudinal in direction and repeated themselves metamerically. The appearance of these, in the form of mesodermic somites, is one of the earliest post-gastrular stages in the embryo, and is the first suggestion of segmentation. In the lower vertebrates the muscle somites develop through the longer process of the formation and extension of pairs of lateral diverticula and the subsequent separation of the epimeres, as in the theoretical sketch given above [Chap. III], but in the Sauropsida and Mammalia, through acceleration of development, the epimeres are separated from an indifferent mesoderm in the form of approximately cubical blocks, arranged in pairs on each side of the nerve-cord and notochord, and were long considered to represent "primordial vertebra," a name occasionally used even at the present time, although its literal meaning has been long since discarded. From these the myotomes develop through the formation of longitudinal fibers, and the mesenchyma of the intervals between the mesodermic somites becomes transformed into the myocommata, to which, on either side, the muscle fibers are attached.

There is thus formed the primitive system of axial muscles, a condition which is retained with but little modification in the primarily aquatic vertebrates, that is, in Amphioxus, cyclostomes, fishes and tailed amphibians. In the first of these the myocommata are not in the form of planes, but are bent in the middle at an acute angle, the point directed forwards, and are set into one another so that several consecutive myocommata would be cut in a cross-section through the body at any plane. In many fishes the myocommata become still more complex and consist of several pairs of cones, those of successive myocommata being set into one another like nested cups. This explains at once the shortness of fiber of fish meat, and also its curious division into sets of concentric circles, when cut in cross section, two phenomena with which everyone is familiar. In the urodelous amphibia the myocommata are almost planes, 
and cut the body nearly at right angles to the longitudinal axis, thus presenting a much more primitive appearance than that found in most fishes, and one that corresponds closely to the embryonic condition.

Above the urodeles the development of more massive limbs and limb-girdles, the same element that produces such profound regional differentiation in the vertebral column, is operative here also in modifying the simple succession of typical myotomes, a condition which was unaffected by the delicate fins and weak girdles sufficient for their primitive aquatic environment. The hip-girdle produces the most direct change through the intrusion of the ilium between two successive myotomes, following the course of a myocomma. By this the axial myotomes become divided into those of the trunk and those of the tail, and the gradual increase in size of the ilium and its extension along the vertebral column, as well as the formation of an immovable sacrum, widens the space between these two groups of muscles, while the differentiation of function between trunk and tail effects profound changes in the muscles themselves. The principal effect of the shoulder-girdle in this regard is an indirect one, for while it presents no intruding process, as in the case of the ilium, it establishes the anterior point of support and causes the differentiation of the region between it and the skull into a neck, which becomes in some cases extremely mobile.

In most reptiles and in mammals, however, in spite of these modifications, the succession of myotomes remains distinct, especially dorsally, and through all the regional differentiations there may still be seen the segmental character of the musculature; but birds and turtles represent two types of extreme specialization in which this continuity becomes broken through local reduction. The cause of this in turtles is the formation of the carapace, in which all free movement of the vertebral elements and ribs is lost through a complete anchylosis, and in this region the trunk muscles, though laid down in the embryo, are early atrophied and become lost. The only axial muscles retained are those of the neck and tail, together 
with a powerful retractor system, lying on the ventral side of the vertebral centra and employed in drawing back the head and neck. This is undoubtedly homologous with the prevertebral muscles of the cervical and thoracic region in mammals (longus colli, etc.), and belongs with the ventral division, innerved by ventral branches of the spinal nerves. In birds, correlated with the lack of mobility of the trunk vertebræ, the corresponding muscles are greatly reduced, but as this loss of motion is not a complete one as in the turtle, so also is the reduction of the muscles not as extreme. The muscles of neck and tail, on the other hand, are extremely well. developed, thus emphasizing by contrast the almost rudimentary condition of the muscles of the back.

In taking up the differentiation of the axial muscles more in detail, their division into dorsal and ventral misses must be emphasized, for, although both are derived embryologically from the epimeres, that is, the originally dorsal portions of the mesodermic somites, yet the distinction is of fundamental importance topographically and morphologically, because they are innerved respectively by the dorsal and ventral branches of the spinal nerves, a criterion which may always be relied upon to help out the homology in doubtful cases, where a muscle lies near the boundary between them or where an extreme degree of differentiation has changed the primary location. These divisions may be taken up in order, beginning with the ventral, which are less complicated than the other.

Throughout the course of these ventral axial muscles four distinct regions may be distinguished, and the same layer, when it is possible to trace it from one of these regions to the other, becomes modified to partake of the features of the inusculature characteristic of each. These are the cervical, thoracic, abdominal and caudal, the three former continuous, the latter more or less separated by the interposition of the hipgirdle and the cloaca. In the abdominal region, even in fishes, there is seen a tendency for the musculature to break up into layers, each with a definite direction of fibers, distinct from that of the others. In amphibians these layers consist pri- 
marily of an obliquus intermus, an obliquus extermus derived from the former, and a rectus abdominis, which runs along each side of the midventral line and is differentiated from fibers belonging to the other two. To these are added during the metamorphosis, an obliquus externus superficialis, formed from the primary externus, and a transversalis, differentiated from the primary internus. These muscles, with the wellknown differences in the direction of their fibers, persist in all higher vertebrates. The obliquus externus superficialis of the amphibians is no longer present as an abdominal muscle, but seems to be identical with the two serrati posteriores, superior and inferior, usually treated as muscles of the back although innerved by ventral nerves. They appear in rodents and in some other mammals as a continuous sheet, but in man the two muscular-portions (superior and inferior) are separated by a considerable interval, although occasionally connected by a thin tendinous sheet, the rudiment of the intermediate portion.

The rectus abdominis is the only one that retains the primitive longitudinal direction of its fibers, and, undoubtedly cor"related with this, is another primitive character, that of the persistence of some of its original myocommata, the "tendinous interscriptions" of human anatomy. Connected with the pubic end of the rectus in mammals is a small muscle, of uncertain occurrence in man, the pyramidalis. This muscle is a rudiment of the pouch muscle of marsupials, in which animal it is extremely well developed, extending from the marsupial bones, upon which it arises, as far anteriorly as the sternum. The cremaster muscle of the mammalian scrotum, noticed more particularly in connection with the decensus testiculorum in Chapter IX, is a derivative of the obliquns internus. The quadratus lumborum belongs also in this place, although its derivation has not yet been worked out. It appears first as a distinct muscle in reptiles and birds, where it is represented by a few fibers associated with the transversalis. In mammals it is large and important.

The essential elements of the abdominal musculature may be 
followed into the thoracic region, although here a new character is introduced by the presence of the ribs, which occupy the place of the myocommata, of which they are direct derivatives, and thus impart to the layers a segmental character. The obliquus externus (profundus) and internus are thus continued forward as the extemal and internal intercostals respectively, and the transversalis is represented by the transversus thoracis (triangularis sterni), in man very variable in extent. The levatores costarum appear as a new element, peculiar to the thorax, but they undoubtedly belong with the external intercostals and the external oblique.

In the cervical region the reduction of both ribs and boriy cavity necessitates another series of modifications of the original elements. There belong here, as ventral muscles of the axial system, the scaleni, and the muscles of the prevertebral group. Of these the scalenus posterior is a continuation of the levatores costarmm, and the other two scaleni, anterior and medius, belong to the system of the intercostals and are attached to those portions of the transverse processes of the cervical vertebræ which are in reality anchylosed ribs. In the same category belong the short lateral muscle, rectus capitis lateralis, and the "anterior" (ventral) series of intertransversarii, which are innerved from ventral branches and are also attached to the rib elements of the cervical vertebra. The prevertebral muscles, lying on the latero-ventral side of the vertebra, consist of the longus colli, and its anterior extension, longus capitis (rectus capitis anticus major), also of the little rectus capitis anterior (rectus capitis anticus minor), which extends between the skull and the atlas, near the rectus lateralis. The history and serial homology of these latter muscles, other than the fact that they belong with the ventral axial muscles, is not known.

Both ventral and dorsal axial muscles are continued beyond sacrum and cloaca along the caudal vertebra, where their development varies as much as does the tail itself. As it is more convenient to treat of the caudal musculature as a single subject, the discussion of the ventral muscles of this region will 
be postponed until later when both dorsal and ventral caudal muscles will be considered together.

The dorsal axial muscles are much more restricted in extent than are the ventral ones, and are mainly confined to the

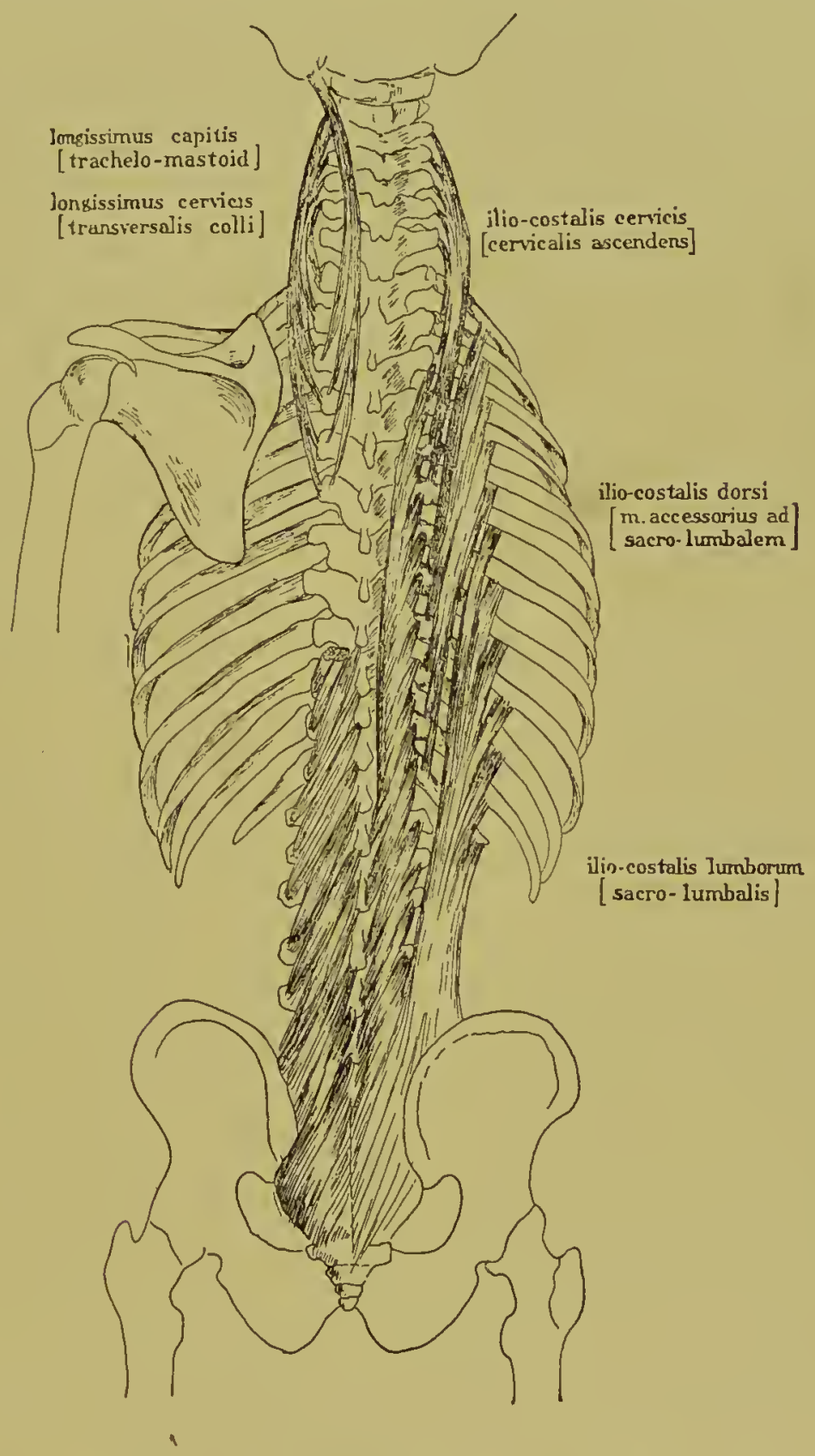

FIG. 53. Muscles of the human back.

The superficial layers belonging to the appendicular system have been removed. Upon the right are shown the three portions of the ilio-costalis system externallw, and within this the longissimus dorsi. Upon the left below is the multifidus; and above, the remainder of the longissimus system. 
triangular prismatic spaces located on either side of the micldorsal line, between the spines of the vertebrx and the transverse processes and ribs; they are, however, far more complex in structure, and are characterized by the presence of almost numberless tendons that repeat one another metamerically and are attached to corresponding parts of successive vertebræ or ribs. The muscular elements are also less differentiated from one another than in other parts of the system, and in many cases the subdivision into separate muscles is an arbitrary one.

But little has been done with the muscles of this group from the standpoint of comparative anatomy, and thus their morphological history cannot as yet be attempted. It must suffice here to treat them to a rapid review as they exist in man, with some attempt at a morphological arrangement of their several elements, after which may be considered what is known of their history.

In man the back is covered superficially with several layers of muscles which are dorsal in a topographical sense only, having secondarily invaded this territory from other places of origin. The most superficial of these, trapezius and latissimus dorsi, form a thin covering over almost the entire back, effectually concealing all beneath them. These belong to the appendicular system and appear in the urodeles as small, fanshaped muscles which extend dorsally from shoulder and humerus respectively, and cover but a small portion of the trunk myotomes. With the gradual increase in the size and importance of the limbs which they supply they have enlarged to the extent found in man and in most of the mammals. The rhomboidei, lying beneath these and of less extent, likewise belong to the appendicular group. Still deeper, beneath all of the appendicular muscles, are the two serrati posteriores, the remains of a continuous sheet in certain primitive mammals. Their innervation from ventral branches of the spinal nerves shows that they, too, are originally strangers to the dorsal region and belong rather with the ventral axial muscles, under which head they have already received treatment.

The removal of all the above exposes the genuine dorsal axial muscles, the derivatives of the trunk myotomes, the 
system innerved by the clorsal branches of the spinal nerves. They lie lodged in the space embraced by the spinous and transverse processes of the vertebræ and run in the main in a longitudinal direction. They are beset with metameric series of tendons, which attach themselves to the corresponding portions of successive vertebræ or ribs, and plainly suggest a segmental origin. Proceeding inwards from the more superficial series they may be divided as follows, although it must be remembered that the muscles are often closely attached to one another and are not as distinct as in most other regions.

I. Spino-transversalis System.-This system, the fibers of which arise from spinous and insert on transverse processes, consists of a single muscle, the splenius, confined to the anterior portion of the trunk, and unrepresented posterior to about the middle of the thoracic vertebrx. In form it is a thin sheet of oblique fibers, a portion of which, splenius cervicis [colli], inserts into the transverse processes of cervical vertebræ, while the remainder, splenius capitis, inserts into the base of the skull.

II. Sacro - transverso - transversalis System. - This arises from a large mass of muscular fibers filling in the space between the hip-girdle and the most posterior ribs. These fibers take their origin from sacrum and ilium, and from the lumbo-dorsal fascia. As the fibers issuing from this origin are not sufficient to supply the entire vertebral column, they are reinforced by others which arise from the transverse processes of the vertebrx, beginning with the lowest lumbar. The metameric insertions are in two longitudinal rows, or series, the outer into the ribs, and the inner into the transverse processes. A bundle of the more anterior fibers of the latter inserts into the base of the skull. The system may thus be designated sacro-transverso-transversalis, the first two elements designating the origin, the third, taken in the broad sense and including the ribs, the insertion.

The outer series begins posteriorly as the sacro-lumbalis, and becomes continued in the region of the ribs by a series of muscular slips arising from lower ribs and inserting in upper, musculus accessorius ad sacro-lumbalem. A still further con- 
tinuation, which takes the series into the cervical region, is formed of slips that arise from the upper ribs and insert on the transwerse processes of the lower cervical vertebra, the corvicalis ascendens. As these three muscles, the names of which have come down to us from premorphological times, are clearly portions of a single series, they are best referred to under the name of ilio-costalis, the three portions being distinguished respectively as ilio-costalis lumborum, ilio-costalis dorsi and ilio-costalis cervicis. [BNA.]

The inner series, posteriorly blended with the former, ascends along the lumbar and lower thoracic regions under the name of longissimus dorsi, and is reinforced anteriorly by the transversalis colli and the trachelo-mastoid, the latter inserting on the mastoid process of the skull. In placing the nomenclature on a morphological basis the series may retain the name of its most important member, the longissimus, the parts of which are longissimus dorsi, longissimus cervicis and longissimus capitis. [BNA.]

In tabular form the parts of this system with their synonyms are as follows:

Sacro-transverso-transversalis System. Outer SERIEs.
ilio-costalis lumborum (sacro-lumbalis)
ilio-costalis dorsi (accessorius ad sacro-lumbalem)
ilio-costalis cervicis (cervicalis ascendens)

INNER SERIES.

longissimus dorsi

longissimus cervicis (transversalis colli s. cervicis)

longissimus capitis (trachelo-mastoideus. s. complexus minor)

III. Spino-spinalis System.-This is a single series lying beneath the preceding close to the median line and consisting of slips that arise from more posterior spinous processes and insert on more anterior ones, skipping at least one vertebra between origin and insertion. This series is, like the two preceding, an interrupted one, and is divided into two constant portions, spinalis dorsi and spinalis cervicis. A few origins from 
spinous processes of cervical vertebra and associated with the scmi-spinalis of the next system, are of occasional ocurrence, and represent a spinalis capitis.

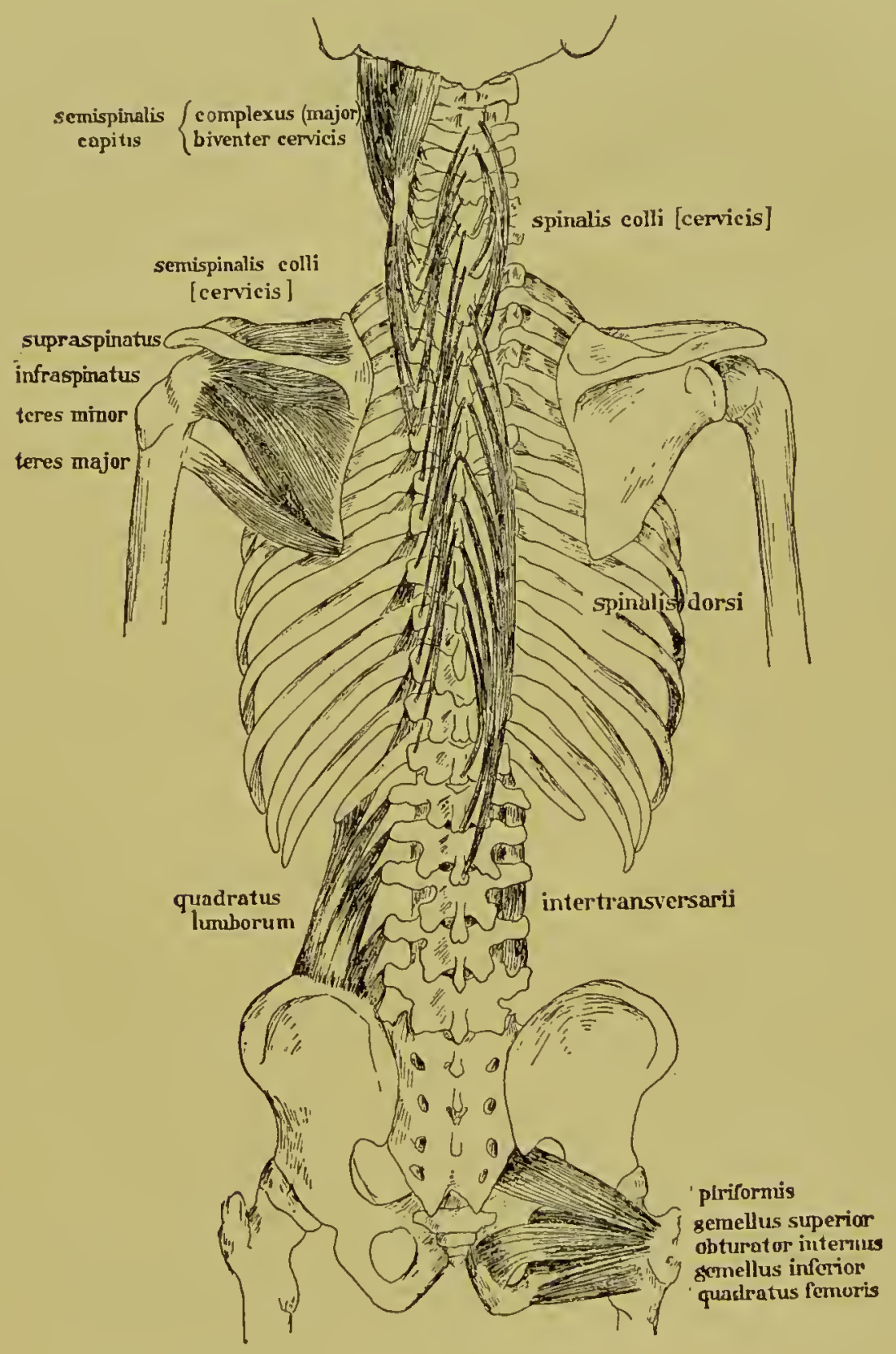

Fig. 54. Muscles of the luman back; the muscles lying beneath those shown in Fig. 53.

Certain of the decper museles of shoulder and hip are also shown. 
IV. Transverso-spinalis System.-This is a complex system, the elements of which arise from transverse processes and insert upon the spinous processes of vertebræ situated more anteriorly. It is in close contact with the previous system, by which it is covered, and may be imperfectly divided into three series or layers, superficial, middle and deep, called respectively semi-spinalis, multifidus and rotatores. These layers differ not merely in position, but in the course of their separate slips, since those of the first pass over 4-6 vertebræ between origin and insertion, those of the second pass over 2-3, while those of the deep layers are themselves subdivided into two series, rotatores longi et breves, of which the outer pass over a single vertebra, while the inner attach to adjacent vertebræ.

The semi-spinalis is divisible into three portions, dorsi, cervicis and capitis. The last of these, semi-spinalis capitis, is a large and well-developed muscle, divided longitudinally into a median and a lateral bundle; the inner one of these is traversed by a myocomma, dividing the muscle into two fleshy bellies, from which comes the older name of biventer cervicis. The outer portion forms the complexus (major) of the older anatomists.

V. Intervertebral System.-Still beneath the multifidus and rotatores are several series of shoft muscles, stretching between adjacent vertebræ, and probably representing a few fibers of the primitive myotomes, which have remained in their original condition. Of these the most extensive are the interspinales, lying on either side of the median line, and extending between adjacent spinous processes. Typically associated with all the intervertebral intervals, they occur in man mainly in the cervical and lumbar regions, including the first and last of the dorsal vertebræ, and are wanting through the middle of the back. A second set, the intertransversarii, between adjacent transverse processes, occur in lumbar and cervical regions, but in the thoracic are represented by tendons without contractile fibers. The intertransversarii of the lumbar region are divided into medial and lateral portions, a division probably without morphological significance; on the other hand, those of 
the cervical region are divided the other way into ventral and dorsal portions, which belong respectively to the like-named divisions of the axial musculature and are hence morphologically distinct. The ventral cervical intertransversarii are innerved by ventral branches, and are attached to the ventral or rib element of the complex "transverse process" of the cervical vertebra. They are thus in the same series as the intercostals, and have been already treated in that connection. The

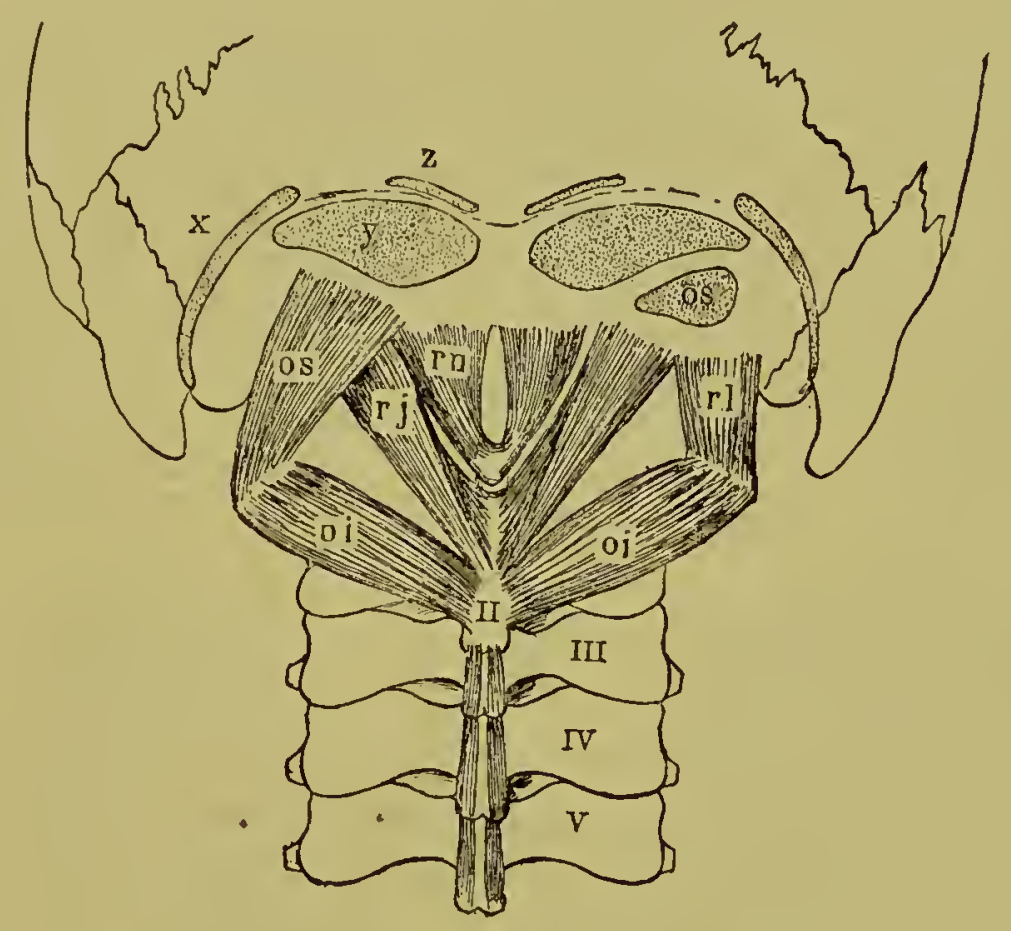

FIG. 55. Muscles of the posterior cervical region (human).

$r j$, rectus capitis posterior major; $r n$, rectus capitis posterior minor; os, obliquus capitis superior; oi, obliquus capitis inferior, $r l$, rectus lateralis. Areas of origin on the skull are indicated as follows: $x$, splenius; $y$, semi-spinalis colli; $z$, trapezius; os, obliquus capitis superior. The cervical vertebræ are indicated by Roman numerals.

dorsal cervical intertransversarii, on the other hand, are innerved by dorsal nerves and are thus serially homologous with both portions of the intertranswersarii of the lumbar region and with the tendons which have replaced them in the thoracic region.

Between the axis and the skull, corresponding to the specialized motions needed in this place, there has developed a complex group of little muscles, which are scemingly differentia- 
tions from the intervertebral system. The rectus capitis posterior minor, in the form of a pair of small slips, extends between the ruclimentary spine of the atlas and the occipital bone, and a similar but somewhat larger pair, rectus capitis posterior major, extends from the spine of the axis to the occipital bone, embracing the other pair. An obliquus capitis inferior extends from the spine of the axis to the transverse process of the atlas, and an obliquus capitis superior continues from this point to the occipital bone.

This entire group develops from what appears in reptiles as a single mass. The dorsal branch of the second cervical nerve runs through this mass and divides it into medial and latera! portions. From the former both recti (major and minor) develop, through a longitudinal separation of their fibers, and from the latter arise both obliqui, which become separated from one another through the outward growth of the transverse process of the atlas, which has divided the muscle across its fibers, a perfect example of the principle designated above as I (b)., p. Ig8.

Posterior to the pubo-ischiadic symphysis and the cloacal orifice, structures which make an hiatus in the ventral series of axial muscles, both dorsal and ventral masses become reduced in size and taper down to form the musculature of the tail. But little morphological research has been devoted to this region, and even in mammals, where the conditions seem the best known, there is much to be done to complete the subject. In long-tailed mammals there are typically two extensores caude upon each side of the mid-dorsal line; an extensor caude medialis, which is a direct continuation of the multifidus and an extensor caudce lateralis, which appears between multifidus and longissimus, but does not seem to be a continuation of either. Upon the sides are two abductores caude, dorsalis and ventralis, the former being short and of lesser functional importance, the latter assuming the function of the principal abductor. Ventrally there is a single pair of flexores (dcpressores) caudc. Of the above, as shown by the innervation, the extensors and dorsal abductors belong to the dorsal 
system, the ventral abductors and the flexors to the ventral. Aside from these there is found a series of intertranswersarii caud $c$, presumably belonging to the dorsal system as in the case of the lumbar muscles of the same name.
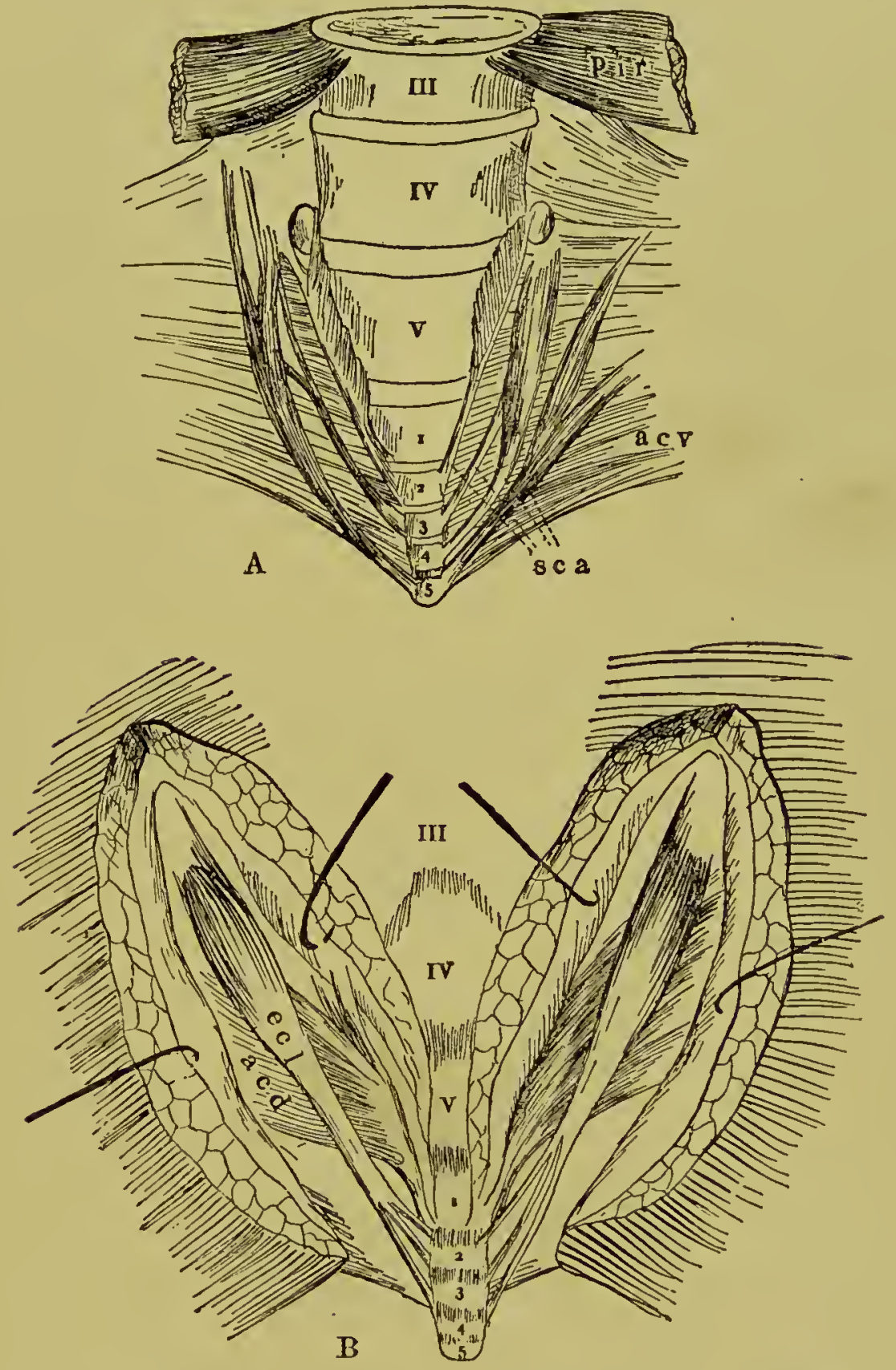

Fig. 56. Human caudal muscles. [After LARTSCHNEIDER.] (A) Ventral. (B) Dorsal.

pir, piriformis; acv, abductor cauda ventralis; acd, abductor caudx dorsalis; ecl, extensor caudx lateralis; sca, sacrococcygeus anterior (= hexor caudx). The lumbar vertebra are designated by Roman numerals, the coccygeal by Arabic. 
In the tailless apes and in man, corresponding to the great reduction of caudal (coccygeal) vertebrx, the muscles are greatly reduced, and of uncertain occurrence, yet traces of all except the intertransversarii have been detected, and certain of them are fairly constant. The sacro-coccygei posteriores (extensores coccygis) are found upon the dorsal side of sacrum and $\operatorname{coccyx}$, and in individual cases may represent either of the two extensors, or the dorsal abductor, or any combination of these. Thus in roo human bodies the medial extensor occurred 53 times, the lateral extensor 43 times and the dorsal abductor 87 times, there being but six cases in which indications of the group are wholly absent. Upon the ventral side of the coccyx are found rudiments of the flexores caudce, described under the names of sacro-coccygei anteriores, s. curvatores coccygis, and of the ventral abductor, here called the coccygeus or abductor coccygis. The former was found in IO2 out of I Io cases, the latter appears to be constant, although often tendinous.

Two further caudal muscles, pubo-coccygeus and ilio-coccygeus, are found in long-tailed mammals, stretching partly across the pelvic floor, and sustaining some connection with the rectum at its termination. These muscles, although rather small, possess much morphological interest, since, in the anthropoid apes and in man, they come to lie transversely across the posterior pelvic opening and form the levator ani, a muscle, which, in connection with the coccygeus, is the principal element concerned in the construction of the so-called diaphragma pelvis. This is a muscular and tendinous floor or partition, closing the posterior outlet of the pelvic cavity, and its formation is unquestionably an adaptation to the erect position of the anthropoids and man, thus strengthening what would otherwise become from this position a point of weakness.

Although the exact homologies of pubo- and ilio-coccygeus (levator ani) are still somewhat obscure, they probably belong to the ventral axial system, and are not connected otherwise than by contiguity with the other muscles of the perinaum, and the genital organs, such as the sphincter ani, ischio-cav- 
crnosus, etc., which, like certain of the pharyngeal muscles, are derivations of the muscular layer of the intestinal wall, and belong primarily to the involuntary system.

Although, as previously stated, the morphological history of the dorsal trunk muscles is almost unknown, there are yet a few points of interest which may prove suggestive. Among the amphibians there is a suggestion of a longitudinal subdivision of this mass into a medial and a lateral portion, a change which in the reptiles becomes complete and definite. In the mammals this condition is still evident, with but a few secondary modifications which tend to obscure the plan somervhat. Thus the spino-spinalis and transverso-spinalis systems, the latter with all of its subdivisions, belong to the medial portion, while the sacro-transverso-transversalis system, on the other hand, with its two main subdivisions of ilio-costalis and longissimus, may be referred to the lateral portion. Of these two systems it may be said in general that the separate slips of the medial portion are inclined towards the median line and become inserted into spinous processes, while those of the lateral portion are inclined outwards (laterally) and become inserted either into transverse processes or into ribs, the two being genetically the same. The most important exception to this is seen in the longissimus, which does not indeed violate the principle just laid down, but which possesses a few origins from spinous processes, and hence from the median line; these, however, have been proven to be of secondary origin, arising through an association of a part of the longissimus with the spinalis, a relation that is not established until during a fairly late embryonic period. The only other doubtful case is that of the splenius, which arises from spinous processes as though belonging to the medial portion, but inserts in part laterally. This muscle, however, is found only in mammals, and may thus be considered to have developed long after the division into the two portions had become established. A similar relationship in the obliquus capitis, which, although belonging to the medial portion, has its origin from a spinous process and its insertion into a transverse process, has been explained above as 
a secondary condition induced by an extension of the transverse process of the atlas.

To complete the discussion of the axial muscles there still remains a group to be considered, and that is, the muscles of the eyeball, for the proper estimation of which one must look to embryology, especially that of selachians and amphibians. During development certain pairs of mesodermic somites appear in the head as well as throughout the trunk and tail, and in embryos of such forms as those named, in which the repetition of the early stages is best given, these epimeres appear for a time as hollow cavities, the so-called "head cavities." With several of these head cavities dorsal (motor) elements from the cranial nerves become associated, and although certain of them become abortive, after continuing up to the stage of possessing a nerve supply, three pairs remain, those associated with the third, fourth and sixth pairs of cranial nerves respectively, all motor nerves. From these develop the muscles of the eyeball, in accordance with their innervation; that is, from the walls of the head cavity associated with the third nerve (motor oculi) develop rectus superior, rectus intermus (medialis), rectus inferior and obliquns inferior; from that associated with the fourth nerve (trochlearis) there develops the obliquus superior alone; and from that supplied by the sixth nerve (abducens) arises the rectus externus (lateralis). The retractor bulbi, a muscle which appears first in the amphibians, develops from rectus cxternus and is consequently innerved by the sixth nerve; it lies enclosed by the recti and often develops into a hollow cone or a system of slips which act almost as independent muscles. It is well developed in most mammals, but is rudimentary or lacking in the primates.

Asicle from the head cavities from which the muscles of the eyeball are derived, a matter which is in many points still controversial, there are several other pairs of head cavities which come to nothing, and although there is at present no generally accepted theory concerning the primary number of cephalic myotomes, or the exact relationship of those that produce the eyeball muscles, it is evident that primarily the 
head as well as the trunk possessed a series of successive myotomes, that certain of those furnished materials from which the eyeball muscles were derived and that all other muscular elements thus disappeared at a very early period. [Cf. Fig. I 26, A, and the text accompanying it.]

The appendicular muscles are the direct derivatives of certain axial metameres, as is especially well shown by the ontogenetic repetition seen in selachian embryos. Here the first indication of a lateral fin is in the form of a mass of tissue, appearing externally as a slight, shelf-like projection, flattened dorso-ventrally. That this may be considered as a remnant of a once continuous fin-fold, which included both lateral and median fins, has been explained above in the chapter on the endoskeleton. Into this mass there grows from the ventral portions of the myotomes a series of what have been aptly termed myotomic buds, becoming, through bifurcation, two for each somite. These continue their development until the fin is supplied with a segmental series of muscle bundles, in number twice that of the somites from which they are derived. The actual number of myotomes thus involved differs in different fishes, but in all there are certain similar principles which may be brought out by the more careful study of the case before us. By comparing the three diagrams in succession (Fig. 57) it will be noticed that of the contribution of the first trunk myotome only the second of the two buds succeeds in developing, and that that one becomes abortive and fails to furnish a permanent contribution to the fin. Beyond this the contributions appear chronologically in regular order from the front backwards, so that in A the two buds of the seventh myotome and the first of the eighth have reached the fin, while the second of the eighth and those of the ninth are still some distance away and less completely developed. The second figure, $\mathrm{B}$, shows the complete disintegration of the contribution of the first myotome and the further development of those from the second to the sixth inclusive, that is, buds II-XIII, while buds XIV-XVII, or those of myotomes 8 and 9 , have barely reached the goal. In $C$ is seen a single bud 

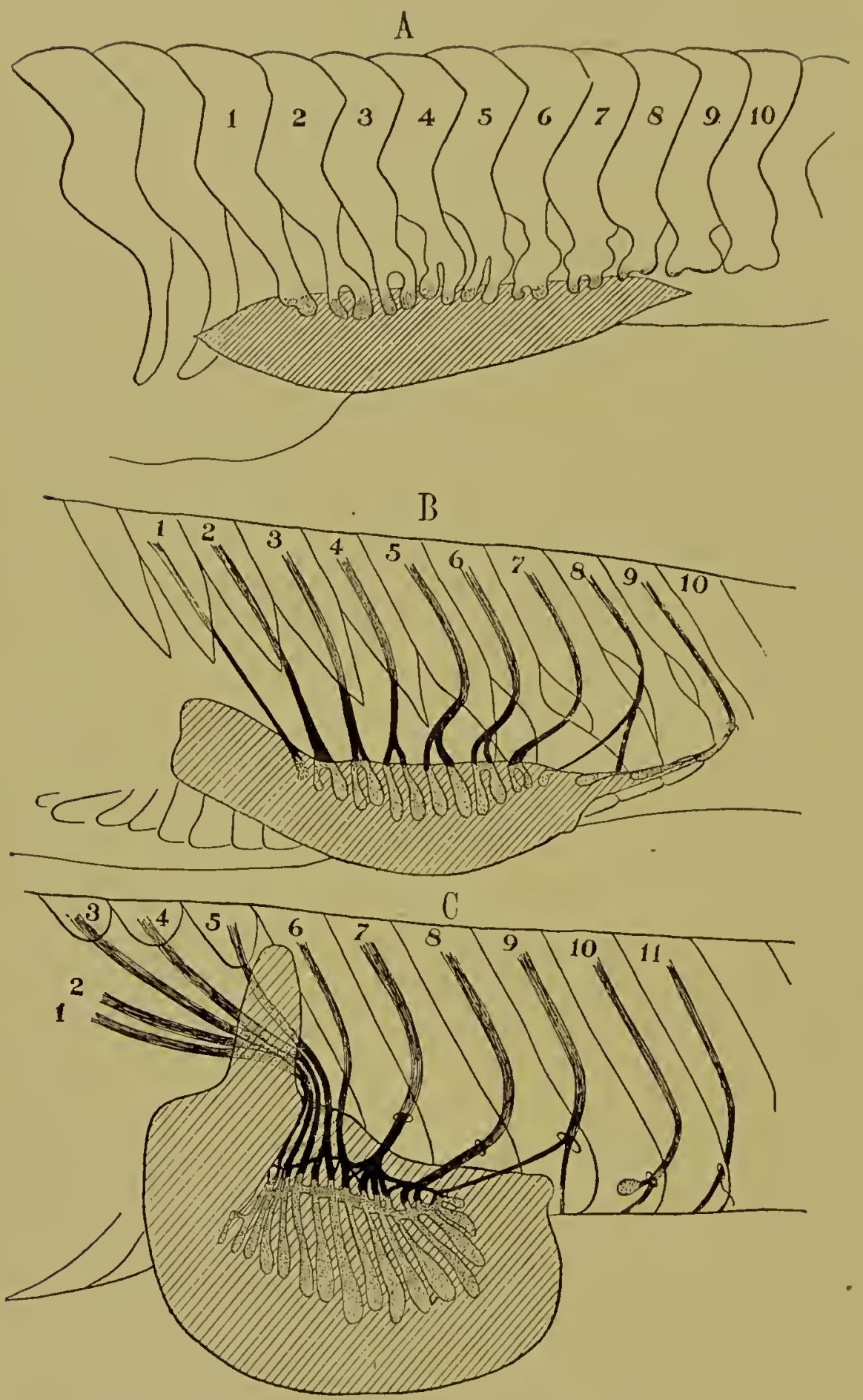

Fig. 57. Development of the anterior (pectoral) fin in the dog-fish [After Braus.]

The shaded portion represents the anlage of the fin skeleton, into which the muscle elements are growing in the form of myotonic buds. 
belonging to the tenth myotome, which seems here far from the fin, but even this eventually reaches the fin, as does also a second one from the same myotome, forming buds XVIII and XIX respectively.

The cause of this apparent struggle to reach the fin on the part of the most posterior myotomic buds, is one which explains also certain other characteristic features of the development, and is found in the unequal rate of growth of fin anlage and of body axis, the latter considerably surpassing the former. There results from this the concentration or bunching together of the nerves of the free limb, especially noticeable in Fig. 57, $\mathrm{C}$, a circumstance favorable to the formation of a nerve plexus, and as this concentration of a number of pairs of nerves to form those supplying the limbs is also seen in the case of all higher vertebrates, it is a convincing proof of the derivation of the limb muscles from a more extensive series of myotomes than that indicated by the adult size of the limb.

From this sketch of the development of a limb as seen in the selachians it becomes apparent that, were it possible in each group of vertebrates to trace the derivation of each limb muscle to a given myotomic bud, or, in other words, were it possible to follow the later history of each separate myotomic bud to the complex conditions of higher forms, a sure and certain homology of the limb muscles could be carried out; as a matter of fact, however, the primitive history in the development of limb muscles is found only in fishes, which, in their adult state, are scarcely beyond the last of the three stages shown here, while in all higher vertebrates, from the urodeles on, these early stages are dropped out completely, and in a developing limb, in which for a time the cells seem exactly alike, and roithout differentiation of any kind, the first indication of any definite arrangement is the collection of these apparently indifferent cells into masses that suggest the parts as they exist in the adult.

In such a case, then, the only recourse lies in the comparison of adult forms, and here, owing to the complexity of the subject and the technical difficulties in the way of such investigation, 
much remains to be accomplished. There has, incleed, been a large amount of anatomical work done on the subject, but little has as yet been attained in the study of the phylogenetic development of the separate muscles or muscle groups, and the morphological history of the limb muscles is as yet far from complete.

The key to the interpretation of the muscles associated with the hand type of limb (chiropterygium) must be found, if at all, among the tailed amphibians, which are the first animals to possess a true chiropterygium, that is, a free appendage furnished with digits instead of fin-rays, and here, in fact, are found many highly suggestive conditions, showing many of the most characteristic muscles of the higher type still in partial connection with the myotomes from which they have arisen. On the other hand, a direct comparison of these muscles with those of man and other mammals is by no means impossible and yields many interesting results, since the distance between these two groups of animals is much less than is commonly supposed, and the intermediate stages do not include either the tailless amphibians, the birds, or even the majority of reptiles, since all these have specialized along lateral lines. Indeed, man himself is far more primitive in the condition of his limbs, with their ancient inheritance of pentadactylism, than are either the salient Anura, with their four anterior digits and their specialized hip-girdle, or such reptiles as the turtles, in which both girdles have become much modified in connection with the formation of carapace and plastron.

Probably the most primitive living vertebrate, above the fishes, is a large aquatic salamander, Necturus, generally distributed throughout the United States, except the Northeastern States, and the extreme South. It may thus be assumed to represent in the muscles of its free limbs the earliest condition of chiropterygial musculature yet remaining to us, and is consequently of the utmost importance in the present inquiry. It will be remembered that in most vertebrates there exists a certain close correspondence in the skeletal parts of anterior and posterior limbs, a so-called serial homology, and in many 
cases this correspondence is at least suggested in certain details of the muscular system. Here, in Necturus, however, the correspondence of the free limbs from elbow or lnee down is practically an exact one, and includes, not only the skeletal parts, but the muscles, arteries and nerves, precisely what would be expected in this primitive form if the serial homolog! is really fundamental and not due to secondary modification through a similarity of use.

Proximal to the elbow and knee, however, there is little if any correspondence or even similarity in the musculature, corresponding in this respect to the great differences in the two girdles, and therefore for descriptive purposes the limb may be divided into unlike proximal portions, to be treated separately,

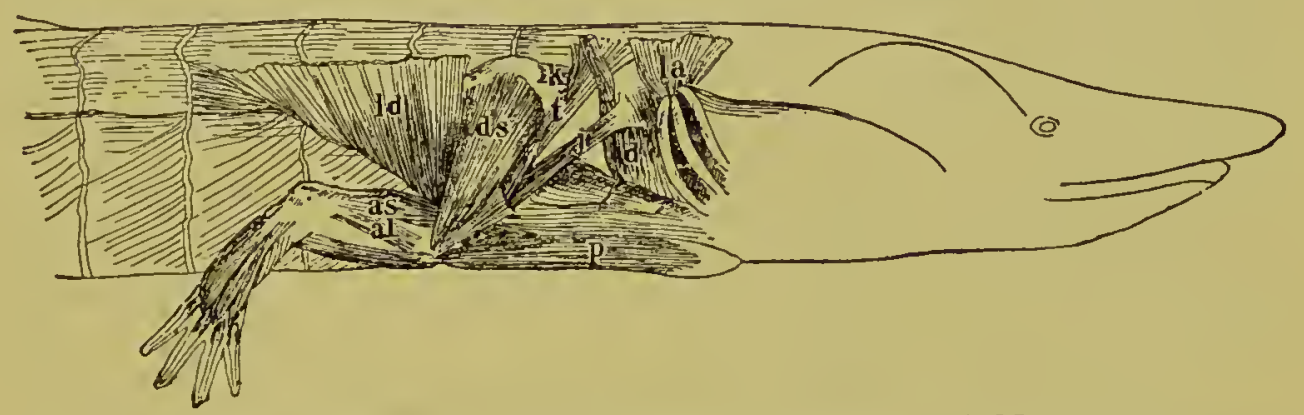

FIG. 58. Lateral view of shoulder muscles of Necturus.

$l d$, latissimus dorsi; $d s$, dorso-scapularis; $t$, trapezius; $j$, omohyoid; $k$, levator anguli scapulx; la, levatores arcuum; $d$, dorso-trachealis; $p h$, procoraco-humeralis; as, anconeus scapularis: $a l$, anconeus lateralis.

and similar distal portions, which are almost identical and may thus be consiclered together. The first will include the girdle and the proximal joint of the free limb, the upper arm or thigh, and the second the remainder, or that from elbow or knee to the end.

The principal muscles of the proximal portion of the anterior limb in Necturus are shown in figures 52 and 58 . Of these certain are extrinsic and extend from trunk or head to the appendicular skeleton; others are intrinsic, both origin and insertion being upon the latter part.

Conspicuous among the first is the latissimus dorsi, certain parts of which are still seen to arise from myocommata in the form of elements in the act of separating themselves from the 
axial musculature, while other fibers, the anterior portion, no longer show their segmental origin. Anterior to the girdle lies the trapezius, now, like the anterior part of the latissimus, showing no trace of myotomic origin, but undoubtedly from that source originally. Ventral to these are seen two slips clearly derived from the long superficial rectus, and still farther ventral, covering the chest region, lies the voluminous pectoralis, still in part composed of slips attached to the myocommata. Beneath these superficial layers are deeper muscles, like the levator scapulce and the serratus magnus, here plainly derived from the axial muscles in the form of separating slips.

Turning to the intrinsic muscles, it is seen that the outer surface of the three portions of the girdle is covered with fanshaped sheets that converge to the head of the humerus, where they insert near together. Of these the dorsalis scapulce covers the scapula, the procoraco-humeralis the procoracoid, and the supracoracoideus the coracoid.

Distal to these come the muscles, the bellies of which occupy the region of the upper arm, and which may thus form a third group. Of these there are three that occupy the flexor aspect, and a complex one with several heads that lies upon the extensor aspect. Of the first the two coraco-brachiales, longus and brevis, arise from the coracoid and insert on the humerus. These lie on the medial side. On the outer or lateral side lies the humero-antebrachialis, which arises along the humerus and inserts by a tendon into the proximal end of the radius. The complex muscle on the extensor side, the anconeus, is constant in the character of arising from several heads and in the insertion of all by a common tendon into the olecranon process of the ulna, although the name of "triceps," applied to this muscle in man, is objectionable, since the number of heads is variable and three is by no means the typical number. Thus here in Necturus there are four, a central superficial one from the scapula, a median and a lateral one from the humerus and a median one from the coracoid. The term anconcus, bearing no suggestion of the number of points of origin, but referring to its location alone ( $\dot{\alpha} \gamma x \dot{w} \nu$, elbow, ulna), is much preferable. 
In mammals, corresponding to the great difference of structure shown among the different Orders, there is a great diversity in the appendicular musculature, but if there be taken for comparison with the above any of the more primitive pentadactylous quadrupeds, such as a marsupial, a rodent or a lower primate, the majority of the muscles in the two can be readily homologized (Figs. 59, 60). Latissimus dorsi and trapezius have greatly increased in extent; the former, having reached the mid-dorsal line, no longer possesses a free dorsal margin anteriorly, and posteriorly shows no trace of the primitive myotomic slips of which it was originally composed. A slip, segmented off from its anterior edge, has become a separate muscle, with the name of teres major. The trapezius extends from the occipital region of the skull, a point which it attains in the higher salamanders, along the mid-dorsal line, to a point considerably posterior to the scapula, where it overlaps the latissimus. It may either be divided into three distinct slips, anterior, middle and posterior, or may be in the form of an unbroken sheet; and in climbing arboreal forms, like the monkeys and apes, and in man, is of enormous extent, the two covering the entire upper half of the back and prolonged posteriorly into a median point like a monk's hood, whence the alternative name of cucullaris, employed by European anatomists. From its anterior margin a bundle of fibers is set off and becomes the sterno-cleido mastoideus, a muscle running obliquely across the side of the neck from the anterior end of the sternum to the skull just behind the ear, and conspicuous in man.

The deeper layer of extrinsic muscles, levator scapulce and serratus anterior [magnus], have increased meanwhile, probably by the addition of intermediate slips that arise from the myotomes between the two, and become in most mammals a continuous layer, the primary metamerism being expressed in its slips of origin, which form "digitations," or separate pointed slips that arise from the successive ribs, or from their equivalent processes in the cervical region. In man these muscles are again separated into two by the failure of certain 


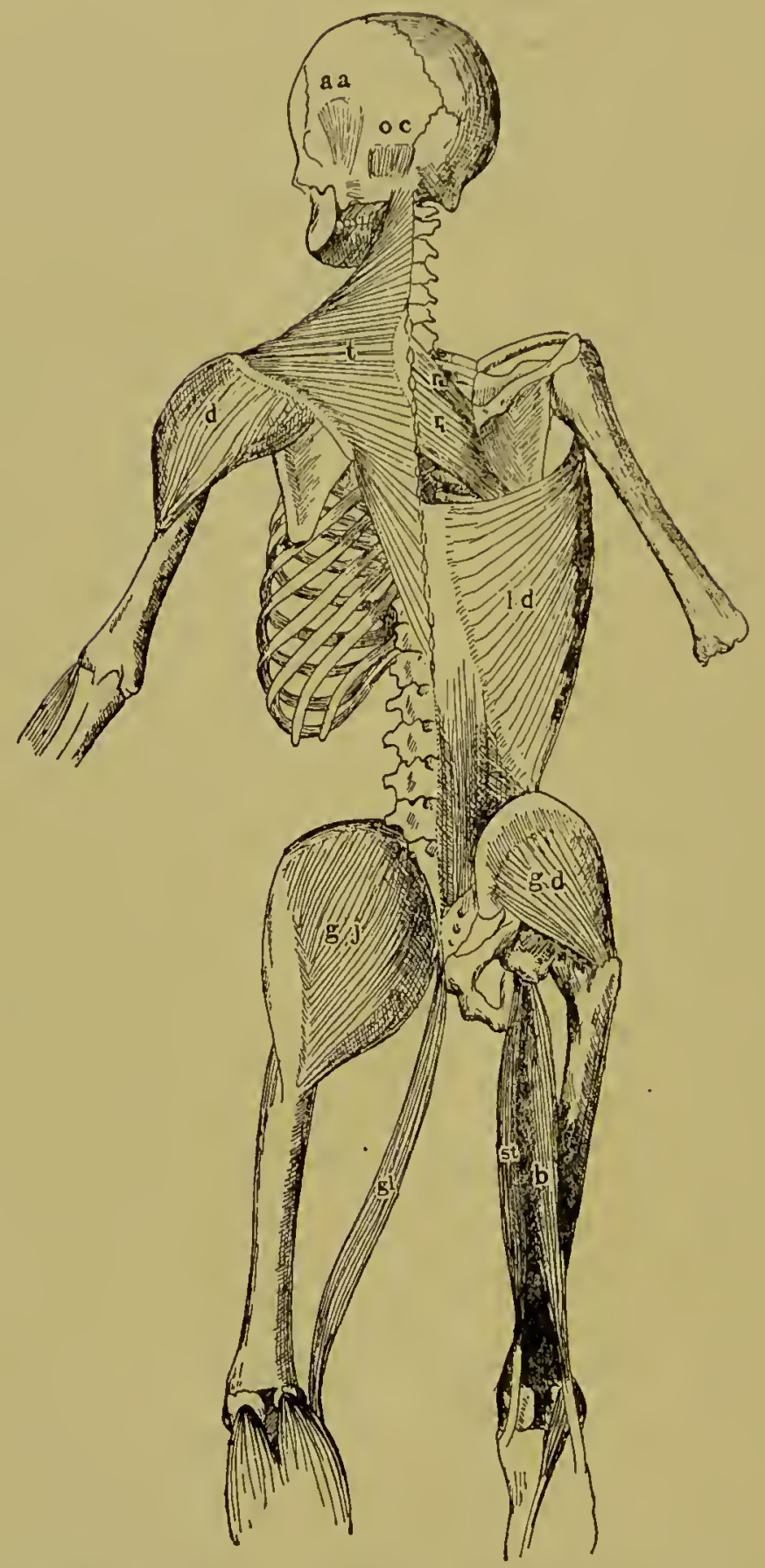

FIG. 59. Diagram of human muscles, showing their relation to the skeleton. [After Eustachius.]

This figure and the next were drawn originally by the gifted Italian anatomist, Bartolomeo Eustachio, who died in I574. His very numerous anatomical drawings, embracing all parts of the body, were neglected for nearly two ccnturies, and wcre finally collected and published, first, by Lancisi in 1714 , and later by Albinus in 1761 . These figures are taken from the latter edition.

$a a$, attollens auris; $o c$, occipitalis; $t$, trapezius; $d$, deltoid; $r r$, rhomboideus, major and minor; $l d$, latissimus dorsi; $g j$, glutrus maximus; $g d$, glutxus medius; $g l$, gracilis; st, scmitendinosus; $b$, biceps fcmoris. 


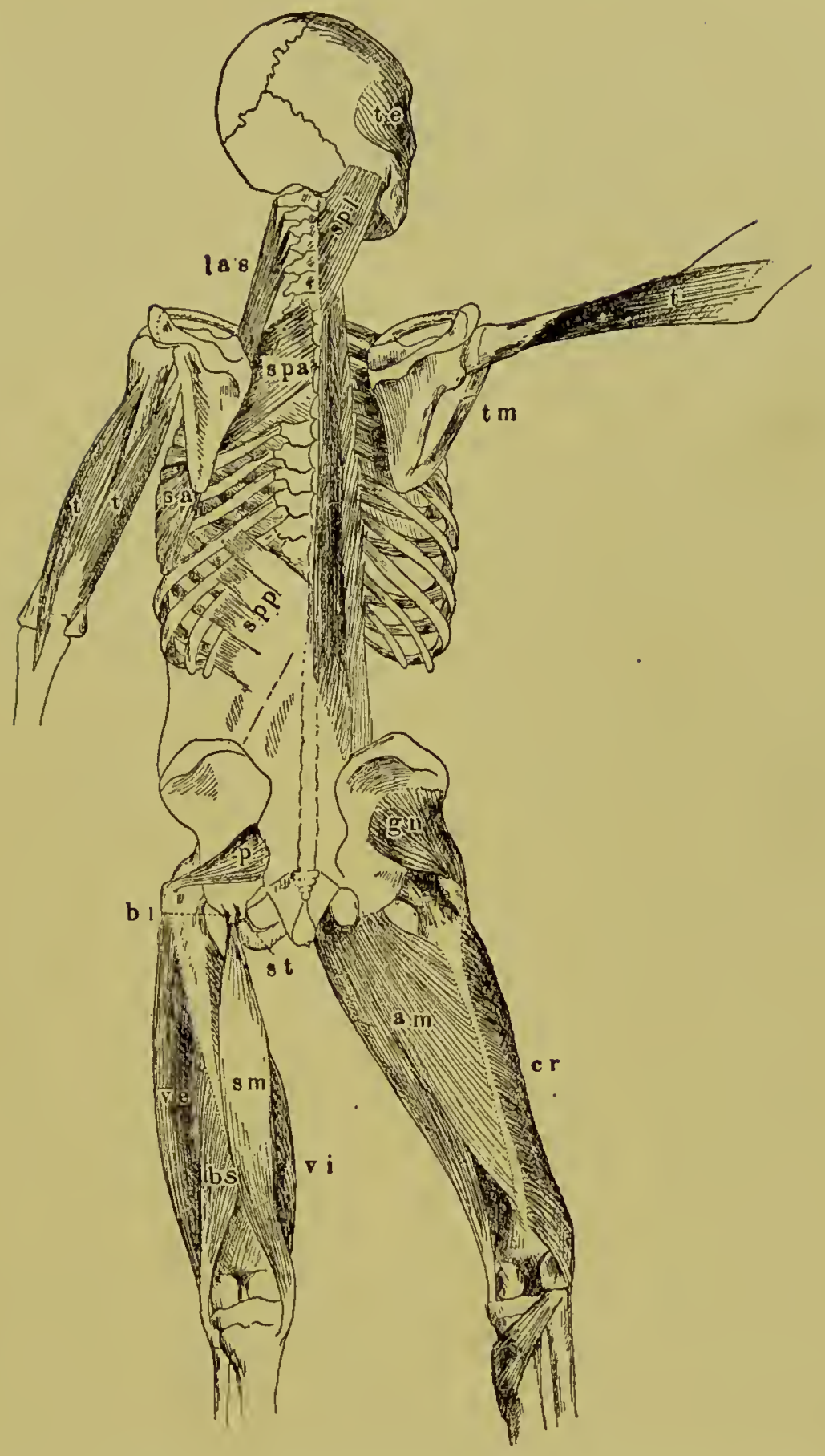

Fic. 60. Diagram of human muscles, showing their relation to the skeleton. [After Eustachius. See explanation of Fig. 59.]

te, temporalis; spl, splenius; las, levator anguli scapulx; spa, serratus posterior superior; spp, scrratus posterior inferior; $s a$, serratus anterior (magnus); $t$, triceps; $\mathrm{im}$, teres major; $g n$, glutaus minimus; $p$, piriformis; st, semitendinosus, origin; $b l$, long head of biceps femoris, origin; $b s$, short head of biceps femoris; ve, vastus lateralis; vi, vastus medialis; sm, semimembranosus; am, adductor magnus; $c r$, vastus intermedius; po, popliteus. 
of the intermediate slips. Aside from these a second series of slips, more superficial than the above, appears in the higher amphibians, and these develop in mammals into the rhomboidens system. This inserts into the scapula and consists primarily of a slip from the occipital bone, rhomboideus capitis, and one from the vertebral spines in the interscapular region, rhomboideus dorsi. Both of these occur in most mammals, but in man rhomboideus dorsi alone is normally present, subdivided into two slips, major and minor, while rhomboideus capitis appears only as a rare anomaly. The pectoralis in many mammals forms a complex system of distinct and semidistinct portions, showing at least a superficial and a deep layer. In man and the arithropoids these two layers are represented by two muscles, pectoralis major and minor respectively. The subclavius is a differentiation from the deeper layer.

Of the intrinsic group the dorsalis scapula becomes mainly the deltoid, often divided into several portions, spino-deltoid, acromio-deltoid, etc., but single in man. A small portion of this muscle becomes the teres minor, topographically associated with the teres major, derived from the latissimus. The supracoracoideus is probably represented by the supra- and infraspinati, which have extenderl dorsally over the scapula, pushing their way beneath the deltoid, as this muscle has gradually lifted itself up from the general outer surface of that bone. The procoraco-humeralis seems to have become lost, together with the axial slips that insert into the procoracoid.

The anconeus, the extensor muscle of the upper arm, varies mainly in the number and position of its heads, and not in its insertion or general position. Its identity with the human triceps has been already commented on. On the flexor side of the upper arm the history is not as plain. In the mammals there are two long muscles that insert into the forearm, the biceps brachii, that arises from the shoulder girdle and inserts by a tendon into the proximal portion of the radius, and the brachialis [anticus] that arises along the shaft of the humerus, and inserts into the proximal end of the ulna. Asicle from these, there is a coraco brachialis, from the coracoid process 
to the shaft of the humerus. These do not homologize readily with the muscles of the same region in urodeles, but the last muscle, which appears in some mammals as two, compares well with those of like name in Necturus. This leaves the humeroantebrachialis to be compared with both biceps and brachialis, and it may wel! be the ancestral form from which both have originated. In origin it is like the latter, and shows no similarity with the biceps, which arises, usually by a single head, from the scapula and the coracoid process; it is, however, precisely like the biceps in its mode of insertion, and must be at least in part homologous with this latter muscle.

The second region to be considered is the proximal portion of the posterior limb and includes the muscles of the pelvic girdle and thigh. As in the skeleton there is in the musculature little suggestion of serial homology between the two pairs of appendages, although in Necturus the two limbs closely correspond in the distal portion. A fundamental difference in the muscles of the two girdles is that in the posterior limb they are nearly all intrinsic, and arise from the appendicular skeleton, while in the anterior limb an extensive system of extrinsic muscles controls in part both the girdle as a whole and the proximal part of the free limb. This difference is undoubtedly correlated with the definite attachment of the posterior girdle to the axial skeleton through the formation of a sacrum, while in the anterior girdle there is either no attachment to the vertebral column, or a freely movable one through clavicle, sternum and ribs.

In Necturus (Fig. 6I), in which the pelvic girdle is in the form of a flat pubo-ischiadic plate and a narrow ilium, the muscles are naturally divided into those of the outer (ventral), and those of the inner (dorsal) side of the plate, and, thirdly, those which arise from the ilium. Of the first there are two; forming as many layers on the outer side of the plate; the pubo-ischio-tibialis, which runs down the inner side of the leg, passes the femur and inserts into the proximal end of the tibia, and the pubo-ischio-femoralis externus, which inserts into the femur. Upon the inner side of the plate there is a single large 
muscle, the pubo-ischio-femoralis internus, the fibers of which part to accommodate the ilium, but reunite again upon the outer sicle of this obstacle. This also inserts into the femur. Aside from these, a narrow band, the pubo-tibialis, arises from the lateral edge of the pubo-ischiadic plate, and inserts in the tibia. From the ilium arise an ilio-extensorius, which inserts into the femur, an ilio-femoralis, also to the femur, and

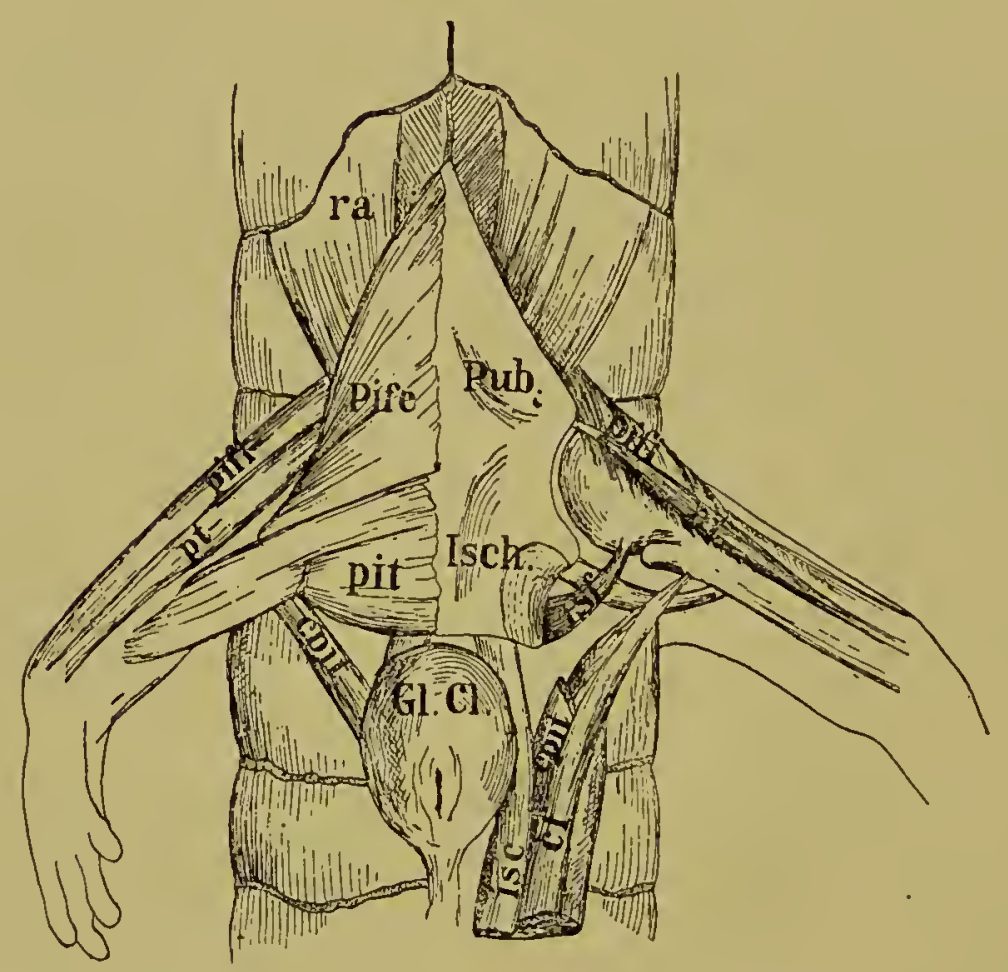

FIG. 6r. Ventral view of the pelvic muscles of Necturus.

pife, pubo-ischio-femoralis externus; pif, pubo-ischio-femoralis internus; pit, puboischio-tibialis; $p t$, pubo-tibialis; ist, ischio-femoralis; isc, ischio-caudalis; cpit, caudalipubo-ischio-tibialis; $c f$, caudali-fermoralis; $r a$, rectus abdominus. Other designations: gl, cl., cloacal gland; Pub., pubic portion of pubo-ischiadic plate; isch., ischiadic portion of the same.

an ilio-fibularis, a narrow band, which inserts into the proximal end of the fibula. A femoro-fibularis, also band-like, arises from the flexor side of the femur, and inserts into the fibula, near the last. The only extrinsic muscles are found in a set of three caudal muscles, which arise along the sides of the tail not far below the pelvis, and run in a sheath anteriorly to the 
posterior limbs, inserting the one into the ischium, another into the femur, and the third, into the margin of the puboischio-tibialis.

Unlike the anterior limb, in which the most of the muscles occurring in Necturus may be readily recogniized in mammals, the homologies of the proximal portion of the pelvic limb are all more or less doubtful. As a beginning, there are found upon the outer side of the pubo-ischium in mammals the obturator extermus, the adductores, the gracilis, which belongs with the adductor group, and possibly the sartorius. Of these the obturator is probably the homologue of the pubo-ischiofemoralis extermus, and the remainder may be derivatives of the pubo-ischio-tibialis, in spite of the difference in respect to insertion. The pubo-ischio-femoralis intermus secms to give rise to the obturator internus with the two associated gemelli, as well as to the ilio-psoas complex, which appears first as a distinct muscle in reptiles. The ilio-extensorius is probably the prototype of the great complex of the front of the thigh, quadriceps femoris, composed of the three vasti, externus, medialis [crureus] and internus, and the rectus femoris. The glutei are probably derived from the ilio-femoralis.

Of the muscles of the posterior aspect of the thigh, enormously developed in mammals, the inner ones, $M m$. semimembranosus and semitendinosus, are probably also derived from the pubo-ischio-tibialis, while the two heads of the outer one, the biceps femoris, come from two distinct sources, and in many mammals are separate muscles. The derivation of the long head is uncertain, but it may be homologous with the iliofibularis of urodeles, in spite of the difference in origin. The short head, on the other hand, is derived from the glutæal group, and is identical with the long narrow band, described in many mammals as the tenuissimus. Since it is associated with the long head to form a "biceps" muscle in a few mammals only, including man and several apes, this slip is best considered as a separate muscle of the glutaal group, under the name of glutco-cruralis. The caudal group of muscles, 
which extends in urodeles from the sides of the tail to the ischium and femur, persists with some modifications in manmals; in man and the higher anthropoids, in which the reduction of the caudal vertebræ restricts the origin, the group is represented by a single muscle, the piriformis, extending from the coccygeal region across to the femur.

The muscles of the distal portion of the vertebrate chiropterygium, that is, from elbow or knee on, aside from the modifications imposed upon them by the varying shapes of the limbs themselves, and the great difference in their use, are, in their essential features, quite similar in all living forms, and in their differences show the modifications of a primary type due to environment rather than the suggestions of an historic development of that type. The study is, therefore, one mainly of the adaptations of a given set of elements, rather than a phylogenetic history, which latter, as is the case also with the bones of the same region, must be sought in the gap separating fin and hand, that is, in the phylogenetic stages represented by lost forms of ganoids, stegocephali, and their allies. The salamander Necturus, probably the nearest approach to this series represented by living fauna, offers in its distal muscles some few suggestions of an earlier phylogenetic stage, and is thus of fundamental importance in the present inquiry. The well-nigh complete correspondence in the fore and hind limb as regards not only bones and muscles, but other parts as well, has been commented on above and offers strong support for the doctrine of serial homology, to be considered later. There are, also, as is the case with higher forms, some traces of a correspondence between the dorsal and ventral surfaces of a single paw, giving a suggestion of the derivation of the chiridial musculature from a fin-like precursor, in which the jointed rays (digits) were supplied by similar muscular elements applied both dorsally and ventrally, as in present-day fishes. The following description is that of the anterior limb, but with the substitution of the terms tibia and fibula for radius and ulna, tarsus for carpus, and so on, it will be found almost equally applicable to the posterior one. In a few cases a muscle 
which is well cleveloped in the anterior limb is small or wanting in the posterior, and thus the former is a little more typical.*

The dorsal aspect of the antebrachium (Fig. 62, a and b) is largely taken up superficially by a single muscular mass, $M$. dorsalis antebrachii (da.) which arises from the distal end of the humerus. This separates, spreads out over the antebrachium, and divides distally into four slips, three for the inter-digital spaces and one for the ulnar side of digit V. Each of these in turn divides into two, which insert by tendons into the bases of the adjoining metacarpals. The muscle is thus an abductor-adductor complex, furnishing the digits with lateral motions, but without any power in extending them. The radial aspect of digit II is alone unsupplied from this system, and this deficiency is made up by the supinator $(s)$, a muscle which underlies the former, arising from the ulnar side of the carpus. It crosses the limb obliquely, and inserts into the internal or free aspect of metacarpal II. Extension of the digits is effected by four short muscles, $M m$. extensores breves $(x, x)$, which arise from the distal row of carpalia and become continued into tendons that lie along the dorsum of the separate digits and insert into the bases of the terminal phalanges. Partly along the sides of the dorsalis, and partly covered by it, thus forming a deeper layer, are two long muscles, associated respectively with radius and ulna, $M m$. extensor radialis and cxtensor ulnaris (er. and cu.). These arise from the humerus with the dorsalis and insert, the one along the shaft of the

* In one point the free limb of Necturus diverges from what is generally believed to be the typical chiropterygium, and that is, it possesses but four digits in each extremity instead of the canonical five which is usually considered primitive. Since the nearest ally of this species, the cave form, Proteus, exhibits a still greater reduction of digits (anterior, 3; posterior; 2), it has been presumed that this is in both cases a secondary reduction. Certain facts, however, lead one to think that the first land vertebrates possessed a smaller number of digits than five, and if this be so, the condition in these two salamanders is primitive, and not a secondary reduction. According to the reduction theory digit $\mathrm{I}$ is assumed to be the one lost, and in accordance with this the four digits present arc designated here, both in text and illustrations, as II-V. 
radius and into certain of the radial carpals, the other along the ulna and into ulnar carpals.

The ventral (palmar) aspect of the limb is more complicated in respect to its muscles. These are covered superficially by a dense palmar fascia or aponeurosis $(f p)$, to which many of the
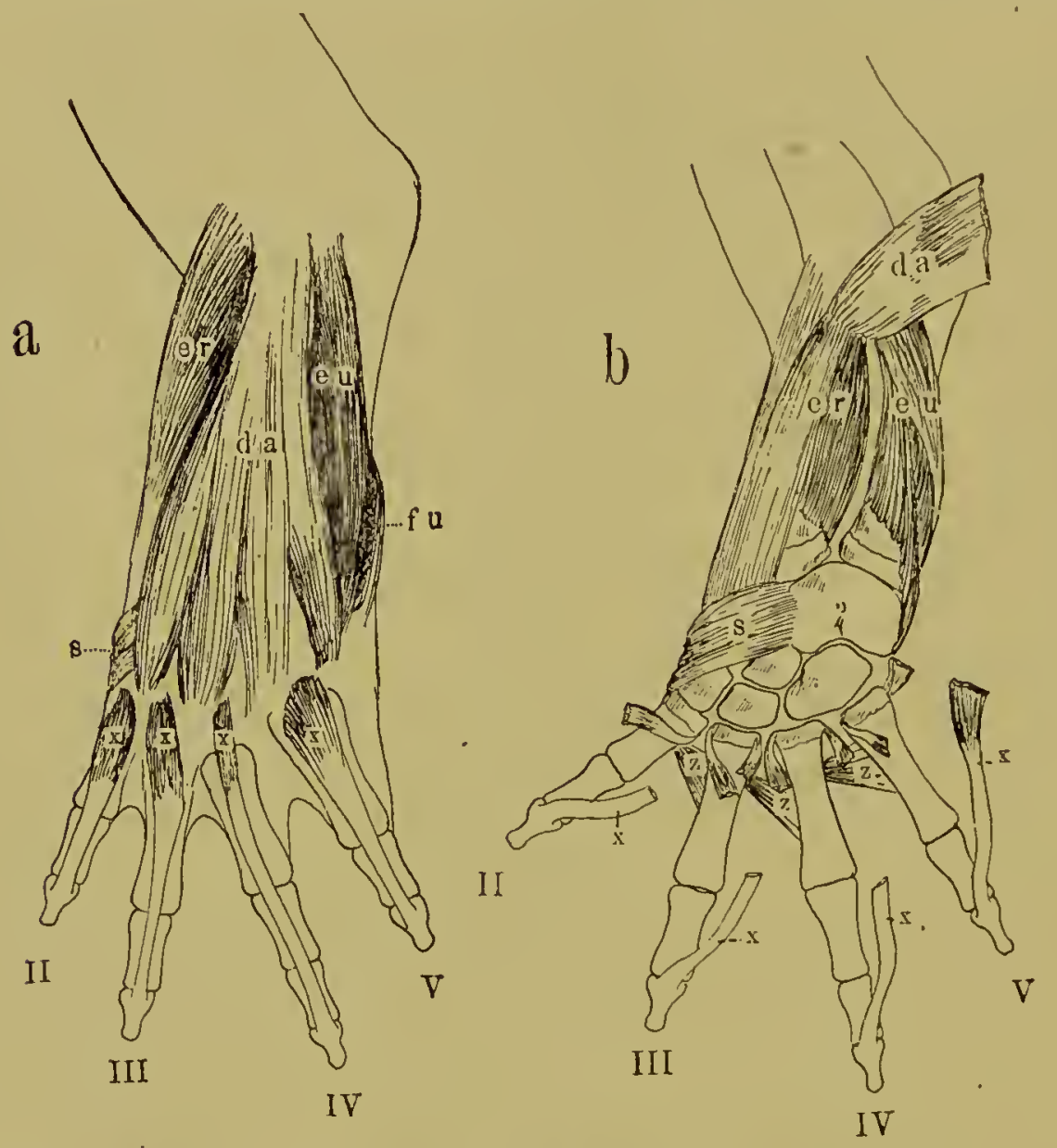

FIG. 62. Muscles of the fore-paw of Necturus; dorsal aspect

(a) Superficial muscles. (b) Deeper muscles.

da, dursalis antebrachii, its separate insertions into the metacarpalia are shown in (b); er, radial extensor; $e u$, ulnar extensor; $f u$, ulnar flexor, showing from the otlier side; $x x$, extensores breves; $s$, supinator; $z$, intermetacarpales.

ventral muscles are attached. This aponeurosis is a continuation of the fascia covering the ventral muscles of the forearm and appears at its thickest and densest as it passes over the carpal and metacarpal regions. At the separation of the digits this aponeurosis is also divided into four bands which run along the ventral surface of the separate digits and insert into the 
terminal phalanges. These slips are functionally and probably morphologically the long flexor tendons ( $t$ t.), and correspond in a way to the long extensor tendons of the dorsal side, but it must be remembered that here they are the continuation, not of muscular bellies, but of a non-contractile aponeurosis. The entire aponeurosis, however, is caused to move by serving as point of insertion of several muscles, more proximally placed,
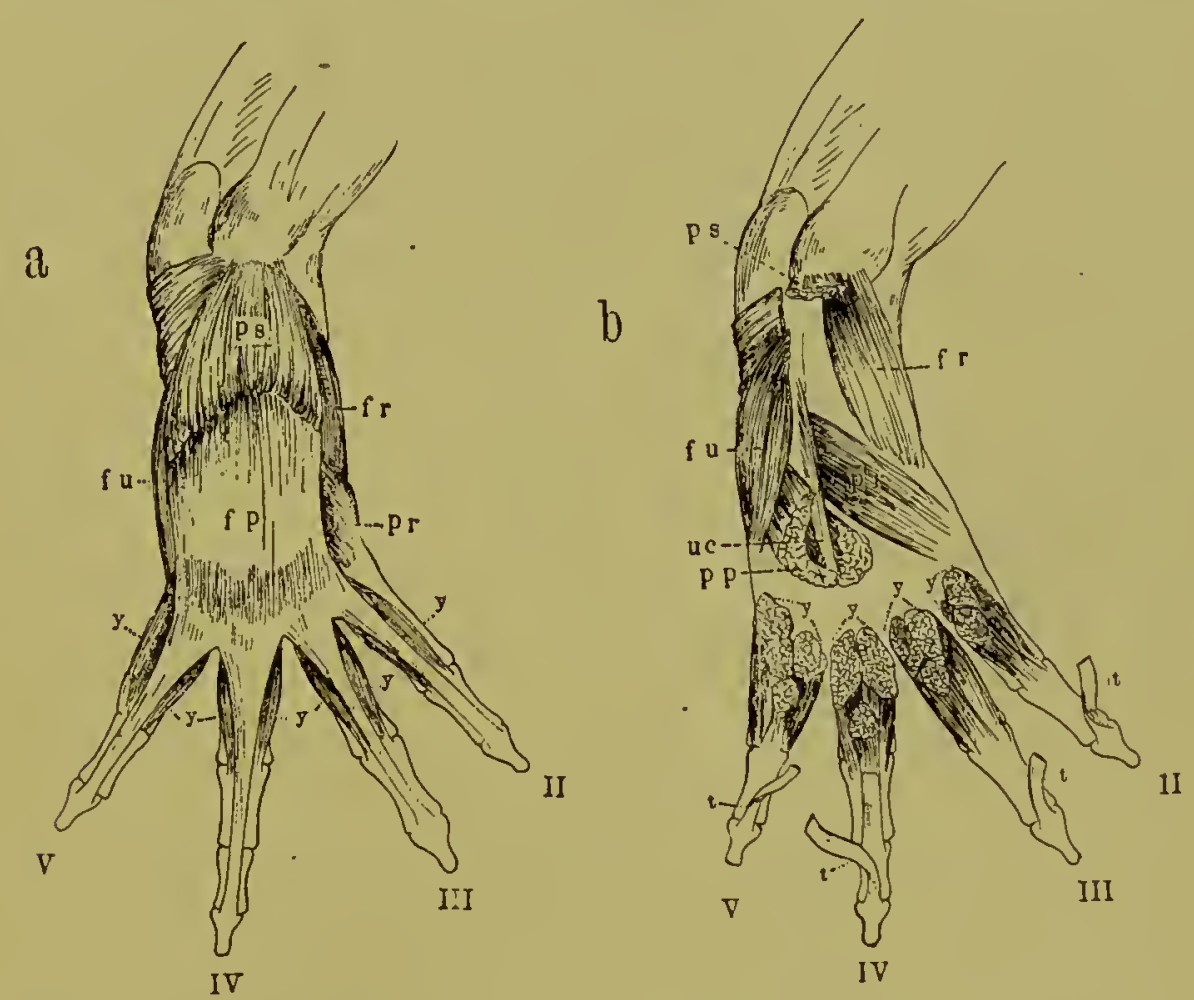

FIG. 63. Muscles of the fore-paw of Necturus; ventral aspect.

(a) Superficial muscles. (b) Deeper muscles.

$f p$, palmar fascia; $p s$, palmaris superficialis; $p p$, palmaris profundus; $u c$, ulno carpalis; $f u$, ulnar flexor; $f r$, radial flexor; $p r$, pronator; $y y$, flexores breves super. ficiales; $t t$, terminal tendons of the palmar fascia.

the palmaris superficialis ( $p s$ ), which inserts along its proximal edge, or, more exactly, between its two layers, which thus invest the muscle, and the palmaris profundus ( $p p)$, which is entirely covered by the aponeurosis and inserts into its dorsal (internal) side. The action of these muscles upon the aponeurosis causes it to act indirectly, through its digital slips, upon the separate digits, and cause a complete flexion. Aside from the palmaris system, the physiological action of which is that 
of a system of flexors, there are two sets of short flexors, $M \mathrm{~m}$. flcrores breves superficiales and flexores breves profundi, each consisting of four muscles, one to each digit. The superficiales arise from the distal row of carpalia, and pass into tendons, which, encountering the long slips of the aponeurosis, divide into two lateral tendons and insert upon the sides of the penultimate phalanges. The flexores profundi lie close to the bone, arise beneath the former, and insert into the bases of the proximal phalanges. There are here also, as on the dorsal side, two long muscles which arise from the humerus and insert along the shafts of radius and ulna and into the corresponding sides of the carpals, serving as flexors of antebrachium and manus as a whole. These are respectively the flexor radialis and flexor ulnaris ( $f r$ and $f u$ ).

The ventral muscles thus far enumerated, act either directly or indirectly as flexors, but beneath all of these is a set of short abductors and adductors of the metacarpals, abductores and adductores breves, which correspond in function to the large muscle mass of the dorsal aspect, $M$. dorsalis antebrachii, with its abductor and adductor tendons. These extend across the interval between the distal carpalia and the metacarpals, and like those of the dorsal mass, supply both sides of the two inner digits, III and IV, and the inner sides of II and V. As in the case of the abductor and adductor system of the dorsal side, $M$. dorsalis antebrachii, the internal (radial) side of digit II remains unsupplied from this system and the deficiency is made good by the pronator $(p r)$, a muscle which lies obliquely across the antebrachium and is related to the skeletal parts precisely as is the supinator of the dorsal side. Like the latter it arises from the shaft of the ulna and passes obliquely downwards to the radial side of the limb, where it inserts by a tendon into the radial side of the base of metacarpal II.

Deepest of all, beneath the short abductors and adductors, and reached equally well from either dorsal or ventral aspect. a set of three intermetacarpales stretch their fibers across the interspaces between the separate metacarpals and act either as 
abductors or adductors of the separate digits (Fig. 62, b, z ).

Reviewing the conditions in this, probably the most primitive chiropterygium now left to us, several interesting points become manifest. The digits are moved in two ways, either flexed and extended or moved sideways, but while the system which provides for this latter form of motion is extremely well perfected, that for flexion and extension is not. For abduction and adduction there are typically five separate muscles for each digit, that is, two ventral, two dorsal and one intermetacarpal, while for flexion and extension, aside from the system supplied by an aponeurosis, and evidently a newly introduced feature, there are but three. This extreme perfection of the siderays movement of the digits in the most primitive chiridium known, together with the weak and makeshift arrangements for bending and straightening the digits, strongly suggest the derivation of the chiridial type from one in which the digits (fin-rays?) required to be constantly opened and shut by lateral movements, precisely as in the case of the fins of most fishes.

During later phylogenetic history there is an evident tendency to increase the efficiency of the flexor-extensor system and diminish that of the abductors and adductors, except in the case of the two digits that form the ends of the series ( $I$ and $V$ ), and the most of these changes have already occurred among the higher urodeles. Thus in Cryptobranchus the dorsalis antebrachii, which in Necturus serves as an abductor-adductor system and terminates at the base of the metacarpals, is continued into four long tendons, which insert into the terminal phalanges, and thus becomes the extensor communis digitorum, although in the hind limb at least, from the sides of the long tendons, small lateral slips extend still to the sides of the metacarpals, the remains of the abductor-adductor system. The short extensors become more complicated than in $N c c$ turus, but insert along the proximal part of the digits and are no longer continued into long tendons to the ter- 
minal phalanges, as that function has been usurped by the other muscle.

Upon the ventral side a more intimate connection between the tendons of the palmar aponeurosis and the associated muscles gives rise to the system of long flexors, as found in the higher vertebrates. In the arm, where this history has been most completely followed, the palmaris muscles, superficial and deep, uniting with the palmar fascia and its long tendons, form the two long flexors characteristic of mammals, flexor digitorum sublimis and profundus. In the monotremes the bellies form a common mass, flexor communis digitorum, although with double tendons to the separate digits, a deep tendon which inserts on the terminal phalanx and a superficial tendon which forks. The two resulting parts insert upon the edges of the penultimate phalanx, and allow the deep tendons to pass through between them. A later differentiation of the belly divides it into a flexor profundus, continued into the deep tendon, and a flexor sublimis, continued into the superficial tendon. The most superficial of the fibers separate into the somewhat inconstant "palmaris longus." The Alexor pollicis longus of man belongs with the profundus.

The tendons of the short superficial flexors of amphibians become mainly employed in the formation of the profundus tendons, while the bellies degenerate, but those associated with digits $I$ and $V$ develop in the mammalian hand and foot into special muscles connected with those digits, such as the abductors of pollex and minimus, the opponentes of the same, and the flexor brevis minimi digiti. The short, deep flexors of the amphibians, flexores breves profundi, become the manmalian lumbricales; and the still deeper set of abductors and adductors, together with the intermetacarpales, become the two sets of interossei, palmares and dorsales, the latter arising upon the ventral aspect and coming through to the dorsal side during development.

The four muscles which in Necturus lie along the ulnar and radial sides of the antebrachium on both dorsal and palmar 
aspect, and furnish a flcxor and an cxtensor for each side, are continued with some modifications in higher animals. The origin from the distal end of the humerus remains the same, but the insertions along the shafts of ulna and radius are given up, and are either confined to the carpal bones of the corresponding sides or a new tendinous insertion is acquired which extends to the base of some metacarpal, the muscles becoming flexor carpi radialis, extensor carpi ulnaris, and so on. The e.rtensor carpi radialis of mammals becomes divided into two similar muscles, longus and brevis, which insert into the bases of metacarpals II and III respectively.

A final group of limb muscles are the pronator and supinator, which give the limb the power of turning about its axis, thus crossing or tending to cross the two bones of the forearm or lower leg. Of these the pronator lies upon the flexor, the supinator upon the extensor aspect of the limb, their fibers extending diagonally across from one bone to the other. In Necturus there is one upon each aspect, the character of which suggests their derivation from the primary system of abductors and adductors.

The striking correspondence in many features between the anterior and posterior limb, especially shown in cases in which the two are used in a similar way, has naturally led to the theory of the serial homology between them, that is, an original homology, not between different animals, as is usually meant by the term, but between different parts of the same animal. The theory presupposes a time at which both sets of limbs were exactly alike, part for part, and thus the final results, however unlike in the two cases, are referable to a single ground plan from which both have been derived. It would be thus possible to homologize bone for bone, muscle for muscle, and to extend the parallelism to the vessels and nerves as well.

A strong proof of this is afforded by the close similarity of the two limbs in the lowest of the amphibians, as stated above, for here, as shown especially in that form which is probably the most primitive of all, the correspondence is very remark- 
able. It must be noted, however, that this serial homology is clear only in the case of the distal portion of the limb, the part beyond the elbow or knee, while in the portion proximal to this point, there is very little suggestion of such a parallelism. From this may be drawn the following conclusions: Granting that both limbs have arisen from a similar origin, and were alike at the start, it is allowable to suppose that the distal portions, being used in a similar manner, have either retained their primitive structure, or have differentiated alike, up to the point exhibited by the present-day urodeles; the proximal portions, facing from the start radically different problems connected with the poise of the body, the varied action of different parts of the trunk, and other differentiating factors, have become modified along different lines, and have attained results that suggest little of the original homology.

The fin-fold theory of the origin of the limbs, given in the previous chapter, throws but little light upon the theory of serial homology, and, it must be confessed, even stands somewhat in the way of such an hypothesis, since, although in its primitive form, the fin-fold may be considered to have been made up of similar elements, repeating themselves metamerically, and appearing probably as skeletal rays supplied with muscles from the trunk musculature in the form of " myotomic buds," yet there is no suggestion that an identical number of these elements was originally taken in the case of the two sets of limbs or that the strictly pentadactylous character of the hand form could have been in any sense primitive, or could have existed at the time at which the two limbs might be stipposed to have been strictly identical.

However, as opposed to all theory in the matter, and it must be remembered that the fin-fold theory itself rests upon very little actual evidence, there is the fact of the actual close correspondence in the fore and hind limbs of urodeles in general, and especially in the case of Necturus, the particular form which from other reasons is considered especially primitive, perhaps even the oldest living representative of all animals possessing the hand form of appendage.

Among manmals the limbs of the primates, even in the 
slightly modified form possessed by bipedal man, are quite primitive, and, together with those of the allied insectivores and rodents, retain the typical five digits, a character in which most of the Carnivora and nearly all of the ungulate groups show a much greater specialization. Thus the distance separating primates from the amphibians is not very great, and it is therefore not surprising that in the distal portion an homology, not merely of the bones, but of the muscles as well, is still quite evident. In the chapter on the endoskeleton the close correspondence was pointed out between the bones of the arm and hand and those of the leg and foot; here the similar correspondence may be considered between the muscles of these parts, the subject being confined in this case, however, to the distal portion, that is, the portion from the elbow or knee on.

Take in the first place the set of four radial and ulnar muscles, which in their final form in the primates become five, namely, the flexor carpi ulnaris, flexor carpi radialis, extensor carpi ulnaris and the two extensores carpi radiales, longus and brevis.

Their homologues in the leg have naturally become modified through the difference in function and the formation of a heel, and their determination is perhaps the least clear of any of the distal limb muscles. There are, however, to correspond to the two flexors, the tibialis posterior and the soleus-gastrochnemius complex, of which the first, a tibial flexor, represents the radial one, and the second, primarily a fibular flexor, the corresponding muscle on the ulnar side. Upon the extensor aspect, the tibalis anterior may be the serial homologue of both radial extensors, while the ulnar extensor is represented by the two peronxi, longus and brevis. In tabular form the above homologies appear as follows:

ARM

LEG

Flexor carpi radialis............... Tibialis posterior

Flexor carpi ulnaris................ Soleus-gastrochnemius

Extensor carpi radialis longus $\}$

Extensor carpi radialis brevis $\}$....... Tibialis anterior

Extensor carpi ulnaris............... Peronaus $\left\{\begin{array}{l}\text { longus } \\ \text { brevis }\end{array}\right.$ 
The set of supinators and pronators may next be considered, and in Necturus, in which in each limb there is a single supinator on the extensor side, and a single pronator on the flexor side, the homologies are evident. In all cases they extend from a more proximal origin upon the outer side (ulnar or fibular) to a more dorsal insertion upon the inner (radial or tibial). In the human arm there are two pronators, teres and quadratus, but as these are continuous in many marsupials and carnivores, they may be considered as derivatives of the single urodele muscle. Their homologue in the leg is undoubtedly the popliteus. Upon the dorsal side most works on human anatomy record two supinators, longus and brevis, but as the longus [=brachioradialis BNA] is really a portion of $M$. brachialis, and belongs with the upper arm, the only true supinator is the one designated brevis [= supinator, $\mathrm{BNA}$ ], undoubtedly the same as that in the urodeles. Its homologue in the leg seems to have disappeared.

The remaining muscles, those controlling the action of the separate digits, are still more in accord, and that too in spite of the great difference in use between the hand and foot, especially in civilized man, suggesting the conservatism of these parts, and the fact that it is easier to keep a complicated structure, when once obtained, even when not used in all its parts, than to replace it with a simple structure without unnecessary parts, provided only that the more complicated structure is in no case detrimental to the effective working of the organ in its simplified function. It is often presumed that the reason why such changes as these have not occurred is that the time has been insufficient to effect it, but this is not the case. The only reason for an adaptation lies, not in the lapse of time, which in itself is powerless to effect even the slightest change, but in the question of expediency for the animal, that is, whether the part comes within the power of natural selection or not. In the present case the foot of man and his immediate ancestors has borne its present shape from pre-glacial times, a period given by conservative estimates at 50,000 to $\mathrm{ro0}, 000$ years, and has as yet undergone but little change along the line of reduc- 
tion. A needful progressive change has indeed taken place, namely, the development of the peroncus tertius, a muscle for lifting the outer edge of the foot and thus counteracting the

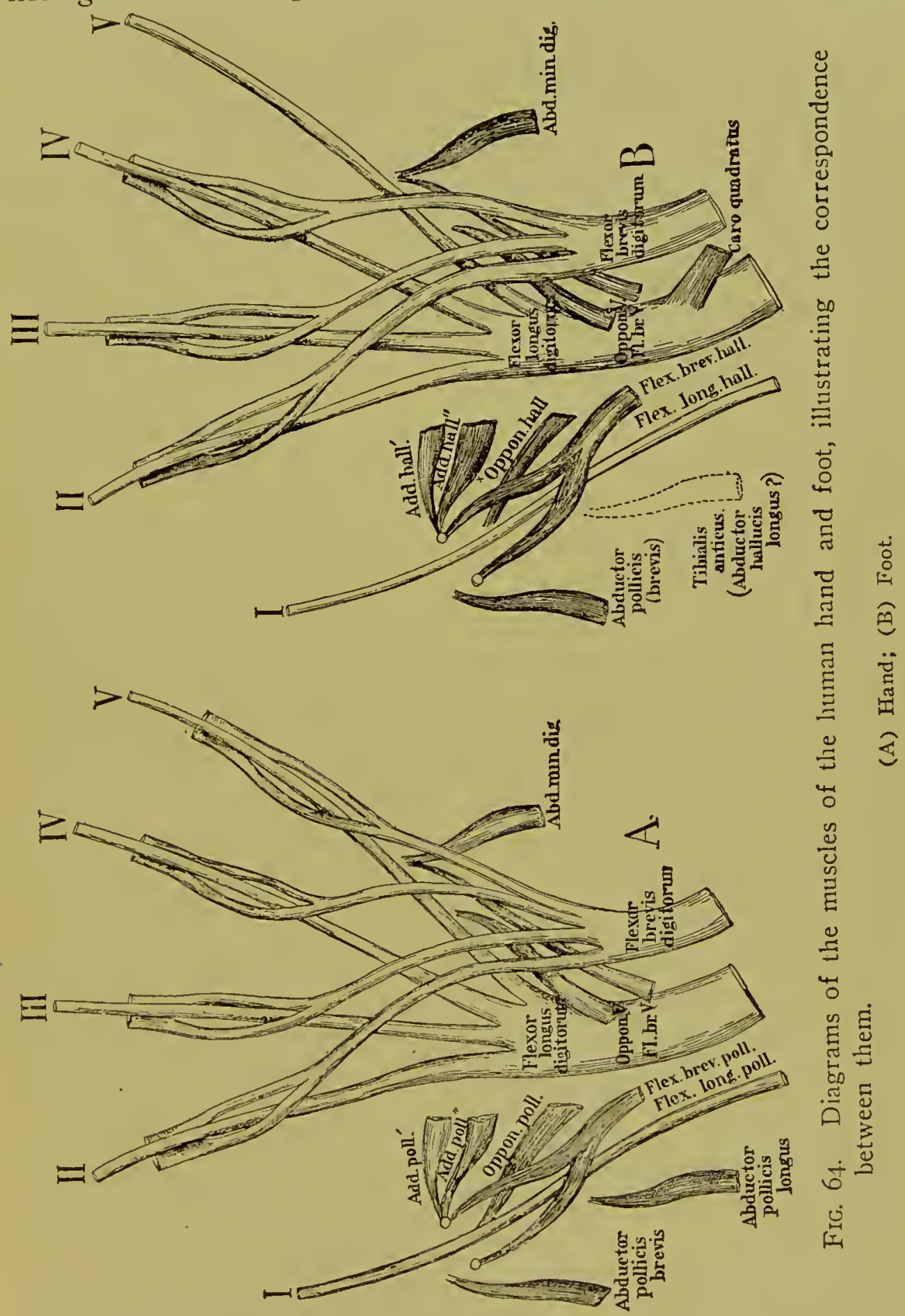

tendency to walk exclusively upon this portion; but the change in the line of reduction of a needless complexity of parts has 
been but slight, simply because there has been no need of such a modification.

To review the complete homology of the muscles of the hand and foot would prove too long a task for the present work, but a large part of the correspondence may be presented in the form of a diagram (Fig. 64), which gives all the muscles of the flexor surface, excepting the lumbricales and the interossei. In both there is seen a double system of flexor tendons, perforantes and perforati; the first digit has the perforans alone, in both hand and foot, and in the foot a perforatus is wanting in digit $\mathrm{V}$, possibly a regressive change. $I_{1}$ the anterior limbs both of these systems are long muscles, their bellies lying along the forearm, while in the foot the belly belonging to the three perforated tendons of digits II to IV is a short one, confined to the foot region. The two outer digits are richly supplied with individual muscles, in which there is a remarkable correspondence between hand and foot, and that too in spite of the loss of independent action in the case of the little toe. This fact of the rich supply of muscles to the marginal digits, I and $\mathrm{V}$, is made much of by supporters of the theory of the pre-pollex and post-minimus, theoretical digits that may have once existed at either end of the present series of five. The muscles in question are interpreted as the musculature of these extra digits, remaining after the loss of the skeletal parts to which they were originally attached. It is noteworthy also that the opponens hallucis is absent in man and has to be supplied in the diagram from the orang and other apes in which it is present, and that a similar loss or, at least, lack of individuality, is observable in the appearance of the little toe, two further regressive characters suggestive of a slight simplification through reduction.

The subject of the homology of the limbs cannot be complete without reference to the various methods of comparison which have been proposed by numerous investigators, and which depart more or less radically from the one given here. Thus the torsion to which the limbs have been plainly subjected appears to many a hindrance to a direct comparison of similar 
parts as given above and leads them to make the comparison in other ways; thus, in the earliest of these theories, more than a century ago, the right arm was compared, not with the right leg, but with the left; the thumb became thus the homologue
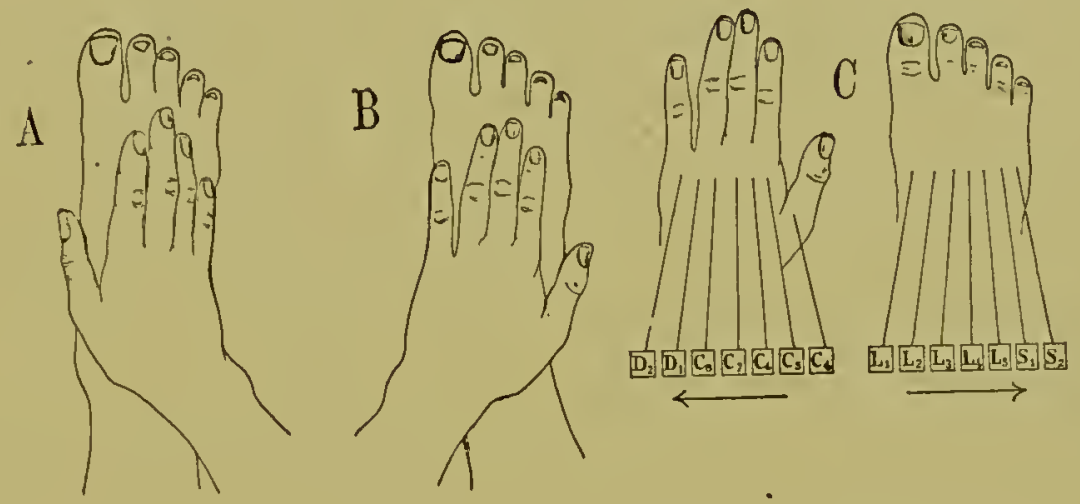

$\mathrm{D}$

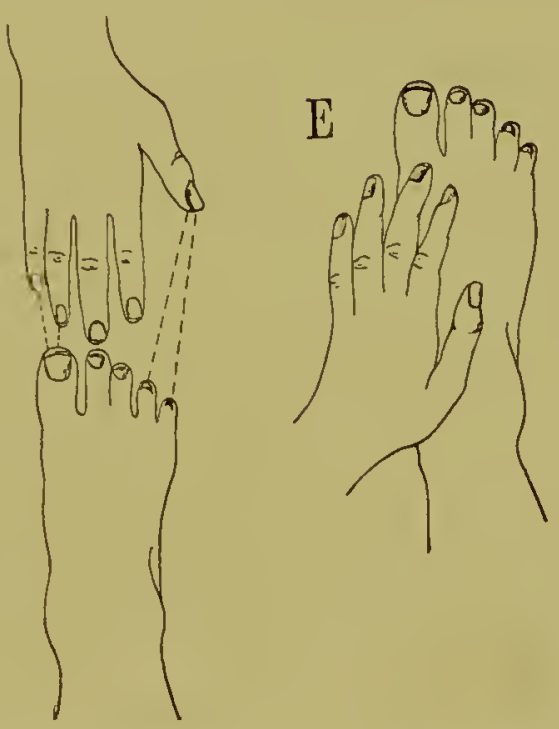

FIG. 65. Diagrams explanatory of various theories of limb homology.

(A) Syntropist theory,-members of the same side homologized. (B) Antitropist theory,-members of opposite sides homologized. (C) Homology between the spinal nerves involved in the antitropist theory. The ccrvical and dorsal nerves going posteriorly, are compared with the lumbo-sacral nerves going anteriorly. Thus, the fourth cervical nerve $\left(\mathrm{C}_{4}\right)$ is the homolog of the second sacral $\left(\mathrm{S}_{2}\right)$, and so on. (D) Theory of Foltz, 1863. The first digit of each limb is bivalent, and the equivalent of digits $4+5$ of the other. (E) Theory of EISLER, 1895 . Here the relation is antitropic, but the homology applies to the three inner digits only in each mcmber, Icaving no homologie for digits 4 and 5 in each case.

of the little toe, radius was compared with fibula, and ulna with tibia (Fig. 65, B). That this theory, fantastic as it may seem, is not merely a vague speculation, but one to the aid 
of which many facts may be invoked, is shown by its persistence in one form or another even to the present day.

In fact, the theorists on the subject of limb homology have been well divided into two schools, syntropists and antitropists, the former making a direct comparison of the limbs of the same side, with the digits in their usual order, the latter changing the order either by reversal, by comparing the limbs upon opposite sides of the body, or by some other unusual means. Of this there is every possible variation; one theory considers in the first place the limbs of the same side to be the symmetrical equivalent of each other, and that thus the ulna is the homologue of the tibia and the raclius that of the fibula, and considers also the three radial fingers to be the equivalent of the three tibial toes, but in the reverse direction, as indicated in the diagram at $\mathrm{E}$. This leaves the two outer digits of each member without correspondence in the other. Another theory compares the digits, also in the reverse order, but considers both the thumb and the great toe bivalent, that is, equal to two digits, and thus compares each with two other digits of the other member $\mathrm{D}$. This comparison of the digits in the reversed direction, however, when carried to its conclusion, leads also to the homologizing also in the reversed direction, of the spinal nerves that supply the limbs. Thus the nerves of the brachial plexus proceeding posteriorly, must be the homologues of the nerves of the lumbo-sacral plexus, proceeding anteriorly, as in C. Perhaps the most recent of the theories in which there is a reversal of any part is one in which the limbs of the same. side are taken for the comparison, and in the normal position as far as the knees, but which assumes that in the distal portion there has been a torsion of both arm and leg, thus causing the original extensor muscles to become flexors and vice versa. This homologizes the flexors of the upper arm with the extensors of the thigh, but allows in the distal portion a direct comparison of the flexors with flexors and extensors with extensors.

As already suggested, the theories just enumerated are not mere vague surmises, but rest in most cases upon careful study 
of the anatomical details; as they are not in accord with one another, however, they cannot all be right, and the remarkable degree of correspondence in bones and muscles, not merely in the salamanders, but also in many mammals, including man, a correspondence that is obvious and easily apparent, is a strong argument in favor of a natural syntropic comparison, as given here. The embryological history, moreover, so far. as it is given, shows no sign of such a torsion or reversal as is demanded by the antitropists, but presents as the first stage of the fore and hind limb, two pairs of lateral flaps, each with a cranial and a caudal border and a dorsal (outer) and a ventral (inner) surface. Of these the cranial border becomes respectively the radial and tibial side of the future limb. the caudal border the ulnar and fibular. The muscles of the original dorsal and ventral surfaces remain in their primary position and may be compared in the two limbs; in the distal portion the dorsal muscles become extensors, the ventral, flexors, in each limb. The embryological history thus furnishes a definite proof in favor of the hypothesis of syntropism, or that of direct comparison, limb with limb, in the normal position, and this theory is espoused at the present time by the majority of investigators.

The visceral musculature differs from the axial-appendicular, thus far considered, in its derivation from the ventral portions of the mesoderm, that is, from the hypomeres instead of from the epimeres. The skeletal parts with which it is associated are those of the visceral arches and their derivatives, including the jaw and the hyoid, and, in the higher forms the numerous cartilages of the larynx and the auditory ossicles. This system has thus its most extensive though perhaps not its most specialized development among the fishes, for here the gill-arches are functional and need to be regulated by systems of levators, depressors, constrictors, dilatators and so on, which often attain a high degree of complexity. In the amphibians, where, in spite of the existence of gill-slits, at least in the larva, there is but little need of controlling the movements of the separate arches so precisely, the visceral musculature appears in a 
greatly simplified form, and the few muscles that persist enter into the service of aerrial respiration and regulate the opening and closing of the pharyngeal cavity and the larynx. Among them appear two well-defined series of muscles, the one dorsal and the other ventral to the visceral arches, that act respectively as levators and depressors of those parts. Their condition in urodeles, together with a diagram representing an hypothetical

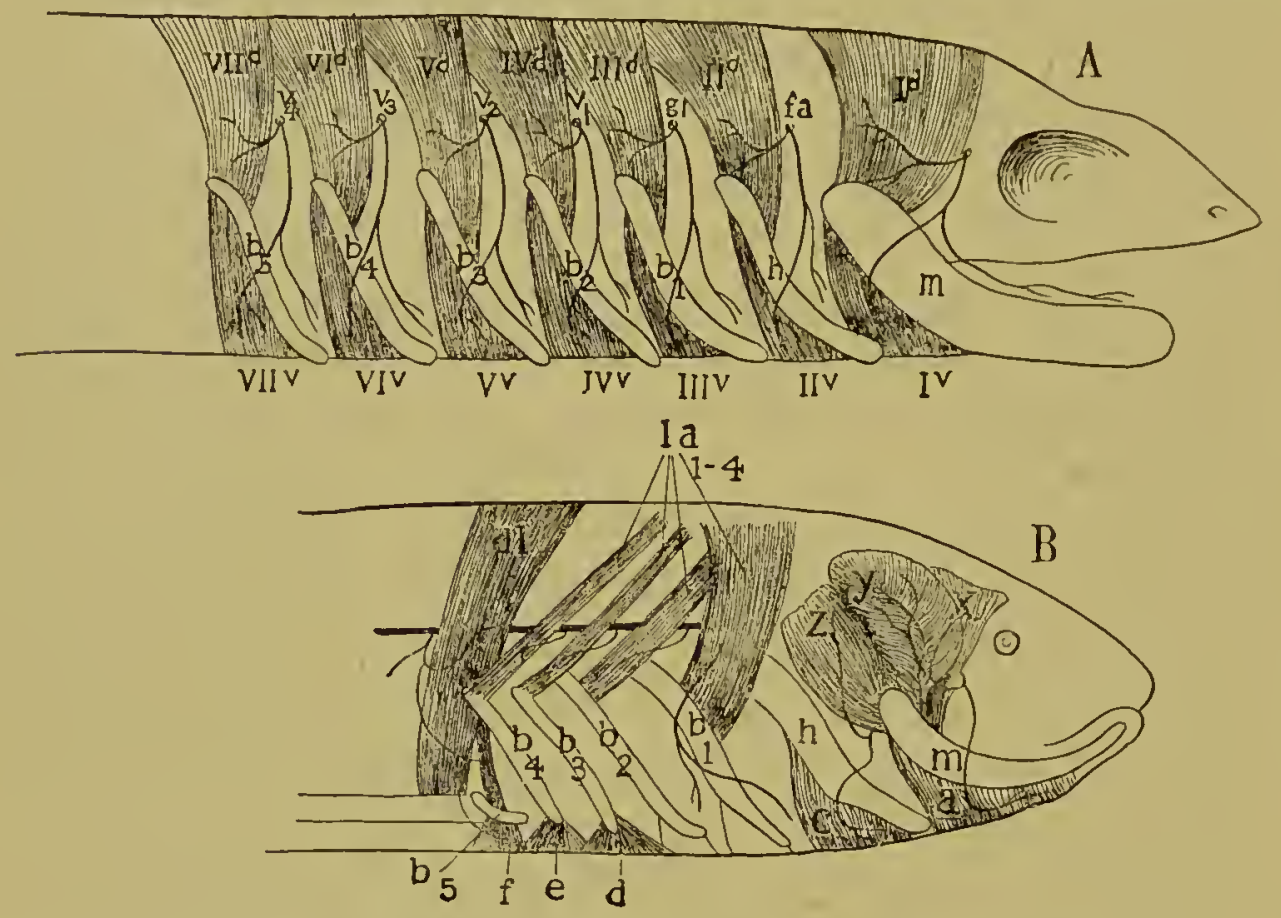

FIG. 66. Diagrams of primitive visceral muscles.

(A) Typical form, hypothetical. (B) Condition based upon that of the urodele Siren, with a few details supplied from Necturus.

$I \cdot V I I$ d, levators of the arches; I.VIIv, depressors of the arches; $m$, mancibular arch; $h$, hyoid arch; $b_{1}$ to $b_{7}$, branchial arches; $t$, trigeminus; $f a$, facialis; $g l$, glossopharyngeus; $v$, to $v_{4}$ vagus (pneumogastric) elements; $x$, temporalis; $y$, masseter; $z$, digastricus; $l a-1-4$, levatores arcuum; $d l$, dorso-laryngis and dorso-trachealis; $a$, intermandibularis anterior; $c$, intermandibularis posterior; $d$, hyo-pharyngeus, anterior portion; $e$, hyo-pharyngeus, posterior portion; $f$, laryngei.

ancestor from which may have been derived, are given in Fig. 66.

In the diagram $A$ the seven visceral arches, including the mandible, are given in order, representing as many somites, with their motor nerve supply. For the first or mandibular somite this latter is the mandibular branch of the trigemimus; for the second or hyoid, the facialis; for the third, the glosso- 
pharyngeus; and for the remaining four, a like number of branches from the vagus, which is a complex of several original elements. The dorsal muscles attached to the arches are lecators, the ventral depressors. In the second figure, $\mathrm{B}$, is shown the actual condition in urodeles, the derivation of which from the first is obvious.

Beginning with the levator series, the first becomes the equivalent of the adductor mandibulce of fishes, here differentiated into temporalis and masseter, the muscles of mastication. The second, having its primary connection with the hyoid arch, becomes also attached to the mandible, but in such a way that it opens it, thus acting as the antagonist to the first. This muscle is usually referred to as the "digastric," a name taken from human anatomy, but it is probably homologous with the posterior belly alone of the mammalian muscle of the same name.

The next four muscles are those associated with the first four gill-arches, and function in the lower urodeles and in the larvæ of the more specialized ones as the levatores arcuum; the next and last belongs plainly in the same series, but as its arch has become specialized as the primary laryngeal cartilage [Chapter $\mathrm{V}]$, it extends ventrally to meet it. On account of this relationship it has received the name of dorso-laryngeus.

The ventral series consists of flat sheets, arising from the mid-ventral line, where they meet in pairs. Of these, the first two, the intermandibulares, anterior and posterior, form the muscular floor of the mouth and are attached respectively to the mandible and the hyoid. Of the next two, those associated with the two first gill-arches, there is no trace, and the next, the fifth, is present only in Necturus and its ally, Proteus, the lowest of the urodeles. The sixth, under the often inappropriate name of hyo-laryngeus, is generally present, and the seventh, stretching between the two lateral laryngeal cartilages, becomes a set of true laryngeal muscles, the dorsal and ventral laryngei (laryngeus dorsalis and laryngens ventralis).

Above this stage the further phylogenetic history of the visceral muscles has been followed only in part, and the conclusions drawn are those which are the most obvious. 
The two masticatory muscles, temporalis and masseter, occur in all higher forms and are homologous throughout, save that two farther slips, the pterygoideus externus and intermus, become differentiated from them, probably from the original masseter. In mammals a small slip from the pterygoideus internus, becomes the tonsor tympani of the middle ear. The second levator becomes associated with a muscular slip from the first depressor, intermandibularis anterior, and forms the digastricus of mammals, the two elements being uniter by a tendon. The diploneuric character of this muscle, that is, the innervation of the anterior belly from the trigeminus and that of the posterior from the facialis, receives thus an explanation. A portion of the posterior belly, that is, of the second levator, becomes separated from it in reptiles, and follows the stapes into the middle ear, whence it becomes the stapedius muscle, innerved by a special branch of the facialis. The ventral muscles of these same first two segments are perpetuated, the first in part as the anterior belly of the digastricus just mentioned and in part as the mylo-hyoidcus; the second as the stylohyoideus. Beyond this, however, the history is not clear. In the previous chapter it was shown that the various gill-arches, beginning posteriorily, become associated with the original pair of laryngeal cartilages to form the complicated larynx of higher forms, but whether the muscular elenents primarily associated with them assist in the formation of the musculature of the final organ, or whether this musculature is derived entirely from the muscles primarily belonging to the seventh arch, that is, dorso-laryngeus and the laryngei, cannot yet be definitely stated.

The musculature of the tongue, especially its extrinsic muscles, such as hyo-glossus, genio-glossus, stylo-glossus, etc., is probably derived from the visceral muscles, but here another element is introduced, and that is the muscular layer of the anterior end of the alimentary canal, which, although of mesenchymatous origin, and primarily composed of unstriated cells, involuntary in their action, are yet capable of acquiring strix and of becoming at least semi-voluntary. From this 
layer are derived the plaryngeal constrictors, and it is probable that the intrinsic muscular fibers which make up the nass of the tongue and known as the lingualis, come from the same source.

Superficial to the muscular systems already described, and lying directly beneath the integument there are found in many vertebrates muscular elements, usually in the form of sheets or layers, and connected with the integument, which thus acquires locally some power of movement. These muscles form what may be conveniently termed the integumental system, although there are included here contributions from several wholly unrelated systems, independently developed in the different groups of animals to subserve special functions and therefore restricted in their occurrence. These integumental muscular elements have arisen from whatever prëeristing muscles happen to be adjacent to the location where such a part is needed, and thus they may be in their origin either axial, visceral or appendicular, or may represent a combination of these systems. They usually possess at one end a firm attachment to some skeletal part or at least to skeletal muscles, while at the other end, or perhaps along an extended surface, they adhere to the inner side of the integument, thus furnishing the skin area involved with the degree of motion required.

In both birds and mammals certain shoulder muscles furnish an important contribution to the integumental system, but, as would be expected, the two cases are totally independent of one another. In birds the integumental area involved is. the patagium or web, extending across the angles of the axilla and elbow and increasing the resisting surface of the. wing. This is regulated by a series of patagial muscles, strictly integumental in their relations, but derived from the varions muscles of shoulder and arm; of these the most important are $M$. propatagialis, derived from the anterior portion of the pectoralis, and an associated slip from the biceps.

In mammals an extensive layer, derived from latissimus and pectoralis, spreads over the side of the body, and in some cases the two extend to the mid-dorsal and mid-ventral lines, 
encasing the trunk in a sub-cutaneous muscular sheet. This is the panniculus carnosus, and is primarily employed in moving and wrinkling the skin as a defense against insects. In the monotremes the portion derived from the pectoralis extends over the entire ventral aspect of the body, and where it meets the marsupial pouch and the cloacal orifice forms

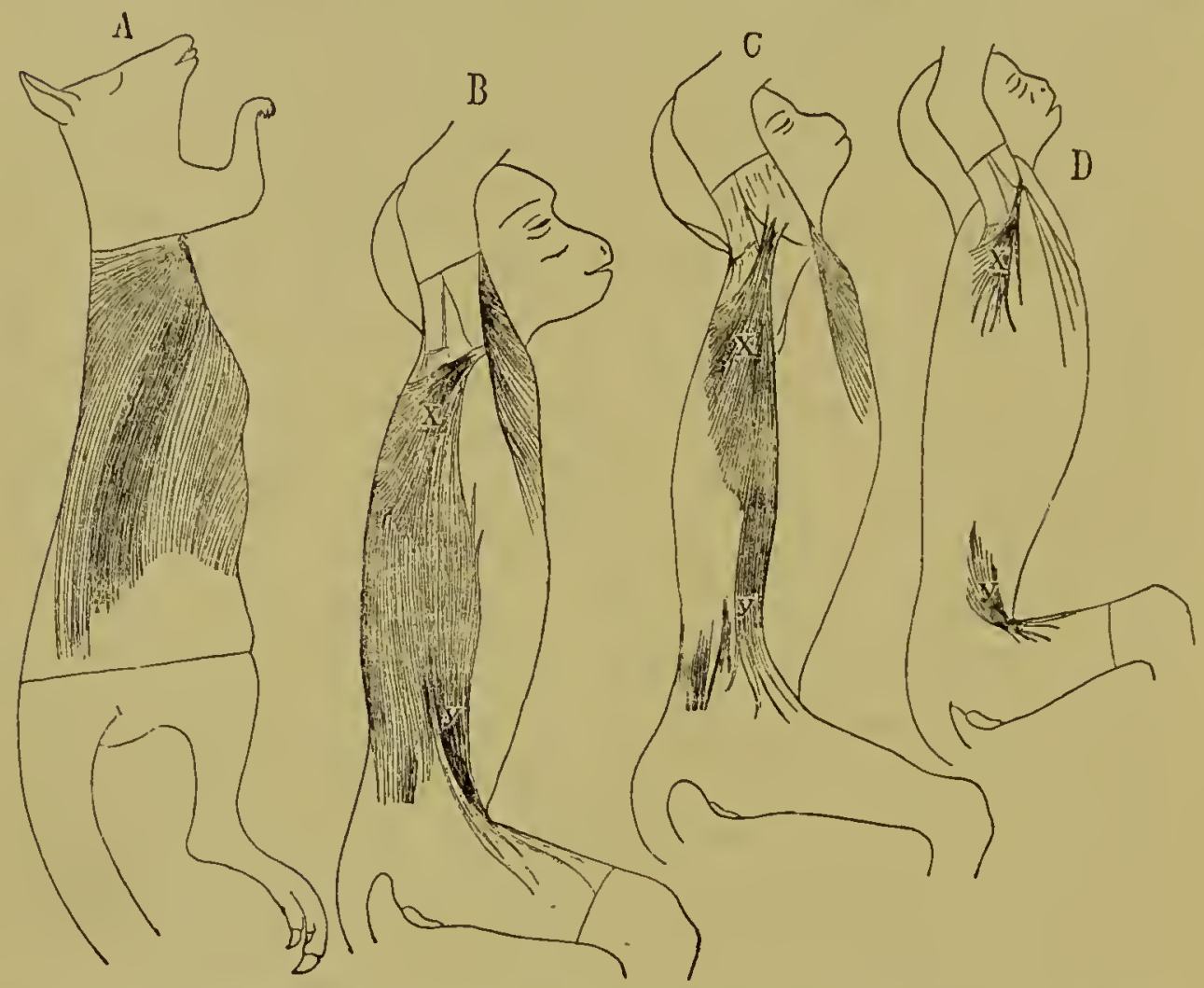

FIG. 67. Phylogenesis of the panniculus carnosus. [After ToBLER.] (A) Macropus bennett (kangaroo). (B) Cynocephalus hacmadryas. (C) Cerco.
pithecus sabaeus. (D) Cercopithecus cephus.

from its fibers certain more specialized slips to serve as sphincters (sphincter marsupii and sphincter cloace).

A panniculus carnosus, perhaps here mainly a contribution from the latissimus, is also present in marsupials (Fig. 67, A) and covers the flanks with fibers that converge to an insertion into the humerus. From these it is directly continued to the. Insectivora and Carnivora, and to other Orders 'of mammals. Its action is seen in the shaking of the skin of a wet $\log$ or the twitching along the outer portion of the legs of horses and cattle when these surfaces are stimulated by the bite of an insect. In the lower primates, the panniculus appears as 
a broad sheet upon each side, much as in marsupials (Fig. $67, \mathrm{~B})$, but within this Order it is seen to separate into axillary and inguinal portions (Fig. 67, C and D) and in the anthropoids, the former alone remains, much reduced in size (Fig. 68). In man there appear to be normally no traces of

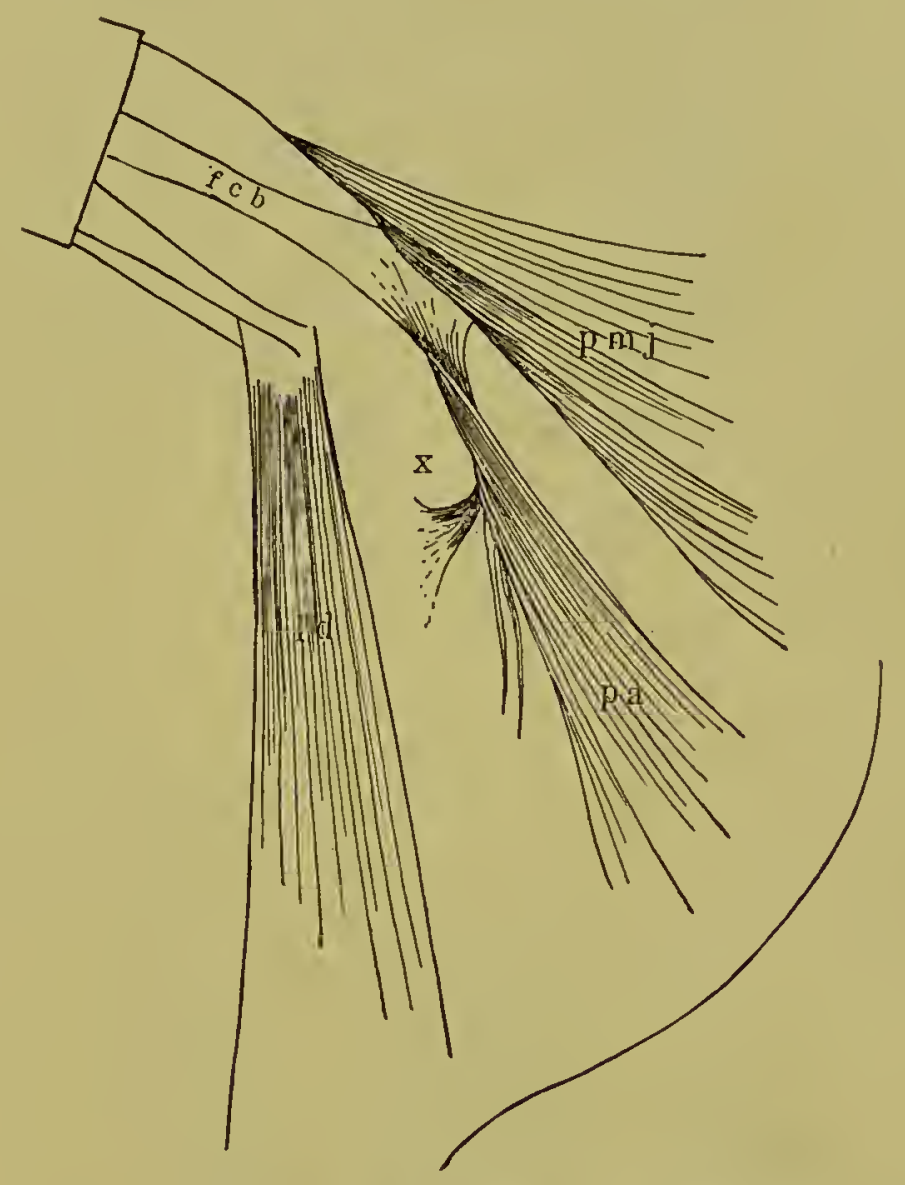

Fig. 68. Anterior remnant of the panniculus carnosus, "achselbogen," in the gorilla. [After TOBLER.]

$p m j$, pectoralis major; $f c b$, coraco-brachialis fascia; $l d$, latissimus dorsi; $p a$, "pectoralis quartus," a part of the panniculus; $x$, tendinuous fibers from the latter.

this muscle, but there occurs occasionally a system of slips in the axillary region, the axillary arch ("Achselbogen") associated with both latissimus and pectoralis and very variable in appearance, a typical characteristic of a rudiment. In association with this, there occasionally develops a more posterior pectoralis slip, the pectoralis abdominis, a relic from a remote part. Still another rudiment of the pamiculus system is seen 

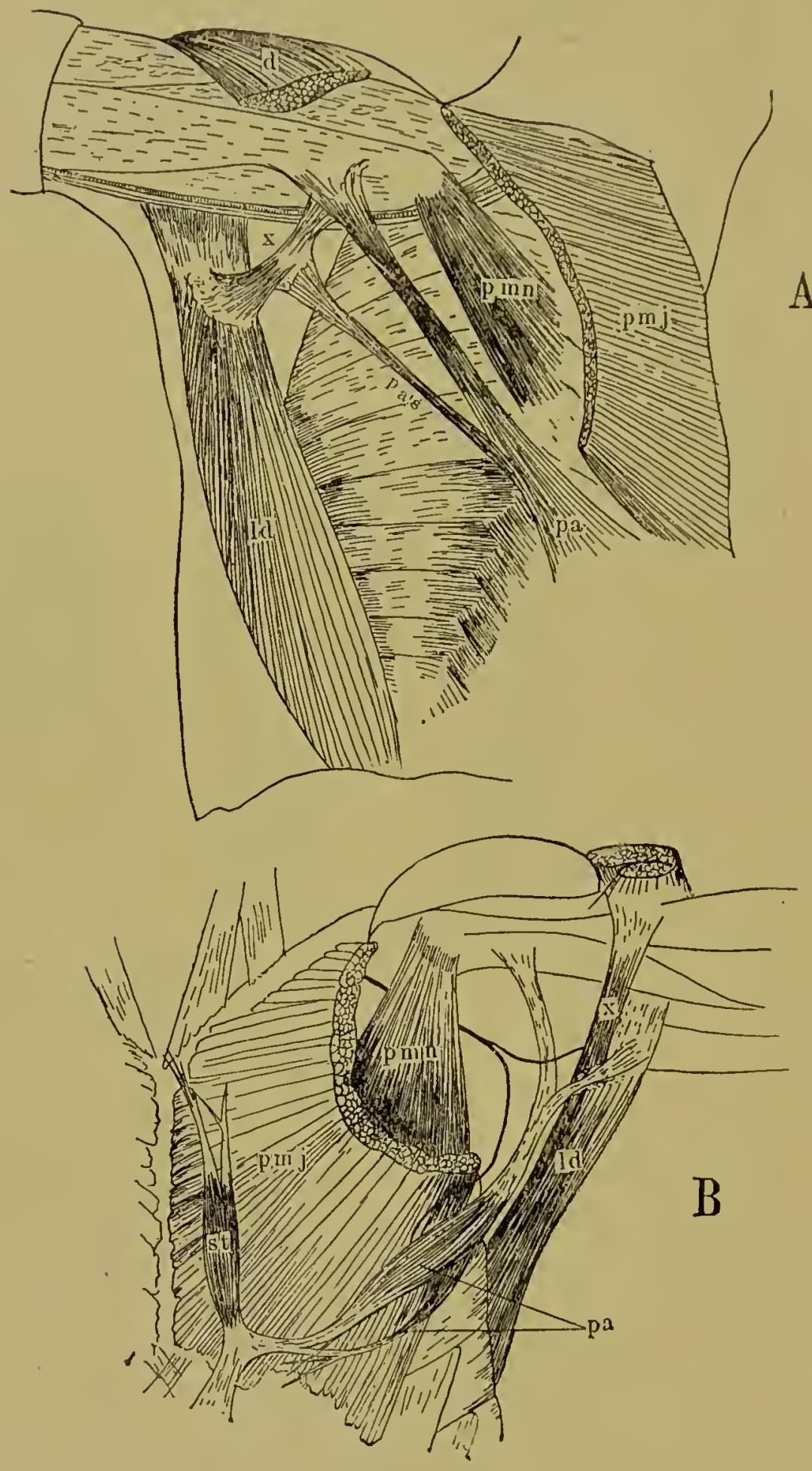

FIG. 69. Two cases of axillary panniculis rudiments in man.

(A) [After Torler]. (B) [After Gerrry]. pmj, pectoralis major; pmn, pectoralis minor; d, deltoid; Id, latissimus: pa, "pectoralis quartus," a part of the panniculus; $x$, the definite "achselbogen"; st, tho sternalis, a rare muscular anomaly, also a part of the panniculus. 
in the stcmalis muscle, an clement of very rare occurrence and proven to belong here by its occasional relationship with both pectoralis abdominis and the elements of the axillary arch, as well as by its innervation from the anterior thoracic nerve, in common with the foregoing (Fig. 69). The sternalis muscle has been recently shown to occur much more frequently in the Japanese than in Europeans ( 3 per cent. against 4 per cent.). It lies superficial to the pectoralis major, and when well developed may be so contracted as to be plainly visible from the exterior.

Another system of integumental muscles is derived from the visceral musculature and appears in its simplest form in the sphincter colli of amphibians, reptiles and birds, and is itself a direct descendant of a selachian muscle, the superficial constrictor. The fibers of this sheet enwrap the neck region and in turtles and birds the muscle is well developed and covers the entire neck. In mammals this sheet differentiates into two layers, a more extensive superficial layer, the platysma, and a smaller and deeper layer, which retains the original name of sphincter colli. The fibers of these two sheets run primarily at right angles to one another, those of the platysma being directed upwards and towards both snout and ear, those of the sphincter in more nearly the original direction across and around the neck.

In following the phylogenetic series through marsupials and lemurs to primates, a considerable extension of both of these layers over the face and head is noticed, and as they meet the eyes, nose, ears, and lips there is seen a pronounced tendency to form special slips for the regulation of these parts, a tendency precisely similar to that of the ventral panniculus in the case of the marsupial pouch and the cloaca of the monotremes. There is thus formed the extensive system of facial muscles, often termed the "minetic" muscles, which become so highly differentiated in the apes and in man, and this gradual differentiation can be clearly followed in the phylogenetic series (Fig. 70).

The superficial sheet or platysma extends upwards across 

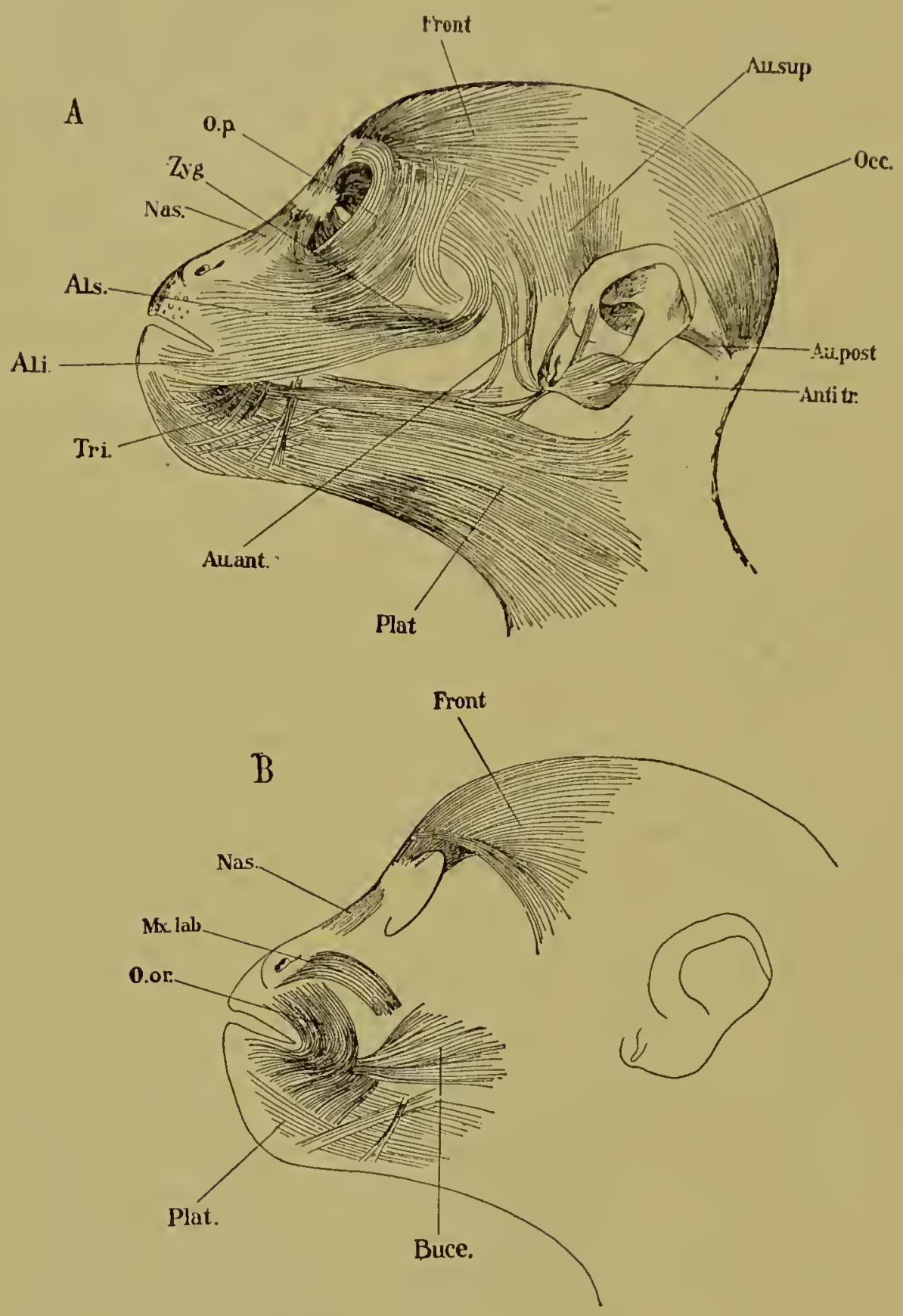

Fig. 7o. Mimetic muscles in Ateles (a South American monkey). [After Ruge.]

(A) Superfieial layer. (B) Dcep layer.

Front, frontalis; $A u_{.}$sup, auricularis superior; $O . p$, orbicularis palpebrarum; $Z y g$, zygomaticus; Nas, nasalis; $A, l, s$, auriculo-labialis superior; $A$. l. i., auriculo-labialis inferior; $A u$. ant, auricularis anterior; $A u$. post, auricularis nosterior; Antirr, antitragicus; Plat, platysma myoides; Occ, occipitalis; Tri, triangularis; Mr.x. lab, maxillolabialis; $O$. or, orbicularis oris; Bucc, buccinator. 
the side of the neck, and, reaching the ear, divides into two sheets, dorsal and ventral. The dorsal sheet, auriculo-occipitalis, subdivides once more and furnishes the auricularis posterior [retrahens auris] and the occipitalis (i.e., the occipital portion of the "occipito-frontalis" of human anatomy), and from the latter are derived the intrinsic muscles of the dorsal surface of the ear-flap, rudimentary in man. The ventral or facial portion gives off along the sides of the mandible the two slips, levator menti and quadratus labii inferioris [depressor labii inferioris], which latter becomes attached to the bone, and is continued over the face as $M$. sub-cutaneus faciei. The ultimate differentiations of this latter portion are quite complex and concern three portions into which the sheet divides itself. Of these an auriculo-labialis inferior furnishes the intrinsic muscles upon the ventral or forward surface of the ear-flap and an auriculo-labialis superior differentiates into the zygomaticus [major], the orbicularis oculi [palpebrarum] and the levators of the lip and side of the nose. Finally a third element, the orbito-auricularis, furnishes two of the extrinsic ear muscles, auricularis anterior and superior [attrahens and attolleus auris], and the frontalis, which in the apes comes nearly in contact with the occipitalis previously mentioned, the two becoming connected by a fascia. The gradual lifting of the cranial dome and the formation of a forehead, culminating in man, spreads apart the two muscular elements of this occipitalis-frontalis sheet and extends the intervening fascia to become the galea aponeurotica, so extensive in Man. That portion of the platysma which covers the sides of the neck in Man remains in its original undifferentiated condition, and, although quite variable in its occurrence and in the control over it, is yet often capable of throwing the skin into longitudinal folds, its original function.

The deeper layer, the sphincter colli proper, extends also to the face, but is mainly confined to the region about the mouth, where it gives rise to orbicularis oris, canimus [levator anguli oris], buccinator and the intrinsic muscles of the nose.

The original sphincter colli, as found in reptiles and monotremes, lies within the province of the seventh cranial nerve 
and is wholly supplied from this source. Exhibiting a superb example of the constancy of a muscular innervation, the branches of this nerve expand and differentiate into the muscle which it supplies, and with the migration of the latter to the face there comes also the nerve; and it thus happens that this element, originally the motor nerve of the hyoid region, comes to be called the "facialis," corresponding to the region in which it is met with in Man, whose anatomy first attracted especial attention.

That this system of facial muscles was primarily developed for the purpose of regulating the orifices of the mouth and the organs of special sense there can be no question, but the high degree of specialization attained in the higher primates suggests a totally distinct function, that of communication of the moods of the animal to its associates, that is, a language. That some outward expression for the developing power of thought should show itself pari passu with the development of brain was to be expected, and it appears that at about the point at which this muscular differentiation became apparent, that is, among the lemurs, the various cries produced by the larynx became insufficiont and were supplemented by the development of mimetic muscles, through the medium of which far more subtle shades of meaning could be expressed. For a time, therefore, in the anthropoid precursors of man, both forms of intercommunication must have existed side by side, and have been of about equal value or with some advantage in favor of the mimetic muscles, as in the apes of the present day; but when, by the shortening of the snout and the consequent flattening of the dental arcade a greater differentiation of articulate sounds became possible, these latter became more and more employed as the better medium of intercommunication, and the help of the facial muscles became less and less necessary. Corresponding to this change, many of the mimetic muscles, such as those of the ears, the nose, and the scalp, show in man less power than in the apes, while those of the cheeks and lips, employed as auxiliary to the production of articulate sounds, have reached a still higher degree of development. 


\section{CHAPTER VII}

\section{THE DIGESTIVE AND RESPIRATORY SYSTEMS}

"Wie in jeder Wissenschaft aus den Thatsachen Schlïss sich ergeben, welche das werthvollste Ergebnis der Forschung darstellen, so sind auch für die vergleichende Anatomie die geistige Verwerthung der Thatsachen durch ihre Verknüpfung das wissenschaftliche Ziel. Was kann es nutzen, unendlich die Organisation betreffende Erfahrungen zu sammeln, wenn daraus nicht eine Einsicht in jene erwächst, ihr allmähliches Werden verständlich wird, indem es sich in mannigfachen, aber auseinander hervorgegangenen Zuständen darstellt, die ihre Verwandtschaft unter einander in der Organisation zum Ausdruck kommen lassen."

Carl Gegenbaur, Lehrbuch d. vergl. Anat. I898 ed., p. 27.

The first step in the evolution of the Metazoa from protozoan cell colonies, that is, the procedure which initiated the transformation of a colony of similar, one-celled organisms into a single organism of many cells, was the inpushing of its walls at a given point, resulting in the formation of a twolayered cup, the gastrula. From that moment on, the inner and outer cells become placed in a different position relative to the entire organism, and were thus subjected to different experiences. The outer layer was interposed between the organism and the external world; the inner dealt entirely with the material received into the cavity formed by it and used for food. It is obvious that this difference of experience would result in a physical differentiation of the cells, and such was, indeed, the case. The cells of the outer layer, the ectoderm, became in part protective and in part receptive of external stimuli, differentiations later to result in the formation of an exo-skeleton and a nervous system; those of the inner layer, the endoderm, developed the power of obtaining, ab- 
sorbing, and assimilating the nutritive qualities of the food, and thus formed the digestive cavity, the first portion of the organism to differentiate as a distinct system. This digestive cavity, or gastrocole, remains in the lower invertebrates as a blind cavity with but a single opening, and first among the worms (Vermes), it becomes converted into a complete canal by the formation of an anal orifice, thus obviating the necessity of employing the same orifice for both the intaking and the expulsion of the contents of the cavity.

A further advance in the development of the endodermic portion of the organism is seen in the higher invertebrates (articulates, echinoderms, etc.), and in the vertebrates, where certain lateral diverticula become divided off from the primary alimentary canal, and form a definite body cavity, the calom, so that the ultimate alimentary canal of these animals is but a part of the canal of the lower organisms. In vertebrates the canal suffers a still farther loss by the formation and later separation of the notochord. Another departure from the primary condition is seen in the mouth and anus of vertebrates, which are shown by their development not to be homologoits with the similarly named cavities of lower forms but new formations, involving other morphological relations, and formed by contributions from the ectoderm. In the development of many invertebrates the primary mouth of the gastrula becomes the permanent one of the adult organism and an anus is formed by continuing the blind end of the gastrular invagination until it meets the surface ectoderm at a point opposite that of the mouth; in the vertebrate embryo, however, the gastrular mouth lies postero-dorsally with reference to the future animal and thus bears no relation to either mouth or anus of the perfected form (Fig. 7I, A). During the development of the nervous system, however, there comes a curious and inexplicable connection between the lumen of the neural tube and the gastrular mouth, which effects a temporary connection between this cavity and that of the gastrocœle through the so-called neurenteric canal (Fig. $7 \mathrm{I}$, $\mathrm{B}$ ), but this connection is only transitory and the entire struc- 
ture soon disappears, leaving the gastrocœle as a closed sac, with neither oral nor anal orifices. These are formed secondarily through inpushings of the ectoderm, the blind ends of which come in contact with the endoderm and later break through at the point of contact, thus completing the canal (Fig. $7 \mathrm{I}, \mathrm{B}$, in and an). The functional alimentary canal

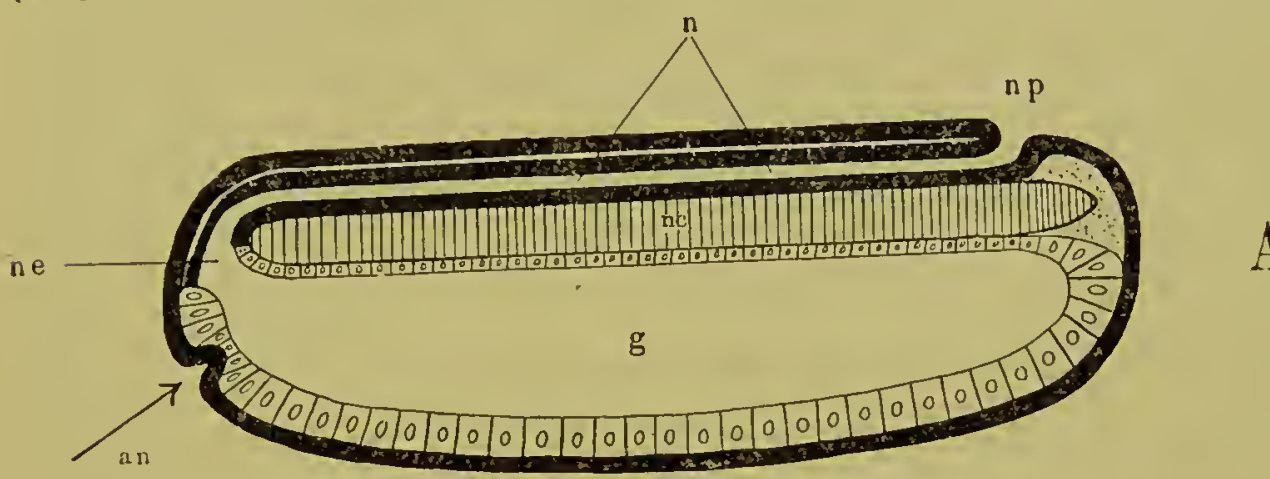

A

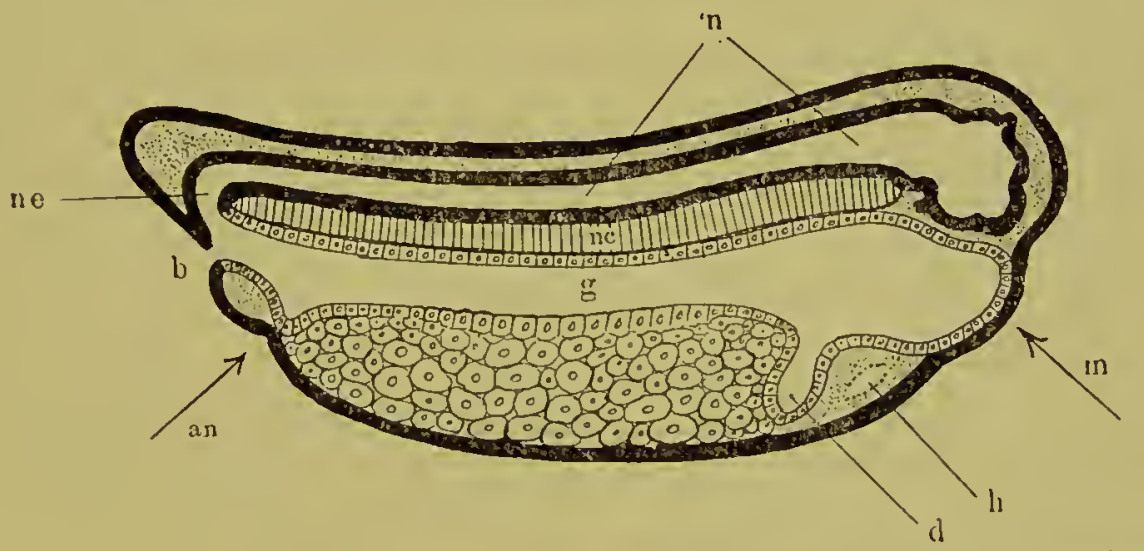

$\mathrm{B}$

FIG. 7I. Diagrams showing the formation of the vertebrate alimentary canal and nerve cord, and the early relation between them.

(A) Early embryo, immediately after the gastrular stage, based on Amphioxus. Compare this with Fig. 13 (c). (B) later stage, based on the embryo of the frog.

g. gastrocœle (=cavity of alimentary canal); $n$, neuroccle (Ecavity of the neural tube); $n e$, neurenteric canal; $n p$, neuropore; $b$, blastopore; $a n$, developing proctodxum; $m$, point where the stomatodxal invagination will take place; $d$, liver invagination; $h$, heart; $n c$, notochord.

thus comes to be formed of three elements, an anterior ectodermic one, the stomatodaum, a middle endodermic one, the mesodcum, and a posterior portion, also ectodermic, the proctodaum. In the articulates (crustaceans, insects, and spiders), in which the alimentary canal is formed in much the same way, the proportion of the functional digestive tract 
formed by stomato- and proctodæum, and therefore ectodermic, is extremely large, and the mesolxum, though voluminous, is employed mainly in the formation of laterally placed digestive glands; but in the vertebrate the canal is mainly mesodxal, and therefore endodermic, the ectodermic oral and anal contributions being much restricted.

With the exception of a small number of auxiliary organs like the jaws, teeth, and tongue, the entire digestive system is derived from this simple tube, and all the parts which appear in even the most complicated cases develop from this by means of such mechanical principles as increase in length, local enlargement, foldings, outpushings, and inpushings, in short, such principles as are employed in developmental history everywhere. More than this, from the anterior portion of this canal there develop the two principal respiratory systems of vertebrates, the branchial or gill system for aquatic breathing, and the pulmonary or lung system for air. This close association between digestive and respiratory systems is essentially a vertebrate characteristic and is hardly known among other animals, save in the cases of Amphioxus, the tunicates and the Enteropneusta, which in other respects also show their close affinity to the Vertebrata. [See Chap. XII.] Although so closely related anatomically, the digestive and respiratory systems are best disassociated in treatment and will be considered separately as far as possible.

Although essentially and in its origin an endodermic organ, the alimentary canal always becomes reinforced by other tissues which form layers outside of the primary endodermic one. Including the latter the layers are usually considered four in number, named in order, beginning with the inner one: mucosa, submucosa, musculosa, and serosa. The nucosa is the primary agent in digestion and develops glands for the production of the various necessary digestive juices; it also contains a thin layer of involuntary muscular fibers and is permeated with blood and lymphatic vessels for absorbing and carrying away the nutriment when in a proper condition for assimilation. The submucosa is a thin layer of connective 
tissue, needed to give support to the more delicate mucous layer. The musculosa, or muscular coat, may vary much in the different regions, but consists typically of involuntary muscular cells arranged in two layers, circular and longitudinal, the former internal. By the contraction of the circular layer the caliber of the tube is lessened and its length increased, while by the contraction of the longitudinal fibers the tube is shortened and thickened. Various combined actions of these fibers produce the peristaltic movements which occur during active digestion, and furnish an important mechanical aid in the process. The serosa, or serous covering for the tube, is in reality a reflexed portion of the peritoneum, which lines the cœlom, and which is attached in such a way that, besides covering the canal itself, its reflexions form broad, supporting membranes known as mesenteries, which attach the tube loosely to the body wall and hold it in place.

In no living vertebrate is the canal, when fully developed, in the form of a straight, undifferentiated tube, but becomes modified in several ways. In the first place, through the normal process of digestion, it necessarily becomes divided into regions, each of which is devoted to the performance of a certain physiological function, either mechanical or chemical. These portions are furthermore differentiated from one another in shape and size, and vary from long, attenuated tubes, to short and wide sacs; some grade into one another without definite boundaries; others are quite sharply set apart by a sudden change of external shape, by a localized restriction in the caliber of the tube, or by the entrance at a definite point of some new digestive juice.

A second cause for modification in the primary simple digestive tube lies in the mathematical law of the ratio of surface to mass, whereby the surfaces of two homologous solids are as their squares, the masses as the cubes, of their homologous dimensions. If, for example, an animal possessing a straight alimentary canal with a smooth mucous lining were to increase to twice its original length, its bulk would be increased eight times, but the square surface of the in- 
terior of the alimentary canal but four times; in other words, it would have but half as much digestive power, and would be in danger of starving were not some means employed to proportionately increase its digestive surface. As the physiological digestive membrane is the mucosa, this layer is the one primarily concerned in these modifications, and increase in its surface is gained, ( I) by lengthening the entire canal and allowing it to fold or coil in some more or less definite fashion, (2) by the formation of diverticula, or blind pockets, usually long and narrow like the canal itself, and (3) by various methods of folding or wrinkling the mucosa itself, with or without the other modifications. Independently of the above law, variations in the amount of digestive surface, and especially in the capacity of those portions of the canal used as temporary reservoirs, are dependent upon the quality of the food habitually taken, an innutritious food requiring a greater capacity and probably a greater mucous area than a more concentrated one.

A third necessary tendency of the mucosa is the formation of glands for the elaboration of the various digestive juices needed in the case of different kinds of foods and in different stages of the process; and in this are shown again the principles of gland formation as treated above in the case of integument. Thus there is a widespread occurrence of bcaker cells and of simple tubular glands, which dip a short distance below the surface, and as they are usually placed close together they form a thick mucosa, the thickness of which is that of the length of the glands composing it. In more complicated cases the glands may become too large to be included within the mucosa and push their way outward to the serosa, beneath which they appear as localized swellings, as in the case of the pancreas of many fishes; a still farther extension of this principle produces an accessory organ like the liver or like the pancreas of higher vertebrates, beyond the bounds of the alimentary canal, but connected to it by one or more ducts and still invested by the serosa (peritoneum).

The application of these principles and the gradual attain- 
ment of a complicated alimentary canal is shown by the comparison of various phylogenetic stages (Fig. 72). At first the canal does not much exceed the body in length, and its windings consist of a few open curves, although in actual cases the length may vary as the quality of the food, and it may thus happen that in two closely allied forms considerable differ-
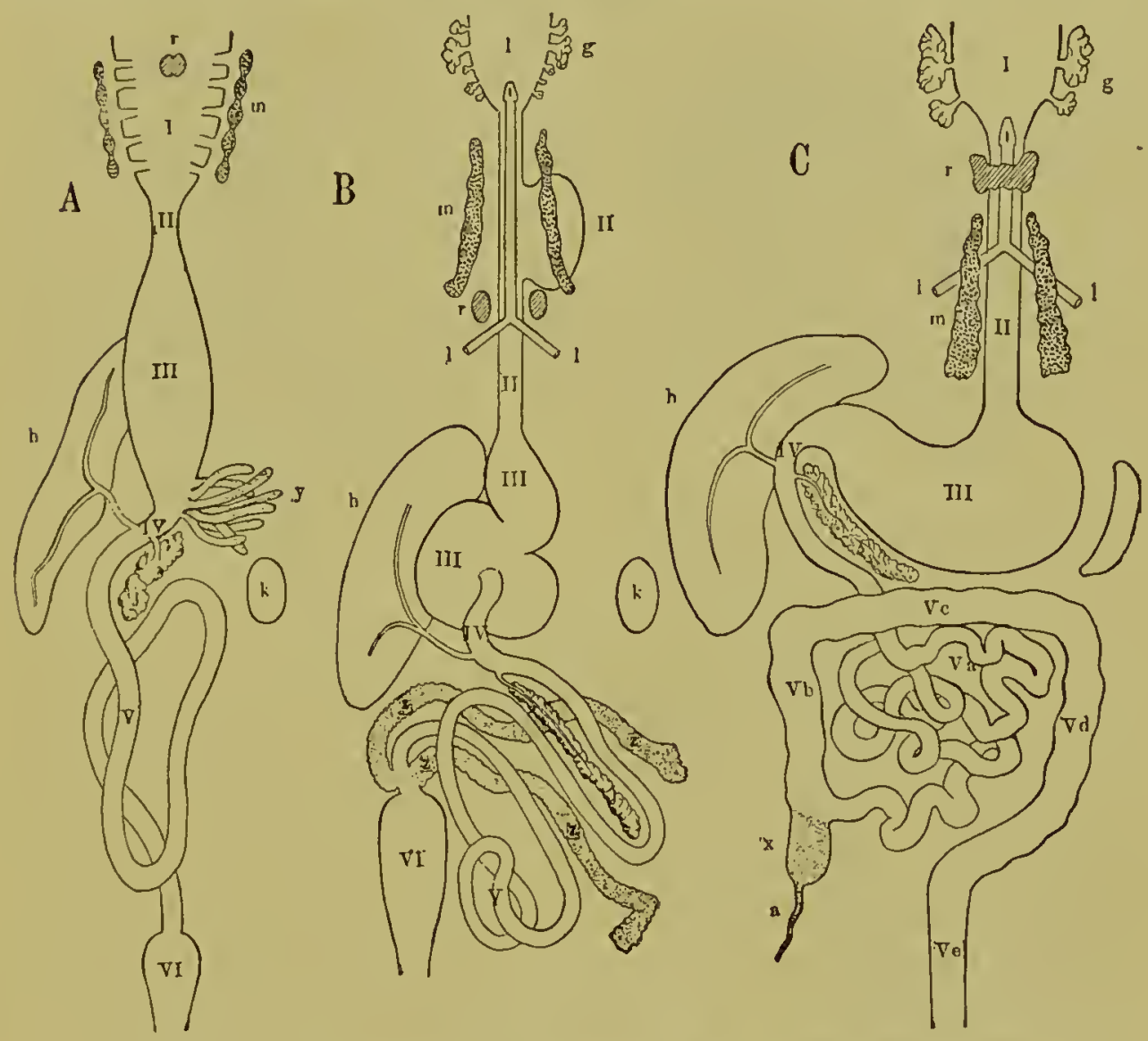

FIG. 72. Comparative diagrams of the alimentary canal.

(A) Fish. (B) Bird. (C) Mammal.

I, pharynx; II, œsophagus; III, stomach; IV, duodenum; V, intestine; VI, cloaca; $g$, salivary glands; $r$, thyreoid gland; $m$, thymus gland; $l$, bronchi, leading to the lungs; $h$, liver; $j$, pancreas; $k$, spleen; $y$, pyloric diverticula (in fishes); $z$, cloacal diverticula (in birds); $x$, intestinal diverticulum, the cœcum (in mammals); $a$, appendix (the narrowed free end of the latter in man). In B the stomach, III, is in two parts, glandular and muscular; in $\mathrm{C}$ the intestine is differentiated into $\mathrm{Va}$, small intestine; $\mathrm{Vb}$, ascending colon; $\mathrm{Vc}$, transverse colon; $\mathrm{Vd}$, descending colon; and $\mathrm{Ve}$, the rectum.

ence may be seen in this particular. Indeed, a great contrast in the length of the canal may occur in various stages of the same animal, as in the frog, the tadpole of which possesses a spiral, much coiled intestine, while that of the adult shows 
but a few windings, the change taking place within a few weeks or even days in correlation with a change of food (Fig. 73). Allowing for a few isolated cases, however, the canal of fishes and amphibians is short and becomes considerably lengthened in the Sauropsida and Mammalia, where the in-
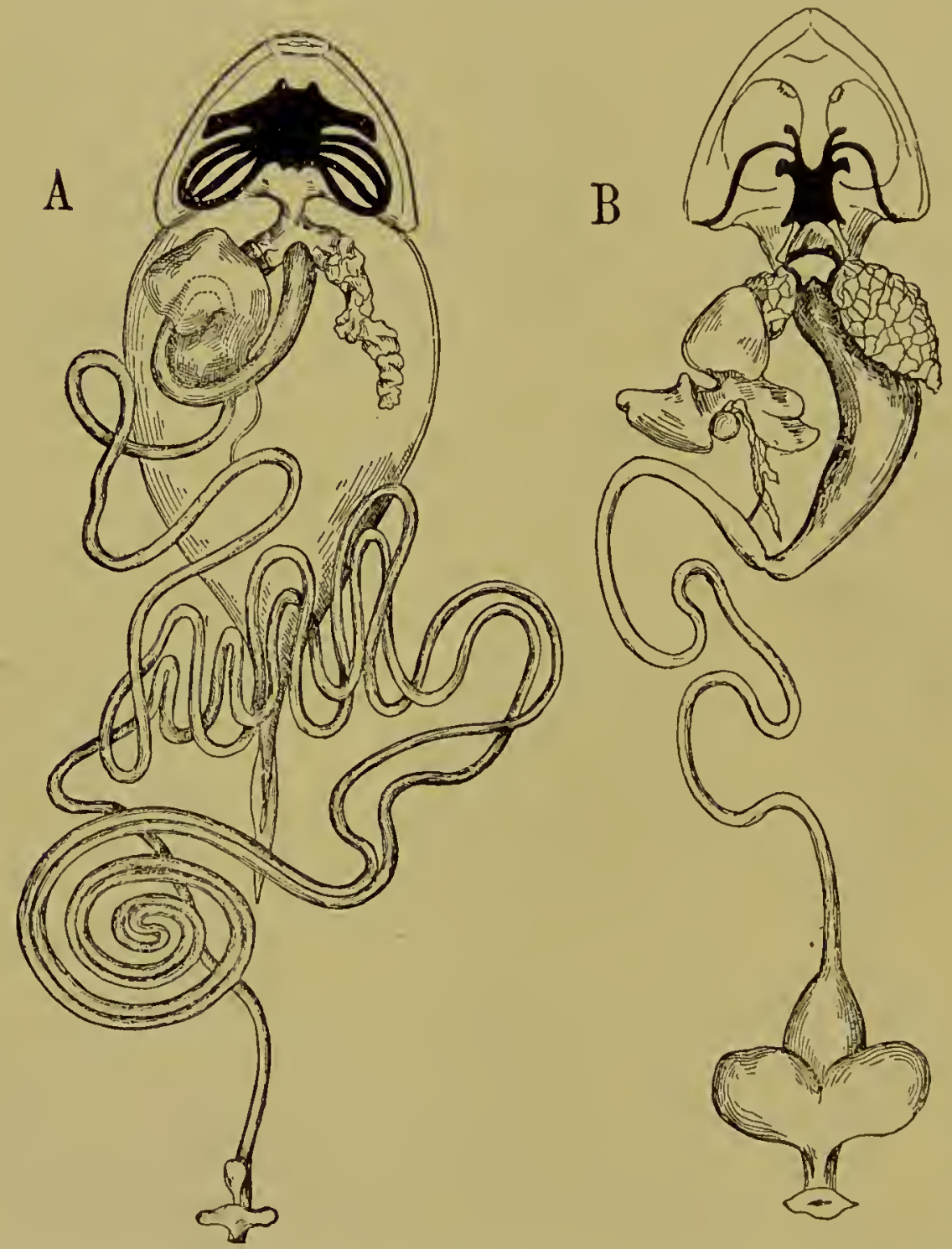

FIG. 73. Comparison of alimentary canal in (A), tadpole and (B), adult frog. [After the LeuckarT wall charts.]

testine, the part mainly involved in the increase of length, becomes disposed in complicated folds and windings. These, although apparently wholly irregular in their disposition, may be referred to a definite system, as may be made clear by a 
study of the various steps in the process. In a simple, straight intestine, the starting point of all forms, the tube is enwrapped by the peritoneum (serosa), which becomes reflected along the mid-dorsal line, the two layers becoming applied to one another to form a suspensory ligament, the mescntcry, which in turn is attached along the medial line of the bocly wall ventral to the vertebral column.

As the tube elongates it lengthens the free edge of this mesentery, the effect of which is to throw it into sinuous curves directed alternately to right and left, which in extreme cases fall from side to side in the form of long and narrow loops. The intestinal windings are never seen in this typical form, however, owing to the peristaltic movements of the musculosa which cause the folds and loops to constantly change their position so that their disposition in an animal is never the same at two intervals of time.*

The subdivisions into which the canal is divided anatomically for descriptive purposes depend upon localized enlargement or constrictions, the formation of diverticula, or the presence of definite digestive glands, and become more definite and numerous in higher forms as these features gradually appear and become more emphasized. The first portions to differentiate are the pharynx and stomach, the former being a funnel-shaped enlargement of the anterior end, characterized by pairs of lateral diverticula, the pharyngeal pouches, which may break through to the exterior and form slits; the latter a spindle-shaped or sac-shaped compartment for the reception of food of all sorts in about the condition in which it is swallowed. The narrowed portion between these forms the œsophagus. At its lower end the stomach is bounded by a restricted portion, the pylorus, which, by a specialization of the circular

* This constant change of appearance and endless varicty in arrangement is exactly suited to the demands of divination, which always depends upon a large amount of chance variation of some object; and it is very probable that the Roman augurs, who manufactured prophecies from the inspection of the cntrails of the sacrificial animals, were possessed of as definite a system as is seen to-day in the case of palmistry, a "science" founded like the other upon the erroncous and utterly baseless assumption of the causal relation of two unrelated sets of phenomena. 
muscles, forms a valve capable of closing, thus converting the stomach temporarily into a closed sac.

The remainder of the canal may be comprehendingly designated intestine, the regional differentiation of which does not appear as early and is never so marked as in the anterior portion. A little below the pylorus it develops from its mucosa two enormous glands or gland-complexes, liver and pancreas, which grow out far beyond the limits of the walls of the canal, retaining their connection with their place of origin through ducts. The portion of the intestine between the pylorus and the orifices of these ducts receives the special name of duodenum. A second early and better marked subdivision of the intestine is a terminal enlargement which generally receives the openings of the urinary and reproductive systems, and is hence termed the cloacal chamber, or simply cloaca. This portion retains its importance in fishes, amphibians and the Sauropsida, but in mammals it plays a subordinate rôle, appearing as a distinct organ in the lowest forms alone, the monotremes.

In certain definite portions of its length the alimentary canal shows a tendency to throw out diverticula, sac-like or tubular in shape and designed apparently to increase the general surface. Of those the most important are the lateral pharyngeal pouches previously mentioned; the pyloric caca, found in fishes, and often very numerous; colic caca, at the beginning of the large intestine in mammals; and cloacal coca, found in birds.

Following this general sketch of the alimentary canal, its development and its differentiation, the separate portions may be considered in greater detail [Cf. Fig. 72]. The most anterior of these are the mouth cavity and pharynx, usually fused into one, the stomato-pharyngeal cavity, although in mammals the development of the soft palate forms an inconnplete separation between the two. This cavity opens to the exterior through the mouth opening or stoma, which appears to be of two types in accordance with its surroundings and equipment.

The first of these, the cyclostoma, is that seen in the lam- 
prey and allied forms, which, on account of it, are termed the Cyclostomata. This type is similar to that of Amphioxus and may be related to it; it is circular in shape and equipped with horny, epidermic teeth.

The second, or gnathostoma, is furnished with a movable pair of skeletal jaws, equipped with teeth formed of dentine overlaid with enamel. In origin these jaws are a pair of visceral arches and the teeth are locally modified placoid scales (See Chap. III.), and it may be held either that the gnathostoma or jaw-mouth is the same as the first, to which the gill-arches with their associated teeth have become added; or that it is a new opening, originally a pair of gill-slits, which have become fused in the mid-ventral line, and that the first mouth has become lost. In favor of this latter view is the position of the gnathostome in the selachians, where it may be expected to show the most primitive condition, and where it is not at the anterior end but on the ventral side with a long rostrum anterior to it. Later on, as is shown in some ganoids, it attains secondarily an anterior terminal position.

If from the evidence presented an hypothetical sketch be permissible it may be allowed that in early vertebrates there was a circular jawless mouth, provided with a hood and situated at the anterior end of the body; that in some form midway between the lamprey eel and the shark the habit arose of seizing and taking in food by the anterior gill-slits, the edges of which, provided with sharp, pointed scales, served better for the retention of their living prey than did the oral hood and horny teeth of the actual mouth. The continuance of this habit would perfect the tools employed, which in this case were movable gill-arches armed with placoid scales, and the new mouth, formed by the ventral fusion of two lateral slits and furnished with superior organs of prehension, entirely usurped the function of the old one, which thus became reduced and finally disappeared. In one point alone, that of position, was the old mouth superior, and the final step in the perfection of the new one was its gradual migration to the anterior end, the various steps in the attainment of which may be seen among the ganoids. 
Behind the mouth on each side there develops a row of outpushings, the plaryngeal pockets, which meet a corresponding set of inpushings from the outside (Fig. 74). In fishes these break through at the points of contact, and form the gill-slits, a series of permanent openings (4-8 in number) that form a communication between pharynx and exterior and allow the

a

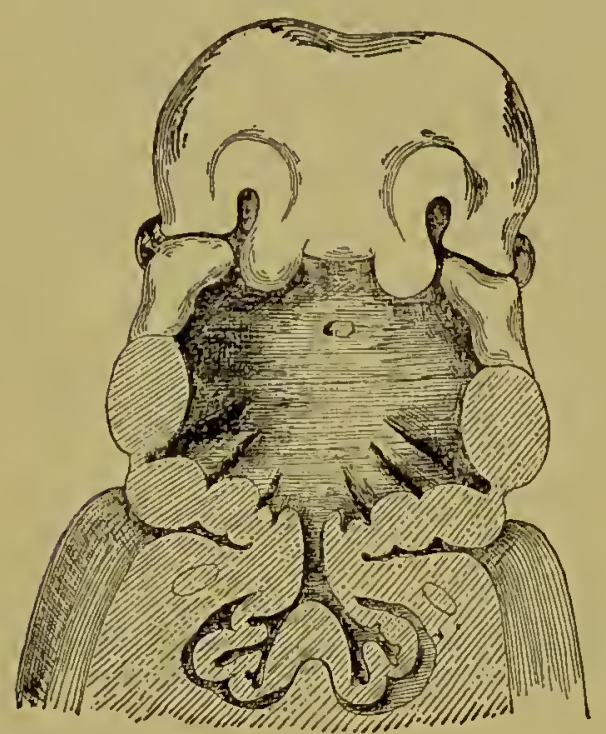

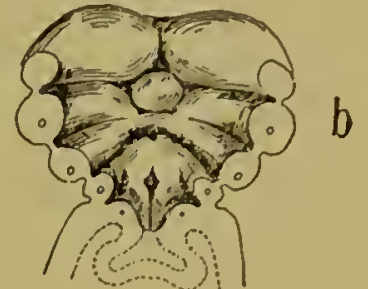

b

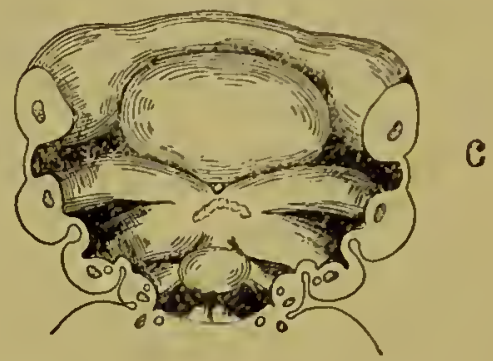

Fig. 74. Pharynx and visceral arches in human embryo. [After His.]

(a) Ventral aspect of early embryo, with the front part of the lower jaw and the gill arches removed. (b) Inner view, looking ventrally, of the lower jaw and gillarches, corresponding to the part removed from (a), but taken from a somewhat younger embryo. (c) Similar to (b), but taken from an older embryo, not far from the age of (a).

In (a) the most anterior of the arches sectioned is the mandibular (=lower jaw), succeeding which are the gill-arches in order, three being definitely formed. An. terior to the sectioned arches are seen the two superior maxillary processes, which, by their later union form the upper jaw. Between and a little above these is the fronto-nasal process. In (b) and (c) the most anterior arch is the mandibular, with the gill-arclies succeeding it in order. The round median mass is the tuberculum im. par, which, with the eminences immediately behind it, form the tongue. The thyreoid anlage and the glottis and epiglottis are seen in (c).

escape of the water constantly taken in at the mouth and used in respiration. One or more of these slits appear in amphibian larvæ and in a few forms persist throughout life, but in reptiles, birds, and mammals, although the pharyngeal pockets and their corresponding external depressions form during embryonic life, but two or three ever break through and 
then for a very short period. The most anterior of these, however, which, in selachians, appears as the spiraculum, or blow-hole, persists in all higher vertebrates, as the Eustachian tube [ tuba auditiva $B N A]$ and the middle ear. The remaining pockets disappear as such, but various accessory structures, such as cartilages, muscles, arteries, and glands, arise in the embryo in association with them and afterwards become modified to subserve numerous important purposes.* A not uncommon malformation in man, a few cases of which have been reported in other mammals, is that of a cervical fistula, which forms an open communication between pharynx and exterior, usually upon one side alone. This is nothing more or less than a permanent gill-slit and may be considered as a case of arrested development, or the retention of what is designed to be a transitory stage.

The nasal cavities, which lie above the anterior part of the stomato-pharyngeal cavity, are in fishes quite independent of the latter, but come into direct communication with it in Amphibia by the formation of a pair of openings, the posterior nares or choance, which appear in the roof of the mouth. This communication was apparently one of the changes inaugurated during the transition from water to land, and allows the ingress and egress of air to the pharynx and thence to the lungs without opening the mouth, since this action, although harmless for an animal immersed in water, would soon cause the drying up of the mucous membrane lining the mouth cavity if resorted to in air with anywhere near the same frequency. In the case of the nasal cavities this is prevented in part by the small size of the external openings, but still more by the formation of slime glands capable of producing an abundant secretion. The waste lacrimal fluid conveyed from the eyes to the nose is undoubtedly also of assistance in this respect.

The posterior part of the pharynx shows a strong tendency to form median diverticula, either dorsal or ventral, which ex-

* Cf. Chap. V under Visceral skeleton; Chap. VI under Visceral muscles, Chap. VIII under Arterial arches; and the present chapter farther on under Thymus and Thyreoid. 
pand into large sacs or reservoirs and either retain or lose their communication with the parent cavity. Such are the various sorts of air-bladders found among fish; these are generally situated dorsally with respect to the pharynx, but ventral ones occur in a few ganoids. Occasionally these reservoirs possess an attenuated pneumatic duct, communicating with the pharynx, and the supply of air is regulated by the fish coming to the surface and making a snapping or swallowing movement; but in the majority of cases the air-bladder is a closed sac, filled by gases extracted from the blood. In terrestrial vertebrates there appears in the embryo a midventral diverticulum which opens from the floor of the pharynx, grows posteriorly and forks into two lateral branches. This forms the pulmonary system, the lateral sacs becoming lungs and bronchi, the median duct the trachea, and the opening in the pharynx the glottis, which becomes regulated by a series of cartilages and muscles derived from the visceral system and forming the larynx.

The idea naturally suggests itself that this ventral pulmonary system is a direct inheritance from a similarly situated air-bladder, such as actually occurs in some ganoids, and although there is no direct proof of this, it seems very probable. It has also been noted that the diverticulum which produces it lies immediately back of the converging line of pharyngeal pockets and it has thus been interpreted by some as a continuation of the system, the forking into the two lungs being taken as proof of the formation of the median diverticulum from the confluence of two lateral pockets. The first of these theories seems by far the more probable, especially since the air-biadder of certain ganoids is richly supplied with respiratory blood-vessels, and thus forms a better lung physiologically than that of the more primitive amphibians, which are often simple sacs, yet a direct continuity from one to the other cannot be traced, since the lungs are always paired and an airbladder is always single.

The nasal cavities are separated from the pharynx by an approximately flat plate of bone that forms the roof of the 
mouth, or hard palate. In fishes and amphibians this is formed in great part by the anterior end of a single extensive median bone, the parabasal, but in higher forms this element is reduced and its function is assumed by the palatines and pterygoids and by horizontal plates directed inward from the maxillaries and premaxillaries. These latter elements are all lateral and arise in the embryo upon the sides of the head and grow towards the center, finally uniting in the median line. In some groups of birds the two halves remain disassociated, thus forming a palatine cleft by which a direct communication is established between the nasal and pharyngeal cavities. This failure to unite may occur as an abnormality in mammals, producing the malformations known as hare-lip and cleft palate, malformations thus attributable to the principle of arrested development. In certain mammals, as the cat and dog, the remains of the closure are permanently shown in the form of a deep median groove, the philthrum, which partly divides the upper lip and runs along the septum of the nose externally.

In mammals the bony palate (hard palate), which is conposed of horizontal processes from premaxillaries and maxillaries and a portion of the palatines, is continued posteriorly into a membranous soft palate or velum palati. This is a duplication of the pharyngeal mucous membrane and is supplied with semi-voluntary muscular fibers from the sheets surrounding the pharynx, which are themselves probably derivatives of the musculosa of the anterior portion of the alimentary canal.

Accompanying the stomato-pharyngeal division of the alimentary canal are several important auxiliary organs derived from various sources. These are the teeth, tongue, tonsils, the glands of the mouth cavity, and the glands of the pharyngeal pockets, which will be considered in the order given.

Excepting the horny formation in the mouth of the Cyclostomata, which are isolated structures of epidermic origin, the tecth of the vertebrates are strictly homologous organs throughout. They were originally placoid scales which difered in no respect from those that cover the exterior of the 
present-day selachians, and have been modified in form through the change of function due to their position in and about the mouth cavity. Teeth and placoid scales correspond closely in structure and development, and consist of a basis of dentine, or "ivory," as it is often called, overlaid by enamel, the first being formed from the corium, the latter from the epidermis.

In their original distribution as seen in fishes and amphibians they occur not only along the edges of the jaw, but also in patches over the roof and floor of the mouth cavity, coextensive with the stomatodrum; but in most reptiles and in mammals they are confined to a single row in each jaw. Corresponding to their origin the most primitive arrangement is that of an imbricated pattern as in other scales, a condition shown in many of the areas within the mouth cavity, and in the jaw teeth of many selachians, where they appear in several rows. Primarily, at the stage in which the jaws and skull are cartilaginous, as in modern selachians, the teeth are separated from one another, but in a slightly higher stage the bases fuse for mutual support, thus forming a flat plate of bone upon which the separate tooth elements appear as projecting points or cusps. This proceeding is repeated ontogenetically in a few cases, as in the paired vomers of the frog; although usually by a shortening of the development the stage at which the scales are separate is dropped out and the bony plate develops as a single structure, so that in cases where the cusps have become lost there is no indication of their dental origin. Thus are formed the flat bones that line the mouth cavity, such as the vomers, palatines, pterygoids, and parabasal, which in lower forms often retain their dentigerous character; such is also the origin of the premaxillaries and mavillarics that form the upper jaws, as well as that of the splint-like dentare, and perhaps the angulare, that enclose Meckel's cartilage and form the mandible. (See Chap. V.) The cusps are thus originally an integral part of the bony splints which bear them, and in fishes, amphibians, and most reptiles the two remain continuous, but in crocodiles and mammals, as well as in the fossil toothed birds, the two become detached, and the 
teeth are inserted by what is termed a thecodont articulation into deep pits in the bone called thece or alveoli (Fig. 75).

That portion of the tooth which fits into an alveolus is termed the root and is covered by a sort of bone called coment, while that which appears above the gum is called the crown, and is the only part overlaid by enamel.

All teeth are hollow and contain a central pulp cavity, which encloses a nutrient corium papilla; in cases where the cusp is a part of the bone the pulp cavity is open along one side to admit the passage of nerves and blood-vessels. In thecodont teeth, however, the latter come up through the bottom of the theca and enter the pulp cavity at the inner end of the root.

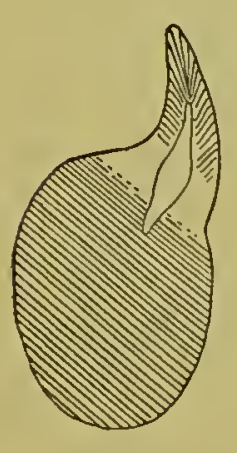

a

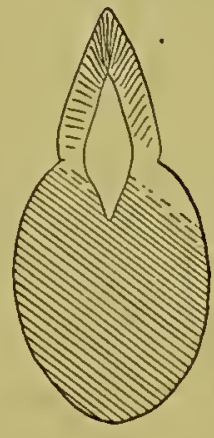

b

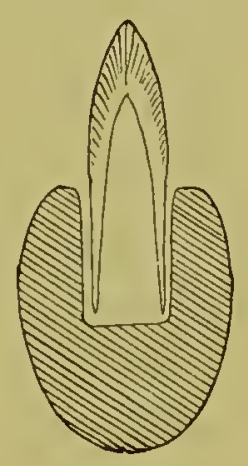

$\mathrm{C}$

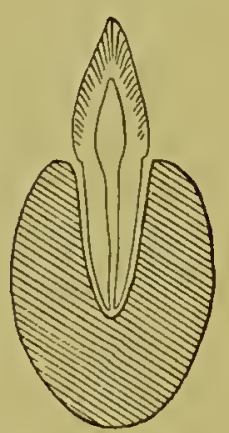

d

FIG. 75. Types of teeth.

(a), pleurodont; (b), acrodont; (c) thecodont with open root; (d) thecodont with closed root.

In this respect there are two kinds of roots, open and closed, in the first of which the root is widely opened and not distinct in structure from the crown, while in the latter the root is nearly solid and its lumen is restricted to a fine canal through which the vessels may reach the pulp. In both cases layers of new dentine are constantly, though slowly, added to the rest through the agency of a layer of cells called odontoblasts, which cover the pulp and are firmly applied to the inner walls of the pulp cavity. In the first type, in which the root is widely open and the entire tooth fits like a cap over the pulp, the tooth is gradually pushed upward by the addition of new layers underneath, and is thus continually elongating, while in the second or closed type the addition of new layers 
merely diminishes the size of the pulp cavity without increasing the length of the tooth as a whole. Both methods are in a way a provision against the loss of substance due to constant use; in the first type the outward growth and the loss through wear usually balance one another so that the tooth remains of about the same length throughout life; in the second the tooth grows gradually shorter while the addition of new layers beneath merely protects the sensitive pulp by keeping a constant thickness of dentine between it and the free surface. Thus, in man, whose teeth are of the second type, the chewing surface in old age is at a level which in youth would lay bare the pulp. Illustrations of the first type are seen in the teeth of the hippopotamus, the tusks of swine and elephants, and the chisel-like incisors of rodents; in the last instance the front side only is covered with enamel, and as this substance is harder than dentine, it wears away more slowly, and constantly presents a projecting edge, thus keeping the teeth sharp. Such teeth depend upon constant use in order to be kept at the proper length, and in abnormal cases in which some irregularity of the jaw prevents the meeting of opposite teeth, they grow past one another and become eventually disposed in coils and other eccentric forms which may prove the death of their possessor through an inability to feed properly. Similar, although here perfectly normal, instances are those of the ornamental tusks of elephants and wild swine, and in one of these latter, the babyroussa of the East Indies, an upper tooth upon each side bores upward through the lip and erects a curved point high above the snout.

The primitive form for a tooth is that of an elongated cone, a type which occurs with slight variation in proportions among all the lower vertebrates. In mammals, however, the teeth are characterized by the introduction of numerous modifications, sometimes very complex in nature, resulting in a large number of variations, not merely in different species, but in different regions of the same jaw, which correspond to a differentiation in use between the front teeth, that are in a position for grasping the food, and the back teeth, which 
come under the immediate control of the masticatory muscles. In the Cetacea alone the teeth are all alike (homodont) and of the simple conical type, and here it may be considered certain that this condition is a secondary one and that the teeth have lost the usual complexity through disuse; since an aquatic animal cannot chew and employs its teeth merely for the purpose of holding and retaining the food. In all other mammals the teeth are heterodont, and possess several distinct forms, which have been conveniently divided into three types, incisors, canines, and molars, with a possible subdivision of the last into premolars and molars proper.

Of these types the incisors are the most anterior and consist in the upper jaw of those borne by the premaxillary bones and in the lower jaw of those opposite the former. There may be five of these in each upper half jaw, and four in each lower half, but the usual number is three or two. Corresponding to the most frequent function of teeth thus situated their typical shape is that of chisels for cutting or biting, a form easily derived from the primitive conical forms by a flattening in a labio-lingual direction. Beyond these there is in each half jaw a single canine, typically in the form of a pointed cusp elongated beyond the level of the other teeth and best preserving the primitive shape. The canines of the lower jaw lie anterior to those of the upper jaw and in the case of elongated canines slip past one another. As their chief policy is that of piercing and tearing, they become reduced or are entirely wanting in animals in which this function is superfluous. The remaining teeth may be classed together as molars, or cheek-teeth, or a distinction may be made in most cases between an anterior group of premolars, in which the first teeth are usually replaced by a second set, and a posterior group of true molars, which develop later than the rest and are not replaced. That this distinction is in part an artificial one and often difficult or impossible of application is shown by the study of the subject of replacement, considered below. Of premolars the greatest number that occurs in mammals is four in each half-jaw, of definite molars, five. 
The number of each kind of teeth occurring in a given mammal is briefly and graphically expressed by a dental formula, which may be written in a varicty of ways. Thus the entire dentition may be expressed as in the following formula for the cat, in which the upper and lower rows represent the two jaws, the mid-ventral line is marked by the short perpendicular and the number of each group of teeth is designated by a digit:

$$
\frac{I-2-I-3}{\mathrm{I}-3-I-3} \mid \frac{3-I-3-\mathrm{I}}{3-I-2-I}
$$

This formula shows at once that there are in each jaw six incisors flanked on each side by a canine, and that there are four cheek teeth above and three below on each side, only the last one of which is not replaced and is therefore a true molar.

Except for the sake of symmetry, however, one half alone may be given, as in the following for the Bovidae, the family to which cattle and sheep belong, a formula showing a total loss of canines and of upper incisors:

$$
\frac{0-0-3-3}{3-0-3-3}
$$

From the study of the dentition in all mammals and especially that of marsupials and the more primitive placental mammals, the following hypothetical dentition has been deduced for the ancestral type, to which all existing dentitions may be referred:

$$
\frac{5-4-I-5}{5-4-I-5} \mid \frac{5-I-4-5}{5-I-4-5}
$$

This formula provides for ten incisors and eighteen cheek teeth in each jaw, with a total of 60 teeth, 30 in each jaw. This formula is nearly attained by some marsupials, where the most primitive condition would be expected, but in placental mammals there is always a reduction of incisors and molars, the former being never more than three upon each side. Thus, in the opossum (a marsupial) the dental formula is:

$$
\frac{5-I-7}{4-I-7}
$$


the last figure representing the total number of cheek teeth, without attempting a distinction between premolars and molars. Among placental mammals the Insectivora show the most primitive dentition; for example, that of Talpa (the mole) is:

$$
\mid \frac{3-\mathrm{I}-4-3}{3-\mathrm{I}-4-3}
$$

Certain cases, on the other hand, show an extraordinary reduction, as in the mouse-like rodent, Hydromys, where the formula is :

$$
\mid \frac{\mathrm{I}-\mathrm{O}-\mathrm{O}-2}{\mathrm{I}-\mathrm{O}-\mathrm{O}-2}
$$

The greatest reduction is found among some Cetacea, and in certain edentates (Myrmecophaga). In certain of the first group the tooth germs never develop but remain within the gums, while feeding is carried on through the development of horny fringes (the so-called "whale-bone") depending from the upper jaw, and forming a filter, which transforms the mouth into a scoop-net for the apprehension of shoals of marine creatures of various sorts. In the duck whale, $H y$ peroödon, there are but four teeth, all in the lower jaw, the anterior ones of conical shape, behind which are two imbedded in the gums; in the narwhal, Monodon, there are numerous small teeth that fall out before maturity, leaving the jaw toothless, but in the male a single upper tooth, usually that of the left side, develops into a long, projecting horn so large that it renders the skull asymmetrical. In certain of the smaller Cetacea, dolphins and porpoises, the jaws are furnished with conical teeth, nearly or quite homodont, which often surpass in number those of any other mammals (in the Dolphin, Delphinus, 47-65 in each half-jaw). Both this excessive number, which is at variance with the general formula for mammalian dentition, and their homodont character, must be looked upon as secondary adaptations to aquatic conditions.

In the primates there are two incisors upon each side and a well-developed canine. In the monkeys of the Western 
Hemisphere (Platyrhini) there are three premolars and either two or three molars, but in those of the Eastern Hemisphere (Catarrhini) there are always two of the former and three of the latter, giving the constant formula of:

$$
\mid-\frac{2-1-2-3}{2-1-2-3}=32
$$

Of these the five medial teeth of each jaw are replaced by a second set, the three molars not, the formula for the milk dentition being:

$$
\mid \frac{2-I-2-O}{2-1-2-O}=20
$$

In these points man corresponds completely with the other Catarrhini.

Regarding the evolution of the various shapes of mammalian teeth from the primitive conical type, the canines and incisors present a simple problem, since the first retain almost their typical form, and the second show merely a labio-lingual flattening. The derivation of the complex cheek teeth, however, presents a serious problem, for the solution of which two main theories have been offered. According to the first, or tritubercular, theory, the fundamental postulate must be laid down that every tooth, no matter how complex, represents a single primary element, and that the modifications are due to the development of additional cusps for the purpose of insuring a better articulation with the opposing surfaces of the teeth of the other jaw. The number of cusps which may thus develop on the contact surface of a tooth is six, the first of which to appear is the protocone, or primary cusp. Associated with the protocone are two secondary ones, the paracone, which is anterior to it, and the metacone, which is posterior (Fig. 76, I and II). These may become connected by crests or ridges and the tooth may become still more complicated by the bending of the crests and cusps into a $V$-shaped figure, the trigon (Fig. 76, III). A tooth, or a dentition, in which the three cusps, para-, proto-, and meta-cone, still lie in a 
straight line following the line of the jaw, is called triconodont, one in which the bending has taken place is trigonodont. This tritubercular tooth, still under the influences of the opposing tooth surfaces, may develop a lateral spur, the hypocone, upon which $\mathrm{r}-3$ tertiary cups, the conuli, may develop, or else the hypocone may form the point of a projecting spur, the talon. The nomenclature given here is that of the upper teeth, the corresponding parts of the lower jaw being distinguished by the addition of the suffix -id, thus: protoconid, hypoconid, talonid, etc. In favor of this theory may be urged its complete applicability to all known forms, both living and fossil, as a system of nomenclature, and its correspondence in sequence of stages to that shown by the extinct forms in consecutive geological periods. The
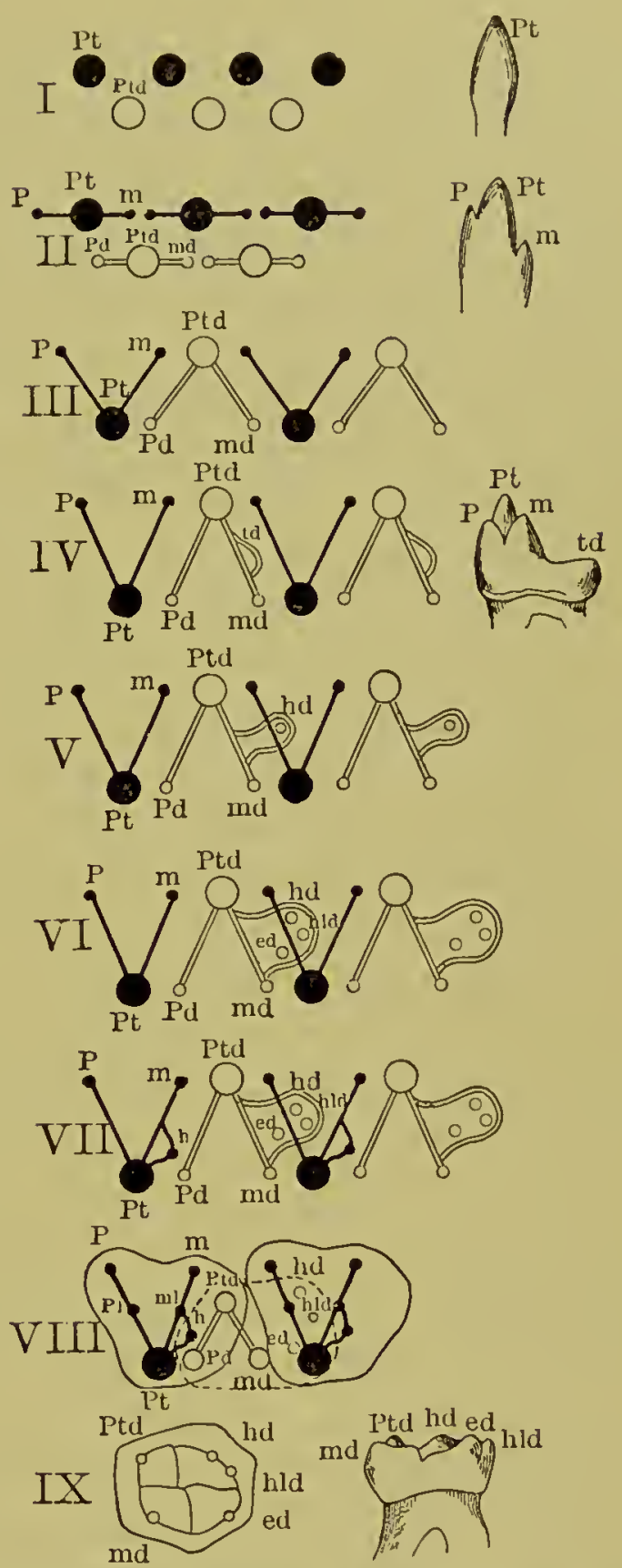

FIG. 76. Phyletic history of the molar cusps. [After H. E. OsBorn.]

I. Reptilian stage, haplodont; Permian. II. Triconodont stage. III. Tritubercular stage. IV. Tritubercular-tuberculo-sectorial; lower Jurassic. $V$. The same, upper Jurassic. VI. The same, upper Cretaceous. VII. The same, Puerco, lower Eocene. VIII. Sexitubercular-sexitubercular, Puerco. IX. Human lower molar.

$P t$, protocone; $P$, paracone; $m$, metacone; $h$ hypoconc; Ptd, protoconid; $P d$, paraconid; $n l$, metaconid; hd, hypoconid; hld. hypoconulid; $c d$, entoconid. 
embryological record, also, with a few exceptions, which may be explained in other ways, is in accord with it.

A second theory to account for the complex form of molars is that they are in reality, as they are often termed, "double teeth," and arise from the fusion of several primary germs. This concrescence theory is much older than the first, and had been generally abandoned in favor of the other when its probability in the case of certain forms was recently reasserted, owing to the testimony of embryology. It is thus possible that both theories may be true as appiied to different cases, the tritubercular method being the more general.

A widespread phenomenon among mammals is that of the replacement at a definite period of certain of the anterior teeth by a second set, and in respect to this power mammals are classed as diphyodont, in which such a replacement occurs, and monophyodont, where but one set appears. A1though in man and in many domestic animals, in which this procedure was first studied, the distinction between the two sets is clear and definite, such is not the case among certain of the mammals, and careful embryological records which show numerous cases of rudimentary tooth germs both preceding and succeeding definite teeth, has caused a revision of the entire subject. The matter becomes more comprehensible by referring to the lower vertebrate Classes, especially reptiles, where the papilla of each physiologically active tooth is associated with a succession of additional tooth germs in different stages of maturity, and designed to replace the functional tooth in case of injury (Fig. 77).

In a vitally important tooth, as in the poison fangs of serpents, at least one replacement tooth, nearly ready for use, lies continually by the side of the functional one, and may develop almost at a moment's notice in case of the loss of the latter. This power, limited to a single generation of replacement teeth, restricted to that part of the jaws anterior to the true molars, and arranged to assert itself at a certain definite period in the case of each tooth would result in the two dentitions of the typical diphyodont Mammalia (Fig. 78, A), and 
such has been undoubtedly the origin of this phenomenon, save that it is not necessary to assume that all the tecth of cither set belong to the same original generation, especially as they do not develop simultaneously. Thus some have seen in the succession of mammalian teeth the remains of five separate tooth generations, individuals from several uniting in a'specific case to form a set, either the milk or the permanent one. Others, admitting that each of the two definite sets represents a single tooth generation, find in certain cases traces of tooth germs
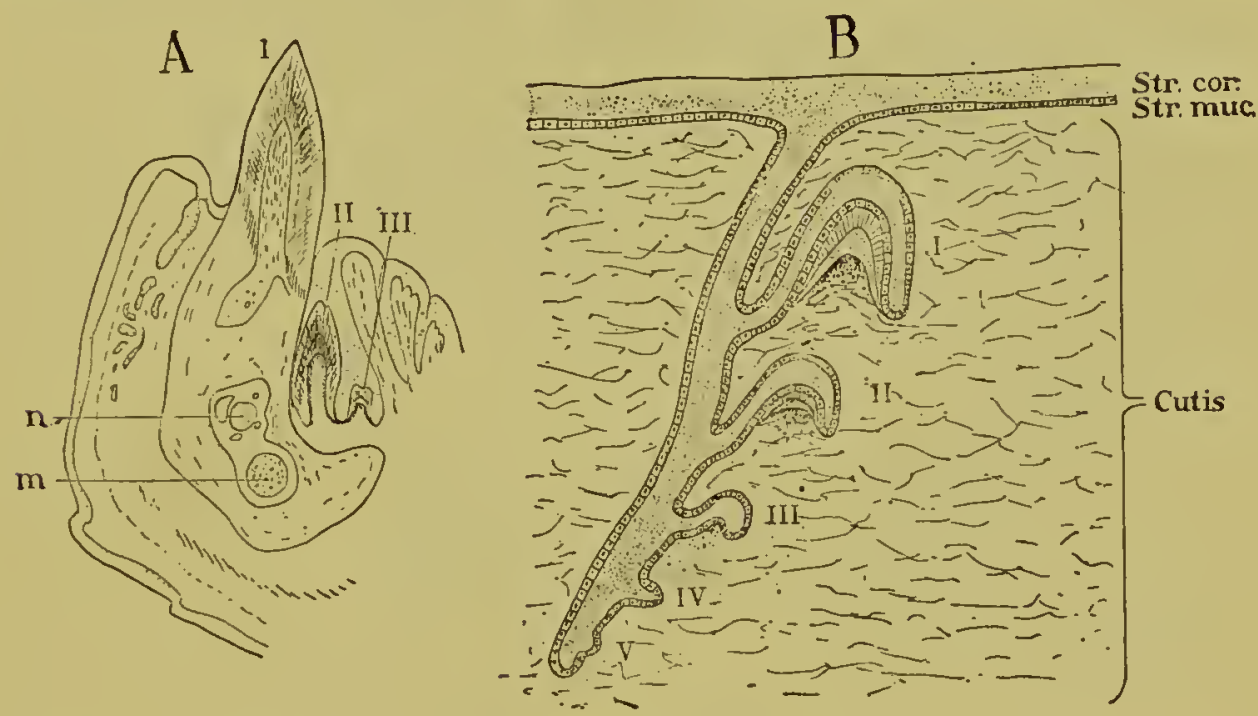

FIG. 77. Succession of teeth in reptiles.

(A) Section through jaw of Plyyllodactylus (a lizard) showing functional tooth (I) and several replacement teeth $(I I, I I I) ; m$, Meckel's cartilage; n, ncrve. [After GeGenbaur.] (B) Diagram of integument of gum, to explain the succession of teeth. str. cor., stratum corneum; str. muc., stratum mucosum; I, II, etc., the succession of tooth germs.

that precede the milk dentition, and others that succeed the permanent set; they thus consider that mammals have inherited from their reptilian ancestors four tooth generations, prelacteal, lacteal, permanent and post-permanent, of which the second and third have become generally established.* The first developed in the premammalian ancestors, the last may come to development in the future.

Regarding the occurrence of the two dentitions there is

* It is asserted that in Nasodon. a Tertiary ungulate, a pair of prelacteal incisors becomes developed. 
much variation. In certain marsupials but one tooth is replaced, the last upper premolar, this tooth alone constituting a second set, while otherwise the milk set is retained through life.
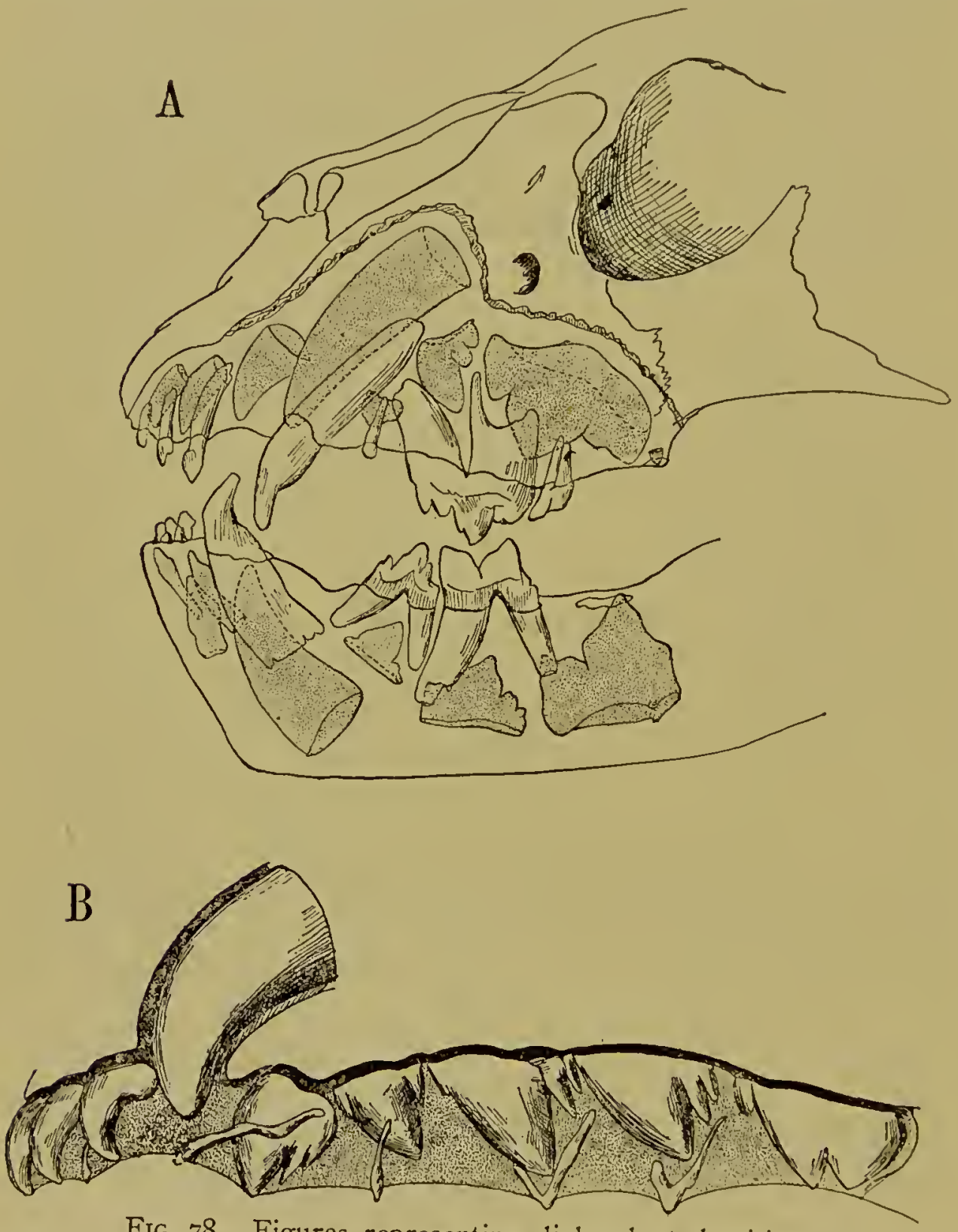

FIG. 78. Figures representing diphyodont dentition.

(A) Dentition of a lion eub of six and a half months. [After Weber.] Milk dentition funetional, replaeement teeth still within the jaw and shown as shaded areas. (B) Upper jaw of new-born seal, with replacement teeth almost ready for funetion. The milk dentition is redueed to useless rudiments cast off immediatcly
after birth. [From HerTwig, after BurckHARDT.]

They are thus almost monophyodont, with a permanent milk dentition, and are in sharp contrast to other monophyodont 
mammals in which the milk set is represented, by useless rudiments that are either absorbed before birth or expelled soon after. In view of all facts thus far presented it seems that the monophyodont condition has been secondarily acquired as a special adaptation in certain forms by the suppression of one or the other of the two typical generations (Fig. 78, B).

Although fishes possess no functional tongue the material out of which a tongue is to be constructed is present in the form of the anterior part of the hyo-branchial apparatus. The anterior border of this complex lies in the floor of the mouth cavity, following the outlines of the jaw, and by certain actions of the visceral muscles may be projected upwards so as to form a noticeable elevation. When in amphibians these parts become relieved from the gill-bearing function, they form the basis of the tongue, often fusing into a complex, moved by muscles and supporting a fleshy organ of some sort. In that type of tongue which leads to the higher vertebrates the skeletal basis consists of two to four of the visceral arches, beginning with the hyoid, and, although admitting of many varieties, possesses as essential a median basi-branchial piece, called here the os entoglossum, and two posteriorly projecting cormua. The tongue of the Sauropsida is a direct continuation of this, and in both cases the principal motion is a protrusion and withdrawal of the organ as a whole, which is effected by means of the two posterior cornua, which lie in sheaths from which they may be everted. When the tongue is unusually long the sheaths and the enclosed cornua are of corresponding length, and their disposition when retracted becomes a problem variously solved in different cases. Thus in a certain salamander, Spelerpes fuscus, the sheaths of the cornua run down the sides of the body and are attached to the ilia, and in the woodpecker they come around the occipital region, pass over the top of the head and terminate at the base of the upper beak, near the anterior nares. In these cases the ends of the cornua are attached to the bottom of the sheaths and as they are withdrawn the sheath is turned inside out and adds to the total length. 
In mammals the hyo-branchial support of the tongue is reduced to a complex composed of a basi-hyal (body) and two pairs of cornua, of which the anterior are typically the lnnger and are formed by a chain of four skeletal pieces, cerato-, epi-, stylo- and tympano-hyal, the latter attached to the tympanic region of the skull. The posterior cornua consist each of a single piece, thyreo-hyal, connecting the body with the thyreoid cartilage of the larynx. In man the anterior cornua show an unusual modification, the tympano- and stylo-hyals are fused with the otic region of the skull, forming the styloid process, the hypo-hyal is reduced to a rudiment which connects with the styloid process by a ligament in which no trace of the cerato-hyal is seen. Thus the anterior cornua, though typically longer and more complex than the others, are spoken of in man as the "lesser," an inheritance from the earlier anatomical science in which comparison with other mammals played no part.

Inserted upon this skeletal complex as a basis there is developed in mammals a fleshy tongue composed of interlaced muscular fibers, a part of which are intrinsic and belong to the tongue itself, while others are extrinsic and consist of the terminal fibers of other muscles. This organ is thus a structure totally unlike the tongue of most other vertebrates, in which the skeletal support reaches through the organ and in which motion is confined to a simple protrusion and retraction, and resembles rather the fleshy lobe which appears in a few cases appended to the other structure, as in the frog. Moreover, in some mammals, notably marsupials and lemurs, there exists, beneath the fleshy organ, an accessory tongue-like structure, the sub-lingua, which possesses many attributes of the tongue of the Sauropsida and like it is supported by a cartilaginous piece which may represent the os cntoglossum. In man the sub-lingua is reduced to a transverse fold, the plica fimbriata, readily seen if the mouth be opened and the tongue elevated. If this organ be taken as the homologue of the sauropsidan tongue, as its structure and position seem to indicate, the fleshy tongue of mammals is a new structure, de- 
veloped upon the dorsal side of the former, and gradually usurping its function. An opposing opinion rejects the claim of the sub-lingua as homolog of the sauropsidan tongue, and finds the rudiment of the os entoglossum in the septum linguce, a band of connective tissue running lengthwise through the tongue and serving as an attachment for the muscles, or more definitely in the lyssa, a vermiform structure composed of connective tissue, fat, cartilage, and muscle fibers, which occurs in the tongue of certain mammals, and is often associated with the septum.

Distinctive glands in the mouth cavity other than simple mucous cells are not found in fishes or wholly aquatic amphibians and appear only as an adaptation to the terrestrial life for the primary purpose of keeping the surface moist. Such glands are thus constantly present in terrestrial vertebrates and become secondarily reduced in those that become readapted to the water, as in the case of the Cetacea. These glands are often voluminous, occur in all available positions, and are named accordingly, buccal, labial, lingual, sub-lingual, sub-maxillary (more properly sub-mandibular), etc. Although the primary function of all these was undoubtedly that given above, they have in numerous instances assumed more special functions and have altered the chemical nature of their secretion accordingly. Thus the intermaxillary glands of frogs and toads, which open into the roof of the mouth, secrete a viscid fluid by means of which the tongue is rendered adhesive for the apprehension of insects, the lingual glands of many salamanders and lizards have a similar function, and certain buccal glands in poisonous serpents become the elaborators of the venom, which is inoculated into the victim by means of the poison fangs. These teeth are provided either with a groove along the external surface or possess a minute lumen through the center as in the case of a hypodermic needle. Another secondary function extensively employed is that of lubricating dry food to render it more easily swallowed, and in association with this the secretion often develops ferments, of use in the digestion of starch, and forms the saliva. The 
glands specialized to produce this fluid may be termed salivary, without reference to their position, and consist in mammals of the parotid, sub-mandibular [sub-maxillary BNA], sublingual, and retro-lingual, the last associated with the submandibular and not found in all mammals. There also occur other voluminous glands, with a structure similar to the others, that secrete a clear fluid without salivary attribute. Such are the molar glands of ungulates, or the voluminous orbital gland of the dog family (Canidæ), which opens by a duct into the mouth cavity in the region of the last upper molar.

Associated in origin with the pharyngeal region are certain organs of more or less uncertain significance and varied destiny, the most prominent of which are the thymus and thyreoid glands. These, although not connected in function with the digestive system, may be treated here because of their origin. In the cyclostomes, which furnish the first, or most generalized stage in this history, there develop seven pharyngeal pockets on each side, each with a dorsal and a ventral recess. About each of these there develops a proliferation of epithelial cells, forming an organ or organ-anlage. These anlagen appear alike in structure, but in the higher forms the differentiations from the dorsal series become collectively known as the thymus, those from the ventral as epithelial corpuscles.

The later history of the thymus anlagen shows three tendencies, (I) to become early separated from their layer of origin, (2) to fuse upon each side to a single long organ, often showing its segmented origin, and (3) to become restricted in number, the more anterior ones being the first to disappear. These are all seen in the accompanying diagram (Fig. 79), which shows the phylogenetic steps in the process. Thus, beginning with the seven anlagen of the cyclostomes, the teleosts, which possess six pharyngeal pockets, show but four, and these the most posterior. The first pocket has none, that of the second is represented by a rudiment, which early disappears. In the urodeles, in which five pockets appear, 

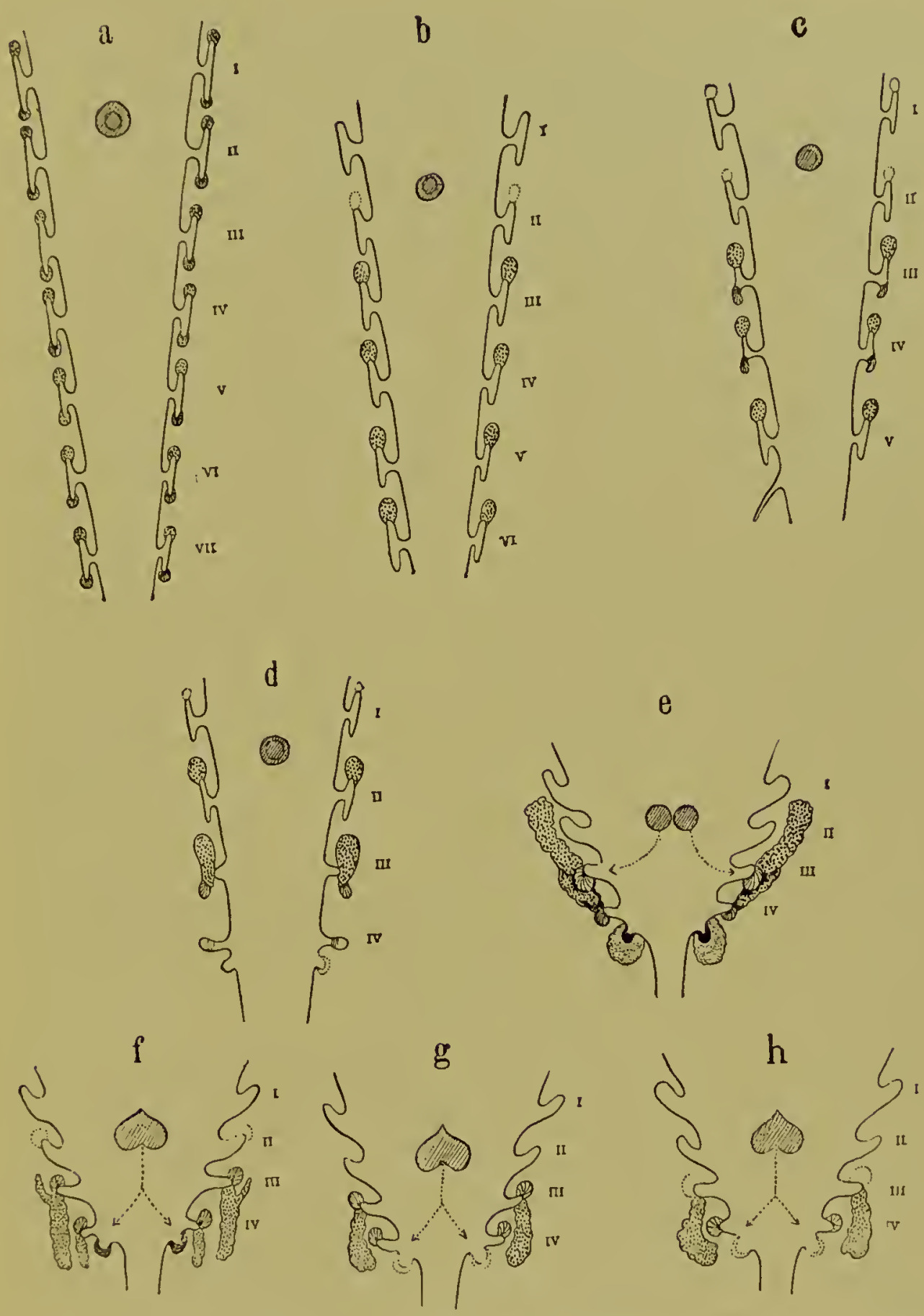

Frg. 79. Embryonal anlagen of the glands associated with the pharynx * and pharyngeal pockets. [a-d, after MAURER; e-h, after VERDuN.]

Thymus anlagen are indicated by dots, thyreoid by diagonal lines, epithelial corpuscles by radiating lines, and post-branchial (supra-pericardial) bodies in black. The pharyngeal pockets arc designated by Roman numcrals.

(a) Cyclostome. (b) Teleost. (c) Urodele. (d) Lizard (Lacerta). (e) Chick. (f) Cat. (g) Man. (h) Mole. 
only the last three possess definite thymus-anlagen, while those of the first two are transitory rudiments. In the lizard, with four embryonic pharyngeal pockets, the first has a ruclimentary thymus-anlage, and the second and third functional ones. The fourth is without one. In mammals the definite thymus is formed either wholly from the anlage associated with the third pocket, or else mainly from this with the addition of a small contribution from the fourth.

The fate of the ventral anlagen, that form the epithelial corpuscles, is known only in part, but they form at best only small nodules, of a more or less glandular nature, and never attain the bulk of a thymus. In amphibians that of the second pocket becomes associated with the carotid artery and forms the so-called "carotid gland," especially conspicuous in frogs and toads. Those of the third and first pockets are developed as epithelial corpuscles. In the lizard the only ventral anlage that persists is that of the third pocket, which forms a carotid gland. In Echidna epithelial bodies appear, associated with pockets two and four, that of the second becoming a carotid gland as in amphibians. In other mammals pockets three and four, or pocket four alone, are provided with such anlagen. These may remain associated with their respective thymus anlagen, or may become detached, but possess little if any physiological significance.

A third system of organs associated with this region appears typically as a single pair of evaginations, which arise from the floor of the pharynx, in all cases immediately behind the last gill-slit. From their secondary relation to the pericardium in selachians they have received the name of suprapericardial bodies, but they are better termed the post-branchial bodies, a name which expresses a fundamental and universal relationship. In selachians both members of the single pair of these organs develop, but in urodeles and in lizards the left alone completes its development, while the right one remains in a rudimentary condition, and eventually disappears. The occurrence of post-branchial bodies in birds and mammals is uncertain, but some identify with these a pair of 
evaginations that in the latter class arise from the same region and become eventually lost in the lobes of the thyreoid gland, the so-called parathyrcoid bodics. Comparing the significance of the post-branchial bodies, they are looked upon by some as the rudiments of a pair of pharyngeal pockets posterior to the last definite ones, a point of view which has suggested for them the name of ultimo-branchial (rather than post-branchial) bodies; but the probability that the last apparent pocket in the various vertebrate Classes is not always the same one introduces a valid objection to this hypothesis.

Still another organ associated with the pharynx as primarily an evagination from its walls is the thyreoid gland. This, in its first appearance, is constant from the selachians to the mammals, and arises as a median outpushing from the floor of the pharynx at a level corresponding to the interval between the first and second pockets. In its later development it becomes, like the thymus, a compact glandular organ, without a duct, and equally uncertain and indefinite in its function, but in the larva of one of the cyclostomes (Petromyzon), we catch a glimpse of its past history, for here for a time it appears as an open trough, lined with cilia, and in open communication with the pharynx. In this condition it corresponds closely in structure and position with the hypo-branchial groove, or endostyle of Amphioxus, an organ which furthers the passage of the food down the pharynx by producing a slimy secretion and by furnishing a ciliated track along which the food may be propelled. The thyreoid gland was thus primarily a digestive organ and deserves a place in this chapter for reasons more fundamental than a mere topographical relation. In the true vertebrates, however, its function, with its structure, becomes completely changed, and it no longer assists in digestion, but presumably possesses some regulating effect upon the blood, especially that supplying the brain. There is yet much that is problematic in this, but that the association between the thyreoid and the brain is an intimate one is shown both by the occurrence of cretinism, a curious developmental malformation associated with the thyreoid and 
in which varying degrees of idiocy play a principal part, and by the frequency with which insanity has followed the extirpation of the thyreoid.

The pre-vertebrate history of the thymus-anlagen is less certain, but attempts have been made to homologize them with the "tongue bars" of Amphioxus, gelatinous rods used to support the gill-clefts. If this be true the change of functioni seems even greater than in that of the thyreoid, for there is more similarity between a gland-lined groove and a compact glandular organ, than between the latter and a skeletal rod. In its development among the vertebrates the thymus is still more problematical than is the thyreoid; it is usually voluminous, and in many mammals extends along the anterior mediastinal space between sternum and heart as far as the diaphragm. In the human species it is large in childhood, but suffers regressive changes; during middle life it is inconspicuous, and in old age is reduced to a few rudiments. -

Beyond the pharynx the alimentary canal becomes narrowed and forms the asophagus, below which it again enlarges to form the great expansion known as the stomach, usually the most conspicuous portion of the entire tract. The œsophagus varies in length in proportion to the length of the neck region, one extreme being represented by frogs and toads, in which it is reduced to a simple constriction like the neck of a bag, the other by such cases as a long-necked bird. In birds that are graminivorous, or those in general which subsist upon hard or dry food, the middle portion of the œsophagus expands to form a crop (ingluvies), into which the food is first collected. Aside from its use as a receptacle in which food may become softened by soaking, the crop is probably in part a provision for the safety of the birds, allowing them to greatly shorten the period of feeding, a time during which they are preoccupied and thus in especial danger from their enemies.

The muscular fibers of the alimentary canal usually change from voluntary to involuntary in the upper part of the œsophagus, but in some mammals striated fibers, probably 
partly under the control of the will, extend farther down, and in ruminants, where the food is voluntarily disgorged in the form of cuds for a second chewing, the entire osophagus is thus equipped.

The stomach is originally a simple, spindle-shaped enlargement of the canal, extended lengthwise and indefinitely separated from the osophagus, although more completely limited below by a restriction, the pylorus, which forms a valve for the purpose of temporarily converting it into a closed sac. This typical form is seen in fishes and tailed amphibians, but an attempt to increase its efficiency both in digestive surface and in capacity causes the formation of a curvature extcnding to the left, so that there is a longer outline upon its left side and a shorter one upon its right, the greater and lesser curvatures respectively. As the same tendency continues the stomach turns, still to the left, in such a way that ultimately its longitudinal axis lies across the body, which places the upper or cardiac end on the left, the lower or pyloric end, on the right, the lesser curvature above and the greater curvature below. Below the cardiac orifice the left end of the stomach usually bulges out laterally to form the fundus, which in some cases becomes a more or less distinct receptacle for the food when first received; and beyond approximately the middle the stomach tapers toward the pyloric end (right) and forms an upward curve at the culmination of which is placed the pylorus. This may be considered the typical form of mammalian stomach and is seen in Primates, Carnivora, Insectivora, and Edentata, these being in other respects also most primitive of placental mammals (Fig. 8o, a).

Modifications of this primary form are due, first, to an attempt to localize and define the different portions of the stomach and specialize their functions, and, secondly, to various attempts to increase the general surface and thus develop a greater physiological efficiency, usually in connection with innutritious food or with the necessity of taking in a large amount in a short space of time.

Progress in the first of these directions is shown by such a 
stomach as that of the mouse (Fig. 8n, b), in which the condiac and pyloric halves are separated by a marked restriction, and this tendency reaches its extreme in the ruminants (Fig. So, h), where each of these two primary sub-divisions is again divided, forming a stomach of four compartments, in two pairs. The cardiac portion is divided into a voluminous pannch (rumen), which receives the food when first taken in, and a small, round honey-comb stomach (reticulum), in which the food from the paunch is made up into cuds. The pyloric por-
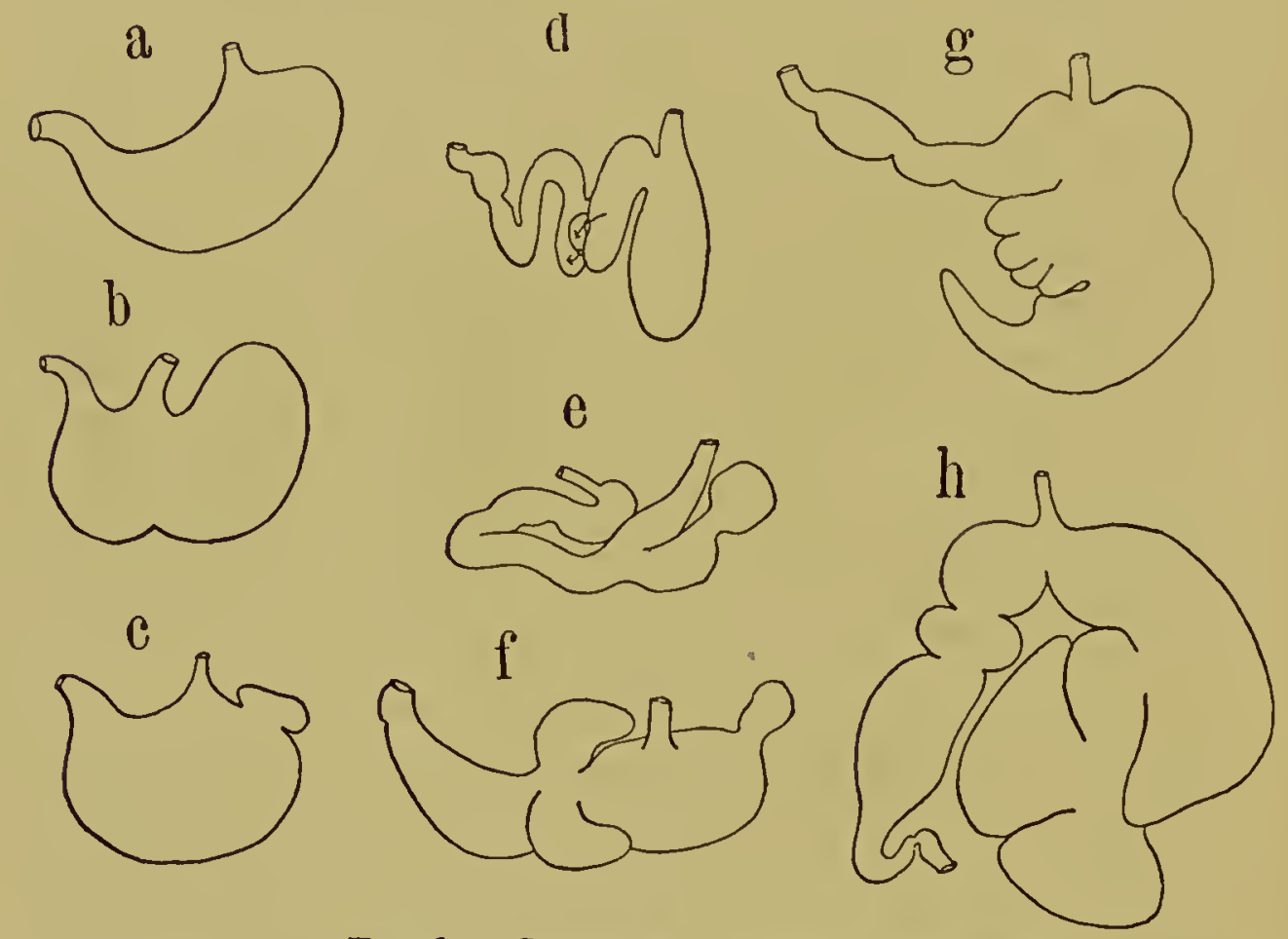

FIG. 80. Stomachs of various mammals.

(a) Man; (b) mouse; (c) pig; (d) seal; (e) vampire bat; (f) manatee; (g) sloth; (h) sheep.

tion is divided into an omasus and an abomasus, into which the food passes in succession when swallowed a second time.

Local enlargements of surface frequently appear in the form of a prolongation of the fundus into one or more diverticula (Fig. 8o, e, $\mathrm{f}$ and $\mathrm{g}$ ). There are two of these in the hippopotamus; three in Tarsipes, a small, insect-eating marsupial; and in the vampire bat, Desmodus (Fig. 8o, e), the fundus is elongated to form a cœcum of twice the length of the body, used as a reservoir for blood. 
It is of interest in this connection to trace the changes in the mesentery of this region, more precisely termed the mesogastrium, as they appear in successive stages in the mammalian embryo which are undoubtedly of historical significance (Fig. $\mathrm{SI}_{\text {I }}$. The formation of the initial curvature to the left naturally broadens the corresponding part of the mesogastrium, an effect still further increased by the lateral torsion of the entire stomach. At this point the widened mesentery comes under

I

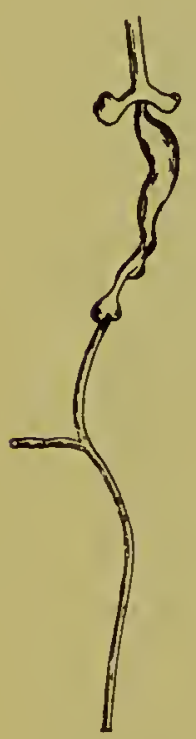

II

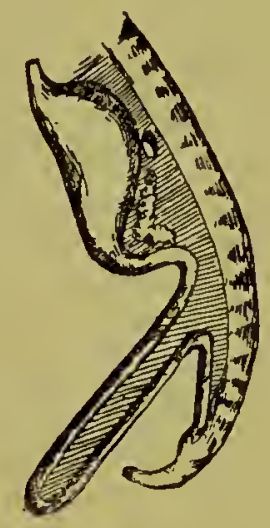

III

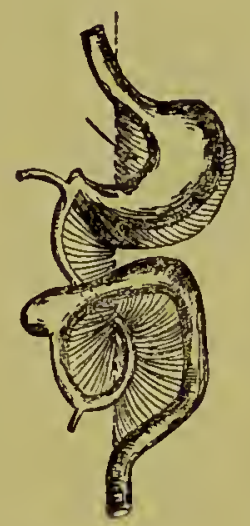

IV

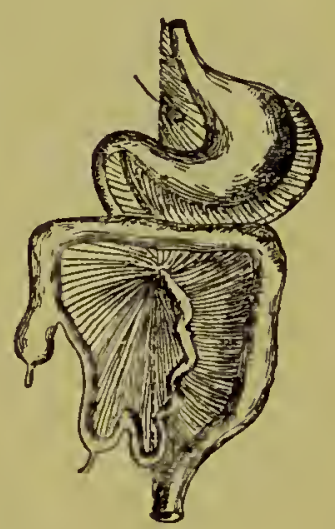

Fig. 81. Development of the peritoneal folds and of the windings of the alimentary canal in the human embryo. [From HeRTwig, Figure I after His; Figure II after ToLDT.]

Figure I shows the spindle-shaped stomach, the lung anlagen, and the beginning of the liver in the form of a median diverticulum; in Figure II the peritoneum is shown, with pancreas and spleen; figures III and IV show the development of the omentum and the lesser peritoneal cavity.

the influence of the spleen, which develops within it and by its weight produces a fullness which sags down behind (dorsal to) the lesser curvature, while attached to the greater; and the continuation of this tendency causes the free lower fold of the bag-like extension to hang down behind the contour of the stomach.

This fold is the greater omentum (omentum majus), which, as all mesenteries are essentially double, consists of four layers 
of serous membranes, applied two and two, each pair holding between them the blood and absorbent vessels naturally belonging to a mesentery. The cavity of the bag is the lesser peritoneal cavity of human anatomy, and its mouth, opening into it behind the stomach, is the foramen epiploicum [foramen of Winslow]. In most mammals the bag is widely open, but in man the foramen is much reduced in size and the layers forming the pendulous fold are fused together, and form a four-layered apron that hangs below the stomach and covers the intestinal folds.

The remainder of the canal below the pylorus forms the $i n$ testine, and although this has been divided for convenience into several more or less definite regions, they are for the most part artificial in character. The most definite of these are the cloaca of Amphibia and Sauropsida, and the large intestine of mammals [intestinum crassum], both enlargements of the posterior portion of the intestinal tract, but probably not equivalent to one another; in distinction from this the remainder is termed the small intestine [intestinum tenue]. In this latter the most definite subdivision is defined by the entrance of the bilary and pancreatic ducts; and the space between the pylorus and this point is designated the duodemum. This portion often forms a conspicuous loop, consisting of ascending and descending limbs, which enclose the pancreas between them.

The liver and the pancreas, the two digestive glands associated with this region, are derived from the mucosa of the intestines, from which they arise as evaginations, the former ventral, the latter dorsal. As they increase in size they protrude beyond the intestinal walls and force their way between the two layers of their respective mesenteries, as a result of which relation they become invested with a serous membrane continuous with that covering the intestines (peritoneum), and remain attached by ligaments both to the latter and to the body wall. These relations are clearly shown in the accompanying diagrams (Fig. 82), which show the origin of these organs from the intestine, their serous investment and their dorsal and 
ventral mesenteries. In spite of the great increase of size these typical relations remain in the case of the liver, and its two suspensory mesenteries become the ligamentum hepatogastricum [ $\left.\mathrm{y}^{\prime}\right]$ (sometimes termed the lesser omentum), and the ligamentum suspensorium hepatis $[x]$. While primarily the entire length of the alimentary canal that passes through the colomic region becomes attached by both a dorsal and a
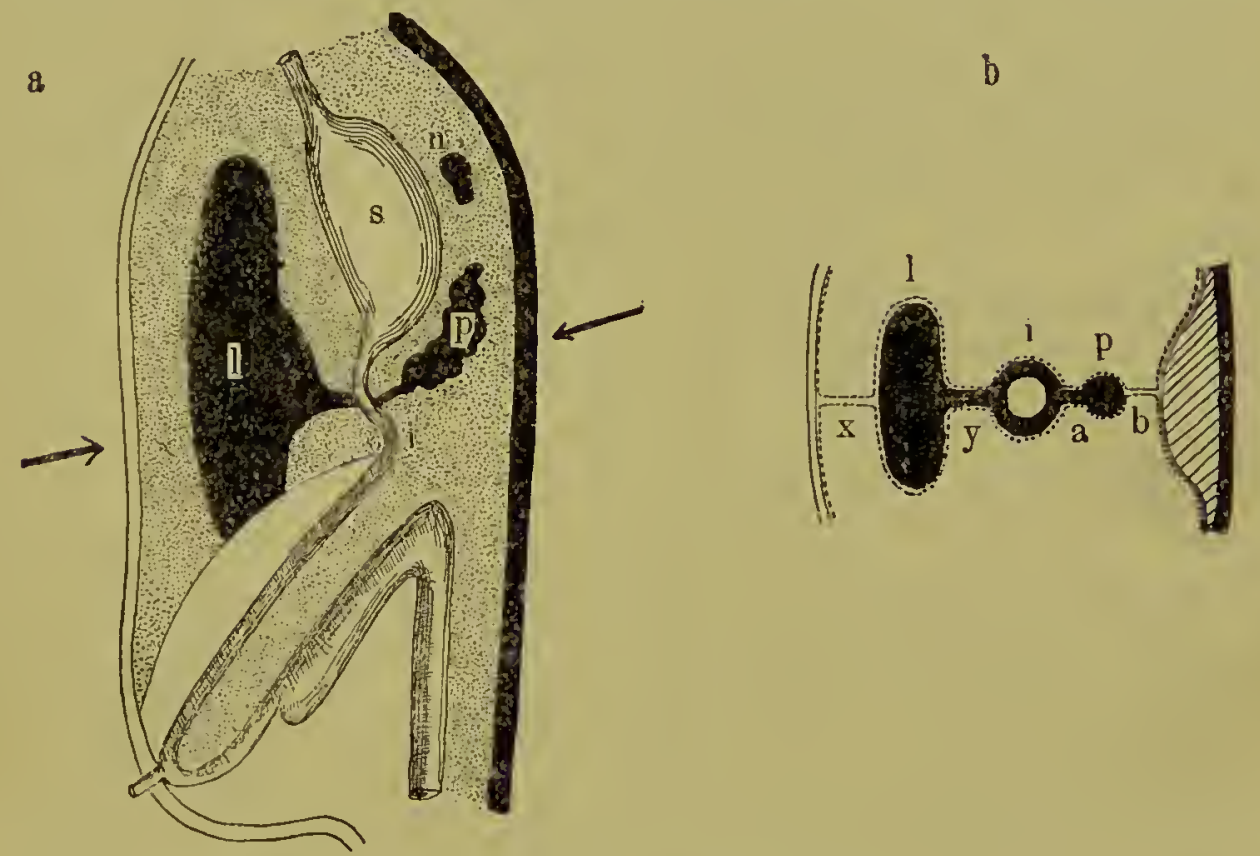

FIG. 82. Diagrams showing the relation of the liver and pancreas to the peritoneum. [After HeRTwIG.]

(a) Lateral view with ventral surface towards the left. The organs are seen lying within the peritoneum, which is represented in a vertical plane stretched across from mid-dorsal to mid-ventral lines. (b) A cross-section. The place through which it is taken is indicated approximately in (a) by the arrows.

Organs: $s$, stomach; $n$, splcen; $l$, liver; $p$, pancreas; $i$, intestine. Ligaments: $x$, ligamentum suspensorium hepatis; $y$, ligamentum hepatogastrium (=lesser omentum); $a$ and $b$, parts of the mesogastrium which form the pancreatic ligaments similar to those of the liver.

ventral mesentery, the ventral one becomes lost below the region of the liver, thus leaving a sharp ventral edge to the two hepatic ligaments.

The gall-bladder is formed as an enlargement of the hepatic duct and is by no means of universal occurrence; it develops rather in response to certain conditions, much as in the case of the crop, and its slight physiological importance is shown by its occurrence in one of two allied animals and its ab- 
sence in the other. A good example of this is that of the pigeon and the common fowl, in the latter of which a welldeveloped gall-bladder occurs while absent in the former. The pancreatic duct is normally without such a resevoir, but a pancreatic bladder has occasionally been observed as an abnormality in the common cat, existing side by side with a normal gall-bladder, the two exhibiting about the same size and proportions.

The terminal portion of the alimentary canal in Amphibia and Sauropsida, and in some fishes (e.g., selachians), enlarges into a cloacal chamber which bears within its walls the outlets of the urinary and reproductive organs, and receives their products as well as that of the intestines. In the Sauropsida and in monotremes the terminal portion of this serves as the functional cloaca and receives also the urinary and reproductive products, but in all mammals except these last the urogenital outlets are emancipated from the alimentary canal, which thus terminates in a rectum instead of a cloaca, and its external opening is a true anus and not a cloacal orifice.

At the junction of the small intestine with the large, there is a strong tendency to form one or more ceca, or blind sacs, which often become digestive organs of great physiological efficiency. The characteristic form in reptiles is that of a single rather short and wide cocum, symmetrically placed. In birds there are usually two symmetrical ones, which attain great length in scratching birds (e.g., the common fowl), and in ducks and geese, but are quite rudimentary in certain others (woodpeckers, parrots, etc.). Ostriches possess a single cœcum of great length ( 7 to 8 meters) and furnished with an internal spiral partition, which greatly increases its effective surface.

In mammals a single cœcum is developed,* which varies greatly in size and functional importance. Rudimentary in

* There are two very short cœca in the arboreal ant-eater, and in the manatee a single cœcum bears two supplementary diverticula. In Hyrax, in addition to a moderately large cœcum, there are two smaller diverticula situated farther down on the colon. 
edentates, most insectivores, and bats, it frequently attains an enormous size in herbivorous or graminivorous forms. In certain rodents (e.g., muskrat, woodchuck), its total capacity equals or exceeds that of the remainder of the alimentary canal, and in the marsupial Phascoloarctus it is three times the length of the body. In the rabbit it is provided with an internal spiral valve; in certain other rodents and in the higher apes and man, the free end becomes rudimentary, restricts its lumen, and forms a worm-like process, the processus (appen$d i x$ ) vermiformis, which, like all rudimentary organs, is subject to a large amount of individual variation.

Thus in the human subject the appendix varies in length between the limits of $2-23 \mathrm{~cm}$., the average for an adult being $8-9 \mathrm{~cm}$. It is longest proportionally during fetal life, its length relative to that of the large intestine being $\mathrm{I}: \mathrm{IO}$, while in adult life it is $I: 20$. It is longest absolutely between the ages of ten and twenty, after which it shows a slight reduction.* Its status as a rudiment of slight functional value is shown by the tendency towards the obliteration of its lumen, a tendency which increases steadily with age.** Furthermore, these two characters, reduction in length and obliteration of the lumen, go hand in hand, short appendices being usually solid, while large ones are apt to possess a lumen.

The position and arrangement of the colon varies considerably among various mammals. In man it begins low down

* Zuckerkandl tabulated the length of the appendix in I6I bodies, with the following result:
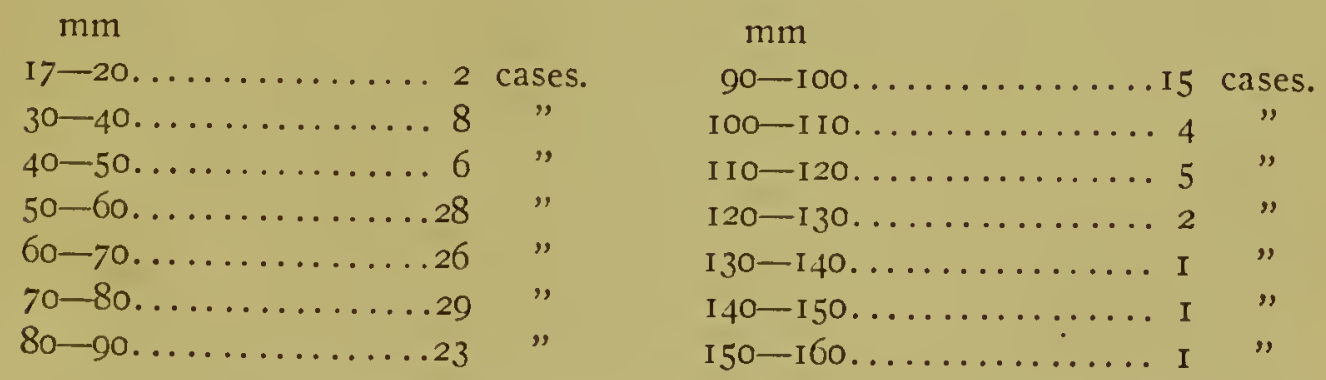

** Wilhelm Müller, from data obtained from I,005 bodies dissected at Jena between 1895 and 1897 , found the amount of obliteration, partial and total, to be as follows: (See table on p. 298.) 
on the right side, from which there proceed in orcler an ascending, transverse, and descending portion, connected with the rectum by a sigmoid flexure, through which the tube attains the median line; a similar disposal is seen in many other anthropoids, in lemurs and rodents, the majority of carnivores, and a few others. A more complex condition than this is produced by the formation of long, narrow loops along the course of either the ascending or transverse colons, or both, and these loops may remain simple or roll into spirals. Such colon labyrinths are seen in ruminants, in certain rodents as the lemmings and jumping mice, and in a few lemurs (Figs. $8_{3}$ and $\left.8_{4}\right)$.

From this brief review of the alimentary canal and its modifications the impression is gained that in this array of enlargements, elongations, diverticula, spiral valves, and other devices, we have to do, not with a consecutive anatomical history, but with numerous special cases of physiological adaptations, developed in response to need; and that a similarity in one of

Continued from p. 297.

\begin{tabular}{|c|c|c|c|c|c|c|c|}
\hline \multicolumn{4}{|c|}{ MALES } & \multicolumn{4}{|c|}{ FEMALES } \\
\hline $\begin{array}{l}\text { Age in } \\
\text { Years }\end{array}$ & $\begin{array}{l}\text { No. of In- } \\
\text { dividuals }\end{array}$ & $\begin{array}{c}\text { No. of } \\
\text { Cases of } \\
\text { Oblitera- } \\
\text { tion }\end{array}$ & $\begin{array}{l}\text { Per- } \\
\text { cent- } \\
\text { age }\end{array}$ & $\begin{array}{l}\text { Age in } \\
\text { Years }\end{array}$ & $\begin{array}{l}\text { No. of In- } \\
\text { dividuals }\end{array}$ & $\begin{array}{c}\text { No. of } \\
\text { Cases of } \\
\text { Oblitera- } \\
\text { tion }\end{array}$ & $\begin{array}{l}\text { Fer- } \\
\text { cent- } \\
\text { age }\end{array}$ \\
\hline o & $4 S$ & o & 0 & 0 & 46 & 0 & o \\
\hline I & $7 \mathrm{~S}$ & 0 & 0 & I & $5^{8}$ & o & 0 \\
\hline $2-10$ & $5 \mathrm{I}$ & I & 2.0 & $2-10$ & 41 & 0 & 0 \\
\hline I $1-20$ & 39 & 2 & 5.1 & II -20 & I9 & I & 5.4 \\
\hline $21-30$ & 47 & 3 & 6.4 & $2 \mathrm{I}-30$ & 23 & 2 & S. 7 \\
\hline $3 I-40$ & 55 & 7 & I 2.7 & $31-40$ & $3^{8}$ & 9 & 23.8 \\
\hline $41-50$ & $S_{4}$ & 22 & 26.2 & $4 I-50$ & 46 & I6 & 34.8 \\
\hline $51-60$ & 73 & I5 & 20.5 & $5 \mathrm{I}-60$ & 60 & I8 & 300 \\
\hline $61-70$ & $5^{\bar{S}}$ & 17 & 29.3 & $61-70$ & 48 & 24 & 50.0 \\
\hline $71-80$ & 3 I & 12 & 38.7 & $7 \mathrm{I}-80$ & 30 & 8 & 26.6 \\
\hline \multirow[t]{2}{*}{$\mathrm{SI}-90$} & I5 & 8 & 53.3 & $8 I-90$ & 17 & 9 & 52.9 \\
\hline & 579 & & & & 426 & & \\
\hline
\end{tabular}

Comparison of the percentage columns shows that in women there exists a greater tendency towards obliteration than in men. The few discrepancies in the table, for example, the smaller average given for men between 50 and 60 , and in women between 70 and So, are doubtless due to the small number of individuals examined. 
these particulars implies, not genetic relationship necessarily, but a similar demand responded to in a similar way. The main object to be achieved in all cases is to regulate the amount of digestive surface to the demands offered by the various kinds of food, and as there is but a limited number of mechanical or architectural devices possible, the same ones are employed in unrelated groups of animals, having arisen independently in response to a similar physiological need. This phenomenon of parallel development (or " analogical resem-

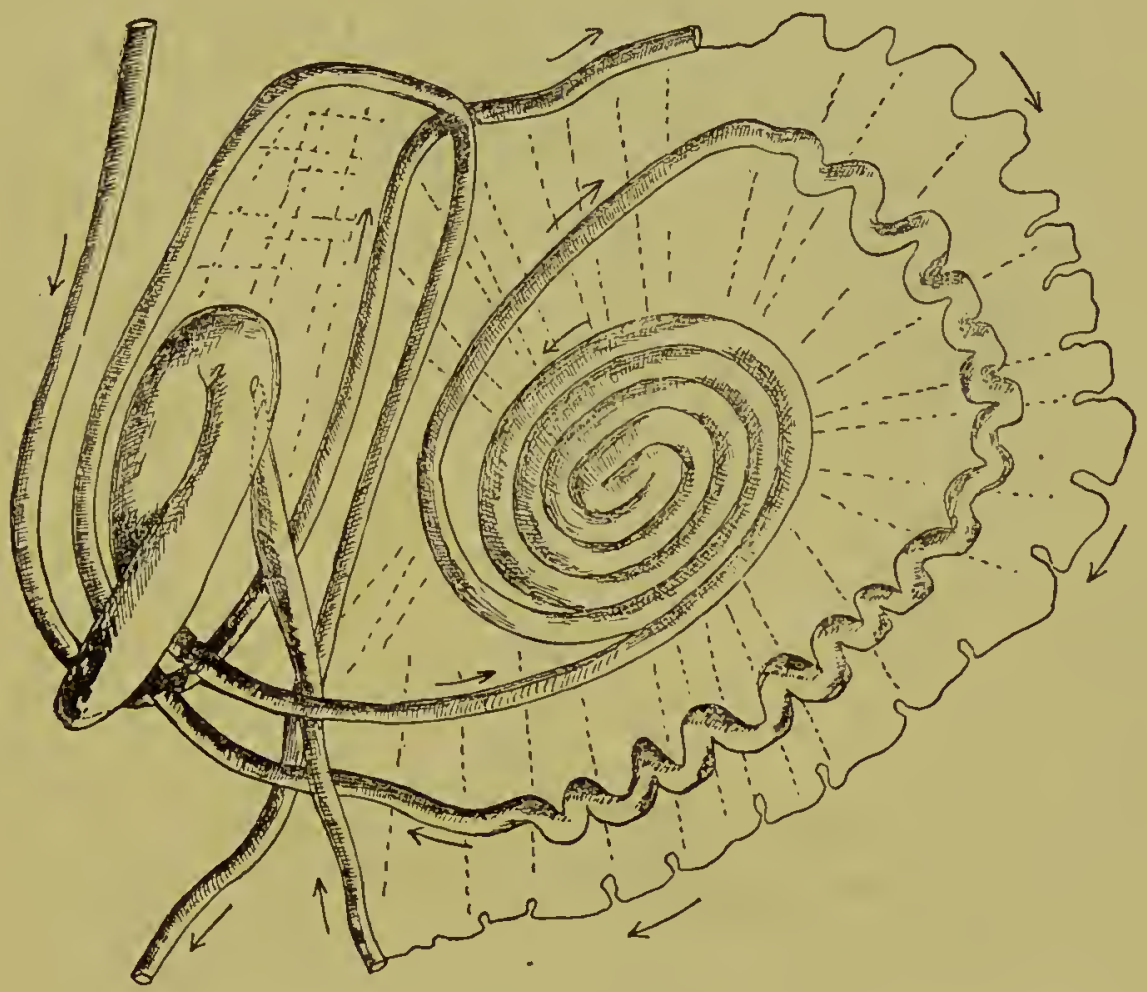

Fig. 83. Colon labyrinth of Cervus canadensis. [After Weber.]

blance," as Darwin calls it), may appear in any system or part and has been a frequent source of error in the estimation of the inter-relationship of animals.

The relation of the total length of the intestine to the kind of food has been frequently emphasized, the idea prevailing that it is short in carnivores and long in herbivorous forms, in accordance with the difference in nutrient qualities and the ease of digestion in the two sorts of food, but this statement is to be accepted only in a general way, as it is subject to 
modifications through the compensation furnished by other factors, such as the special devices just considered. Thus in the ox the length of the entire intestine, small and large, in proportion to the length of the body taken as unity is $20: I$,

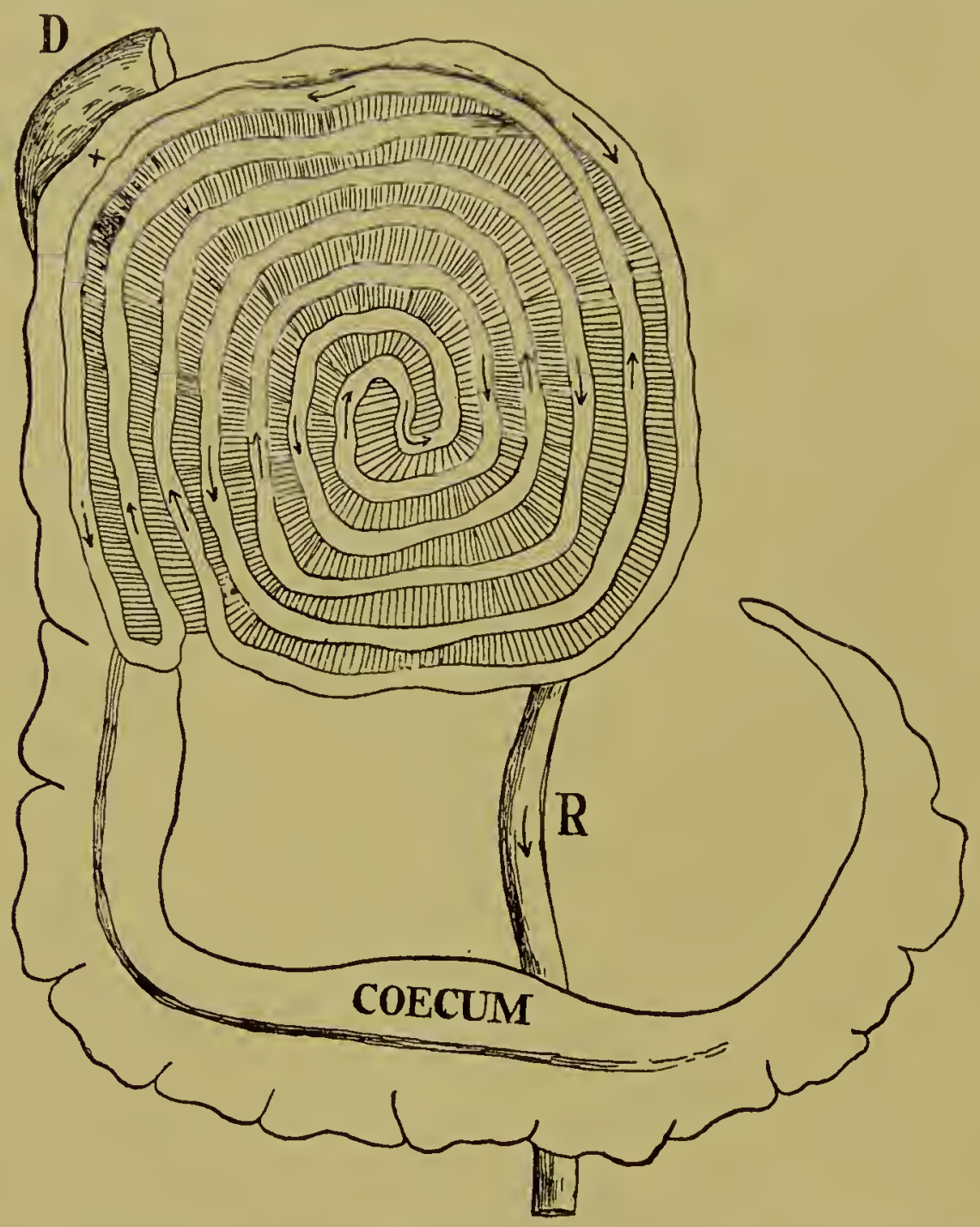

FIG. 84. Colon labyrinth of Propithecus diadema. [From Weber, after VAN LOGHEM.]

while in the horse, which eats similar foods, it is but I $2: \mathrm{I}$, but in this latter animal an enormously developed cœcum furnishes a compensation for the reduction in length of the main canal. Perhaps the greatest extremes of variation within the same Order are shown within the limits of the Cetacea, where the proportionate length varies between $4: 1$ and $32:$. This last, 
probably the largest among mammals, is reported to be that of Pontoporia, a South American dolphin, while the shortest mammalian intestine is that found in certain insectivorous bats, the proportion of which to the body length is 2:I. A change in the length or volume of an organ is, however, so easily effected even during an animal's lifetime, that it is probable that members of the same species may show considerable difference in length of intestine, especially if a comparison be made between specimens from quite different localities where the diet is different. Thus in man, the intestinal canal of the Japanese, whose food is largely vegetable, exceeds in average length by one-fifth that of Europeans; and in whites of medium stature the length of the intestine proper, from pylorus to anus, averages $960 \mathrm{~cm}$., while the average length of the same in nine negroes was but $866.7 \mathrm{~cm}$.

This whole subject, therefore, gives but little indication of phylogeny and is valuable in the present inquiry mainly as an example of the complete correlation between environment and structure. In the examples given mammals have been purposely emphasized and instances of adaptations in the other groups of vertebrates have been omitted as far as possible, since their inclusion would convey the subject far beyond the proper limits of this work.

The function of respiration is the simplest of the major functions, since it consists primarily of an interchange of gases through osmosis, and involves in itself nothing save a moist membrane, with air or aërated water, on one side and blood on the other. The blood must be constantly renewed through some form of circulation, and there is usually some auxiliary mechanism to create a current in the respiratory medium also. It is also imperative that the osmotic membrane be kept moist, a matter of no difficulty in an aquatic animal, but one involving some little additional apparatus, usually an interior chamber with a regulated outlet, in terrestrial forms. Thus in aquatic invertebrates the respiratory membrane is usually external, often a modified portion of the integument. In many minute forms in which the integument is thin, respiration takes place 
through the general surface without the formation of localized organs for the purpose, and in larger forms effective organs of respiration are produced by the formation of external folds or other outpushings of the integument. These are formed in the embryo when the skin is still soft and thin and remain in the adult state unaffected by the process of chitinization which involves the surrounding integument. Such organs are called gills, a general term for all aquatic respiratory organs. These present numerous mechanical devices for increasing the surface; they may be in the form of single plates, sets of plates placed parallel to one another, dendritic structures formed by the repeated branching of simple diverticula, sets of parallel tubes for the blood with interspaces for the water, and so on, and are in most cases provided with accessory structures. some for protection and others for producing a current of water.

In a terrestrial animal, on the other hand, the respiratory system must be internal in order to secure the proper con. ditions of moisture, and as all terrestrial animals are the descendants of aquatic ones that succeeded in adapting them.selves to the difficult environment of land, with its many disadvantages, it forms an interesting study in adaptation to compare the respiratory system in each terrestrial group with that of the animals which most nearly represent their aquatic ancestors. In some cases the old respiratory organs are retained by sinking them into deep recesses kept moist by glands. in others they are discarded in favor of new ones, formed, perhaps, by the transformation of some ready-to-hand cavity, which is lined with blood vessels and made to communicate with the exterior through some regulated outlet. Still another principle is seen in the tracheal tubes of insects, which are branching tubes lined with chitin and leading from a series of external openings into the interior, ramifying all the internal organs. These, as shown by their development, are in origin integumental folds, like the plate-like gills of their ancestors, which, as they develop, turn in instead of out, thus satisfying the conditions of aerrial respiration. 
Essentially different in principle from all of the respiratory methods thus far mentioned is that which utilizes for the purpose some portion of the wall of the alimentary canal, a method employed sporadically among invertebrates, especially the echinoderms, and forming the essential system in vertebrates and allied forms. In this, which, by using the term in its most comprehensive sense, may be called intestinal respiration, the function is usually located near one end of the alimentary canal, for the purpose of obtaining the respiratory medium, and the wall at this place is richly supplied with capillaries, through which the interchange of gases takes place. The respiratory medium, which may be either air or water, is kept in motion by a system of involuntary or semivoluntary muscles, and the motion thus generated is usually rhythmic in character.

In the vertebrates, as well as in those invertebrates that probably represent their ancestors, the respiratory function is located in the pharynx and the respiratory current is primarily taken in at the mouth and driven out through a series of lateral openings, the gill-slits.*

These latter, as seen in the worm-like Balanoglossus, and in Amplioxus, as well as in the embryos of true vertebrates, are seen to be metameric in character, a pair for each somite, and to be arranged in a single row along each side; but in the more specialized group of Tunicata, these rows of slits which appear in the larva become secondarily modified by the formation of numerous cross-bars, so that ultimately the entire pharynx comes to resemble a grating or a loosely woven basket.

The number of these slits is very large in both Balanoglossus and Amphio.rus, but has suffered a considerable reduction

* Aside from the respiration at the anterior end of the canal there are 2. few isolated instances of respiratory action in other parts of its extent. Thus in the teleost Cobitis, an Eastern Hemisphere carp, some respiratory function is possessed by the intestine; and in turtles, two lateral bladders, opening from the cloaca in association with the median, or allantoic bladder, are used for aquatic breathing. 
in true vertebrates, so that even in the lowest of the fishes no more than nine pairs are ever indicated, and this number suffers a constant reduction in higher forms, the loss being progressively from behind forwards. In the lower vertebrates also the effectiveness of the system is increased by the formation, in the endoderm lining the pharynx, of soft structures, richly supplied with blood vessels, which border the gill-slits and form the true respiratory organs, the definite gills or branchice. When, in the history of the race, vertebrates came out of the water and adapted themselves to a terrestrial element, they substituted for this branchial system a pulmonary one, enploying as lungs a pair of sacs which open into the floor of the pharynx a little behind the last gill-slits, and which were undoubtedly in existence at the time of the change, employed as air bladders. In the gradual perfection of this secnnd respiratory system many of the parts of the old one obtained employment, and were one after another selected and modified to add to its efficiency.

This history of the sudden replacement of one system by another, and of the gradual perfection of the second by making over to its own use the material of the first, forms one of the most interesting although most complex bits of anatomical history, and one of which the record has been especially well preserved. As it involves, however, the entire region and includes skeletal parts, muscles, nerves and other elements aside from those which may be strictly termed respiratory organs, much of the history will be found in the chapters devoted to those other parts. Here an attempt will be made to outline the history of the parts as a whole, with special reference to the function of respiration.

The fish type of respiratory apparatus is presented in its most primitive form in the sharks and dog-fish, since numerous modifications which have been acquired in the more specialized fish are absent. It is a type that looks both ways, and, while in many respects similar to that of Amphioxus, from it may be clearly derived the branchial respiratory system of higher forms. Like all special respiratory organs of vertebrates, it 
is essentially pharyngeal and consists primarily of a series of lateral pockets in the walls of the pharynx, which break through to the exterior and form slits. These openings are metameric in arrangement and are paired, each pair corresponding to a single metamere as expressed in the associated organs, but show considerable reduction in number from those found in Amphioxus, the functional slits in most cases being limited to five pairs. In two especially primitive genera, how-
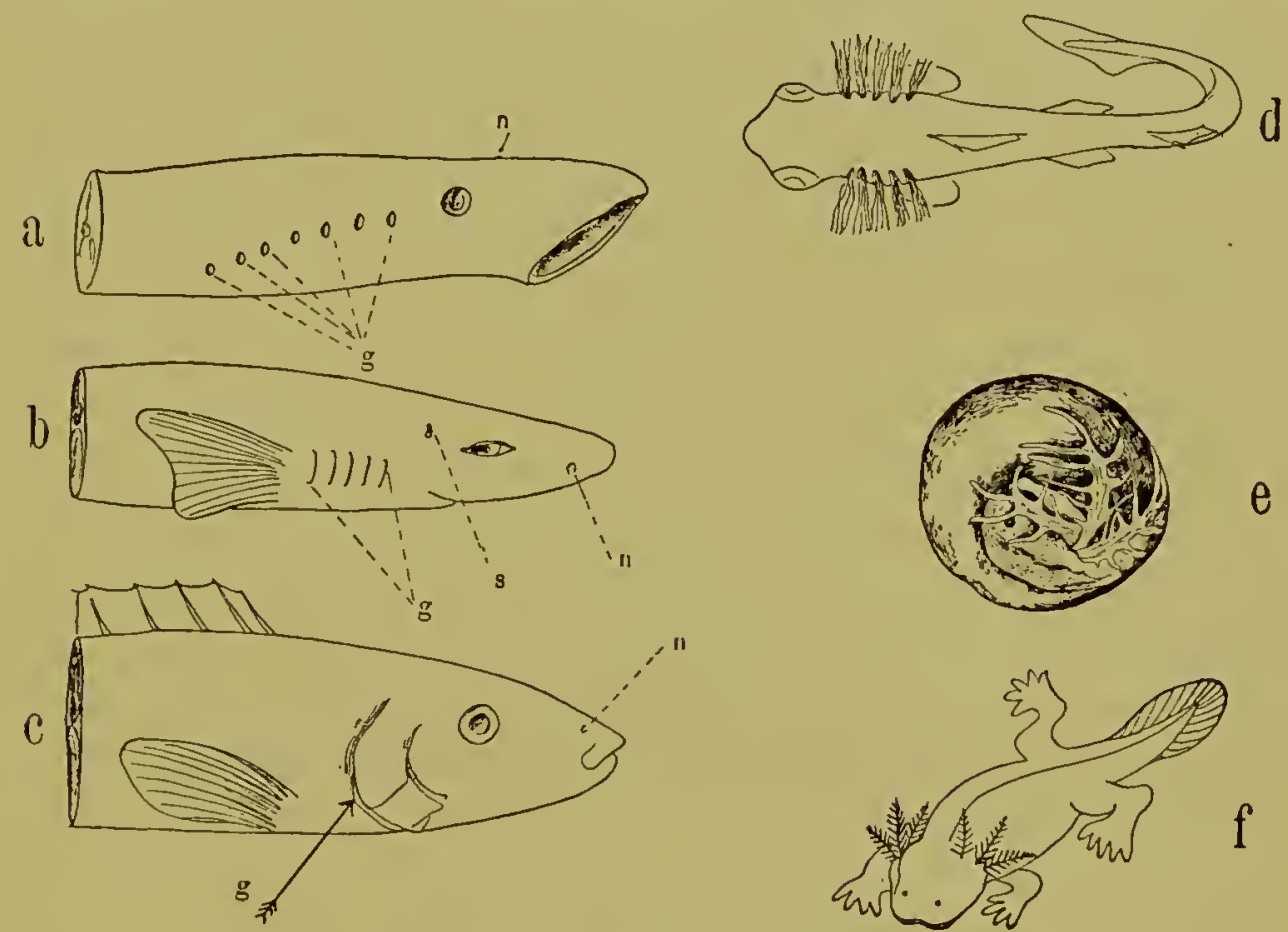

FIG. 85. Respiratory organs.
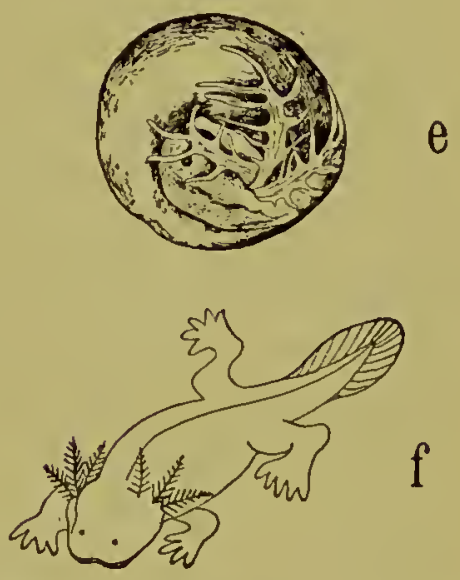

(a) Cyclostome. (b) Selachian. (c) Teleost. (d) Selachian embryo. (e) Am. phiuma larva. (f) Cryptobranchus larva [from a Japanese print].

$n$, nostril; $s$, spiracle; $g$, gill-slits.

ever, Hexanchus and Heptanchus, there are respectively six and seven, facts which suggest that the number at present represents a reduction from a previously more extensive series, the reduction being from behind forwards. Upon the pharyngeal side of these slits there develops a series of soft organs in the form of fringes or tubes, which consist of localized elaborations of the pharyngeal wall, the gills or branchice. These are profusely vascular and are supplied with a rich capillary network developed between two sets of branchial arteries, the 
afferent branchials, which bring the bluod directly from the heart to the gills, and the efferent branchials, which collect the blood from the gill capillaries and unite to form the main aorta. Since the blood is aerated in the capillary network of the gills it follows that the blood coming from the heart through the afferent vessels is impure, while that in the efferent vessels is pure; and since these latter unite to form the main aorta, this vessel, the branches of which supply the entire body, contains only aerrated blood, while the heart is employer merely to receive the venous blood which returns from all parts, and to send it to the gills. This is the primitive type of vertebrate circulation, and obtains not only in all fishes, but reappears as the early form in all vertebrate embryos, thus proving its fundamental character as an historic stage.

The essential respiratory cavity is thus the entire pharynx, through which a current of water is kept in constant flow by being taken in at the mouth and exhaled through the gillslits; and while in earlier forms, as suggested by Amphioxus, the capillaries lie in the unmodified pharyngeal wall in the vicinity of the gill-slits, the selachians show a considerable advance by the formation of definitely localized organs, with a large increase of surface, and thus physiologically more efficient.

In other fishes this gill-system, which is essentially similar to the foregoing, exhibits several secondary modifications, the most apparent of which is the formation of a large gill-flap (operculum), which arises in front of the most anterior slit and extends backwards, and as the slits become closely approximated and are reduced in number to four, the operculum becomes capable of closing entirely over them, meeting a ridge of integument behind the last slit (Fig. 85, a). The current of water is directed by rhythmic respiratory movements, which consist of opening and closing the mouth and operculum, the motions of the two alternating with each other.

With the fishes true internal (endodermic) gills pass away, but in the permanently aquatic salamanders and in all larval amphibians one or more slits break through, usually two to 
three, in connection with which certain integumental structures arise which are gills physiologically, but are unrelated to the former. The most widely distributed form of these is that of the crternal branchice, three in number upon each side, and attached to the cartilaginous gill-arches. In structure they are usually plumose or dendritic (Fig. $8_{5}$, e and $\mathrm{f}$ ), although in a few cases they are thin and leaf-like. The slits appear between these, with occasionally an additional one in front of the first, and the animals obtain fresh water for respiration in part by forcing a current through these slits in the manner of fishes, and in part by waving the branchiæ up and down by means of special muscles with which these organs are furnished. As stated above, external branchiæe are characteristic of the larvæ of all amphibians, and are found permanently in a few aquatic salamanders, which are either more primitive than the rest, or are pædogenetic, that is, they retain the larval form while becoming sexually mature. These salamanders are called perennibranchiate in distinction from those in which the branchiæ become lost, the caducibranchiate salamanders. A second form of gills which are external in origin but become internal in position, occurs in frog larvæ, where they replace the former, which appear at first. As these are plate-like and are attached to the gill-arches, they have often been considered exactly homologous with the gills of fishes, but their ectodermic origin renders such a conclusion impossible.

Aside from the two sets of branchiæ most amphibians possess definite lungs, which arise in the larva and exist for a time side by side with the external branchiæ, usually replacing them in later life. These are often in the form of simple sacs, without any formation of internal partitions, and even when in their highest development, as in frogs, are far from complex. It thus seems probable that, although they are true lungs anatomically, they play a subordinate rôle in respiration, and are perhaps primarily used either for regulating the specific gravity of the animal in the water, or in the production of the voice, since the larynx is often very large and curiously 
specialized, and is of considerable importance in the production of sexual calls. The slight respiratory importance of the lungs in amphibians is further emphasized by the fact that in a large number of species of salamanders, both lungs, trachea and larynx are entirely wanting, although in a few cases rudiments of these parts attest the former presence of these organs.

The question will naturally occur at this point: what are the means of respiration in adult amphibians if they have lost their branchix and yet possess either no lungs at all or those of slight functional importance? The answer to this lies in the fact that amphibians have developed two other systems, neither branchial nor pulmonary, the assumption of which shows how great may be the systematic response to a physiological need, and suggests also the trying period of transition when vertebrates first essayed the terrestrial environment, and when attempts were made in all possible directions to adapt themselves to the new respiratory medium. These two systems are the integumental and the pharyngo-esophageal, and as both of these demand for their highest efficiency a moist environment with an occasional submersion in water, they are successful in amphibians with their semi-aquatic mode of life, but in higher forms have been discarded in favor of the pulmonary system, which enables its possessor to leave the marshes and inhabit the dry land.

The origin of the assumption of a respiratory function by the amphibian skin may be traced to the abundance of integumental glands, inherited from the fishes and used to protect the surface from the action of the water. The presence of these glands necessitates the formation of a superficial network of capillaries to supply them with nourishment, and the integument becomes thus transformed into an organ that possesses the qualities necessary for a respiratory organ, that is, a moist surface bathed by the respiratory medium and supplied with a rich capillary net-work. Thus apparently by accident, as in all morphological changes, an organ which becomes modified for a certain function shows a capability of assuming a second one, not intended in the original plan, and 
the moist, glandular skin becomes an effective respiratory organ.

The pharyngo-œsophageal system appears to be a special compensation for the loss of the lungs, and is present in only those salamanders in which the pulmonary system has been lost (Fig. 86). Here again the incentive towards the formation is a moist membrane richly supplied with capillaries,

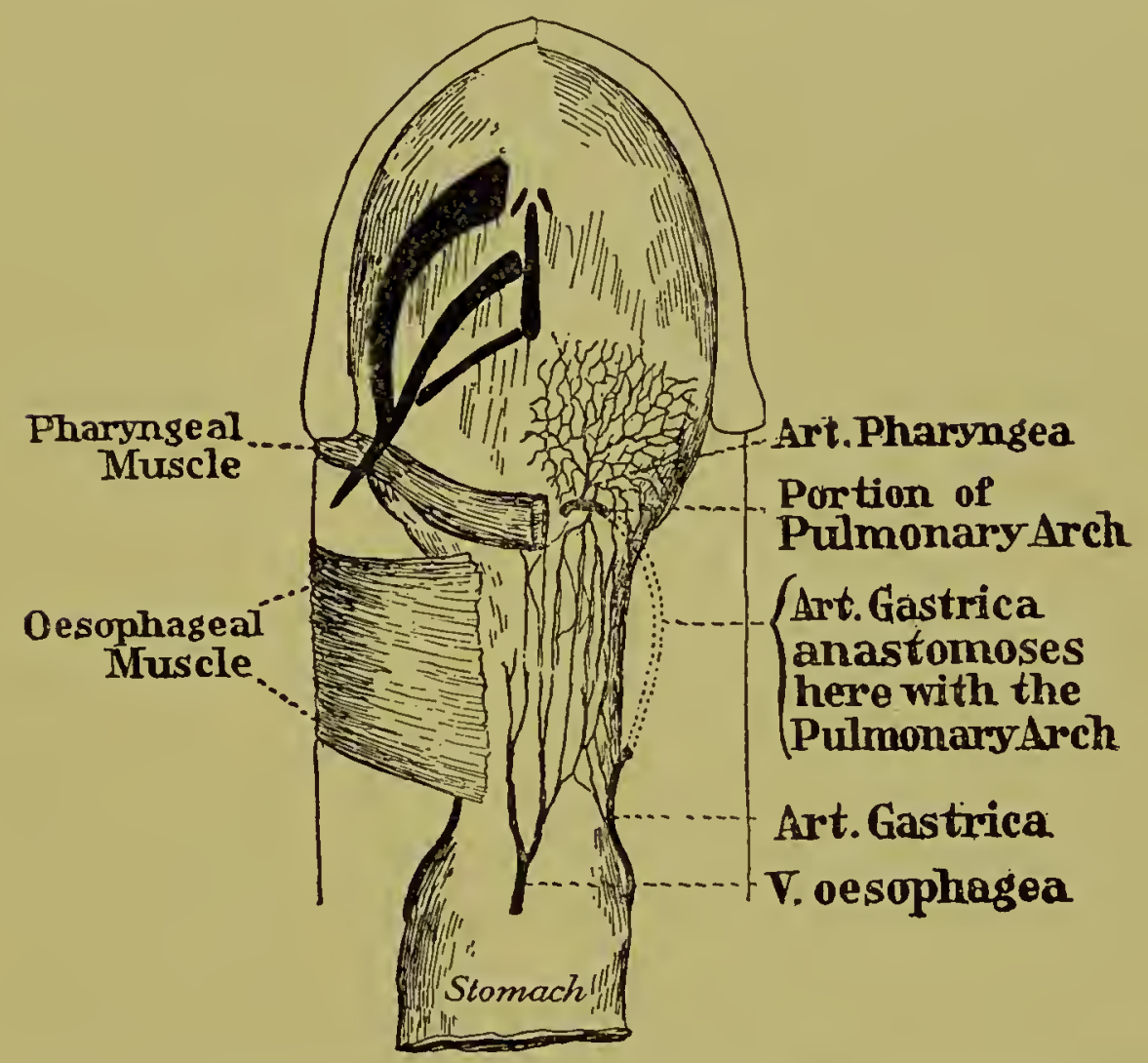

FIG. 86. Pharyngo-œsophageal lung of Desmognathus, showing pharyngeal and œsophageal muscles, and the net-work of blood-vessels in the walls of the pharynx and œsophagus.

in this case, the mucous lining of the pharynx and œsophagus. The natural vascularity of this structure has been considerably increased, while the capillaries themselves have become more superficial and even invade the external epithelial layer, the only case known. The muscles of the lost pulmonary system have been in part retained, and through their aid, together with that of others which are developed for that purpose, the 
pharynx and osophagus are dilated and contracted in association with the usual respiratory movements of nostrils and floor of the mouth, and the anterior part of the alimentary canal thus becomes a functional lung with the power of inspiration and expiration, forming doubtless a more efficiont organ than the simple air-sacs which these salamanders allowed to atrophy.

Above the amphibians, which, with their numerous methods of respiration, suggest the experimentation of our early ancestors in their attempts to occupy what must have been at first an unnatural environment, the pulmonary system becomes supreme, and its further development is shown principally in the increased efficiency of its two main organs, the lungs and the larynx. The later history of this system is quite well known, especially that of its development in terrestrial vertebrates, but the origin of the system is still in part obscure, and rests upon surmises rather than upon actual proof.

The history begins with the period represented by fishes, during which the pharynx exhibits a tendency to throw off median diverticula, sometimes dorsal and sometimes ventral, for the purpose of forming pneumatic cysts or air-bladders to add to the buoyancy and thus aid in swimming. In many cases these become closed and depend upon the adjacent blood vessels for the gases with which they become distended, but in others the original connection with the pharyngeal cavity is retained and the two are kept in communication through a small duct. In this latter case the cyst is filled with air, which is expelled and renewed through the mouth when the fish is at the surface of the water, a proceeding that demands some sort of regulator at the orifice of the duct, an opening to which, by an extension of meaning, the term glottis may be applied. Such an apparatus, which consists of muscles and fibro-cartilage, is a functional larynx, of which there must be two distinct organs, a larynx dorsalis, and a larynx ventralis, in accordance with the position of the pneumatic cyst. That cysts in these two positions cannot be homologous is evident; indeed, those in the same position in fish not closely related are not neces- 
sarily the same, yet until the subject has been thoroughly investigated, the latter may be assumed to be the case.

In several ganoids either one or the other of these pneumatic systems becomes complex in character and serves as an accessory respiratory organ. The air-bladder functions as a lung; it becomes honeycombed with connective tissue partitions, and is profusely vascular, thus forming an organ of far greater functional activity than the definite lungs of many amphibians; corresponding to this its larynx, the regulator of the air supply, develops an extensive set of muscles and masses of fibro-cartilage. That such a structure, when dorsal in position, as in Lepisosteus, cannot be the precursor of the final pulmonary system of higher forms is self-evident, but when such an organ is ventrally placed, thus corresponding exactly to the embryonic stages of the latter, as in Polypterus, such an homology, although not definitely proven, is very likely. As for the dorsal system, there is no indication that it is represented in any way above the fishes.

If, however, the ventral air-bladder of Polypterus is identical with the paired lungs of higher forms (which begin as a single median diverticulum that divides later into two branches), the larynx can be the same only in respect to its opening, the glottis, since the accessory parts, that form the functional organ, are derived from two totally different sources in the two cases. In the fish larynx the hard parts are derived from the adjacent connective tissue, and are composed of fibro-cartilage, which represents as it were the first stage in cartilage formation and differs but little from a compact form of simple connective tissue. The muscles are evidently slips differentiated from the muscular walls of the pharynx. That this forms a very effective organ cannot be denied and, had no better material for a laryngeal mechanism becn furnished, that of the ganoid with its fibro-cartilage and slips of pharyngeal muscle rould have undoubtedly developed to fill all the necds of a pulmonary system, eron including the functions of roice and speech. It chanced, however, that at this period, the fifth branchial arches with their accompanying muscles, 
emancipated from all respiratory function, and employed in a desultory way as tooth-bearing structures or as parts assisting in deglutition, were lying in the immediate neighborhood, one on each sicle of the glottis but a little anterior to it, and equipped with well-differentiated muscles; and it may well have happened that little by little these parts may have usurped the function of the other apparatus, being better fitted for the purpose.

Be that as it may, when, after a succession of forms that have become lost, the curtain rises upon the lowest of the amphibians, this very pair of arches is seen lying, one upon each side of the glottis, and forming with its muscles a primitive though very effective larynx. These cartilages are proven to be the actual 5 th pair of gill-arches through the identity of their nerve supply, and the weak point in the story is the identity of the two pulmonary systems, that of the ganoid and the definite one found in terrestrial vertebrates, a point not yet proven; but, granting this, a theory which seems extremely probable, the rest must follow. In all events the history of both lungs and larynx from the amphibians on is a continuous one, and the latter organ, equipped at the start with the 5 th branchial cartilages and their associated parts, becomes more complex by the gradual addition of other arches, proceeding from behind forwards, each accommodating itself in shape and position to the especial function desired in each case.

The simplest amphibian larynx is that of the perennibranchiate salamander Necturus, where the two cartilages in question are in the form of flattened triangular pieces, the lateral cartilages, placed one upon each side of the glottis (Fig. 87, a). A short membranous trachea, entirely without cartilaginous support, leads to the bag-like lungs. In an allied form, Proteus (Fig. 87, b), a slight advance is seen in the fact that the posterior angles of the lateral cartilages are more prolonged and appear as slender processes which are applied along the sides of the entire trachea as far as the bronchi. These in adult animals show a tendency to separate from the main mass. This differentiates the cartilaginous 
pieces into an anterior pair of arytcenoids, upon either side of the glottis, and a posterior pair of tracheal picces. Within the Class of Amphibia there are no new pieces formed beyond these, but they exhibit a great variety of forms, and become especially complex in the Anura, where they are employed in the production of various sorts of notes used as sexual calls (Fig. 87, c-e). The muscles associated with these skeletal elements consist originally of a pair of dilatators, which are attached to the outer edges of the cartilages and serve to draw them apart, and a double pair of adductors, the laryngei, which stretch across from one to another and serve to approximate them. These give rise in many of the more complicated

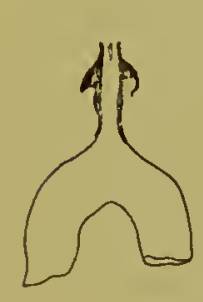

a

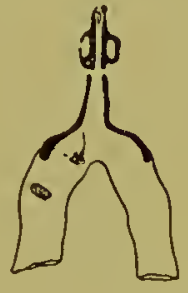

b

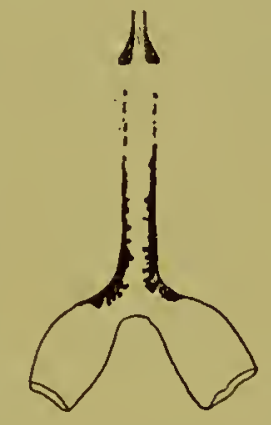

c

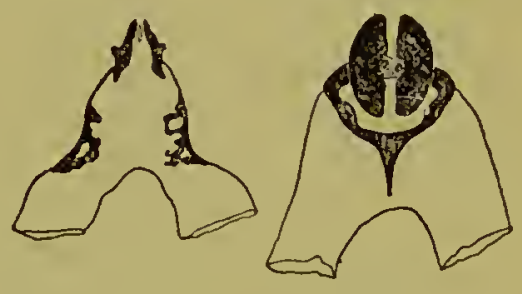

d

FIc. 87. Laryngeal cartilages of various Amphibians.

(a) Necturus (mud-puppy). (b) Proteus. (c) Amphiuma. (d) Triton (Newt). (e) Rana (frog).

cases to an entire system of muscles, mainly connected with the arytænoid cartilages, which form the essential skeletal organ of the larynx, and to which the vocal cords in the form of mucous folds become attached.

In the Sauropsida there are two conspicuous points of advance; the one concerns the larynx, the other the trachea. The first consists of the addition of the $4^{\text {th }}$ pair of branchial cartilages, which become reduced in size, unite in the middle and form a triangular flap, the epiglottis; this, during passive breathing, stands erect above the glottis but shuts down over the latter during the act of swallowing, thus preventing the entrance of solid food into the trachea. The second advance consists of the presence of a series of rings of approximately 
equal size, which embrace the trachea and protect it from collapse. These are deficient behind, where the trachea comes in contact with the osophagus, as a provision to allow the passage of a large mouthful, but are strongly developed in front and serve to keep the trachea distended. The most anterior of these rings is much heavier than any of the others and is probably formed by the fusion of several of them. It is known as the cricoid cartilage and is topographically considered a part of the larynx. The tracheal rings must have been developed in some way from the tracheal pieces that segmented off from the lateral cartilages, but the manner of their formation is not known. Similar rings occur in the trachea of the Gymnophiona, the rare Order of subterranean amphibians, but whether these are homologous with those of the reptiles and birds has not yet been determined.

There is but little variation in laryngeal form among the representatives of the Sauropsida, and this in spite of the great differentiation of voice in the case of the birds, since in these the voice is produced, not by the larynx, but by a special organ, the syrinx, or lower larynx, situated at the forking of the bronchi and not found outside of the group of birds.

In mammals a conspicuous addition is seen in the thyreoid cartilage. The origin of this piece is not apparent in placental mammals, in which it appears as an extensive shield, covering the ventral surface of the entire organ, but in the more primitive monotremes, instead of the single shield-like piece, there are two pairs of narrow bars which from their origin and their similarity to the more anterior ones, as well as from their mode of development, are clearly seen to be branchial arches, evidently the 2nd and 3 rd (Fig. 88). This leaves only the first arch, which in this Order unites with the true hyoid arch to form the hyoid complex ("hyoid bone" of human anatomy), to which it contributes its posterior cornua, the thyreo-hyals. The cricoid cartilage is much as in Sauropsida and is manifestly the result of the consolidation of certain of the upper tracheal rings.

The development of the lungs is mainly along the lines of 
physiological efficiency through a repeated subdivision of the interior. This results in the production of small chambers, commonly known as "air-cells," more properly alveoli, which are connected with the bronchi by means of numerous smaller branches. The walls of these alveoli are covered with a network of capillaries, thus making them the ultimate organs of respiration to which all other parts are accessory. Primarily there are no cartilages in the lungs themselves, but in reptiles they may be seen to develop along the course of

a

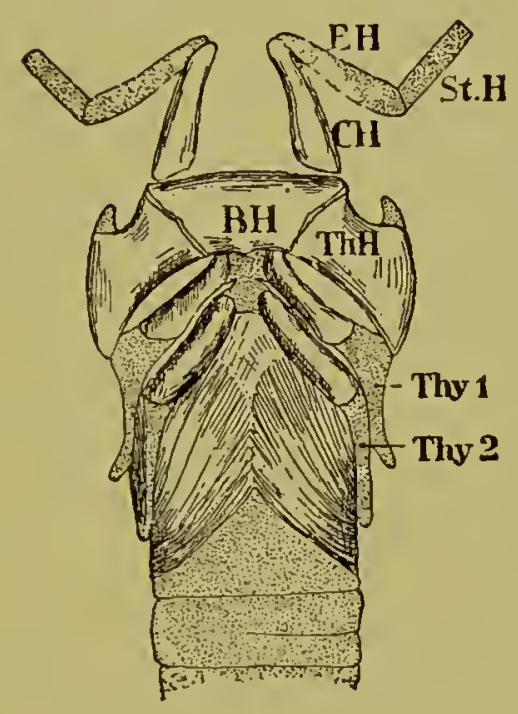

b

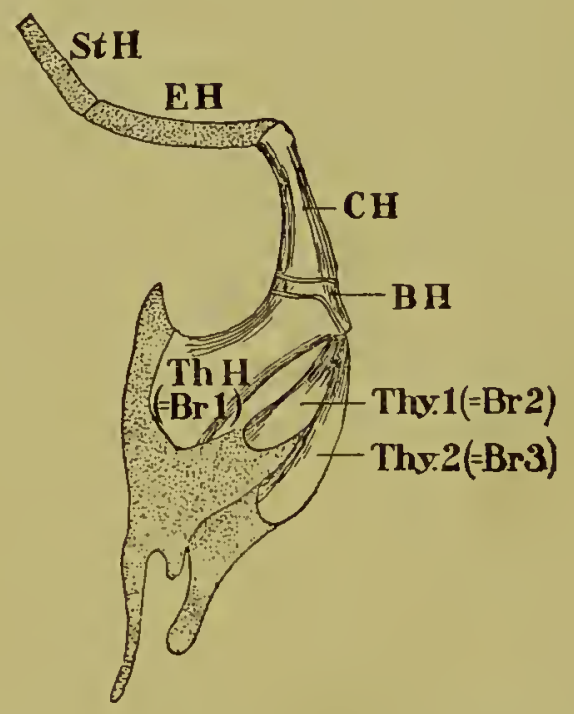

FIG. 88. Larynx of Echidna (monotreme). [After GøPPERT.]

(a) Ventral. (b) Lateral.

St. $H$, stylo-hyal; $E H$, epi-hyal; $C H$, cerato-hyal; $B H$, basi-hyal; $T h . H$, thyrohyal; Thy, I, first thyreoid cartilage; Thy, 2, second thyreoid cartilage.

the bronchi and invade the lungs; in mammals the smaller bronchial tubes are similarly equipped, almost as far as the ultimate branches, although in the course clownwards the rings become less complete and are finally reduced to irregular pieces lying in the sides of the tubes. The smallest tubules, which are without cartilaginous pieces, are termed bronchioli.

In birds and in many mammals the lungs are subdivided by grooves into lobes, but in other cases the grooves are shallow, and the lobes become hardly more than slight protuber- 
ances. Although quite constant in number and arrangement in a given mammal there is the greatest variation of the lobes in forms not closely related; and that these parts are of slight physiological importance is shown by their complete absence in mammals quite unlike structurally and occupying different environments. Thus the lungs are without lobes in the Cetacea, Sirenia, and some seals, thus suggesting a modification due to an aquatic life, but on the other hand the lungs are similarly undivided in sloths and ant-eaters, and in certain rodents, as mice and squirrels. The left lung in the elephant is also without lobes.

The development of a diaphragm in mammals separates the general body-cavity into thoracic and abdominal portions and cuts off the pleura, which invests the lungs and lines the thoracic cavity, from the peritoneum, which stands in similar relationship to the abdominal viscera. These changes cause some variation in the mechanism of breathing, in which the diaphragm becomes a powerful accessory organ. 


\section{CHAPTER VIII}

\section{THE VASCULAR SYSTEM}

"However, if we consider that all the characteristics which have been cited are only differences in degree of structure, may we not suppose that this special condition of organization of man has bcen gradually acquired at the close of a long period of time, with the aid of circumstances which have proved favorable? What a subject for reflection for those who have the courage to enter into it!"

LAMARCK in Recherches sur l'Organization des corps vivans. I802. Transl. Packard, Igor.

A VASCULAR system of some sort occurs in all cœlomate animals, except in some reduced parasitic forms, and consists essentially of a cavity, or series of connected cavities, in which a fluid circulates, containing detached cells of one or more kinds. Both fluid and cells are concerned in metabolism and act as carriers of material both to and from the various tissues. In many Metazoa, especially the smaller and less highly organized ones, the system is lacunar, and the circulating medium, here often termed the perivisceral fluid, occupies everywhere the irregular spaces between the organs, and its circulation is furthered by the movements of these latter and of the entire body; in other cases the lacunar system becomes reinforced, or largely replaced, by the formation of definite channels in the form of branching tubes, through which the fluid circulates. Such a circulation is said to be closed, in distinction from the lacunar or open type first mentioned, and in such a system, deprived as it is of the propelling power insured by the movement of external parts, dependence must be placed upon some intrinsic force within the vascular system itself, and thus there arise pulsating vesscls, 
certain localized portions of the system of tubes, the walls of which are caused to dilate and contract rhythmically through the development of a layer of involuntary muscles.

Vertebrates possess the tubular or closed type of vascular system, reinforced by a few definitely localized lacuna, and indirectly aided by the various serous cavities of the body like the colom and the capsules of the joints. Both anatomically and physiologically this system is divided into two subordinate systems, homal and lymphatic, of which the first is the one principally emphasized, while the other bears to it the relationship of an important auxiliary. The tubules of the first system are divided into the heart, a localized pulsating vessel with enormously hypertrophied muscular walls; arteries, in which the current flows from the heart; veins, in which the current flows toward the heart; and lastly capillaries and simsoids, two forms of the minute vessels which extend between the arteries and veins and supply every tissue of the body. To these, which collectively bear the name of blood-vessels, there are associated a few definitely bounded lacunce, here spaces limited by membranes, and mainly differing from the rest of the system, into which they are continued, by the absence of walls of their own. The circulatory medium contained in this system is termed blood, and consists of two main types of cells, the erythrocytes or "red blood corpuscles," and the leucocytes or "white blood corpuscles," suspended in a liquid plasma.

The auxiliary system consists primarily of lymphatic vessels, which in distinction from the veins and arteries are small and thin-walled, and of lymph glands, which are not glands in the usual sense, but storehouses for leucocytes. With the lymphatic system are associated the serous cavities of the body (cœlom, capsules of joints, bursæ about the larger tendons, etc.), with which numerous lymphatic vessels communicate so that, by a physiological though not a morphological right, these cavities have been considered as expanded lymphatic vessels. In the lower vertebrates a number of definitely located pulsating organs, or lymph hoarts, further 
the circulation of the liquid medium, which is here termed lympll and consists of plasma containing leucocytes alone.

These two latter elements constantly escape from the blood through the walls of the capillaries during the process of feeding the tissues, and it is one of the functions of the lymphatic system to collect these by means of its smaller vessels and eventually to return them to the blood. The other main function of the lymphatic system is to aid in the extraction of digested foods from the alimentary canal and convey them also to the circulatory system.

In no system of the body does the embryonic record tell the story of the race development so completely as in the case of the circulatory system, and although the change in vertebrate history from water to air, replacing one set of respiratory organs by another, has profoundly modified the blood-vessels, yet even this change is repeated with great fidelity in the individual life of each of the higher vertebrates. This might be expected of the amphibians, in the most of which the actual change of external environment is individually experienced, yet a similar metamorphosis in the circulatory system takes place in Sauropsida and Mammalia, although it is confined to embryonic life.

Since this is so, the best introduction to the history of the circulatory system is that furnished by embryology, the early part of which may be here given in the form of a general sketch, which, althoigh not intended to represent the details of development in any one animal, or even of any one group, yet is based rather more upon the development of the higher vertebrates, since in these alone is the story complete. In beginning this sketch a certain characteristic of nearly all vertebrate embryos must be emphasized, since it is closely connected with the circulatory system, especially in its earlier stage, and that is, the extra-embryonal yolk-sac, which develops a set of blood-vessels for the purpose of feeding the embryo.

In this is seen a probable reason why the history of this system is retained in so much more perfect condition than 
are most of the others, and that is, because this system is actively functional almost from the beginning of embryonic life, while in other cases the parts lie passive and let themselves. gradually assume the final shape without contributing anything to the functional life of the organism, a condition most favorable to the suppression of intermediate stages.

The early vertebrate embryo, during its cleavage stages, appears most frequently as a circular disc of rapidly proliferating cells floating on the surface of a spherical or spheroidal yolkmass; and although at first these cells possess sufficient energy within themselves to continue development, there soon comes a time at which they become dependent upon the nutriment stored in the yolk, and it is thus one of the earliest cares of the organism to develop blood-vessels for the purpose of carrying yolk granules to the embryonic area.

These blood-vessels first appear as irregularly branching spaces on the surface of the yolk beyond the limit of the definite embryo; these spaces soon form themselves into a capillary net-work and unite upon each side of the embryo into a vitelline vein.

Within the embryo a similar process lays down the first blood-vessels and the entire system appears as in Fig. 89, A. The two vitelline veins unite into a median vessel, the future heart, situated ventrally with reference to the embryo, and immediately back of the future gill region. Further anteriorly the median vessel divides and forms two lateral loops, the first arterial gill-arches, which continue around the pharynx until they come in contact with one another upon the ventral side of the notochord, from which point they run backwards, forming two aorte.

At a point a little posterior to the entrance into the embryo of the vitelline veins, the aortæ pass mainly into the formation of two vitelline arteries, which spread out over the yolk. but the small vessels which continue into the posterior end of the embryo form morphologically their real continuation. During later development the posterior aortc fuse into one and increase greatly in size so that the proportions between 
them and the vitelline arteries become reversed; and, as this part of the embryo expands and develops legs, tail, and pelvic organs, these latter become supplied by secondary branches from this main trunk. A similar arterial supply to the head region is furnished by the artery which develops anteriorly from the arterial gill-arch. In the figure it appears as a mere stump, but is destined to become the carotid artery, which in-
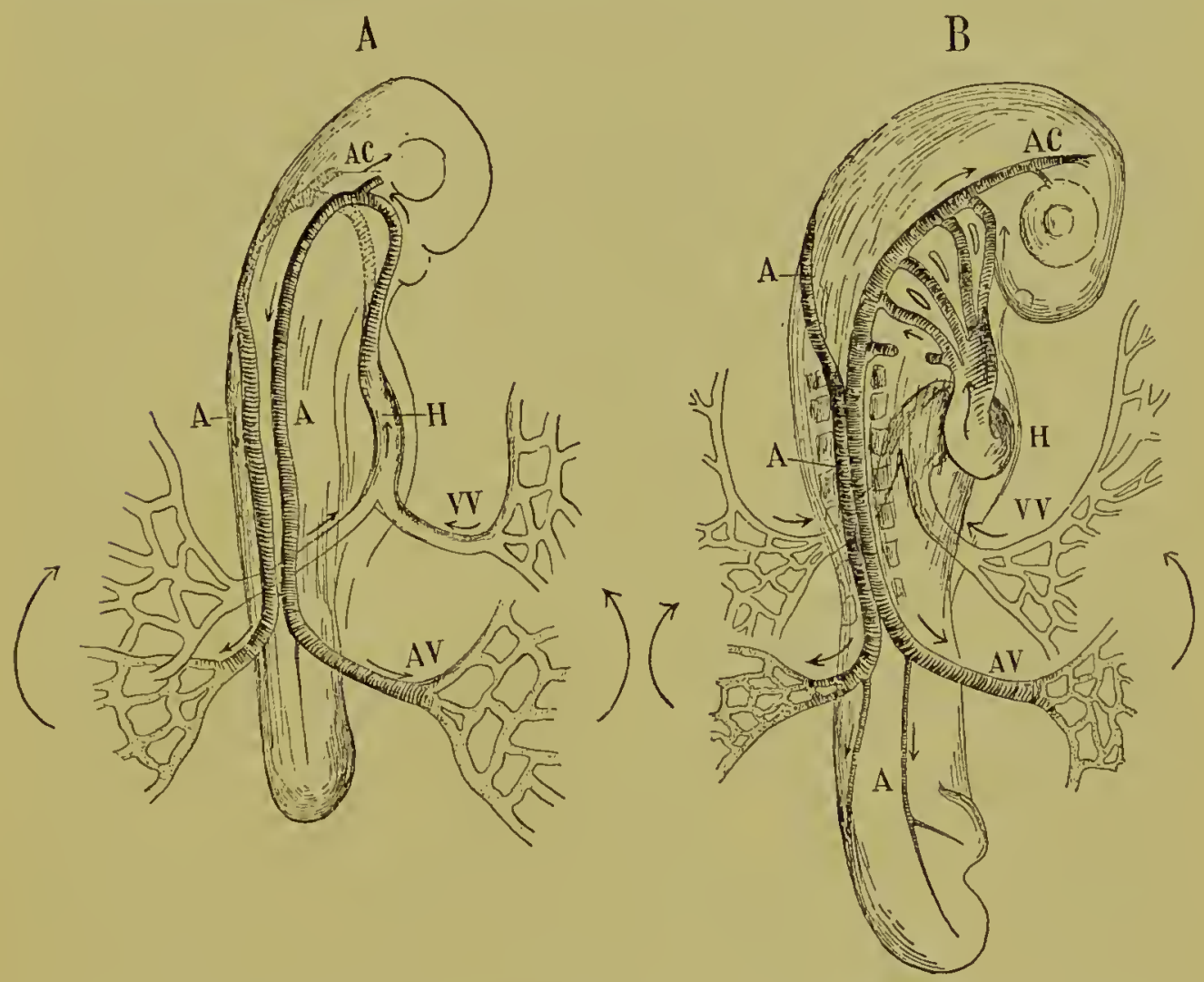

FIG. 89. Early circulation of vertebrate embryo.

(A) First appearance of definite vessels. (B) Later stage, during the formation of gill arteries. heart.

$A C$, carotid artery; $A$, dorsal aorta; $A V$, vitelline artery; $V V$, vitelline vein; $H$,

creases in size and the complexity of its branches in exact proportion to the development of the part which it supplies. As these last two vessels, carotid artery and posterior aorta, distribute the blood ouside of the main channel, a new set of vessels must be developed to bring it back again and thus complete the circuit. Those appear in the form of the four cardinal veins, two anterior and two posterior (not shown in 
the figure), which collect the blood sent to the growing tissues of the embryo by the arteries and return it into the main channel. The anterior and posterior cardinals of each side unite opposite the heart and form a lateral vessel, the duct of Cuvier (ductus Cuvieri), which enters the heart from the side immediately after its formation through the union of the two vitelline veins. In this system of vessels is seen the first systemic venous system, the function of which is to collect from the body the blood supplied it by the arteries and return it to the heart.

A considerable advance is seen in Fig. 89, $\mathrm{B}$, which repre.sents a somewhat older embryo. The heart has increased both in caliber and in length, which has caused it to assume a somewhat contorted attitude, the prelude to those later changes which will result in the formation of a compact organ with definite compartments. To the single arterial loop which forms the first arterial arch in the gill region others have been added in a posterior direction, the general method of formation being shown by the last one, in this figure the 5 th. The appearance of limbs has caused the development of arteries to supply them, subclavians for the anterior, and iliacs for the posterior; these are duplicated by veins associated with the cardinal system.

At about this stage a striking change, but one the significance of which is mainly embryonic, consists in the development of the bag-like allantois with its accompanying bloodvessels, the allantoic (or umbilical) veins and arteries (see Fig. I7). This appears indeed in amphibians as an evagination from the ventral wall of the cloaca, where it functions as a urinary bladder, but here it never surpasses the limits of the body; in Sauropsida and Mammalia, however, it develops into an enormous extra-embryonal organ of great functional importance. It begins in the form of a small sac that pushes its way out from the embryo, and is supplied with two arteries from the posterior aorta, and two veins which enter the heart in association with the vitelline vein, but it soon increases greatly in size, and its blood-vessels increase proportionately. 
Ultimately, in Sauropsida, animals with very large eggs encased in a porous shell, the allantois comes to line the entire shell and serves as the embryonal respiratory organ; in mammals it forms the main part of the placenta and umbilical cord, and functionally replaces the yolk sac, which is here a useless rudiment, although equipped with its full complement of blood-vessels. In both cases the allantois is cast away from the embryo at birth, hæmorrhage being prevented by an atrophy of the blood-vessels at the point at which they leave the body.

Further important modifications of the circulatory system are caused by the development of liver and kidneys and by the increase in bulk of the intestine. Owing to an original continuity between the yolk sac and the intestine, the veins from this latter organ empty into the vitelline veins, forming a compound vein, composed of intestinal and vitelline branches, the omphalo-mesenteric. Of these the right one does, not develop beyond a certain point, and the main, and ultimately the entire, duty falls upon the left. About this the developing liver grows, and in such a way as ultimately to include it within its substance, and as a result of this that part of the vein which runs through the liver becomes divided into a system of capillaries. The result of this is that the blood coming from both yolk and intestine has no longer any way of getting directly into the heart through a large vessel, but must first pass through the capillary system of the liver, and be re-collected upon the other side. From this stage on the single omphalo-mesenteric vein, that originally of the left side, becomes known as the portal vein, and the collecting vein upon the other side of the liver, which brings the blood from that organ into the heart, forms the hepatic. Throughout this portion both of the original vitelline veins are preserved, and it thus happens that there are two hepatic veins, but only one portal.

A similar change is that inaugurated by the development of the embryonic kidney. The blood comes back from the tail in a median caudal vein, which, posterior to the cloaca, divides 
into two branches. These pass along the outer sides of the kidneys and are resolved entirely into a set of small branches, the vence renales advehentes, which enter these organs and break up into capillaries. From these the blood is recollected by veins which emerge from their inner edges, the vence renales revehentes, which unite to form the posterior cardinals.

There is thus formed a portal system similar to that of the liver, and called the renal portal, in distinction from the latter, the hepatic portal. This relationship is a permanent one in fishes and amphibians, but in the Sauropsida and Mammalia the kidneys in which this portal system is developed function as such only in the embryo, and become eventually replaced as kidneys by a new organ in connection with which no such portal system becomes developed.

Thus far in the development of the circulatory system all Classes of vertebrates agree, allowing for slight differences in the relations of the extra-embryonal parts, such as the relative development of the allantois, or the amount of yolk; one Class, the lowest, that of fishes, remains permanently at this stage, while the others progressively modify the fundamental plan. It may be well, then, to consider the condition in the adult selachian, which is practically that of the foregoing sketch, after which the later development of the various parts of the system may be taken up one after another.

The circulatory system of the selachians is represented in the accompanying diagram (Fig. 90), in considering which the reader may begin at the heart and trace the vessels anteriorly. The heart is situated very far forward, immediately behind the gills, its embryonic position in higher animals, and consists of four chambers arranged in a single longitudinal row along the median line. The most posterior of these, the sinus venosus, is the receptacle into which is brought the impure blood from all parts of the body. Next in order, into which the blood passes in succession, are the atrium, the ventricle, and the comus arteriosus. This last and most anterior compartment is prolonged into an arterial trunk (truncus arteriosus), which breaks up into 
paired lateral branches, the afferent branchial arteries. These pass along the cartilaginous gill-arches and supply the gills, dividing into very fine branches for the purpose. Thus far

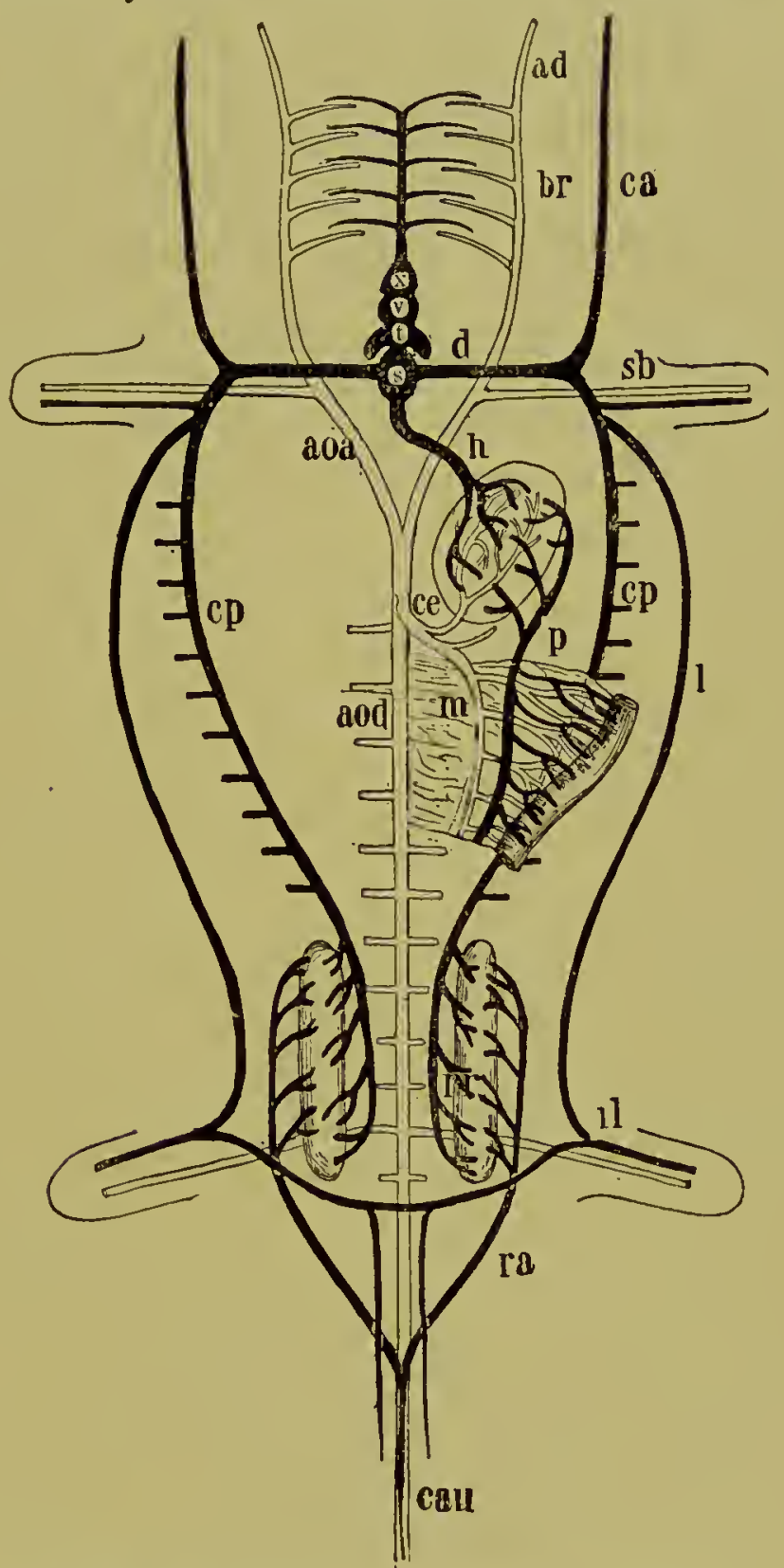

FIG. 90. Diagram of primitive vertebrate circulation, based on the condition found in selachians.

$s$, sinus venosus; $t$, atrium; $v$, ventricle; $x$, conus arteriosus; $b r$, branchial artcries: $a d$, carotid artcry: ao a, aortic arch; aod, dorsal aorta; $c c$, coliac axis, consisting of (m) mesenteric, and licpatic and splenic (unmarked) branches; can, caudal arteries and veins; $i l$, iliac arteries and veins; $s b$, subclavian arteries and veins; ra, rcnales advchentes; $r r$, renales revchcntes; $l$, lateral vein; $c p$, postcrior cardinal vein; $c a$, antcrior cardinal vein; $p$, hcpatic portal vein; $h$, hepatic vein.

the blood is impure, in the state in which it was received from the body, but at this point there intervenes a system of capil- 
laries, in which the exchange of respiratory gases takes place, and when it is re-collected into the efferent branchial arteries, corresponding in number to the afferent branchials, the blood has become aërated. These latter arteries converge to the median line, where they unite to form a median aorta, which lies upon the ventral side of the vertebral centra, and gives off the main arteries of the body. Before the arches of the two sides unite they give off the carotid arteries, which supply the head and brain; and then, not far from the point of union, the subclavians, to the anterior paired limbs (pectoral fins). Lower down appear branches that supply the body walls and the viscera; and the posterior paired limbs (ventral fins) are supplied by the iliacs. As these branches are given off, the aorta diminishes in size and terminates at the end of the tail as a mere thread, protected throughout the caudal region by the hæmal arches of the vertebræ.

The entire body is thus supplied with aërated blood from a single main channel with its branches, but on its return its course is not so simple, and involves three distinct venous systems connected with one another by capillaries. The first of these consists of four great longitudinal veins, the two anterior and the two posterior cardinals, which collect the blood from the head, the anterior fins, and the walls of the trunk. As in the embryological sketch, the anterior and posterior cardinal veins of each side unite into a ductus. Cuvieri, which enters the sinus venosus. Associated with the posterior cardinals are the two large lateral veins which lie in the body wall and were perhaps originally situated along the bases of the lateral fin-folds, from which the paired limbs have been derived. They arise as very small vessels along the sides of the tail and enlarge rapidly as they proceed anteriorly through the assumption of tributary branches from each somite. In the cloacal region the two lateral veins communicate by numerous anastomosing branches, forming a cloacal plexus (represented in the diagram by a single vein), and receive the iliac vcins from the posterior fins. Anterior to this they still receive metameric contributions from each somite and finally empty 
into the posterior cardinals near their fusion with the anterior ones to form the ductus Cuvieri. The subclavian vein from the anterior fin enters either the lateral vein or the posterior cardinal near the entrance of the latter. In the former case, which may be considered the more primitive, we have the suggestion of the early relation of the lateral vein to the fin-fold, for this condition suggests strongly a primitive one in which the lateral vein received a branch from each metameric element of the fin-fold. When the definite limbs were established by the hypertrophy of an anterior and posterior region and the loss of the intermediate portion, the veins corresponding to the regions retained became large and important, while the rest were somewhat reduced. To account for the retention of a single vein for each appendage, rather than one from each somite represented, one may suppose either the retention of one and the loss of the others, or the fusion of several. Since, in the pelvic fin of the skate, there are, in addition to the principal iliac vein, one or two small vessels which open independently into the lateral vein, the former alternative is the more probable.

The second system begins by a median caudal vein, which starts at the lip of the tail and runs within the hæmal arches, upon the ventral side of the aorta. When near the cloaca this vein divides into two lateral branches, which run along the lateral margins of the long and narrow kidneys, and give off to these organs numerous lateral branches, the vence renales advehentes. These break up into a capillary system within the substance of the kidneys and form the renal portal system. From this capillary net-work the blood is collected along the medial margin of the kidneys by numerous vence renales revehentes, the union of which into a common trunk forms the origin of each posterior cardinal.

The third, or hepatic portal system, is exactly as given in the embryological sketch. It collects the blood from the intestines and stomach into a common trunk, the portal vein, which enters the liver upon its dorsal side and becomes resolved into capillaries, as in the former case. From this organ the blood is re- 
collected by one or more hepatic veins, lying more on the ventral side of the liver, and is cmptied into the sinus venosus. Thus all the impure blood, through one channel or another, finds its way into this most posterior chamber of the heart, from which it passes in succession through atrium, ventricle, and conus arteriosus, and finally into the gills, where it becomes aërated.

It thus happens that the heart contains only impure or venous blood, since it is not purified until it reaches the gills, which suggests that the terms "arterial" and "venous," as applied to pure and impure blood respectively, are not applicable in the case of the lower vertebrates, and are much better dropped, since they are often misleading. Furthermore, in Amphibia and Reptilia these two kinds of blood are not sharply defined, since both sorts are often allowed to mingle, forming a mixed blood of varying degrees of purity. All confusion on this point, however, may be avoided if the terms artery and vein and their corresponding adjectives are used in their anatomical sense only, arteries being, as previously defined, those vessels in which the blood flows from the heart, and veins those in which the blood flows towards the heart. The physiological distinction which designates pure blood as arterial and impure blood as venous is taken from its condition in the two sets of vessels in birds and mammals, and even here in the case of the pulmonary system the conditions are reversed and physiologically arterial blood flows in the veins, and physiologically venous blood in the arteries.

The history of the arterial arches is shown in synoptical form by the accompanying series of diagrams (Fig. 9I), which present the facts as deduced from the combined study of both the adult anatomy and embryological development of representatives of each Class of vertebrates. The diagrams represent the adult conditions in each case, the relationship being morphologically. interpreted by the help of the development.

There are typically six pairs of arterial arches, which lie along the sides of the pharynx and extend from a ventral vessel that proceeds directly from the heart to a dorsal one that col- 
lects the blood from the arches and conveys it to all parts of the bocly, the ventral and dorsal aorte respectively (Fig. 9I, a). In all the diagrams the parts of both sides are shown, viewed ventrally and flattened out so that the ventral aorta lies in the middle and the dorsal aorte converge from the outer sides. In selachians (Fig. 9I, b) five of these arches are present and functional; each arch is clivided into an afferent and an efferent branch, between which respiration is effected by means of capillaries spread out over soft endodermic gills. From the anterior portion of the efferent system the carotics are given off, vessels which include the only remnants of the first arterial arches.

In the urodelous amphibians (Fig. 9I, c) the first two arterial arches disappear in the embryo, leaving four functional arches." Of these arch III unites with remnants of $I$ and II to form the carotids, IV and $\mathrm{V}$ on
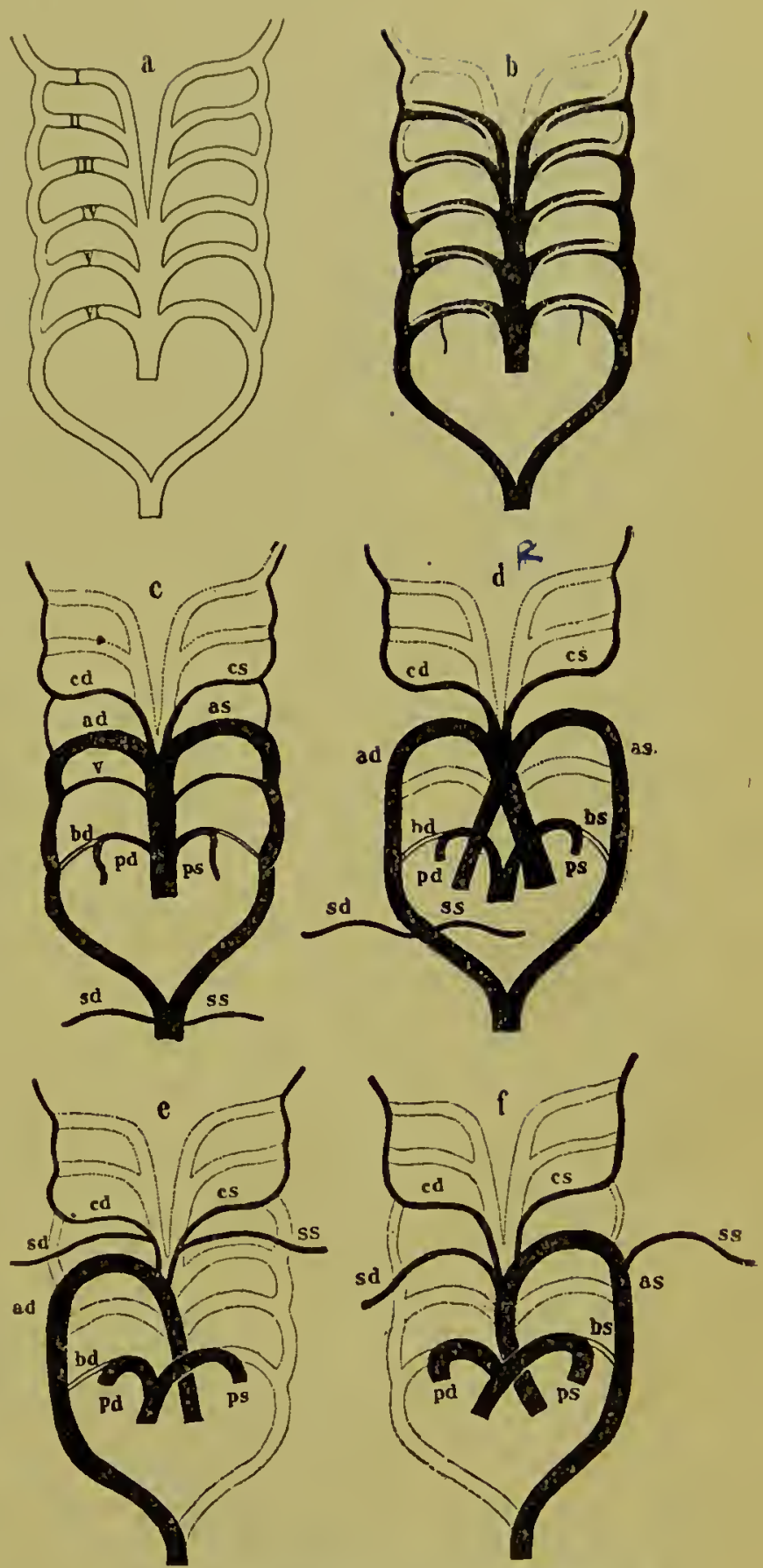

Fig. 91. Diagrams showing modifications in the arterial arches of Vertebrates.

(a) Typical, embryonic. (b) Fishes. (c) Amplibians. (d) Reptilcs. (e) Birds. (f) Mammals.

1, II, III, IV, V, VI, arterial arches; $c d$, Art. carotis dextra; cs, Art. carotis sinistra; st, Art. subclavia dextra; ss. Art. subclavia sinistra; ad, Aorta dextra; as, Aorta sinistra; $b d$. Ductus Botalli dexter; bs. Ductus Botalli sinister: $p d$. Art. pulmonalis dextra; $p s$, Art. pulmonalis sinistra. 
both sides form complete arches and unite to form the dorsal aorta, and VI becomes the pulmonary (here the pulmo-cutaneous). Of the two aortic arches IV is the principal one and $\mathrm{V}$ is a subordinate, and is of such slight functional importance that in the higher Classes it is destined to disappear altogether. These arches are usually continuous, and are not as a rule interrupted in the midst by the interposition of respiratory capillaries as in fishes; in larval urodeles, however, and in a few adult forms, the perennibranchs, a branch from the ventral side of the arch supplies the external gill-bushes with capillaries, from which a collecting branch returns the blood to the arch at its dorsal end. When such a gill-bush is of much functional importance these lateral branches are large, and in extreme cases it is possible that practically all the blood of a given arch may pass through these indirect channels. In most cases the external gills, and with them their supplying branches, disappear at the expiration of larval life, and the arches form continuous vessels, as in higher forms. The sixth arch is in the larva a complete one, and joins the dorsal aorta, as do the two preceding; with the development of the lungs and the integumental respiration a small branch, which arises from this arch near the middle, becomes engaged in supplying the lungs and skin, and increases so much in size that it ultimately transmits all of the blood that enters the arch, leaving the distal half of the arch without employment. This part then closes its lumen and is retained as a connecting band, the ligamentum arteriosum [Botalli], extending along its old path between the pulmonary artery and the dorsal aorta. A similar ligament, or in many cases a small pervious artery, is also retained between the carotid arch and the main aortic arch (III and IV).

In reptiles (Fig. 9I, d) the metamorphosis of the arterial system is pushed back into embryonic life, and, from this point on, no longer appears after birth. In other words, the transition from water to land, an historic scene actually enacted during the post-natal existence of amphibians in the form of the metamorphosis, with all the changes involved, not only in 
the circulatory, but in other systems as well, is pushed back among the stages that are recapitulated in the embryo; there is a metamorphosis in reptiles and mammals just as truly as in the case of amphibians, but it is embryonic. Here, as in amphibians, arch III, with rudiments of I and II, forms the carotids and its connection with arch IV disappears. This latter becomes the aortic arch, and is retained on each side, as right and left arches, the two uniting dorsally and back of the heart to form the main aorta. Arch V, which in amphibians is practically superfluous, is given up in reptiles, and from this point on is seen no more, save in the embryo, where it often appears as a rudiment. A pulmonary artery develops from the sixth arch of each side, as in amphibians, leaving a right and left ligamentum arteriosum [Botalli]. The subclavian arteries, that supply the fore-limbs, which in most fishes and amphibians arise from the dorsal aorta after the union of the two lateral arches, possess a more anterior origin and arise from the right aortic arch. Crocodiles and turtles present an exception to this, and in these, as in birds, the subclavians arise from the base of the carotids, an origin so radically different as to lead morphologists to believe that these vessels are not the subclavians at all, but are secondarily developed arteries (subclavia secundaria) which have functionally replaced the true subclavians.

/ In birds and mammals (Fig. 9I, e and f) but a single aortic arch comes to development; in birds this is the one on the right side, in mammals the one on the left, a convincing proof, if proof were wanting, of the independent development of these two Classes. There is thus, in each case, but one ligamentum arteriosum, connecting the pulmonary and aortic arches. In the mammalian fetus, in which pulmonary respiration is not assumed till the moment of birth, this vessel is functional and is known as the ductus arteriosus [Botalli]. It is still pervious at birth, but the lumen closes within a few days by the rapid thickening of the wall of the vessel. There is here to be noted an important difference also in the subclavians; in birds, as in the turtles, these vessels are repre- 
sented by sub-clavic secundaric, branches of the carotids, but in mammals they are the primary vessels, homologous with those of typical reptiles. There is, indeed, a new morphological distinction between those of the two sides, for while the left one, that of the side which furnishes the aortic arch, arises from this arch, that of the right is the equivalent, not only of that of the left side, but of the fourth arch as well. It is this relationship which causes the intimate association upon the right side between the subclavian and carotid, and the short common trunk, arteria anonyma [innominata], is thus the region once common to arches III and IV. Thus, while these vessels on the right side are superficially similar in both birds and mammals, they are morphologically totally different. In the former the "subclavian" is a secondarily formed branch of the carotid, with the true subclavian probably suppressed; in the latter the "subclavian" is the fourth arch plus the true subclavian that once branched from the point where this arch joined an aorta, as on the other side.

In this relationship is seen also the explanation of the curious asymmetry in the origin of the human carotids and subclavians, a condition which is undoubtedly a primitive one. This becomes more complicated in many other more specialized mammals by various secondary approximations and fusions. Thus, in the Carnivora the left carotid fuses near its base with the other and produces the phenomenon of three of the four arteries in question arising from a common stump, while the left subclavian is alone distinct: in ruminants this latter also shifts its position forward and fuses with the others, so that a single median trunk arises from the crest of the arch. This gives off first the two subclavians, and then, after continuing forward a little, divides into the two carotids.

Ontogenetically the most anterior of the six arterial arches is the first to appear, and this, with the ventral and dorsal aortæ, forms a lateral loop directed forwards. The other arches are successively added through the formation along the course of the aortæ of buds or sprouts that meet and join. In fishes all but the first of the arches are retained, but in higher 
forms, where the first two become lost, these begin to degenerate before the more posterior ones appear. In the mammalian embryo there are in the appearance of these arches two important points to notice; first, the successive supremacy in size and function of each arch down to the fourth, and, second, the extremely rudimentary condition of $\operatorname{arch} \mathrm{V}$, amounting in some cases to a complete suppression. This is shown in the rabbit embryo in the four states given in Fig. 92. In $a$ arch $I$ is the principal, or, in fact, the only functional one, and II and III are forming from approximated dorsal and ventral buds. In $b$ arch $I$ has become disintegrated, while the chief function is assumed by arch II: the ventral bud of arch IV is also seen. In $c$ both first and second arches are lost and their remnants appear in part as continuations of dorsal and ventral aortæ and in part as stumps of vessels from which important branches of the carotid system are to be developed. Arch $\mathrm{V}$ appears at about its maximum here and in the next figure, and the ventral bud of arch VI has become well developed. In $d$ arch IV, to be later 'the permanent aortic arch, is assuming good proportions, and arch VI, the future pulmonary arch, is completed. From the dorsal side of the dorsal aorta appear the beginnings of certain transitory arteries that correspond to the head somites.

The complete ontogenetic history of the carotid system in mammals shows a curious shifting of branches from one source to another, and the development and decay of transitory elements. In this the remnants of arches I and II, long supposed to be lost, play a prominent part, and unite with the main carotid arch (III) in the formation of the system. This history, together with that of the other arterial arches, is seen in the accompanying set of diagrams (Figs. 93 and 94), based, with the exception of the last one, upon the embryology of the rat. In $a$ is seen the first arch [Cf. Fig. 89, $a$ ], which starts from the conus arteriosus $(c a)$, proceeds forward, and returns as the dorsal aorta $(a d)$, giving forth a carotid, the arteria carotis cerebralis $(c c b)$, just before returning. The ventral bud of arch II has also appeared. In $b$ arch II has 


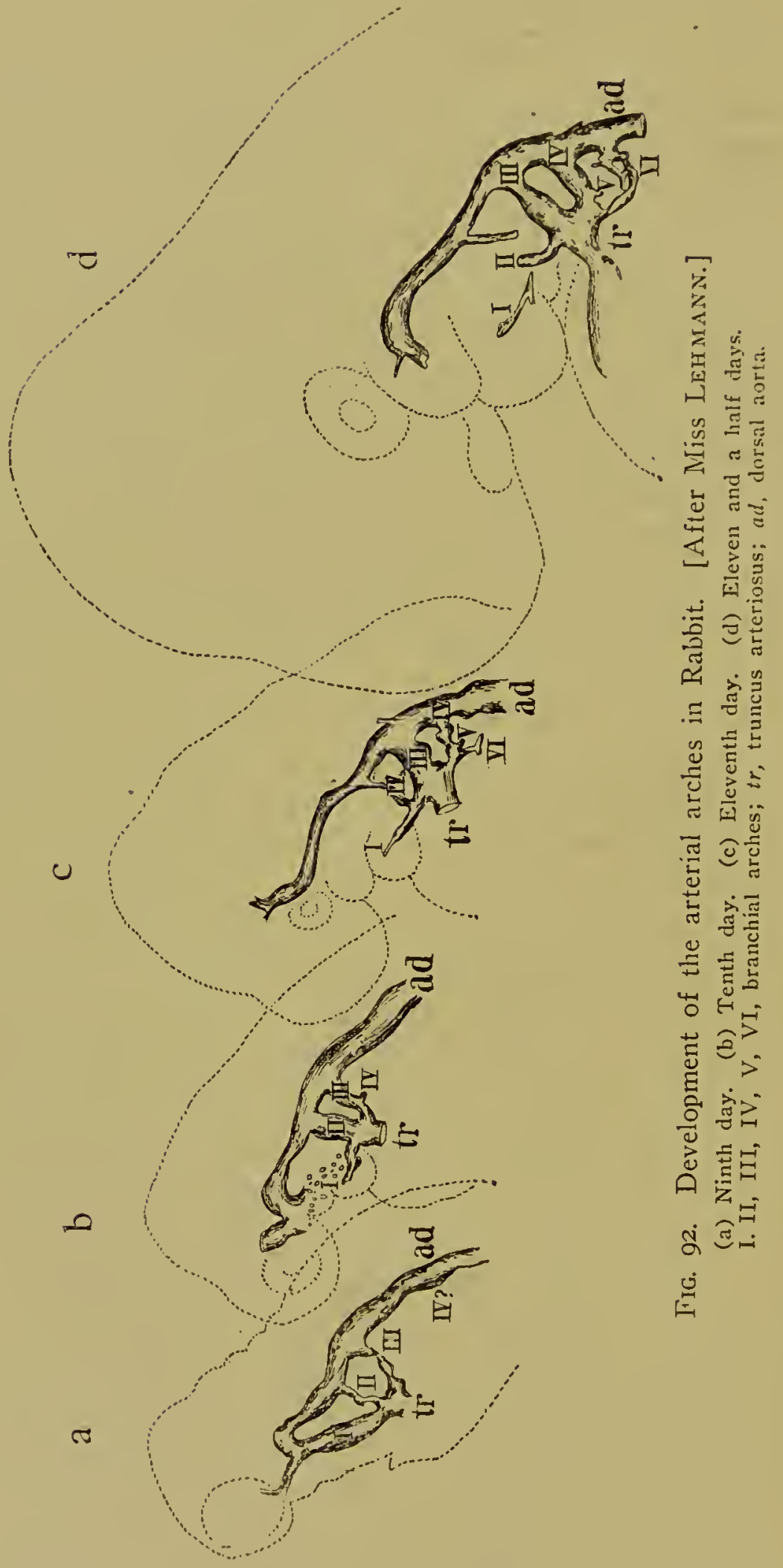


become completed and arch III is forming from ventral and dorsal buds, and in $c$, arches I, II, and III are complete, with a ventral bud forming for arch IV. Arches IV and VI are both formed in $d$, with several "islands" in the former, which probably have no especial significance. Arch I has broken, leaving dorsal and ventral stumps.

From the dorsal aorta appear segmental arteries $(s)$ which soon disappear; of these the lowest is the hypoglossal (hy). Below this are other segmental arteries, of which the first cervical $(e c)$ is figured here. Stage $e$ shows but little change save in proportions and the loss of segmental arteries. A pulmonary artery appears, arising from arch VI. The human embryo at about this stage shows a well-developed arch V. The segmental arteries of the head have disappeared. In $f$ arch II has also broken through, leaving stumps, and of these the ventral becomes closely associated with that of arch I, and both are borne by the anterior end of the ventral aorta (av), a part destined to play an important rôle later on. Arch III has become large; arch IV is very large, and a rudiment of arch $\mathrm{V}$ has appeared. The dorsal stump of $\mathrm{I}$ has divided into two branches, the maxillaris ( $m s$ ), which goes to the developing upper jaw, and the mandibularis $(m d)$, which becomes distributed to the lower jaw.

In stage $g$ the maxillary artery just mentioned has divided again into a supra-orbital (o) and an infra-orbital ( $i$ ), thus giving three terminal branches of the dorsal stump of arch $\mathrm{I}$. From the free end of the ventral aorta $(a v)$ appears a branch that goes to the tongue-anlage, the lingualis $(l)$. The point especially to be noted here is that of the two buds, $(x)$ and $(y)$, which arise from the dorsal stumps of arches I and II, respectively, and grow toward one another. The formation of the arteria vertebralis cerebralis $(v c)$ by the union of the hypoglossal and first cervical arteries with one from much further forward is also to be noticed, but is without special interest. In stage $h$ the buds $(x)$ and $(y)$ have united the dorsal buds of I and II, and the significance of this step is seen by comparing this with stage $i$, for here the portion connecting the com- 

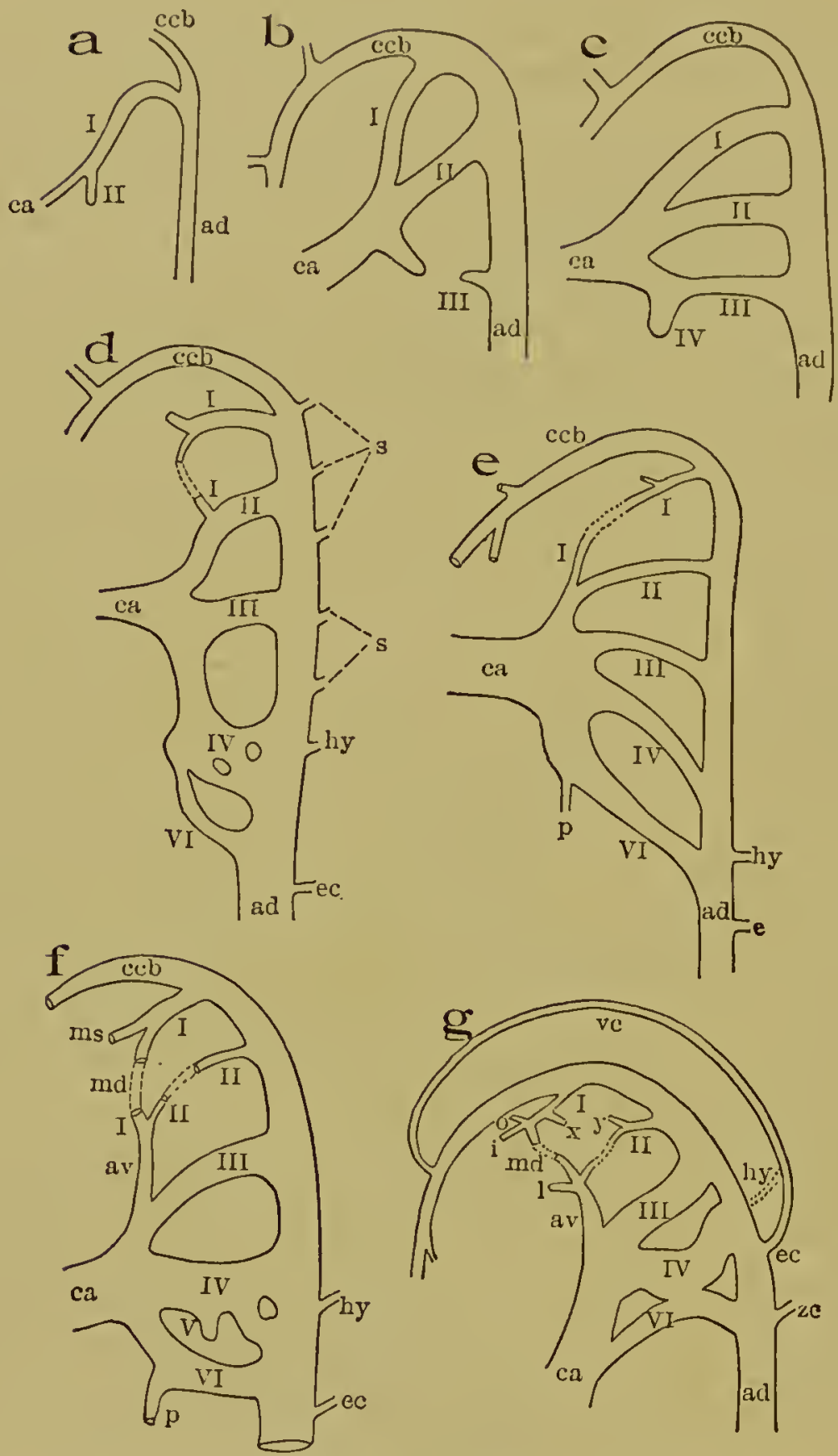

FIG. 93. Development of arterial arches in Rat embryo. [After TANDLER.]

I, II, III, IV, V, VI, represent the respective arches or their rudiments; $a$, conus arteriosus; $a d$, dorsal aorta; $a v$, ventral aorta (truncus arteriosus); $c c b$, art. carotis cerebralis; $p$, artcria pulmonalis; $e c$, first cervical artery; $s$, segmental arteries, $h y$, hypoglossal artery; $m s$, maxillary artery; $m d$, mandibular artcry; $o$, supraorbital artery; $i$, infra-orbital artery; $l$, lingual artery; $v c$, art. vertebralis cerebralis; $x, y$, stumps which ultimately join, and form the stapedial artery, (st.) 
mon origin of the supra- and infra-orbital and mandibular arteries has become lost and their source of supply has become transferred to the dorsal stump of II. The artery thus formed penetrates the mass of cells destined to become the stapes and forms the foramen characteristic of this bone in the higher
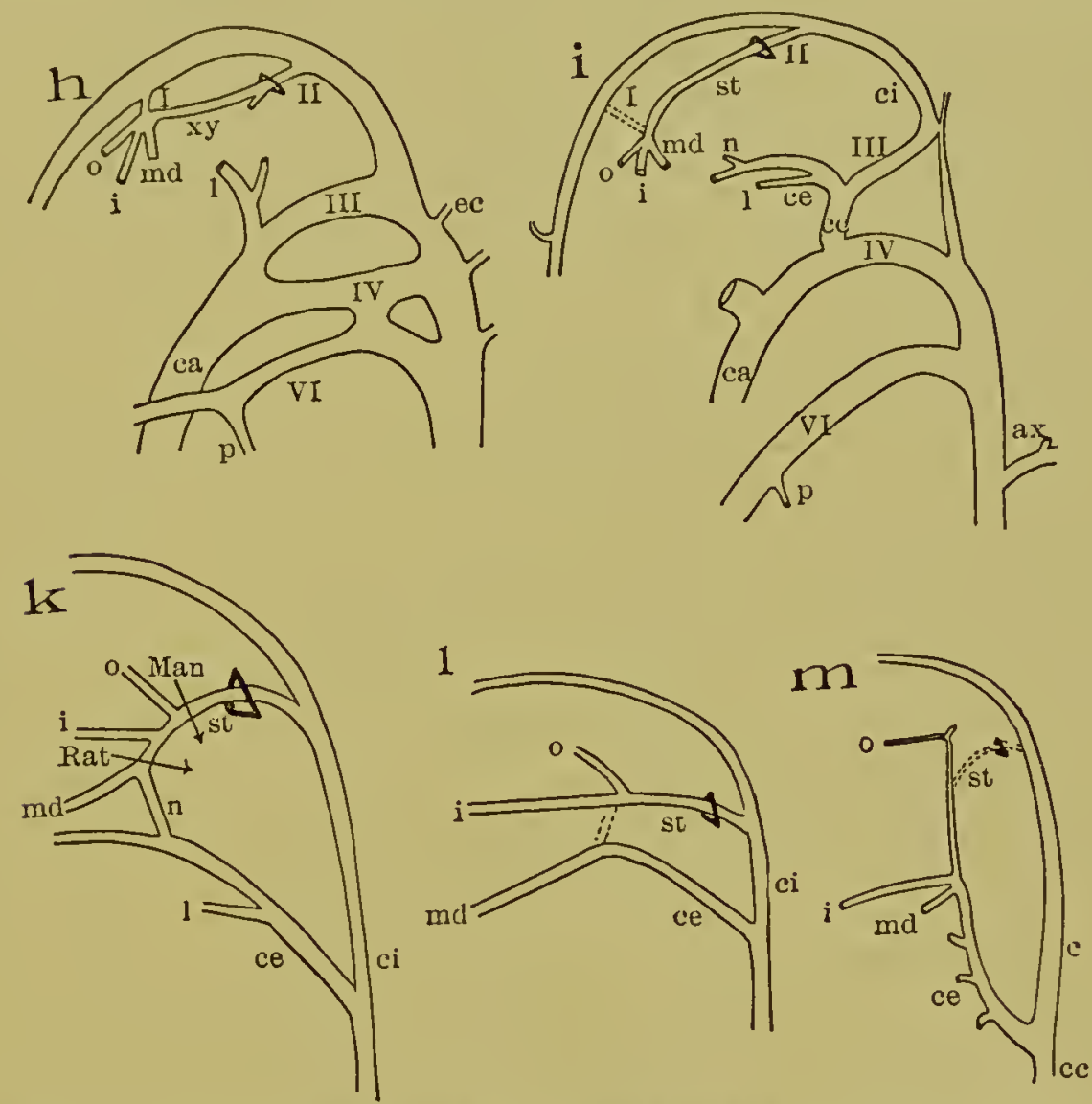

FIG. 94. (h)-(1), Continuation of the series given in Fig. 93. (m), Ultimate condition in Man, for comparison with (1). [All figures after TANDLER.]

$x y$, the artery formed by the union of $x$, and $y$, in the previous figure; $c c$, common carotid artery; $c e$, external carotid artery; $c i$, internal carotid artery; $s t$, stapedial artery; $n$, anastomosing branch between the external carotid and mandib. ular arteries. The other abbreviations are given under FIG. 93 or are explained in the text.

Mammalia. In the monotremes, where this action does not take place, the bone is columnar, and without a foramen. From now on the artery formed by the dorsal stump of arch II, the anastomosing branch $(x y)$, and a bit of the dorsal stump of arch $\mathrm{I}$, becomes called the stapedialis $(s t)$, through its relation- 
ship to the stapes. How it comes to bear the three important branches of the later external carotid has been already seen.

Between stages $h$ and $i$ a second important change has been inaugurated in the reduction of that part of the dorsal aorta which connects arches III and IV. This finally effects a complete separation of the two arches in this place, and causes the third arch to become a common carotid artery ( $\mathrm{c} c$ ) which divides into two branches, an external carotid (ce) which was formerly the anterior part of the ventral aorta plus the ventral stumps of arches I and II, and an internal carotid (ci), the main third arch plus the original arteria carotis cerebralis.

One more change in relationship is to be effected, and that is inaugurated through the growth of another anastomotic branch ( $n$ in stage $i$ ) which enters the side of the mandibularis (or, perhaps, the continuation of the stapedialis) and forms a complete circuit, as in stage $k$. From this point on the history differs in the rat and in Man, as is indicated by the two arrows, with their respective designations. In the rat the circuit breaks at the point between the infra-orbital and the mandibular, and in Man at a point above the supra-orbital. The two results of these are seen in diagrams $l$ and $n$, which represent the adult condition of this detail in the rat and in Man, respectively. In the former $(l)$ the stapedial artery, a branch of the internal carotid, bears both supra- and infra-orbital arteries, while the external carotid becomes continued mainly into the mandibular. In the latter the external carotid bears all three of the branches in question, while the stapedial artery, being of no further use, disappears, and leaves in the stapes the hole through which it formerly ran, thus accounting for the particularly curious shape of this little bone, which attracted the attention of the early anatomists, but for which they had no explanation. In considering the details of the development of any part of the circulatory system, the process is seen to be a metamorphosis, correlated with the changes in the parts supplied by the blood-vessels under consideration. Such a metamorphosis is like the changes in the roads and 
paths of a given district, due to a shifting of the centers of population, and the development or decay of any points of human interest. Changes like these set the traffic now over one, now over another, series of roads, which increase or decrease in width and degree of development in exact proportion to this use, certain ones becoming highways and others lanes, solely through the functional importance of the localities which they connect. Even the atrophied rudiments have their counterpart in the ancient roads, entirely overgrown and lost to all save the antiquary.

The branches of the aorta posterior to the arterial gillarches and their derivatives are sufficiently similar in all vertebrates to be easily recognized, but it may be said in general that, as is the case with other systems, these branches show many more indications of metameric arrangement in the lower vertebrates, and are accordingly more numerous. Instances of this are seen in the numerous lateral and dorsal branches which supply the muscles of the body wall and are segmentally arranged in fishes and amphibians, while in higher forms their number is much reduced, forming the intercostal and lumbar arteries. It is again strikingly shown in the mesenteric arteries which, in lower forms, are very numerous and suggest a metameric series, while in higher forms they are collected at their origin into a common trunk (Fig. 95).

The relative size of the various branches varies directly with that of the parts which they supply, a fact especially noticeable in the case of the subclavian and iliac arteries, which are small and unimportant in fishes, with small lateral fins, but which become excessively developed in connection with the hypertrophy of one or both pairs of limbs. The caudal aorta, like the other elements of the tail, retains its primitive character and gives off metamerically arranged branches in the case of well-developed tails, in which the other parts are sufficiently emphasized to allow it. In Man the caudal artery becomes reduced to the insignificant arteria sacralis media, in which the earlier anatomists failed to see the continuation of the aorta. This is in part due, however, to the enormous development 
of the legs correlated with the erect position, which has developed the iliac arteries out of all proportion, giving the erroneous but inevitable impression that these latter arteries form the real continuation of the aorta, which becomes bifurcated, and that the arteria sacralis media is an unimportant median branch arising from the point of bifurcation and supplying the coccygeal region.

In the adult selachians, which in their venous system represent practically the starting point of the history so far as vertebrates are concerned, the two sets of cardinal veins, an-
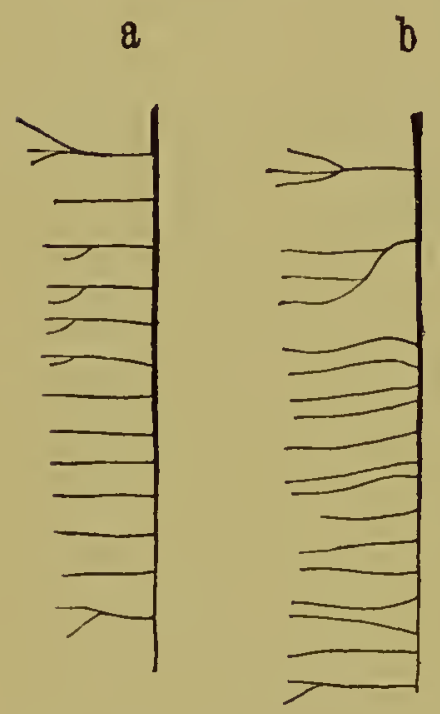
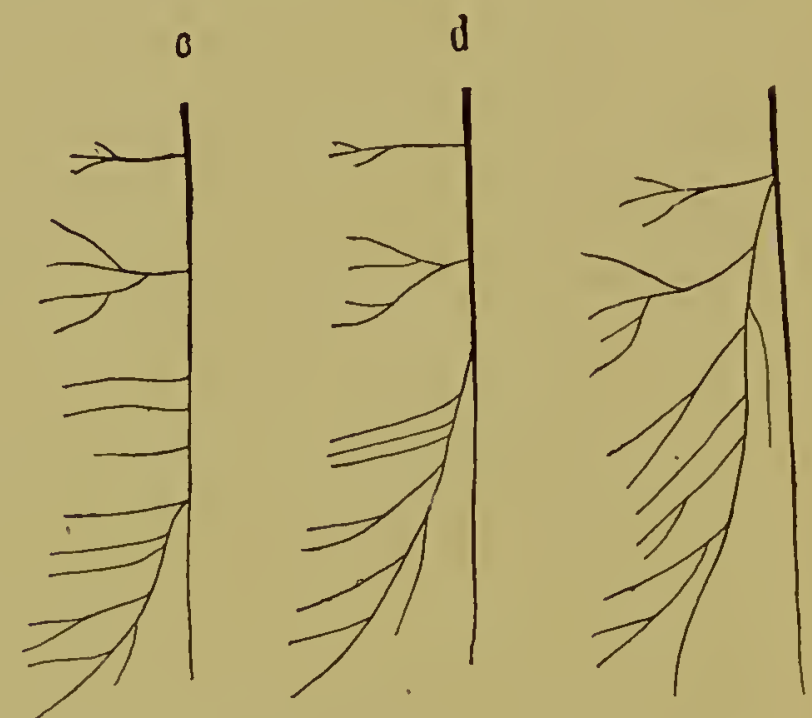

FIG. 95. Metamerism in the mesenteric arteries of Amphibia. [After KLAATSCH.]

(a) Siren. (b) Necturus. (c) Cryptobranchus. (d) Cryptobranchus (a second specimen). (c) Anura.

terior and posterior, are in control of the venous blood, except that from the alimentary canal, and return it from all parts of the body to the simus venosus. However, during the embryological development of these animals one catches glimpses of a still earlier systemic vein, the sub-intestinal, which, here embryonic and transitory, must have preceded the cardinal system historically, and have been totally replaced by the latter before the advent of true vertebrates as we now know them. It appears soon after the establishment of the two yolk veins, always for practical reasons the first to appear in vertebrate embryos, and extends from the left yolk vein, from which it 
arises, to the tip of the tail, lying in the median line, just ventral to the intestine. At the very first it consists of a pair of fine vessels running very near one another, but these soon coalesce into a single median vessel, much as in the case of the aorta. At the level of the cloacal opening the two original elements remain distinct, and run along the sides of the intestine, but fuse again posterior to it, forming a loop or ring.

Previous to this the cardinal system has begun its development in the form of minute vessels which grow out from the sides of the simus venosus, and as they extend farther and become of larger size the free ends of the posterior cardinals form several anastomoses with the subintestinal vein anterior to the cloacal ring and at the place about which the kidneys (mesonephros) are to develop. This connection furnishes two large lateral channels for the blood from the subintestinal system, a change which has two direct results, first, the gradual usurpation of function of that part of the subintestinal vein which lies anterior to the anastomoses, a relationship that leads to its ultimate disappearance, and second, the retention of the part posterior to the connection as the caudal vein, now become a part of the cardinal system. At the point where the original anastomoses occur, the development of the kidneys causes the formation of a rich capillary net-work, a process which ends in the establishment of the renal portal system with caudal veins for conveying the blood to the kidneys and posterior cardinals for re-collecting it and conveying it to the heart.

Although we know that stages like those just described no longer exist in living adult animals, it is quite certain that in these embryonic changes an early phylogenetic history is recapitulated; that in some past group of animals, dimly foreshadowing the vertebrate type, a well-developed subintestinal vein existed, and that the usurpation of its function by the cardinal system, repeated with great faithfulness to detail in selachian embryos, was once actually experienced and slowly worked out in adult animals through the action of natural selection or whatever other forces are and have been in operation for the gradual improvement of organisms. 
As has been shown above, the Class of fishes comprises forms which have remained at the stage last described, the one in which the cardinal system holds the supremacy; but by the time the amphibians are reached there has been another usurpation in that part of the body. posterior to the heart, and the posterior cardinal system has, in its turn, become subordinated to a third system, that of the vena cava posterior. or, more briefly, the postcava. How this appears in full functional activity is seen in the diagram representing the main venous channels of the urodele (Fig. 96, pc), where it has secured nine-tenths of the traffic between the kidneys and the heart, and allows but a small part to be conveyed by the posterior cardinals, formerly completely in charge of this territory. Still another rival of the cardinal system has appeared in the abdominal vcin $(a b d)$, which begins as two lateral veins that issue from the iliacs, run along the ventral abdominal wall until they meet in the median line, and continue as a single vessel until opposite the liver, when the vessel leaves the body wall, and enters this latter organ, forming a part of the hepatic portal system.

The origin of the vena cava historically cannot be now learned from adult anatomy, since it undoubtedly took place in those forms which successfully achieved the transition from an aquatic to a terrestrial life, or to, at least, a paludic one, and, having left for their descendants this new world with its opportunities, perished and left no trace save in the perfected parts which render a terrestrial life possible.

Here again, however, embryology furnishes us with some information concerning at least the place and mode of origin of this new vein, as may be seen by a comparison of the diagrams given in Fig 97, $d$ and $e$, where is shown the development of the postcava in the lizard. During the early stages in the development of the liver and its extensive system of capillaries, developed in association with the portal system to be considered later, the postcava appears as a partially distinct element in this capillary system, and becomes gradually more definite. This vein grows posteriorly and ultimately reaches the renal 
portal system and the anastomoses between the caudal vein and the posterior cardinals. Here it is united with the posterior ends of these latter vessels and annexes them as well as the caudal vein to itself, thus establishing a single path

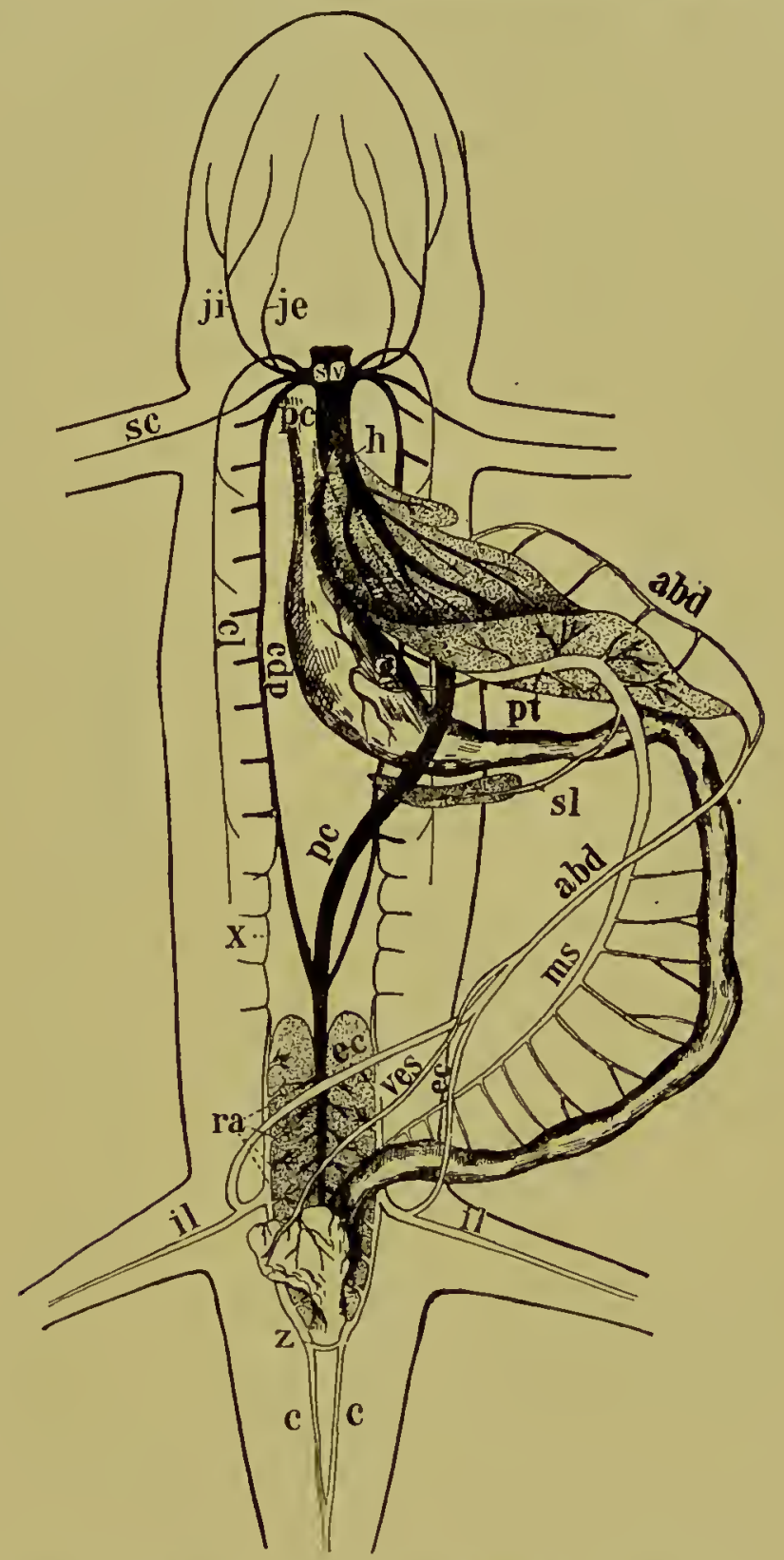

FIG. 96. Venous system of urodeles, based on that of Desmognathus. [In part after (Mrs.) finNe Barrows SEelye.]

$j i$, internal jugular; $j c$, external jugular; $s c$, subclavian; $p c$, postcava; $h$, hepatic; pt, portal; $g$, gastric; $s l$, splenic; $a b d$, abdominal; $2 c s$, vesical; $c c$, epigastric; $m s$, mescntcric; $i l$, iliac; $c$, caudal; $z$, anastomotic brancl between the two caudals; $r a$, venic renalcs advelicntes; $c l$, lateral cutaneous; $c d p$, cardinalis posterior; $x$, anastomotic brancli betwcen postcardinal and renal. The systcmic vcins arc given in black; tlic portal systcm is in outline. 
from the end of the tail, between the kidneys, to the heart. The remainder of the posterior cardinals, anterior to the connection with the vena cava, becomes reduced in proportion to the loss of function and the two remain either as small but continuous vessels, as in the Amphibia, or as the azygos veins, which continue to play a subordinate rôle by collecting
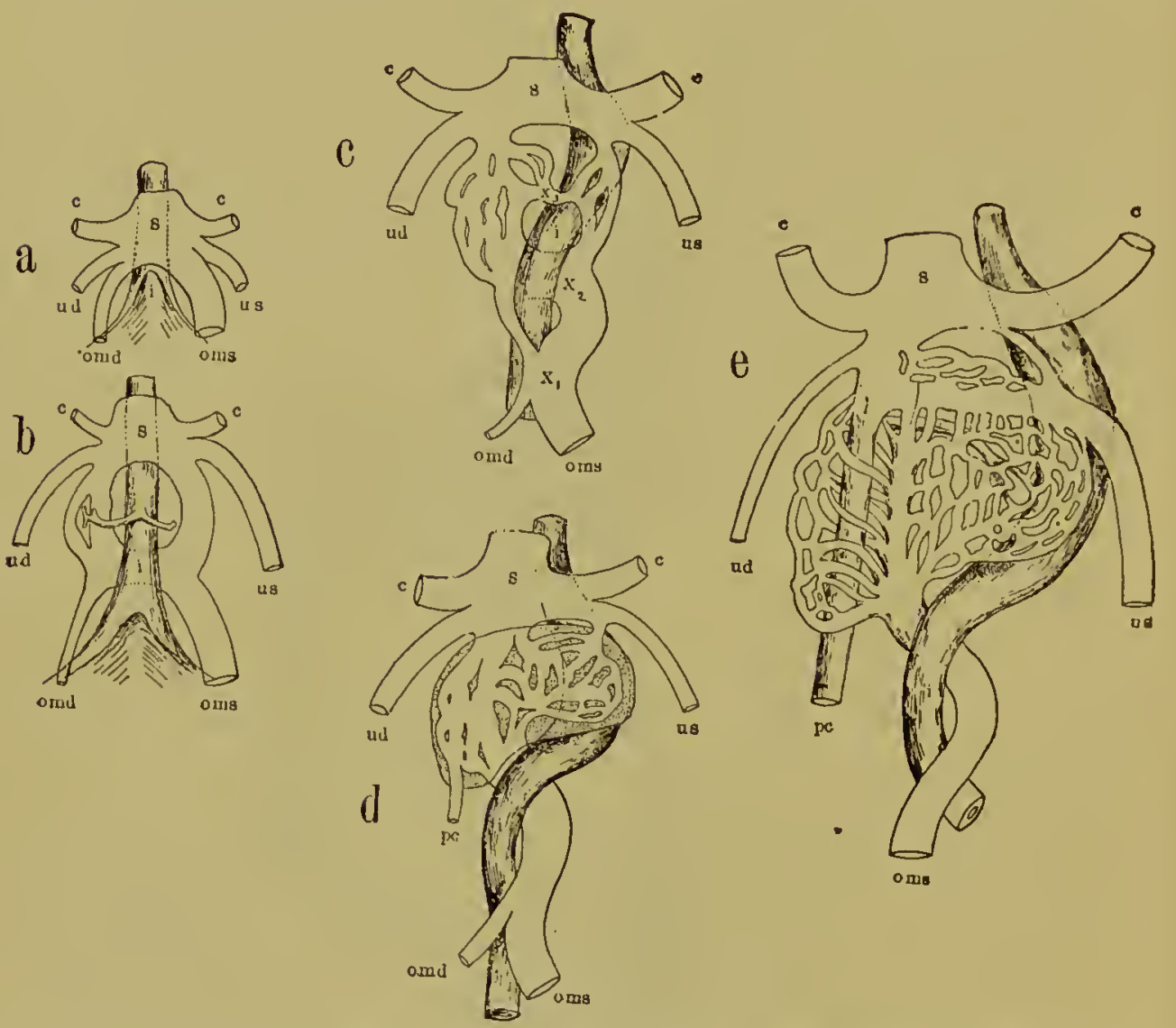

FIG. 97. Development of the postcava and the hepatic portal system in the lizard (Lacerta). [After Hochstetter.]

The figures (a) to (e) represent consecutive stages of development. $s$, sinus venosus; $c$, $c$, ductus Cuvieri; $u d$, right umbilical vein; us, left umbilical vein; omd, right omphalo-mesenteric vein; oms, left omphalo-mesenteric vein; $p c$, postcava; $i$, intestine; $x_{1}, x_{2}, x_{3}$, conmissures between the veins of the two sides.

the blood from the sides of the trunk, especially from the intercostal spaces, a function which they exercise in sauropsids and mammals.

Fig. 98 shows the development of the postcava in a mammal in which the part played by the posterior cardinals is especially emphasized. In some details the developmental 
history in reptiles, birds, and mammals differs a little; there may, in fact, be slight differences within the limits of each group, but the essentials are in all cases as given above. In this specific case the posteriorly developing postcava enters the right of two small veins developed in the (here transitory) renal portal system (stage $a$ ). Stage $b$ is developed from stage $a$ through the formation of a transverse anastomosis between this vein and the two posterior cardinals, with an accompanying increase of size in these parts. This anastomosis, $x$, divides the original posterior cardinal into two parts, $y$
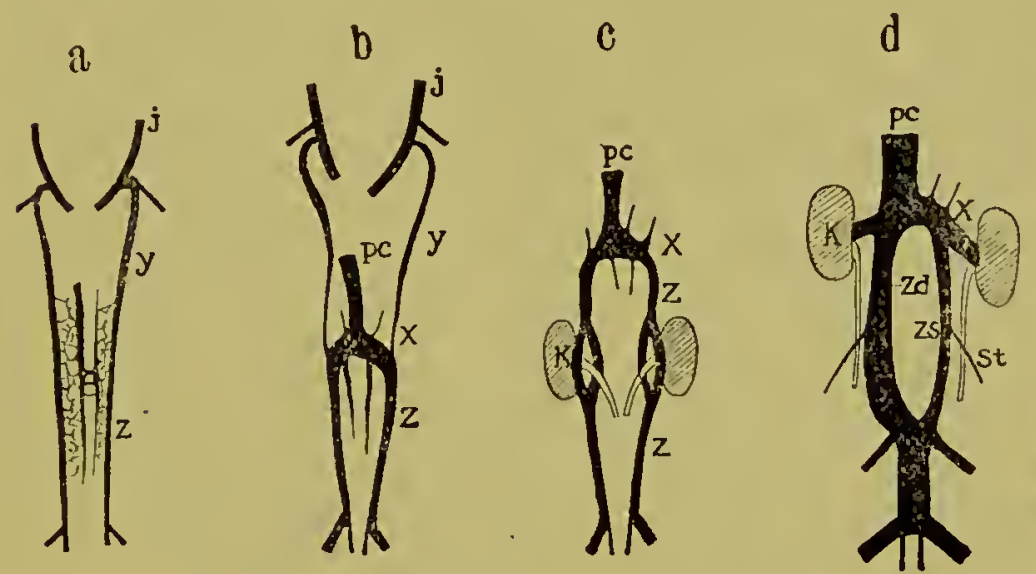

e

FIG. 98. Development of the postcava in manmals. [After HocHSTETTER.] (a) - (d), Rabbit; (e), Man.

$j$, anterior cardinal (jugular); $y, z$, the two parts of the posterior cardinal, divided by the anastomotic vessel $x ; p c$, postcava; $z d, z s$, right and left posterior cardinals, between the commissure $x$ and their union posteriorly; in (e) the left one of these atrophies in part, the remainder becoming a portion of the spermatic vein $s t ; k$, kidney; $s$, suprarenal body.

and $z$, of which the former becomes reduced and forms the azygos, while the latter develops as part of the postcaval system. In stage $c$ the permanent kidneys have formed, the ureters from which run through a temporary ring in the part $z$, a relation without special significance.

In stage $d$ an important change is effected, first by the fusion of the two lateral elements, once the caudal ends of the posterior cardinals, and, second, by the precedence in size and function established by the right portion of the part $z$ anterior to this fusion. The kidneys have also moved anteriorly, and have developed the renal vcins. The final condition is shown 
in stage $c$, in which the left limb of the loop ( $z s$ of stage $d$ ), has for the most part disappeared, while the right has become nearly median, and has thus straightened the entire vessel. The left spermatic (or ovarian) vein, which in stage $d$ enters the left limb of the loop, has caused the retention of that part through which its connection with the main system was originally established, while the right spermatic vein enters the postcava directly, since this was originally the part to which it was attached.

This last diagram $(e)$ is taken from the human embryo, since in man the relation of the iliacs differs considerably from that in the rabbit, from which the other diagrams of this series are taken. In other respects there is no appreciable difference between the two forms.

This embryological history explains the composite structure of the postcava as seen in the adult. Anteriorly a sprout from the liver capillaries, it is composed more posteriorly of a vein connected with the embryonic kidney and a portion of the right posterior cardinal, and to this is added, still more posteriorly, the caudal vein, primarily a portion of the subintestinal.

Concerning the abdominal vein, which seems to appear in the amphibians as suddenly as does the postcava, the embryology of urodeles shows it first in the form of paired lateral vessels lying in the body wall and emptying into the ductus Cuvieri without connection with the liver. Its embryonic position and relationships thus render it probable that this vein is the same as the lateral vein of fishes, which likewise runs in the body wall and empties into the ductus Cuvieri or near it. The connections of this vein with the iliacs posteriorly and with the liver anteriorly appear later on in embryonic development and are thus shown to be secondary modifications, and not features of the original vein.

Above the amphibians there is nothing which at first sight resembles an abdominal vein, but the tro lateral elements of which it is composed are probably identical with the similarly related umbilical voins, which in the embryo supply 
the allantois. This membrane is itself the amphibian urinary bladder extended beyond the limits of the embryo, and there is little doubt that the two veins which lie along its sides and enter the liver, are the primary lateral elements which in adult amphibians fuse to form the median abdominal vein. In the later history of the umbilical veins, the right one becomes early recluced, and in advanced embryos the left one alone remains; this collects all the blood from the entire allantois, enters the body at the umbilicus, and conveys the blood from that point to the liver.

At birth, in the case of the mammal, and upon hatching, in reptiles and birds, the extra-embryonal portion of the allantois, together with its blood-vessels, becomes pinched off at the umbilicus, but the umbilical vein, extending from the anterior body wall to liver, is retained as a ligament (lig. teres s. hepato-umbilicale).

The portal vein, previously described, which conveys the blood from the intestine to the liver, is a constant factor in vertebrate circulation from cyclostomes to mammals, and as it is essentially similar in all cases there is but little history shown by the comparison of adult forms. The early embryonic development shows, however, the method by which this portal system becomes established, and is thus valuable in explaining the relation between the original morphological elements and the adult structures. The formation of the hepatic-portal system occurs always in connection with the two first veins that appear, the vitelline or omphalo-mesenteric, that lead in from the yolk and unite just posterior to the heart. The intestinal canal runs between them, and from its ventral aspect the liver buds out in the form of a connected group of diverticula, which surround the veins in question and cause them to develop a capillary net-work. In fishes and amphibians the process is a fairly simple one, but in Sauropsida and Mammalia the matter becomes somewhat more complicated by the addition to this very region of the two umbilical veins, which come in from the allantois. This development, in its more complex form, is shown in Figs. 97 and 99, 
which are taken from the lizard and mammal, respectively. In $a$ of either figure are seen the primary elements which enter into the process, namely the two omphalo-mesenteric or yolk veins, the two umbilical veins, and the alimentary canal. The ducts of Cuvier, the cardinal veins and the sinus venostus are also shown, but they are not directly concerned here.

The initiative is taken by the two omphalo-mesenteric veins which form successively three connecting bands that unite them to each other $\left(x_{1}, x_{2}\right.$, and $x_{3}$ in Fig. 99). Of these, $x_{3}$ is the most posterior, and lies ventral to the intestine; the next, $x_{2}$, is dorsal; and the third, $x_{1}$, also the most anterior, is again ventral. The result of this is the formation of two rings, forming a figure 8 , through which the intestine is threaded (Fig. 97, c, and Fig. 99, c). The subsequent suppression of the left side of the anterior ring and the right side of the posterior ring, as indicated in Fig. 99, c, produces a single large trunk, eventually the portal, which twists in a spiral about the intestine (Fig. 97, $d$ and $e$ ). Meanwhile, the liver has formed about the two omphalo-mesenteric veins anterior to the rings, and the necessity thus thrust upon them of supplying it with blood-vessels results as seen in Fig. $99, b$ and $c$. Each sends off lateral branches from the posterior side of the liver and gathers them up from the anterior side until they are resolved into a mass of capillaries, which permeate the liver substance in all directions. As a temporary necessity, to be removed at the end of embryonic life, there develops a vessel running diagonally through the liver and extending from the left omphalo-mesenteric vein posteriorly to the right one anteriorly, the ductus venosus Arantii. Through this the blood passes while the liver tissue is still embryonic, and before the capillary system within it is fully established, but with the approach of birth the duct atrophies and the hepatic portal system becomes fully established.

The two umbilical veins, which appear in all these figures, take no part in the formation of the hepatic system and may rank thus as structures wholly embryonic. It is remarkable, however, that, as in the case of the omphalo-mesenteric veins, 
the two become reduced to a single one, not through so complicated a process as in the former case, but through the complete suppression of the right vein. The anterior portion of the left, also, shares the same fate, and the umbilical vein

a
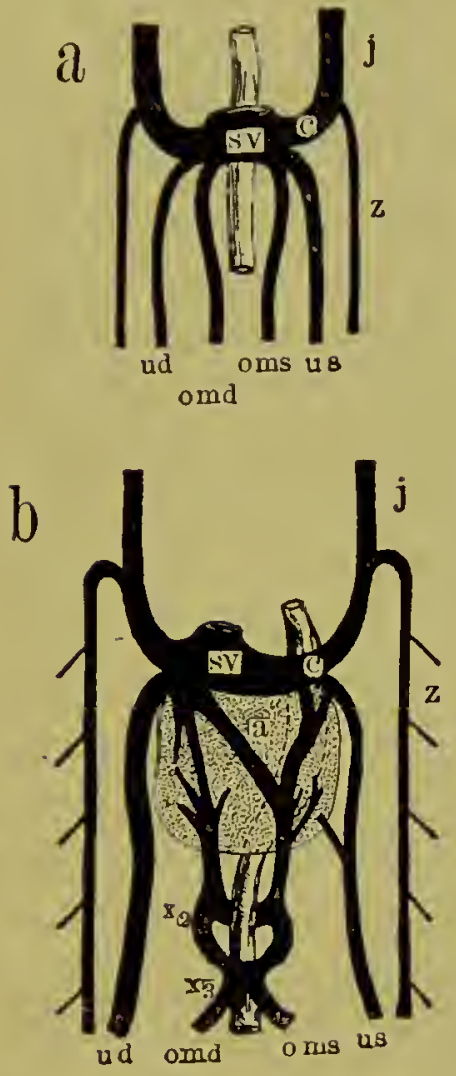

c

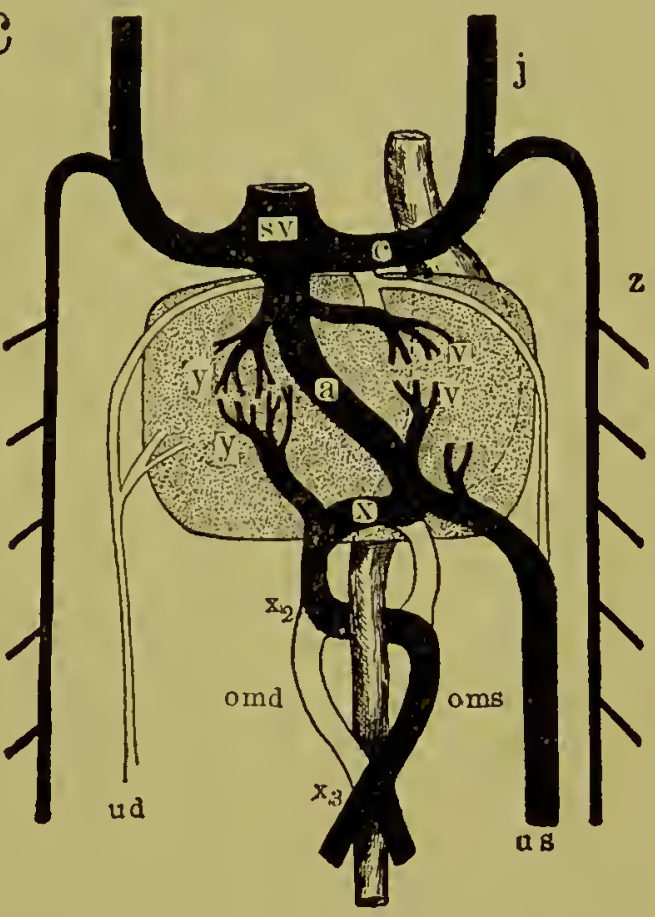

FIG. 99. Development of the hepatic portal system in mammals. [After HochstetTER.]

$j$, anterior cardinal (jugular); 3 , posterior cardinal; $c$, ductus Cuvieri; omd, oms, right and left omphalo-mesenteric veins; $u d$, $u s$, right and $l \mathrm{cft}$ umbilical veins; so, sinus venosus; $x_{1}, x_{2}, x_{3}$, commissures between the omphalo-mesenteric veins of the two sides; $y, y$, and $v, v$, beginnings of the hepatic and portal capillaries in the liver; $a$, ductus venosus Arantii.

finally establishes a direct connection with the heart through the ductus venosus Arantii (Fig. 99, c).

The fate of the veins anterior to the heart, when compared with that of those posterior to it, is a very simple one, for while in the latter region three veins in succession have held the supremacy, the sub-intestinal, the posterior cardinal, and the postcava, anteriorly the first to appear are the anterior cardinals, and it is these very vessels which in the higher mam- 
mals, under the name of jugulars, continue to serve in the same capacity as at first. The changes in these parts are comparatively slight, and consist mainly in the establishment of two definite branches on either side, the external and internal jugulars, and in the greater development of the subclavians, correlated with that of the fore-limbs, which gains for it an anatomical rank equal to that of the anterior cardinal itself, and suggests the name vena anonyma for that part of the original anterior cardinal below the entrance of the subclavian, since it appears to be formed by the union of two equal veins.

These changes of nomenclature, it will be seen, are purely anatomical, and express merely the apparent differences die to change in the caliber or the relative position of the separate portions. There are, however, a few genuine morphological changes in the higher Classes, doubtless rendered necessary by modifications in related parts. The most striking of these occurs in Man and some other mammals and consists of the formation of a connection across the middle line between the right and left jugulars, and the more or less complete atrophy of the right vena anonyma. The left innominate vein thus has to convey all the blood from both sides of head and neck and from both anterior limbs, and as it is greatly increased in size through the assumption of this double task, it early received the name of superior vena cava (anterior or precava), in comparison with the inferior vena cava (posterior or postcava), which enters the right atrium near it.

A simple modification takes place in the mammalian posterior cardinals. The formation of a transverse connection between the two allows one of them to assume the function of conveying to the heart the blood collected by the metameric branches, and permits the other to sever its anterior connection with the heart. Although there is much variation in this, the more common condition in Man consists of the preservation of the posterior cardinal on the right side in its entirety, into which the other empties by means of the transverse connecting vein, and is deprived of all direct connection with the heart. The first, or complete vein, was called the azygos, 
or unpaired, vein, from a mistaken early notion that it had no mate on the other side; the other, the incomplete one, was named the hemiazygos. What litthe applicability these names may possess, however, is confined to Man and allied forms, since in many other mammals quite different results obtain. Thus, in rabbits, the main trunk of the hemiazygos entirely disappears, and the azygos receives the intercostal veins from both sides, while in the pig the reverse is the case and it is the hemiazygos which persists. These relations are extremely variable, even in Man, where the occasional conditions classed as anomalies receive their complete explanation through the morphological history of the region.

As a review of the venous system of Man, with the morphological significance of the principal parts, there may here be presented Fig. IoO, which shows the course of the main trunks in the adult, the older parts which have

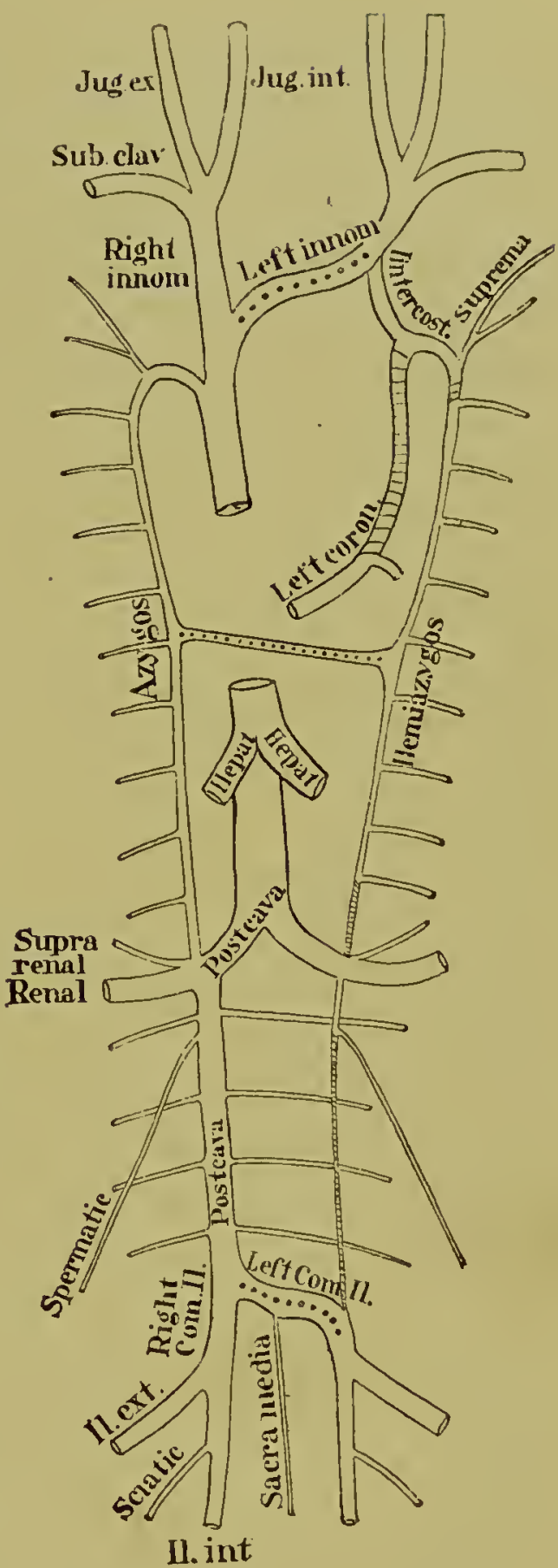

FIG. I00. Diagram showing the history of the venous system in Man. [Modified from THANE, in QUAiN's Anatomy.]

Primitive vessels that become atrophied are marked by cross lines; those secondarily established are marked by rows of dots. 
atrophied and the new connections winich have been added. In the background may be seen the primitive cardinal system of fishes, and perhaps in the minute caudal vein, even a trace of the still earlier sub-intestinal system. Here, as elsewhere, we receive the distinct impression of the constant modification of old relations to fit new conditions, and we see the numerous mechanical difficulties which are the inevitable result of such a process. Here and there, where a difficulty is sufficiently great to interfere seriously with the preservation of the race, it is overcome, if possible; if not, the race dies out; but generally the adaptation is fairly complete, and, while we may never. know of the countless forms which were lost in the sifting process, those that survive are not seriously handicapped by the circuitous paths through which their organs have arrived at their final condition, and the atrophied rudiments form no serious disadvantage to the organism.

As one example of the slowness of the adaptation where the disadvantage is inconsiderable, we have the case of the vena anonyma of the left side. Although the plan by which all the venous blood is received upon the right side of the heart is inaugurated by the amphibians, there is, in the anterior cardinal system, no anatomical recognition of this throughout amphibians, reptiles, and birds, all of which still possess symmetrical vence anonyma, and the left one is forced to bring its blood over to the right side. First among the mammals comes the formation of an obliquely placed transverse connective, which allows the establishment of a true vona cava anterior, and rectifies the slight mechanical disadvantage. The mere fact that this condition continues so long without readjustment suggests that the disadvantage must be exceedingly slight, far too inconsequent to come under the direct control of Natural Selection; and the bettered condition in these mammals cannot fail to suggest the result of mechanical causes, operating continually for a long time, and always in the same direction.

The heart is in origin nothing but a localized portion of a large blood-vessel, the walls of which develop a thickened 
muscular layer, transforming it into a pulsating engine to promote the flow of blood. Similar pulsating vessels occur in all animals furnished with a closed circulation, usually a single one, but in some cases several in number. Thus, in arthropods and molluses there is a single median heart, located dorsally upon the main blood channel in that region, but in annelids several of the lateral commissures are enlarged and function as hearts. In vertebrates the hypertrophied region which
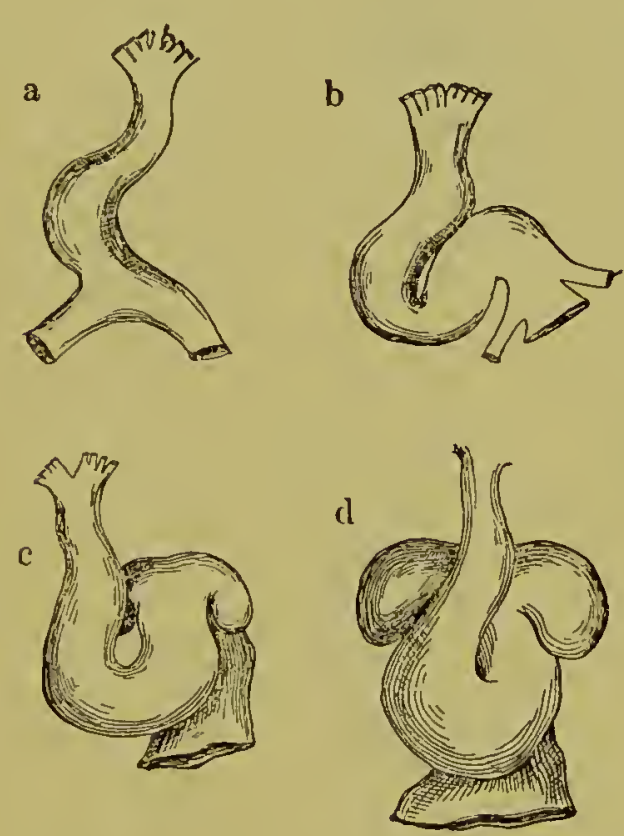

FIG. IOI. Four stages in the development of the amniote heart. forms the heart is located along the median ventral blood-vessel formed by the union of the two vitelline veins, and involves its posterior portion. This brings it topographically very far anterior, just back of the gill region, and this primary position is, indeed, that permanently retained in fishes and amphibians; but in reptiles, birds, and mammals it suffers a considerable change of location in a posterior direction and comes to lie in a thoracic cavity, formed by the ribs and sternum, with some participation of parts of the shoulder-girdle.

In its first stage, as shown by Amphioxus and in early embryos, the heart is still a straight tube, formed posteriorly by the joining of the hepatic veins, and, in true vertebrates: the two ducts of Cuvier and the two vitelline veins also, the last being embryonic and transitory. The chamber into which these vessels empty soon differentiates off from the rest as the sinus venosus, and, in like manner, by the formation of transversely placed constrictions, there are added successively an atrium, a ventricle, and a conus arteriosus, the latter being continued into the median artery that supplies the gills.

The next advance is seen in a flexion of the axis of the 
heart into the form of an S-shaped tube, the bending being in such a way that the sinus venosus is dorsal and the conus arteriosus ventral, the atrium anterior and the ventricle posterior, a stage represented in adult fishes and in the embryos of higher forms. The atrium increases in width more than the other parts and forms two lateral recesses or broad diverticula, which, from the ventral aspect, appear on either side of the conus arteriosus, suggesting the division into two separate compartments, which is, in point of fact, the next advance. Furthermore, the several parts brought into contact by the flexion become permanently adherent to one another and the heart becomes molded into a more compact organ.

In the tailed amphibians a new physiological moment is introduced by the reception for the first time of arterial blood, which comes from the skin and lungs through the great pulmo-cutaneous veins and enters the left side of the atrium (Fig. 102, B). The anatomical response to this consists of the division of the atrium into right and left portions, the former for the reception of impure, and the latter for pure blood.* From now on the sinus venosus plays a subordinate rôle and consists merely of a vestibule of entrance for the systemic veins, applied to the dorsal side of the right atrium. In birds and mammals it is no longer distinct. The ventricle is still undivided in the urodeles, but in the tailless forms, probably as a further response to the new physiological condition, a partial septum appears in this, which suggests a division into right and left ventricles, but contains a large opening through which the two kinds of blood still mingle (Fig. I02, C). In these animals also, the complete differentiation of the third and fourth arterial arches as aorta and pulmonary artery, respectively, leads to a longitudinal division of the conus arteriosus by means of two longitudinal folds placed opposite to one another which grow from the inner

\footnotetext{
* In the urodeles the scptum atriorum is not complete, but possesses a few secondary perforations. In the lungless salamanders the left atrium, into which the pulmonary veins would empty under other circumstances, is suppressed.
} 
walls, meet, and fuse. This causes a complete separation of these main vessels as far back as the ventricle, and their exits from this chamber are placed in such a way that the blood from the right side, which is mainly impure, passes
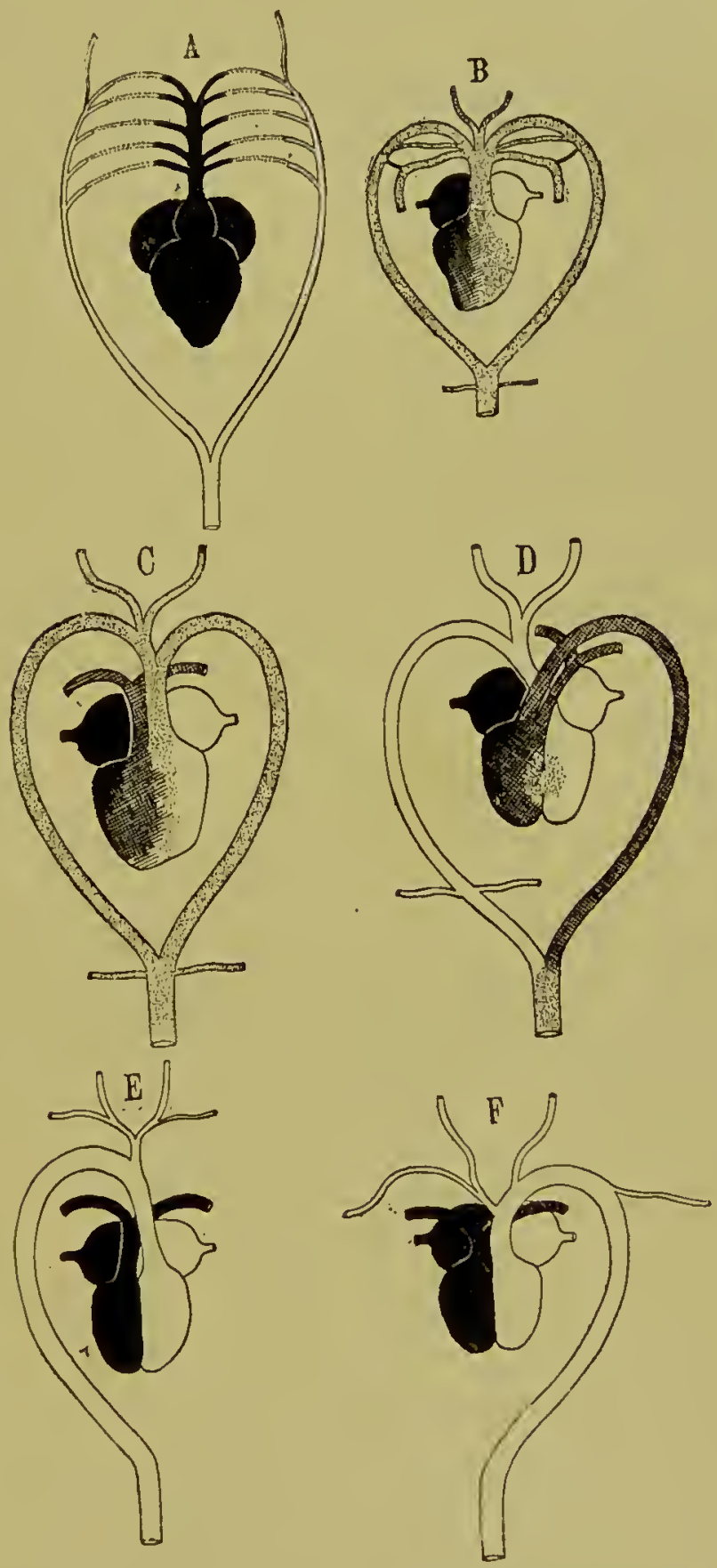

FIG. 102. Diagrams of the heart and its compartments in the different vertebrate Classes. [In part after Wiedersheim.]

(A) Fish; (B) Urodele; (C) Anuran; (D) Reptile; (E) Bird; (F) Mammal. 
into the pulmonary artery, a condition which continues as a permanent one from this point on. The relation of the two aortic arches, however, is not so perfect, for they cross in such a way that while the right one contains mainly pure blood, the left one, in company with the pulmonary artery, collects, in part, impure blood from the right side. This causes a mixture of blood in this arch as well as in the main aorta, and the result is that the blood is never wholly aërated, a condition which does not allow the establishment of a constant bodily temperature, but compels the animal to be "coldblooded," or more correctly, poikilothermous, that is, changeable in temperature in more or less accordance with its surroundings.

The heart of reptiles (Fig. I02, D) is similar to the last save that the ventricular partition is more extensive, though still incomplete, therefore the relation of these animals to external temperature is the same as in amphibians; in birds (Fig. I02, E), however, the opening between the ventricles closes, thus, for the first time, completely separating the two kinds of blood. Probably correlated with this is the complete atrophy of the left aortic arch, leaving the right one as the only connection between heart and median aorta.

Mammals (Fig. IO2, F), although but indirectly related to the birds, have accomplished the same separation of pure and impure blood, corresponding to the left and right halves of the heart, respectively, a relation which is still further emphasized by the main blood-vessels, the systemic venous trunks assuming a position on the right, in connection with the atrium of that side, and the main aorta being on the left, instead of the right. In both birds and mammals, then, the tissues are supplied with pure blood and are thus enabled to maintain a constant body temperature, which fluctuates but a few degrees, and is usually higher than the external temperature. There thus comes to be developed in the two most highly specialized Classes of vertebrates, birds and mammals, a complete and almost symmetrical double heart, of which the right half is associated with the venous, the left with the arterial, blood. 
In both the symmetry is not a primary, but a secondary one; the heart begins and ends with four chambers, it is true, but they do not at all correspond, the latter form resulting from the suppression of two of the first and the subdivision of the remaining two*.

The lymphatic function, that which cares for the blood components which become infiltrated into the tissues, and returns them to the general circulation, is performed, not only by spaces and vessels primarily formed for that purpose, but, in the lower forms at least, by the most of the spaces and lacunze of the body that form parts of other systems. In fishes and amphibians a system of lymph channels becomes developed in the loose connective tissue that forms an external sheath for the larger blood-vessels, and here the lymph, not confined within special walls, is intercellular and circulates freely within the meshes formed by the branching connectivetissue cells. One of the largest of these lymph channels is the subvertebral space, which enfolds the aorta. The lympatics that collect the digested food (chyle) from the intestine communicate, either directly or indirectly, with this channel, which thus forms a very primitive thoracic duct.

A second series of lymphatic spaces is found immediately beneath the membranes lining the great serous cavities of the body; such are the sub-peritoneal spaces in the walls of the cœlom, the sub-dural and inter-dural spaces connected with the membranes that invest the central nervous system, and the peri- and endo-lymphatic spaces of the inner ear. Still others

* During fetal life there is, in the mammalian heart, an opening in the interatrial septum, through which the blood of the two atria freely mixes. This is, however, a secondary condition, developed in adaptation to the fetal circulation, as is shown by the fact that the septum is first completed before the opening is formed. In the embryos of the monotremes and marsupials there are several small foramina instead of one big one; which is similar to the condition found in urodeles, where the perforations are also secondary. The large foramen in the mammals, the foramcn ovale, persists normally in the human infant for a few days after birth; but is occasionally permanent, producing the condition known as cyanosis, in which the inclividual suffers continually from the presence of venous blood in the arterial system. 
are intermuscular or sub-fascial, their locations being designated by their names, and in tailless amphibians there is found an extensive series of sub-cutaneous lymph-sacs, some of great extent.

These various spaces are in communication with one another and usually communicate with the venous system in four places: anteriorly with the two jugulars at or near their union with the subclavians; and posteriorly with either the caudal vein or the posterior cardinals near the entrance of the iliacs. It is to be noted that these four points at which the lymphatic and circulatory systems communicate are associated with the four limbs, and although the number of these points of communication is decreased in the higher vertebrates through a suppression in the adult of certain of these, there are no new ones formed, and the lymphatic system, even in its most specialized form, still follows in this particular the lines laid down for it from the first.

In fishes the lymphatic vessels, near their entrance into the veins, enlarge into thin-walled sinuses, organs which in tailless amphibians develop into pulsating sacs with muscular walls, the so-called lymph-hearts, the action of which furthers the flow of the lymph (Fig. I03). Each lymph-heart possesses a single venous ostium, by which the sac communicates with the vein, and several lymphatic ostia, through which the sac receives the fluid from as many lymphatic vessels. The former opening is equipped with two semi-lunar valves to prevent filling the sac with blood during its expansion, but the lymphatic ostia are without special valves. The tailed anphibians seem to lack the anterior pair of lymph-hearts, but here, in addition to the posterior pair, a series of small pulsating sacs occur along the lateral line.

Progress in the history of the lymphatic system among the higher vertebrates is shown along two directions: first, in the formation of more and more vessels with walls of their own, the definite lymphatics, and, second, by the reduction of the lymph-hearts. Thus in reptiles only the posterior lymphhearts persist in the adult, and the same appear in birds dur- 


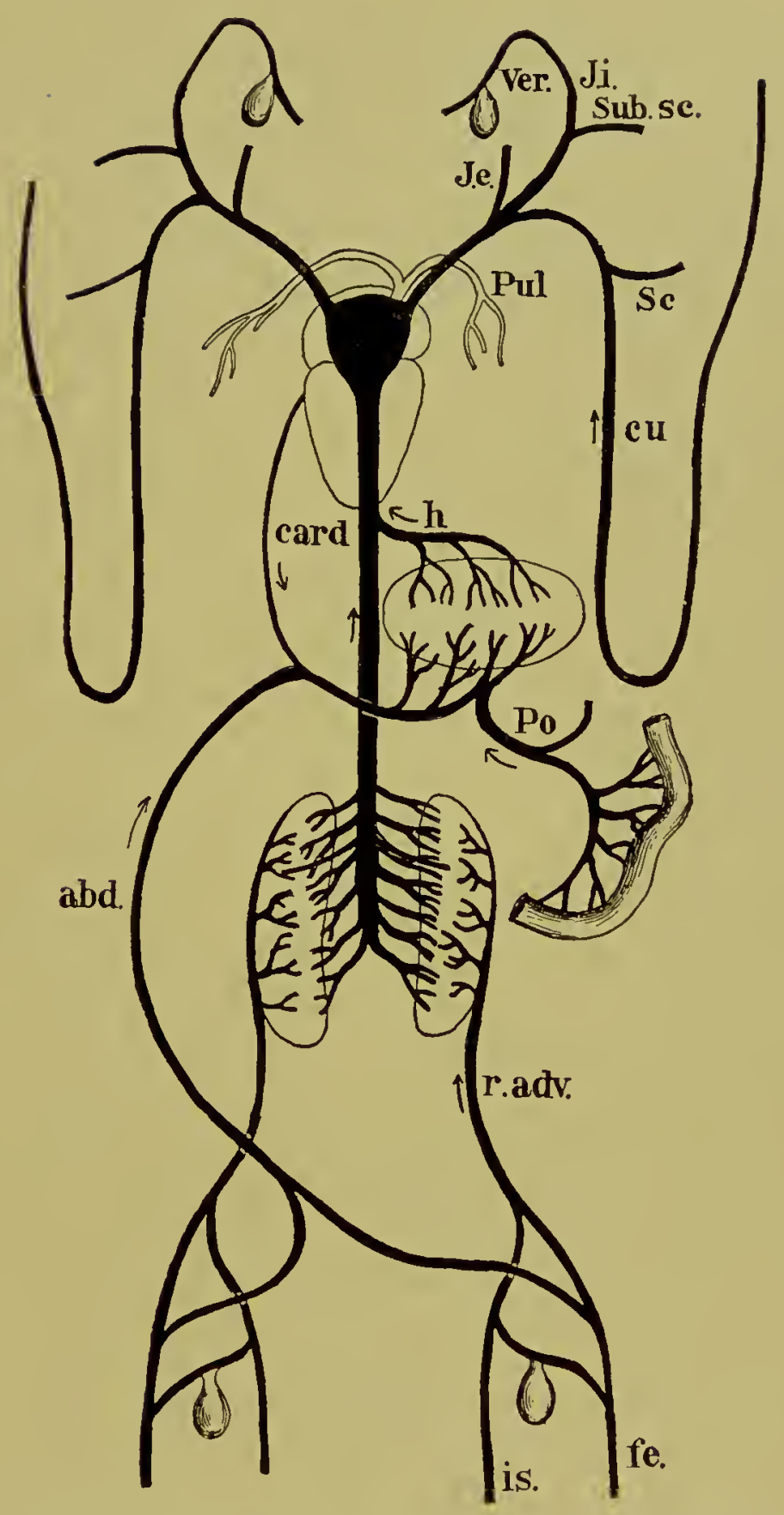

Fig. I03. Venous system of frog, to show the two pairs of pulsatile lymph-hearts; an anterior pair opening into the vertebral veins, and a posterior pair opening into a cross vein between the ischiadic and femoral veins.

$j i$, internal jugular; je, external jugular; ubsc, subscapular; $s c$, subclavian; cu, cutancous; pul, pulmonary; h, hepatic; card, cardiac; po, hepatic portal; abd, abdominal; $r$. adv, afferent renal; is, ischiadic; fe, femoral. 
ing development, but are here transitury structures and do not survive embryonic life. The division of the subvertebral space into two lateral thoracic ducts is inaugurated in crocodiles and turtles, and becomes definite in birds; in these the chyle from the intestines is collected and emptied into the veins at the juncture of jugular and subclavian. There are also in all Sauropsida posterior connections with the venous system, but only in reptiles do pulsating hearts persist at these points.

The above phylogenetic history of the lymphatic system is well recapitulated during the embryonic development of mammals, and the adult condition is best understood by tracing the steps in this development (Fig. IO4). As in the case of the circulatory system, the preservation of so many phylogenetic steps in this developmental history is doubtless due to the continual functional activity of this system from an early embryonic period, its usefulness at all stages preventing the customary degeneration of transitory structures.* The lymphatic system first appears in the form of a pair of tiny diverticula which bud out from the venous system at the angle formed by the meeting of the jugular and subclavian veins. From these develops an anterior pair of lymph-hearts, in location similar to those of lower forms, but without muscular walls; and from these chambers as centers, definite lymphatic vessels begin to develop, growing from their free ends and gradually invading the surrounding tissues. At a slightly later period a pair of posterior hearts appears, and from these in the same way there grow out branching lymphatics.

It is at this period that the thoracic ducts make their appearance, starting from the vessels connecting the lymphhearts with the veins and growing posteriorly, following the aorta. There are two of these, which at first are about equal in size, but soon the left one gains the superiority, and branches to supply each side, while the right one remains small. The

* An embryo in which the lymphatic system is not in full functional activity becomes œdematous, and in extreme cases the result is a spherical ball, without indication of the normal shape. 

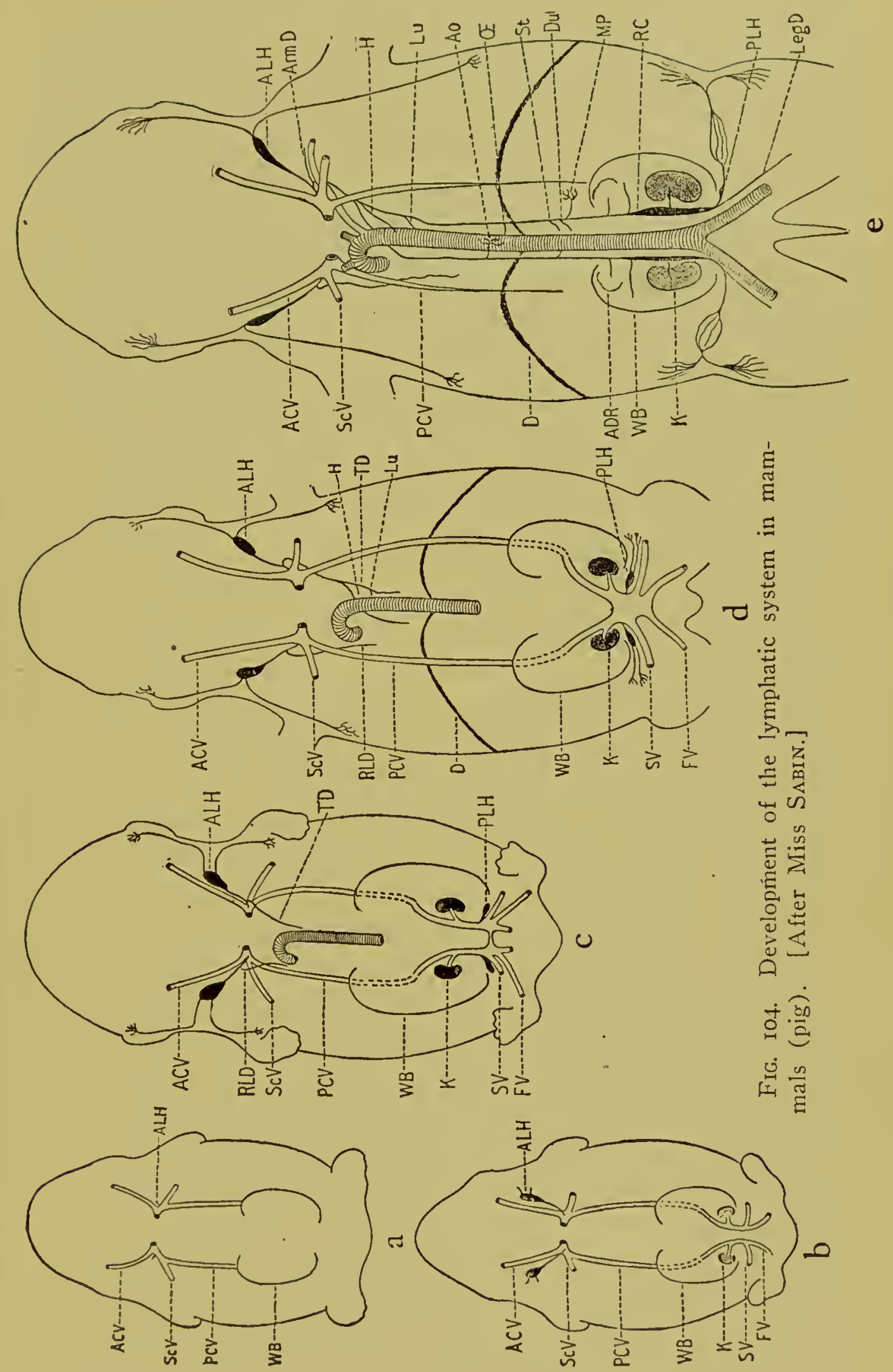
left duct thus comes to furnish two long lateral vessels, one on each sicle of the aorta, which extend posteriorly until they enter the posterior system near the lymph-hearts, thus uniting the two systems; and the severance of the original connection between the posterior lymph-hearts and the posterior cardinals, now the postcava, renders the posterior system dependent upon the anterior connections. During later development several important changes take place. The lymphatics gradually spread from the four primary centers over the entire body, the original anterior and posterior systems communicating at every point until all distinction between the two becomes lost; the lymph-hearts lose their identity; two posterior enlargements, the cisternce [receptacula] chyli, appear in the posterior parts of the lateral thoracic ducts; and the growing intestine becomes supplied with lymphatics from the left lateral thoracic duct. During the growth of the lymphatic vessels numerous secondary centers are formed, from which several vessels radiate in various directions, and about these, through participation of the connective tissue and the blood-vessels, there develop the characteristic lymphatic nodes or "glands." Similar centers, developed in the course of the left lymphatic duct as it branches within the mesentery, form the mesenteric glands. In these glands the physiological unit seems to be a tuft of blood capillaries surrounded by lymphatic vessels, the whole packed in a loose connective tissue, the lymphoid or adenoid tissue. Such a structure is called a lymph follicle, and a node may consist of a large number of such structures. Within the interstices of the lymphoid tissue occur large quantities of lymphocytes, or wandering cells that appear to be identical with the leucocytes or white blood corpuscles, and although proof is thus far wanting, it is probable that the lymphatic nodes form one of the localities in which these cells are formed.

In the walls of the colon of mammals appear aggregations of nodules, similar to those connected with the lymphatic system; and forming large areas known as noduli lymphatici aggregati [Peyer's patches]. Many attempts have been made 
to connect these with the lymphatic nodes, the extreme theory being that here is the center of origin for all the latter, and that they migrate from these patches during development, first invading the mesentery and forming the mesenteric glands; thence passing from these along the lymphatic channels to all parts of the body. As the nodules of Peyer's patches are endodermic in origin, it would follow that, with such an origin, all of the lymphatic nodes, wherever found, must be also endodermic, and it was thus held that we had here an example of elements, originally endodermic, wandering over the entire body and invading practically all the tissues. The more recent exposition of the development of the lymphatic system, as given above, renders such theories no longer tenable and show's the lymphatic system, at least in mammals, to be a definite system, budding out from that of the circulation, and, like it, mesenchymatous in origin. Whether this is the case in the lower Classes of vertebrates and whether the various spaces utilized by the lymph can be thus derived cannot be ascertained until the development of the lymphatics in these forms is as well known as it is in mammals; but with our present knowledge it seems probable that certain definite channels that possess walls of their own, like the sub-vertebral space, are produced as outgrowths from the blood-vessels, and that these enter into secondary communication with numerous irregular spaces which can well be utilized as adjuncts of the lymphatic system until their function can be supplied by definite lymphatic vessels.

Aside from the solitary and aggregated nodules, both of which appear to be centers of origin of leucocytes, there are numerous other places in which the cellular constituents of the blood are developed. Many of these, as in the case of the aggregated nodules of the intestine, are developed within the wall of the alimentary canal and are therefore endodermic in origin. These include the tonsils, the thymus, and thyreoid glands, the associated epithelial bodies, and perhaps, the spleen. The marrow of the bones is especially important in this respect, and develops large quantities of the blood-cells, espe- 
cially erythrocytes (red blood corpuscles). Although lymphatic vessels secondarily invade these organs, and are hence found in the adult in close association with them, they are not to be considered parts of the lymphatic system any more than one would consider the kidneys a part of the circulatory system because their tissues are invaded by special forms of blood-vessels.

In their function as formative nidi for the cellular elements of the blood and lymph these organs form physiologically important auxiliaries to the vascular system as a whole. but belong elsewhere in their anatomical and developmental affinities. 


\section{CHAPTER IX}

\section{THE URO-GENITAL SYSTEM}

"We do not draw conclusions with our eyes, but with our reasoning powers, and if the whole of the rest of living nature proclaims with one accord from all sides the evolution of the world of organisms, we cannot assume that the process stopped short of Man. But it follows also that the factors which brought about the development of Man from his Simian ancestry must be the same as those which have brought about the whole of evolution."

August Weismann, The Evolution Theory, Authorized translation. Vol. II, p. 393.

THE two systems included under this compound name are those concerned with the very diverse functions of the elimination of liquid waste and the formation of new individuals. They are, however, closely associated topographically and usually possess certain parts in common, so that they belong together anatomically, though not physiologically. They possess also important relations to the body cavity or colom (more strictly, metacole), and as the latter is in by no means a primitive condition in either Amphioxus or the cyclostomes, recourse must be had to early embryonic stages and also to invertebrates in order to reconstruct the early period of the history of these organs, a knowledge necessary for the explanation of many of the existing relationships. To begin with, let us suppose an animal built on the plan of a gastrula, but with a space left between the endoderm and the ectoderm (Fig. I05, A; also Plate I). Such an animal consists of two tubes, one inside the other, and two carities. The two tubes are, of course, alimentary canal and body wall, and the two cavities are the digestive cavity (gastrocole) and the primary body cavity (protoccele). Within this protocole are contained two sets of organs, each opening to the exterior, excretory tu- 
bules (nephridia) and germ glands (gonads). (Fig. IO5, B.) If the animal is unsegmented, $i$. $e$., consists of a single segment, there is a single pair of each ; if it is multisegmented, there is a pair of each for each segment.

Each nephridium consists of a free tubule whose function is to extract from the protocole certain waste products in liquid form, a function which it performs in part by a ciliated funnel-shaped opening, the nephrostome, and in part by the physiological action of the cells of which its walls are composed. In its simplest form it is straight or slightly curved, but it is more usually coiled in order to increase its length,
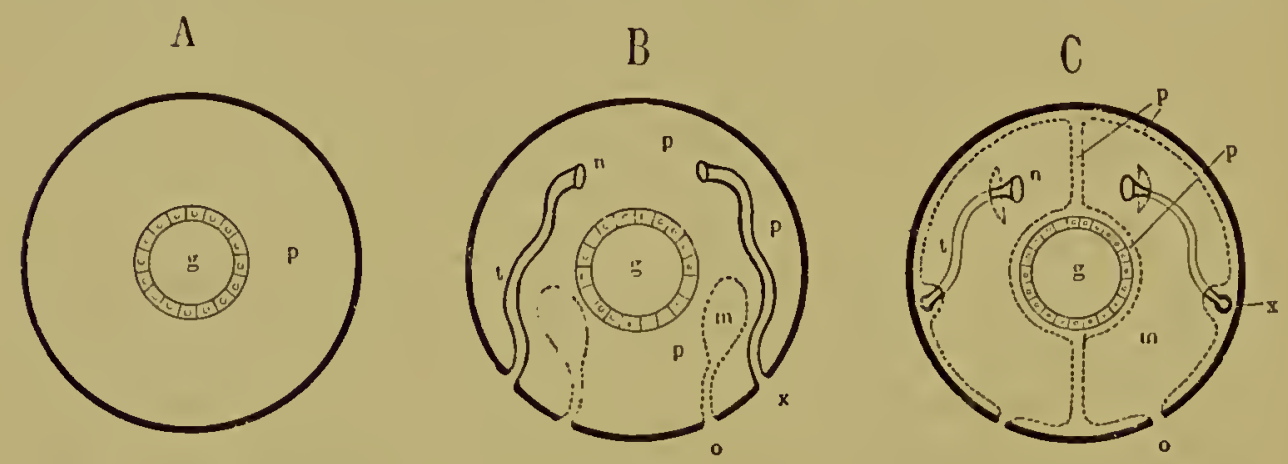

FIG. 105. Diagrams to illustrate the prevertebrate history of the nephridia, the gonads and the colom.

(A) Stage of gastrula, with two germ layers, a gastrocole $(g)$, and a primary body cavity ( $p$ ). (B) Here a third layer has appeared in the form of paired gonadic sacs $(m)$, and paired nephridial tubules $(t)$; with external openings at $o$ and $x$ respectively. The nephridia are furnished with an inner opening, the nephrostom $(n)$. (C) In this the gonadic sacs $(m)$ have expanded and form the definite colom, limiting the primary body cavity to a series of small spaces in all parts of the body. The nephridia open internally into these sacs, and their outer ends open into a longitudinal duct $(x)$.

and hence its functional efficiency within the prescribed limits. Nephridia of this type are frequent among invertebrates.

The other sort of organ, the gonad, has the form of a simple epithelial sac, with a narrow duct. Its walls are constantly proliferating and furnish cells which project into the interior and finally become free, passing out through the duct. These are the germ-cells, and may be either ova or spermatozoa, the product respectively of female and male parent individuals. Gonads of this character are frequently found among invertebrates, often in quite typical form. 
Taking now an animal of the type shown in Fig. I05, B, with a continuous protocœle and with a metamerism marked by several successive pairs of associated nephridia and gonads, imagine the result of a gradual and equal expansion of the latter until they attain the furthest possible limits (Fig. I05, $\mathrm{C}$; also Plate II). The protocœle becomes suppressed and in its place exists a series of paired chambers, metacœles, each pair in contact with the previous and succeeding ones and enclosing between them the alimentary canal. This latter part is thus hung between dorsal and ventral partitions, the mesenteries, each double and composed of the walls of the gonadic sacs; also each pair of cavities is separated from the next by similar double partitions which form intersegmental diaphragms or dissepiments. Each lateral metacole opens to the exterior by the opening which was once that of the gonadic duct. Thus far no provision has been made for the nephridia, which, with the suppression of the protocole, find themselves deprived of their original function. Their fate is, however, simple and obvious, for they receive an investment of the gonadic wall, and although lying between this and the outer body wall, project into the metacœlic cavity, with which they communicate through the ciliated nephrostome at their free end. But one further modification is necessary to adjust matters to the new conditions, and that concerns the walls of the metacoles. When in their original condition as the walls of small sacs employed for the production of germ cells, every portion of their surface is needed for the production of these latter elements, but when expanded to their final dimensions, they become mesenteries, dissepiments and the lining membrane of body walls, and form a thin and firm membrane, the peritoneum, while their original reproductive function is confined to certain restricted areas, situated near the dorsal mesenteries. These, by a slight evagination, produce rounded elevations that project into the lumen of the cavity and form specialized germ glands, the ovaries and the testcs. Their products, when mature, separate from their place of origin and wander freely about in the metacœlic cavity. From this they 
have two avenues of escape, the nephridia and the original openings of the gonadic sacs, and while so far as is known no animal exists that utilizes both methods, either one may become specialized to subserve this function. Furthermore, in an animal with many somites it is not necessary that ovaries or testes should develop in each pair of metacœlic sacs, but these may be confined to a few pairs or even a single pair, in which either the nephridia or the gonadic openings develop into special excurrent ducts for the liberation of the germ cells.

The conditions of this second hypothetical historic stage are realized in almost every detail by the annelid worms, allowing for a few modifications. [Cf. Fig. I39.] To some this indicates the conclusion arrived at independently by the consideration of other systems, that these animals lie very near the main stem of vertebrate ancestry, but to others this is no more than a case of parallel development, in no way more remarkable than countless other adaptive resemblances, such as the instance of the eye in cephalopods and vertebrates. However this may be, the example of the annelid is most useful in showing us that animals can exist in precisely the condition of the hypothetical form indicated by the study of vertebrate embryology and constructed from the data thus furnished.

From this second stage, which must be very near the actual condition in the ancestor of modern vertebrates, the final type may be reached by the introduction of a few slight and very natural modifications. The first of these concerns the metacolic sacs and consists, first, of the breaking down of the dissepiments between the body segments, thus throwing all the sacs of each side into one, and secondly, a similar loss, at least in part, of the ventral mesentery, making the two lateral sacs confluent below the intestine and allowing this latter to swing free in the cavity, suspended dorsally.

Thus, for the first time is reached a single secondary body cavity or metacale (the definite "colom"), lined acith peritoneum, which is reflected along the mid-dorsal line and 
furnishes an investment and suspensory ligament for the intestine. The calom is formed, as has been shown, by the expansion and later fusion of numerous pairs of gonadic sacs, and is thus an expanded gonadic cavity, while the peritoneum is identical with the walls of a great compound gonadic sac. The many pairs of gonadic openings are lost and appear either as a single pair (the pori abdominalis of cyclostomes and selachians) or are entirely lost (all higher vertebrates). Owing to this reduction either the second method for the liberation of germ-cells is employed, the utilization of nephridia, or else secondary ducts are developed to serve the purpose. The proliferating masses of germ-cells project from the peritoneal wall and become suspended in band-like ligaments like the mesenteries of the intestine, the mesovarium or mesorchium, and may either remain in their original location or become displaced and assume a secondary position. Finally the nephridia become confined to a certain region of the body, where they may form a pair of single definite masses, the kidneys, the units of which no longer open externally by independent openings, but become attached to a common excurrent duct, which opens, either independently, or, more usually, into the terminal portion of the alimentary canal.

Turning now from theory to fact, we may take up the urogenital organs as they actually exist in the various vertebrate groups, thus tracing a history by means of which the condition found in Man may receive at least a partial explanation. The condition in the cyclostomes is difficult to interpret; the teleosts seem not to come into line with the rest and represent a side branch, which, perhaps, presents an independent solution of the problem; but from the selachians and certain ganoids directly to the amphibians, and from them to the Amniota the history is a fairly continuous one. For the sake of clearness it will be best to consider separately the two systems involved, beginning with the urinary.

We have already learned that organs performing the same function in the different groups of vertebrates are not necessarily homologous, and are familiar with such phenomena as 
the two tongues, the two sternums, the two sets of ribs and the possibility of two mouths, but here we enter into a greater complexity, for the history of the urinary organs involves three kidneys, pronephros, mesonephros, and metancphros, each with its associated parts, which represent as many successive dynasties of organs that have replaced one another. In cases like that of the two respiratory systems, where the branchial system becomes replaced by the pulmonary, many of the parts of the first become employed by the second, often in quite a new capacity; but in the present case an added element is introduced on the part of the neighboring reproductive system, which not only employs at times portions of one of the urinary systems, but retains them in its service long after the system of which it formed a part has disappeared.

The first, or pronephrotic, system, appears in the embryo of all vertebrates; it functions during the larval life of some fishes and amphibians (Fig. I06), and in a few teleosts persists as a functional organ in the adult, but in other fishes and in all higher forms it becomes reduced to a few rudiments. It thus strongly suggests the assumption that it once formed the functional kidney in some vertebrate ancestors, from which it has been inherited. It consists of a few nephridial tubules, strictly metameric in arrangement, that is, a pair for each of several successive somites, situated very far anteriorly, often involving the first of the trunk somites. The nephridia of each side become associated together to form a single kidney, the pronephros, and enter a common pronephrotic duct, laterally placed, and opening either directly to the exterior in the vicinity of the cloacal opening or, more usually, within the cloaca itself by means of a papilla which projects from its dorsal wall.

This duct is, for the most part, like the nephridia themselves, mesodermic in origin, although in some of the lower forms the posterior portion arises from the ectoderm, giving to the entire duct a double origin. This strange condition may be in part accounted for if we consider that originally there was a larger number of nephridia and that each opened 
by itself directly to the exterior. It may then be supposed that for the better disposal of the excretory fuid the separate openings became connected by a groove which continued to the side of the cloacal orifice and deepened posteriorly into a trough, from which by a further continuation of the process an internal tube would be formed, opening either at the margin of the cloaca or just within it. The mesodermic anterior portion may be the result of the fusion of the outer ends of the succesive nephridia, each one contributing that portion belonging to its own somite.

Typical pronephridia (Fig. IO7, A), the units of the pronephros, closely resemble the one given in the theoretical description above. They possess at the inner end ciliated nephrostomes and show a greater or less tendency to coil, suggesting a former condition of considerable physiological efficiency. Aside from this, they show the beginning of a relationship essentially vertebrate, and carried out in greater detail in the mesoand meta-nephrotic systems, namely, an association with capillary blood-vessels, enabling the nephridia to extract waste material directly from the blood. This association is here very slight,

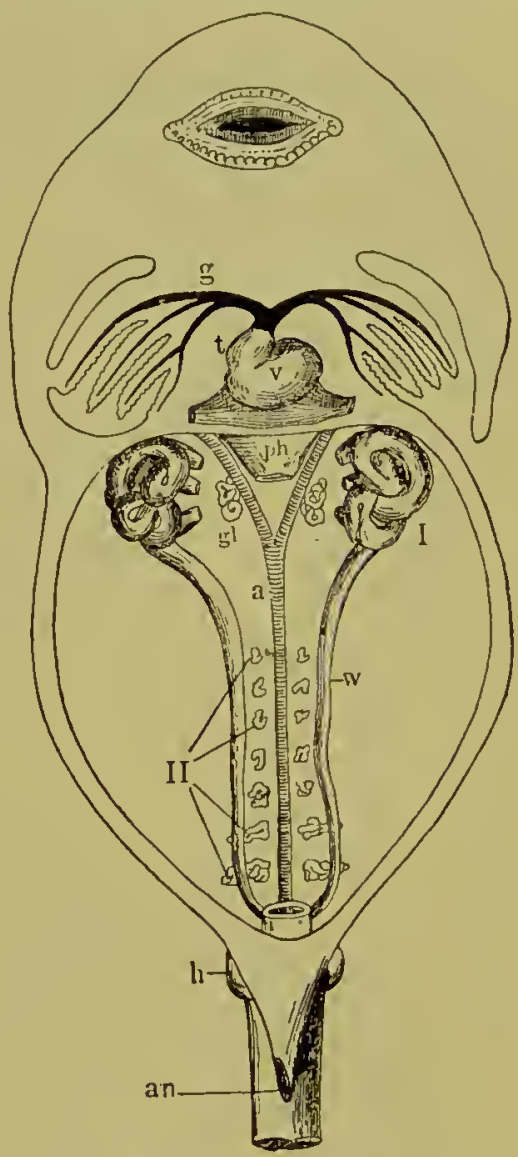

FIG. 106. Frog tadpole with functional pronephros and developing mesonephros. [After MARSHALL.]

$v$, ventricle of heart; $t$, truncus; $g$, gill arteries; ph, pharynx; $a$, aorta; $h$, anlage of hind limbs; $a n$, anus; $I$, pronephros; II, mesonephros; $g l$, glomerulus; $w$, Wolffian duct. and consists of segmentally arranged tufts of capillaries, glomeruli, which protrude into the colomic cavity and form rounded elevations covered by the peritoneum. These are located opposite the nephrostomes, and the excretory fluid, which passes from the glomeruli to the cœlomic cavity, is 

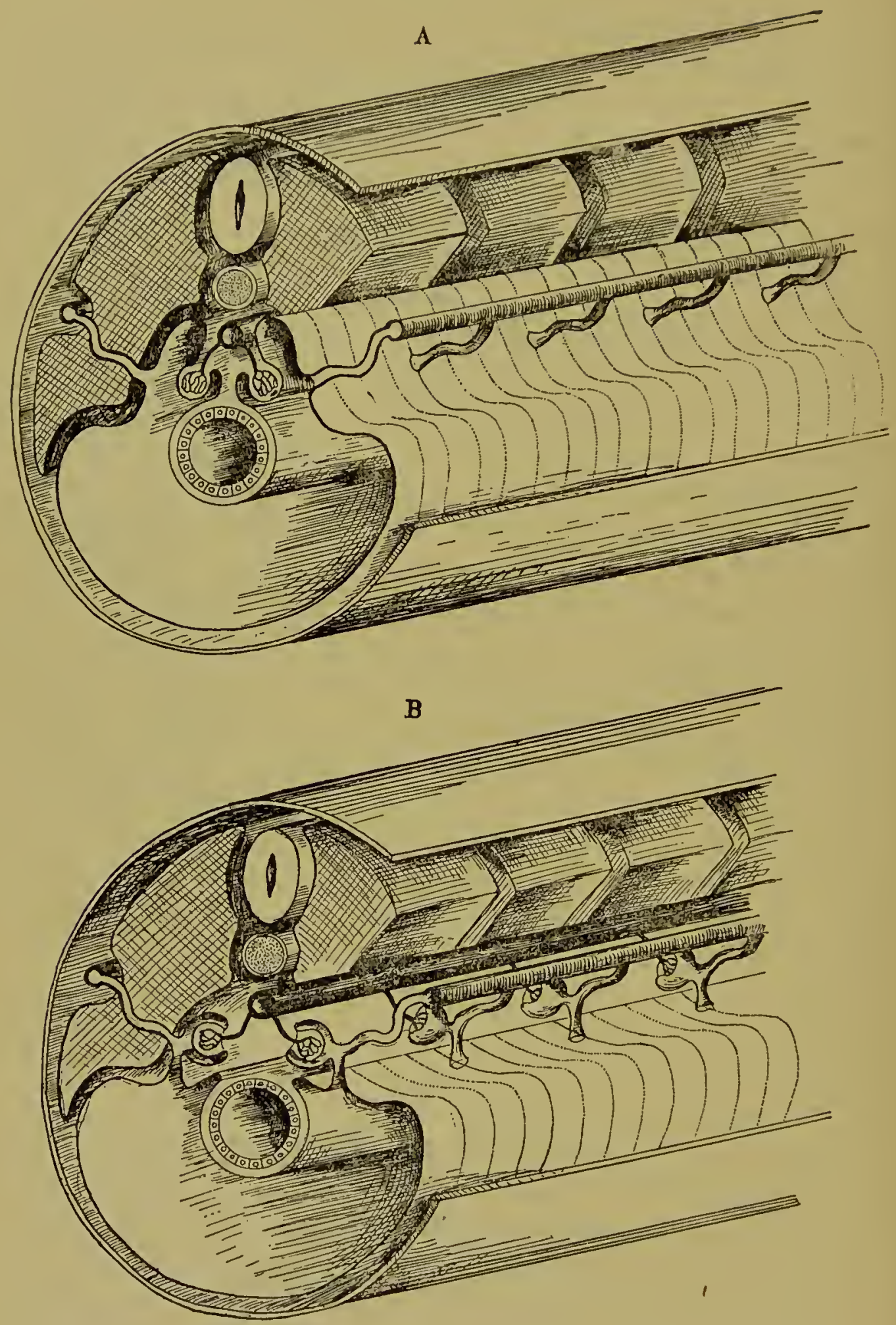

FIG. 107. Diagrams illustrating the two forms of nephridia characteristic of pro- and meso-nephros, respectively.

(A) Proncphros. (B) Mesoncphros. Farther cxplanation in text. 
taken tup by these latter organs. There is no direct connection between glomerulus and nephridium, although in several instances both the elevation containing the former and the nephrostome become included within a recess of the cœlom, an arrangement which furthers the mutual action of these parts. The number of pairs of nephridia involved is usually small (3-4), but in the Gymnophiona, in which the pronephros functions for a considerable period, there may be as many as IO-I3. Naturally the pronephrotic system is seen in its most complete state among the lower vertebrates; in Amniota it is often quite rudimentary and variously modified.

The pronephros, even when best developed, possesses but a temporary existence and becomes supplanted by the mesonephros, the kidney of the second or mesonephrotic system. This organ is formed from nephridia which are, like the first, segmental in origin and arise from somites posterior to those associated with the previous system. It forms the permanent kidney of fishes and amphibians, and in the embryo of Sauropsida and Mammalia it is large and prominent and has been known as the "Wolffian body," named in honor of its discoverer.

The separate units of this system, the mesonephridia (Fig. I07, B), differ in one essential particular from those of the pronephros, namely, in their closer association with the arterial glomeruli.

In the case of the pronephridia these capillary tufts were merely brought into close relation to the nephrostomes, but each mesonephridium surrounds a glomerulus with a thinwalled evagination from its side, which fits about it like a double cup and forms what is known as a Boroman's capsule. The entire structure thus formed, including both the capsule and its glomerulus, forms a renal [Malpighian] corpuscle. Otherwise the mesonephridia are like those of the former system, and possess nephrostomes and coils. They develop no duct of their own but utilize the pronephrotic duct, becoming secondarily connected with it posterior to its connection with the pronephridia. Later on both pronephridia 
and that portion of the duct anterior to the connection with the mesonephridia become atrophied, and the duct thus becomes the mesonephrotic, the "Wolffian duct" of an earlier nomenclature. On account of this utilization of the pronephrotic duct by the mesonephrotic tubules it has been held by some that both belong to one system and that the latter are merely later appearing elements of the pronephrotic series, but this is discredited by others on the ground of the difference in the time of functional activity of the two systems and also on account of the several somites without nephridia of either kind that intervene between the two. The structural difference between the two types of nephridia, one with a Bowman's capsule, the other without, may be also employed as an argument in favor of the distinctness of the two systems, but this argument is weakened by a consideration of the manner of formation of this new part, and by the assumption of the existence of almost every grade of transition between the two. Thus in its more usual form a pronephrotic tubule is related to the accompanying glomerulus much as in Fig. Io8, A, but in some cases the projection bearing the glomerulus becomes partly enclosed in a recess of the colom, and the nephrostome opens into this instead of into the main cavity (Fig. Io8, $B$ ). The development of cilia at the narrow passage which leads into the recess, and the loss of them around the margin of the original nephrostome, would convert the entire apparatus into a mesonephrotic tubule, in which the added portion, including the new nephrostome and the Bowman's capsule, is a contribution from the peritoneum. These diagrams seem absolutely persuasive, but unfortunately do not correspond with the actual facts, since, although in special cases the glomeruli of the pronephrotic system are related much as in the second diagram, the Bowman's capsules of the mesonephrotic tubules develop directly as evaginations from their walls, and there is thus no indication of either the participation of the cœlom or of the formation of a new nephrostome.

The mesonephrotic system may be found in full functional 
activity in any adult fish or amphibian. Originally involving a large number of somites the mesonephros extends, usually as a pair of long, narrow organs, along a large portion of the trunk, lying close up against the vertebræ and ribs. The original nephridia become greatly multiplied and lose more or less of their original segmental arrangement, the nephrostomes appearing irregularly along the ventral surface, that is, the surface turned toward the colomic cavity. The mesone-
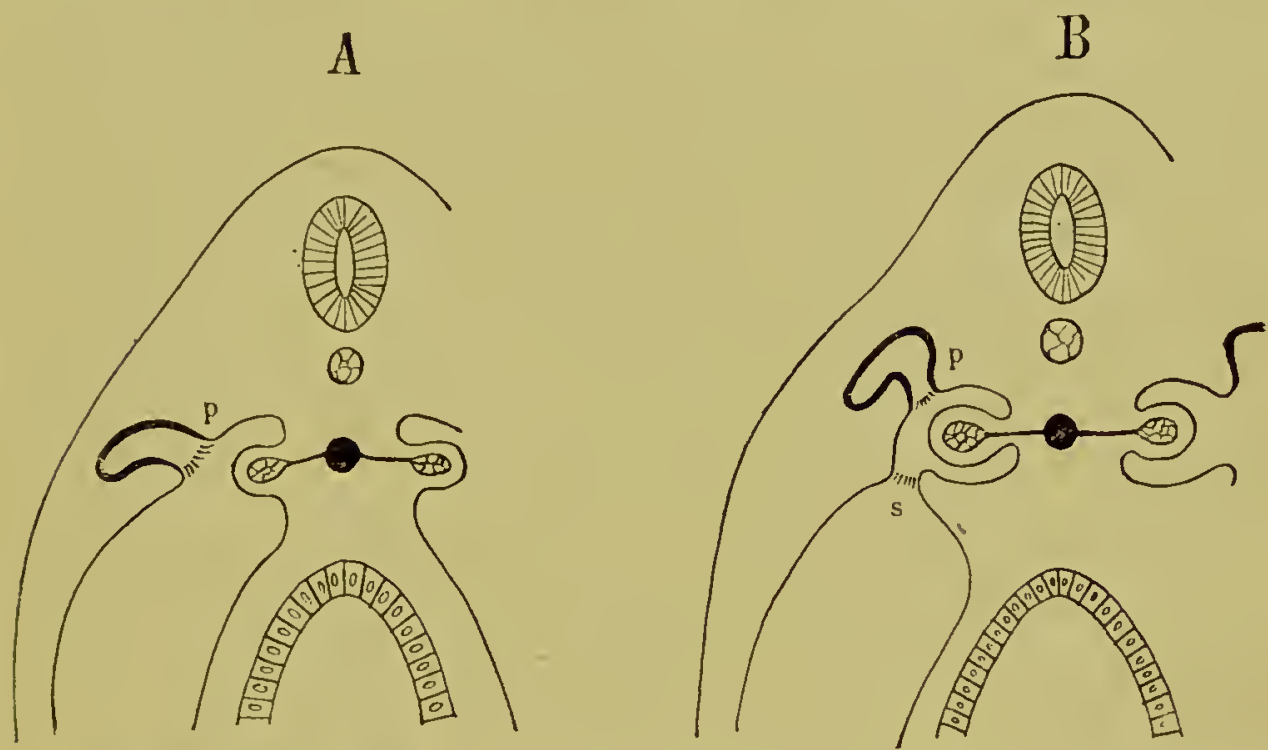

FIG. I08. Diagrams to illustrate a theory of the relationship of the pro- and meso-nephrotic tubules to each other. [After GEGENBAUR.]

(A) Stage of the pronephros. Its neplirostome $(p)$ is placed opposite the glomerulus, but with a small portion of cœlom interposed between them. (B) Stage of the mesonephros. Here by the formation of a new nephrostome at $s$ the interposed piece of the coelom has become included in the nephridium, forming a Bowman's capsule.

phrotic (Wolffian) duct lies along its outer side and sustains that relationship to the cloaca noted above under the pronephrotic system, usually opening by a urinary papilla into the dorsal wall of the cloaca.

The third urinary system, the metanephrotic, arises directly from the second, and thus the relation between the two is closer than that between the second and the first. It is the definitive urinary system of the amniotes, and in all reptiles, birds and mammals ultimately replaces the mesonephrotic, although this latter system is well developed during embry- 
onic life. The metanephrotic system is not laid down at first in the form of nephridia as in the other cases, but arises as a blind canal or evagination from the mesonephrotic duct near its lower end. This evagination, medial at its origin, comes to lie dorsal to the mesonephrotic duct and develops anteriorly until it comes in contact with the dorsal wall of the cœlomic cavity, where it meets a mass of indifferent cells proliferating from it. From the differentiation of this cell mass develop numerous nephriclia of a type similar to but in certain characters distinct from either of the other types, and from the repeated branching of the anteriorly growing canal there develop collecting tubules with which the nephridia unite. The expanded bases of the terminal branches of the tube form a pocket or pelvis, which collects the fluid from the tubules. The nephridia and collecting tubules form together the definitive kidney, the metanephros, while the main tube, beginning with its expanded pelvis, becomes the ureter.

Each elementary unit of the metanephros, a metanephridium, is like that of the previous system without the nephrostome. The connection with the circulatory system through the glomeruli, which when first introduced was clearly a secondary function of the nephridia, becomes in the mesonephros of primary importance through the development of a Bowman's capsule, and in the metanephros all direct connection with the colom is given up. Here, in addition to the association with the circulatory system through the Bowman's capsules, the tubules themselves become very long and attenuated, and, as they are accompanied by a rich network of capillaries, they are enabled to extract the waste products through their entire length as well as at the localized renal corpuscles.

This history of the development of the metanephrotic system, so different in origin from that of the other two, and yet so similar in its results, has led to much speculation. It cannot be supposed for a moment that nephridia so nearly alike as those of the meso- and meta-nephros can have developed independently, for that would involve also an independent 
origin in the two cases of such complicated structures as the renal corpuscles. The primary location of the metanephros, posterior to that of the mesonephros, or at least to that of its functional portion, leads to the idea that the nephridia of this system were originally a part of the mesonephrotic series, belonging to its more posterior somites, and that their development from a structureless mass is a case of shortened development, in which the primary segmental arrangement has become lost. The necessity for the development of a new ureter is easily seen in the employment of the older one (the mesonephrotic or Wolffian duct), as a ductus [vas] deferens, a point to be brought out later in connection with the reproductive system.

The external form of the metanephros varies considerably. This in the Sauropsida is in accordance with the form of the dorsal skeletal wall, to which it is closely applied. In structure it is usually distinctly divided into lobes that correspond to the terminal branches of the ureter. This is characteristic of the kidney of most mammals, and the compact form found in Man is attained considerably after birth, and is met with in only a few cases.

As may be followed from the development, the ureters terminate posteriorly in the mesonephrotic ducts and may be expected to share the common outlet into the cloaca. This is actually the case in snakes, crocodiles and birds, which consequently never perform urination as a distinct act, but in other reptiles and in mammals there is found a terminal resevoir, the urinary bladder, with which the ureters become secondarily connected. This opens at first directly into the cloaca, but its narrowed neck develops in the higher mammals into a distinct canal, the urethra, which in the male comes into direct association with the ductus deferens. The urinary bladder is no new formation, but is the remnant of the inner end of the allantois, an extensive embryonal membrane, which passes out of the body at the umbilicus and becomes in the Sauropsida an external respiratory organ, and in mammals furnishes the essential parts of the umbilical cord and placenta. 
After birth a portion of this becomes shut within the body by the closure of the umbilical connection, and as this portion is in the form of an open bag leading out from the cloaca, it is easily converted into a reservoir for urine, the greatest change necessary being a slight shifting of the terminal portions of the ureters. Only the lower portion is actually utilized for this purpose, and the remainder atrophies into a ligament, which extends from the apex of the bladder to the umbilicus. Approaching the cloaca the bladder becomes narrowed to a small neck which is continued as a.median duct or canal, the urethra, and opens, in common with the genital ducts, into the urogenital sinus.

A structure called a urinary bladder is present in amphibians. This is in the form of a collapsed bag leading out from the ventral wall of the cloaca and is without direct connection with the urinary system. This seems to represent morphologically an undeveloped allantois, and is thus really homologous with the bladder of the Amniotes. Its function is not wholly understood, as it never appears to contain liquid, but the occasional presence within it of excretory salts suggests a subordinate use in connection with the urinary system.

The second of the two associated systems to be considered is that of reproduction (generation), and consists primarily of the germ glands, in vertebrates a single pair, together with some definite avenue of escape for the mature germ cells. To these may be added secondarily external parts to insure the union of the two sorts of germ cells.

The germ glands, the essential organs of reproduction, develop as localized areas on the peritoneal wall of the cœlom, and are primarily located dorsally, one on either side of the vertebral column, in about the middle of the trunk region. This similar origin, from a layer which otherwise forms nothing but investing membranes and suspensory ligaments, is easily explained by the theory given above, which considers the entire cœlom as the result of the fusion of a series of expanded gonads, a theory perfectly in harmony with all the related facts. The germ glands, primarily patches of germinal 
epithelium, become reinforced from behind by the proliferation of connective tissue, containing nerves and blood-vessels; and thus are formed mounds projecting into the cœlom, covered with germ cells. This association becomes more intimate through the intrusion of the germinal epithelium into the interior in many places, where the cells receive the nourishment necessary for their complete development.

The germ cells themselves are of two sorts, ova and spermatozoa, and their differences in form and size necessitate a more or less apparent difference in the organs that produce them, the ovaries and testes respectively. In certain cases among cyclostomes the same germ gland produces both sorts of germ cells, although at different times, but with this exception the sexes are normally separate. The occasional occurrence of a few cells of one sort in a gland which normally produces the other, as the development of a few ova on the side of a testes, or vice versa, occurs as an anomaly among many of the lower vertebrates, and this phenomenon, taken in connection with the cases among the cyclostomes just cited, has led to the possible theory that the ancestors of vertebrates were hermaphroditic, as is the case in many invertebrates, but there is little else to indicate this. Reported hermaphrodites among higher vertebrates are usually if not always apparent rather than real and are in fact malformations due to some error in development affecting mainly the external parts.

All that is essential for the production of a new organism is the complete and intimate union of the two germ cells, one of each sort, but the varied environment of the parents often makes it a problem to arrange the means by which this may be accomplished. It offers the least difficulty in the case of aquatic forms, for all that is here necessary is to liberate the cells of both sorts into the water, in which the union can be easily effected, since the water furnishes the fluid medium necessary for the locomotion of the spermatozoa. Often, too, such animals associate in pairs and develop elaborate instincts which insure the discharge of the two products in close proximity to one another. 
This absolute necessity of a fuid medium causes the devclopment in land forms of a number of accessory parts. Thus there develop in the male special glands to supply a vehicle for the spermatozoa, forming a spermatic fuid; and as this cannot be allowed to dry up, it must be conveyed directly to the female by an internal copulation, necessitating again certain modifications of the cloacal margin, from which develop the various external organs. Although it is evident that the development of the process of copulation is here diue solely to the terrestrial life, there are sometimes other conditions that develop it, for although, on the one hand, it is universal among terrestrial forms, invertebrates as well as vertebrates, it is occasionally found among aquatic animals, notably in this connection the selachians; that, however, it is here an independent development is shown by the source from which the copulatory organs are derived, namely, from the inner margin of the ventral fins, and not from the rim of the cloaca, as in higher vertebrates.

There are thus two groups of accessory reproductive organs to be considered, (I) those which furnish an outlet for the germ cells, and (2) those which are concerned in internal copulation. These may be taken up in order.

The conception of the peritoneal cavity as an expanded gonadic sac demands that the germ cells generated in its wall should break loose and float about within the cœlom, until finally expelled either through some direct channel of communication with the exterior, the original gonadic ducts, or else by utilization of some part of the nephridial system; and as a matter of fact all conditions found in vertebrates, with the possible exception of that of teleosts, may be directly referred to one of these methods.

The most primitive condition is that seen in cyclostomes, in which the peritoneal cavity communicates directly with the exterior by means of a pair of pori abdominales, canals which begin at the posterior part of the cœlom and open along the sides of the cloacal orifice. The germ cells, when matured, become freed from their place of origin and float about in the 
peritoneal cavity until discharged through these abdominal pores. The urinary organs have no direct connection with this system other than through the nephrostomes which open into the peritoneal cavity, and these are not specialized to receive the free germ cells.

There is thus showen the original condition of gonads and their excurrent ducts, slightly modified by the fusion of all the gonads into one and the reduction of the gonadic ducts to a single pair. Otherwise the primitive physiological functions are carried on as they were before the gonadic cavities became converted into a metacole.

It will be noticed that in the above description the nephrostomes open directly into the cœlomic cavity and thus suggest the possibility of the use of nephridia for the exit of the germ cells. Such is actually the next stage in the history of these organs, for in the selachians certain of the nephridia are so employed while the pori abdominales, although they still exist, are no longer used for their original purpose. In the male the testes lie in close proximity to the anterior portion of the kidneys, and enter into direct connection with the nephridia of this region through the development of a series of tubes, the vasa efferentia, which extend from the testes and enter the nephridia a little beyond the nephrostomes. The original function of this part of the kidneys is not impaired, and during the greater part of the time it exercises the urinary function alone; but during the periods of sexual activity the nephridia involved become filled with the spermatic fluid and deliver it directly from the testes to the mesonephrotic duct and thence to the cloaca. From there it is received into a channel formed by the approximation of the inner modified portions of the ventral fins, and delivered within the cloaca of the female by an internal copulation, an unusual method among aquatic animals. That there is no genetic connection between this act and that developed among terrestrial vertebrates may be seen from the employment of very different organs for the purpose in the two cases and from the fact of the interposition, in the direct line of descent, of forms that 
do not develop any such method. Through this close connection between the originally distinct reproductive and urinary systems it results that both the anterior part of the mesonephros and the mesonephrotic duct become, apart from their urinary function, accessory reproductive organs, the former serving as a "sexual kidney," and the latter as a ductus deferens, or excurrent seminal duct.

In the female selachian a different modification takes place, seemingly not due to association with the urinary system, but proven to be so by the developmental history of the parts. The ovary of the adult occupies about the same position as do the testes of the male, but shows no direct connection with the anterior part of the kidney. In place of this there appears on each side a long tube running along the side of the mesonephrotic duct and opening posteriorly into the cloaca beside that of its associate. This is the oviduct, or "Miiller's duct" of many writers. At the free anterior end, which extends to almost the forward limits of the colom, it opens by an expanded mouth, ostium tubce, directly into this latter cavity and receives into this the mature ova which become released from the ovary and wander about in the colom in the primitive fashion. The oviduct arises in the embryo as a tube segmented off longitudinally from the mesonephrotic duct by the common method of the development of two longitudinal folds opposite one another, and thus points to a period at which the ova as well as the spermatozoa were conveyed to the cloaca through the mesonephrotic duct. The ostium is probably an enlarged and specialized nephrostome, associated with a single nephridium, * and it is thus easily imagined that the primary conditions in the female corresponded closely to that of the male, but, that owing to the greater size of the products to be

* The not infrequent occurrence, even in the human subject, of two ostia upon one side may possibly be the result of the retention of two nephrostomes instead of a single one, or it may be simply an anomaly like the multiplication of digits on other parts. If it be the first it concerns a very ancient bit of history, and suggests an extreme degree of reversion. 
transmitted, a single nephridium with its nephrostome became differentiated for this purpose, and that later on there came a longitudinal splitting of the primary mesonephrotic duct, beginning above and progressing gradually, for the better accommodation of the sexual products. The employment of a nephrostome instead of vasa efferentia is quite a fundamental difference, but rudiments of these latter vessels are to be detected in association with the ovaries, and thus the use of the former may have been a later adaptation.

The uro-genital relations of the selachians seem to have been inherited directly by the amphibians (Fig. Iog, $a$ and $c$ ), for the two correspond closely; in the male there is the same relationship between testes and sexual kidney, and the mesonephrotic duct is a common ureter and ductus deferens. A rudimentary oviduct tapering anteriorly to a blind end, is usually found attached to the side of this latter tube. In the female the oviduct is often very long and convoluted, and its walls are often glandular and furnish membranous and gelatinous encasements for the eggs. In a few instances the lower part of the tube is expanded into a uterus for the retention of the larva.

Corresponding to the lack of internal copulation there are no external organs, but there are various instincts developed which have for their purpose the mingling of the sexual products. Thus the males of some aquatic salamanders produce conical spermatophores, which rest upon the sand at the bottom of the pond and are taken up by the cloaca of the female; a similar purpose is seen in the amplexation of frogs and toads, in which the males embrace the females during oviposition and void the seminal fluid over the egg masses as soon as laid.

A fundamental change of relationship is seen in the Amniota, caused by the appearance of the third kidney, the metanephros. This organ, which possesses a separate ureter and is thus a complete urinary system in itself, assumes the entire control of this function, and leaves to its predecessor, the mesonephrotic system, nothing but reproductive functions. 

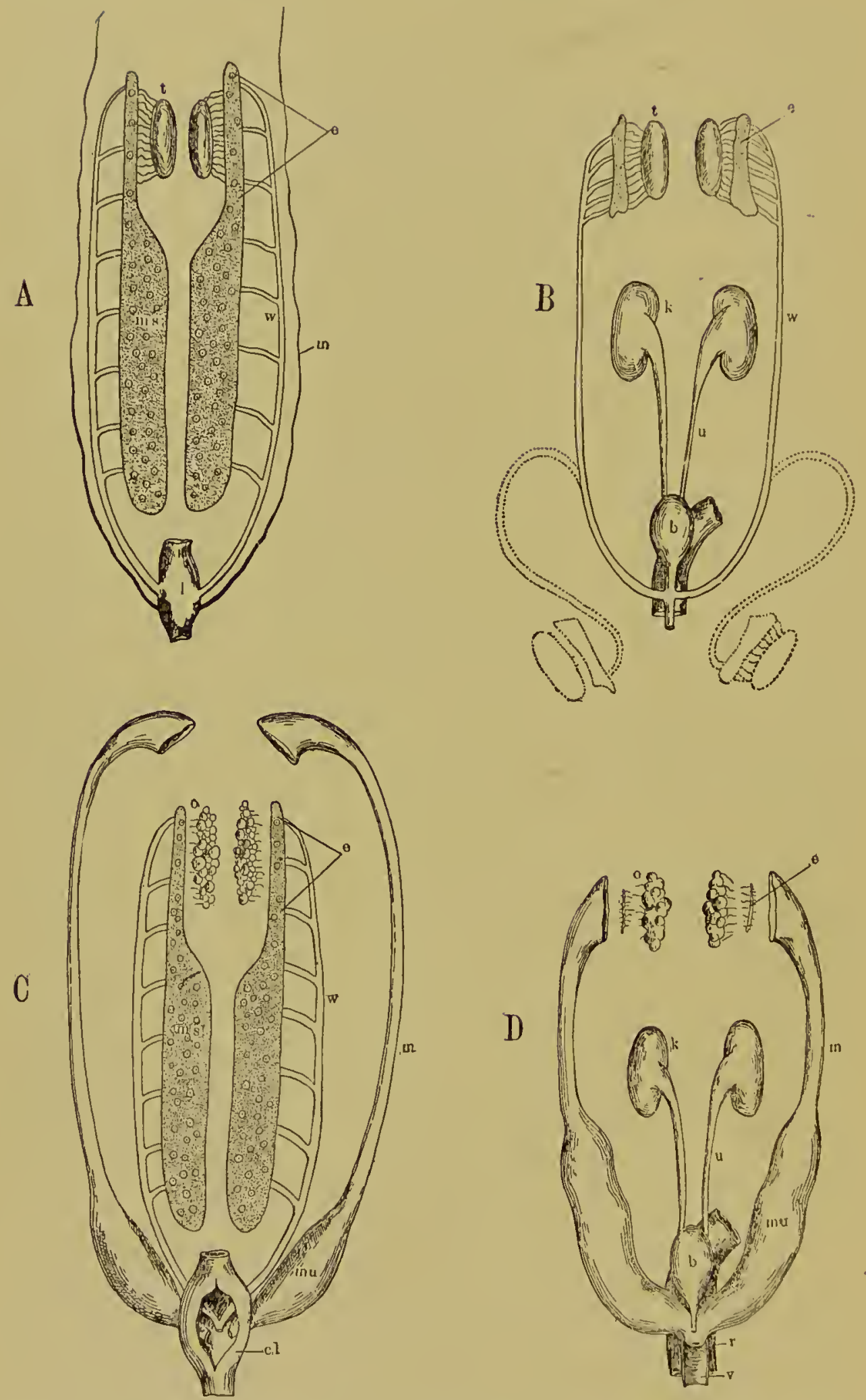

FIG. I09. Comparison of the urogenital system in Anamnia and Amniota. (Continucd at bottom of p. 385.) 
As a result of this, those parts of this latter system which have been previously employed for reproductive purposes are retained and even become more highly specialized, while the parts that were wholly urinary disappear, with the exception of a fere vestiges.

In this the two sexes are affected differently, as may be made clear by a reference to Fig. I09, in which $a$ and $b$ show the changes produced in the male, $c$ and $d$ those in the female. In the male amphibian the sexual parts are the testes, the vasa efferentia and the mesonephrotic duct; in the male amniote these parts are retained while the remainder of the mesonephrotic system has disappeared, being replaced by the metanephrotic. The mesonephrotic duct, released from all urinary function, becomes the definite ductus deferens, and the remaining portion of this system, including vasa efferentia, sexual kidney and collecting efferent tubules, becomes closely associated with the testes under the name of the epididymis. In the female amphibian the reproductive system has become practically independent of the urinary through the development of a separate excurrent duct, the oviduct, and thus, with the rise of the metanephrotic system, that of the mesonephros becomes reduced to a few functionless vestiges; yet the more conservative embryonic history records the fact that both oviduct and ostium were originally portions of the mesonephrotic system, and, although with a different history, both sexes are in reality about equally indebted to it for their accessory organs.

Although the reproductive organs, as given in the above sketch, are the common heritage of all amniotes, the separate groups of reptiles, birds, and mammals have been left to work out the details in accordance with their own necessities. In each there is a metanephrotic urinary system, with kidneys and ureters distinct from the reproductive system except for intimate topographical relationships at their outlets; in the

(A) Male anamnian. (B) Male amniote. (C) Female anamnian. (D) Female amniotc.

$t$, testis; $o$. ovary; ms, mesonephros; $c$, that part of the mesonephros which is associated with the germ gland, (in male amniotes this becomes the epididymis); $w$, Wolffian duct (ductus defcrens); $i$, Müller's duct (oviduct; mu, utcrus); $k$, mctanephros; $b$, bladder (of the Amniota); $u$, ureter; $r$, rectum; $v$, vagina. 
male the testes are accompanied by an epidirlymis and a ductus deferens, respectively the anterior portion of the mesonepinros and the mesonephrotic duct; and in the female there is an oviduct with an enlarged ostium, into which the wandering ova are received. In the present treatise the details of these parts in reptiles and birds cannot be considered further, but the history that is shown in mammals is of much importance, as it includes the human conditions.

In the mammalian embryo the mesonephrotic system attains a high degree of development, and the mesonephros, under the name of the "Wolffian body," is large and conspicuous. In the marsupial young of monotremes and marsupials it forms the functional kidney, and as this is but one of several organs that become profoundly modified or replaced during later life, the development may be rightly considered a true metamorphosis in which the marsupial young represent a larval stage. In placental mammals a similar replacement of urinary systems takes place, but as the intra-uterine life is here made longer than in former cases and includes also approximately the period passed by lower mammals in the marsupial pouch, there is no free larva, and the changes are considered a part of the embryonic development.

As both the stage of the functional mesonephros and its later reduction are of importance in understanding. the adult conditions, they may be first studied by the aid of the accompanying Plate. During the period designated as that of sexual indifference, which includes all the early development and continues until the embryo is quite well matured in many other particulars (up to 70 or 80 days in the human species), the sexes, although definitely determined, show absolutely no difference in the general appearance of the uro-genital organs. [Plate III, a]. The mesonephros is large and functional and stands out freely from the dorsal wall of the abdomen, held in place by a suspensory fold of peritoneum, the mesoncphrotic ligament. This fold becomes prolonged posteriorly beyond the limits of the Wolffian body and forms the inguinal ligament, a part of great importance in subsequent relationships. 

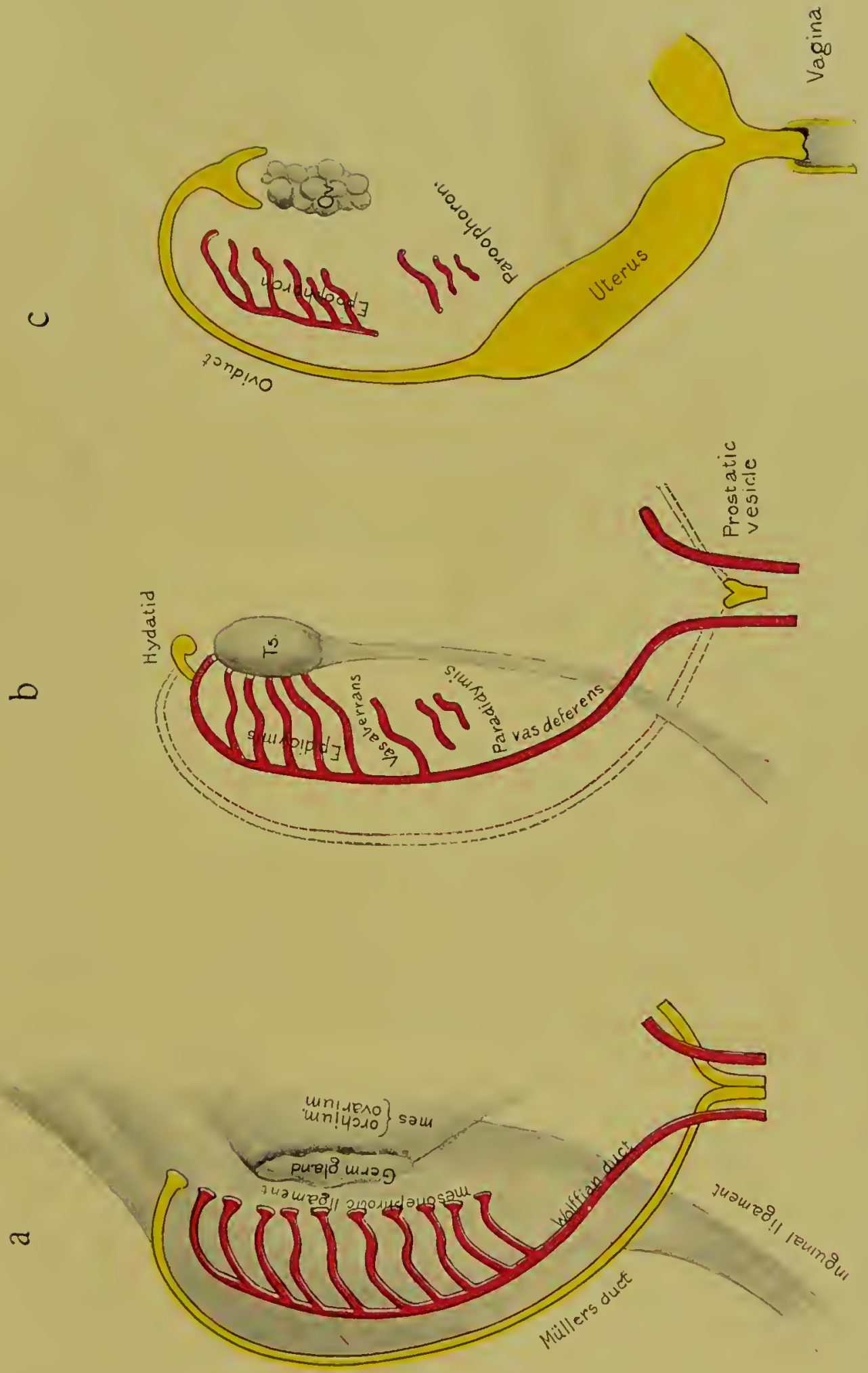

Upon the inner side of each mesonephros appears a longitudinal fold of its peritoneal investment, along the free edge of which the cells become proliferated and form the germ gland; the remaining, or basal portion of this fold is later to form the mesorchium or mesovarium, the suspensory ligament of the mature testis or ovary respectively. The Wolffian (mesonephrotic) duct lies along the free edge of the mesonephros, and not far from this is Müller's duct, suspended in a fold which projects from the ventral surface of the mesonephros. These two pairs of ducts are brought together at their distal ends and form a common chamber, into which all four empty, the uro-genital sinus.

From this stage the conditions found in the female are readily developed. [Plate III, c.] Its most important organs are the germ gland, which becomes the ovary, and Müller's duct, the upper part of which becomes the oviduct [uterine (Fallopian) tube], and the lower part, the uterus. The entire mesonephrotic system, since it is in no wise concerned in the reproductive function and. since the urinary function is wholly assumed by the metanephrotic system, disappears except for a few useless vestiges; its loss allows the mesonephrotic ligament to become continuous with that of Müller's duct, and thus to extend as the broad ligament of oviduct and uterus from the dorsal body wall to the latter organs. The round ligament is formed from the posterior extension of this latter, the ligamentum inguinale.

Müller's duct gives rise to the oviduct, uterus and vagina, which are thus seen to be nothing more than differentiations of the various regions of a single tube. The ostium is much nearer approximated to the ovary than in Sauropsida, and as it opens and partly surrounds the latter during ovulation, the entrance of the ova into the oviduct is practically assured. In some mammals there is a special arrangement in the form of a recess or pocket of peritoneum, the bursa ovarica, in which the ovary lies, covered by the ostium, and in a few cases the fusion of the edges of ostium and bursa convert the latter into a capsule which may either open to the coelom through a small 
foramen or may be absolutely closed. In the primates, including Man, the connection is not as intimate as this, and the ova occasionally escape into the colomic cavity, as is normally the case among lower forms. Here, however, they usually disintegrate and become lost, but in rare cases a fertilized egg escapes in this way and may even attain considerable development through the formation of a sort of placenta, attached to the cœlomic wall.

A uterus is more or less an adaptive organ, related to Müller's duct much as the crop is to the œesophagus; it is primarily nothing but a localized enlargement and develops whenever needed, in some cases appearing in a given form, while absent in a closely related one. Thus in vivaparous sharks (e.g., Squalus), the expanded lower portion of each Müller's duct becomes enlarged and forms a uterus in which the embryos are retained until they reach practicaliy the adult form; and the same is true in the case of a certain salamander (Salamandra atra). In none of these cases, however, is the organ more than a container or brood cavity, and there is no placenta formation or other direct connection between embryo and uterine wall. The same is true of the lower mammals, the marsupials and monotremes, in which there is no placenta, and the young are produced in a very immature state; but in the higher, or placental, mammals, the w'all of the uterus becomes differentiated for the purpose of the nutrition of the embryo, and thus becomes a definite physiological organ.

There are numerous types of uterus among the mammals, depending on the degree of fusion between the Müller's ducts of the two opposite sides; and these types consequently present a regular graded series between two distinct lateral uteri and a single median one (Fig. IIO).

In the first type of this series, that seen in monotremes, the two ducts are entirely distinct from one another. They are short, thick walled, and of rather large caliber, and may be termed oviducts or uteri, according to the taste of the writer, although the former term is more usually applied to them. They open separately into a common urogenital sinus in close 
association with the openings of the ureters. There are no vaginx, although the urogenital sinus serves functionally as one. In the oviducts the small oval eggs, $3.5 \times 4.0 \mathrm{~mm}$. in diameter when they leave the ovary, are retained for some time and increase in size to about I 2 XI $5 \mathrm{~mm}$. through the absorption of nutrient fluids secreted by the oviducal walls.

The next stage is that of the marsupials, in which the two ducts are still distinct, but each shows a differentiation into oviduct, uterus and vagina. The two vaginal orifices open

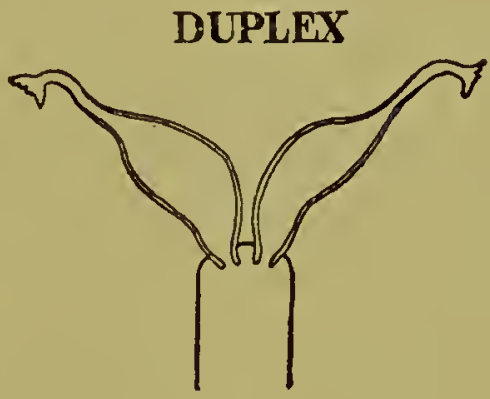

BIPARTITUS

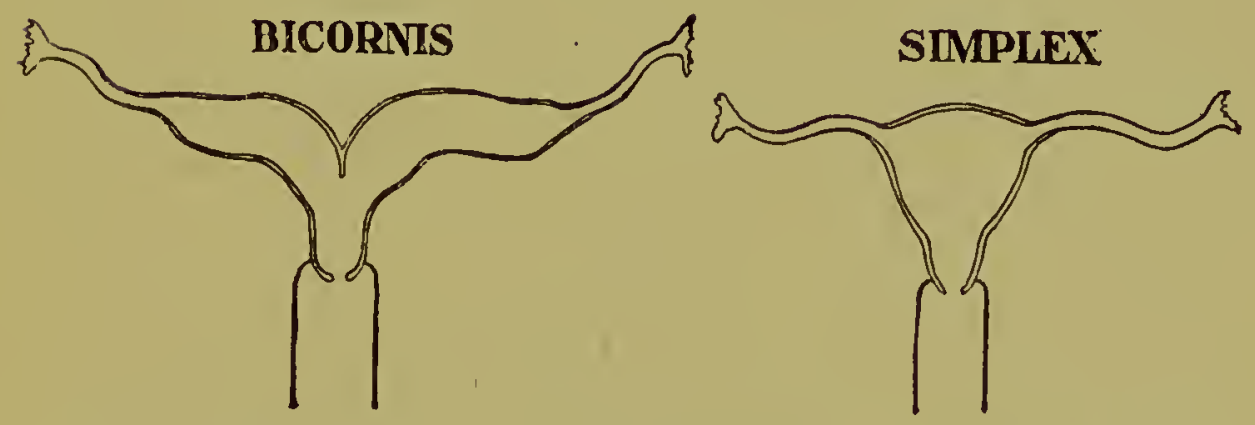

Fig. IIo. Different types of mammalian uterus: explanation in text.

into a common urogenital sinus, which is here prolonged into a canal, and opens to the extcrior separately from the rectum. Here for the first time the complcte separation of these two canals, urogenital and alimentary, is met with, since in monotremes, although internally distinct, there is externally a common cloacal orifice. The partition separating the two becomes thicker and of more importance in the higher mammals, and forms the perincum.

Above the marsupials the two ducts fuse into one at their 
terminal portions, and form a single vagina, though usually with two lateral uteri, various stages of the fusion of which form the successive steps known as uterus duplex, viterus bipartitus, and uterus bicornis. The first of these types possesses two distinct openings for the uteri (ora uteri), which open into a common vagina; in the others there is a single os, but two uterine compartments. In all cases the vagina is single, but an indication of its former duplicity is observed in a few animals (e.g., Equus), in the form of a median longitudinal fold.

The duplex type occurs in Procavia (Hyrax), an isolated group of small mammals found in Western Asia and in Africa; the bicornis is widely distributed and occurs in ungulates, cetaceans, most bats, and other forms; the bipartitus occurs in carnivores, the pig, and a few bats.

When several embryos are developed simultaneously, as in most small mammals, the two uterine halves become drawn out into long tubes, and the embryos are fixed at approximately equal intervals, each half containing about the same number. As the embryos develop, the portions of the tube in which they lie become greatly enlarged, while the intervening parts are restricted, giving the whole the appearance of a necklace or a string of sausages.

The uterus simplex, characteristic of man and the apes, represents the extreme degree of fusion of the two parts. In this nearly all signs of its double origin are lost and the uterus assumes the form of a balloon-shaped or piriform organ, somewhat flattened dorso-ventrally, and possessing two oviducts, which open at the slightly prolonged antero-lateral angles.

Uterus and oviducts are supported in all mammals by two principal suspensory ligaments, the broad and the round (ligg. latum et teres), which are easily explained by comparison with the indifferent condition. [Plate III, a.] Here the two layers of peritoneum that form the mesonephrotic ligament contain between them the mesonephros and its duct, and become continued along the ventral surface of the mesonephros as a low 
longitudinal fold which contains in its margin the Mïllerian duct. The germ gland (here the ovary) is attached to this along the inner side of the mesonephros by means of a narrow mesovarium. Imagine now the two important changes which actually occur, namely, the complete reduction of the mesonephrotic system and the development of the Müllerian duct into oviduct and uterus, and we have as a result the broad ligament, arising from the body wall and extending to the oviduct, which it enwraps along its free edge. The round ligament is formed from the posterior slip of the original mesonephrotic ligament, the ligamentum inguinale.

The vestiges of the mesonephrotic system are found exactly where they would be expected, lying in the broad ligament not far from the oviduct, between it and the insertion of the mesovarium. They consist of three portions, epoöphoron, its longitudinal duct, and paroöphoron. [Plate III, c.] The first of these ("organ of Rosenmüller") consists of a series of blind tubules attached to a common duct, and plainly represents the vasa efferentia and the upper portion of the Wolffian duct, in other words, the "sexual kidney." Below this are a few scattered tubules, forming the paroöphoron and representing the lower or urinary portion of the mesonephros. The longitudinal duct of the epoöphoron ("Gärtner's duct") is the remnant of the main part of the Wolffian duct, and lies imbedded in the wall of the uterus; it occasionally joins its upper part and thus completes the representation of the mesonephrotic system. It occurs quite regularly in the pig, the horse and in ruminants, but is only occasional in Man.

The original direction of the mesonephrotic ligament, that is, the direction which it has in the embryo, and that which is retained in adult Sauropsida, becomes changed in mammals and comes to lie transversally across the dorsal wall as though laid over laterally from above, the lower part remaining as at first. The principal effect of this is to remove the ovaries, and with them the oviducts, from their primary position in the lumbar region and located them near or within the brim of the pelvis, not far from the inguinal region. This occa- 
sions several compensatory changes, such as the lengthening of the ovarian nerves and blood-vessels, which are contained in the mesovarium. The significance of this process, known from its principal feature as the decensus ovariorum, is unknown, but it seems to correspond in part to a somewhat similar but more extensive descensus of the testis, found in the male.

To comprehend the relationships of the male organs in mammals, it is best to begin again at the indifferent stage [Plate III, a], which is thus seen to furnish the starting-point for the explanation of the reproductive organs in both sexes. While the accessory organs in the female are mainly the product of differentiation in the Müllerian duct, the Wolffian duct becoming vestigial, in the male it is the Wolffian duct that is emphasized, together with the upper portion of the mesonephros, while the Müllerian duct is reduced to a few rudiments. [Plate III, b.] As in male selachians and amphibians, the anterior tubules of the mesonephros serve as vasa efferentia for the conduction of the spermatozoa, but here they are used exclusively for this purpose, while the nephrostomes, Bowman's capsules, and all parts of those tubules once associated with the urinary function, are no longer developed. The tubules are much convoluted and form a compact mass, closely associated with the testis, the epididymis. The remaining mesonephrotic tubules, those of the posterior, or exclusively urinary portion in lower forms, never develop into functional organs, but one or two of them, with blind free ends, may retain their connection with the Wolffian duct, and form the so-called vasa aberrantia, while the remainder, without connection at either end, form a rudiment termed the paradidy'mis ("Organ of Giraldés"), the homologue of the paroöphoron, of the female. The Wolffian duct, freed from all association from urinary functions, becomes the exclusive spermatic duct, the ductus deferens (vas deferens). The Müllerian duct is lost along the greater portion of its extent, but leaves rudiments at either end. The anterior end is represented by the appendix testis [hydatid of Morgagni], a knobbed body at- 
tached to the epididymis; the posterior by a median vesicle which leads from the dorsal wall of the urethra and lies imbeclled in the prostate gland, the prostatic vosicle, sometimes referred to as the utcrus masculinus.

The history of the urogenital organs, as thus far considered, with their correspondence in the two sexes, may be conveniently shown in a table, in which the first column gives the part in its primary morphological significance, while the second and third state their ultimate fate in the male and female Amniota respectively. Vestigeal parts are given in italics. This table may be studied in connection with the one at the end of the chapter, in which the external parts are considered in the same way.

In the monotremes the ductus deferentes [vasa deferentia] open into a urogenital sinus, the ventral recess of 'a common cloaca, in common with the urethra or excurrent duct of the urinary bladder; in all higher mammals, however, with the formation of a perinæum or division between this uro-genital sinus and the rectum, the ductus deferentes are received by the much prolonged urethra so that the distal portion of this is a common duct for both urinary and reproductive products, a resumption of early conditions under another form.

While in the monotremes and in certain placental mammals the testes remain throughout life in or near the original position, in others they experience a more or less marked change of location. This is termed the descensus testiculorum, and is more or less comparable to a similar descent on the part of the ovaries, although the procedure involves different parts, and is quite likely of a different historical significance. Aside from the monotremes, no appreciable descent takes place in elephants and in certain insectivores, while in sloths and anteaters the testes descend considerably and take up a final position in peritoneal folds between bladder and rectum, but still within the pelvic cavity. The only other placental manmals in which there is no external manifestation of this process are the armadillos, related to these last, and the two aquatic orders of Cetacea and Sirenia, in aihich the condition is plainly a 
secondary modification due to the needs of an aquatic life. In all remaining mammals the process is connected with the formation of an inguinal canal, a subcutaneous evagination of the body wall involving muscles and peritoneum, and the testes pass into this either periodically, in association with sexual activity, or permanently. The former condition occurs

\begin{tabular}{|c|c|c|}
\hline $\begin{array}{l}\text { MORPHOLOGICAL DESIGNATION } \\
\text { (Embryonic or phylogenetic) }\end{array}$ & $\begin{array}{l}\text { MALE } \\
\text { AMNIOTE }\end{array}$ & Female amxiote \\
\hline Germ gland & Testis & Ovary \\
\hline Mesonephros (upper portion) & Epididymis & Epoöphoron (in part) \\
\hline Mesonephros (lower portion) & $\begin{array}{l}\text { Ductuli } \\
\text { aberrantes } \\
\text { Paradidymis }\end{array}$ & Paroöphoron \\
\hline Mesonephrotic (Wolffian) duct. & $\begin{array}{l}\text { Ductus de- } \\
\text { ferens }\end{array}$ & $\begin{array}{l}\text { Epoöphoron (in part) } \\
\text { Longitudinal duct of } \\
\text { epoöphoron }\end{array}$ \\
\hline Müller's duct & $\begin{array}{c}\text { Appendix } \\
\text { testis } \\
\text { Vesicula } \\
\text { prostatica }\end{array}$ & $\begin{array}{l}\text { Oviduct } \\
\text { Uterus } \\
\text { Vagina }\end{array}$ \\
\hline Urogenital sinus & $\begin{array}{l}\text { Morphologi- } \\
\text { cal urethra, } \\
i . e . \text { the por- } \\
\mathrm{t} \mathrm{i} \text { on b e - } \\
\mathrm{t} \text { w e en the } \\
\text { bladder and } \\
\text { entrance of } \\
\text { the ductus } \\
\text { deferentia }\end{array}$ & Urethra (entire) \\
\hline
\end{tabular}

among many insectivores and rodents, and in the bats; the latter is characteristic of the land carnivora, ungulates, most lemurs and the primates.

In the majority of animals coming under this latter head, that of permanent descent, the testes lie in a special integumental sac, the scrotum, but in some cases, as tapirs, rhinoceros, etc., there is no definite scrotum, and the testes lie beneath the 
integument of either the inguinal or perinæal regions. The scrotum is originally double, furnishing a separate sac for each testis, but ustally the two are fused into single median sac in which the suture of union is usually apparent. In relation to the penis the scrotum is originally anterior to it, prepenial, as in all marsupials that possess one, but in placental mammals these relations are reversed and the scrotum becomes postpenial through the migration of the penis in an anterior direction.

No definite cause for the descensus is known, either phylogenetic or physiological, and the phenomenon has gained rather than lost in complexity through recent researches which show the coöperation of several distinct elements previously not taken into consideration. Formerly a mechanical explanation was found in the gradual contraction of the band of peritoneum which extends from the testis to the inguinal region (Plate III, b), and termed the gubernaculum in reference to its supposed function, but the matter is not as simple as that, since this band itself is composed of several originally distinct elements, and, furthermore, can hardly be considered to exert the tension ascribed to it. The initiative in the process seems to be a slight invagination of the abdominal wall at the point of insertion of the inguinal ligament. Through a subsequent evagination followed by a second invagination a conical body is formed, the conus inguinalis (Fig. I I, A), which involves the muscular layers, and by a final outpushing of this and the surrounding structures a subcutaneous muscular pouch is formed, the bursa inguinalis, in the bottom of which lies the conus, which serves as a point of insertion of the inguinal ligament. The bursa is lined by a pocket of peritoneum, the processus vaginalis, which is reflected up over the conus. The inward development of the conus absorbs and shortens the inguinal ligament, and eventually the testis comes to lie in the bursa, covered internally by the reflected peritoneum. As shown above, the bursa may or may not become placed in a scrotal sac, but when it does, a scrotal ligament (chorda gubernaculi) extends from the bottom of this sac to the base of the conus. 
In a complete, or typical descensus, in which the bursa is contained in a scrotal sac, the parts are related as in Fig. Ir I, B. The processus vaginalis of the peritoneum, continuous beyond the sac with that which lines the abdominal wall, wraps itself partly around testis and epididymis, thus forming a membrane, the tunica vaginalis propria, with a parietal and visceral layer, and a serous cavity included between them. This serous cavity is naturally continuous with the main abdominal cavity, the colom, and the passage between them remains open in those mammals in which the external appearance of the testes is periodic; in those, however, in which the descent is final and definite, it closes up during late, often post-natal, development, and all communication between the two cavities is lost. The vessels and nerves of the testis, to which is added the ductus deferens, become united by connective tissue into a single structure, the spermatic cord, which escapes from the testis along the side not invested by peritoneum, becomes recurved and enters the abdominai cavity by running along the wall of the pouch, covered by the parietal layers of peritoneum i.e., the tunica vaginalis propria.

Outside of this come three layers which represent the abdominal muscles and their fascia; in order, beginning from within: I, the tunica vaginalis communis, i.e., common to both testis and spermatic cord, a continuation of the fascia transversa; 2, the cremaster muscle, a continuation of the transversalis and internal oblique, and 3 , the fascia cremasterica [Cooper's], which represents the external oblique, but is without muscular fibers.

Beyond this comes the integument, although this is often differentiated into two layers through the development of its involuntary muscular fibers into a layer of integumental muscles, the tunica dartos, which occasions a wrinkling of the surface in response to slight stimuli.

The external reproductive organs have arisen as one of the adaptations required by the assumption of a terrestrial cristence, the ultimate cause being found in the non-suitability of the air as a medium for the transmission of the spermatozoa. 
These delicate motile cells can exist only in a liquid medium, and from this cause alone arise in all terrestrial animals the necessities, first, of secreting a liquid to serve as a vehicle for the male germ cells, and second, of developing organs through which this liquid may be directly transmitted to the cavities of the female organs, without suffering from the drying action of the air. That this necessity was not immediately apparent
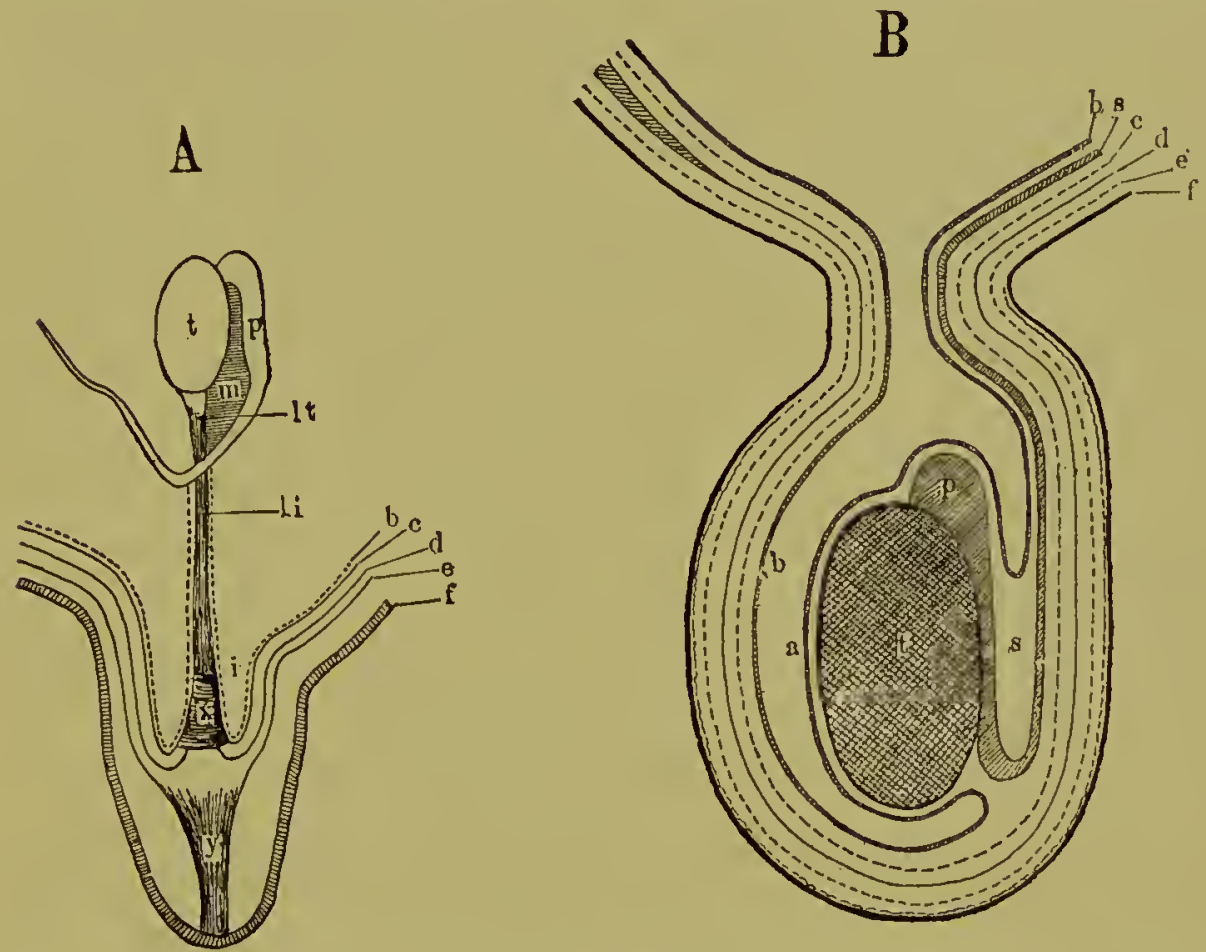

Fig. III. Diagrams illustrating the descent of the testes in mammals. [After WEBER.]

$t$, testis; $p$, epididymis; $s$, spermatic cord; $m$, mesorchium; $l t$, ligamentum testis: $l i$, ligamentum inguinale; $i$, bursa inguinalis; $x$, conus inguinalis; $y$, chorda gubernaculi (=ligamentum scroti); $a$, tunica vaginalis propria, visceral layer; $b$, the same, parietal layer; $c$, tunica vaginalis communis, continuous with the fascia trans. versa; $d$, cremaster (=transversalis-obliq. int. abdom.); $e$, fascia cremasterica Cooperi (=obliq. ext, abdom.); $f$, integument, including the tunica dartos and involuntary muscular layer.

is due to the semi-aquatic habits of most amphibians, even the most terrestrial of which resort to the water at the breeding season and are thus able to dispense with any external mechanism; yet here, notwithstanding the absence of external organs, there have arisen numerous habits, such as the love antics of salamanders and the amplexation of frogs and toads, which are designed to secure a greater likelihood of fertiliza- 
tion and thus form the prelude to the development of a genuine copulation.

It is evident, however, that with the complete relinquishment of an aquatic life, and the subsequent impossibility of employing an external vehicle for the conveyance of the spermatozoa, some method must be found by means of which the seminal fluid may be conveyed direct from the male to the female; and this process, beginning with the most natural stage of the approximation of the two unmodified cloacæ, would develop first a temporary evagination of a portion of the inner cloacal wall, and then a permanent modification of this evaginating portion; a development which would naturally take place in the male alone, as the producer of the fluid to be transferred. There thus arises for the first time in vertebrates an intromittent organ or penis, three distinct types of which are found these appear to have arisen independently, although in all cases by a modification of the cloacal wall. The first is seen in those highly specialized burrowing amphibians, the Gymnophiona, and consists of a protrusible tube worked by muscles; the second is that of lizards and snakes, and is in the form of two lateral protrusible sacs, the walls of which are often cornified, and possess a spiral groove for the conveyance of the spermatic fluid; the third occurs in its simplest form in turtles and crocodiles and suggests a terrestrial origin for both groups. This latter is the type from which the penis of both birds and mammals is derived, and may be described more at length. Owing to the imperfectly understood law of sexual homology which obtains among vertebrates, this organ, sometimes termed the phallus to distinguish it from the other types, exists also in the female in a much reduced form, and is termed the clitoris. Although useless as an intromittent organ, it reflects the peculiarities of the male organ and in the various groups often shows in a reduced form the characteristics developed by the latter.

The phallus develops from the ventral wall of the cloaca and consists of a longitudinal thickening of fibrous tissue, the corpus fibrosum, upon which rests a mass of cavernous (erec- 
tile) tissue in the form of two lateral ridges, the corpora cavernosa, with a median groove between them, the seminal groove. This entire organ is somewhat tongue-shaped and free at the tip, and is capable of considerable protrusion beyond the cloacal orifice. The urogenital sinus, bearing the openings of the ductus deferentes, opens into the seminal groove near its proximal end.

Although the phallus of these reptilian forms seems at first sight quite distinct from that of mammals, and although there exist at present no transition forms among adult animals, the development of these parts in mammals supplies the missing portions of the history and substantiates the homology. The essential change is that of the conversion of the spermatic groove into a complete tube, which is accomplished by the increase in size of the lateral ridges and their subsequent fusion, a process repeated during early development. The failure to complete this produces the condition known as hypospadias, and is thus seen to be a case of arrested development, the retention of the reptilian stage.

The relative position of the penis changes completely during its mammalian history from a post-scrotal one with the free end directed posteriorly to one that is pre-scrotal and directed anteriorly. The first of these positions is similar to that of the turtles and crocodiles and is seen in the monotremes, and to a lesser extent in marsupials; the latter position is characteristic of placental mammals. This change may be made clear by the accompanying diagrams (Fig. I I 2 ).

In the monotremes the conditions are still essentially reptilian. There is a common cloaca and the penis projects a little from its ventral wall. The ureters, ductus deferentes and urinary bladder form a common duct which under normal conditions serves merely as a passage for the urine. This duct is morphologically the urethra as far as the entrance of the ductus deferentes and ureters; beyond this point it is morphologically the urogenital sinus. The erection of the penis, through the slight lengthening of its inner end, closes the entrance into the cloaca, but continues the urogenital canal 
into its own lumen, thus forming a direct outlet from the ductus deferentes to the exterior. At the same time the free end becomes protruded from the cloacal orifice, and the organs,
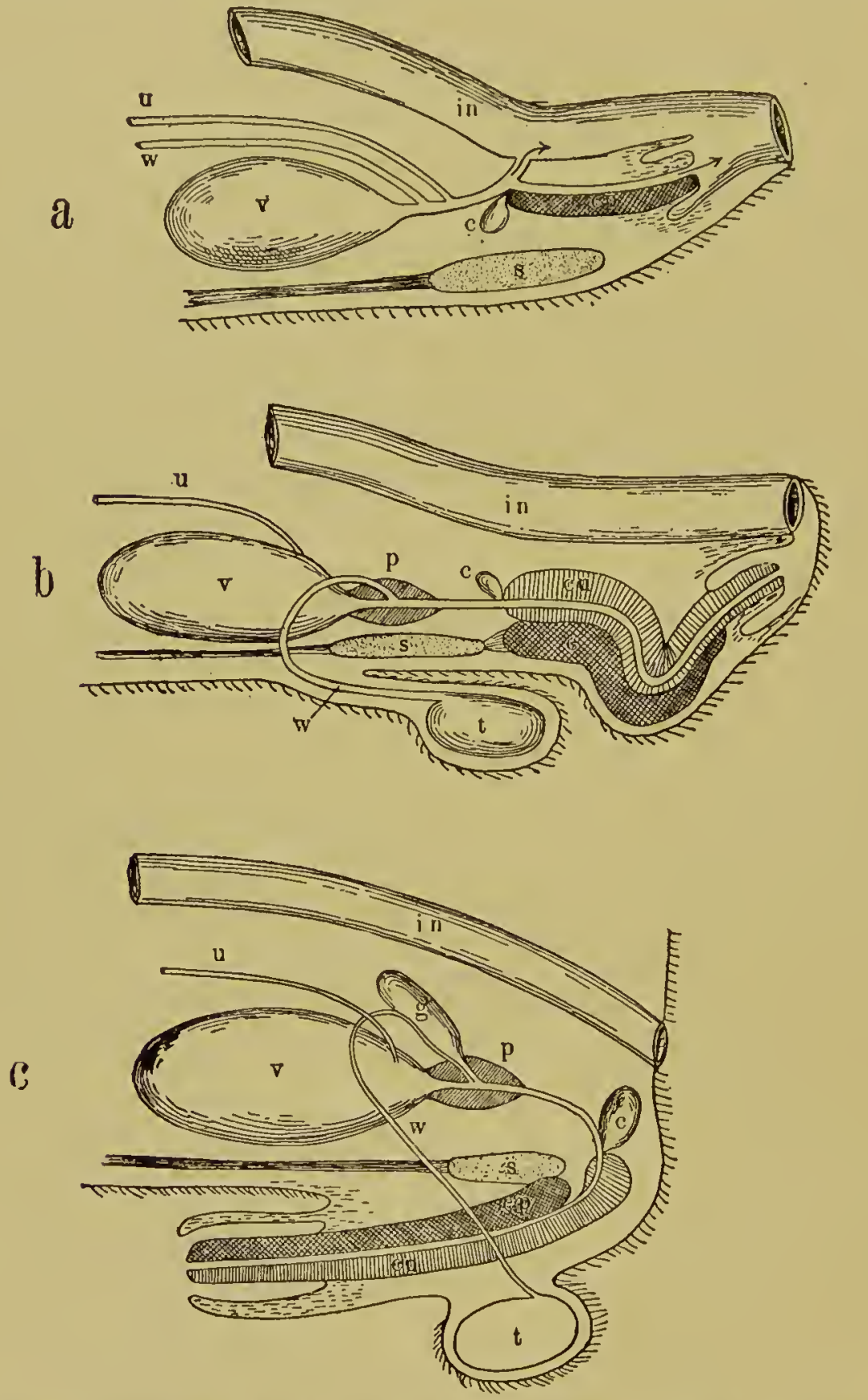

FIG. II2. Relationships of the male urogenital organs in mammals. [After WEBER.]

(a) Monotremcs. (b) Marsupials. (c) Placental mammals.

$s$, symphysis pubis; $i n$, intestine; $v$, urinary bladder; $u$, ureter; $t$, testis; $w$, ductus deferens; $p$, prostate; $g$, vesicular gland; $c$, bulbo-urethral gland; $c p$, corpus cavcrnosum penis; $c u$, corpus cavernosum urcthra (=corpus spongiosum). 
usually wholly subservient to the urinary function, become for the time being wholly reproductive.

The marsupials show an intermediate condition by which the transition to the placental mammals can be explained. The cloaca has been divided by a perinæum and the alimentary and urogenital outlets have become entirely separated. The testes show a marked descensus and usually come to lie in a scrotal sac, which is prepenial in position. The penis is posterior to the testes and is still directed backwards as in monotremes and sauropsids, but becomes attached at its proximal end to the posterior margin of the os pubis.

The true urethra is very short, as the ductus deferentes enter the tube soon after its origin, but the urogenital tube thus formed is permanently continuous with the lumen of the penis, forming a long urogenital canal. This condition is essentially that found in placental mammals except for the relative position of the penis, which in the latter animals, retaining its proximal attachment to the lower margin of the os pubis, turns about and becomes directed anteriorly, thus changing its apparent relations with the testes, which are now post-penial.

Connected with the penis are various sorts of glands, employed mainly for the purpose of furnishing a liquid vehicle for the spermatozoa. They are thus the most widely developed in mammals of marked fertility, like rodents and insectivores, and may be arranged in five groups, each associated with a definitive part of the spermatic tract (Fig. II3). The glandula ductus deferentis are thickenings of the wall of the ductus deferens, and are situated near its entrance into the urogenital canal. The glandula vesicales are large and evident glands, which open near the latter. These have often been considered as receptacles for the spermatic fluid, and are hence usually called seminal vesicles, but they are clearly glandular in their nature, and their cavities contain spermatozoa only by accident. Of the remaining three, which open into the urogenital canal, the primitive condition is seen in the urcthral glands, tubular glands occurring in the walls of the above canal, especially along its proximal portion. From 
such elementary structures are derived the two other sets, the prostate and the bulbo-urethral [Cowper's]. Of these the former are more proximal in position, the latter more distal.

The function of these five sets of glands seems in all cases that given above, and their occurrence in the various mam-
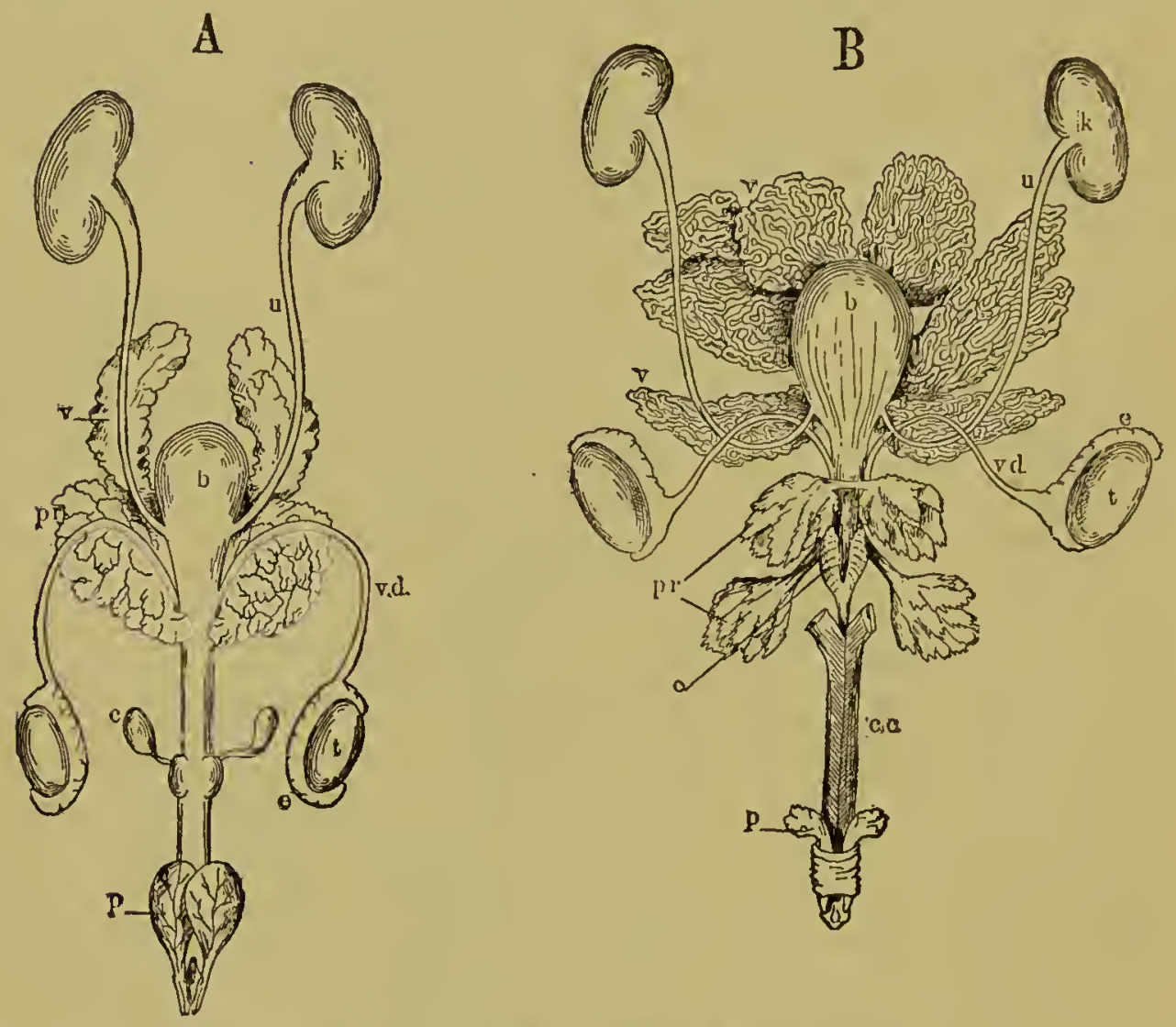

FIG. II3. Penis of placental mammals.

(A) Mouse (Mus musculus). [Combined from RAUther and OPpel.] (B) Hedgehog (Erinaceus curopaeus.) [From OPPEL, after SEUBERT.]

$k$, kidney; $u$, ureter; $b$, bladder; $t$, testis; $e$, epididymis; $v$. $d$., ductus deferens; $c c$, corpora cavernosa; $v$, vesicular glands; $p r$, prostate glands; $c$, bulbo-urethral (Cowper's) glands; $p$, preputial glands.

mals is such that the large development of one is compensatory for the small size of another.

Thus in monotremes and marsupials there is no prostate gland, but the urethral glands are very abundant; the vesicular glands are wanting in carnivores, but large and well developed in primates. In Man the most important of these is the prostate, but the vesicular are also well developed. The bulbourethral glands are evident but not voluminous. In addition 
to the above glands, the function of which is to furnish a liquid vehicle for the spermatozoa, occur certain modified integumental glands, like the preputial, the function of which is to lubricate the parts.

The external organs of the female are but slightly developed and appear to represent the various elements found in the male, though retained permanently in a reduced and almost embryonic condition. This is best shown by a comparison of the two as they appear in development, differentiating from an indifferent condition common to both, as in the case of the internal parts. As this history begins with a simple cloaca and develops the external parts from its walls and margin, the history recapitulates also, in a very complete fashion, the stages shown phylogenetically in the preceding pages (Fig. I I4).

In an early human embryo the cloacal orifice is approximately circular in shape and is surrounded by a rounded and somewhat elevated margin, the genital ridge. From within its ventral wall, and projecting a little beyond the cloacal orifice, rises a conical papilla, the genital tubercle $[g]$, which is really in the form of an inverted trough, enclosing the urogenital sinus and freely open along its ventral aspect, thus forming the genital cleft $[r]$. At a later stage the cloacal orifice becomes more prolonged dorso-ventrally, and the genital ridge has become more pronounced along the edges, forming two lateral ridges $[h]$, instead of a circular lip. The genital tubercle has also developed and projects conspicuously from the ventral margin of the orifice; its groove is still conspicuous, but not so widely open, and its lateral lips take on the aspect of rounded folds $[c]$. The terminal end of the rectum has become visible and forms an anus, distinct from the genital parts, but almost continuous with them.

Thus far the conditions in the two sexes are precisely alike and the stages are termed indifferent, although we have reason to believe that the sex determination is made at a far earlier period than the first one considered here, probably even in the fertilized egg previous to segmentation. 
At about this point, however, sexual differences begin to appear, as may be seen by a comparison of the remaining figures. The female organs, which remain nearer the embryonal condition, are not essentially different, save in proportions, from the last stage common to both. The genital cleft remains open, forming the introitus vagino, into which empty the united Müllerian ducts (uterus) and the two ureters. The genital folds form the corpora cavernosa (labia minora or
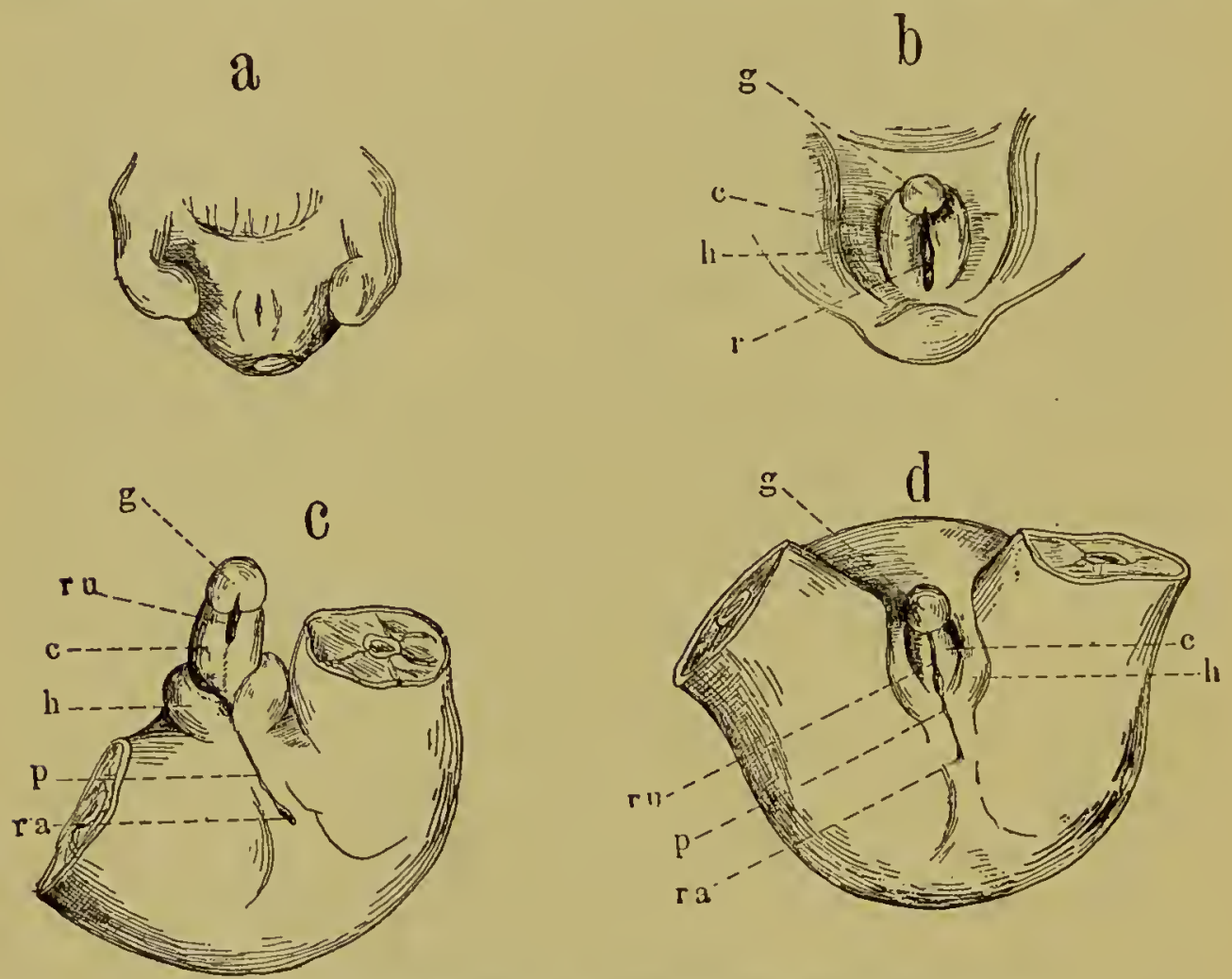

FIG. II4. Development of the external genitals in Man.

(a) and (b) Indifferent stages. (c) Early stage of the male organs. (d) Early stage of the female organs.

$g$, genital turbercle; $c$, genital folds; $h$, genital ridges; $r$, genital cleft; $r a$, anus; $p$, perinæum.

nympha) and the free tubercle itself forms the clitoris. The external lips of the cloaca, the lateral genital ridges, form the greater labia (labia majora).

In the male the genital tubercle develops into the glans and corpus cavernosum urethre [corpus spongiosum], and the genital folds become the corpora cavernosa penis. By the fusion of these latter the groove becomes converted into the uro-genital canal, which becomes continuous with the urethra, 
and into which the ductus deferentes empty. . The lateral genital ridges unite to form the scrotal sac, and the point of union between these is marked by a raphé. A median line of dark pigment lies along the under side of the penis continuous with the latter and marks the fusion of the lips of the genital groove. Special muscles, also in part sexually homologous, develop in connection with the external organs of both sexes. These are composed of striated fibers and are more or less under the control of the will.

The elements of the indifferent stage and their differentiations in the two sexes may be expressed in the following table, which shows the sexual homologies. This table, taken in connection with the one given above, for the internal parts, will form a brief synopsis of the entire subject.

\begin{tabular}{c|c|c}
\hline Embryonal Part & Male & \multicolumn{1}{c}{ Female } \\
\hline Genital ridges & $\begin{array}{c}\text { Sorpus cavernosum } \\
\text { urethræ } \\
\text { Glans penis }\end{array}$ & Clitoris \\
\hline Genital cleft & $\begin{array}{l}\text { Pigmented line } \\
\text { Corpora cavernosa } \\
\text { penis }\end{array}$ & Labia minora \\
\hline
\end{tabular}




\title{
THE NERVOUS SYSTEM
}

\begin{abstract}
"Indeed, while Nature is wonderfully inventive of new structures, her conservatism in holding on to old ones is still more remarkable. In the ascending line of development she tries an experiment once exceedingly thorough, and then the question is solved for all time. For she always takes time enough to try the experiment exhaustively. It took ages to find how to build a spinal column or brain, but when the experiment was finished she liad reason to be, and was, satisfied."

JoHN TYler, The Whence and Whither of Man, p. I73.
\end{abstract}

THE central nervous system begins its history as a straight tube lying along the mid-dorsal line just beneath the integument. Anteriorly this tube ends blindly and exhibits a series of three vesicular enlargements, the beginnings of the brain; posteriorly it ends blindly also and tapers to a point, although there are certain mysterious indications in the embryonic record of a former connection with the lumen of the alimentary canal, indications which have not as yet received any satisfactory explanation, and which may be after all merely developmental necessities, without historic significance. Through modification of this simple neural tube without the addition of extraneous elements save as auxillary to this, there arise in all vertebrates the brain and spinal cord, which, even in their highest and most complicated form, appear to the morphologist as still tubular; the walls, enormously thickened in places and often folded, give rise to such solid masses as the cerebellum or the cerebral hemispheres, the lumen persists as the ventricles of the brain, and their continuation through the spinal cord as the canalis centralis.

All nervous systems have arisen in the beginning in response to stimuli from without, and hence developed originally upon 
the surface of the body, especially upon that portion which, through the customary position of the body, is pot exposed to such stimuli. Such superficial systems are still found among lower invertebrates; in sessile radiate forms equally developed on all sides of the projecting rim or upon the tentacles, in freeswimming forms as an apical plate located upon the point which first comes in contact with external objects. That such was also the case with the unknown ancestor of vertebrates is suggested by the embryonic history of the neural tube, for it is formed here by the rolling in of the external dorsal surface of the early embryo. This process is inaugurated by the formation of two longitudinal medullary folds, one upon either side of the middle line, and as these are united around the anterior end and diverge posteriorly, they form for a time a figure not unlike that of an ordinary hair-pin. The area enclosed by these, which consists of a strip along the dorsal surface, becomes somewhat sunken, and as the two medullary folds, beginning anteriorly, approach one another, and finally unite, the area becomes the bottom of a trough, and eventually the inner surface of a tube.

The complete coalescence of the folds and the pinching off of the trough are the final steps in the process, which results in the formation of the neural tube as described above, the ańlage of the central nervous system. If we may take this process as a recapitulation of pre-vertebrate conditions, a view sustained by its universality and the reasonableness of the conuclusions, it suggests that the primitive ancestor of vertebrates was exposed to external stimuli mainly over its dorsal surface, a supposition which in its turn suggests a slightly flattened, worm-like form, with the ventral side resting upon the ground, here undoubtedly the ocean bottom. The greater development of the anterior portion of this tube, even from the first, suggests a locomotive habit, which would thus favor the anterior end in this regard. As this superficial nervous system became more highly developed, and hence more sensitive, it was protected in the most natural way for such a system, by the formation of elevated ridges along its lateral borders, thus form- 
ing a dell or trough, in the bottom of which lay the sensitive surface. Such a method of protection, once inaugurated, could have but one logical outcome, the gradual formation of a tube through the increase in height and the approximation of the protecting lateral folds, until in this way the form was attained with which all the present-clay vertebrates are equipped. We must here not lose sight of the fact that the original external, and hence the sensitive, surface is not that of the exterior, but that of the lumen of the tube, which explains the fact, to be developed later, that in all lower vertebrates the central or ganglion cells, which form the "gray matter," are situated along the lumen, and not along the external surface, a condition retained throughout in the more conservative spinal cord, although secondarily in the higher forms large masses of gray matter develop also over the external surface of parts of the brain.

A central nervous system, by thus sinking into the interior and becoming entirely covered up by a much less sensitive surface, gains the protection which it seeks, but, in order to retain its functic $n$ as a receiver of external stimuli, a function upon which its very existence as a nervous system depends, it must retain its connection with the exterior through sets of secondary cells which remain external and are yet continuous with the central organ.

These are the sensory cells, which, when grouped over a certain area and specialized to receive a certain form of stimulus, become definite scnse-organs. These are connected with the central system by sensory nerves, in which the direction of the impulse is always from without inward, that is, afferent or centripetal. As the sensory cells become themselves more specialized and hence more sensitive as well as more vital to the organism, they themselves need protection, which they obtain either by the formation of an external non-sensitive horny layer, the epidcmis, which protects the sensory cells scattered over the general surface while it still allows the transmission of the stimuli; or, in the case of such special sense organs as the patches of sensory ceils that form the es- 
sential organs of vision and hearing (retina and acoustic maculc) elaborate series of protective organs become developed, while at the same time the special stimuli are intensified by various accessory organs.

Thus, while this secondary system of sensory cells, like the rank and file of a modern army, meets the external world with its hazards, the central nervous system, and more especially the brain, like the general staff, remains in safety, though in constant communication with the front. The eyes see, the ears hear, the outer surface receives constant evidence of the external world, while the brain, immured within a dense wall of bone; sits in utter darliness and silence. It neither hears nor sees; no ray of light ever penetrates its obscurity, and even when exposed through injury or operation it is found to have no power of direct perception or even of sensation; and yet it directs the entire mechanism with the utmost intelligence, sending its messages to the motor system, and causing the entire body to act in the strictest harmony with the external conditions. In the performance of this function it has developed a complexity immeasurably in excess of that of any other organ, and even far beyond that of its own senseorgans, since these latter attain a high degree of development among fishes, while the brain continues its development through amphibians and reptiles, becomes larger and more complex among the mammals, especially along the line leading to the anthropoids, and attains its highest point in the human species, a member of the latter Order, not otherwise to be especially distinguished from the remainder of the group. It is thus to be concluded that the remarkable development of brain characteristic of mammals in general, and the Anthropoidea in particular, has not been brought about through a greater perfection of the sense-organs, but rather by increasing its own power of receiving the sensory impressions and of recording them through the formation of association paths; and this, like all other structural advances, has been gradually brought about through the working of natural law, as a more perfect adaptation to environment. 
The material history of this advance appears to the morphologist as the gradual modification of the simple neural tube described above, a development which is traceable alike in the comparison of adult animals, Class by Class, and in the embryological record of a single animal, the lower forms preserving in greater detail the early stages of the history, the higher

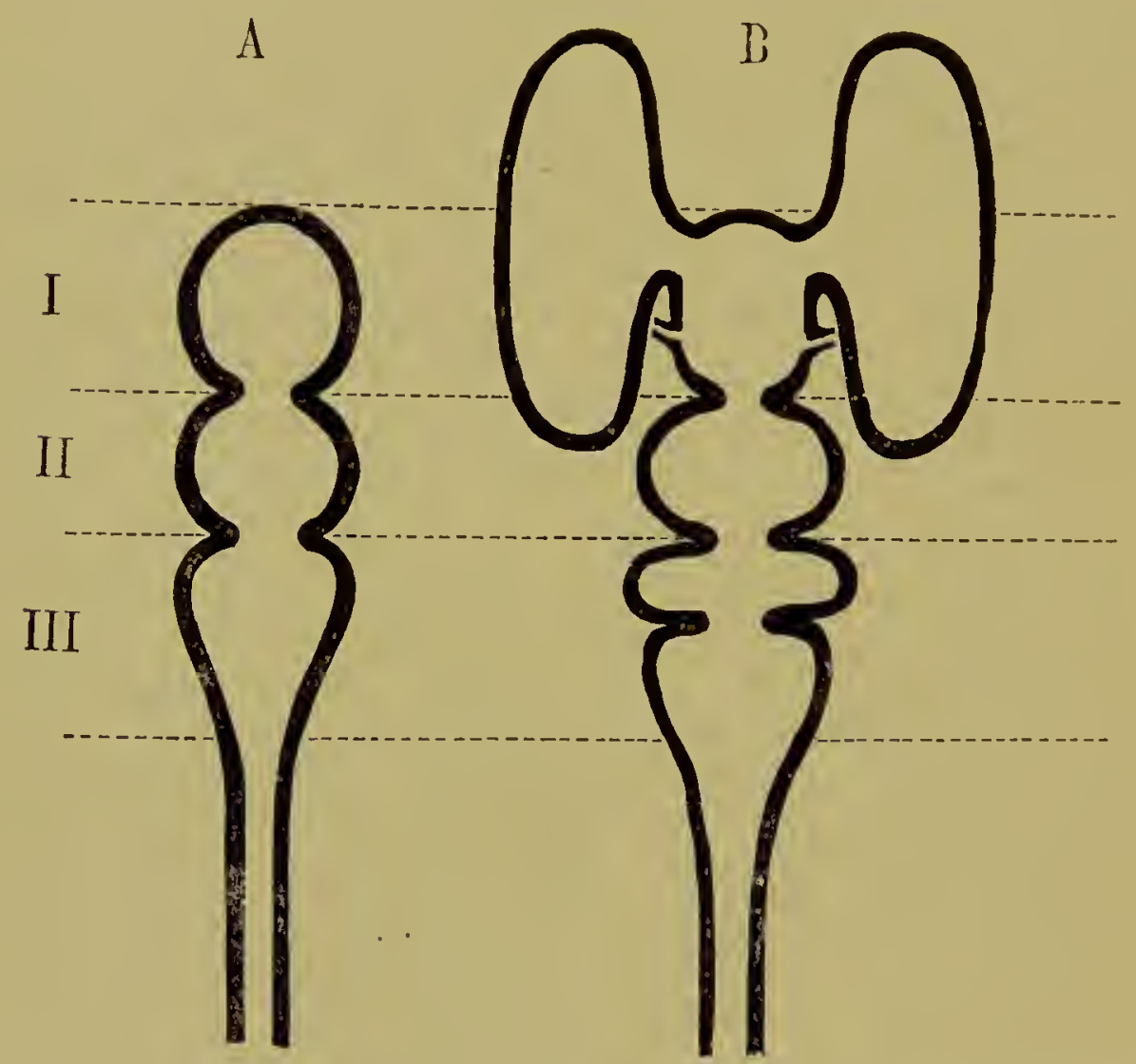

FIG. I15. Diagrams of the primary and secondary cerebral vesicles.

(A) The primary vesicles. (B) The typical form of brain of vertebrates as derived from $A$.

The correspondence between the two is indicated by the horizontal dotted lines, which mark off the areas of the primary vesicles, I, II, and III.

forms recording the later stages. Completed in this way from the numberless fragmentary records presented to the investigator, the history of the neural tube in its progressive modifications is as follows:

It begins, so far as records go, with a form in which the anterior part of the tube, that corresponding to the head of the 
animal, is somewhat enlarged and divided by two transverse constrictions into three successive vesicles (Fig. II $5, \mathrm{~A}$ ), the fore-, middle-, and hind-brain, or, more technically, prosencephalon, mesencephalon and metencephalon, each with its cavity or primary ventricle. Of these the first two seem to represent the very rudimentary cerebral vesicle found in $A m$ phioxus and may be termed the archencephalon, or primordial brain, while the third may be considered the anterior end of the spinal cord, which becomes added to the brain at some point between Amphioxus and the cyclostomes. This cerebral vesicle of Amphioxus bears two rudimentary sense organs, an olfactory groove and a pigment spot; it may be more than a coincidence, then, that in the higher forms the first two original vesicles furnish but two pairs of nerves, olfactory and optic, while the other nerves are derived from the primary third vesicle.

Of all vertebrates the cyclostomes alone possess a brain which may be interpreted as still consisting of three primary vesicles; in all others several modifications take place (Fig. I I $5, \mathrm{~B})$. The prosencephalon becomes modified by the formation of two diverticula, which are thrown out from the sides and grow anteriorly, often reaching a point considerably beyond the anterior limits of the primary tube. These are the two lobes of the telencephalon (the cerebral hemispheres of the higher vertebrates), in distinction from which the unpaired remainder is designated as the diencephalon.

Internally the primary first ventricle becomes divided into the two lateral ventricles and the one naturally denominated the third; the latter communicates with the two first through a passage which is inclined to become narrow, the foramen interventriculare [foramen of Monro].

The mesencephalon is the most conservative of the primary vesicles, and other than a lateral expansion which sometimes forms a pair of prominent optic lobes, suffers no marked change. Its ventricle is often large and obvious, but has received no special name or number.

The third primary vesicle, the metencephalon, shows a 
greater or less differentiation of its anterior portion, which forms the metencephalon in a restricted sense (the cerebellum of higher forms), while the posterior portion, which tapers indefinitely into the spinal cord, is distinguished as the myelencephalon or medulla.* The ventricle of the third primary vesicle, or more especially that of the myelencephalon, is a large and conspicuous cavity in lower vertebrates and in the embryo of the higher ones, and is known as the fourth ventricle. That part of the lumen which lies between this and the third ventricle, including the ventricle of the mid-brain, forms in Man a small tube or duct, and has consequently received the name of aqueductus cerebri [Sylvii], or the "iter a tertio ad quartum ventriculum."

The original three primary cerebral vesicles, by a secondary subdivision of the first and third, thus become increased to five, and form a fundamental plan to which the brain of all higher vertebrates may be referred. In the development of the many forms of adult brains from this ground plan certain mechanical principles are involved which it is well to consider separately before continuing the special history. These mechanical principles are as follows:

\section{Increase in the thichness of the wall over a definite area.}

* The ease with which the German anatomists have translated into the vernacular the somewhat ponderous Greek terms for the parts of the brain (Vorderhirn, Zwischenhirn, Mittelhirn, etc.) has led to various attempts on the part of English-speaking scholars to emulate their example, but with varied success. Thus "fore-brain" and "mid-brain" for prosencephalon and mesencephalon respectively are convenient although somewhat mediæval, and these, together with the inelegant "hindbrain," are now in general use. The forms "after-brain" for myelencephalon (Ger. Nachhirn) and "twixt-brain," or "tween-brain" for diencephalon (Ger. Zwischenhirn) are less happy, and it is doubtful if they will ever receive general favor. The Greek terms are, on the whole, the most satisfactory, and are more in accordance with our usage than are their rather crude Anglo-Saxon equivalents.

The numbering of the cerebral ventricles is that of an old enumeration and does not at all correspond with the morphological value of the parts. They are most conveniently named in accordance with the vesicles of which they form the cavities, thus: telocales, diacole, mesocalc, metacale and myelocale. 
This is seen almost everywhere, but the extent of the development in thickness varies much. It is well shown by the thickening of the floor of the telencephalic lobes, forming the corpora striata, or by that of the roof and sides of the same parts in the higher vertebrates, forming the cerebral hemispheres.

2. The retention of the embryonal thimness over a definite area, forming a place where the lumen is separated from the exterior by merely a thin, often a transparent, membrane. Such places are extremely puzzling, and misled anatomists until within a generation. The physiological purpose of such a thin place is to allow the blood to communicate with the lymph of the ventricles and to nourish the inner surfaces without violating the integrity of the original neural tube. A plexus of blood-vessels is thus the constant accompaniment of such a thin place, and the relations usually become still more complicated by the sinking into the cavity of the entire structure, although each loop of capillaries is covered and veiled by the membranous wall, and thus the integrity of the tube is never violated.* In extreme cases almost the entire thin area, covering a network of capillary loops and following its intricacies, may come to lie within the cavity of a ventricle and form a socalled chorioid plexus.

The most important of these organs are (I) those of the lateral ventricles, formed by the invagination of a thin area in the inner wall of each, (2) a similar one in the third ventricle, invaginated from its roof, and (3) one formed from the roof of the fourth ventricle immediately behind the cerebellum.

* As the only exception to this rule there have been described in Man and in certain other mammals one or more small perforations in the roof of the fourth ventricle, the foramina of Majendie, which form a direct communication between the lumen of the neural tube and the subarachnoid space. The existence of this communication, which violates the morphological principle of the complete integrity of the walls of the neural tube, has given rise to much discussion, but it seems now probable that, while these foramina certainly do occur occasionally, it is an individual peculiarity, like the epitrochlear foramen of the humerus, and of no especial significance. 
In other cases localized thin areas push out instead of in and form evaginations of more or less importance in the formation of various organs, which are supplementary to the actual brain. In this way are formed the retina of the eyes, a portion of the hypophysis, and one or two problematic structures arising dorsally from the diencephalon.

3. Folding or creasing of a certain part of the wall. This mechanical device means here as elsewhere an increase of surface, and hence of physiological efficiency, without a corresponding increase in bulk. Its best manifestation is, perhaps, that of the cerebellum, morphologically formed from roof and sides of the metencephalon. In some forms, as in the adult dog-fish, these folds, three or four in number, are seen with the clearness of a diagram; in others, as in adult birds and mammals, the original creases become shallow by a coalescence of the applied surfaces of adjacent folds, but the structure is still marked by the characteristic dendritic arrangement of the white matter, which marks the core of each fold. In some cases a secondary or even tertiary folding is thus marked.

4. Flexure, or the bending upon itself of the entire longitudinal axis of the neural tube (Fig. II6). The possible flexures are three in number, apical flexure, flexure of the pons, and cervical, the two first appearing in birds and quadrupedal mammals, the last found only in Man, caused directly by the erect position and the consequent bending of the skull over an angle of nearly $90^{\circ}$. The reason for the other flexures is undoubtedly the same as in the case of the foldings of the surface, since by folding the parts on themselves a larger brain may be accommodated within the length limits of a given skull. The gradual formation of these flexures may be well seen during the embryonic development of a bird or mammal, preferably, however, in Man, in which alone the third or cervical flexure is involved. They occur in the order of their position, beginning anteriorly, the first being dorsal, the second ventral, and the third dorsal again, in accordance with the natural law of folded objects.

With the above principal in mind, the further history 

a

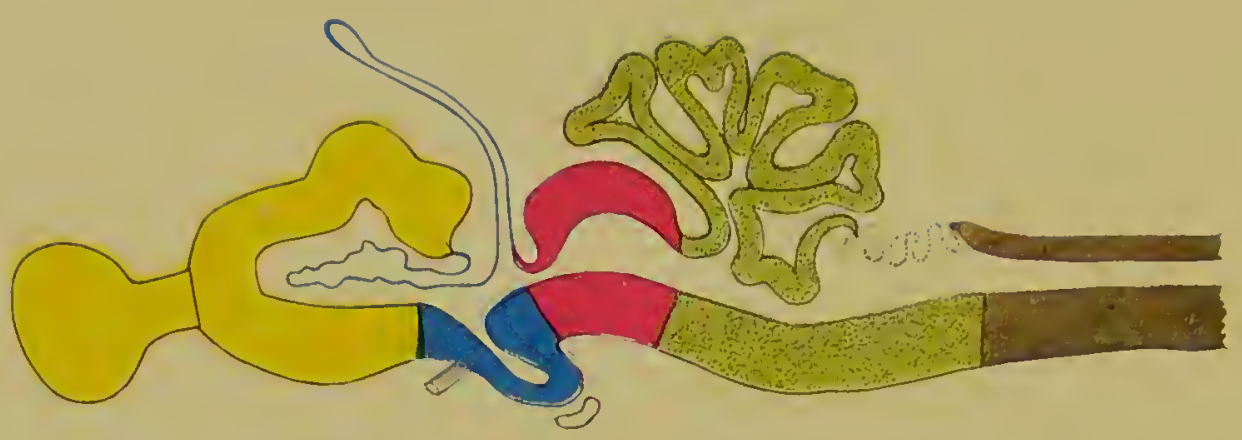

b

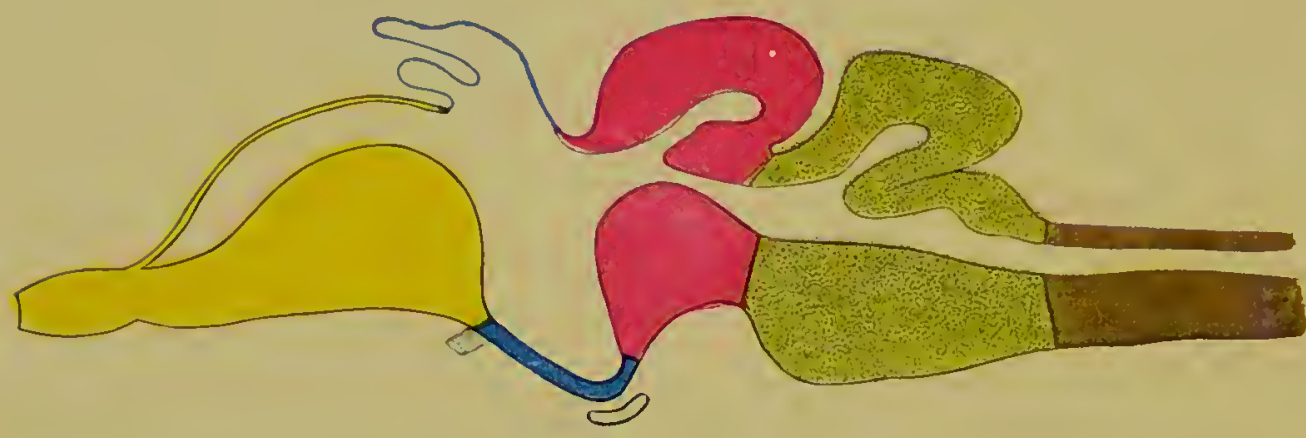

C

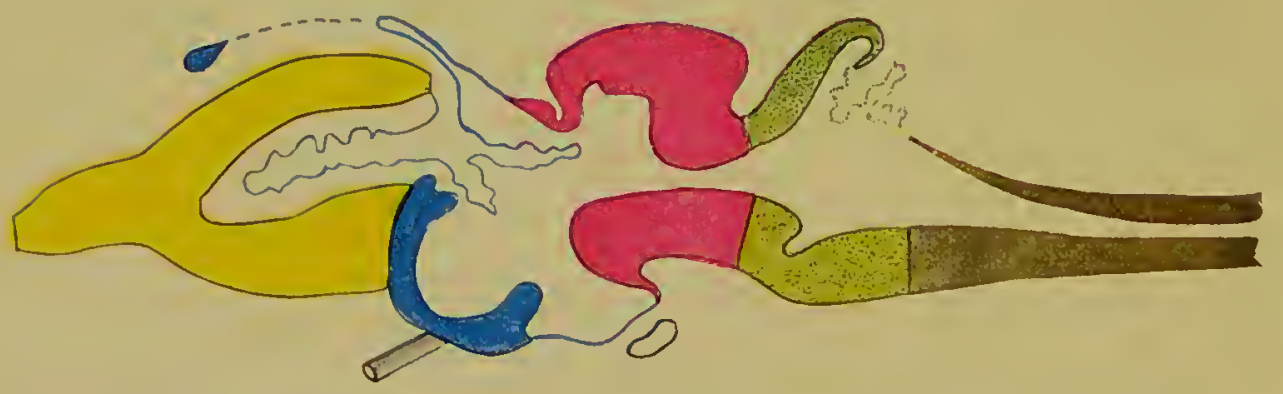

Plate IV. Longitudinal median sections of Vertebrate brains corresponding to the first half of the series in Fig. II 7 in the text. [(b) and (c) after EDINGER. ]

(a). Selachian; (b). Teleost; (c). Amphibian.

Color Scheme: yellorv, telencephalon; blue, diencephalon; rod, mesencephalon; green, metencephalon; brown, myelencephalon and cord. 

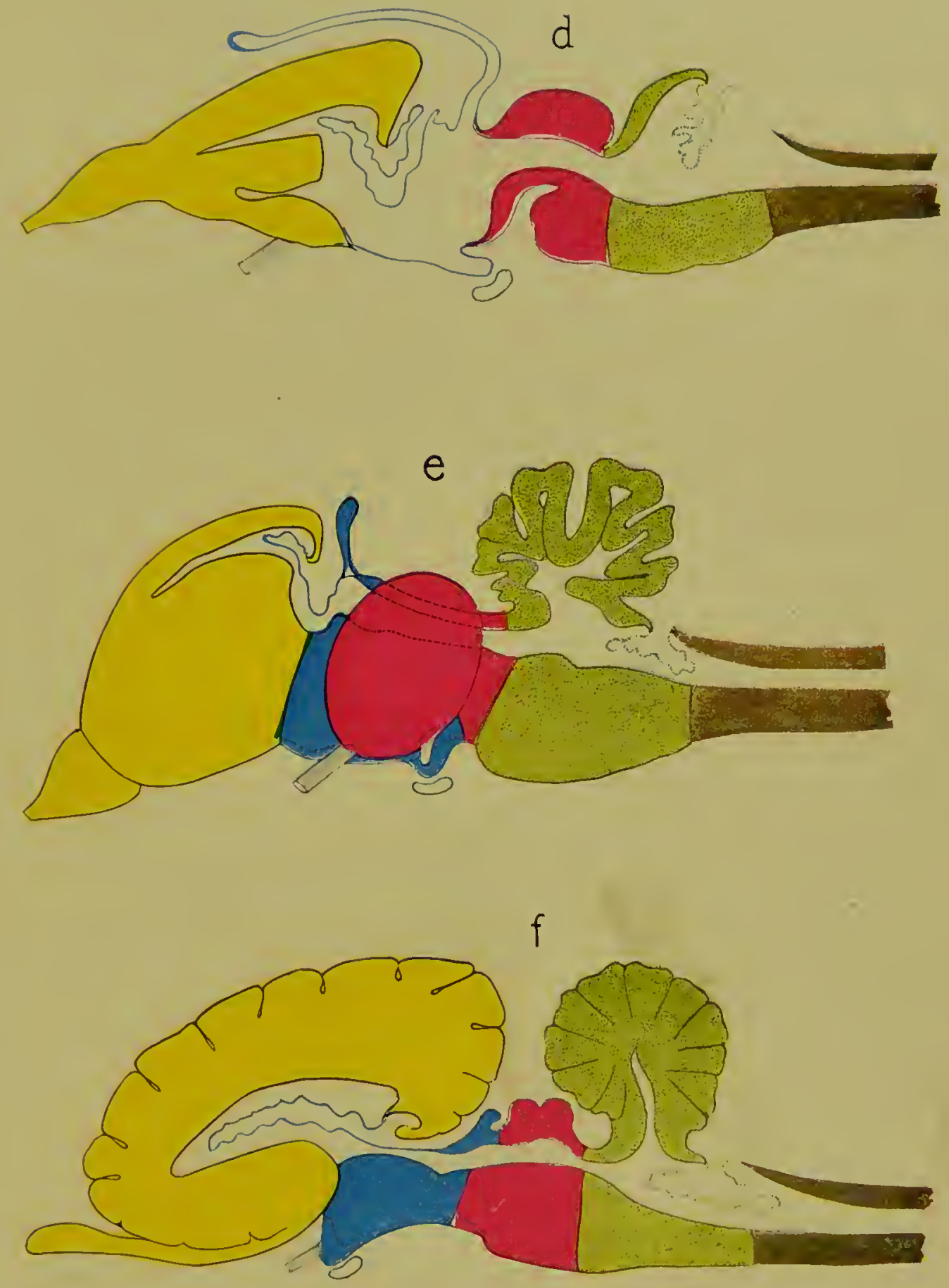

Plate V. Longitudinal median sections of Vertebrate brains corresponding to the second half of the series in Fig. II7. [After EDINGER.]

(d). Reptile; (e). Bird; (f). Mammal.

Color Scheme: yellorw, telenceplialon; hlwr, dienceplialon; red, mesencephalon; green, metencephalon; brown, myelenceplialon and cord. 
of the development of the five areas of the brain, as shown in the different vertebrate Classes, may be studied by the help of the accompanying diagrams [Plates IV and V], which represent the adult brains of a dog-fish, a teleost, an amphibian, a reptile, a bird and a mammal, sectioned sagittally in the median plane and viewed from the inner aspect. The comprehension

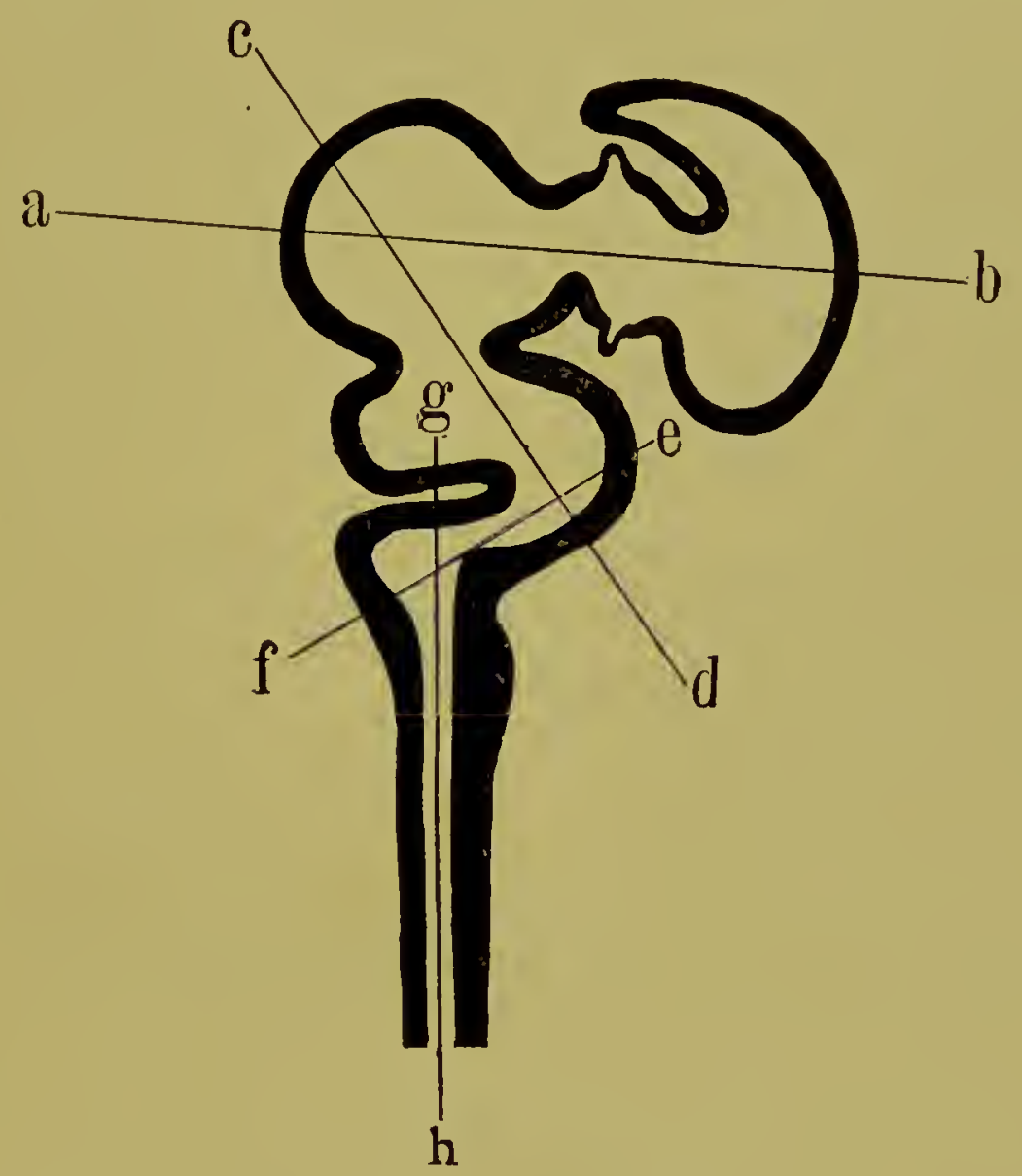

FIG. I16. Diagram of the cerebral flexures.

Angle between axes $a b$ and $c d=$ apical flexure. $\Lambda$ ngle between axes $c d$ and $e f=$ flexure of the pons. Angle between of and $g h=$ cervical flexure.

of these will be facilitated by comparing them with Fig. I I 7 , which shows the dorsal aspect of the same series.

The telencephalon in the dog-fish is inconspicuous in size but of a considerable thickness, which is approximately uniform save at the posterior part of the roof, where a considerable area retains its membranous character and invaginates to form 
three chorioid plexuses, one for each telencephalic lobe and one for the diencephalon (third ventricle). The thickening in the floor forms the area to be known later as the corpus striatum; that of the roof and sides is the potential cercbrum. The two telencephala thus represent the cerebral hemispheres together with the corpora striata; their cavities are the lateral ventricles in which lie the two plexus chorioides, the entrance of which into the ventricles is effected through the interventricular foramina. The anterior portion of each telencephalon forms an extensive olfactory lobe (rhinencephalon), which is here voluminous and stalked. This latter portion is in reality nothing less than the "olfactory nerve," which, when stalked as here, and especially when prolonged, as in some lizards and in birds, gives the appearance of a true cranial nerve. It is here seen not to be a genuine cranial nerve, but an element of the brain.

The teleosts and ganoids show a unique development of this part; the entire roof and sides remain membranous, but the corpora striata are enormously developed. Since the membranous portion, which is here called the pallium, or mantle, is absolutely transparent and extremely delicate, it is usually lost in dissection, or if retained, seems of no importance; and as the corpora striata are very large and convex, they seem to the casual observer to be the true cerebral hemispheres. The rhinencephala are well developed and appear as the direct continuation of these latter parts.

The telencephalon of amphibians and reptiles is not unlike that of the selachians (dog-fish), of which it seems a direct descendant. The rhinencephalon is proportionately smaller, although in many lizards it becomes greatly extended, in adaptation to the prolonged snout.

In birds there is again, as in teleosts, an enormous development of the corpora striata, which makes up the bulk of the cerebrum, although the roof and sides have some thickness and are not reduced to the condition of a pallium. In the mammals the telencephalon reaches its highest development, when it ust1ally greatly exceeds in bulk the remainder of the brain. This 
excessive development is mainly that of the roof and outer side of each of the telencephalic lobes, which form enormous hemispheres that extend forward over the rhinencephalon and backward over di- and mesencephalon, usually coming almost in contact with the cerebellum (metencephalon), from which they are separated merely by a membranous or bony partition, the tentorium. In, addition to increase in bulk there is also an important histological change, namely, the appearance of large masses of ganglion cells over the outer surface, arranged in definite layers and constituting the most important nervous element, the seat of the highest faculties. This ganglionic tissue forms a definite layer of gray matter of considerable thickness, the cortex ccrebri. In lower mammals, such as the marsupials and rodents, the outer surface of the hemispheres remains smooth, but in the higher Orders, such as the ungulates, the carnivores, and especially the primates, it becomes folded up into irregular rounded elevations, the gyri or convolutions, separated from one another by grooves, the deeper of which are termed fissures, (e. g., latcral cerebral fissure [fissure of Sylvius]); and the others, sulci (e.g., sulcus centralis [fissure of Rolando]).

This folding of the surface has the evident effect of still further increasing the physiological efficiency of the cerebral cortex by extending its surface area within the same mass limits.

While the main mass of the hemispheres is derived from the roof and the outer side of the telencephalic lobes, the inner side, remaining thin at first, makes a contribution in the form of a longitudinal invagination which thickens, and forms a ridge that encroaches upon the lateral ventricle. This is the hippocampus [hippocampus major or Ammon's horn]. It attains a considerable development in Man, where it forms a conspicuous elevation upon the inner side of the floor of the ventricle and becomes prolonged posteriorly into a free rounded end, terminating in digitations [pes hippocampi]. This intrudes itself upon the thick outer portion and lies imbedded in it. covered by the temporal lobe. 
Still further down, ventral to the hippocampus, and partly enclosed by the surrounding parts, the same inner walls of the two hemispheres come in contact and form a thin double partition known as the septum pellucidum. The two walls enclose a small space to which the name "fifth ventricle" was formerly given. It is unnecessary to state that this is not a true ventricle and has no connection with the lumen of the neural tube.

The telencephalon of all higher mammals is further distinguished by the formation of an extensive bridge or commissure across the middle line between the two lobes. This lies dorsal, and is easily seen by drawing the hemispheres a little apart and looking down from above. It is called the corpus callosum, and consists of fibers of white matter that form a medium of intercommunication between corresponding parts of the two hemispheres and insure harmony of action. Aside from this extensive commissural system, which has evidently arisen in mammals in connection with the added needs coming from larger hemispheres, there are three smaller transverse bundles, common also to the brain of lower forms, the anterior, middle and posterior commissures. Of these the first alone comes within the province of the telencephalon, the others are respectively $\mathrm{di}$ - and mesencephalic.

The diencephalon, never an extensive element in the vertebrate brain, becomes nearly or wholly covered dorsally and laterally in the higher forms by the excessive development of other parts, but though small and of subordinate interest in itself, it is especially characterized by the formation of secondary organs, either as in-or out-pushings. Some of these latter become of fundamental importance while others appear to be more or less vestigial, presumably inherited from prevertebrate ancestors and of problematic significance.

Several of these formations occur along the dorsal aspect, where over a considerable area of debatable territory between tel- and di-encephala the roof remains thin. The most anterior consists of an extensive invagination into the third ventricle, which lies just beneath this region. This invagina- 
tion is accompanied by blood-vessels, and by division forms three chorioid plexuses, a median one for the third ventricle (the tela chorioidea of human anatomy) and the two laleral

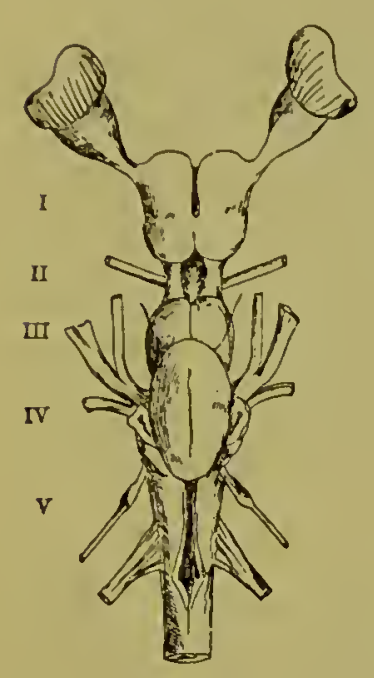

a

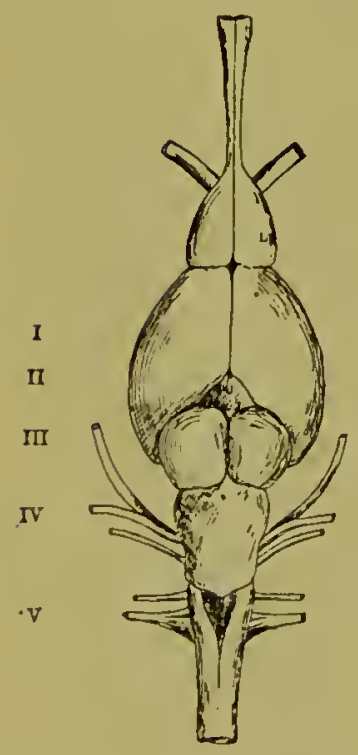

d

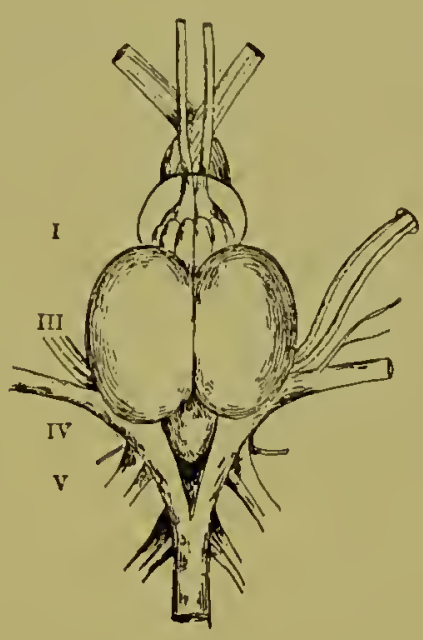

b

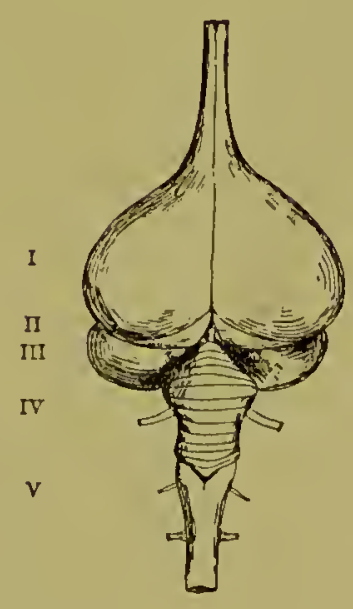

e

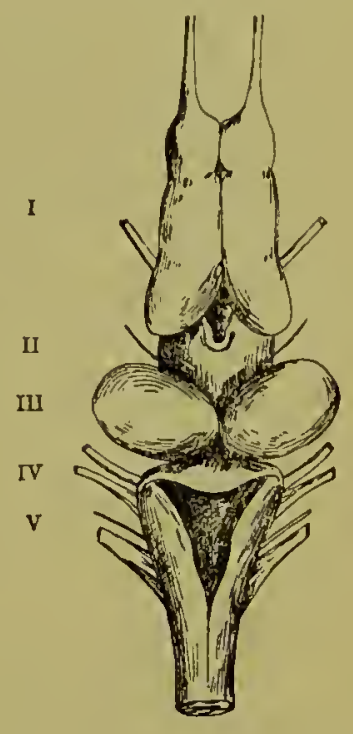

c

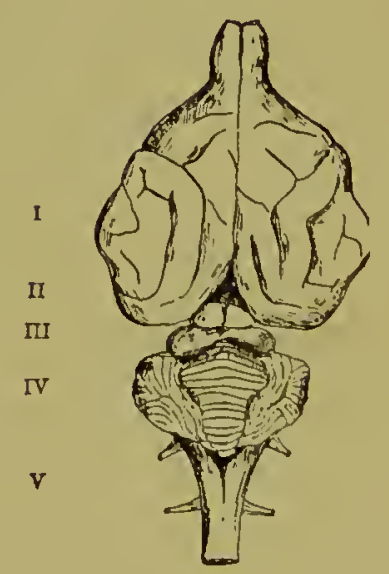

f

Fig. II7. Dorsal views of vertebrate brains, corresponding to the longitudinal sections given in plates IV and $\mathrm{V}$.

(a) Sclachian (dog-fish). (b) Telcost (sculpin). (c) $\Lambda$ mphibian (frog.) (d) Reptile (turtle). (c) Bird (sparrow). (f) Mammal (ent).

V. myclencephalon; II, dicncephalon; III, misencephalon; IV, metencephalon; cxpose the mid-brain. 
ones already mentioned (tania chorioides), which pass through the interventricular foramina and supply the two lateral ventricles of the telencephalon.

Behind the plexuses there appear in the mid-dorsal line typically two median diverticula, which, owing to the many grades of development under which they appear, as well as to the fact that they have long been treated as identical, have received a large number of distinct designations. The more anterior of these is best known as the paraphysis, the posterior one the epiphysis, but the former is also correctly known as the parietal organ, the latter as the pineal organ. Both show a tendency to pass through the skull and attain a position directly beneath the skin in the middle line, developing there a rudimentary sense organ of uncertain nature, but probably an eye in each case.

In the cyclostome Petromyzon, both structures attain considerable development, and the optical structure of the epiphysial organ is evident through the occurrence of pigment in what may be well a vestigial retina. The paraphysial organ is smaller, but of similar structure. In no other form are both of these structures so well developed, but in several cases one may attain an even higher development while the other is rudimentary. In some instances the highest point in development is reached during embryonic life, while in others it is exhibited by the adult. Thus in the selachians, the epiphysis passes through a minute foramen in the skull and reaches the surface; its terminal organ is visible externally, but the paraphysis is not developed at all. In frogs and toads the paraphysis attains a development similar to that of the epiphysis in the former case, while this latter part has not been found. The paraphysial organ, here known as the "frontal organ," is plainly visible externally, but in the adult is entirely separated from the brain by the retrogression of its stalk. The highest development of either organ is reached among certain lizards, where it is the epiphysis that is thus favored. The terminal organ here lies in a socket (parietal foramen) formed in the interparietal suture and represents a fairly good eye, with pig- 
mented retina, a more or less makeshift lens, and a well-developed nerve connecting the terminal organ with the brain. Above this, on the surface, is situated a transparent scale, surrounded by a ring of smaller opaque ones, making a con-
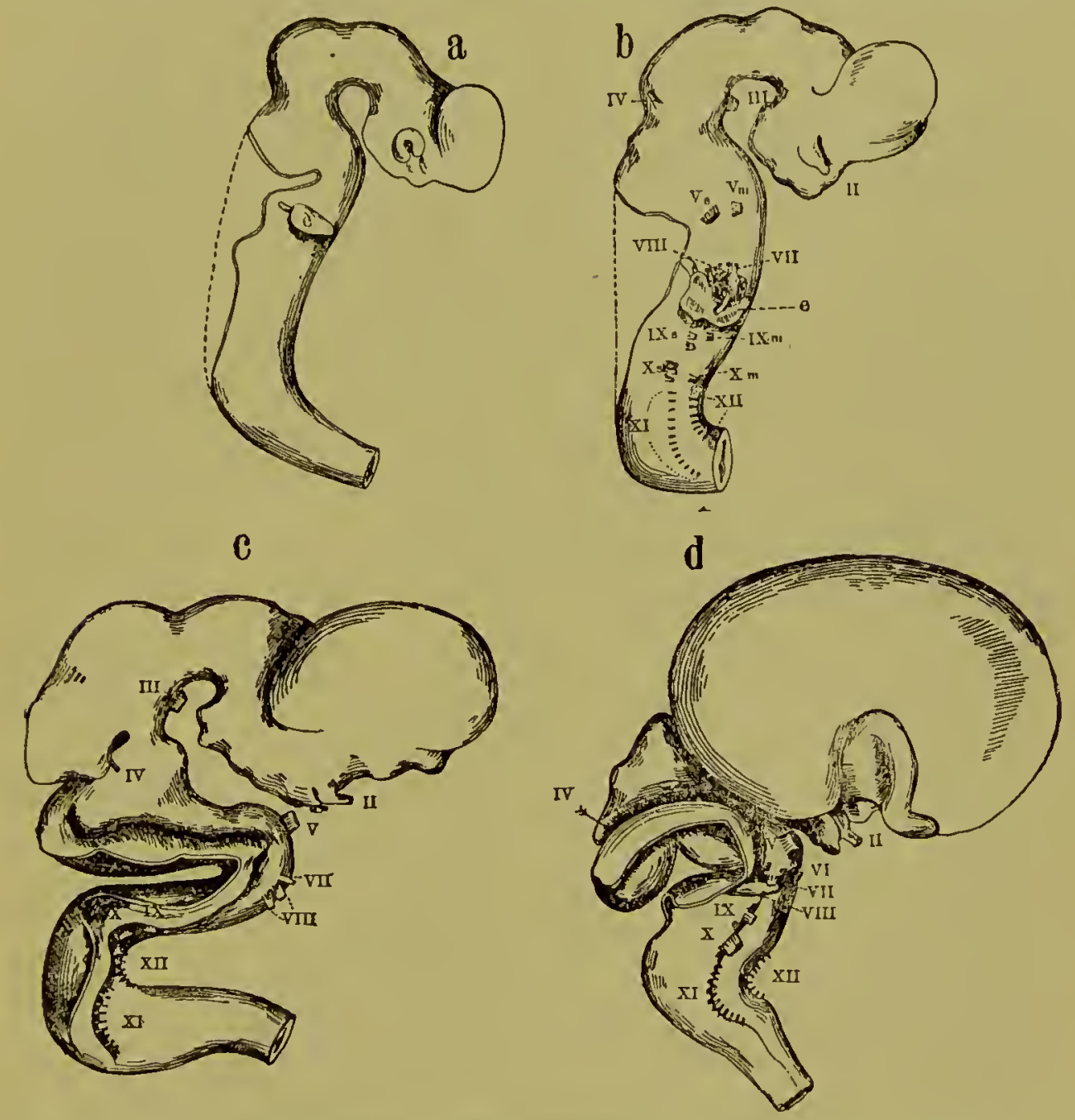

FIG. II8. Lateral views of the developing human brain; drawn from wax models by F. Ziegler after WM. His.

The cranial nerves are indicated by roman numerals; exponent letters $m$ and $s$ denotes respectively motor and sensory branches; $e$, the otic vesicle

spicuous object on the heads of these forms. The paraphysis appears to be associated with this epiphysial structure. In birds and mammals there seems to be no trace of a paraphysis, while the epiphysis is reduced to the form of the so-called "pineal gland," pushed backwards from its original position 
by the growth of other parts. In man it lies so hidden that the early anatomists, finding it as it were in the innermost penetralia of the organ of life and individuality, deemed it the seat of the soul, a view from which the morphologists of the present day have escaped only by substituting one mystery for another.

The sporadic occurrence of these vestigial sense organs, paraphysis and epiphysis, which, save perhaps in the case of the parietal (epiphysial) eye of the lizard, cannot be of the slightest use, points definitely to the presence of similar organs in a functional condition in some remote ancestor. That these parts were organs of vision there can be but little doubt, and there are certain indications which lead us to think that they were once paired, although always close together. Beyond this, investigation has as yet shown nothing, and the whole subject remains at present one of those half-completed histories, of which the record consists of a few poorly preserved fragments.

Far more satisfactory is the history of the diverticula which develop laterally from the sides of the part under consideration, for, although we do not have adult animals which show the steps in the development, they are yet traced in perfect agreement during the embryological history of every vertebrate, a procedure familiar to all students of embryology. These appear at an extremely early age, often beginning before the completion of the telencephalic lobes, and soon assume the form of spherical vesicles, connected with the brain by narrow stalks, and almost in contact at their outer surface with the external germ-layer, the surface ectoderm. By an invagination of this outer surface the vesicle is transformed into a double-layered cup, and in this one may recognize the fundamental elements of the eye. The primary vesicle is hence called the optic vesicle, the transformed cup-like figure, the optic cup. [See Fig. I 36.]

Of this the invaginated layer, now lining the cup, becomes the retina, certain cells of which give rise to the rods and cones, the essential nervous elements of the organ; the other layer, now forming the covering of the cup, develops pigment and 
becomes the tapetum nigrum, a layer which, together with the blood capillaries later to be associated with it, will become the chorioid coat. The stalk, although not directly transformed into the optic nerve, forms the path along which it develops and thus marks its final position. (For the details of this cf. the last part of Chapter XI.)

During the time at which the optic cup has been forming by a turning in of the outer part of the vesicle, an associated process takes place in the ectoderm directly opposite the cup. This process consists of an inpushing from without on the part of this ectoderm, the inpushing going rapidly through the stages of a simple depression, a depression with a narrowed neck, and finally that of a spherical vesicle entirely cut off from its layer of origin. That this may once have been the essential sense organ to supply the needs of which the diverticulum from the brain may have originated, seems likely from the similarity of its early development to that of certain actual sense organs, especially the otic capsule, which develops into the inner ear. This latter, as will be shown later, appears to have been at first merely a single unit of the system known as the "lateral line organs," and the lens, although no longer sensory in function, may with some probability be referred to the same source. In all present-day vertebrates, however, it is no longer sensory, but develops into an auxiliary though essential organ of the eye, the crystalline lens. This is accomplished by an enormous thickening of the inner wall of the vesicle, which finally fills up the entire lumen, leaving the outer wall to fit over it in the form of a protecting epithelium. During later development the eye receives its vitreous humor, its blood-vessels, its sclerotic coat and other essential parts from the surrounding tissue, mainly the mesenchyme, and develops into the adult form.

But one other diverticulum arises from the diencephalon, and that one is directed clownwards from the middle of its floor. Like the lateral eyes, it does not form a complete organ in itself, but unites with a similar diverticulum which develops upward from the roof of the mouth, and together they form an organ of slight functional importance, in respect to which the 
elaborate method of development, involving as it does two distinct elements, is disproportional. It is thus generally supposed that we have a vestigial organ like those developing dorsally and laterally from the same region, and that it, like them, represents the remnant of an organ of considerable importance in some unknown ancestral group. This organ is a noticeable feature of the ventral aspect of all vertebrate brains, and bears the noncommittal name of hypophysis, literally that which grows beneath, in allusion to its position. In most skulls, especially in the more completely ossified one of the amniotes, there is a distinct depression for its lodgment (the sella turcica of human anatomy), and, as the hypophysis is often connected with the brain by a narrow stalk around which the bone may fit quite tightly, it is seldom removed in its entirety with the brain, and hence its true relations are apt not to be wholly understood.

The portion contributed by the diencephalon is in the form of a hollow cone or funnel, the infundibulum. About this the invagination from the mouth cavity, which is glandular in its nature, and termed pituitary body, becomes developed, and by the secondary loss of the original connection between this latter and the roof of the mouth, through the development of the palate, the hypophysis is made to appear like a simple organ, attached to the brain.

Although there is no feeling of certainty among morphologists concerning the original form of this organ, the opening of the pituitary portion into, or rather from, the exterior in the more primitive forms, suggests that this part may represent the rudiment of an earlier mouth, the palcostoma, with which, as shown by other data, the prevertebrate ancestors seem to have been equipped prior to the development of the definite vertebrate mouth, the neostoma.*

Aside from these diverticula and the organs found in asso-

* The pituitary diverticulum arises in gnathostomes from the ectoderm of the stomatodæal invagination, but in cyclostomes is beyond the limits
of the mouth and pushes in from the external surface of the head in close of the mouth and pushes in from the external surface of the head in close
association with the medial nasal invagination. For farther details and theories concerning this part see Chapters VI, XI and XII; also Fig. 129. 
ciation with them the diencephalon develops in mammals a pair of lateral ganglionic masses, the thalami optici, which arise as thickenings of the sides of the vesicle beneath the optic stalks. These are to be sharply distinguished from the lobi optici (optic lobes), under which name the lateral halves of the mesencephalon are usually described.

The mesencephalon is the most conservative of the elements of the brain: it develops very little that is new througnout its entire history, and in Man and the other mammals, although suffering little or no actual diminution in size, it becomes reduced proportionately to a very small portion of the brain through the excessive growth of the surrounding parts. This is made clear by the diagrams, in which the mesencephalon may be followed through fishes, amphibians and reptiles with but little change. Its roof and outer sides are moderately thickened and usually divided along the mid-dorsal line by a longitudinal groove, thus forming a bilobate organ, the corpora bigemina or lobi optici. In many fishes they form a conspicuous part of the brain which, so long as the cerebral lobes remain but slightly developed, must be of great functional importance. In some teleosts, for example, in which the cerebral hemispheres are represented merely by a non-nervous membrane, they furnish at least two-thirds of the dorsal surface, and thus perhaps functionally replace the former. In amphibians and reptiles the gradual development of the cerebral hemispheres reduces the importance of the optic lobes, although in birds, forms not in the direct line of human ancestry, they again reach a certain prominence; thus when the enormously developed corpora striata and the small and thin walls of the hemispheres are taken into consideration, birds are seen to be as unique in their brain development as they are in their skeleton and their general form.

In mammals the mesencephalon is to be looked for between the two greatly hypertrophied elements, telencephalon and metencephalon (cerebrum and cerebellum), and here the bilobed organ has become transformed into one with four lobes, the corpora quadrigemina. The beginning of this 
change may be found in some reptiles, where the development of a pair of small subordinate lobes posterior to the main ones makes it clear that the four-lobed form in mammals is due to the development of a new pair of lobes posterior to the others, and not merely to the formation of a crosi-furrow. Subordinate lobes like those of reptiles are found also in birds.

The floor of the mesencephalon is thickened in all cases and is of considerable functional importance. Through this region pass the fibers of connection between the cerebral lobes and the medulla, and as the hemispheres increase in size, these bundles become greater and form the pedunculi cerebri [crura cerebri], especially conspicuous in mammals, as would be expected.

During the process of phylogenetic development the roof and sides of the metencephalon become selected as a region where a large part of the work of the central nervous system is accomplished. This part, the cerebellum, is thus almost always large and voluminous, and often, even in fishes, becomes folded up into several plicæ, thus emphasizing its great functional importance.

It has already been shown how both the corpora striata and the lobi optici, although of supreme importance in some fishes, eventually become, except perhaps in birds, entirely subordinated to the cerebral hemispheres; the cerebellum, on the other hand, has retained from the first an office of great importance, and in mammals becomes subordinated to the hemispheres alone. There are occasional exceptions to the general importance of this part, as in the case of the singularly small cerebellum of the frog, but such cases are very few. The floor of the metacephalon is utilized in part for the location of commissural fibers between the two lateral halves of the cerebellum, and in mammals, corresponding to the increase in size of this organ, this commissural bundle becomes large and conspicuous, forming a broad loop around the base of the medulla, the pons [Varolii].

Although this region of the myclencephalon is perhaps the most complex of any part of the brain, this complexity lies in 
the minute structure rather than in the external form, in which latter respect it is singularly simple and uniform throughout all vertebrates. Its sides and floor, which alone come into consideration as a nervous organ, together with the crura cerebri and the pons, form the central system of commissures for the entire nervous system, receiving the fibers from all other parts and forming the necessary connections of these with one another and with the spinal cord. That these connections become vastly more complex in the higher than in the lower vertebrates is evidenced both by the gradual growth of the various parts of the brain in size and complexity, and by the results as seen in the behavior of living animals. A rhomboidal area which includes the greater part of the roof of this part remains membranous and forms an important chorioid plexus, that of the fourth ventricle (tonia ventriculi quarti). As this thin place and its subjacent rhomboid cavity (fossa rhomboidalis) are extremely conspicuous objects in all embryos, this portion of the brain is often conveniently termed the rhombencephalon.

Morphologically the medulla is the anterior continuation of the spinal cord, and the nerves that proceed from it resemble the spinal nerves more than do those which arise farther forward. In fact the line of division between medulla and cord is an artificial one, the first being considered as coterminous with the skull in all cases. Similarly those nerves in that region which obtain their exit through a foramen in the skull are termed cranial and are accorded to the medulla. The artificial character of this distinction involves confusion at one point at least, namely, the varying limits of the skull between amphibians and reptiles due to the absorption of a vertebra. (See Chap. V.) In this way the hypoglossal nerve (XIIth), a spinal nerve in amphibians, becomes added to the list of cranial nerves in the Amniota, although this case involves rather more than the simple addition of a single pair of spinal nerves, and is still a somewhat obscure point.

Beyond the medulla the neural tube becomes the spinal cord. which, although it often shows some little regional differentiation, is far more conservative than the anterior portion and 
consists essentially in all cases of a tube with a minute lumen (canalis centralis) and extremely thick rvalls. It consists of both ganglion cells (gray matter) and connecting fibers (white matter) and, as the latter usually form the greater part of its bulk, it is to be considered in the main a great central nerve bundle proceeding from the brain and distributing its fibers to all parts. This distribution takes place through the formation of pairs of spinal nerves, which are arranged metamerically, a pair for each body somite.

The proportion of the spinal cord in weight as compared with that of the brain may be said in a general way to decrease as we ascend the scale of vertebrates, but this is due rather to the increase in the size of the brain than to a decrease in that of the cord. There is, however, another principle, that of progressive cephalization, which tends to shorten the cord and concentrate the nervous system at the anterior end, and it is through this that the changes may be best explained. This principle appears equally well among many groups of invertebrates and is shown in (I)) a tendency to shorten the body axis, and (2) to concentrate and hence shorten the longitudinal nerve axis. The results of this process may be especially well followed among such a group of animals as that of insects, in which the central nervous system originally consists of a pair of small ganglia for each somite, this condition running through the entire body.

Thus in the myriapod (Fig. II9, A), an ancestral form, the primitive condition is still realized; in such a low form of insect as the dragon fly or grasshopper the concentration of ganglia has commenced, and in the fly the highest cephalization is reached. That these stages are passed through during the development is shown by a comparison of the nervous system of the fly in its various stages, that of the larva still showing a quite primitive condition (Fig. I I9, cf. B and C).

This principle is shown in vertebrates by the progressive shortening of the spinal cord in a series of gradually cephalizing forms, but can be used as a criterion of development only within the limits of a single group. Thus the frog, with 
its extreme shortening of the cord, exhibiting but ten pairs of spinal nerves, is not to be compared with Man, in which there are more than thirty, but with the long-bodied salamanders,
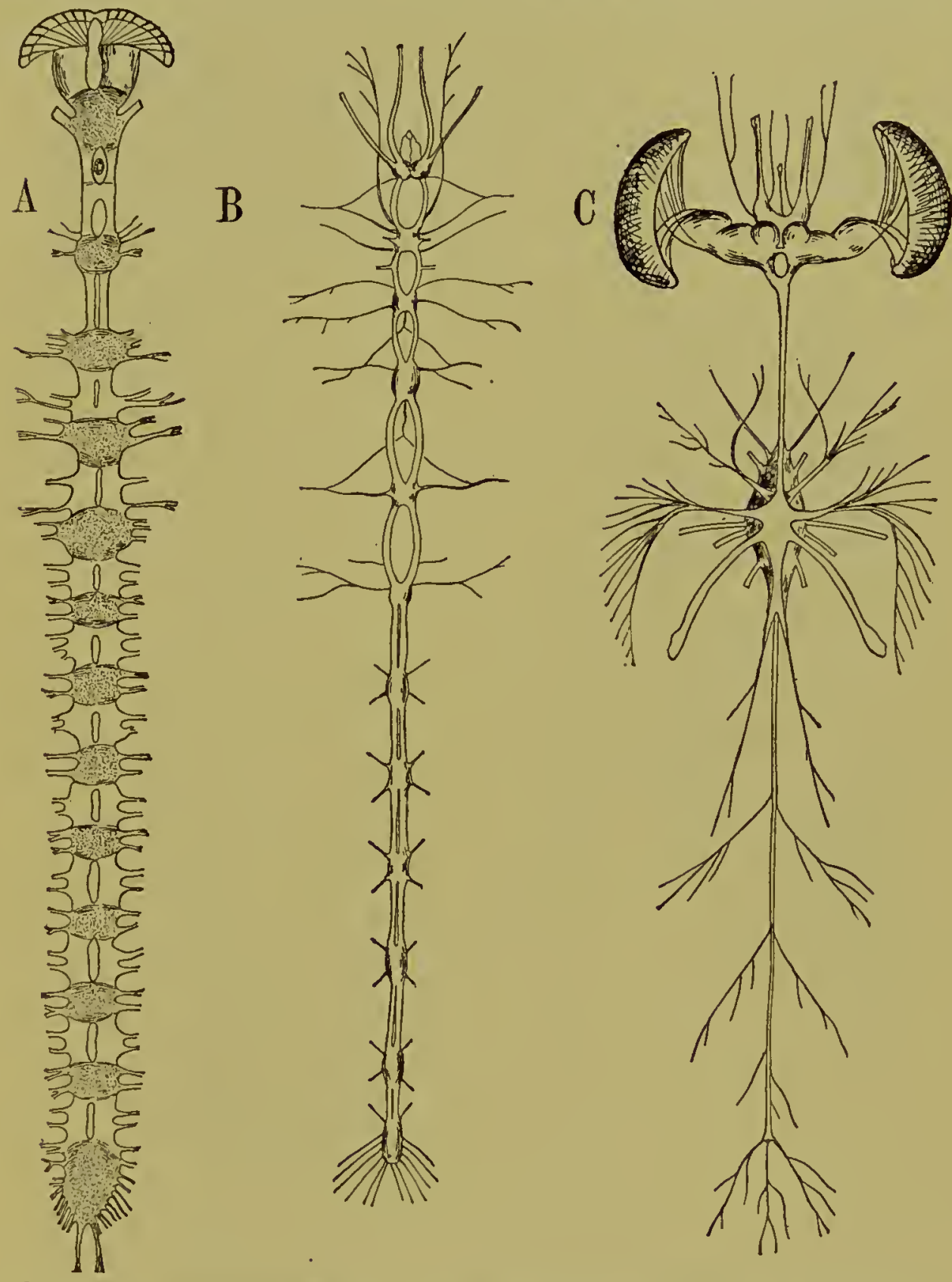

FIG. IIg. Nervous systems of invertebrates, showing the principle of concentration. [A, from LANG, after Oudemans; $B$ and C after LowNE.]

(A) Nervous system of the myriapod Lithobius, showing a connected chain of approximately equal ganglia. (B) Nervous system of the larval Chironomus, the "harlequin fly," showing a long chain of ganglia as in A. (C) Nervous system mid-thoracic. 
animals in its own class, in which the cord is nearly coterminous with the tail (Fig. I20). Indeed, an almost absurd result of this is shown in a certain teleost, Orthagoriscus (Fig. I I I, b),
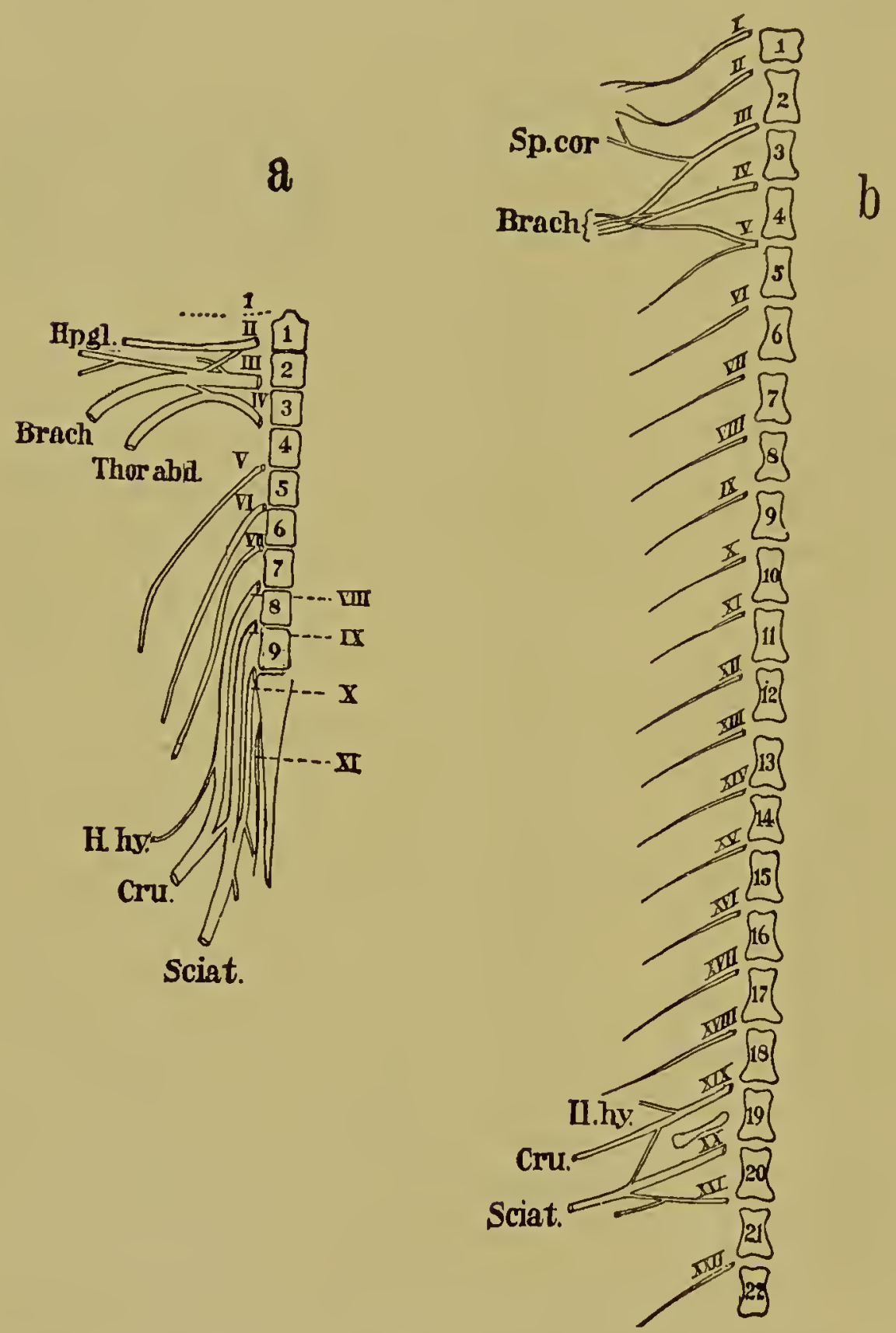

FIG. I20. Spinal nerves of two amphibians, showing differences in the degree of concentration.

(a) Frog. [After GAupP.] (b) Necturus. [Combined from drawings by WarTE.]

Hpgl, hypoglossal nerve; Brach. brachial; Thor. abd, thoraco-abdominal; Sp. cor, supracoracoid; Il. hy, ilio-hypogastric; Cru. crural; Sciat, sciatic. The vertebrae are numbered by arabic numerals, the spinal ncrves by roman. 
in which the entire cord is perhaps a little shorter than the brain. In all cases in which a shortening has occurred two connected phenomena may be observed at the posterior end of the cord: first, the thick, functional portion terminates more or less abruptly and the cord is continued as a tapering thread known as the filum terminale, without function as a nervous organ, and secondly, the shortening is usually so great that the posterior portion of the cord is drawn up considerably ahead of the parts which it supplies, compelling the nerves involved to turn around at a progressively sharper angle until the most posterior ones run in a longitudinal direction parallel to the filum terminale. This bundle of approximately longitudinal nerves which appears thus to terminate the cord, is known collectively as the cauda equina, and is often a noticeable object, as in the frog and in Man (Fig. I2 I, c). In the higher vertebrates, especially in mammals, the relation of spinal cord to tail becomes quite different from that of the lower forms, a change that is correlated in an inter-
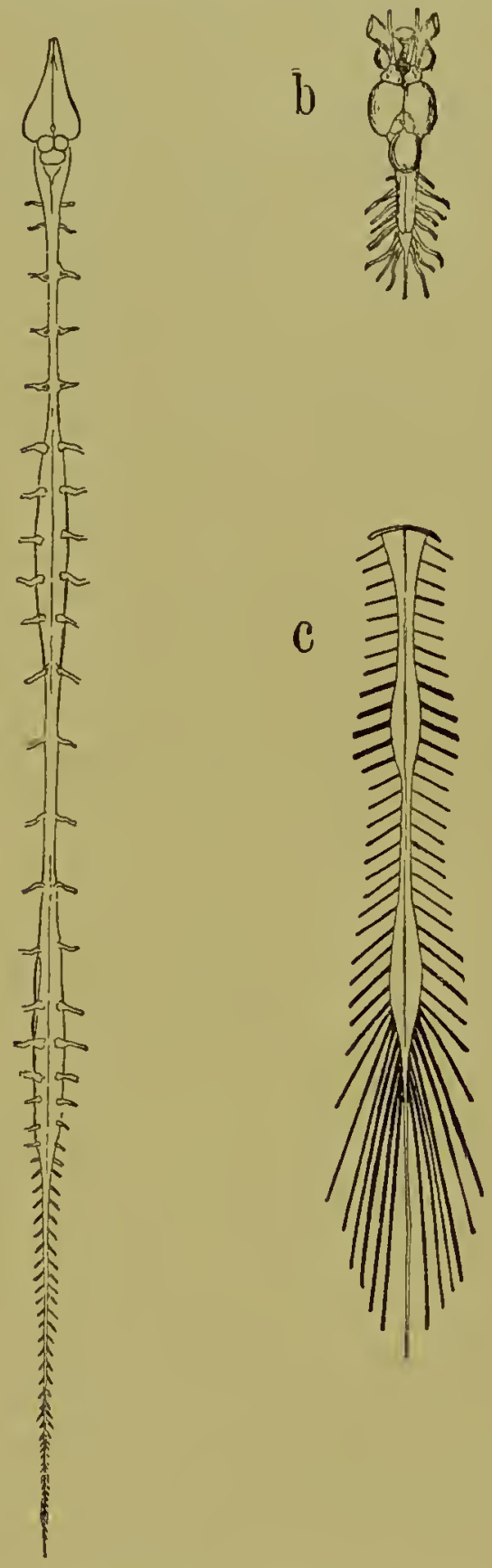

F1G. I2I. Spinal cords, showing the intumescentia, also a marked length variation.

(a) Turtle. [After BoJanus.] (b) Orthagoriscus (telecost). [From GeGeNbaUR after B. Haller.] (c) Human spinal cord without the brain. [ $\wedge$ fter Wredersieim.] 
esting way with changes in the musculature of that part. In such forms as fishes and salamanders, the metameric musculature is not discontinued at the cloaca, which marks the posterior limit of the body cavity, but is continued in a gradually reducing series to the extreme tip of the tail. Each of these caudal metameres is supplied with a pair of spinal nerves, to furnish which the cord must of necessity be continued quite or nearly to the end. In mammals, although in some cases the tail is long and extensive, its metameric muscles have been given up except those of its most anterior somites, and the tail is moved by a complex system of tendons proceeding from these latter. The only nerves necessary for the tail, then, are those of its anterior metameres, which are easily supplied from the cauda equina, thus obviating all necessity on the part of the cord for extending very far posteriorly. Indeed, with the exception of the primitive Ornithorhynchus and a few rodents, the spinal cord of mammals fails to reach even the sacrum.

In much the same way as the development of the caudal muscles conditions the point and manner of termination of the cord, so is its caliber modified in other places through the relative amount of muscular development in the various body regions, especially in the case of the limbs. In such a form as Amphioxus, where the successive metameres are practically alike, the spinal nerves, and consequently the cord, are of about an equal caliber throughout the body, gradually tapering to the end of the tail, and for the same reason in forms like eels and snakes, which have secondarily lost their metameric differentiation, the cord is correspondingly simple; where, however, a certain metamere, or a series of successive ones, becomes greatly developed, the nerves which supply this part are necessarily increased in caliber, and this causes a corresponding increase in the cord at or near the point from which they originate.

The most conspicuous example of this principle is, of course, that of the limbs, which are often excessively developed in the higher forms and cause a corresponding increase of size in 
the metameric nerves from which they receive their supply, as well as in the cord of the region from which these nerves proceed. It will be remembered that each vertebrate limb above those of the fishes is a development of a few (not more than 5-6) metameres, and thus involves primarily a corresponding number of nerves. There are thus in the spinal cord two swellings (intumescentice), cervical and lumbar, corresponding respectively to the anterior and posterior limbs. These sivellings are directly proportionate to the amount of development in each pair and are markedly unequal in such forms as bats, with their exaggerated fore limbs and reduced hinder pair, and in the ostrich, in which the development shows the reverse tendency.

In snakes, in which the limbs have been lost, the intumescentiæ are also absent; on the other hand, in turtles, members of the same class, the disappearance of the most of the trunk muscles has caused a considerable reduction in the size of the cord between the intumescentix, making the latter, which are well developed, seem still greater by contrast (Fig. I $2 \mathrm{I}, a)$.

The most exaggerated development of these spinal intumescentiæ seems to have been among the extinct dinosaurs, in which the excessive development of the hind limbs, on which they supported their enormous weight, caused a proportionate exaggeration of the corresponding swelling, the intumescentia lumbalis. As shown by the cavities in the vertebræ (neural canal), this intumescence was often considerably larger than the entire brain, exceeding that organ some twelve times in Stegosaurus.

In shape, as seen best by cross-sections, the spinal cord varies somewhat in the different regions of the body, and considerably more in the various vertebrates, especially the lower as compared with the higher. In the cyclostomes it is strongly flattened, convex dorsally and concave ventrally. In amphibians it is elliptical, flattened from above downwards, and with a noticeable, though not very deep, ventral furrow. In mammals, by the addition of a dorsal and two lateral furrows, 
all longitudinal and parallel, the well-known form of a fluted column is produced, with a dorsal, lateral and ventral column upon each side [posterior, lateral, anterior, $B N A]$. The shape of the mass of gray matter as seen in section varies from that of a symmetrical triangle in lower forms to that of a figure like a clouble crescent in the higher; in all cases it retains the primitive position, bordering the lumen of the tube, the original external surface.

All parts of the body are in constant communication with the central nervous system through the medium of the peripheral nerves, which are in structure essentially the same as the white matter of the brain and cord, as seen in the various commissures of the former and in the columns of the latter, save that here there is added a connective tissue element, which not only forms an external sheath for each entire nerve (perineurium), but also a delicate wrapping about each nerve fiber (neurilemma). These nerves issue in pairs from both brain and cord, and, although in form and character the transition from one group of nerves to the other is a gradual one, the two groups are distinguished for convenience as cranial and spinal.

The latter, which are the less modified, issue from the cord at approximately equal intervals and are metameric in arrangement, a pair corresponding to each metamere or body somite, as expressed in the muscles or the skeletal parts. This metameric arrangement, which is often expressed with great clearness in the trunk, is not distinct in the head, and the craniai nerves, although showing indications of a former metameric orcler, cannot be satisfactorily resolved into their separate elements.

According to their use nerves are sharply divided into two groups, sensory and motor. The first are distributed chiefly to the external surface and are the media by which the central organ receives intelligence concerning external stimuli. These terminate in many cases in special sense organs arranged to receive certain definite stimuli, but are distributed also over the general surface, where they respond to simple contact. Other sensory nerves supply certain internal parts, as the 
muscles, giving these parts some degree of sensation. In all sensory nerves the impulse necessarily travels from the terminus to the central organ, and these nerves are consequently designated as centripetal or afferent. The other type of nerve, the motor, supplies the muscles and furnishes them with the impulse to contract. In these nerves the current runs from the center to the terminus, and they are thus centrifugal, or efferent. The nerves that regulate the action of other organs,

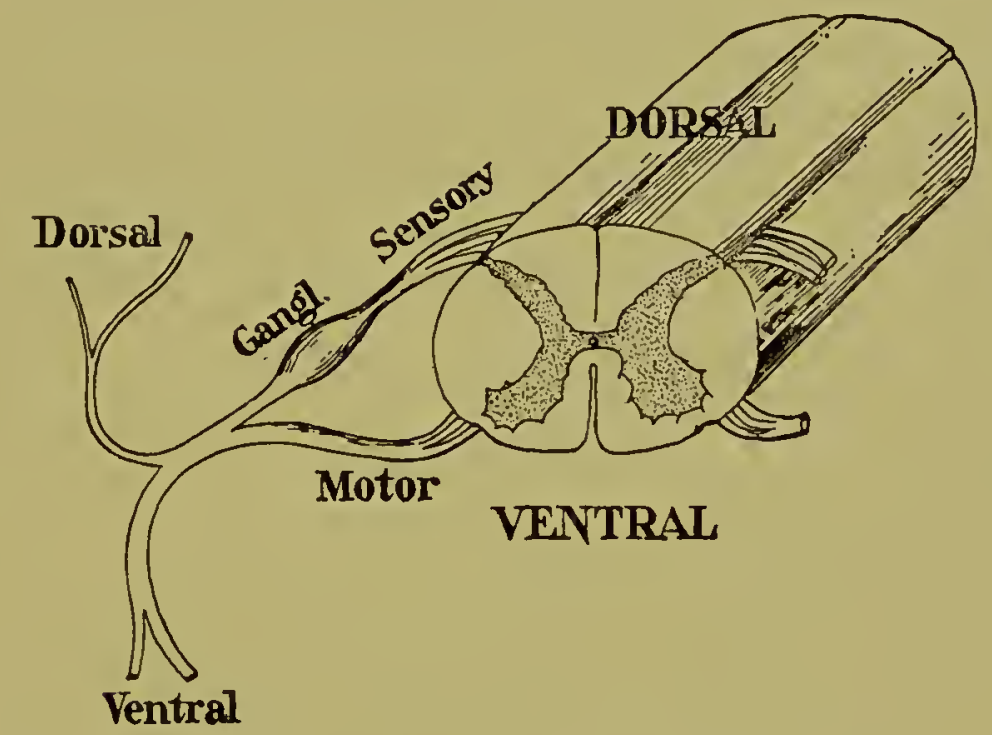

Fig. I22. Diagram of a typical spinal nerve.

such as the secretion of glands, are a subdivision of the motor class.

Nerves issue from the central organ, whether brain or cord, in bundles called roots, each of which contains mainly one type of nerve fiber. The roots are hence called either motor or sensory, but since a given part must usually be supplied with both"motion and sensation, two roots, one of each sort, become associated together, and blend their fibers within a single external sheath, thus forming a mixed nerve. This is true of all of the spinal nerves and of some of the cranial pairs, but others of this latter class arise from single roots and retain their simple character. Each metamere possesses typically a pair of sensory and a pair of motor roots, the sensory situated dorsally, the motor ventrally, thus forming two longitudinal 
rows on each side.* The two roots of the same side unite soon after their exit from the cord into a single metameric nerve, containing botlı sorts of fibers, but then divides again almost immediately into dorsal and ventral branches, each containing both sorts of fibers. These, like all subsequent divisions, are merely topographical, and not physiological, as in the case of the roots. A spinal ganglion appears in association with each pair of roots, usually associated with the sensory root, but in lower forms often connected with both and situated at the point of union.

This typical arrangement of nerve roots and their association in the formation of single pairs of metameric nerves is a constant one in all vertebrates and is already suggested by the somewhat more primitive condition in Amphioxus. In the lower phylogenetic stages, however, the plan is a little less precise, and there is sufficient indication to show that here, too, as elsewhere, the final arrangement has been obtained by a natural development from a less definite one.

Thus in Amphioxus the motor roots consist of a series of fibers distinct from one another; the sensory roots are more definite and are placed in the intervals between the first, in such a manner that the motor roots correspond to the myomeres, the sensory roots to the myocommata. Moreover, since in this singular animal the body somites on the two sides do not match but alternate with one another, the nerve roots do the same, and a sensory root of one side will lie in the same transverse plane as a motor root of the other. The sensory and motor roots do not unite, and the former becomes associated with a subcutaneous ganglion.

This alternate arrangement of the roots, excepting the non-correspondence between the two sides, is continued in most fishes. In the selachians, for example, the motor root passes through a foramen in the side of the vertebra, the sensory root through a similar foramen in the intercalary piece; the latter is thus inter-, the former intra-vertebral.

As we ascend the series the tendency is more and more

* The sensory roots often contain motor fibers. 
towards an intervertcbral cxit for both roots, but even in birds and mammals there are cases of an exit through a vertebra. Thus in the pre-sacral vertebræ of birds there are two foramina on each side in the bodies of vertebræ for the separate exit of the two roots; there are also many instances among mammals of the piercing of a vertebra for nerve exits; for example, the majority of the cervical and dorsal vertebræ in pigs, or the dorsal and lumbar vertebræ among ruminants.

Regarding the union of dorsal and ventral roots; in the cyclostome Petromyzon the two remain separate, although in other cyclostomes, (e.g., Myxine) they unite. In true fishes the union of the two roots takes place outside of the vertebral canal; in the higher forms the union is within it and the united nerves pass through the inter- (or intra-) vertebral foramen. The spinal ganglia, which, in the higher forms, are exclusively associated with the sensory roots, are often in the lower connected with both. They may possibly be homologous with the subcutaneous ganglia found on the sensory nerves of Amphiorus, but their development from a ridge along the spinal cord does not seem to support this idea.

In studying the distribution of the peripheral nervous system there are two fundamental principles to be first considered, (I) that of the exact relation between the size of a nerve and the amount of development of the part to which it is distributed, and (2) that of the permanence of nerve distribution. The first follows from the fact that every cell or related group of cells in a given organ has each its own nerve fiber, and there are thus as many fibers in the nerve bundle supplying the organ as there are such units in the organ itself. If, then, a part reduces or increases its total number of cells, the change is directly indicated in a corresponding reduction or addition of nerve fibers; and, furthermore, as the separate nerve fibers must each reach a central cell in the brain or cord, there are changes there also. These latter are sometimes sufficient to become easily noticeable, as in the case of the intumescentix of the spinal cord, which are correlated with the development of the limbs. 
The second principle, that of the permanence of nerve distribution, has already been referred to in several places, since many of our safest and surest conclusions concerning homologies are based upon it. This principle, more fully expressecl, affirms that a part never changes its nerve supply, and that a given nerve, once associated with a certain organ or complex of organs, will follow it through all its subsequent transformations and even migrations. A good illustration of this is seen in the history of the stapedius muscle of the middle ear, which is supplied by a branch of the facial nerve. This supply is by no means the most convenient, and is reached only through overcoming a series of mechanical disadvantages, yet it is rendered necessary by the fact that the muscle in question was once a part of the digastricus (the posterior belly of the mammalian muscle of the same name), and as such was supplied by the Facialis. Through the application of this inviolable principle numerous homologies have been established, and others, long believed in, have been disproven.

Of undoubted connection with this close correspondence between peripheral nerves and the organs to which they are distributed, as enunciated in the above principles, is the singular phenomenon of plexus formation, seen in the nerves which supply the limbs. These pleruses consist of a more or less intricate set of intercommunications between the spinal nerves that are distributed to the limbs, and are hence two in number, plexus brachialis and plexus lumbo-sacralis, involving the nerves which supply the anterior and posterior limbs respectively. The number of nerves involved in each plexus differs considerably, and reaches a large number in certain fishes, in which the fins are associated with a large number of myotomes, but in animals with the hand form of limb (chiridia) the number varies between two and seven. Of this series one or two, usually the central ones, perform the greater part of the task of supplying the limb, and are consequently the largest; the others grade off above and below to those of normal size. The number and complexity of the intercommunications also reach 
their extremes in the center of the plexus in connection with these larger nerves, above and below which the nerves become gradually less involved, until those are reached which have so slight a connection with the plexus that they are included within it by some authors and not by others. There is, in fact, considerable individual variation in a given plexus, and a debatable nerve may furnish a communicating branch in one specimen which may be absent in another.

The organization of a plexus may be best learned by actual examples, for which the brachial plexuses of two amphibians, two birds, and two mammals may be selected (Fig. I23). From these it will be seen that not only is the number of nerves involved a different one, but that the nerves themselves are not the same, counting from the first. This latter fact is but another way of saying that the girdles shift along the columns in different animals, locating in all cases at the point where the support will be the most effective, a fact brought out in previous chapters in relation to the bones and muscles. It shows clearly that homologies cannot rest upon definite body metaneres, since there is great variation, both in the total number of metameres and in the relative length of each subdivision of the body; neck, trunk and tait. Thus in a frog, with a total of but ten pairs of spinal nerves, the brachial plexus involves the first three and the lumbo-sacral plexus the last four, leaving but three pairs of spinal nerves not involved in plexus formation; yet the metameres thus represented cannot be taken, metamere for metamere, as the homologues of the first ten of other animals, which, in some cases, as in most birds, for example, would be included entirely in the neck; it may rather be said that the ten of the frog are homologous in a general way with the total number of other animals, the two plexuses serving as fixed points for comparison.

In comparing the various forms of plexus with one another, there are, in spite of the great diversity of combinations, certain points of similarity. In the first place, both plexuses are always formed entirely of the ventral divisions of the spinal 
nerves, the dorsal branches being in all respects similar to those of adjacent nerves; secondly, the final outcome of the branching results in the formation of distinct dorsal and ventral

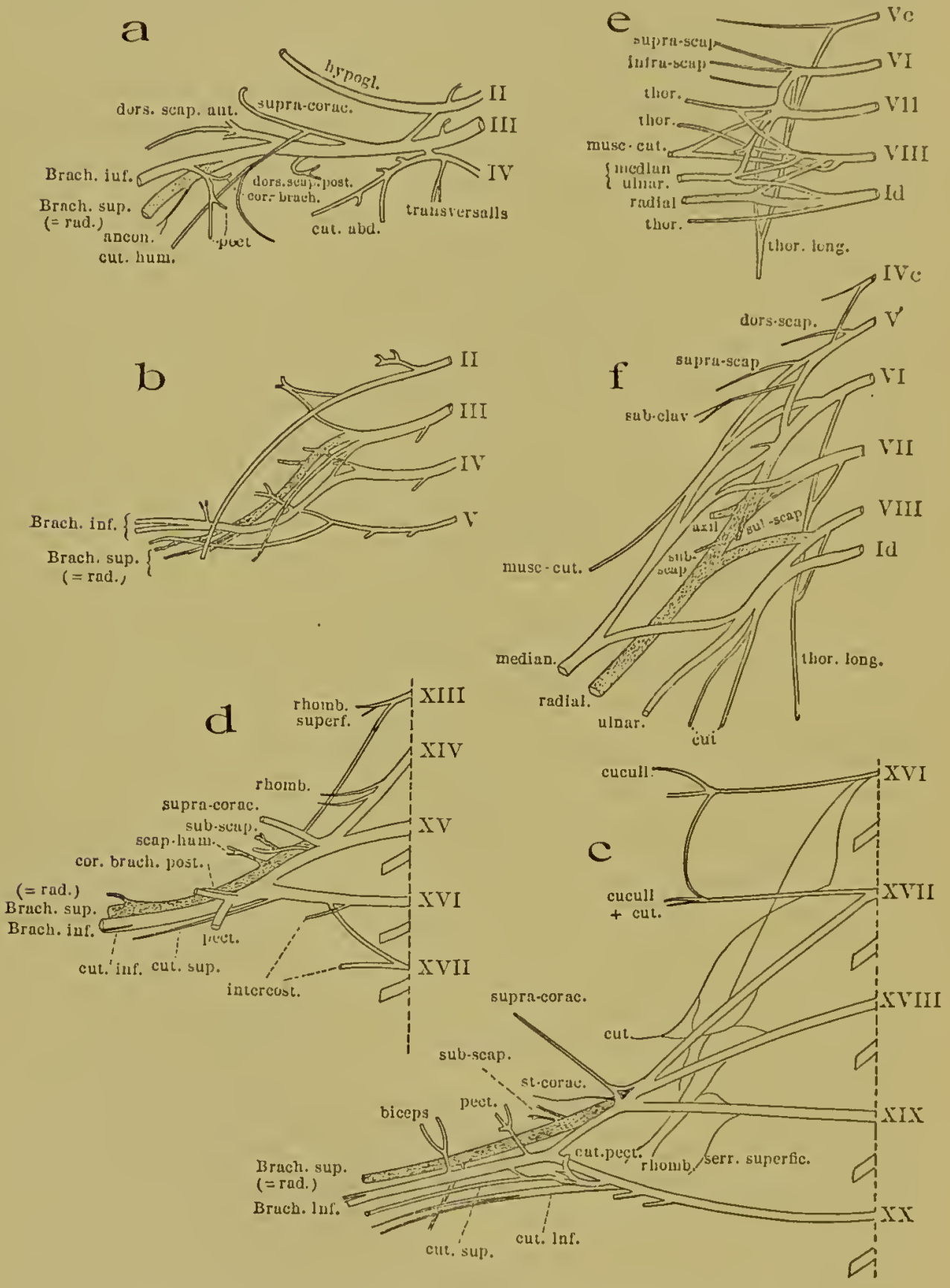

FIG. 123. Brachial plexus of various vertebrates.

(a) Frog. [After Gaupp.] (b) Axolotl (urodele). [Aftcr Fürininger.] (c) Cassowary. [After Fürbringer.] (d) Domestic fowl. [Fürrringer.] (c) Dog. [After Elemierger and Baum.] (f) Man. [Gegenbaur.]

The roman numerals indicate the spinal nerves. The other abbrevintions are suficicntly complete to designate the nerves coming from the plexuses. 
branches, cach one or tico in number, the former distributed along the extensor, the latter along the fle.ror, aspect. In the figures given, which are drawn from the ventral side, the dorsal elements are represented as forming a deeper layer, and are shaded for the purpose of rendering them more distinct. It will be seen, also, that each of these final elements involves more than one root, and also that the same roots furnish fibers for more than one nerve. Furthermore, owing to the embryonal relation of the chiridium to the body, the first digit being anterior in both cases, the nerves supplying the inner (radial or tibial) side of each limb are derived more from the anterior portion of the plexus; those supplying the outer side from the posterior. Based upon the principles given above, the formation of a plexus possesses great morphological significance; for its intercommunications and its branchings are, in part at least, records of the past history of the limbs, records which are so complicated that but little progress has as yet been made in their interpretation. It may be supposed, however, that if any tivo parts, two muscles, for example, each supplied by its own nerve, should coalesce, their nerves would also fuse into a single bundle, at least distally, and even that the extent of this fusion, that is, the distance from the origin at which these two nerves come together, would measure the relative length of time the parts have remained fused. Similarly, if a single part should differentiate into two, a phenomenon constantly occurring among limb muscles, the nerve would branch; and, furthermore, the increase of the differentiation between thenn, that is, a gain in the independence of action, would tend to separate the nerve still more and cause the point of bifurcation to move proximally.

It is thus probable that the plexuses have a meaning for him who is able to read it, the well-known conservatism of nerves in regard to their course assisting greatly in the preservation of these records. This conservatism is well shown in the case of snakes, in which the limbs have been lost, but where there are still traces of the plexuses, a fact attesting the former presence of the limbs. In certain other cases, as in the 
Gymnophiona and in the lost hind limbs of the urodele Siren, not only have the limbs and their girdles utterly vanished, but there is also no trace of a plexus, showing that since the recluction of the limbs a much longer time has elapsed than in the former case, a conclusion in full accord with the relative place of these animals in the system and in their geological appearance.

It is not probable, however, that all the changes in a plexus have a historic significance, since another factor must be taken into consideration, one that is the cause of certain changes, especially those of an individual character. This factor is found in the evident tendency, of certain forms at least, to shift the position of their girdles. This tendency is shown in individual cases by an increase in the size of certain of the nerves involved and a corresponding diminution in that of those either anterior or posterior to them; and, in certain species, by making careful counts of the separate fibers of the main nerves in a large number of individuals of a given, species, the direction in which the girdle is migrating has been definitely established. Thus in the common toad there is shown a tendency to push the shoulder girdle still further anteriorly, and as its present position is extremely cephalic, the continued tendency must be an instance of the inertia of variation through which a line of development, once started, is often carried far beyond the point of greatest efficiency. This procedure involves more generally the posterior than the anterior girdle, and hence the lumbo-sacral plexus is more apt to vary individually. This migratory tendency may result in the establishment in a given species of two or three types of plexus, to which all individual variations may be referred, as has been established in the case of the urodele Necturus, well known also for its variability in pelvic attachment.

The early anatomists, by a careful count of the nerve roots as they were found proceeding from the brain in the human subject, enumerated the following twelve pairs of cramial nerves, that is, of nerves which originate within the cranial cavity and escape through foramina in the bone: 
NAME

I. Olfactorius

II. Opticus

III. Motor oculi

IV. Trochlcaris [Patheticus]

V. Trigemimus [Trifacial]

VI. Abducens

VII. Facialis

VIII. Acusticus [Auditorius]

IX. Glosso-pharyngeus

X. Vagus [Pneumogastricus]

XI. Accessorius [Willisii]

XII. Hypoglossus
Function

special sense, smell.

special sense, sight.

motor.

motor.

$\left\{\begin{array}{l}\text { mainly sensory, with a small motor } \\ \text { root. }\end{array}\right.$ motor. mixed.

special sense, hearing.

mixed.

mixed.

mixed, mainly motor.

mixed.

Of these the first two arise from the primary fore-brain, the tel- and di-encephalon respectively; the remaining ten take their origin from the met- and myelencephala, leaving the mesencephalon without any. It would thus seem that the former may be nerves of the archencephalon or primary brain, laid down in Amphioxis, while the latter belong to the secondary addition from the anterior end of the original spinal cord. The last ten were thus at first spinal nerves, in which, in spite of their extreme specialization, it might be possible to recognize the original spinal elements, each with its sensory and motor roots, its accompanying ganglion, and so on. That the original elements have in some cases become modified is evidenced by several facts, first, the existence among them of wholly motor nerves without sensory fibers and lacking a ganglion; and, again, the fact that some of the nerves in the above list are shown to be composed of several primary nerves by their origin from multiple roots, or from the presence of several associated ganglia. The twelfth nerve is outside of the cranium in fishes, and becomes later included within it, probably by the fusion with the skull of the vertebra with which it is associated. The eleventh is closely associated with the Vagus and appears as a distinct cranial nerve only in mammals.

Aside from the elements found in the above there are traces 
of several other spinal elements originally belonging to the primary anterior end of the cord, which do not survive in the higher forms as definite cranial nerves. These are designated as the spino-occipital nerves, and are first met with in the selachians, where they appear as I-5 pairs, placed very far back, along the medulla. They are spinal in character and not associated with the other cranial nerves, although they are all included within the skull. As this latter part ends abruptly with the otic region in cyclostomes, and is immediately followed by the successive pairs of true spinal nerves, it seems reasonable to suppose that when, in the sela'chians, the cranial cavity became enlarged by an addition at the posterior end, several of the original spinal nerves were included, forming the nerves in question. In the higher cartilaginous fish (Holocephali), and in ganoids, this set of nerves becomes reduced to two pairs, yet a second set, also of I- 5 pairs, has been taken in, presumably in the same way. To distinguish between these two sets of spino-occipital nerves, the first are termed occipital, the second occipito-spinal. Representatives of both sets occur in varying proportions in other fishes, but in the amphibians they seem to have wholly disappeared, and are never seen again as distinct nerves. Although nothing has as yet been definitely proven in the matter, it is probable from other evidence that above the fish the occipital region suffers considerable reduction, during which many of these elements may have become lost, while others may have become established among the root elements of the twelfth nerve, the hypoglossal, since this nerve appears first as a cranial element in the reptiles and continues throughout Sauropsida and Mammalia.

For purposes of description and with reference to their morphology the cranial nerves fall naturally into groups which are best considered separately. These may now be taken up in cletail.

I. The anterior group. (Olfactorius and Opticus.)

These, the two first in the list, are nerves of special sense, the fibers of which are distributed respectively to the nasal- 
mucous membrane and to the retina. The morphological position is doubtful, for, while they are considered by some to be the first true cranial nerves and to belong to a much earlier period than any of the rest, others deny them the right to be called nerves at all, and treat them as parts of the brain, the olfactory lobes (rhinencephalon) and the optic stalks respectively.

Attempts have been made to bring this condition into accord with that found in Amphioxus, for here the archencephalon, a rudimentary brain formed by the enlargement of the anterior end of the spinal cord and possibly the equivalent of the telencephalon of vertebrates, bears two rudimentary sense-organs, the first an olfactory pit and the second a pigment speck. Of these the first is connected with the brain by a short diverticulum, while the second is embedded within the brain wall. Although similarity of function of the two sets of organs in the two cases tempts one to believe in an homology between them, the decision really hinges upon the identity of these sense-organ rudiments and the perfected organs of the higher vertebrates; for if the olfactory groove and the pigment speck are historically the anlagen of the nose and eye, a point not definitely established, then the identity of the nerves with the corresponding parts of the archencephalon naturally follows. In favor of this latter assumption is the fact of the origin of these two nerves from the primary fore-brain, while none of the others arise anterior to the metencephalon. An entirely problematical element belonging to this region is that of a definite pair of nerves, Nerous terminalis, which occur in all selachians, and extend from the anterior part of the telencephalic lobes, where they originate, along the anterior aspect of the olfactory stalks. As they have been but recently discovered they have escaped enumeration with the classical twelve pairs: their origin from telencephalon is also anomalous. Nothing can as yet be predicted of their morphological significance.

II. The motor nerves of the eyeball. (Motor oculi, Trochlearis and Abducens.) 
These three pairs are small and very special nerves, having no other distribution than the six muscles of the eyeball. They are thus exclusively motor, and on the theory that the cranial nerves, excepting, perhaps, those of the preceding group, represent modified spinal nerves, seem to correspond to the ventral roots of three original nerves, the sensory roots of which are either lost, or, more probably, contained in the
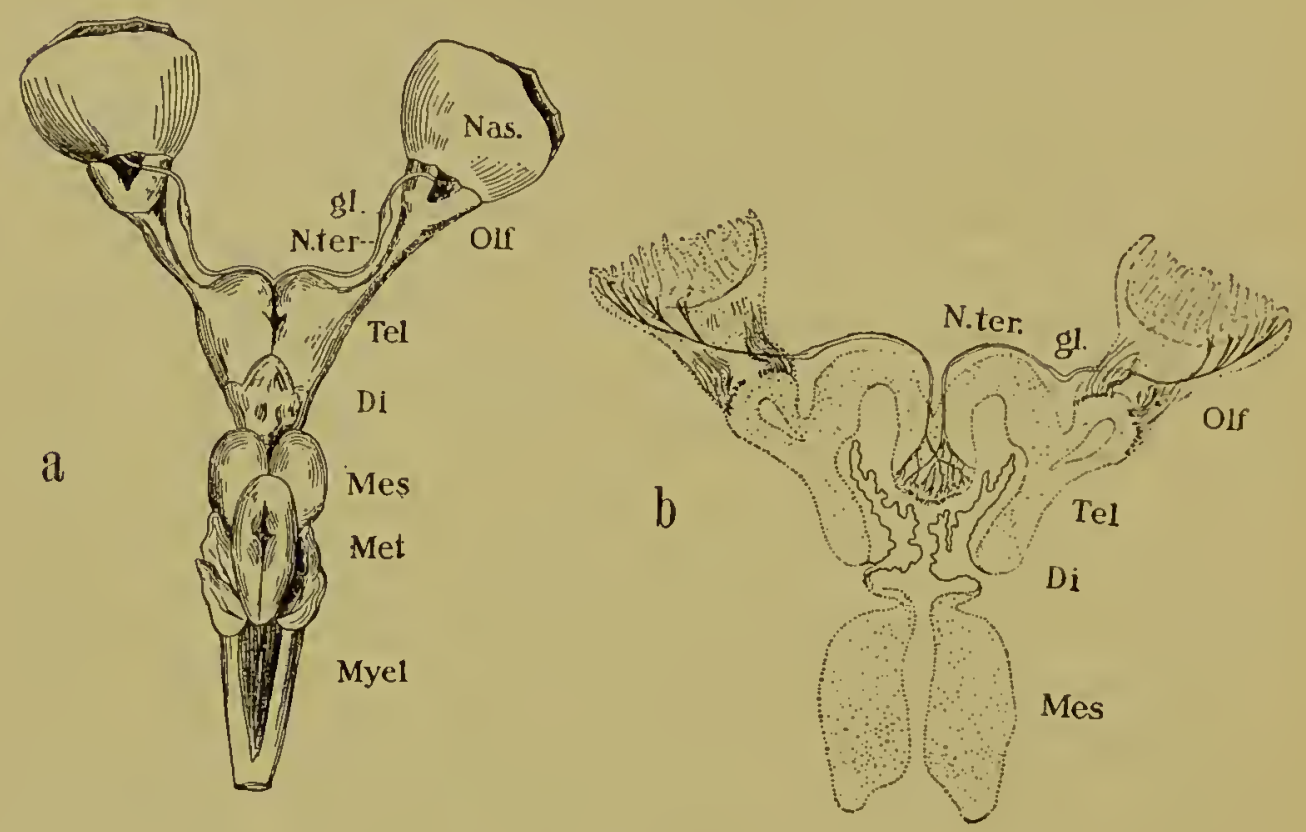

FIG. 124. The Nervus terminalis of the selachians. [After Locy.]

(a) Dorsal view of the brain of the dog-fish, Squalus acanthias. (b) Horizontal section through the anterior part of the same, showing the origin of Nervus terminalis.

$N a s$, nasal capsule; Olf, olfactory lobe; $N$. ter, Nervus terminalis; $g l$, its ganglion; Tel., telencephalon; Di, diencephalon; Mes, mesencephalon; Met, metencephalon; Myel, myelenccphalon.

sensory elements of adjacent cranial nerves such as Trigeminus or Facialis. The fact which suggests this hypothesis most strongly is the strictly metameric character of their field of distribution, namely, the eye muscles themselves, as is shown by their developmental history. In selachian embryos, which have preserved this early history more completely than have the higher forms, there develops in the head a series of myotomes, similar to and continuous with those of the trunk. Some of them soon atrophy, but the first three fold 
about the developing eyeball and furnish it with muscles. From the first arise three of the straight muscles and one oblique, from the second the other oblique, and from the third the remaining straight muscle. These three myotomes are innerved by the three nerves under consideration, and in their natural order of succession, as follows:

\begin{tabular}{c|c|c|c}
\hline Somite & Muscles Developed & Nerve & No. \\
\hline \hline Myotome I & $\begin{array}{l}\text { Rectus superior } \\
\text { Rectus internus } \\
\text { Rectus inferior } \\
\text { Obliquus inferior }\end{array}$ & Motor oculi & III \\
\hline Myotome II & $\begin{array}{l}\text { Obliquus superior } \\
\text { Myotome III }\end{array}$ & Trochlearis & IV \\
\hline
\end{tabular}

These relationships are constant throughout all vertebrates, corroborating the idea that we have here the enumeration of some very primitive morphology. In certain Orders, in response to special needs, other special muscles appear in connection with the eyeball, but these are seen to be differentiations of certain of the above, and retain the same innervation; thus the retractor bulbi* arises from the external rectus, and, like it, is innerved by the sixth nerve.

The relation of these three nerves to adjacent sensory elements and their right to be considered ventral roots are matters concerning which, although much has been done, few definite conclusions may be drawn as yet. The Motor oculi, although its fibers are purely motor, yet becomes connected with the small ciliary ganglion, through which its fibers innerve the ciliary muscles and the iris. This ganglion may have the morphological value of the one belonging to a sensory root now lost, a conclusion which would make this nerve an entire spinal element with a reduction of the sensory root. Other views associate with it as its sensory element a portion of the Trigeminus. The Trochlearis, although essentially a motor nerve, possesses in fishes and amphibians a few sensory fibers,

* This muscle is rudimentary or wanting in the Anthropoidea. 
yct, in spite of this, all are agrecd that the sensory element originally associated with this has become incorporated with the Trigeminus. The sensory portion of the Abducons is probably also a part of the Trigeminus, although certain facts indicate an association with the Facialis.

III. Tire trigeminus-Facialis group. (Trigeminus, Facialis, Acusticus.)

This group and the next are by far the most extensive, and together constitute the main bulk of the nerves of the head. Their relationships differ considerably in fishes and aquatic amphibians on the one hand [Plate VI], and in terrestrial (and secondarily aquatic) vertebrates on the other [Plate VII], a difference largely due to the presence in the one and the suppression in the other of an extensive system of external sense-organs of uncertain function but undoubtedly of assistance in an aquatic life. These organs, variously termed "integumental sense-organs" or "dermal canal system," are visible externally and are arranged in definite lines running about the head and continued in a single (or double) longitudinal row, the lateral line, down the sides of the body. The system of nerves which supplies these is shown in the first of the accompanying diagrams, and consists of three superficial trunks directed forwards and a fourth one directed backwards, the former referred to the Facialis (VII), the latter to the Vagus $(X)$. To each trunk there belongs typically a special ganglionic swelling, placed near its origin; but these are distinct in only a few forms (selachians, dipnoans, a few aquatic amphibians) and in all others become completely fused with the ganglion semilumare of the Trigeminus and are demonstrable only in the embryo. To the compound ganglion thus resulting, which is found in most amphibians and in all the amniotes, may be applied the term Gasserian, long in use in human anatomy for this organ.

The most dorsal of the three Facialis branches of this system is the superficial ophthalmic (ramus ophthalmicus superficialis Septimi), and is accompanied by a like-named branch of the Trigeminus (ramus ophthalmicus superficialis Quinti), 



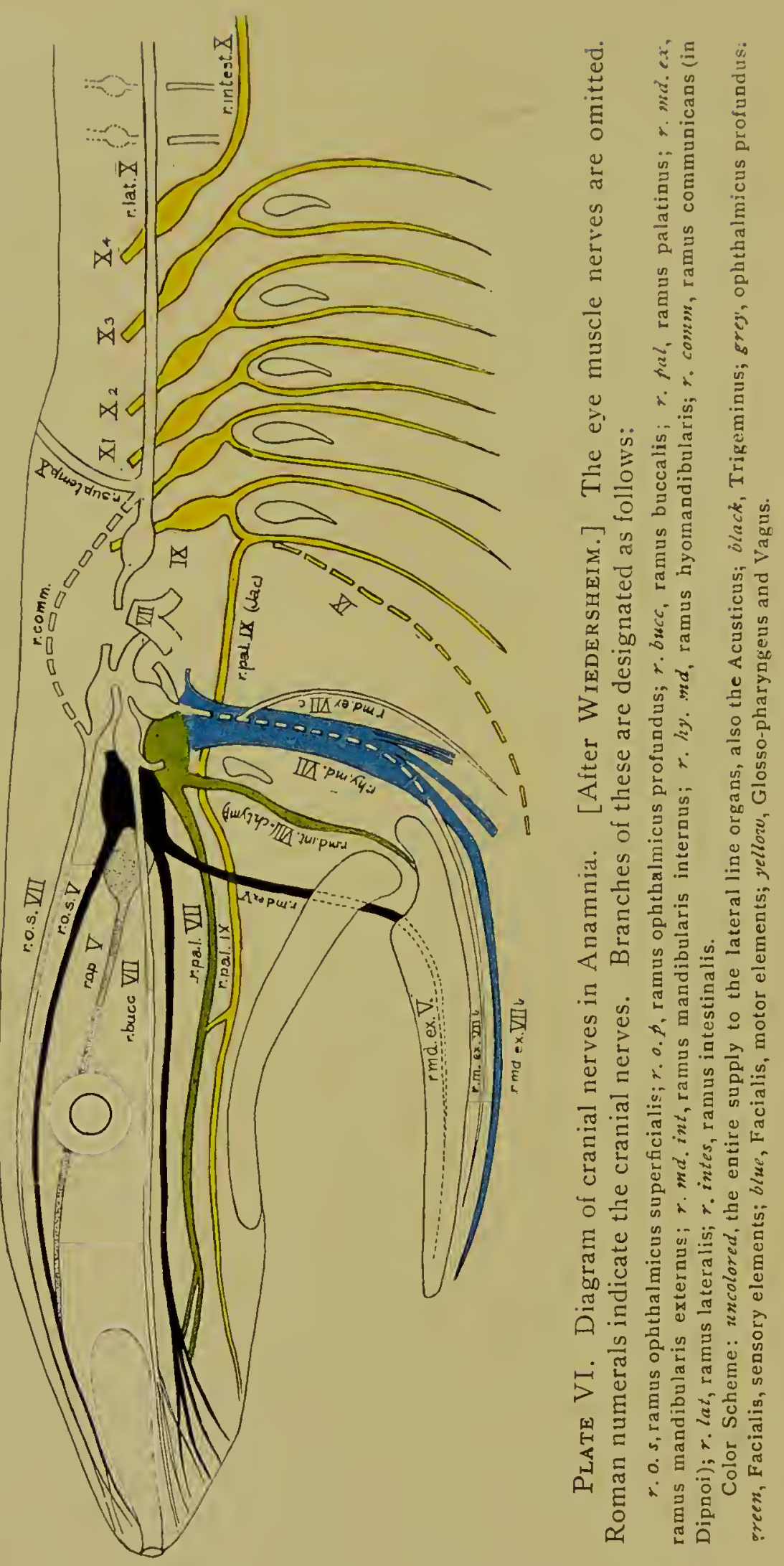




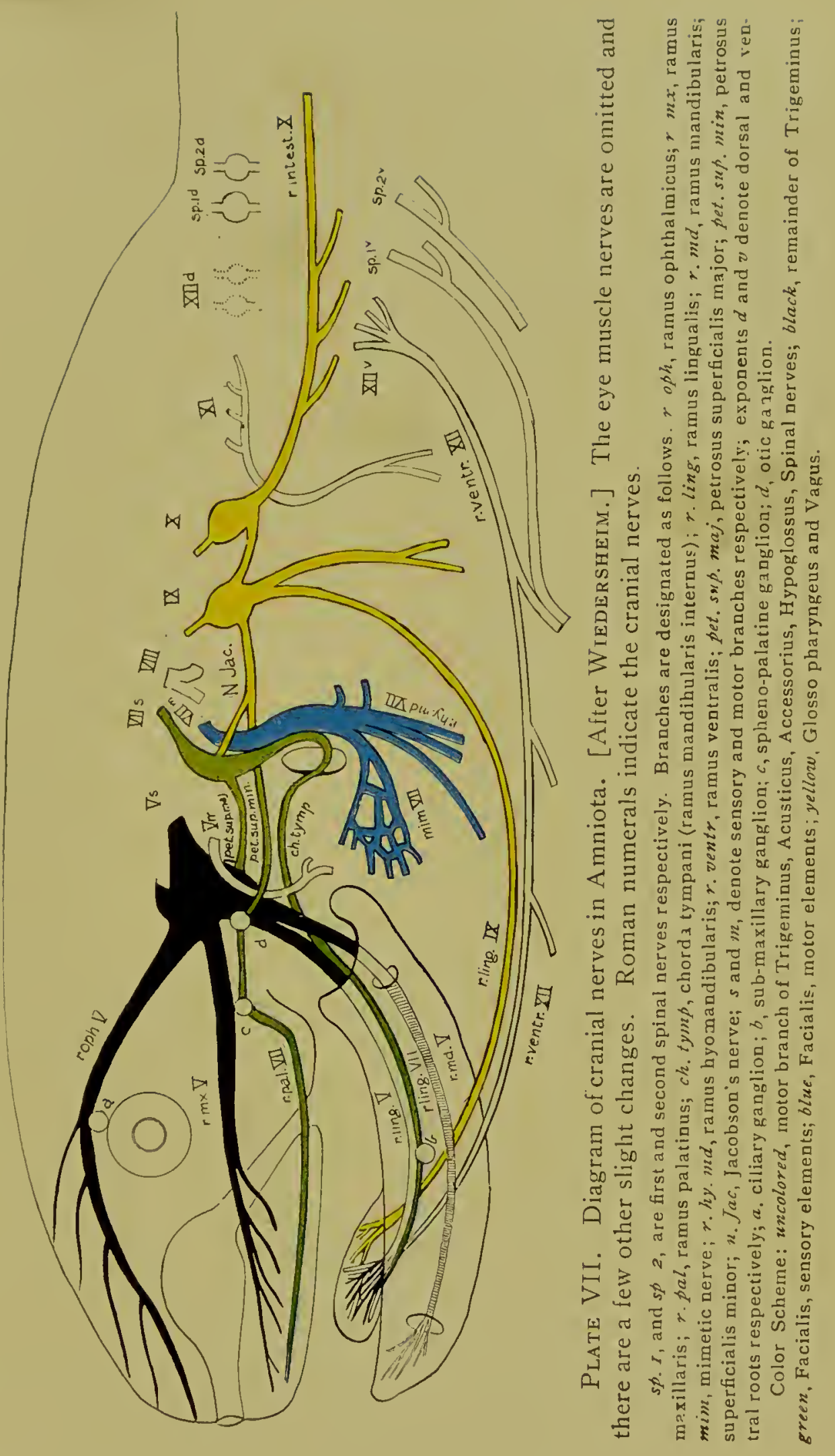



which supplies general sensation to this region. The second branch is the buccal (ramus buccalis), accompanied in its turn by the marillary branch of the Trigeminus. The third is the external mandibular, divided into anterior and posterior branches. The companion branch from the Trigeminus, associated with the anterior of the two subdivisions, is the mandibular of that nerve (ramus mandibularis Quinti). In the Dipnoi there is a communicating branch between this part of the system and that belonging to the Vagus, but this seems to be wanting in other cases.

The remaining branches of the facial nerve are divisible into tivo portions, sensory and motor. The sensory portion possesses at its origin the large genicular ganglion, from which proceed (I) a large palatine branch and (2) a small internal mandibular. The motor portion incorporates within itself the external mandibular branch of the lateral line system given above, and this forms the mixed hyo-mandibular branch which supplies the region of the lower jaw and the hyoid arch.

Much of the Trigeminus has already been described in association with the Facialis. There remain to be mentioned the large semilunar ganglion (often fused with the sensory ganglia of the lateral line nerves of Facialis) which lies at the base of the three branches already described, and the deep ophthalmic branch (ramus ophthalmicus profundus). This latter. possesses a ganglion of its own and issues from the skull by a separate foramen. It is thus semi-distinct from the remainder and may be considered a separate nerve, originally anteripr to the Trigeminus, and secondarily associated with it. There are some indications to show that it may have once been associated with the Trochlearis, as sensory and motor roots, respectively, of the same elementary nerve. The mandibular branch of the Trigeminus possesses a few motor elements, which prevent the nerve from being classed as wholly sensory.

The later history of the parts above considered may be followed from the second diagram [Plate VII], which represents in a general way the terrestrial type, but which in its propor- 
tions and certain other cletails suggests more especially the mammalian condition. The first and most striking change is the loss of the special system supplying the lateral line senseorgans, the entire equipment for which vanishes in amphibians that become terrestrial and never reappears. This causes at once, among other changes to be considered later on, a loss of three branches of the facial nerve, the superficial ophthalmic, the buccal, and the external mandibular. There remain the palatine, the internal mandibular and the motor element of the hyo-mandibular, of which the first two become reduced in size and fuse with Trigeninus elements, while the third loses in one direction but more than compensates for it in another. The palatine, under the name of $N$. petrosus superficialis major, passes through the pterygoid [Vidian] canal (in Mammals) and enters the spheno-palatine ganglion of the sympathetic system, where it meets with fibers of the Fifth nerve, and continues under the name of palatinus major, usually considered as a part of the Trigeminus. The internal mandibular, now known as the chorda tympani, transverses the tympanic cavity, running between malleus and incus. It leaves the middle ear through the Glasserian fissure (in mammals) and blends with the lingual branch of ramus mandibularis $V$., encountering in its course the sub-maxillary ganglion (better, sub-mandibular) of the sympathetic system.

The explanation of these complicated relationships becomes clear when we consider the history of the related parts. Malleus and incus are primarily the condyle of the jaw and the quadrate bone, respectively, and the branch in question runs along the articulation between them. In the Mammalia these osseous elements become drawn writhin the carity of the middle ear, where they undergo a transformation into auditory ossicles; and the nerve, in oraer to preserve its original relationships, must follow them, thus producing a complicated condition, casily explained by their morphological history, but wholly inexplicable otherwise. Furthermore, with the increased importance of the tongue, and more especially with the development of the fleshy part of it in mammals, the orig- 
inal branch of the Trigeminus gains in importance and the two become secondarily associated.

With the reduction in bulk of the hyo-branchial musculature the motor element of the hyo-mandibular, the only portion now remaining, tends to decrease in size, but this is more than compensated for in mammals by the development of the mimetic muscles. These have been shown to originate from the integumental muscular layer of the neck region, innerved by the branch under consideration, and, as this layer spreads up over the neck and differentiates into specialized slips, the innervation increases also and spreads eventually over the entire face, thus gaining its right to the name "Facialis," a right which, curiously enough, it possessed originally, in connection with the lateral line organs, but which it afterward lost until it regained through its motor elements what it had lost in its sensory. The branch to the stapedius muscle of the middle ear, $N$. stapedialis, proceeds also from the hyo-mandibular, and comes originally from the branch supplying the digastric muscle.

The Trigeminus of the second, or terrestrial, type, suffers no reduction through the loss of the lateral line organs, since it has nothing directly to do with them, but the four original branches become reduced to three through the loss of the deep ophthalmic element, which seems, in part at least, to fuse with the superficial branch of the same nerve to form the "first branch" of human anatomy, the ophthalmicus. The maxillaris and mandibularis show but little change and form the second and third branches, respectively, thus giving the reason for the name "Trigeminus," first applied in Man.

The great increase in the size of the lingual branch of the mandibularis has already been noticed; otherwise the most important innovation is found in the new relations of the Trigeminus with the Facialis, the Glosso-pharyngeus, and the sympathetic ganglia. The first of these has already been treated in detail. The Glosso-pharyngeus sends a communicating branch (tympanic [Jacobson's] nerve) to the otic ganglion, which rests upon the base of ramus mandibularis; the 
two nerves also come into indirect contact in the tongue, where the fibers of the gustatory and lingual branches of the two nerves interlace. Four ganglia of the sympathetic system, the entire cephalic group, become associated with the Trigeminus, the ciliary with the first branch, the spheno-palatine with the second, and the otic and submaxillary with the third.

The Acusticus (eighth nerve) is originally a part of that Facialis element which supplies the lateral line, and as the essential part of the ear, the labyrinth, closely resembles in its early development the sense-organs of the lateral line, the suggestion is strongly felt that we have here a case of the local specialization of a single element out of a series of similar parts, and that the Eighth nerve is consequently nothing more than a branch of the superficial sensory system of the Facialis, the region of distribution of which chanced to develop a high degree of complexity as an organ of special sense.

IV. The vagus group. (Glosso-pharyngeus, Vagus, Accessorius.)

This group includes in all vertebrates the Glosso-pharyngeus and Vagus nerves, to which is added in mammals the Accessorius, secondarily derived from the Vagus, and existing in the Sauropsida as a semi-independent slip. This group is primarily associated with the gill-region, but secondary sends branches backwards and forwards which may even reach the extreme ends of the body, thus having a more extensive distribution than that of any other cranial nerves. In the lower forms this group is extremely regular and possesses a wellpronounced metamerism, thus strongly suggesting its origin from spinal nerves, similar to those which form a direct continuation of the series. Taken in connection with the much greater differentiation of the other cranial nerves it seems evident that the acquisition of the anterior end of the primordial spinal cord by the cranium has been a gradual one, and that the Vagus group is less modified than the nerves anterior to it, because it has been annexed later. The primitive condition, that found in fishes, may be first considered by the help of the diagram previously referred to [Plate VI]. The most 
superficial, and at the same time, the most aberrant, is the ramus lateralis, which belongs to the system of lateral line nerves and supplies the lateral line itself, which extends typically to the end of the tail. It is thus the longest nerve in the body, co-ertensive with the spinal cord itself. The lateral nerves of the two sides are connected with one another by the supra-temporal branch, which forms a connecting loop over the top of the head; in the Dipnoi, though not in other fish, lateral communicating branches connect it with the superficial ophthalmic nerve of the Facialis, thus uniting the two parts of the system. The ramus lateralis has at its proximal end a ganglion of its own (ganglion laterale), although it arises in connection with the combined ganglionic mass of the Vagus. In spite of this association, however, it is probable that the ramus lateralis did not originally belong to the Vagus alone, but was built up as a collecting trunk from branches supplied by cach metameric nerve of the body, beginning with the Vagus. The gradual loss of these metameric connections, beginning posteriorly, would, in time, leave the most anterior one alone, the condition found at present.

Ventral to the ramus lateralis appear five elements, similar to one another, each associated with a gill-slit, and possessed of its own ganglion. This extremely primitive condition is seen in a few forms only (e.g., the rays and skates), but these animals are in other respects so primitive, and the condition is so exactly what one would expect as an early one, that it may be taken as undoubtedly the starting point. The first of these elements is more distinct than the others, and forms the Glossopharyngens, treated as a separate nerve; the remaining four are Vagus elements and in all but very primitive forms arise from a single ganglionic mass, the ganglion jugulare, formed of a fusion of the four primary ones. It is with this that the ganglion laterale of the ramus lateralis is associated. Each of these five elements (Glossus-pharyngeus, and the four Vagi) possesses an identical distribution. From the ganglion the main stem passes cownwards, and forks into two branches, including a gill-slit in the fork. The two branches, one in 
front and one behind the slit, are known respectively as rami prec-and post-trematici. In this connection it is interesting to see that the spiracular opening, which probably represents the gill-slit next in order anteriorly to the regular series, is similarly included between the internal mandibular (chorda tympani) and the hyo-mandibular of the Facialis, which thus become, respectively, the pre- and post-trematic branches of that nerve. It is even possible in like manner to consider the maxillary and mandibular branches of the Trigeminus as similarly related to the mouth opening, resting upon the probability of the identification of the mouth with an original gill-slit anterior to the spiraculum. We have thus a character of great value in the resolution of the cranial nerves into their original metameric elements, one which will be considered later in the treatment of this difficult and unsolved problem.

Of these five branchial nerves, the Glosso-pharyngeus, as the most anterior and consequently the most modified (earliest absorbed by the cranium) possesses additional branches not represented in the others, and these have run forward and supply parts anterior to it. One of these is a communicating branch between this nerve and the Facialis and passes from its ganglion (ganglion petrosum) to that of the Facialis (ganglion geniculare). This nerve possesses no special name in lower forms, other than the generic ramus communicans, but in the higher forms it becomes the tympanic (nerve of Jacobson), and forms an intimate means of communication between these nerves and the Trigeminus. Below this is the palatine, lying near the Facialis branch of the same name, and developing a few connections with it. A small lingual branch is present in the Dipnoi.

There remains but one further element to be considered, but this is an extensive one, the ramus intestinalis Vagi. This appears in primitive forms as a separate element, with its own ganglion, but in all other cases it arises with the rest of the Vagus and its ganglion becomes lost in the general mass, the ganglion jugulare. This is the branch which, even more than the lateralis, has earned for the nerve to which it belongs 
the title of Vagus (wandering), since it becomes distributed to the osophagus and stomach, the heart, and, in higher forms, the lungs. In spite of its great length and extensive distribution, however, it is not to be considered, like the ramus lateralis, a compound nerve, but its length is due rather to the extension posteriorly of parts once placed far forward and thus within the legitimate province of the original nerve. 'Thus, the heart has primarily a very anterior position; the œsophagus and stomach were probably once very far forward, and the lungs are diverticula of the primary œsophagus. The wide distribution of this branch is thus a striking illustration of the principle of conservatism of nerve distribution enunciated above, and belongs in the same category as the case of the stapedial nerve or that of the innervation of the mimetic musculature.

In the transition to terrestrial life the Vagus group suffers naturally the loss of the branchial elements in which metamerism was so clearly displayed, but has gained by the greater development of the tongue and the sense of taste, and has differentiated the Accessorius element. The intestinal branch also, corresponding to the higher development of its field of distribution, is still more extensive and complex.

The extreme differentiation of this group may be learned from Plate VII, which here especially, in the separation of the Accessorius and in other points, suggests the mammalian conditions. The communicating branch, the tympanic nerve, becomes somewhat more complicated and forms connections between four ganglia; starting from the ganglion petrosum of the Ninth nerve it runs forward and sends branches to the ganglion geniculare of the Seventh, and to the otic and sphenopalatine ganglia of the sympathetic system. It is also involved in a small plexus of sympathetic nerves which surround the carotid artery. The relationships of this nerve, which are so complex in mammals, become especially so in Man, owing to the shortening of the longitudinal axis of the skull and the formation of the cervical flexure, both of which tend to the shortening of the distance between the nerve roots. The relations found in Man are shown in the accompanying figure (Plate 
VIII), which is to be carefully compared with Plate VII. As special names are often given in human anatomy to parts spoken of by the morphologists under more general terms, a list of equivalent terms is here added, for the better comparison of the figures alluded to.

Terms in human anatomy. Morphological eQuivalents.

Ganglion Gasseri, composed of. $\left\{\begin{array}{l}\text { Ganglion ophthalmicum superfi- } \\ \text { ciale VII. } \\ \text { Ganglion buccale VII. } \\ \text { Ganglion mandibulare VII. } \\ \text { Ganglion semilunare V. } \\ \text { Ganglion ophthalmicum profundum. }\end{array}\right.$

Ganglion geniculare........ $\left\{\begin{array}{l}\text { Sensory ganglion of VII, excepting } \\ \text { the parts belonging to the lateral } \\ \text { line system. }\end{array}\right.$

Ganglion petrosum......... Together form the sensory ganGanglion jugulare IX.........

Ganglion jugulare X...... $\left\{\begin{array}{l}\text { Compound sensory ganglion of } X, \\ \text { formed by the fusion of the } \\ \text { ganglia of all of the original } \\ \text { sensory elements with the excep- } \\ \text { tion of the ganglion laterale of } \\ \text { the lateral line systen. }\end{array}\right.$

N. petrosus superficialis major ........... Ramus palatinus VII.

N. palatinus major V....... $\begin{aligned} & \text { Continuation of the ramus pala- } \\ & \text { tinus VII beyond the spheno- } \\ & \text { palatine ganglion, plus some } \\ & \text { fibers from the Trigeminus. }\end{aligned}$

Chorda tympani................ mandibularis internus VII.

N. tympanicus.............. $\left\{\begin{array}{r}\text { Together form Jacobson's nerve } \\ \text { which is, morphologically, ramus }\end{array}\right.$

N. petrosus superficialis minor. $\left\{\begin{array}{l}\text { which is, morpholo } \\ \text { communicans IX. }\end{array}\right.$

N. petrosus profundus major.
N. petrosus profundus minor.. $\left\{\begin{array}{l}\text { Both included in the brancl of the } \\ \text { above to the spheno-palatine } \\ \text { ganglion. }\end{array}\right.$ 


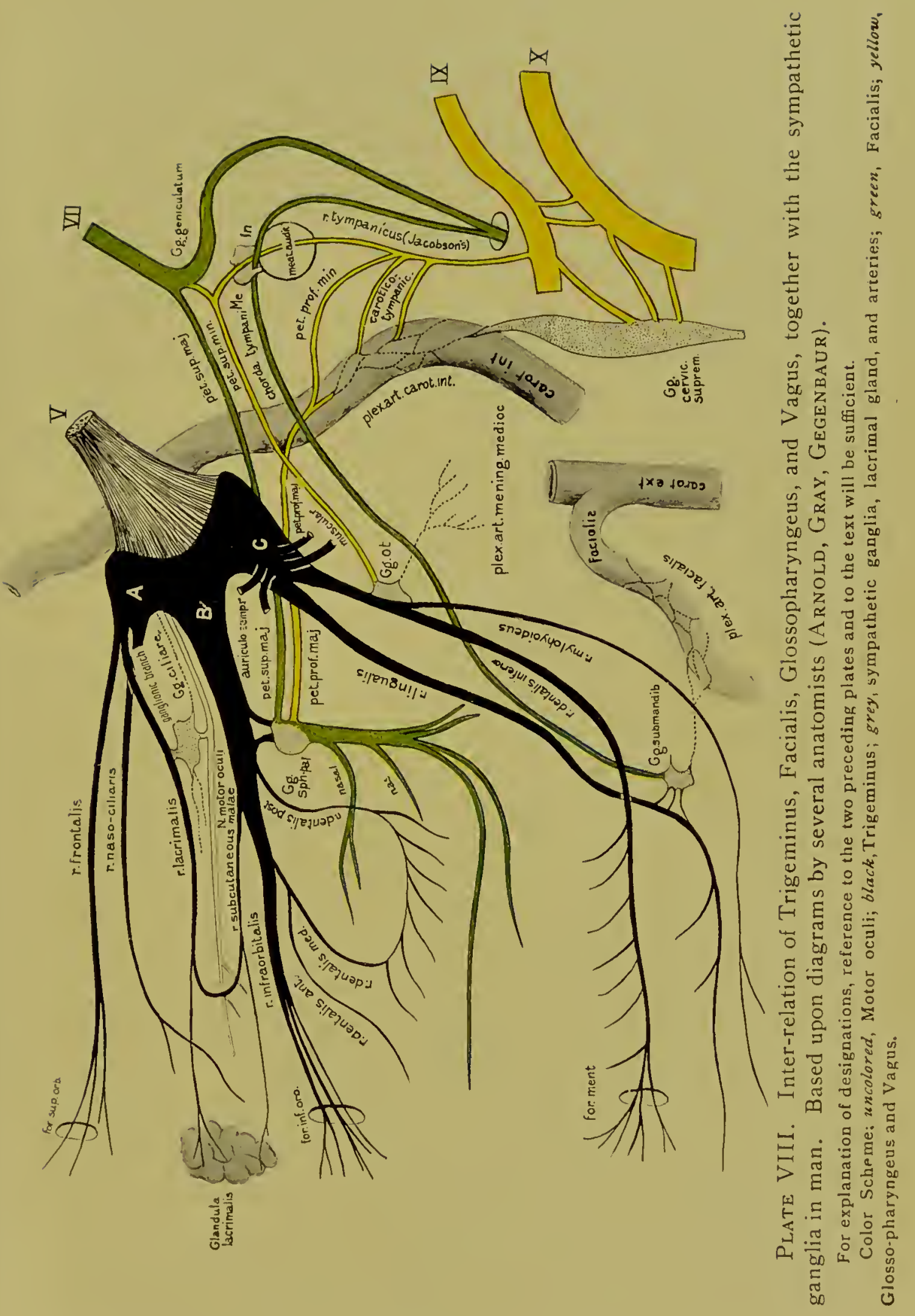



The lingual branch of the Glosso-pharyngeus, which appears first in the Dipnoi, becomes in the Amniota, and especially in the mammals, a large and important nerve, and specializes as the nerve of taste (gustatory). The remaining branch is motor and is distributed to those muscles which are derived from those of the first branchial arch. In the same way motor branches of the Vagus supply the muscles of the larynx and trachea, and the walls of the pharynx.

The ramus lateralis disappears with the advent of terrestrial life, but, on the other hand, the increase in size and complexity of heart, lungs, œsophagus, and stomach so increase the importance of ramus intestinalis that it alone comes to be considered the main nerve (hence the name "Pneumo-gastric"), of which the other elements are considered branches.

The circumstance which leads in Sauropsida to the partial, and in mammals to the complete, separation of the Accessorius is clearly found in the greater development and higher degree of independence of the parts to which it is supplied, the trapezius and sterno-cleido-mastoid muscles, a development due in its turn to the increased importance of the neck. This nerve is still a part of the Vagus in the human embryo and shows the steps of its gradual emancipation during development (Fig. I 25).

V. Hypoglossus.

The Hypoglossal nerve, which appears in the higher vertebrates as the last of the cranial nerves, is plainly one or more adopted spinal nerves, found still in their original office in fishes and amphibians. It is mainly a motor nerve and arises from several roots which belong in the ventral series; this is rendered more certain by the appearance, usually transitory and embryonal, of corresponding dorsal roots, equipped with ganglia, which thus complete the elements necessary for genuine spinal nerves. In Plate VII these latter are indicated by dotted lines; and the two spinal nerves which are shown in Plate VI may be considered to represent the potential hypoglossal still in an indifferent condition. Some of the spino-occipital elements may also enter into the formation of 
the Hypoglossus, but this has not yet been definitely shown. The Hypoglossus enters into connection with one or two of the first cervical nerves, forming the ansa hypoglossi, a union

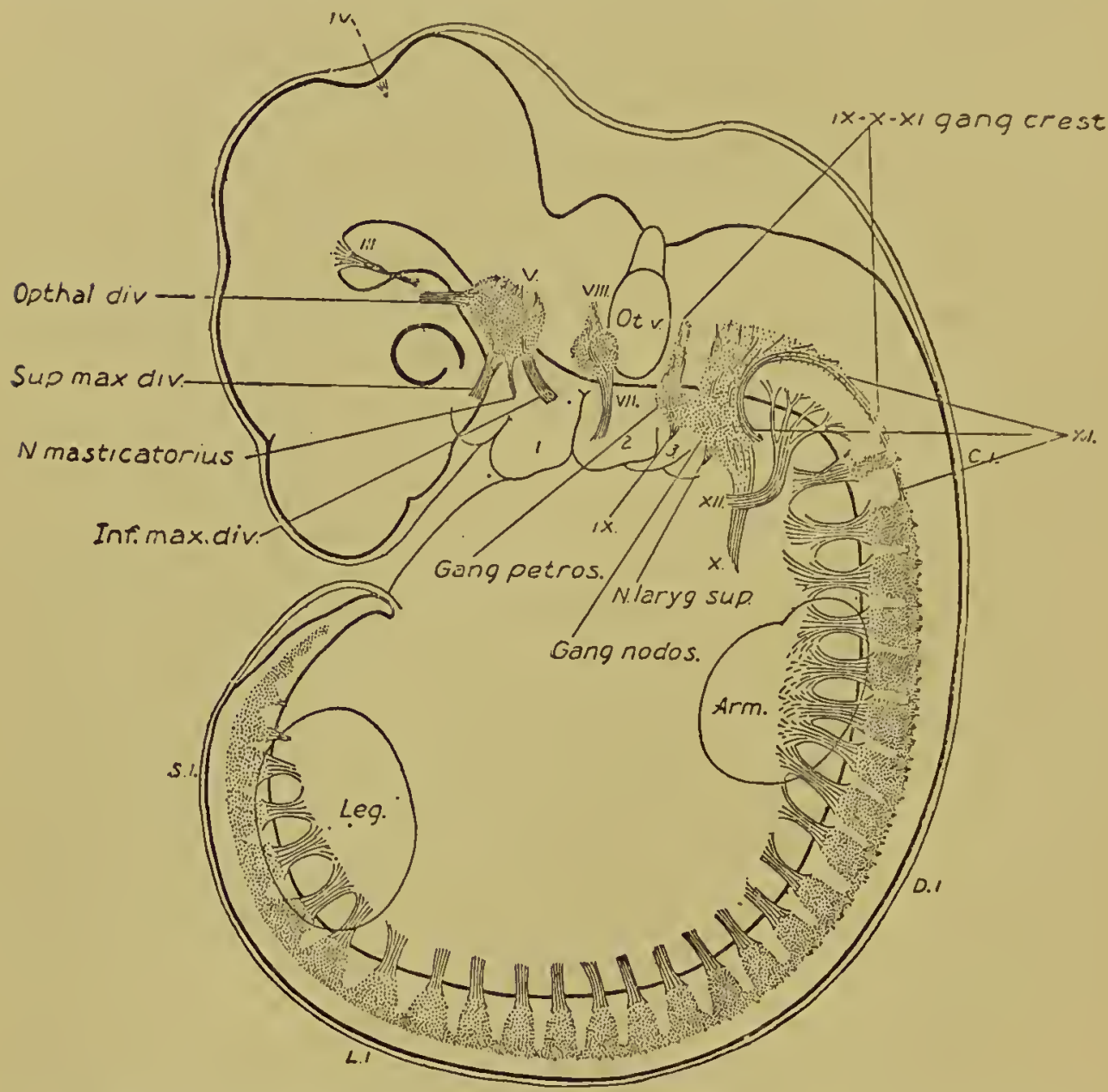

FIG. I25, a. Reconstruction of peripheral nerves in human embryo. [After Streeter.] Four weeks human embryo, $6.9 \mathrm{~mm}$ long.

$N$. tymp, tympanic nerve; $N$. laryg. sup, superior laryngeal nerve; Gang. petros, ganglion petrosum; Gang nodos, ganglion nodosum; Froriep, Froriep's ganglion. The cranial nerves are designated by roman numerals, the spinal by arabic. The other designations are evident.

from which proceed motor nerves to certain of the hyoid and extrinsic laryngeal muscles.

It is now time, after this review of the cranial nerves and their morphological history, to take up the question of the original segmentation of the vertebrate head. and consider what light the nerves throw upon this obscure and much-disputed subject. 


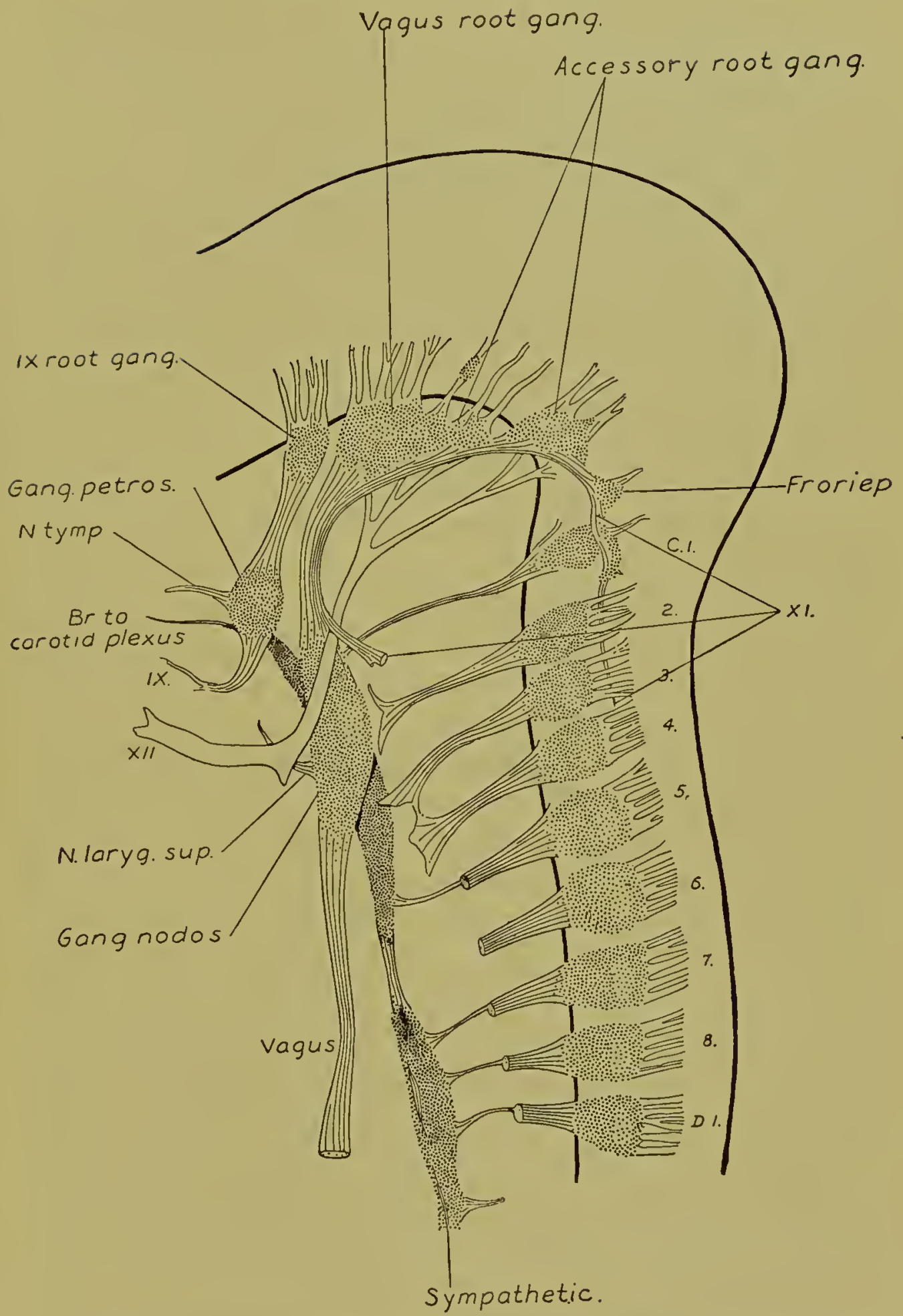

FIG. 125, b. Reconstruction of peripheral nerves in human cmbryo. [After Streeter.] Six weeks human cmbryo, $17.5 \mathrm{~mm}$ long. For abbreviations sce Fig. I25, $a$. 
The question rests upon the assumption that the precursors of the vertebrates were, like Amphiorus, headless but completely segmented, and that the head was first formed by the union of a certain definite, though probably rather small, number of somites, each with its similar set of organs, into a single body unit or complex. A similar process has been postulated in the case of the head of insects, and the two cases have much that is analogous, especially the gradual addition of primarily trunk somites in proceeding from lower to higher forms. In a myriapod, for example, or still better, in an annelid like the earth-worm, the somites are practically alike, each containing one pair of nerve ganglia, one pair of segmental organs (in the latter case), one set of metameric muscles, and one pair of external appendages, and it is by comparing the number of ganglia, of appendages, or other metameric parts, that morphologists attempt to resolve the head complex into its primary somites. In much the same way the body somites of Amphioxus, or, to a lesser degree, of a fish, are also similar, and the pairs of nerves, the gills, the nephridia, and especially the myotomes, are metameric, at least in the embryo. By thus ascertaining the original mumber of each of these metameric elements that exist in the head, as shorun in the embryological record, morphologists have sought here also to reconstruct the carly conditions and translate the head of modern vertebrates into a definite series of somites, each with its metameric parts. Thus far the views of investigators are widely apart, and the suggested number of primary somites varies from three or four to eighteen, or even more; the most usual results agree, however, in placing the number between the limits of nine and eleven.

The posterior portion of the head in fishes, and especially in certain primitive selachians, shows a definite metamerism, marked in the cartilaginous gill-arches, the arterial arches, and the cranial nerves (Vagus group), but anterior to the otic region this becomes effaced, and it is extremely difficult to see here any suggestions of segmentation, even in the embryo. Some, have, indeed, asserted that the prcchordal and parachordal portions of the head, divided at about the hypo- 
physis, are distinctly different in this respect, and that while the latter forms the original anterior end of the primary segmented ancestor and may thus be expected to show traces of metamerism, the former or prechordal portion represents a later addition, gained somewhere between Amphioxus and cyclostomes, and is thus primarily unsegmented. It is more probable, however, that this præchordal portion is primarily metameric as well as the others, and that the indications of this have become more completely effaced, first, because it began to be modified much earlier than the other part, and secondly, that, because of its position, it became naturally the seat of important organs of special sense and became more modified through their influence.

In the consideration of this problem, the cranial nerves offer an especially hopeful material, as the various sensory and motor elements, sensory ganglia and other parts, suggest that they have differentiated from an original series of typical spinal nerves. Thus, as primary sensory roots, each with a ganglion, we may suggest the Trigeminus, Facialis, Glosso-pharyngeus, and Vagus, the latter a compound nerve, capable of resolution into four, or perhaps, five elements. If to these the ramus ophthalmicus profundus be addled with its ganglion as an originally separate element, we have the sensory roots of eight original pairs. The three nerves of the eye muscles are, both in origin and function, motor roots, and in some cases, as in the relation of Abducens to Facialis, they seem to belong with certain definite sensory elements. The tracing out of præ- and post-trematic branches which include a gill-slit as above mentioned assists in locating seven elements, if the mouth opening and spiraculum be included.

The above relations are summarized in the following diagram, which, although not claimed as the ultimate solution of the problem is, at least, suggestive (Fig. $126, \mathrm{~A}$ ). The head is here represented as being composed of nine somites on the basis of nine pairs of "head cavities" (muscle somites or myotomes) found in dog-fish embryos. These are represented by the heavy black rings numbered from I-IX. For the first 
somite the Ophthalmicus profundus represents the sensory, and the Motor oculi the motor root. To the second the remaining portion of the Trigeminus and the Trochlearis are similarly related, the former with præ- and post-trematic branches about the mouth. The third is supplied by the Facialis for a sensory
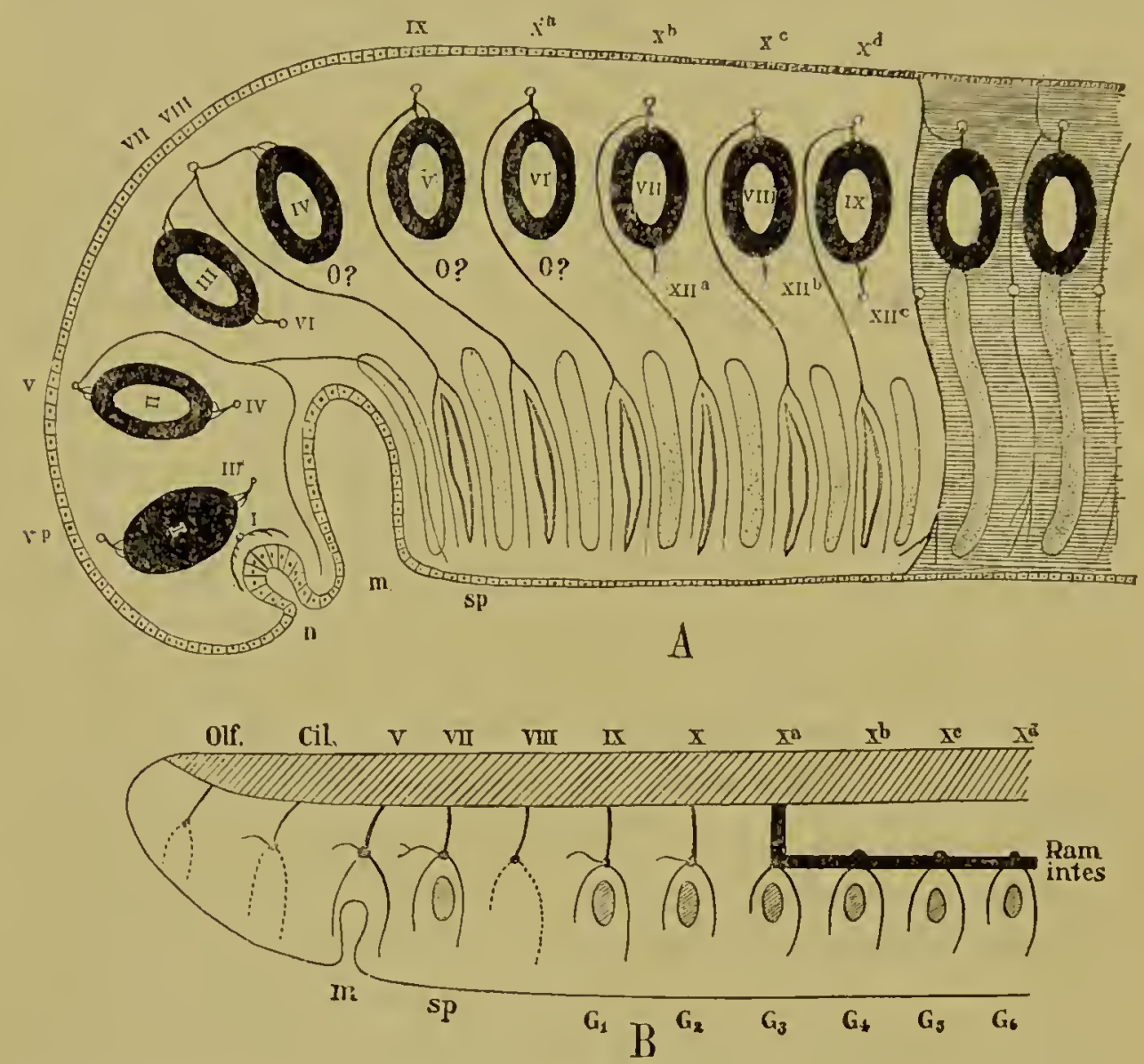

FIG. I26. Two suggestions for the solution of the problem of vertebrate cephalogenesis.

(A) According to VAN WIJHE. (B) According to BEARd.

The roman numerals enclosed in the ovals in $A$ designate the head somites, all other roman numerals refer to the cranial nerves. $m$, mouth; $n$, nasal opening; $s p$, spiracular cleft; $G_{1}, G_{2}$, etc., gill-slits; Olf, olfactory nerve; $C i l$, ciliary nerve.

and by the Abducens for a motor root, but for the fourth we have the Acusticus alone, with the motor root wanting. The remaining five possess the five elements shown in the Vagus group in fishes, while two Hypoglossus roots supply motor elements for the last three somites. Fig. I26, B, shows a suggestion made by another investigator, and based mainly upon 
the relation of prex- and post-trematic branches to their corresponding gill-slits.

Later researches have modified these diagrams somewhat, as, for example, the discovery of transitory sensory roots for the hypoglossal elements, and indications of other nerves anterior to the Ophthalmicus profundus; the spino-occipital nerves, although of unknown value, seem indicative of still other somites in the occipital region and need to be thoroughly explained before the problem of the segmentation of the head can receive its final solution.

There remains to be mentioned an auxiliary system of ganglia and nerve fibers, not directly under the control of the will, but often of great importance in regulating the physiological activities of certain of the internal organs. This is the sympathetic system, often erroneously treated as originally a distinct nervous system coördinate with the cerebro-spinal, thus far the subject of this chapter. As a matter of fact, however, the sympathetic system is an integral portion of the latter, and its differentiation from this may be followed both in the race history as well as during individual development. It attains its greatest degree of individuality only among the higher forms. It consists primarily of a series of ganglia, segmented off from the sensory ganglia of the spinal nerves, although retaining connection with their places of origin through commumicating branches. The ganglia of the two sides, which come to lie ventral to the spinal nerves on either side of the vertebral column, may become secondarily connected with one another by longitudinal connectives, thus forming two lateral trunks.

This appearance of metameric ganglia connected in two longitudinal series, and especially their ventral position, has led the sympathetic system to be compared to the ventral chain of ganglia found in articulates (e.g., insects, crustaceans), a suggestion of homology that is sufficiently disproved by the mode of origin and the fact that the similarity is most perfect in the higher forms. Indications suggest that the separation of sympathetic ganglia began historically in the head, since in 
fishes the system is better developed in this region than in the trunk. In the embryo also the cephalic portion develops before the rest.

The system appears well developed in Amphibia, with its two lateral trunks. In Sauropsida a pair of subsidiary trunks in the neck region accompanies the vertebral arteries, the only instance of a distinct dorsal position for any part of this systenn.

From the ganglia as centers numerous nerve fibers proceed, supplying many of the internal organs, especially the alimentary canal and the arteries, the favorite mode of distribution being an intricate plexus, which spreads over the broader surfaces and enwraps the smaller parts.

Owing to the origin of the sympathetic system from the strictly metameric sensory ganglia of the cerebro-spinal nerves, this system also shows at first a metameric character; this appearance becomes modified, however, in regions of the greatest differentiation, as in the head and neck and the pelvic region. The four cephalic ganglia, ciliary, spheno-palatine, otic, and submaxillary, which in mammals assist in forming connections between certain of the cranial nerves, and which have been treated with these latter parts, belong to the sympathetic system. 


\section{CHAPTER XI}

\section{THE SENSE-ORGANS}

"Die wunderbare und wirklich überraschende Ähnlichkeit in der inneren Organisation, in den anatomischen Structurverhältnissen, und die nochmerkwürdigere Übereinstimmung in der embryonalen Entwickelung bei allen Thieren, welche zu einem und demselben Typus, $z$ B., zu dem $Z$ weige der Wirbelthiere, gehören, erklärt sich in der einfachsten Weise durch die Annahme einer gemeinsamen Abstammung derselben von einer einzigen Stammform. Entschliesst man sich nicht zu dieser Annahme so bleibt jene durchgriefende Übereinstimmung der verschiedensten Wirbelthiere im inneren Bau und in der Entwickelungsweise vollkommen unerklärlich."

ERNST HaEckel, Schöpfungsgeschichte, Kap. III.

IT will be remembered that the nervous system is primarily external, developed in response to stimuli from without, and that, as this system becomes more specialized, and hence of greater importance to the organism, it withdraws in great part into the interior, leaving upon the surface a set of sense-organs, capable of receiving the impressions and transmitting them to the central organ. Taking into consideration the intimate connection between these two portions, external and internal, it might be supposed that the enormous development in size and complexity shown by the brain would be the result of a corresponding degree of differentiation of the external parts, yet such is by no means the case. The sense-organs are early brought to a high state of efficiency and develop but little during the entire vertebrate history. The eye of the fish is almost as good an optical instrument as is that of the mammal, and, save for a few external parts, is as complex; the sense of hearing, although not as early in development as the eye, is yet very acute in reptiles, and, perhaps, in amphibians, and gains in birds and manmmals very little except, perhaps, 
the recognition of musical tones; indeed, the early aquatic forms possess in the lateral line organs an entire system, no trace of which seems to have survived the transition to land, and yet, with 110 especial progress on the part of the senseorgans, the central nervous system, and especially the brain, the receiving organ of the special senses, has increased from a simple condition to one showing a marvelous degree of complexity. The cause of this extreme development must be laid, then, not to the sense-organs, but to the direct and cumulative influence of the impressions received. The motor centers, which have contributed not a little to the complexity of the central nervous system, have also developed in response to the external environment, though rather more indirectly, perhaps, through the necessity of controlling the more specialized limbs and other parts, which, in their turn, were directly influenced by external conditions.

The morphological history of the sense-organs does not, therefore, show the extensive progress exhibited in the case of most of the other systems; but as certain definite changes were necessitated by the transition from water to land, this history is divided into two great stages, (I) that of the aquatic, and (2) that of the terrestrial life.

Throughout the animal kingdom, the elementary type of sense-organ is a single epithelial cell, connected by a nerve with some sensory center. From this as a starting point higher efficiency is gained in three ways: (I) by the association of a number of these elementary units to form a larger sensory area, (2) by the specialization of the cell itself, and (3) by the development of accessory parts.

The area over which a given form of sensory cell may occur may be a general surface of indefinite limits, or it may be restricted and form a definite sense-organ. Most generally the epithelial cells composing such an area are not homogeneous, but are differentiated among themselves into two sorts, sensory cells and supporting cells; the first are the receptive units of the nervous system; the latter are non-sensitive, and are grouped about the sensory cells in such a way as to form 
a support and protection for the essential elements of the senseorgan, which may thus attain a high degree of sensitiveness.

Regarding the differentiation of the sensory cells themselves, they may present at their free end a ciliated or simple surface, or may bear one or more flagella. In the most specialized types the flagella themselves may be modified for the better reception of certain definite forms of impression, as in the case of the rods and cones of the retina, or the acoustic hairs of the inner ear; these types, however, owing to the extreme delicacy of the projecting parts, can exist only upon an external surface bathed by water, as in many aquatic invertebrates, or upon a surface that faces some internal cavity furnished with an artificial fluid or semi-fluid.

Accessory organs for the reception and intensification of the external stimuli or for the protection and care of the essential parts are the rule in the case of the more specialized and complex organs, but are not employed to assist in the reception of general tactile impressions save in certain invertebrates with a thick and hard exo-skeleton which would naturally prevent such impressions from reaching the interior. In this latter case the sensations are transmitted by sensory hairs which are protruded through pores in the external armor, and communicate with underlying sense-organs. In those vertebrates in which the integument is covered by non-sensitive parts, such as scales, feathers, or hairs, often necessary to protect the animal from serious injury, the sensory organs are developed between them, or may be situated about their bases, when they are stimulated indirectly through the movements of the insensitive outer parts, much as in the previous case.

Concerning the actual sensations produced by the different kinds of sense-organs found among vertebrates we know very little, and inferences must be made with extreme caution. Although something can be deduced from the mechanical structure of a terminal organ, especially from that of its accessory parts, and although from the physiological side something can be learned from the behavior and responses of an animal under observation, the only certainty concerns our own sense-organs 
and those formed like them. Thus, we are positive concerning the sense furnished by the eye, since the variations from the human structure are very slight in any case, even in fishes; the ear, however, is somewhat more variable, and presents several problems, since the lower types of ear lack certain parts, like the cochlea, which in Man are essential to the completeness of the sense of hearing as we understand it. In this case we have two alternatives, either that in the different forms the same function is subserved by different parts, as is possible in the case of the brain (cf. the pallium of teleosts and the cerebral hemispheres of mammals), or else that there are elements in the human sense of hearing not perceived by ears belonging to other types.

Aside from the above, the sense of smell seems to be a common possession, and in terrestrial forms the sense of taste also seems general if we are to judge from the similarity in the location and structure of certain specialized sense-organs and the identity of their nerve supply. After these are excepted, however, there remains a large number of types of terminal sense-organs, more or less localized in different areas, the special functions of which are practically unknown, but are included within the comprehensive terms of touch or feeling. That many distinct impressions are involved in this is shown by this very dissimilarity in the structure of the terminal organs, the complexity of which, in certain cases, suggests the possibility of definite senses, at least as distinct as those of smell or taste; but as few of these types occur in Man, and as even here the elementary sensations have not been wholly coördinated with the various forms of nerve terminations, but little can yet be stated on the subject, and the psychology of the tactile sensations of the lower vertebrates remains an unexplored field.

Probably the lowest form of vertebrate sense-organ, and one that is universally distinguished among them, is that of simple sensation, the contact sense, which resides in the epidermis and is thus generally met with over the entire surface. The vertebrate epidermis, which, in contrast to that of invertebrates, is many cells thick, is supplied everywhere by sensory nerves 
which branch repeatedly and with their ultimate fibers form a delicate net-work which permeates the entire layer, stopping only at the most external cells. This type is characteristic of all vertebrate integument, including both cyclostomes and mammals, and furnishes them all with a surface capable of responding to general tactile impressions.

Aside from this general tactile sense vertebrates possess a large number of more or less specialized sensory endings, usually classed also as tactile. These are local in distribution, often confined to a single group of animals, and usually occur upon prominent portions of the body or occasionally, as in the case of certain fishes, upon special papillæ or long filaments. The most extensive of these, and the only one to develop into a definitely organized system, is that of the lateral line organs, referred to above in connection with the cranial nerves and possessed by the primarily aquatic vertebrates (fishes and amphibians). Although this system, as such, together with its nerves, disappears utterly with the assumption of a terrestrial life, it is yet of importance in this connection because of the possible derivatives from it in higher forms, among which have been mentioned, with more or less basis for the claim, the taste-buds, the inner ear, and the mammalian hair.

In its simplest form a lateral line organ consists of a small group of sensory cells, slightly convex in form and protected by a wall of non-sensitive supporting cells. This organ gains its simplest form of protection by sinking slightly beneath the surface, its supporting cells remaining at the general level, or even projecting a little above it. 'By continuing this process the sense-organ comes to lie at the bottom of a flask-shaped cavity, communicating with the surface by a narrow neck.

From this point on, greater complexity may be gained by development in one of two directions, the flask-shaped cavities may either become associated in rows and break down their adjacent walls, forming the slime canals, or else each separate flask may become elongated, bearing the sense-organ at its very bottom, as in the canals of Lorenzini. In the first of these, the coalescence may result in the formation of either a 

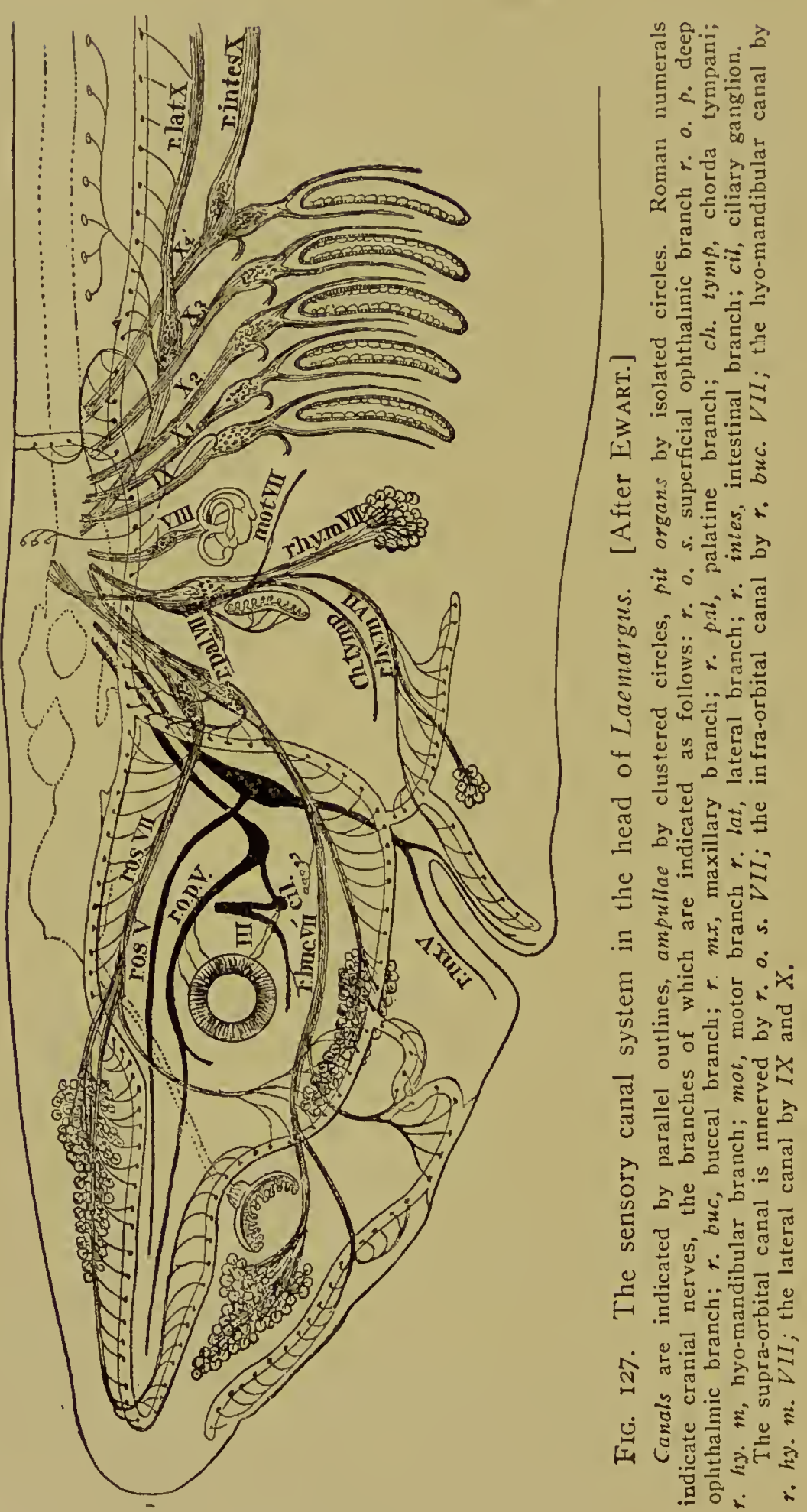
deep trough or, more usually, an enclosed tube, running just below the surface; and in this latter case, each organ may open by its own pore, placed directly above it, or an entire tube may open by a single common pore at one end. These canals are usually filled with a clear mucous or gelatinous material, secreted by the walls, and destined to protect the sensory cells. In the case of the second form of development, there will be produced local groups of associated, though distinct, canals, each beginning superficially at an external pore and running obliquely beneath the surface to terminate proximally in a bulb or ampulla, in which the sense-organ is located. These organs occur in localized masses in the heads of selachians, associated topographically with the mucous canal type, the ampulla with their nerves clustered in such a way as to resemble bunches of grapes (Fig. I27).

That a system so highly developed and so extensive in its distribution as that of the lateral line organs should have wholly disappeared in terrestrial vertebrates, together with its nerves, is a phenomenon of so unusual a nature that numerous attempts have been made by morphologists to find its direct continuation among the parts of higher vertebrates. Thus, one well-known theory associates these organs with the taste-buds, a view arising naturally from the extreme similarity between the two structures. There seem, however, to be no definite data to form the logical steps between the two, and the fact that the nerve supply to the taste-buds comes from the Glossopharyngeus and not from any part of the extensive sensory system associated with the lateral line organs speaks strongly against this homology. As an added evidence in the same direction there are found in the nasal mucous membrane of many fishes and of certain of the lowest urodeles (Siren) groups of cells forming "smell-buds," extremely similar to taste-buds, and yet by no possibility connected with the lateral line system.

A second possible survival of the lateral line organs is seen by some in the hair of mammals. This theory is based upon a certain similarity in the early stages of development of the two structures, that is, the initial procedure in both 
cases concerns the epidermis alone and consists of a concentric arrangement of a small group of cells. If this homology be a true one we must also consider the amphibians as the direct ancestors of mammals, since the lateral line organs do not occur in reptiles. In this comparison the original sense-organ is, of course, the equivalent of the convex hair papilla, which lies at the root, covering the corium papilla, and from which proliferate the cornified cells of the hair shaft. Something analogous to this exists in the so-called "pearl-organs," horny bodies which develop from certain of the lateral line organs in some fishes, showing that there is present in these organs a tendency to produce cornified structures.

Again, the sensitiveness of the hair root, and its abundant nerve supply, especially in cases like that of the vibrissa (whiskers) of the upper lip in many mammals, speaks in favor of such a derivation. The great multiplicity of the hairs, considering that each represents an original sense-organ, and also their almost universal distribution, is paralleled by the adaptive multiplication of other parts, such as the mammæ in some forms or the vertebræ in elongated animals. On the other hand, the test of nerve supply fails to even suggest this hypothesis, since in the earliest terrestrial vertebrates the extensive system of superficial nerves associated with the lateral line organs becomes entirely lost (unless the Acusticus may be looked upon as derived from it) ; furthermore, the close association between hairs, scales, and integumental glands turns the argument in a totally different direction. (Cf. Chap. IV.)

The third possible derivative of the lateral line system, the inner ear, does not come into the same category as the tastebuds and the hair, since if it came from this system at all, it must have separated from it very early, and thus could not in any case be considered a survival of the system as it exists in fishes and amphibians. This theory receives its strongest support from the developmental origin of the Eighth nerve, which has been clearly proven to segment off from that part of the Seventh which supplies the lateral line organs, certainly a strong argument, since, if the Eighth nerve were once an ele- 
ment of this system, the part to which it is distributed must have been so also. A suggestive comparison has also been made between the semicircular canals of the inner ear, each with its own ampulla, and the canals of Lorenzini, the resemblance between which is apparent. The actual value of this comparison is somewhat questionable, and the theory itself, although far better supported by the facts than are either of the others, is seriously opposed by the actual developmental history of the labyrinth; which arises as a vesicle invaginated from the exterior long previous to and not associated with the lateral line organs. The otic vesicle seems rather to form one of a series of very early organs, to which belong also the lens of the eye and possibly the nasal sacs, as well as a few transitory structures associated with other cranial nerves and usually interpreted as lost sense-organs of unknown function. In our present state of knowledge it seems a surer course to believe that the entire lateral line system of the Ichthyopsida, the function of which is in some way associated with an aquatic habitat, disappears completely where the assumption of a terrestrial life renders it no longer necessary.

In connection with the description of the general tactile sense, that of the so-called " free nerve endings," certain more specialized forms of nerve termini were referred to, which in our present lack of precise knowledge are classed under the general head of organs of touch. The most elementary of these are the tactile cells ( Fig. I28, b), which are scarcely more than isolated units of the general type, somewhat more specialized and thus rendered conspicuous. They are first seen in tailless amphibians, where they are associated in groups, forming small areas known as tactile spots. In other cases the ending has a tendency to form a bulb or sphere, composed of many cells, and often of appreciable size; these are termed collectively tactile corpuscles, each different type being designated by the name of the investigator who first made an accurate description of it. These tactile corpuscles show various types of structure and make use of very different mechanical principles. Thus, in Meissner's corpuscles (Fig. I28, c) the terminal cells form 
an oval core, upon which the nerve fibers are wound in an irregular branching spiral. Certain types, on the other hand, seem to possess no epidermal elements, as in Krause's corpuscles (Fig. I28, d), which consist of a globular snarl of nerve fibers like a capillary glomerulus, enclosed within a thin covering of connective tissue. In still another type, shown by Grandry's corpuscles (Fig. I28, e), the nerve terminus is enclosed between two large epidermal cells which seem to pro-
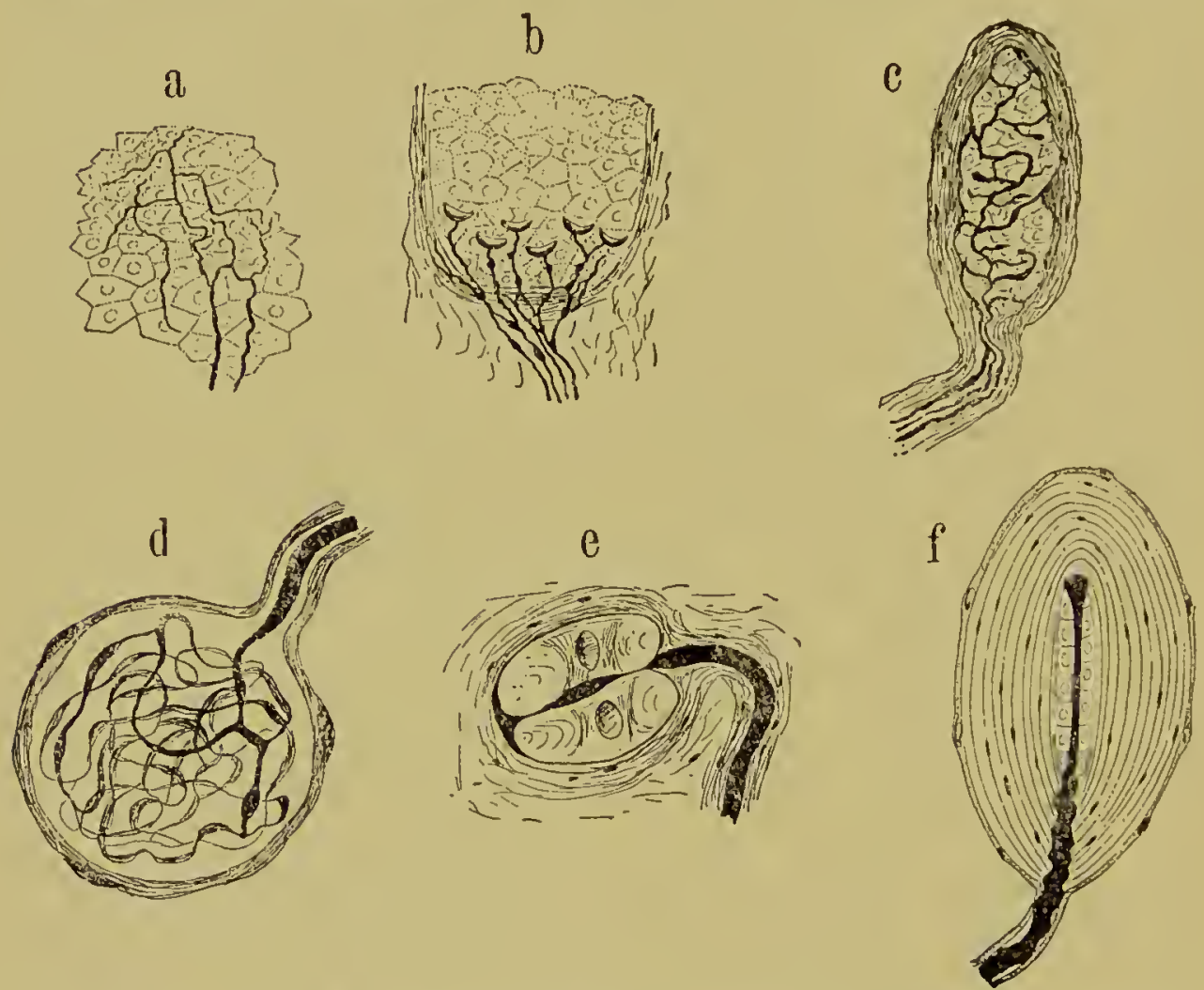

FIG. I28. Various endings of sensory nerves.

(a) Free nerve ending. (b) Merkel's corpuscles. (c) Meissner's corpuscie. (d) Krause's corpuscle: (e) Grandry's corpuscle. (f) Pacini's corpuscle.

duce the stimulus by transmitting any pressure to which they are subjected. This last principle is employed in a more elaborate manner in Pacini's corpuscles, perhaps the largest and most complex of the series, where the nerve terminus is enclosed first by a layer of epithelial cells, then by a series of consecutive lamellæ of connective tissue, something like the coats of an onion, and finally by an external connective tissue wrapping, the continuation of the nerve sheath or neurilemma. 
All of the above types of tactile corpuscles occur in man and other mammals with the exception of Grandry's corpuscles, which are found only in the beaks of various birds. They do not seem to occur over the general hair-covered surface, but are found, often in association with one another, upon such modified hairless surfaces as the palms and soles, the tips of the digits, the lips, the nipples, the external genitals, and, in many mammals, on the end of the nose or snout. The Pacinian corpuscles also occur in such various internal organs as the pancreas, the submandibular gland, and, in the cat, even in the mesentery. This internal distribution has led to doubt concerning the function of these corpuscles as tactile organs, but, on the other hand, their profuse occurrence and large size in such places as the balls of fingers and toes can be accounted for in no other way.

The sense of taste, which in popular estimation is raised to the value of one of the special senses, is, all things considered, but little more than a tactile sensation, and the organs in which it is located are but little differentiated from certain of the foregoing. The organs of taste themselves, cut off from all association with the sense of smell, are restricted in function to the perception of certain elementary qualities of liquid substances, as sweet, sour, bitter, and salt, qualities which are mechanical or chemical in their action and, as such, can be also perceived and, in part, distinguished, by the general tactile sense. To prove this last it is only necessary to bring some acid or astringent liquid in contact with a surface from which the external layer of the epidermis has been removed.

The ultimate organs of the sense of taste, the taste-buds or taste-beakers, are found, from the amphibians on, only within the cavity of the mouth, especially upon the tongue and palate; but in fishes they are far more general in their distribution, and have been found in some species (e.g., bull-heads) scattered over the skin of the external surface. Such fishes are thus probably enabled to taste the water through which they pass. A taste-bud consists of a group of long, spindle-shaped cells, surrounded by a rampart of supporting cells of shape 
similar to the others, but longer, and thus greatly resemble the terminal organs of the lateral line system. At their free ends the sensory cells usually possess one or more modified flagella, which project into a small space, that is formed about them by the supporting cells, and communicates with the exterior through a small opening. In mammals the taste-buds are associated together in groups in connection with several sorts of papilla, especially the circumvallate, and the foliate, the latter not occurring in Man.

The sense of smell is located in a pair of ectodermic cavities, situated anterior to the eyes, thus forming the most anterior of the sense organs. They are thus in the most favored position for organs of sense, and although the data are too insufficient for theories, this fact suggests that they were the earliest to develop and that the primæval habitat was either in mud or in the deep sea where the olfactory sense was of primary importance. Amphioxus gives no clew to this, for here the sense of smell is located in a median ciliated pit at the anterior end and pushed a little to the left side by the development of the median fin. The early stages of the cyclostomes furnish much material for speculation, but unfortunately there is no certainty felt as yet concerning the meaning of the details presented. Here (Fig. I29) there appear at the anterior end two median invaginations, the more posterior of which is the cavity of the mouth (stomatodcum). The anterior depression is that of a median nasal cavity, which would suggest a primitive condition and possibly a kinship with the ciliated pit of Amphioxus were it not for the fact that it is supplied by two olfactory nerves from as many olfactory lobes, showing that the single or monorrhine condition has here been secondarily attained from a previous paired (amphirrine) one. From the posterior wall of this depression there develops a tubular process, which, in Myxine, connects ultimately with the pharynx and thus forms a direct communication between nose and throat, but in the other cyclostomes ends blindly and soon disappears. This passage is of interest as a prophecy of the similar connection to develop later in air-breathing vertebrates, 
and is of still greater interest for its direct connection with the hypophysis, which develops from it. If in this may be seen the remains of an earlier entrance into the alimentary canal,
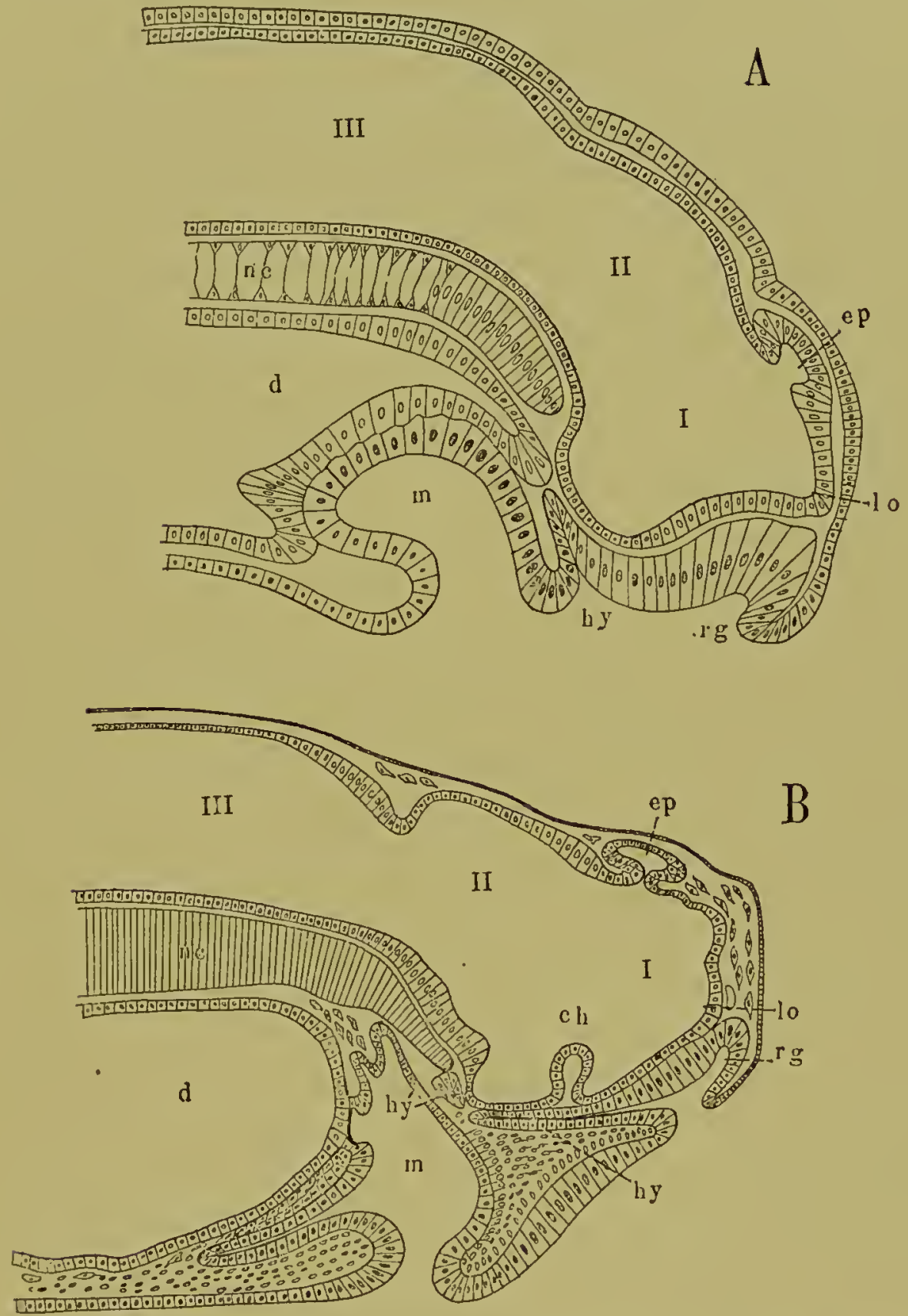

FIG, I29. Median sagittal sections through the head of two stages of the Ammocates embryo of Pctromyzon. [After von KupfFer.]

$I, I I, I I I$, the three primary cerebral vesicles; $d$, intestine (mesodxum); $m$, mouth cavity (stomatodreum); nc, notochord; $e p$, epiphysis; hy, hypophysis; $h y^{\prime}$, portion detached from distal cnd of hypophysis; $\mathrm{rg}$, olfactory pit; 10 , median olfactory lobc; $c h$, optic chiasma. 
then its associated mouth (palcostoma) must have been the later nasal cavity, and its unpaired condition in cyclostomes, in spite of the contradictory testimony of the olfactory nerves, would then be primitive and not secondary.

In all other vertebrates the nose is strictly amphirrhine, that is, it consists of two lateral cavities, symmetrically placed. These cavities begin as localized thickenings of the ectoderm, which invaginate and form nasal sacs, a condition that persists in fishes. In the Dipnoi, the first air-breathers, these sacs become prolonged posteriorly and break through into the mouth cavity, forming the choance [posterior nares], formations which are present also in amphibians and all higher vertebrates. When these are present, the lower part of the cavity is used more or less exclusively for respiration, and the olfactory sense becomes limited to the more dorsal portion, thus dividing the cavity into a pars respiratoria and a pars olfactoria.

Within possible limits the greatest diversity exists in the location of the nasal cavities, and especially their openings, the anterior and posterior nares. The former may be placed ventrally, as in dog-fish, and may occupy all intermediate positions to an extreme dorsal one. Marked adaptations in this respect are seen in those air-breathers which have become secondarily aquatic, enabling them to breath at the top of the water without being seen. Thus in the whales and porpoises the nostrils seem to be moved to the top of the head, the deception being due to very short frontal and nasal bones and to an excessive anterior prolongation of the maxillaries and premaxillaries. In some birds, like the albatrosses and petrels, the nostrils become prolonged into tubes formed by the beak, so that they open near the tip of that organ instead of at its base. An extreme case is seen in the Dipnoi, in adaptation to their annual hibernation within a cocoon of dry clay; for in these animals the openings of the anterior nares lie within the mouth cavity, and the mouth is connected with the exterior during hibernation by means of a long tube composed of slime secreted by the animal.

As regards the choane, their original position is shown by 
amphibians to be very far forward, a position retained, with some variation, by Sauropsida. In mammals this becomes greatly modified by the formation of the hard palate, which develops from the fusion of two lateral shelves, beginning anteriorly. This shuts off from the mouth cavity its own primary roof, including the openings of the choanæ, and therefore pushes back their communication with the mouth cavity to its posterior limit. A trace of the former communication is retained, however, in many mammals in the form of the naso-palatine canal (Stenson's canal), which opens into the roof of the mouth behind the incisor teeth. A rudiment of this duct occasionally occurs in man, lodged in the incisive canal of the maxillaries.

For greater efficiency the olfactory surface may be increased in three ways: (I) by folding the nasal mucous membrane in an oval or otherwise simple cavity, (2) by complicating the walls of the cavity itself, usually by means of ridges or shelves which may themselves become rolled or variously convoluted, or (3) by the addition of accessory cavities within the adjacent bones. The first of these devices is seen in fishes, where the folds are variously disposed, either transverse or longitudinal. This folding of the mucous surface may become extremely complex and thus furnish an organ of considerable efficiency. The second and third methods reach their highest development in mammals and are best treated separately.

The interior of the mammalian nasal cavity, which is usually very large, is by no means a simple space, but is well filled up by projecting folds, composed of thin lamellæ of bone covered by mucous membrane. These are termed turbinalia and come under three categories, in accordance with their relationships to other parts: (I) a naso-turbinal, (2) several ethmo-turbinals, and (3) a maxillo-turbinal.

The maxillo-turbinal lies ventral and usually anterior to the others, in the pars respiratoria, and in mammals has lost all olfactory function, but is often very complicated and forms a filter or screen to intercept the foreign matter in the air taken in, or to temper it if cold. This is the homologue of the 
single turbinal found in certain groups of reptiles, where its function is wholly olfactory. The bone which forms the framework of this part is usually distinct from the ethmoid, and forms the "inferior turbinated bone" of human anatomy. The name "maxillo-turbinal" is to be preferred, as it better expresses its relationship.

The remaining turbinalia, all of which are olfactory, form a set of parallel projecting ridges, arising from the lateral wall of the cavity and arranged in series from above downvards; the most dorsal of these is borne, at least in part, by the nasal bone and is termed the naso-turbinal; the others are ethmoturbinalia, that is, they arise from the ethmoid. The total number of turbinalia, not counting the maxillo-turbinal, is most usually five, but larger numbers are met with up to eleven, the number found in certain edentates. Besides these primary turbinalia which, although arising from the outer wall, yet nearly reach the inner one, there is often present a variable number of secondary and even tertiary turbinalia filling the spaces left free by the first. These relations are shown in Fig. I30, $A$ and $B$, which represent diagrammatic cross sections of nasal cavities; A, with primary turbinalia alone, and $\mathrm{B}$, with secondary and tertiary ones. The primary ones are termed endoturbinalia from their position, to which all the rest are contrasted as ectoturbinalia. The various possibilities which arise from rolling the edges of the laminæ are also shown; the edge may be rolled inwards ( $B, I)$, or outwards $(B, I V)$, or again there may be two free edges either rolled in different ways ( $B$, III), or in the same way ( $B$, II' and $\left.I^{\prime \prime}\right)$. This latter possibility, when viewed from the free inner surface, appears like two separate turbinalia, but is morphologically a single one.

Such involved forms of turbinalia are the rule rather than the exception among quadrupedal mammals, the greatest degree of complexity being reached by the ungulates (Fig. I 30 , C), rodents and carnivores, a structure which gives them a high degree of power in the sense of smell. These structures become much reduced in the anthropoids; and in Man (Fig. 
I30, D-F), but three ethmo-turbinalia are usually represented, and these are of the simplest character. Of these the two largest, II and III, form the "upper and middle conche" of human anatomy. The first, $I$, is a rudiment, and the last.
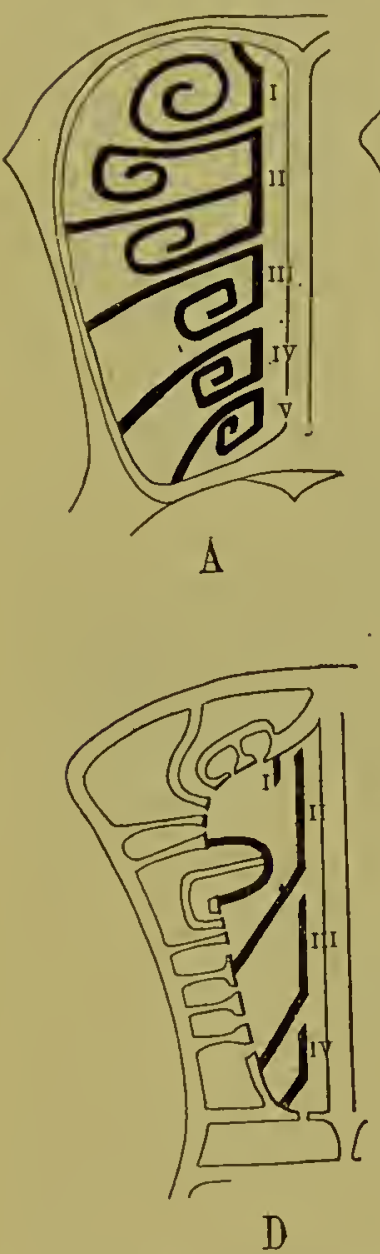

B

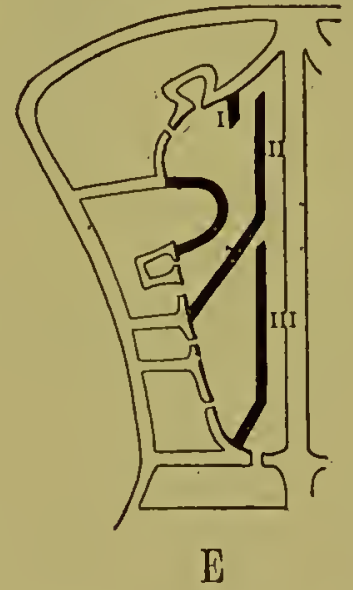

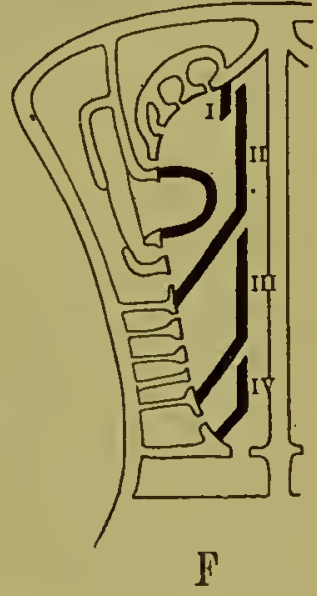

FIG. I30. Diagrams of ethmoturbinals in Mammals. [After PAULLI.]

(A) Type showing endoturbinalia alone. (B) Type with endoturbinalia (heavy lines) and two ranks of ectoturbinalia. (C) Diagram of turbinals and pneumatic cavities in the ox. (D), (E), (F) Diagrams of three actual cases in man, showing individual variation.

IV, is usually absent. The "lower concha" is a distinct bone, the maxillo-turbinal, and is not shown in the diagrams. In the human embryo a larger number of ethmo-turbinals occurs, showing that man's immediate predecessors possessed a much 
more highly developed olfactory sense than appears at present (Fig. I3I).

Still another method for increasing the olfactory surface and thus sharpening the scent seems to be found in the system of accessory cavities, hollowed out in the surrounding bones and communicating with one another and with the primary cavity. These occur in the maxillary, sphenoid and frontal bones, and in animals with the most elaborate nasal equipments are lined with olfactory mucous membrane and may even develop turbinalia themselves. Some of these cavities are retained after the loss of their olfactory function and are lined by simple mucous membrane. The largest of these accessory cavities
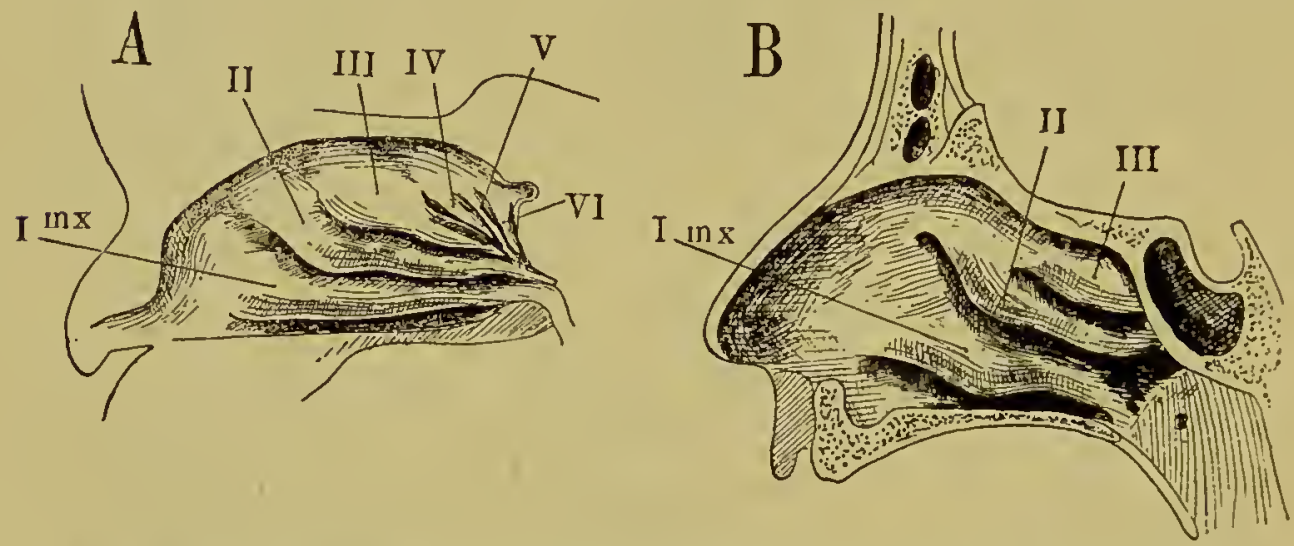

Frg. 131. Lateral wall of human nasal cavity, showing the turbinals.

(A) Embryo, after Killian. (B) Adult, in part after Wiedersheim.

I $\mathrm{mx}$, maxilloturbinal; II-VI, ethmoturbinals.

in Man is the sinus maxillaris [antrum of Highmore] in the maxillary bone; the frontal and sphenoid sinuses also belong to the same system. [Cf. Fig. I3O, C-F.]

This extraordinary development of the organ of smell in mammals is an illustration of the late perfection of a part that has existed as a functional organ for a very long time, yet without the necessity of a high degree of specialization. The need of a turbinal is first felt in reptiles, but here a single one, and that of the simplest pattern, is found to suffice. That the human nose during its own past history once reached a much higher state from which it has since failed through degeneration and loss of the parts once gained is shown by the anlagen 
in the embryo of turbinalia that never develop, and is indicated also by the simple condition of the cavities, and the lack of complexity in the turbinalia.

An interesting bit of morphology in connection with the history of the nose is that of Jacobson's organ (vomero-nasal organ), at first a sinus or pocket leading out of the main nasal cavity, later an independent organ, and finally a rudiment. This organ is first seen in urodeles, where it appears upon the medial side of the nasal cavity and gradually migrates along the floor, attaining ultimately a lateral position, though still included within the nasal capsule. This migration is seen by comparing the lower with the higher members of the order and actually takes place during the embryological development of such a form as Triton, where it may be followed step by step. When its lateral position is fully established, it gradually restricts its communication with the main cavity until it is connected by a small duct in the region of the posterior nares, as in Gymnophiona. In lizards and snakes, where it reaches its highest degree of development, it forms upon each side a tubular, somewhat contorted organ, with a blind anterior end, opening posteriorly into the roof of the mouth by an independent opening, yet still near the posterior nares. Its ventral wall is rolled up into its thickened and strongly convex dorsal one, and this latter possesses olfactory sense-cells. The position of the organ has again changed owing doubtless to the development of related parts, and it lies almost directly beneath the primary nasal cavity, between it and the hard palate, and thus more nearly in its original position near the median line.

In turtles, crocodiles, and birds, Jacobson's organ exists only in the form of embryonic vestiges, but, on the other hand, It is located upon either side of the cartilaginous nasal septum, a definite organ and persisting throughout life in many cases. It is located upon either side of the cartilaginous nasal septum, and is protected by a cartilage of its own, the paraseptal, vomero-nasal, or Jacobson's, cartilage. When well developed the organ is in the form of a short tube, which opens anteriorly 
into the naso-palatine canal, thus retaining its early relationship to the primitive choanæ, but in anthropoids and some other mammals, it is quite vestigial and appears only in embryonic life. In the monotremes it reaches the highest development attained among mammals, and is here entirely encased in its cartilage, through which passes a small branch of the olfactory nerve to supply the organ. From the lateral wall of the cartilage a turbinal process develops, similar to the turbinalia of the main cavity, but very simple in form and covered with indifferent non-olfactory epithelium. Remains of this process are seen in marsupials and even in rodents, forms in which the entire organ is well developed.

If we except such special adaptations as the tubular prolongations of the nostrils which exist in certain fishes and a few aquatic birds, an cxtcrnal nose as a separate organ is a mammalian characteristic. It possesses a cartilayinous framework derived from the primordial skeleton and thus, in part, homologous with the cartilaginous capsule of amphibians, and it is supplied with superficial muscles from the mimetic group. It shows great power of adaptation in the various mammals, sometimes forming a flexible snout or trunk, as in swine, elephants, and moles, and sometimes developing a moist, sensitive surface, as in carnivores and ruminants. In the anthropoids it is reduced in size, corresponding to the lessened importance of the olfactory sense, although its muscles are very mobile and assist greatly in the expression of emotions. These latter powers seem to be undergoing degeneration in Man in spite of the fact that the external nose is more prominent than in most primates.

The ultimate organ of smell is the olfactory membrane, which in fishes and amphibians is distributed over the entire nasal cavity, but which, with the establishment of air-breathing and the setting apart of a respiratory portion, tends to confine itself to the more dorsal region. It consists of a highly differentiated form of epithelium in which occur the terminal olfactory cells surrounded by supporting cells, some or all of which may be ciliated. In certain fishes and in the lower urodeles 
there occur in the nasal mucous membrane definite groupings of the olfactory cells, surrounded-by protecting cells, thus forming olfactory buds, almost identical in form with the tastebuds, which in turn resemble the lateral line organs. Some have seen in this, as well as. in the condition of the primary olfactory pits, which, in the embryo, form the anlage of the nasal cavities, a possible genetic connection with the lateral line organs, but this homology is rendered improbable from other reasons. Of these the most fundamental is the singular relationship between the individual olfactory cells and their nerve fibers, the two being directly continuous, and not, as in all other known cases of sensory cells among vertebrates, simply in contact with one another. This continuity of fiber with terminal cells is, however, characteristic of the sensory cells of the lower invertebrates and suggests that the sense of smell, or at least the primary olfactory membrane, has been inherited from some far-away invertebrate ancestor, and is thus much older than any of the other sense organs. Another possible relationship with certain other parts will be taken up below in connection with the lens of the eye.

The essential organ of hearing is the labyrinth or inner ear, a series of membranous tubes or sacs, the complicated structure of which has suggested its name. In fishes it is placed immediately beneath the bones of the head and in its comparatively superficial position needs no accessory apparatus, but in the higher vertebrates it is located deep in the interior and associates with itself a number of auxiliary parts to aid in the collection and transmission of sound vibrations. Probably the chief reason for this difference lies in the change from water to air, since the denser medium transmits the sound waves with so much more intensity than does the air that the apparatus which develops in adaptation to the former requires an intensifying mechanism when placed in the latter.

The anlage of the labyrinth appears in the early embryo as a slight thickening of the ectoderm over a small lateral a rea at about the level of the metencephalon. As the cells of this area proliferate more rapidly than those of the surrounding 
ectoderm, they gradually fold in and form a deep pit, which, as the process continues, pushes further into the interior, where it expands into an otic vesicle, retaining its connection with the exterior, however, through a narrow tube, the ductus endolymphaticus. In selachians this connection is retained throughout life, and a minute but evident external pore is found near the top of the head which communicates through a small duct with the interior of the labyrinth; but in all other cases the connection with the exterior becomes severed and the endolymphatic
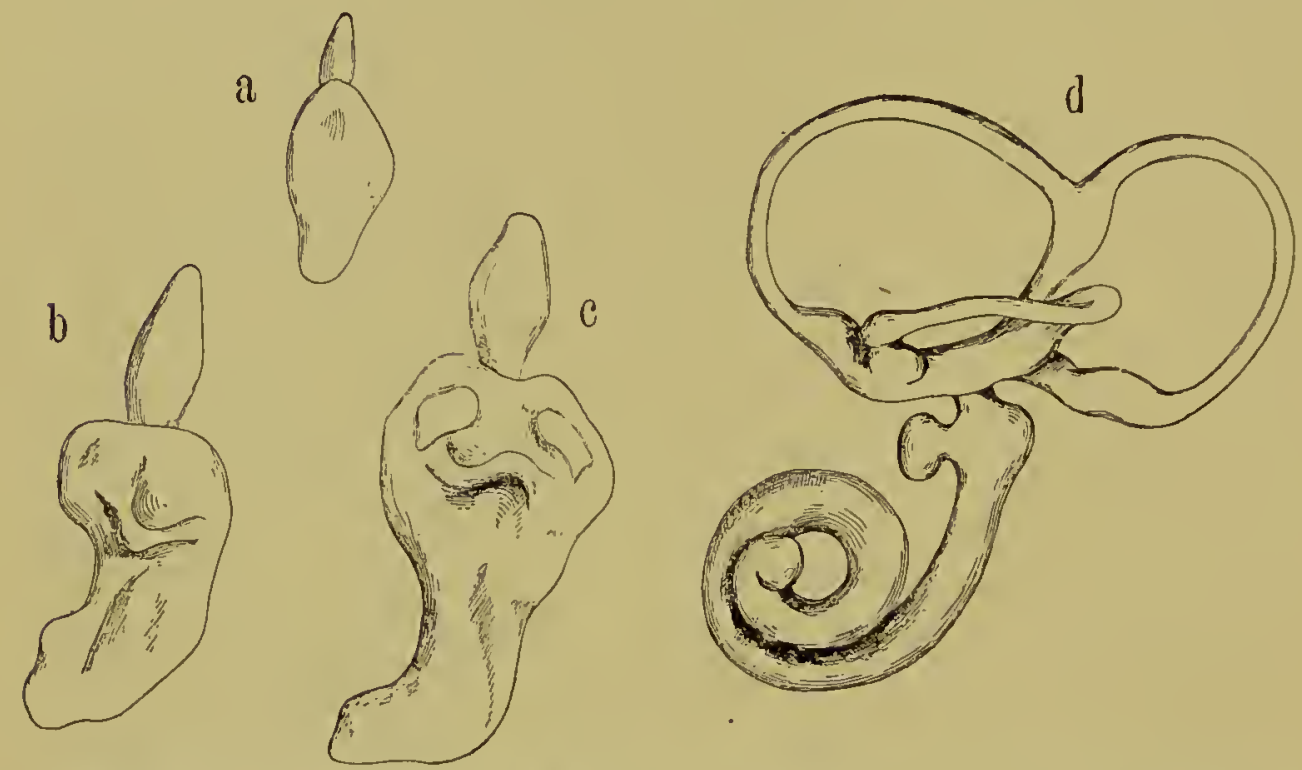

FIG. 132. Development of human otic capsule. Drawn from models by F. ZIEGLER, after WM. HIS.

duct ends in a somewhat expanded blind sac, the saccus endolymphaticus. In mammals the endolymphatic duct is lodged in a canal of the petrosal bone, the aqueductus vestibuli, and enters the cranial cavity, where its terminal sac lies just beneath (i.e., outside of) the dura mater.*

* In some cases the ductus endolymphaticus and its terminal sac attain a high degree of development and come into association with organs remote from its place of origin. Thus in certain teleosts the two ducts unite into a median sinus which is connected with the air bladder by a chain of four ossicles (Weber's apparatus), developed from the ribs of the first four vertebræ. By this means the degree of fullness of the air. bladder may be perceived.

In the Anura the endolymphatic ducts form a common sinus, which 
The otic vesicle itself develops, mainly by the unequal growth of the different regions, into the labyrinth. This develops first into two expanded portions, utriculus and sacculus, with a restricted portion between them, the utriculo-saccular canal. [Cf. Figs. I33 and I34.] From the utriculus, which lies dorsally, develop three flattened folds, which by the adhesion and subsequent atrophy of the middle portion of their walls, develop their marginal portions into tubes. Thus are formed the threc scmicircular canals, which are constant in all vertebrates above the cyclostomes, and vary but little in general appearance or relationships. They are set approximately at right angles to one another in such a way that one lies horizontally and the other two vertically, but at an angle of about $45^{\circ}$ with the bilateral plane of the body. They empty at either end into the utriculus, one end of each being. expanded into a flask-shaped ampulla. These are so placed that the ampullæ of the anterior vertical and horizontal canals open together into a pocket of the utriculus (recossus utriculi), while that of the posterior vertical canal opens by itself into a similar pocket on the other side (sinus utriculi posterior). The two vertical canals unite at their expanded end and enter or form the sinus utriculi superior, a diverticulum which extends directly upward; the unexpanded end of the horizontal canal enters the main body of the utriculus unassociated.

The parts of the utriculus with its derivations, the semicircular canals, appear first in the form described above in the

extends posteriorly, dorsal to the spinal cord and outside of the dura mater, as far as the tail rudiment (urostyle). This extensive sinus sends to the roots of the spinal nerves a series of diverticula which wrap themselves about the spinal ganglia and expand into sacs containing granules of calcium carbonate. In many reptiles the duct reaches the top of the skull and even escapes, its terminal sac being subcutaneous. In snakes this sac contains calcareous crystals, which in some cases may be seen through the skin of the living animal. This apparatus reaches its highest development, so far as reptiles are concerned, in the lacertilian family of the geckos (Ascalabota), where the sac escapes from the skull through the parieto-occipital suture and pushes its way between the muscles of the neck and shoulder as far as the pharynx. It is filled with a soft calcarcous mass. 
selachians and are retained with very little deviation by all higher vertebrates. The sacculus, however, exists in a simple form in fishes and shows considerable advance in the ligher forms. This advance consists of the gradual development of a lateral sac, the lagena, which is situated upon the inner side and which in fishes and amphibians is barely indicated. In reptiles and birds the lagena becomes considerably elongated and curved, and in mammals it becomes spirally wound and, associating certain outside elements with itself, forms the cochlea, here attaining the highest degree of complexity of any part of the labyrinth. In the higher forms also the direct connection between utriculus and sacculus becomes replaced by an indirect one through the ductus endolymphaticus, which arises by two branches, one from each of the two parts [ $\mathrm{C} f$. Fig. I34]. These unite and thus indirectly retain the connection in question.

The labyrinth of the cyclostomes stops at a lower point of development than is represented by any of the gnathostomes and may well represent the permanence of what is, in the higher forms, an early embryonic stage. It consists of a simple oval sac, not yet differentiated into utriculus and sacculus, and possessed of either one (Myxine) or two (Petromyzon) semicircular canals. Its endolymphatic duct, however, is short and - loses its connection with the exterior (Fig. I33,a).

The walls of the embryonic labyrinth are composed of a single layer of epithelial cells of appreciable thickness and all alike; as development proceeds, however, the greater part of the cells become flattened and form a transparent membrane, while over certain definite areas, 6 to 8 in all, the cells are thickened and columnar, forming neuro-epithelium. Certain of these cells form the ultimate organs of hearing and are provided with various sorts of terminal flagella and other similar structures (auditory hairs), which project into the lumen of the labyrinth and are bathed in the endolymph, i.e., the fluid filling the interior. These are supplied with nerve fibers from the auditory nerve. About these are placed various sorts of supporting cells, which are without auditory function and 
serve to hold and protect the others. These spots, which thus show a higher degree of cellular differentiation, are the seat of the auditory sense, and appear from their greater thickness

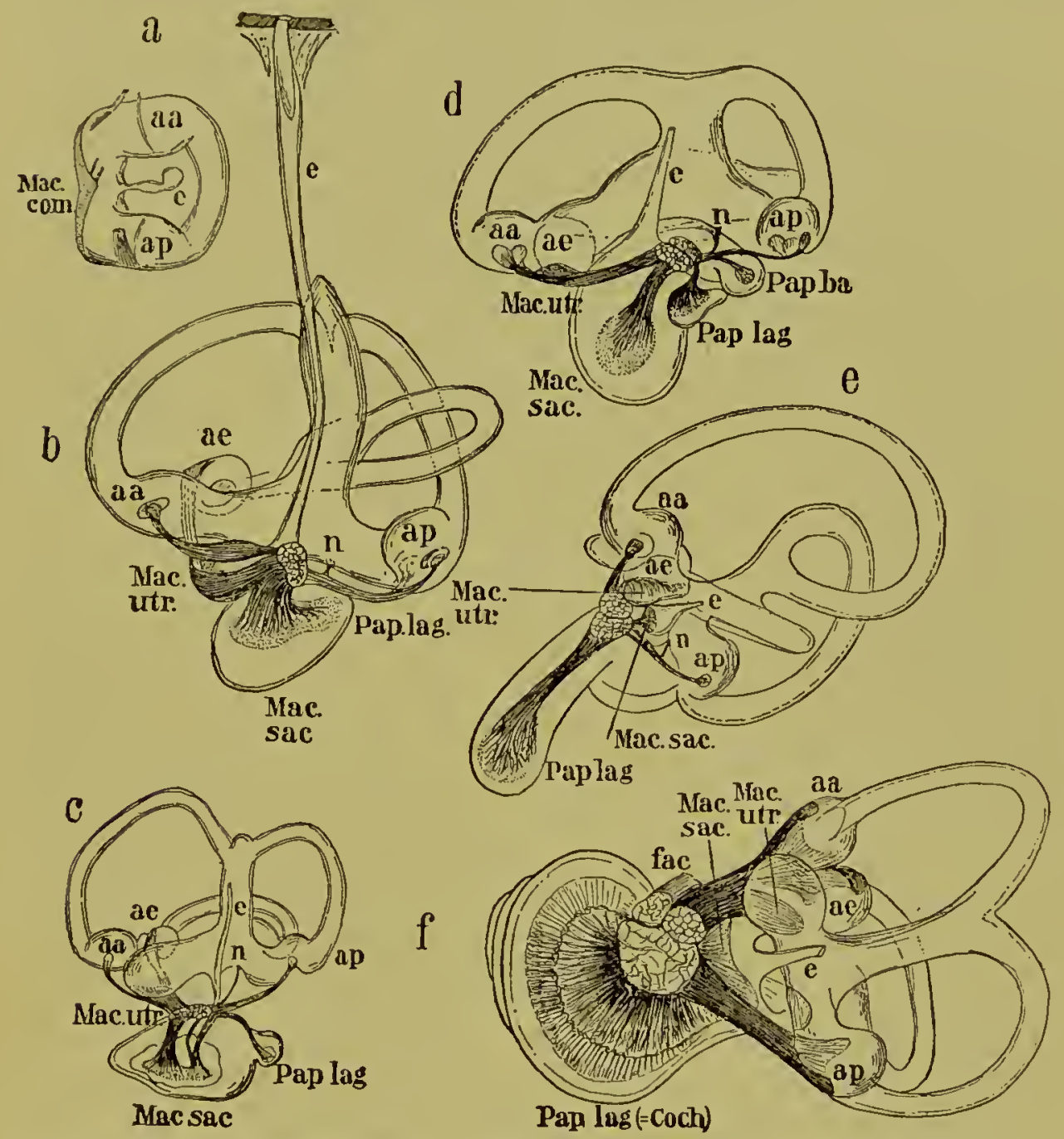

Fig. I33. Ear labyrinths of various Vertebrates. [After RETzius.]

(a) Cyclostome; Myxine glutinosa (hag fish). (b) Selachian; Chimaera monstrosa, (c) Teleost; Anarrlichas lupus (wolf-fish). (d) Amphibian; Rana esculenta (frog). (e) Bird; Bubo ignavus (horned owl). (f) Mammal; Sus scrofa domestica (pig). $a a$, ampulla anterior; $a c$, ampulla extcrna; $a p$, ampulla posterior; $e$, ductus endolymphaticus; $f a c$, facial nerve; mac, macula communis; mac. utr, macula utriculi; mac. sac, macula sacculi; $n$, macula neglecta; pap. lag, papilla lagenx; pap. bas, papilla basilaris.

as white patches in the otherwise transparent wall. They are further indicated by their association with the nerve.

The largest occurring number of such auditory areas is eight, although all do not occur simultaneously. Of these the three which are associated with the semicircular canals are some- 
what different from the rest and are absolutely constant. They are situated in the ampullæ and are in the form of ridges which encircle them and project into the lumen. They are thus ristinguished from the others as cristce acustica. The remaining five are evidently formed by the successive breaking up of a single large area, due to the differentiation of parts of the labyrinth, and the history of this segmentation and later migration is represented in the phylogenetic series (Fig. I33). Thus, in the cyclostomes the acoustic region, aside from the one or two semicircular canals, is represented by a single area, macula acustica communis, covering the bottom of what is here a simple sac (Fig. I33,a). The differentiation of utriculus and sacculus gives a separate area to each, respectively, the macula. acustica recessus utriculi and macula acustica sacculi (Fig. I33,b). With the gradual development of the lagena in fishes there appears an outgrowth of this latter area which finally separates from its place of origin and establishes itself as the auditory area for the newly developed part, under the name of papilla acustica lagenc. This gradual separation of both lagena and its acoustic area is accompanied by a similar separation of the nerve, which splits off a supply branch for the new area. (Fig. I33, cf. $b$ and c.) A small macula is also formed near the utricular macula, the macula acustica neglecta, evidently an offshoot from the latter, although no phylogenetic proof of this appears as in the former case. The fifth and last of the auditory areas, not counting the cristæe acusticæ of the ampullæ, the papilla acustica basilaris, also belongs in the lagenar region, and appears in the higher urodeles as an offshoot of the papilla lagenæ (Fig. I33, d).

To put these points into the form of a phylogenetic history we may take as a starting point the macula communis of cyclostomes, which may be considered to hold all the later elements within itself. In fishes we find a primary macula for each of the two parts into which the labyrinth has become divided, also a macula neglecta, which has presumably separated from the one belonging to the utriculus at some point below the fishes. Within the group of the selachians the 
papilla lagena appears, sometimes as a separate area, sometimes as a lobe of the macula sacculi, thus giving four acoustic macula for most fish. In amphibians the papilla basilaris appears while the others are retained, although there is a slight transposition of the macula neglecta. This condition, with five acoustic areas, is retained by the Sauropsida with some variation, such as the division of the papilla basilaris in certain lizards and a great reduction of the macula sacculi in turtles, points referable to special adaptation and of no general significance. In mammals there are important changes. The macula neglecta has entirely disappeared and the papilla lagena is found only in Ornithorhynchus (monotreme), leaving in this Class but three acoustic areas aside from the three cristæ acustica of the semicircular canals.

The most important, difference in the mammalian labyrinth is the great development of the lagena. The tendency, already shown in crocodiles and birds, to prolong this part and to curve its axis, results here in an excessive elongation which becomes wound into a close spiral, the nerve forming the central axis. The number of complete coils in man is about 3 , but varies among mammals between the limits of $\mathrm{r} \frac{1}{2}$ and $5 .{ }^{*}$ This coiled lagena becomes complicated by the addition of parts of the outer bony labyrinth, to be explained later, which form two additional coiled passages, scala vestibuli and scali tympani, that receive between them the lagena under the anatomical name of ductus cochlearis [scala media]. This entire organ, including both this part of the labyrinth and its accessory organs, is called the cochlea.

The papilla basilaris lies along the floor of the coiled lagena (scala media) and becomes highly differentiated into a number

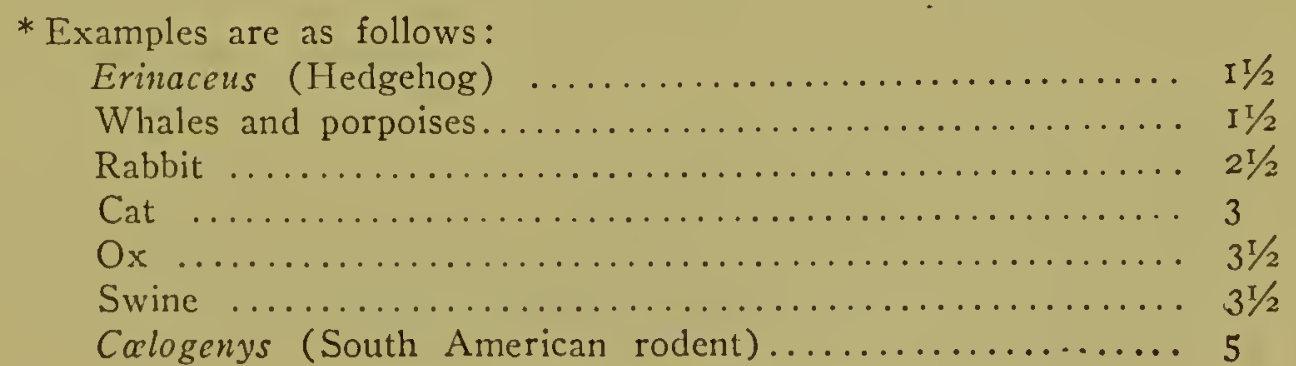


of kinds of histological elements, which change their proportionate size along the course, being the smallest at the base and the largest at the apex. The most important of these cellular elements are certain elongated cells associated in pairs, the rods of Corti, and two groups of cells with specialized terminal organs, the outer and inner hair cells. The ventral portion of the lagena, that is, the floor of the scala media beyond the auditory area, is termed the basilar membrane, and the dorsal portion (roof) is Reissner's membrane; these terms are, however, purely anatomical ones. expressing certain relationships

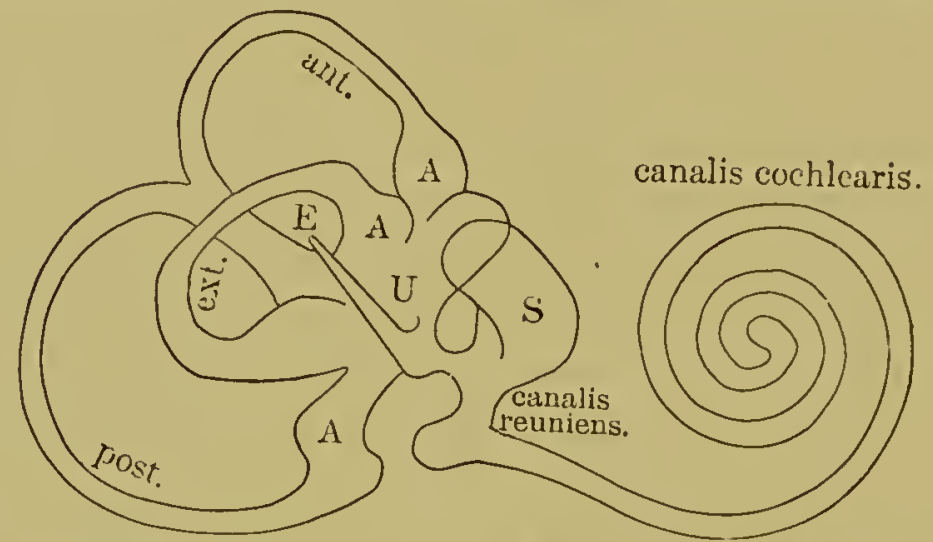

FIG. I34. Diagram of membranous labyrinth of human ear. [From Gegenbaur, after RetziUs.]

$A, A, A$, ampullae; $U$, utriculus; $S$, sacculus; $E$, ductus endolymphaticus; ant, ext, post, the three semicircular canals.

to the surrounding parts and are without morphological significance.

The membranous labyrinth as above described, that is, the higher development of the auditory vesicle of the embryo, becomes surrounded while still embryonic by a gelatinous connective tissue, its first accessory organ. Later on in development this tissue becomes converted either to cartilage or bone, leaving, however, a nearly uniform layer of the original tissue between it and the membrane. There is thus formed a mold which reproduces the membranous labyrinth in its details, the bony (or cartilaginous) labyrinth. The gelatinous tissue becomes soon converted into a serous fluid, called the perilymph, in distinction from the endolymph of the interior, and the cavities involved are conveniently distinguished as the porilym- 
phatic and cndolymphatic cavitics respectively. The membranous labyrinth is held in place by scattered strings of the original connective tissue, which connects it with the bony wall, aside from which the two come into close contact at the places of entrance of the various branches of the auditory nerve. In the lower vertebrates the outer labyrinth remains as a mold imbedded in the petrosal bone, but in higher forms, especially in mammals, the bony labyrinth appears over certain regions, especially the semicircular canals and the lagena, as a thin but very hard wall, with a space between its outer surface and the main mass, thus reproducing from without also, the main details of the membranous labyrinth.

The transition from water to air, undoubtedly the greatest change which vertebrates have ever experienced, and one which demanded modifications affecting every part, affected the organ of hearing directly, for a change was made from a denser medium, which readily transmitted sound vibration, to a lighter one in which transmission was more difficult. This disadvantage was undoubtedly felt by the urodeles, which exhibit a new organ, evidently destined to assist in the reception of less powerful vibrations. In the cartilaginous otic capsule surrounding the labyrinth, that which partly corresponds to the "bony labyrinth" of higher forms, there exists an oval opening with a reinforced rim, closed by an ossicle in the form of a lid, usually with a process projecting from its center [cf. Fig. 39, $o p]$. The opening, which persists in all higher vertebrates, is the fenestra ovalis, and the osseous lid, which is, in origin, a portion cut off from the wall of the capsule, is the operculum. This latter is fitted to the rim of the fenestra ovalis by a membrane, and, as it is nearly subcutaneous, it is set in motion by the impact of sound waves, and thus serves to slightly intensify the vibrations.

This apparatus proves sufficient for urodeles, which are much in the water, but in the tailless forms (Anura), far more terrestrial than the salamanders, the sound-receiving apparatus is much improved by an important addition, the tympanum, or cavity of the middle ear. This is developed from the gill 
pouch of the spiracular opening, the one associated, as will be remembered, with the hyoid arch. The inner portion of this cavity communicates with the pharynx and forms the auditory or Eustachian tube, but direct communication with the outside is prevented by the presence of a circular tympanic membrane at the outer end, just beneath the skin, and usually very evident from the outside.

This membrane, which is covered outwardly by integument and on its inner side by mucous membrane, is a separate formation, usually of connective tissue, but in a few cases it is cartilaginous. It has been doubtfully homologized with the spiracular cartilage of selachians, but this is too uncertain to be definitely asserted. In many cases there exists a second opening in the wall of the otic capsule, the fenestra cochlece [rotunda], filled with a thin membrane, also termed the inner tympanic membrane. This part is present in ali higher vertebrates, thus giving the tympanum the characteristic from which it derives its name, i.e., two drum heads, outer and inner. To complete the likeness of the middle ear to a drum the Eustachian tube represents the opening always present in the cylinder of a drum and employed in both purposes for equalizing the air pressure on either side of the drum heads.

A mechanism, however, which is lacking in a drum, is that formed by the columella, a delicate spindle of bone or cartilage, which extends from the center of the outer tympanic membrane to the operculum. By this means the sound vibrations that impinge upon the former are transmitted directly to the latter, and through it to the perilymph within the otic capsule. Another channel for the transmission of sound waves is furnished by the air enclosed in the tympanic cavity, the vibrations striking the inner tympanic membrane. The apparently new skeletal element, the columella, is probably nothing more than a process of the operculum, but it has been considered by some to be a distinct element and to represent the hyo-mandibular of fishes, employed there as a suspensory piece for the mandible and originally the dorsal segment of the second risceral $\operatorname{arch}$ (hyoid). 
In the Sauropsida there is but little change in the tympanic cavity from that of the Anura. The two Eustachian tubes often form by their union a median duct, which opens into the pharynx in the mid-dorsal line. Such is the case in birds and in crocodiles, and in the latter the tubes under consideration form a complicated system of cavities, many of which are lodged in the bones of the cranium. In other cases similar systems extend out from the main tympanic cavity, and in certain instances the two latter communicate with one another across the median line.

A characteristic and important addition is gained in mammals by the appearance within the tympanic cavity of the articular and quadrate bones, hitherto employed in forming the mandibular articulation. These form respectively the malleus and incus, and become added to the columella to form a chain of ossicles which reaches across the cavity from the other drum head to the fenestra ovalis, thus assuming the function formerly sustained by the columella alone. This latter apparently becomes reduced in size and forms the stapes. (Cf. Chap. V.) The singular and characteristic foramen in this bone, to which it orves its similarity to a stirrup, is caused by the development of a small artery, which perforates the columella. This relation appears only in the embryo in most mammals, including Man, but in some (certain rodents and insectivores) it persists throughout life. (Cf. Chap. IX.) In others still, mainly monotremes and marsupials, the perforation does not take place, but the bone remains in the primitive cylindrical form.

The stapes is supplied by a tiny muscle, the stapedius, which is shown by its embryology to be a slip separated from the digastric muscle, an element primarily associated with the hyoid arch. To the malleus is attached a second small muscle, the tensor tympani. This was originally a portion of the common mass from which the masticatory muscles of the jaw have differentiated, the abductor mandibuli of the selachians. This little slip arises from that portion which forms the pterygoid muscles, and is innerved by a branch from Trigeminus, the nerve associated with the first or mandibular arch. These two 
tympanic muscles have thus had a history as old as the parts to which they are attached, and form here, together with their associated nerves and ossicles, groups of parts which have retained their original relationships through all their migrations and changes of form and function.

Another characteristic mammalian element, not directly within the tympanic cavity but closely associated with it, is the tympanic bone (os tympanicum). This, when in its full development, forms a complete bony ring or frame about the outer tympanic membrane, and often develops in addition a concave osseous shell or tympanic bulla, which forms a conspicuous object at the base of the skull and aids in protecting the delicate parts of the middle ear. Occasionally, too, the bone extends outwards to form an osseous wall for the external meatus. This bone remains distinct throughout life in monotremes, marsupials and a few others, but in the majority of cases, as in Man, it fuses with the petrous elements and becomes eventually lost in the complex designated as the "temporal bone." The homologies of this bone are uncertain, although some consider it the same as sauropsidan quadrato-jugal. It can hardly be a new osseous element, but that it appears here in a new rôle and is thus a new bone physiologically is evident.

The external ear, characteristic of the mammalia, is mainly a cartilaginous structure covered by integument, and consists of a round tube, the external auditory meatus, and an external flap, the auricula [pinna]. The first of these, the meatus, allows the outer tympanic membrane to sink below the surface and still retain connection with the exterior and its curve affords the membrane a more or less complete concealment. In cases where the tympanic bone furnishes a prolonged tube for this purpose, the external cartilaginous element is less extensive, and the two together form the wall of the canal.

The auricula shows a large degree of adaptation, being very large and mobile in cases where acute hearing is desired, for example, in bats, and in most ungulates; and is reduced or entirely wanting in many burrowing or aquatic forms. The characteristic anthropoid car is shaped at the base much as in 
Man, but there is no lobule and little or no recurving to the free edge. This latter peculiarity, which starts at the upper part of the base, is distinctively human, but extends over a varying distance in different individuals, and is often hardly begun in the new-born infant. A rudimentary point, tuberculum auriculi [Darivin], is often retained at the free edge, and is brought ower by the rolling process so as to point forward instcad of backward, its primary position. This is occasionally a conspicuous feature, and in all cases its place can be determined by feeling, being indicated by a thicker, harder area on the outer rim a little below the top of the curve. A lobule is usually present, but is rudimentary or absent in certain races.

Concerning the origin of the cartilaginous elements of the external ear, it becomes evident from the condition found in monotremes that it is largely or wholly derived from the upper end of the hyoid arch, which curves about the tympanic membrane and forms a tubular meatus together with a rudimentary pinna. This leaves unaccounted for a series of protuberances in the integument surrounding the opening of the meatus, which are seen to form in the human embryo and fuse together to build up the external portion (pinna). These protuberances are considered by some to be elements furnished by the first four visceral arches, i.e., mandibular, hyoid and the first two branchial, but this is rendered very improbable by the innervation of the pinna, which is wholly from the Facialis. It may thus prove to be a modification of development, and portions which were originally hyoid elements may here appear in this form. In the Sauropsida the outer tympanic membrane is frequently depressed a little below the surface and provided with small protuberances or flaps which assist in its protection, but these are evidently incidental adaptations and can have nothing to do with the external ear of mammals.

The developmental history of the eye, as given in the previous chapter, shows that this sense-organ, that is, its essential part, the retina, differs radically from all the others in being originally a portion of the brain surface, the cells of which have 
become specialized in form and function so that they respond directly to the stimulus of light vibraiions. To this essential part, which, with a pigmented outer layer, are the only parts derived from the brain, accessory organs are added from two sources to complete the formation of the eyeball; the lens, formed from the ectoderm of the outer surface; the chorioid and sclerotic coats and the vitreous body from the surrounding connective tissue. Aside from the eyeball itself there are many external accessory parts, such as muscles and glands, conjunctiva and eyelids, which come from several sources and aid in the movement and protection of the sensitive organ.

To begin with the essential sense-organ, that is, the retina, if we follow the in- and outpushings of its layer of origin from the beginning, it is clear that the original external surface lines the lumen of the neural tube and eventually forms the outer retinal surface, that is, the surface turned toward the pigmented tapetum. Now in all cases it is the primarily extermal surface that becomes specialized to receive external stimuli, and it is also the original outer or superficial end of the sensory cells that develop the specially modified flagella and other parts. It thus happens that the terminal cells of the sense of vision are not only the outer ones of the retina, which is several celllayers in thickness, but also that their free ends, bearing the terminal rods and cones, point in the same direction, namely, towards the interior of the head and away from the source of light. Moreover, since a sensory nerve must approach its terminal cells from their physiological inner side, this arrangement compels the optic nerve first to penetrate the entire retina and attain the interior of the eyeball, and there spread out its separate fibers, which severally become recurved and pass back again through the retina to supply the terminal cells. Finally, in order that the image, received through the pupil and focused by the lens upon the physiological outer side of the retina, may reach the terminal rods and.cones, all the intervening parts, the nerve fibers and the various layers of retinal cells, have to be perfectly transparent; and, furthemore, the tcrminal rods and cones must needs be buried in the pigment of the tapetum in 
order to stop possible light impressions from coming from without the eycball, i.c., the natural dircction for the receptive cclls.

The necessity of this arrangement becomes clear to anyone who has followed the foldings of the embryonic layers, yet there is scarcely anything in vertebrate construction that seems a greater mechanical mistake, although there are many others, like the appendix and the inguinal canal in man, where a lesser error involves far more serious consequences. This error in the arrangement of the retina, however, becomes still more apparent when a comparison is made with the structurally similar eye of the cephalopod molluscs (squid, devil-fish, etc.), in which the retina is developed directly from the surface ectoderm and is placed in the natural way, with the terminal cells lining its interior and the optic nerve entering it from behind. Notwithstanding the fundamental differences in development between this eye and that of vertebrates, the final results, when compared part by part, are marvelously similar, and the adult eye of each is furnished with retina and crystalline lens, iris and cornea. This case is thus one of the best examples of what Mr. Darwin termed "analogical resemblances"; that is, the production of a similar organ in two unrelated forms and often from entirely different starting points, not through any genetic connection, but because of the same environmental influences, which give rise to the same necessities.

In its histological structure the vertebrate retina shows some similarity to other well-developed portions of the brain, and exhibits several layers of cells, connected with one another by branching processes which interlace and thus continue the communication from one to another. At the exact focal center of the lens all but the terminal sense-cells disappear, and produce a small depressed area, the arca contralis. This is often in the form of a circular pit, forca centralis, but may be oval, or in the form of a broad band or streak. It is, however, not always depressed, and may be entirely wanting. These variations seem to bear little or no relation to phylogeny, since a fovea is present in some fishes and in most Sauropsida, while the area 
seems entirely lacking in many mammals (Insectivora and some rodents). In the anthropoids it is very pronounced, and in man it is designated by a yellow color (hence " macula lutea"). Certain birds possess two such areas, medial and lateral.

To understand the addition of the accessory organs and the formation of the eyeball it is necessary to examine more thoroughly the early stages in the formation of the optic cup. The study of a few actual sections will show that the invagina-

a

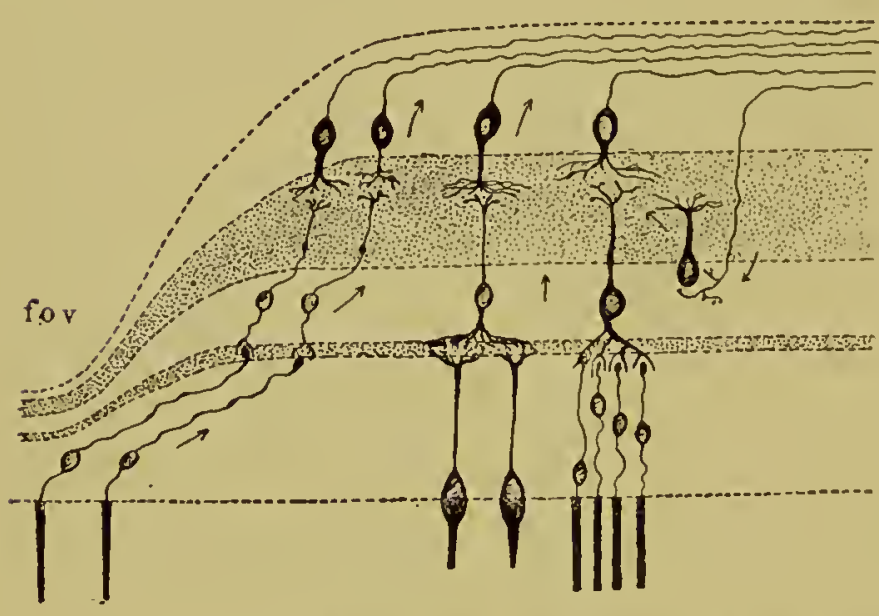

$\mathrm{b}$

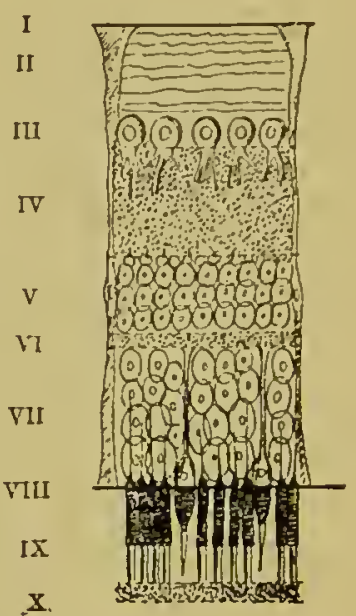

FIG. 135. Diagrams of the retina.

(a) Section including the fovea, showing the separate elements [From GEgenbaUR, after RAMON y CAJAL.] (b) More conventionalized representation of retinal layers. [After GEGENBAUR.]

fov, fovea; $I$, membrana limitans interna; $I I$, nerve fiber layer; $I I I$, nerve cell 1ayer; $I V$, inner granular layer; $V$, inner nuclear layer; $V I$, outer granular layer; $V I I$, outer nuclear layer; VIII, membrana limitans externa; $I X$, rod and cone layer; $X$, tapetum.

tion of the primary outpushing is not a symmetrical one, but is so effected that the cup is deficient for a little space on the ventral aspect, and that this deficiency is continued as a groove along the lower side of the optic stalk. It thus happens that when the lens, which at this time is added to the optic cup, becomes closely applied to its rim, a fissure or oblong aperture, the chorioid fissure, is left, through which communication may be made with the interior of the cup behind the lens. Through this inlet migrate embryonal connective tissue cells (mesen- 
chyma) and form a gelatinous tissue, the basis of the vitreous humor. From similar mesenchymatous elements added externally is formed the vascular network of the chorioid coat, and outside of this is formed the sclera [sclerotic coat]. The anterior portion of the chorioid forms the iris and the corresponding portion of the sclera forms the cornea. This latter stands out from the lens in front and thus forms an anterior chamber, filled with the aqueous humor, a colorless lymph, which serves as a refracting medium. The corresponding chamber of the cephalopod eye is perforated by a foramen communicating with the exterior, and through this it is filled with sea water which serves the same purpose. This expe-
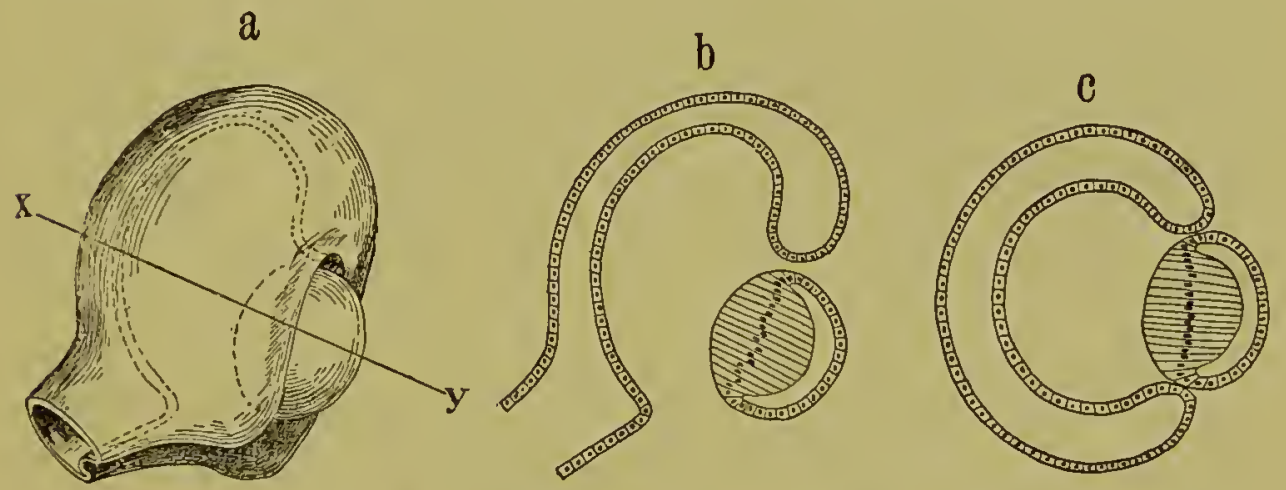

FIG. I36. Development of the optic cup.

(a) Plastic representation. [After Hertwrg.] (b) Median longitudinal section of (a). (c) Cross section in plane indicated by the line $x y$.

dient is comparable with that of the internal ear of selachians, with its direct communication with the exterior through the ductus endolymphaticus.

The crystalline lens, the formation of which has been alluded to elsewhere, is a product of the ectoderm and appears first as a thickening opposite the optic cup. It soon invaginates and pinches off from its layer of origin, at first as a vesicle with a nearly uniform wall and a central lumen. The posterior wall soon thickens and restricts the lumen more and more until this latter becomes entirely suppressed, while the wall itself, becoming lenticular in shape, is covered by the anterior portion as by a cap. This thickening is produced by an extreme elongation of the cells, which remain in the form of a single layer. 
The functional lens is formed by the cornification of these celis, and the mass thus formed is covered anteriorly with a thin epithelium, the original anterior wall of the vesicle.

It will be noticed that there is in this development of the lens a striking similarity with the early stages of both the nose and the ear, and if there be taken in connection with these certain temporarily thickened areas of the external ectoderm in association with the Facialis and the Glosso-pharyngeus nerves, which appear and vanish again during the embryonic life of the lower vertebrates, the idea comes at once to mind that we have here the record of a series of ancient sense-organs laterally placed, perhaps a pair for each metamere, some of which have specialized in various ways while others have become lost. If this be true, the lens was originally, not a refracting medium, but a sense-organ itself, which has given up its primary function entirely and entered the service of another sense-organ, different in origin from that of any other in vertebrate history, namely, a specialization of a portion of brain surface.

The idea of this ancient series of sense-organs suggests many questions. What was the primary function of this series? Did these sense-organs sustain any relation to the lateral line organs? To these questions, belonging themselves to the realm of pure suggestion, we can give but speculative answers. Both the nose and the ear, as we have already seen, have in their structure and development something to suggest a kinship with the lateral line organs; this is especially true of the latter, with its nerve appearing in connection with that element of the facial nerve that supplies these organs in fishes, and with its semicircular canals that resemble the canals of Lorenzini. The eye itself cannot, of course, be included in any series of sense-organs of integumental origin, but the lens can, and there seems no intrinsic difference, up to a certain stage, between the lens capsule and that of the inner ear. The nasal sacs, again, are similar capsules that do not lose their connection with the exterior, and it must be remembered that in the endolymphatic duct of the selachians we see the same retention 
of the original connection. We seem here almost able to reproduce an important bit of lost history, but the proofs are not forthcoming and may always be wanting, since the early phylogenetic stages were probably passed in those lost forms between Amphion ans and the selachians, and concern soft parts, no trace of which is likely to be found in fossil remains.

The absolute size of the eyeball is very variable. In general it is somewhat in proportion to the size of the body, yet the eyeballs of the elephant or the whale, although large in both cases, are not proportionate to their enormous bulk when compared with those of Man, for instance. Again there is a certain proportion between the size of the eyeball and the sharpness of vision, as, for example, the enormous eyes of birds ; but here, again, must be mentioned the small but exceedingly acute eyes of rodents where the decrease of size seems to be due to the excessive development of the masseter muscles, and appears to have no direct influence upon the vision. The eyes are apt to be large in animals with nocturnal vision, like the lemurs, and it is possible that the relatively large eyes of Man, which have encroached upon the nasal cavities, and thus reduced the power of smelling, may be the result of a nocturnal habit in some not very remote ancestors.

Of the organs external to the eyeball which are accessory to the sense of sight the muscles have been treated in a preceding chapter (Chap. VI). There thus remain for consideration only the eyelids and the glands, two sets of structures closely associated with one another. They are both employed in preventing the surface of the eyeball from becoming dry upon exposure to the air, and belong to that series of changes necessitated by the change of environment from water to air. They are consequently found only in the higher vertebrates, and are absent in fishes, and but poorly developed in aquatic urodeles.

In fishes the integument fits smoothly over the region surrounding the eyeball, and is continuous over the latter as a thin skin, usually transparent, but occasionally ornamented in places with pigmented areas, which continue the color scheme of the rest of the skin. The eyclids, which appear first in 
amphibians, form as dorsal and ventral foids of the integument, which may become stiffened, either by connective tissue or by cartilage, as in mammals (tarsal cartilages). That portion of integument which forms the inner face of the folds and is continued over the front of the eyeball is very thin and sensitive, and forms the conjunctiva. A nictitating membrane is formed in some vertebrates by an inner fold of this last; it attains in birds and in some mammals the dignity of a third eyelid; in Man it is represented by the plica semilunaris, a delicate fold situated in the inner corner.

The lubricating fluid, the "tears," is furnished by two groups of glands which arise as invaginations of the conjunctiva and retain their connection with that layer, supplying the pockets formed by the lids. These are (I) the harderian glands, which are located about the anterior (inner) corner and are associated with the nictitating membrane, and (2) the lacrimal glands, located near the posterior (outer) corner. This differentiation is not found in the amphibians where the glands are all alike and are evenly distributed, but appears in reptiles, from which point the two groups are distinct, both in location and structure. The harderian glands are well developed in reptiles, birds and most mammals, but are rudimentary in the Anthropoidea. The lacrimal gland is associated in reptiles and birds with the lower eyelid, beneath which its ducts empty, but migrates in mammals to a more dorsal position and thus becomes almost exclusively associated with the upper lid. In some mammals a few ducts occur in the lower fold; indications of its former location. The lacrimal fuid, supplied by both of these glands, is continually being secreted and is as constantly spread in an even layer over the outer surface of the eyeball by the movement of the lids. The excess is conveyed to the nasal cavities through the nasolacrimal duct, which appears in amphibian larve as an integumental groove extending from eye to nostril. This eventually closes up, sinks into the interior, and gains its independence from the integument, thus forming an internal canal connecting the conjunctival sac with the anterior end of the nasal cavity. 
Aside from these conjunctival glands there appear in mammals certain glands associated with the eyelashes. These are (I) the tarsal [meibomian], which are modified sebaceous, and (2) the ciliary, modified perspiratory glands. These open along the edges of the lids and produce narrow lines of oil which repel the lacrimal fluid and assist in retaining it within the peripheral folds. As the eyelashes are modified hairs, the tarsal glands may be looked upon as the associated sebaceous glands, considerably hypertrophied, and changed somewhat in their relation to the hairs.

This entire lacrimal apparatus, including the glands and the nasolacrimal duct, becomes much reduced in such aquatic mammals as the hippopotamus, seal and otter, and in the pelagic whales and porpoises is entirely rudimentary. In snakes there occurs a singular adaptation, which protects their eyes from the danger of the thick grasses and twigs by fusing the two eyelids together over the eyeball and then rendering them absolutely transparent. There is thus formed a plate, in shape like a watch glass, and serving as a second cornea. This is shed with each successive skin and forms a conspicuous feature of the exuvize, or "snake-skins," objects commonly met with in fields frequented by snakes. A lacrimal apparatus is wholly wanting.

As special protective organs to the eye may be mentioned the long superciliary bristles, which in cats and a few mammals project over the eye and when touched cause the automatic closing of the lids; also the eyebrows of the higher anthropoids, especially Man, the hairs of which point outwards and curve downwards at the outer end to receive the perspiration of the forehead and convey it away from the eyes. 


\section{CHAPTER XII}

\section{THE ANCESTRY OF THE VERTEBRATES}

"Ainsi la plus ancienne couche fossilifère connue nous
montre des représentants de presque toutes les classes
d'Invertébrés. Cela démontre l'existence d'une longue
période antérieure à celle sur laquelle la Paléontologie
peut nous fournir des renseignements et dans laquelle
ont pris naissance presque tous les types actuels.
Parmi ces êtres, dont les formes resteront toujours un
mystére, devaient se trouver les ancêtres sans squelette
des Vertébrés actuels."
DELAGE ET HeRouARD, Les Procordés, I898, p. 357 ,

Previous to the establishment of the modern theory of evolution, which removed each animal and plant from an isolated position unrelated to the rest, and assigned to it a place in a connected chain of organic beings, morphological speculation was limited to ingenious comparisons with little or no logical basis, conjured up to explain real or fancied resemblances. Thus Lorenz Oken, having conceived the idea that the head must possess parts corresponding to those of the trunk, considered the nasal cavity, the cephalic thorax; the mouth cavity, the cephalic abdomen; and the palate, the cephalic diaphragm; to him the halves of the upper and lower jaws represented respectively the anterior and posterior limbs, in which the teeth were the digits. Thus Geoffroy St. Hilaire compared insects with vertebrates, making the exoskeletal rings the equivalent of the vertebra, and the jointed legs that of the ribs. The relation of nerve cord, intestine, and main blood-vessels was made the same by placing the insect upon its back.

Others, like Goethe and Cuvier, sought to base the comparison between different forms upon the assumption of an archetype (Goethe's "Urbild"), of which a certain related group of animals might be considered as so many various modifications. If such an archetype had been considered to have or to have had 
a real cristence, it would have been the ancestor of the group in the modern scnse, but there is little to be found in the writings of these early morphologists to suggest such a relationship, and the archetype seems to have been considered a mere abstraction, a working hypothesis in dcfinite architectural form, employed for the purpose of facilitating comparison. There is often indeed the idea that the archetype, non-existent in its perfection, forms a divinely constructed plan upon which the Creator has modeled each member of a group of organisms, and that Man is able to grasp and understand this plan through his spiritual insight, a faculty akin to that of the Deity himself. Says Goethe, "Sollte es denn eben unmöglich sein, da wir einmal anerkennen, dass die schaffende Gewalt nach einem allgemeinen Schema die vollkommeneren organischen Naturen erzeugt und entwickelt, dieses Urbild, wo nicht den Sinnen, doch dem Geiste darzustellen, nach ihm als nach einer Norm unsere Beschreibungen auszuarbeiten und, indem solche von der Gestalt der verschiedenen Thiere abgezogen wäre, die verschiedensten Gestalten wieder auf sie zurückzuführen?"

This employment of an hypothetical archetype for the comparison of organisms reached its culmination in the marvelous structure reared by the English anatomist, Sir Richard Owen, who first established his great fundamental conception of a typical vertebra, and then described in terms of this all the skeletal parts of every known vertebrate, including here not the vertebral column alone but the skull and appendicular skeleton as well.

His diagram of a typical vertebra, reproduced here (Fig. I37), shows that he, too, as well as so many others, conceived of the typical or primordial form as a symmetrical and perfect

\footnotetext{
* "But should it then be impossible, when once we recognize that the Creative Power has produced and developed the more completely organized natures after a general plan, for tis to represent this Archetype, if not to the senses, at least to the mind, to elaborate our descriptions in accordance with it as with a norm, and, since such archetypes were taken from the forms of different animals, to refer the most varied forms back to it again?" Johann Wolfgang Goethe, "Uher einen aufzustellenden 'Typus zu Erleichterung der vergleichenden Anatomie," I796.
} 
one; the Golden Age idea appearing in Anatomy, but here, as everywhere else, the reverse of actual history. Yet this diagram, although erroneous as an explanation of early conditions, represents in a clear manner the parts that appear in actual cases, especially in the higher forms, and as such has been taken as the foundation of our modern nomenclature. This typical vertebra consists of a cylindrical centrum, furnished with a neurai arch, a hamal arch and several lateral ele-

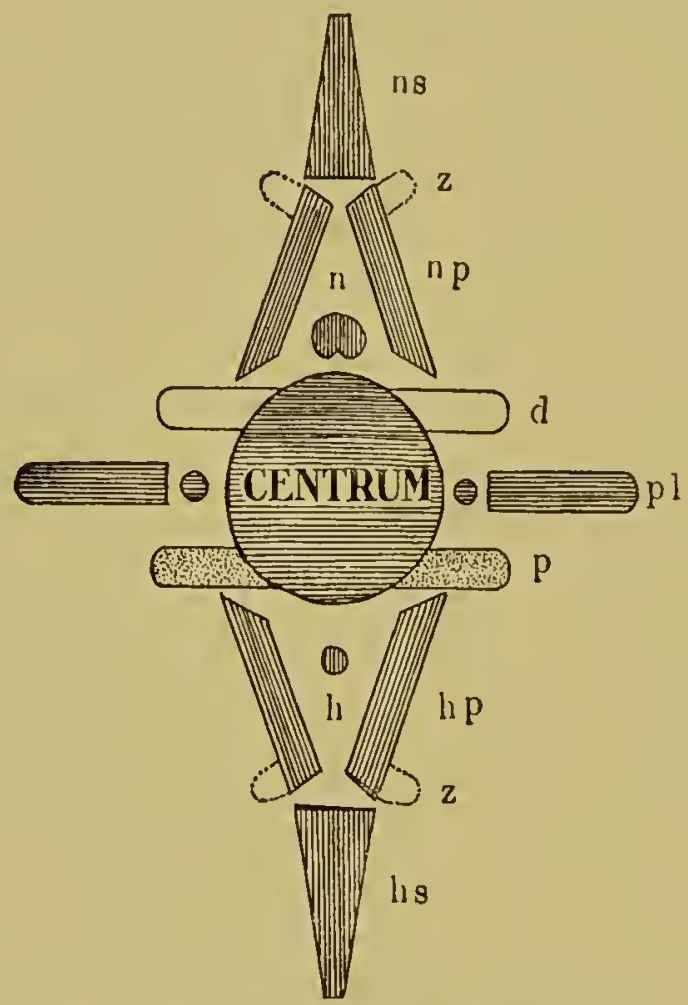

FIG. I37. Owen's original diagram of a typical vertebra, to illustrate his theory of the archetype. [After OwEN.]

us, neural spine; $z$, zygapophysis; $n p$, neurapophysis; $d$, diapophysis; $p l$, pleurapophysis; $p$, parapophysis; $h p$, hrmapophysis; $h s$, hæmal spine; $h$, hæmal canal.

ments. The neural arch consists of a pair of neurapophyses and a neural spine, and bears a pair of articular processes, the zygapophyses; and in like manner the hæmal arch is composed of a pair of hamapophyses, a hamal spine and a second pair of zygapophyses. Of the lateral pieces the central ones are the pleurapophyses or rib elements, sometimes forming free ribs, and dorsal and ventral to these lie respectively the diapophyses and parapophyses, more occasional elements. 
THE ANCESTRY OF THE VERTEBRATES 509

From this typical vertebra Owen was able to explain the skeletal elements in each segment of the body in every vertebrate, and was thus able to construct the skeleton of an Arche-

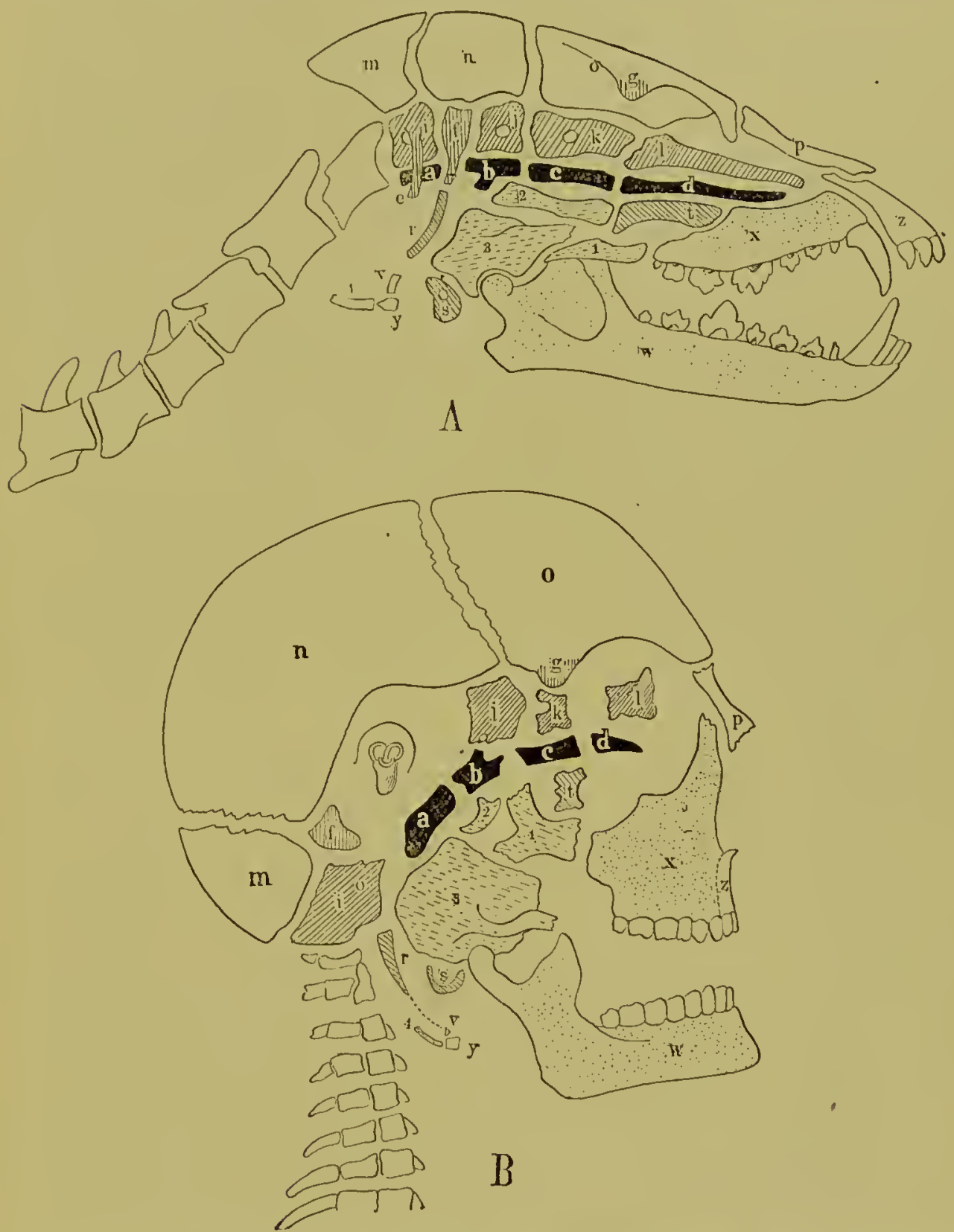

FIG. 138. Owen's interpretation of mammalian skulls. [After OwEN.] (A) Generalized mammal. (B) Man. For explanation see accompanying table given in text.

type, which consisted of a series of such vertebræ gradually tapering and losing their characteristic features in the caudal 
region and somewhat modified also at the anterior end, through the development of the brain and the introduction of the senseorgans.

The skull was formed by four of these typical vertebræ, called nasal, frontal, parietal and occipital. The centra are reppresented by vomer, prespheñoid, basispheñoid, and basi-occipital, the neurapophyses by prefrontals, orbitospheñoids, alisphenoids and exoccipitals, and the neural spines, composed mainly of paired pieces, by the nasals, the frontals, the parietals and the supraoccipital. The postfrontal was the diapophysis of the frontal vertebra, and the mastoid that of the parietal. Pleurapophyses were represented by the palatine, which belonged to the nasal vertebra, the tympanic, which belonged to the frontal, the stylohyals, parts of the parietal vertebra, and the suprascapula and scapula, which were reckoned with the occipital. The homopophyses were respectively represented by the maxillaries, the articularia, the ceratohyals and the coracoids, and the homal spines by the premaxillaries, the dentaries, the basihyals and the episternum. Other parts, such as the squamosal, the thyreohyal, and the free $11 \mathrm{mb}$, formed elements called appendages.

Never was there a more stupendous result of the labor of a single human life than this great work of Owen, and yet of the entire structure reared by his incessant toil all that remains is the large amount of accurate description and a great enrichment of osteological nomenclature. It was a house built upon the sand, and Owen's "typical vertebra" may be placed alongside of Goethe's "Urbild" as the noble attempt to picture the great truths which they felt in spirit and saw but dimly.

The formulation by Charles Darwin in 1859 of the doctrine of animal descent, with the implied conception of actual blood relationship between the different groups, introduced into

The theory may be further elucidated by the help of the following table and by the accompanying diagrams (Fig. $138, \mathrm{~A}$ and $\mathrm{B}$ ). The small letters added to the names of the separate elements in the table correspond to those used in the diagram, so that the former may be used to explain the latter. 
THE ANCESTRY OF THE VERTEBRATES 5II

\begin{tabular}{|c|c|c|c|c|c|c|}
\hline 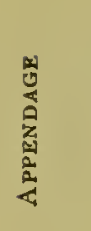 & 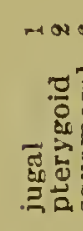 & & 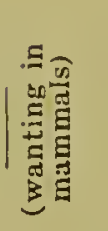 & & + & 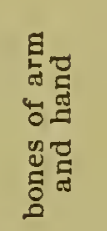 \\
\hline 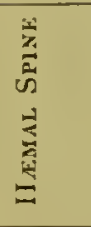 & 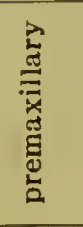 & N & 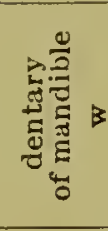 & 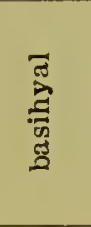 & in & \\
\hline 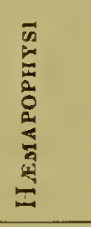 & 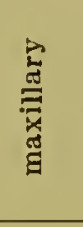 & $\star$ & 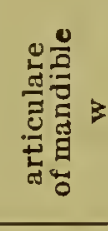 & 䨌 & D & $=$ \\
\hline 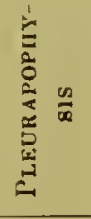 & 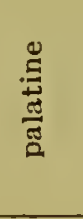 & 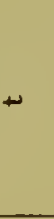 & 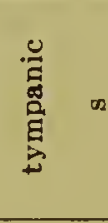 & 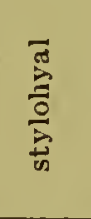 & L & 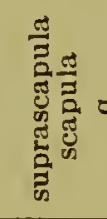 \\
\hline 童 & 兽 & 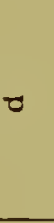 & 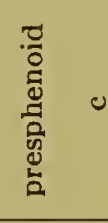 & 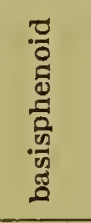 & مـ & 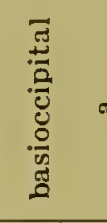 \\
\hline 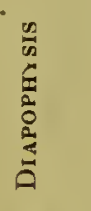 & & $\Rightarrow$ & 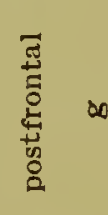 & 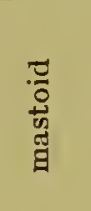 & $\omega$ & 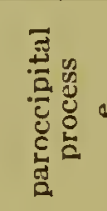 \\
\hline 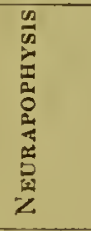 & 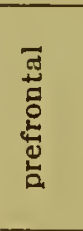 & - & 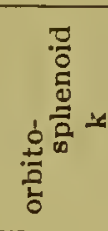 & 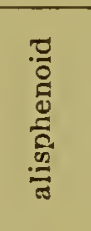 & - & 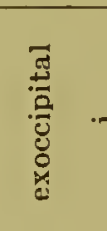 \\
\hline 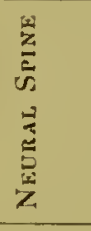 & $\begin{array}{l}\overline{\text { J }} \\
\text { 㟒 }\end{array}$ & 2 & 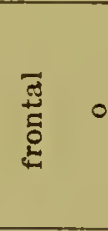 & 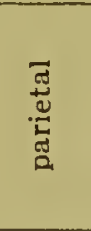 & $a$ & 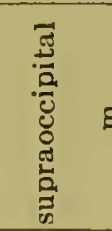 \\
\hline 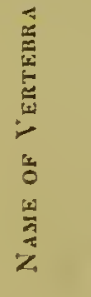 & 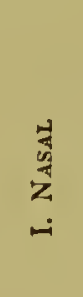 & & 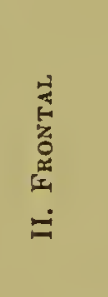 & 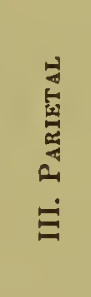 & & 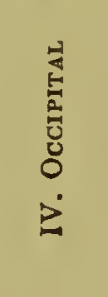 \\
\hline
\end{tabular}


anatomical speculation that necessary principle which had been lacking in the philosophy of pre-Darwinian anatomists, and from that time on actual ancestors took the place of theoretical archetypes. It became then of fundamental importance to establish the true interrelationships of the various animal groups, that the structure of a given form might be explained in terms of the ancestral structure of which it might be considered a modification.

Naturally the intensest interest centered about the establishment of the group from which the vertebrates were derived, and here for a long time the most of the speculation follored the lines laid down by St. Hilaire with his reversed insect. Certainly one of the most characteristic features of a vertebrate, and one of the earliest to appear in the embryo, is the division of the body into somites, and the search for a bilateral segmented ancestor must inevitably lead back to the articulates, which alone of the invertebrates emphasize this characteristic to an equal degree. It is true that in the two groups the arrangement of the internal organs is in the main reversed, for in the one the central nervous system is ventral and the main bloodvessel dorsal, and in the other the former is dorsal and the latter ventral; but the device employed by St. Hilaire to explain this is by no means an absurd one, since what is called dorsal or ventral in a given animal is merely its constant physiological relation to the surface of the earth, and in several cases, like the flounder and the squid, is known to be quite at variance with the condition usual in related forms.

Thus by postulating the occurrence of a change quite in accord with several known instances the differences in the relationships of the different systems in the simplest members of both groups (annelids and selachians) may be brought into almost complete harmony (Fig. I39, $a$ and $c$ ). Even the notochord, perhaps the greatest problem of vertebrate structure, may be compared to the "Faserstrang," a bundle of fibers running along the nerve chain and serving as a support. This and the notochord lie in a precisely similar position in relation to the other organs, and in both cases they are enclosed with 
the nerve cord in a common sheath of connective tissue. In the blood system there are equal points of resemblance, for in each case there are two median longitudinal vessels, one on either side of the intestine. In the vertebrate the dorsal one is the aorta, which sends the blood in a posterior direction, while the ventral one, with a current in the reverse direction, is represented by the embryonic subintestinal vein posteriorly and by the heart anteriorly. In an unreversed annelid it is true that the dorsal blood-vessel sends its blood from tail to head, while in the ventral one the blood flows from head to tail, but by reversing the animal the correspondence in the direction of the current becomes complete. The vertebrate aorta is then represented by the original ventral (now dorsal) vessel in which the current flows backwards, and the subintestinal vein and heart are represented by the original dorsal (now ventral) vessel, in both cases with the current directed forwards.

The most convincing of the many correspondences, however, lies in the nephridial system of annelids and selachians, which in both cases consist of segmentally arranged pairs of tubes that open into the colom at their inner ends by ciliated nephrostomes. There are also close correspondences in the relation of these tubules to the germ glands and to the cœlom. The more primitive type may be considered to be that found in annelids, in which each somite possesses a separate cœlom, or rather a pair of coloms, since those of adjacent somites are separated by transverse dissepiments, and those of the two sides of the same somite by median sagittal partitions, which form dorsal and ventral mesenteries. Each of the compartments thus formed is supplied with a single nephrostome, the tubule from which pierces the posterior dissepiment and enters the next posterior colomic pocket, where it exhibits a convoluted portion and a vesicular enlargement, and eventually opens directly into the exterior by a lateral opening. (Fig. I39, $a$ and b.) The germ glands develop on the anterior walls of the coelomic cavities, and the germ cells become free and float about in the colomic fluid until they are taken up by the nephrostome and find their way to the exterior, through the 

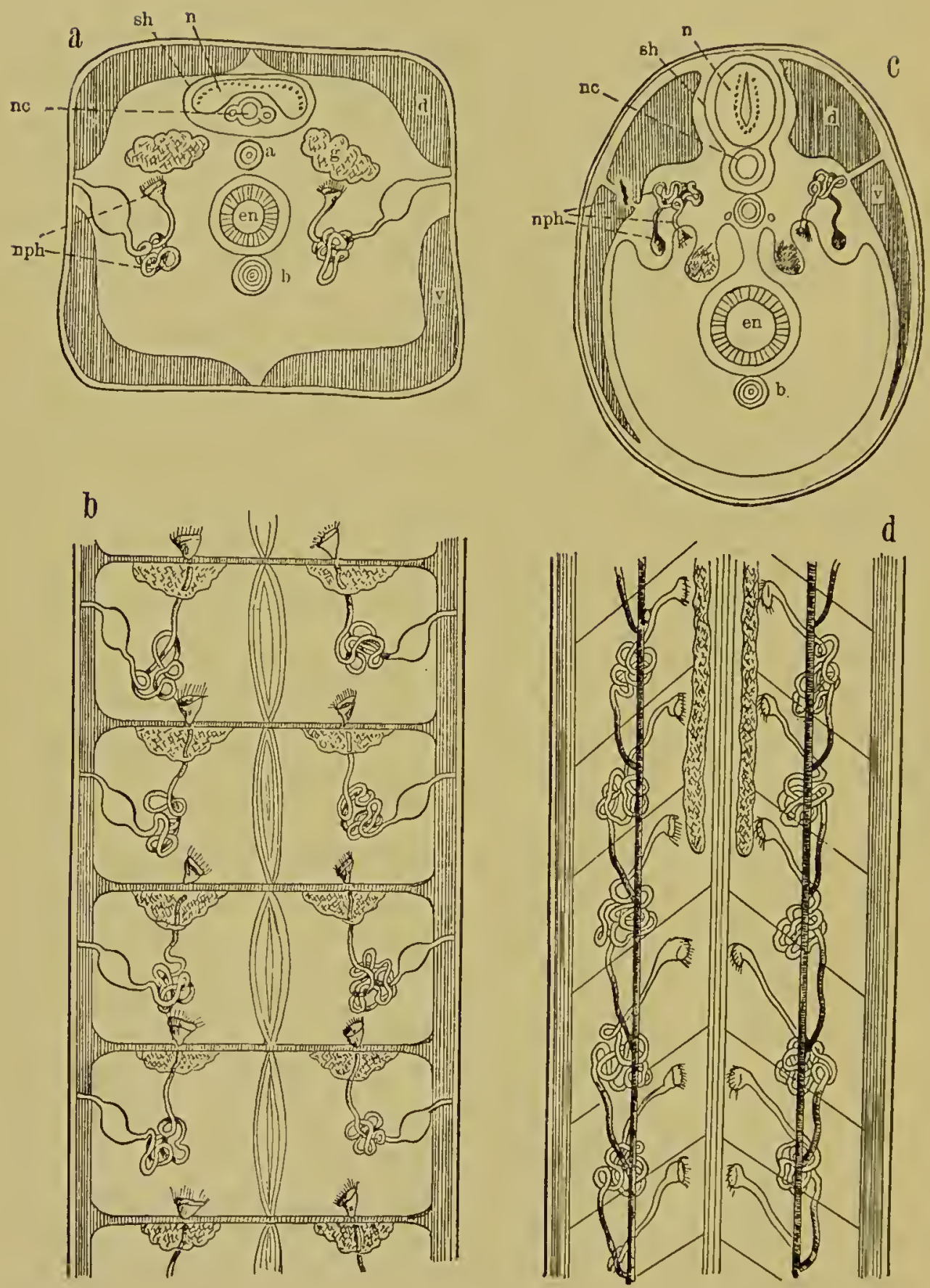

FIG. I39 Figures illustrating the Annelid theory. [After SEMPER.]

(a) Cross section of Annelid (reversed). (b) Longitudinal section of 
nephridium, which thus serves as ductus deferens or oviduct. Typically, as in the diagram, a germ gland belongs with each lateral coelomic cavity, but in actual cases they develop in only a few segments, and the associated nephridia become especially modified for the conveyance of the germ cells from the body.

If, now, the primitive vertebrate nephridia, germ-glands and cœlom, as described in Chapter IX above, be compared with the annelid condition, the similarities are found to be remarkable. Here also the nephridia are at first strictly segmental, although they no longer open to the outside independently, but through the medium of a common Wolffian duct. Since, however, this develops in part from the ectoderm, it may have begun as a simple external trough-like depression which ran along the sides of the animal and connected the several individual openings for the better disposal of their secretion. The segmental subdivisions of the colom are no longer continued in the higher vertebrates but the mesodermic diverticula, which appear clearly in Amphioxus, and in a more imperfect manner in the others, suggest the former presence of transverse dissepiments, and both dorsal and ventral mesenteries actually persist as far back as the posterior boundary of the liver, beyond which the ventral one disappears. Nor can it be said that the transverse dissipiments are wholly lacking, since, although they no longer divide the cœlomic cavity, they are still represented in the body wall by the intermuscular septa (myocommata) with which the nephridia sustain in the embryo similar relationships as in annelids (Fig. I39, d). In both cases the germ glands arise as localized portions of the cœlom (peritoneum), and the presence of a single pair in the true vertebrates may be correlated with the confluence of the several colomic cavities into a single one. The larger number of gonads in Amphioxus indicates the former presence of a much larger number of colomic cavities. That in vertebrates as in annelids the

\footnotetext{
Annclid. (c) Cross section of Selachian. (d) Longitudinal section of Selachian
in region of kidney.

n, nerve cord; nc, notochord; $g$, "faserstrang" of Annelid; sh, sheath surrounding nerve cord and notochord; $d$ and "' muscle masses; $e n$, intestinc; $p h$, nephridiun; $a$ and $b$, longitudinal blood vesscls.
} 
nephridial system is made to furnish channels of exit for the germ cells has been already shown (Chapter IX), and the opening into the oviduct has been homologized with a pronephridial nephrostome, while in the male the entire anterior portion of the mesonephros and its duct becomes utilized for the passage of the seminal fluid.

One of the most fundamental characteristics of vertebrates is the presence of paired gill-slits, extending in two lateral rows along the pharyngeal region and forming direct communications between the pharynx and the exterior; these may be readily derived from nephridia by supposing, first, that the inner ends of these tubes become secondarily connected with the pharyngeal lumen, and secondly, that the tubules become reduced in length until ectoderm and endoderm come in contact. Only in some such way can one explain the embryonic development of gill-slits from a series of ectodermic inpushings that meet a similar series of endodermic outpushings, a mechanical process that necessitates some reason back of that which is apparent in order to account for the accuracy with which these several folds meet one another. In the embryo of the cyclostome Myxine, precisely the form where we would look for the retention of the earliest phases, there still appears at first a fairly long canal between each ectodermic inpushing and its endodermic associate, perhaps a remnant of the nephridial tube. It may also be more than a coincidence that, when genuine nephridia of the pronephrotic system appear immediately posterior to the gill region, none arise in the somites that develop the gill-slits.

Important changes seem to have taken place in both outlets of the alimentary canal, and indications show that vertebrates have acquired both a new mouth and a new anus, although they still retain in the embryo many traces of the older organs. That the mouth of the vertebrates is not the primitive one is shown by a variety of indications, one of the strongest being its exceptionally late appearance in embryonic life. A mouth is one of the most essential of organs, and in other animals, corresponding to its important function, is one of the first to 
appear. In vertebrates, however, the reverse is true; the nervous system is laid down, the brain is differentiated, the notochord is formed, even the special sense-organs appear, and still the alimentary canal remains a sealed cavity, without communication with the exterior. At last a mouth appears, placed very far ventrally, in line with the gill-slits, and in certain fishes appears first as two lateral openings which eventually become confluent. All this seems a complete corroboration of the fact arrived at independently through adult anatomy that the vertebrate mouth has resulted from the confluence of a pair of gill-slits anterior to those now functioning, and still equipped with gill-arches which serve as jaws. There comes, then, the inevitable conclusion that previous to this conversion, and while the mandibular slits were still functioning as gill-slits, the ancestral forms must have had another mouth, traces of which are to be looked for in the earlier embryo. Such a primary mouth is actually found indicated in precisely the place where it would be looked for in the annelid, taking the reversal of the body into account, and this indication appears in the widely open "fourth ventricle" of the nerve cord.

In annelids, as in all Articulata, the mouth is upon the ventral side, and, since the alimentary canal is dorsal to the nervous system, this position is reached by means of an œsophagus, whicn turns downwards almost at right angles to the remainder of the canal, and runs between the two nerve connectives that connect the first and second pairs of ganglia. The first ganglionic pair thus becomes the supra-asophageal, the second, infra-asophageal, and the connections between them form a circum-@sophageal ring through which the œsophagus passes. These relationships will be clearly seen by reversing the accompanying figure (Fig. I4O), which will thus give the conditions as seen in annelids. In all true vertebrates the actual external opening of this early mouth has disappeared, but it may be identical with the neuropore in the embryo of Amphioxus, which forms in this place a direct communication between the lumen of the neural tube and the exterior and is otherwise unaccounted for. Aside from the indications of the early mouth 
and its œsophagus, furnished by fuurth ventricle and neuropore, there is also the hypophysis, or rather, that portion of it that is pushed up from the alimentary canal, for which there is yet no satisfactory explanation. Its origin from the alimentary canal, its constant appearance in all vertebrates, its relationship to the nervous system and its position, all suggest that it also is a remnant of an early œsophagus.

The formation of a new, ventrally placed anus is due to a procedure similar to that which forms the new mouth, although there is here no suggestion of a gilt-slit. The vertebrate anus arises as a mid-ventral inpushing of the ectoderm at some distance from the end of the tail, and thus reaches the primary intestine along its course, leaving beyond it a piece of considerable length, the post-anal gut, which soon atrophies. This

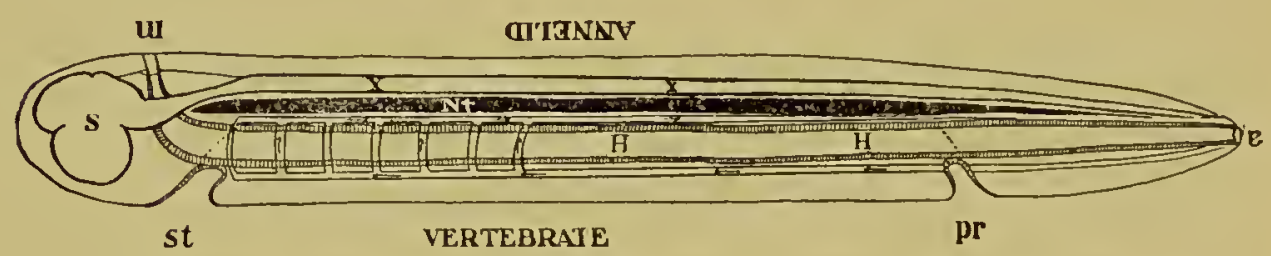

FIG. I40. Reversible diagram illustrating the Annelid theory.

Reversible designations, applying to both forms: $S$, brain; $X$, nerve cord; $H$, alimentary canal. Designations applying to Annelid only: $m$, mouth; $a$, anus. Designations applying to Vertebrate only; st, stomatodæum; pr, proctodæum; $n t$, notochord.

phenomenon, inexplicable by other means, is easily explained by the postulate of an annelid ancestor, for in these animals the anus is at the posterior extremity of the body, and the formation of a new anus in the vertebrate position would actually leave just such a piece as the one in question.

The gills of aquatic vertebrates receive also an adequate explanation through the annelid hypothesis. The annelid gills are external duplicatures of the integument, and occur upon the sides of every somite, attached to the parapodia, or short locomotor organs. In simple forms they are plates, but when specialized they become fringed or dendritic and somewhat resemble the external gill-bushes of amphibians. Although primarily distributed along the entire body, in certain specialized 
forms they are confined to the anterior end. Gills of this sort are well adapted to slow-moving or crawling forms, but when there is a necessity for the development of rapid motion, as is indicated for the direct ancestor of the rapidly moving fishes, such gills, especially if long and fringed, would tend to retard the motion. It would thus be natural to consider that they might wander within the openings of the nephridia, which in annelids lie close to these external gills, and this relationship gives, in its turn, the motive for the secondary connection of such nephridia with the alimentary canal, in order to supply the gills with a current of water.

The increase in the size of the gills would tend to develop some firmer tissue at their base to support them, and in this way there may have been developed a series of cartilaginous arches, which, together with the gills themselves, may have been at first and for a long time coextensive with the body itself, or have extended at least as far as the anus. When at a later period the gills became restricted to a few anterior pairs while the rest atrophied, the arches accompanying the former would be the persistent gill-arches, and form the visceral skeleton of vertebrates, while the remainder, freed from their gills, and repeating themselves metamerically, would become the ribs. It is even permissible to conceive of the limb skeletons as further derivatives of the metameric system of gill-arches; perhaps also the original elements of the primordial skull, the trabecula and parachordals, may be traced to the same source, thus accounting for all parts of the skeleton save the dermal bones, which are integumental, and the notochord, which has already been accounted for.

Convincing as these comparisons seem when taken by themselves, the influence of later investigation has tended rather away from the annelid hypothesis, and at present, although there are many investigators who seek the ancestor of vertebrates in some worm-like form, there are few who wish to definitely assert that this ancestor was an annelid.

The annelid theory rests largely upon the definite body segmentation of both these animals and vertebrates, yet segmenta- 
tion is not in itself as fundamental a character as would appear at first, and may be easily acquired by an animal group in any one of several different ways. It is likely, for cxample, that such a segmentation as that possessed by vertebrates may have been gained through the muscular action of a previously unsegmented form, and the fact that in vertebrate embryos the segmentation first appears in the mesoderm, from which the muscles are derived, furnishes a strong support for this view. The oldest of the annelids, on the other hand, begins life as an unsegmented larva, upon which the somites become developed one after another through a sort of budding, a process totally unlike that in which the vertebrate initiates its segmentation.

A second group of vermian forms from which the vertebrates may have developed is that of the nemerteans, a group of mainly marine worms, of uncertain affinities, but probably allied to the platyhelminths (flat-worms). Here the nervous system is not a ventral one, but consists of two lateral cords imbedded in the body wall, and often a smaller mid-dorsal cord, the three being bound together by commissural nerves which run around the animal (Fig. I4I, A). A branching intestinal nerve proceeds from one of these and is distributed to the sides of the intestine; and from the ventral portion of some of the anterior commissural nerves small nerve branches appear, also distributed to the intestinal wall. The anterior end of each lateral nerve is enlarged into a ganglion, from which a few nerves proceed anteriorly.

The manner in which such a nervous system may become converted into that of a typical vertebrate may be readily seen by a comparison of $A$ and $B$ of Fig. I4I, the first of which has already been referred to. Of the three longitudinal nerves the dorsal one has become the central nervous system, and has expanded its anterior end into a brain, while the two lateral nerves have become subordinated to it, but persist in lower vertebrates as the lateral nerves of the Vagus system, rami laterales $X$, with which the long intestinal nerve is also associated. The original ganglion of the lateral nerves breaks up 
into the various ganglia found in vertebrates, in association with the cranial nerves. As for the commissural nerves, they become alternately sensory and motor in function and associate together in pairs, forming the metamerically arranged spinal nerves of vertebrates, the elements of which are in lower forms still separate and issue from the neural canal through separate foramina. Lastly a sympathetic system is formed by collect-
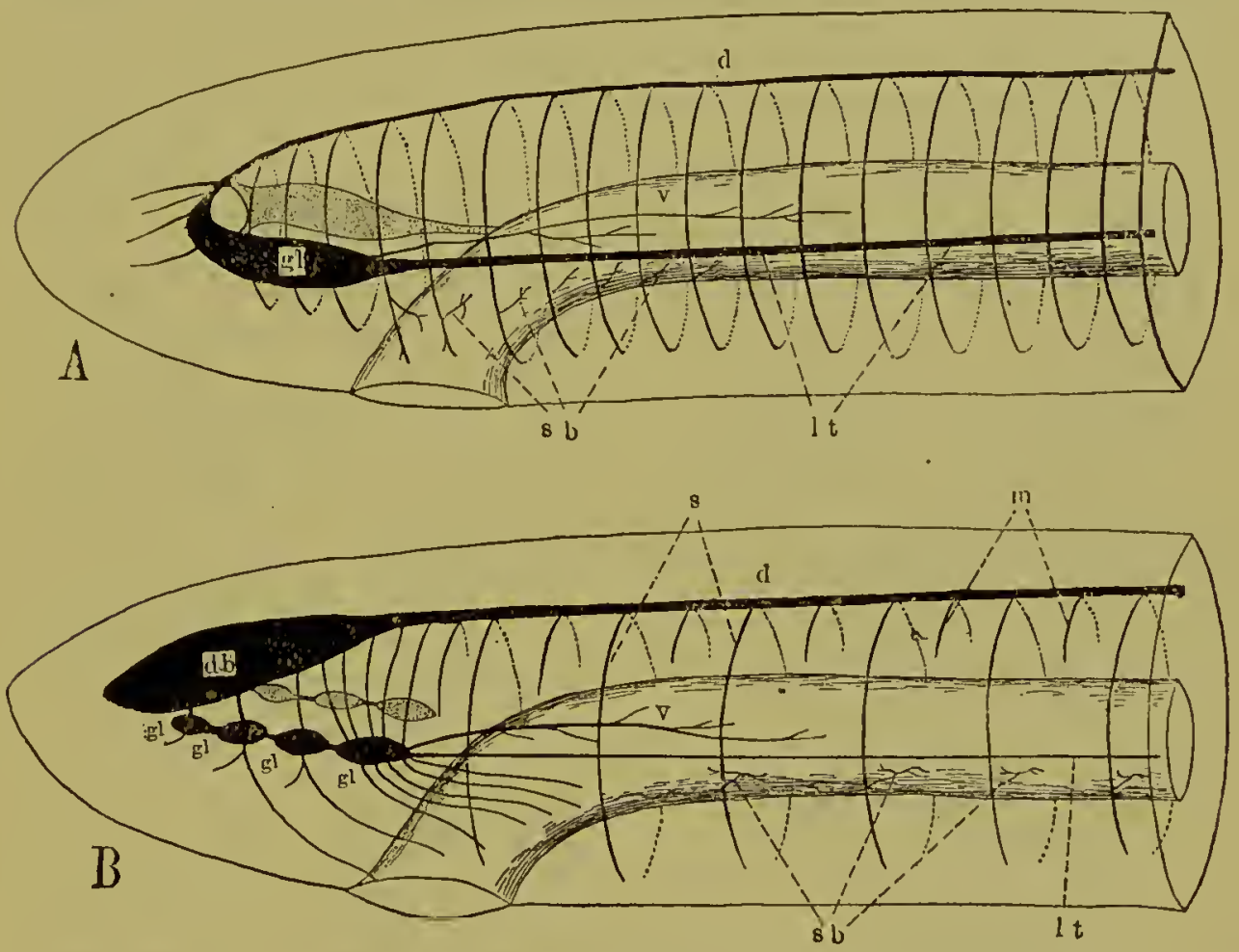

Fig. I4I. Nemertean theory of the origin of Vertebrates. [After HUBRECHT.]

(A) Typical diagram of Nemertean. $d$, dorsal nerve cord; $g l$, ganglion; $l t$, lateral nerve cord; $v$, intestinal nerve; $s b$, small intestinal branches.

(B) Typical diagram of Vertebrate; $d b$, brain; d, dorsal nerve cord; $s$, sensory, and $m$, motor spinal nerves; $g l$, sympathetic ganglia; $v$, ramus intestinalis vagi; $l t$, ramus lateralis vagi; $s b$, sympathetic branches.

ing together the small intestinal branches that come from the ventral portions of the commissural nerves.

Concerning the other systems it is only fair to say that their correspondence is by no means as close as is that of the nervous system, although a characteristic nemertean structure, the proboscis, has been likened to the hypophysis, while its sheath, into which it may be retracted, has been cited as possibly fur- 
nishing material for the notochord. Attention has also been called to the respiratory function of the anterior portion of the intestinal canal in nemerteans.

Aside from all hypotheses which have at their basis the consideration of a worm-like ancestor may be briefly mentioned a recent theory which finds the vertebrate ancestor among the more primitive arachnoids, now represented by such animals as the scorpion and the horse-shoe crab (Limulus) and formerly exhibited by the extinct group of Merostomata. To appreciate this one must at the outset dispose of the cyclostomes and other low forms like Amphiowus as degenerate and without special,significance, and take as the starting point of vertebrates such forms as the ganoids, or more especially the placoderms, which lived in Devonian times and were contemporaries of certain aquatic arachnoids, allies of the horse-shoe crab.

As the starting point in this theory there may be taken a certain series of resemblances between the brain and cranial nerves of vertebrates and the fused cephalo-thoracic ganglionic mass found in such arachnoids as the scorpion and the horseshoe crab. In these forms this central mass is divisible into three distinct portions, comparable to fore-, middle- and hindbrains, with an accessory part corresponding to the medulla. The number of neuromeres, or primary nerve somites of which these parts are composed, $i, c ., 3-\mathrm{I}-5$ for the brain and 2 to 4 for the medulla, also corresponds closely with the conclusion of many specialists concerning the segmental values of those parts of the head in vertebrates. A similarly suggestive resemblance exists in the cranial nerves and the relations of the organs of sense.

Although the anatomy of the soft parts of the Merostomata will never be known, they could not have been very different from the condition found in Limulus and the scorpion, and it may even be supposed that they and modern vertebrates have developed in distinctly different directions from these as common ancestors, and that thus their condition may have been far more like that of the vertebrates than is that of any of the arachnoids now living. Aside from the nervous system, 
numerous other parts are more or less comparable. For example, the eyes of the Merostomata consisted of a pair of widely divergent lateral eyes, and a pair of closely approximated median eyes, a peculiarity seen in the presentday Limulus; in the vertebrates there are the same widcly divergent lateral eyes, and the median pair is well represented by the pineal eye, in the development of which there are many suggestions of its having been double at an earlier period. In Limulus the central nerve-complex is protected by
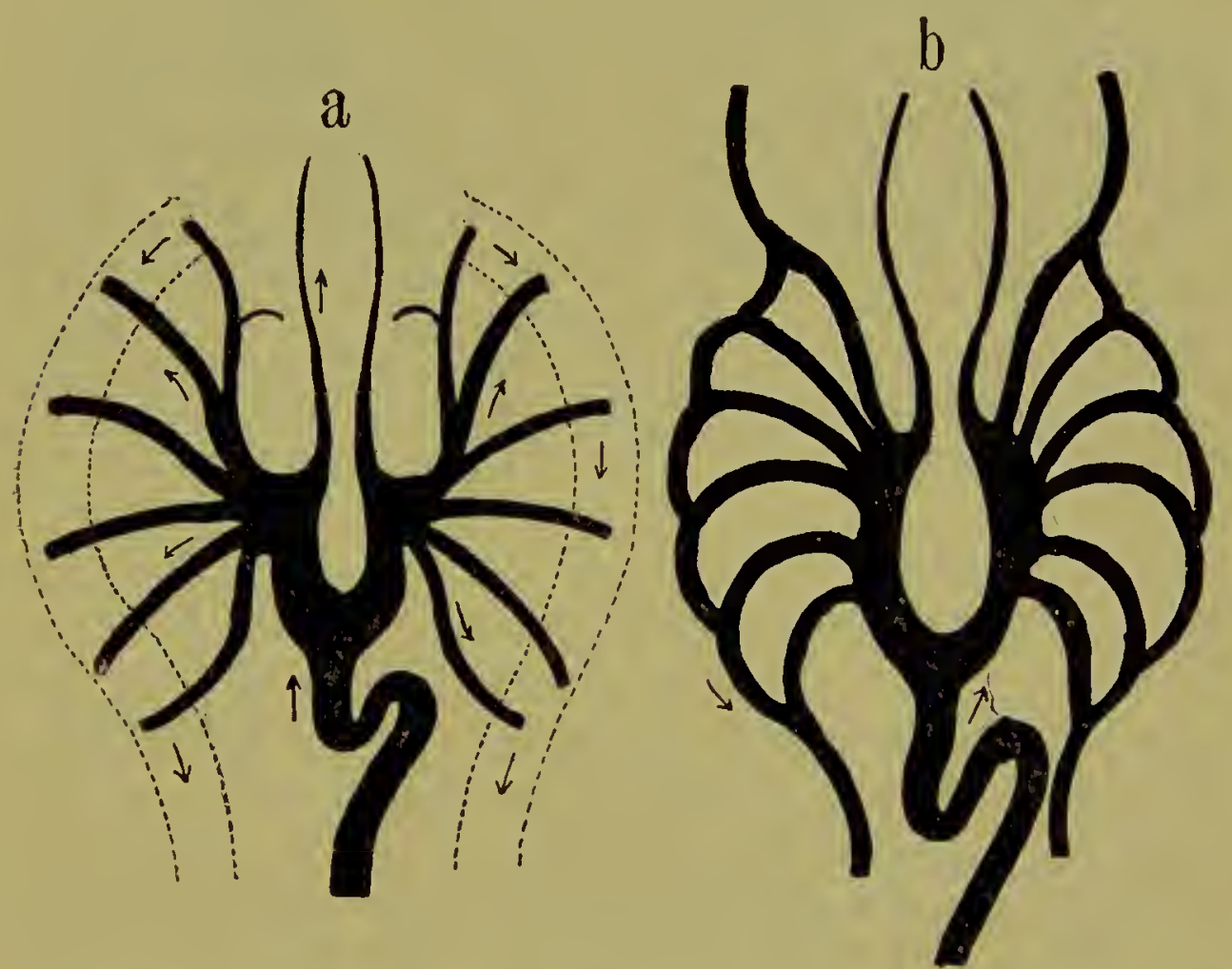

Fig. I42. Comparison of heart and gill arches of (a) Arachnoid, and (b) Vertebrate. [After Patten.]

an internal skeletal piece, variously called "sternum" and "endocranium," which in general form and still more in its relationships to other parts resembles the primordial skull of vertebrates. The arterial system shows in each case a tubular, somewhat contorted heart, an arterial trunk proceeding anteriorly from this, and dividing into pairs of arterial arches, from which, after sending a branch to the head, the blood is recollected into two lateral channels, in the one case a sinus, 
in the other a definite vessel. This relationship suggests a certain homology between the appendages of Limulus and the gillarches of the vertebrates, and as a matter of fact it has been suggested that the second pair of arachnoid appendages, so largely developed in the scorpion, do actually represent the mandibular arch, and that the next pair, or perhaps the next two, may represent the hyoid. The vertebrate gills them-
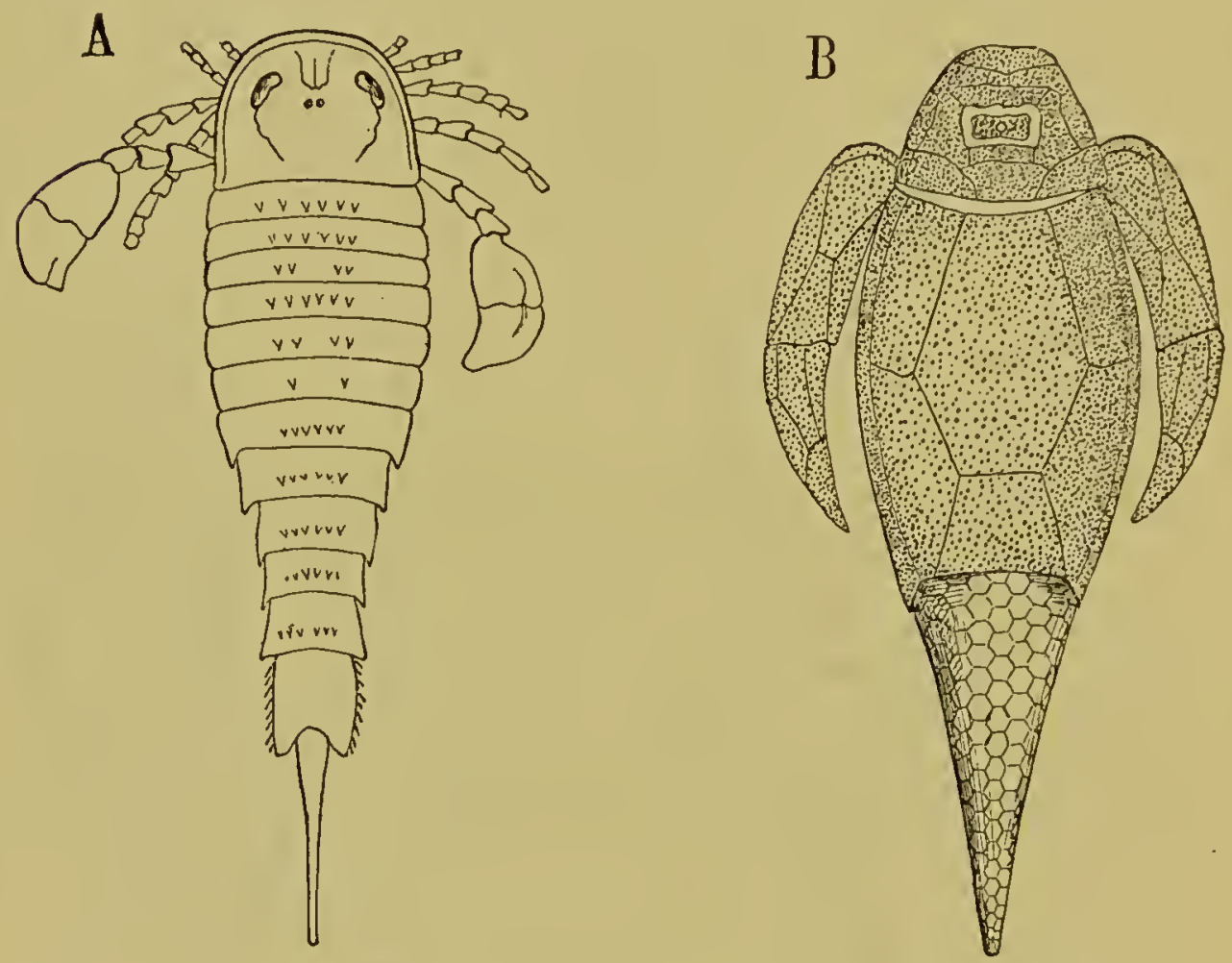

FIG. I43. Comparison between extinct Crustacean and Vertebrate.

(A) Gigantostracan, Eurypterus [after Niedzkowsky]. (B) Placoderm, Pterichthys [after NeUMAYER].

selves seem, however, more nearly comparable in structure with the gill-plates or gill-books of modern arachnoids, and their more posterior position in those latter is accounted for through a backward migration, since the nerves supplying them are precisely the ones which, for other reasons, have been compared with Vagus elements.

Perhaps one of the strong points in this erratic theory lies in the fact that the vertebrates are here derived, not from a primitive segmented form, like an annelid, in which the somites 
are much alike, but from one in which the differentiation of the head somites and their grouping to form complexes has already progressed quite far, and apparently along the same line. The external resemblance between the heavily armored placoderm fishes and their contemporaries among the arachnoids is certainly striking, and the similarity extends also in the head region to the plates of which the shell or cephalothorax is composed (Fig. I 43).

Without subjecting the arachnoid theory to further comment other than to say that it has received very little recognition, we may pass to that theory which places especial weight upon the notochord, the gill-slits and the dorsal position of the central nervous system, and by means of these has traced the line of vertebrate ancestry through a series of invertebrate

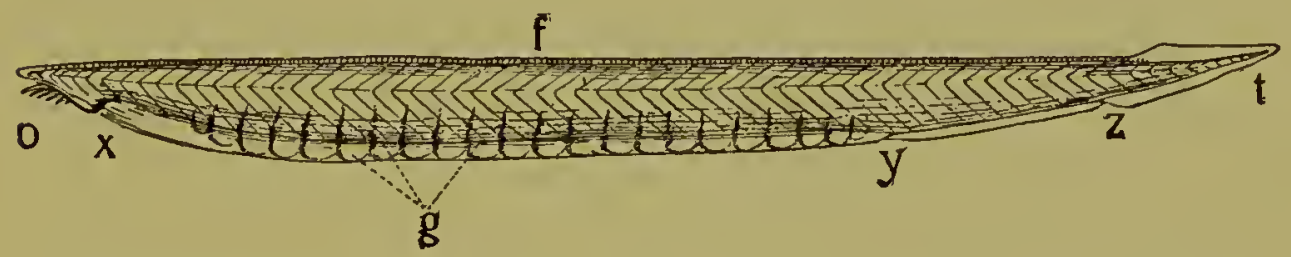

FIG I44. Amphioxus.

$o$, oral hood with cirrhi; $x$, mouth; $g$, gonads; $y$, atriopore; $z$, anus; $t$, caudal fin; $f$, dorsal fin.

torms, externally very unlike one another, and each somewhat isolated in its systematic position. The first of these in descending series, and representing in a way a simplified vertebrate, stripped of everything save the essentials, is the now famous Amphioxus (or Branchiostoma), first described in I778 as a shell-less snail, or slug (Limax lanceolatus). It is a shore form, and, with a few specific differences, occurs on almost all coasts. It is one or two inches in length, flattened laterally, and pointed at both ends; it is thus without a distinct head, but the mouth, which is situated a little ventrally at the anterior end, is equipped with a membranous expansion in the form of a hood. The adult burrows perpendicularly into the sand, leaving exposed only the anterior end, and in this temporarily sessile condition it expands the oral hood and collects the débris that drifts past it, much after 
the manner of other non-locomotive forms. The larva is, however, actively free-swimming, and the adult often changes its locality and thus retains the power of rapid motion.

A striking external feature of Amphioxus is the regular segmentation of its muscular system, which shows through the transparent skin and is marked by lines formed by the myocommata, each bent in the form of a $V$, the point directed forwards. The reproductive organs or gonads, also, are often sufficiently developed to appear through the skin as a succession of square or slightly rounded masses, each corresponding to a segment, yet with those of the two sides placed alternately, as is also the case with the myomeres and the other lateral parts. There is a slightly indicated median fin, supported by minute fin rays, and extending along the entire back, around the tail and upon the ventral side considerably past the anus, throwing this latter opening out of the median line, and dislocating it to the left. Anterior to this and at the termination of the fin is a large and conspicuous opening, the atriopore, through which the water that is continually taken in at the mouth is as continually expelled. The chamber from which the atripore leads is termed peribranchial, and appears at first like an internal cavity; a little investigation, however, shows that it is in reality external and develops in the larva from two longitudinal folds that arise along the sides and grow together ventrally. The region of the body which they enclose is perforated laterally by a very large number of obliquely placed gill-slits, communicating with the pharynx, so that the water taken in at the mouth passes through these slits and thus enters the peribranchial chamber, from which it is finally expelled through the atriopore. A similar device is found in frog and toad tadpoles, and the external outlet, here called the "branchipore," varies in position in the different genera, but appears quite high up on the left side in the true frogs (Rana). As the peribranchial chamber develops during larval life, the gillslits of young larvæ open directly to the outside, as in true vertebrates, and suggest that, as in the frog tadpole, this chamber has been developed as a special adaptation to the 
needs of this particular animal, and is thus not ancestral in character.

Where the two lateral folds that form the peribranchial chamber come together they unite in such a way as to leave their original edges in the form of a pair of parallel metapleural folds, and it is quite possible that either these, or more probably the original folds before they extended far enough to unite ventrally, are identical with those folds from which, at some period in the long history between Amphioxus and the fishes, the two pairs of limbs were originally derived. [Cf. Chapter $\mathrm{V}$.]

Of the internal organs the most conspicuous is the notochord. This is an elastic skeletal rod of gelatinous tissue, surrounded by a firm connective tissue sheath. It extends to the extreme ends of the animal and insures to it a certain grade of rigidity while allowing the body to be extremely flexible. Lying along the dorsal aspect of this rod, and enclosed in a sheath which is continuous with that of the notochord, is the dorsal nervous system, closely resembling that of fishes, but with no brain other than a slight club-shaped enlargement at the anterior end, the archencephalon. An olfactory pit on the left side, and a median pigment spot, are its only definite senseorgans. Beneath the notochord lies the alimentary canal, which expands beyond the mouth into a pharynx, that extends more than half the length of the body and passes into a straight intestine with no especial differentiation of parts. The pharynx is perforated by 6o-8o pairs of narrow gill-slits, placed obliquely, and kept open by an elaborate system of skeletal rods, formed of a material resembling chitin, and thus more like an invertebrate than a vertebrate structure. Both the elaborateness of this skeletal system and the very large number of gill-slits are plainly secondary modifications, like the peribranchial chamber, since they are not found in the larva, and mark Amphioxus as a much modified form, probably that one out of a large class which survived on account of these very modifications.

A characteristic structure runs along the floor of the 
pharynx in the form of a ventral groove. This is the cndostylc, an organ that performs a very important service in the collection and retention of food. Its surface is covered with cilia, which produce a backward directed current, and it is furnished also with gland cells that secrete a viscous fluid. The nutrient particles that enter the pharynx in the water current are engaged by the viscous fluid, and the combined mass is conveyed to the intestine by the motion of the cilia.

The main blood-vessels consist of a sub-intestinal vcin, ventral to the alimentary canal, in which the current is directed forwards, and an aorta, situated between the intestine and the notochord, in which the blood flows posteriorly. Aside from these there is a series of branchial vessels, a pulsating heart, and other vessels, the relations of which are like that found in vertebrates, but simpler.

The general impression given of the structure of Amphioxus is that of a diagrammatic vertebrate modified by several secondary adaptations. Had it been known to Goethe, he would have almost denominated it the realization of the primordial vertebrate, an incarnate "Urbild." In the general arrangement of the essential organs, the dorsal nervous system in tubular form, the notochord, the dorsal aorta, the intestine with its pharynx perforated by gill-slits, it is essentially vertebrate; while in its peribranchial chamber, and its great multiplication of gill-slits it suggests the successful attempt of an animal to survive through the power of acquiring secondary adaptations, the only one out of a large group that has come down to us. The segmentally arranged muscles are essentially vertebrate, and a series of rather complex nephridia in the gill region may be homologous with a pronephros. Even those mysterious organs of true vertebrates, the thymus, thyreoid, and epithelial bodies, the history of which begins in the cyclostomes with a series of segmental anlagen, are probably seen here in an earlier stage, for it has been suggested that the endostyle is the homolog of the thyreoid series, and the numerous thymus anlagen have been rather hesitatingly identified with certain elements of the gill skeleton. 
There seems thus no doubt that in Amphioxus we have a genuine, although somewhat modified, ancestor of the vertebrate group, the sole survivor of a lost race, the more typical members of which were probably simpler and more like the true vertebrates than is their present-day representative. This conclusion serves, however, only to put the question one stage further back, and we now ask it in this form: What is the relation of Amphioxus to other invertebrates? In order to summarize the most fundamental characteristics of Amphioxus, those should be selected which it possesses in common with the vertebrates, since characters possessed by Amphioxus alone may, with great probability, be considered secondarv modifications, and thus unrepresented in, their ancestors.

Thus reduced, search should be made for an animal with a notochord, a dorsal nervous system, and a pharynx perforated by gill-slits, and possessed of a mid-ventral endostyle. Some have included among the essentials a pronounced segmentation, that shows itself in the muscular and nephridial systems, but a study of segmentation in general leads to the opinion that the segmentation of an animal, either expressed in the bodywall, or by the repetition of some of the organs, is not a fundamental character, but one easily acquired, and thus is not to be considered necessarily as an essential characteristic of the group from which Amphioxus and its allies have come.

The direction of the search thus defined, one is led inevitably to another isolated and problematic group of invertebrates, which have been variously classified among molluscs, worms, and other comprehensive and noncommittal groups, which have had, in short, about the same treatment as that accorded to Amphioxus. This group differs from the latter, however, in that, although isolated, it is extremely rich in the species still extant, and is represented by forms so variously modified, and so widely different from one another, that they form several Orders and numerous Families, thus showing all possible modifications of their fundamental plan.

This group is that of the Tunicata, a Class of marine animals, some of which are free-swimming throughout life, while 
others, although active as larvæ, soon settle to the bottom and become sessile. Contrary to expectation, it is the latter which in general have retained the more primitive characteristics, while the free-swimming ones are often greatly modified. In a typical sessile tunicate, like the one shown in Fig. I45, a, the delicate and rather complex organism is shut within a tough and often wrinkled external coat or tunic, in which there appear but two definite structures, an incurrent and $e x$ current orifice, through which the water is continually driven,
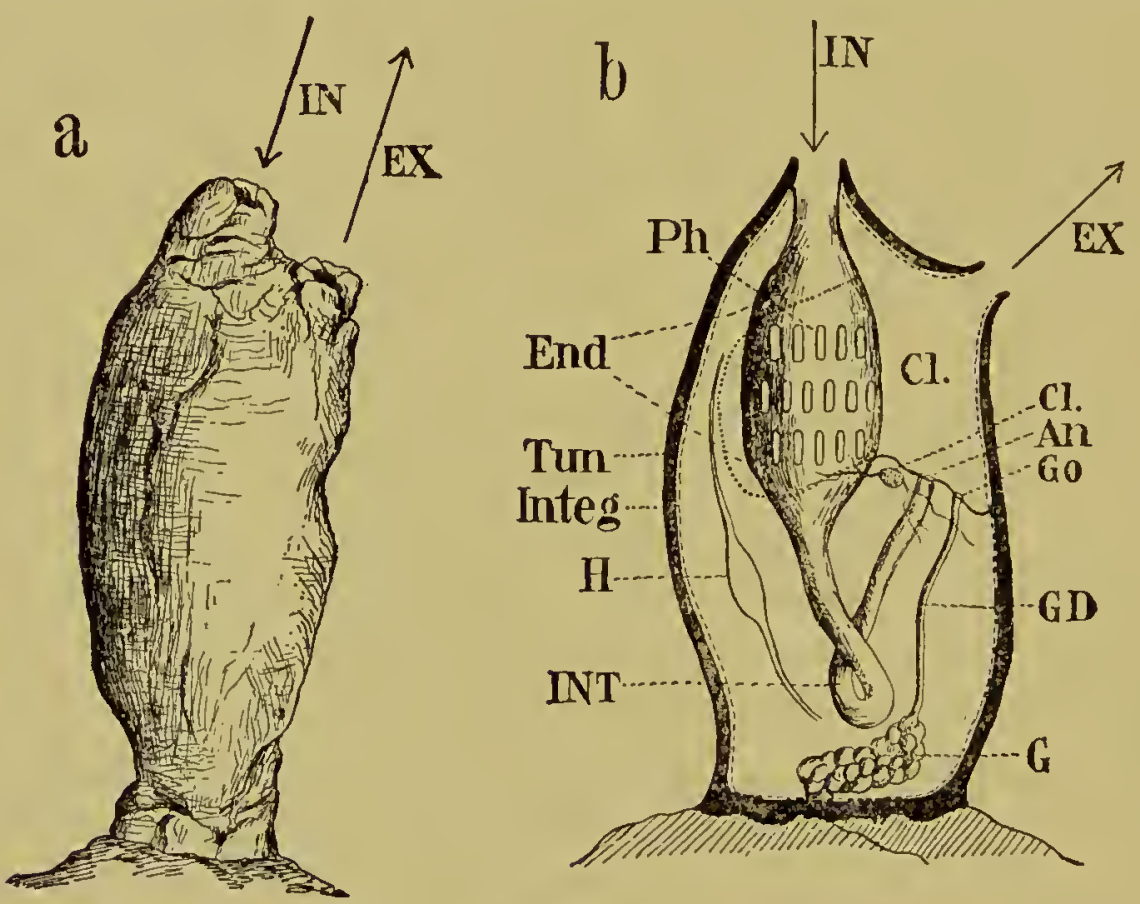

FIG. I45. Typical Tunicate.

(a) External view. (b) Diagram of internal anatomy.

$I N$, incurrent; and $E X$, excurrent canals; $P h$, pharynx; $I N T$, intestine; $A n$, anus; $C L$, cloacal chamber; Tun, tunic; Integ, integument; End, endostyle; GL, ganglion; $G$, gonadic gland; $G D$, gonadic duct; $G O$, gonadic opening; $H$, hcart.

much as in sponges. The incurrent orifice, which is really the mouth, leads into a capacious pharynx, and this continues into an intestine which, as usual in sessile forms, becomes bent upon itself and opens by an anal orifice not far from the pharynx. The anus opens, not directly to the exterior, but into a cloacal chamber, which is really outside of the animal but enclosed within the outer tunic. This same chamber surrounds the pharynx and receives the water taken into the latter through a series of gill-slits, which, in larva, are few in num- 
ber and disposed in lateral rows, but which usually become almost indefinitely multiplied, and in many cases transform the entire pharyngeal wall into a structure resembling basket-work. Thus the cloacal chamber, except for the relation of the anal outlet, is precisely similar to the peribranchial chamber of Amphioxus, the excurrent orifice being the equivalent of the atriopore. The pharynx in the two animals is also similar in the presence and secondary multiplication of its gill-slits, and here also there is an endostyle, which lies along that side of the pharynx which in the free-swimming larva is ventral. The nervous system consists of a single ganglion, placed in the adult between the two arms of the U-shaped intestine, but dorsal to it in the larva, and the vascular system is represented by a ventrally placed heart. The reproductive gonads lie in the bend of the intestine, and open by ducts of their own into the cloacal chamber.

These numerous suggestions of an organization akin to that of Amphioxus which are noticeable in the adult are far more apparent in the larva (Fig. 146, a). In this stage the animal is tadpole-like and possesses a long tail, flattened laterally and provided with a typical notochord, similar in its development to that of Amphio.rus. A series of segmentally arranged muscles, separated by myocommata, is also found here, especially developed posteriorly. The nervous system appears in the form of a prolonged neural tube and expands at its anterior end into a sensory vesicle (brain) which is provided with a pigment speck that forms a primitive eye, and a somewhat problematic organ usually interpreted as an car. although both organs are exceedingly simple in construction. In this larval condition the gill-slits are few in number and simple in arrangement, and the endostyle is in the proper position for comparison with that of Amphioxus. The heart also lies ventrally and just posterior to the osophagus.

The changes that take place during the assumption of the sessile position are shown in Fig. I $46, b$ and $c$, and are explicable by assuming a twist or rotation of the animal towards the left after fixation, by means of two papillac of attachment. 

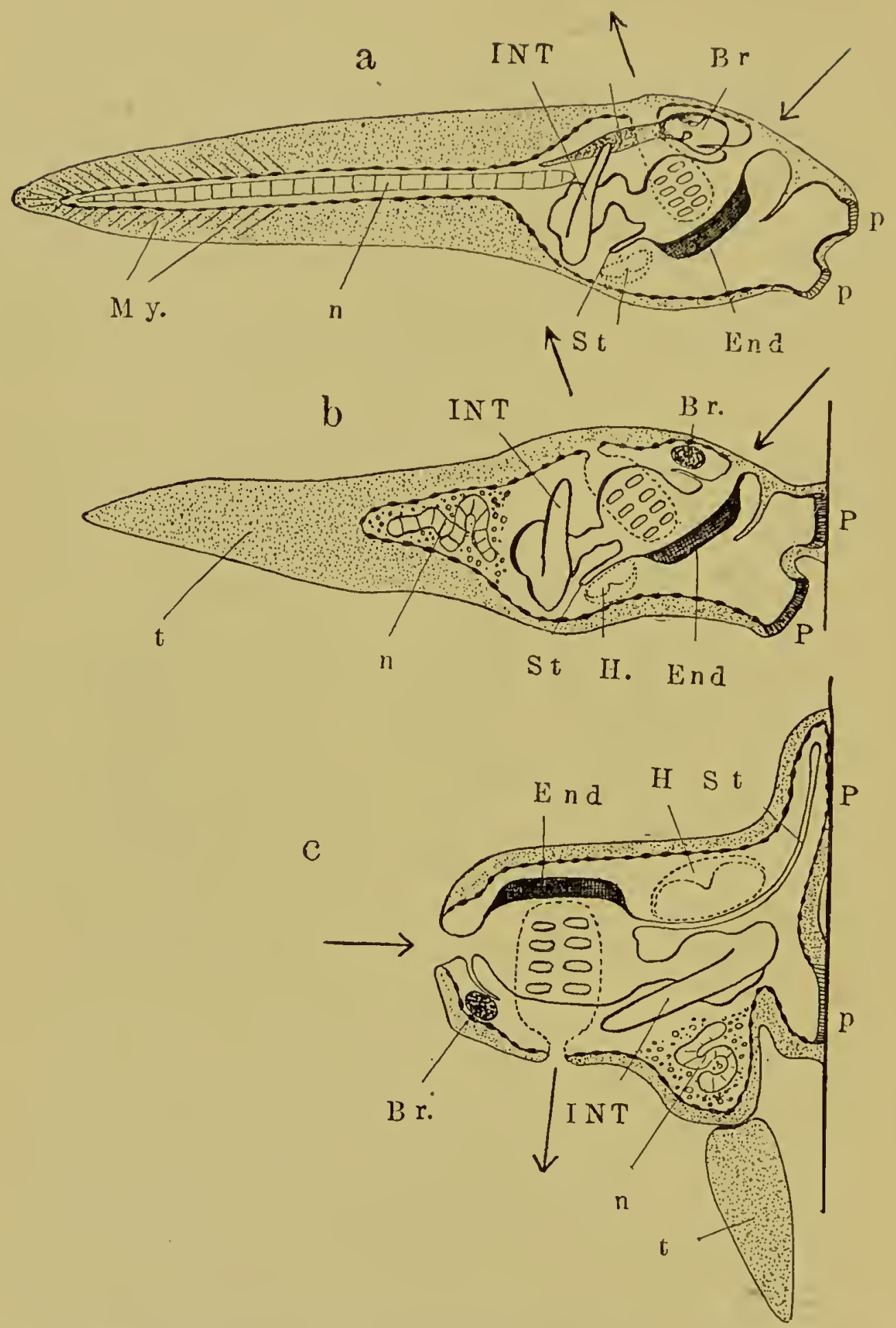

Fig. I46. Development of Tunicate from free-swimming larva. [After SeELiger.]

$p^{\prime}, p$, adhesive suckers; $B r$, brain; $C$, nerve cord; $I N T$, intestinc; $H$, heart; End, cndostyle; St, stolo; $n$, notoehord; $m y$, myotomes; $t$, tail. The orientation is indicated by arrows, whieh mark the ineurrent and excurrent orifices. 
During this metamorphosis, which is a regressive one, the tail, with its notochord and segmented muscles, becomes lost, and the central nervous system becomes much reducẹd. The posterior end of the intestine connects with the cloacal chamber and the adult relationships are gained.

From this sketch of the organization and metamorphosis of the tunicates it is evident that the group is somewhat closely related to Amphioxus, and hence to the vertebrates, but that, since the time of the common ancestor, the Tunicata have followed for a long distance a divergent road of special adaptation, which, although it has allowed them to continue existence, has been one of degeneracy and loss and has masked their true relationships. The ancestor that zue here seek is better seen in the larva than in the adult, and we may believe that there once existed an adult animal with attributes like that of the tunicate larva of the present day, and that this animal was the direct ancestor of that group of which Amphioxus is now the only living representative.

Is there now any record of the history of vertebrate descent back of the tunicate ancestor? Is there any other invertebrate animal possessed of gill-slits, a notochord, and a dorsal nervous system? And as an answer to this, a very incomplete and uncertain one at best, there is only a single animal form, though represented by several closely allied species, an animal as unpromising in its exterior, and here, perhaps, almost as much so in its interior also, as those hitherto considered. This form is Balanoglossus, a marine worm that lives in self-constructed tubes of sand between tide-waters, and, like Amphioxus, has a wide distribution. Externally the animal is wormlike and is possessed of four body regions, a conical proboscis, a collar with a free anterior edge, a flattened gill region and a cylindrical posterior part. The mouth is situated ventrally, immediately beneath the edge of the collar, and receives the sand mixed with nutrient material as it becomes unearthed by the burrowing action of the proboscis. The pharynx is quite extended and communicates directly with the exterior 
through two lateral rows of paired gill-slits, which are supported by a branchial skeleton very much like that of $A m-$ phioxus.

This characteristic of the possession of pharyngeal gillslits is extremely significant, for nowhere else, except in vertebrates and in their allies, above considered, do such organs occur, and investigators have naturally been led through these to seek for the other essential vertebrate characters of a noto-

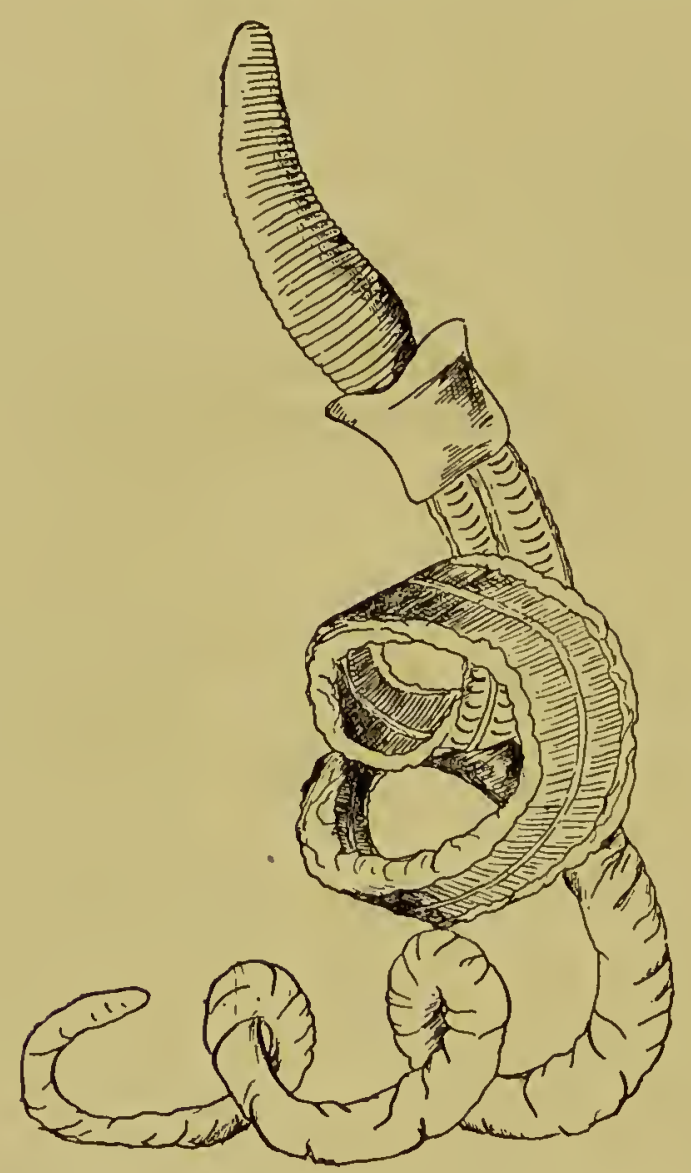

FIG. I47. Balanoglossus. [After Loos, in LEUCKART charts.]

chord and a dorsal nervous system. In this search they have been to a qualified extent successful, although these characters are far from appearing with the same distinctness as in the case of the gill-slits. The nervous system consists in general of a diffuse net-work of nerve fibers lying in the depth of the surface epithelium and occurring everywhere, a very low type of nervous system. This net-work is, however, reinforced and 
formed into four longitudinal cords, slightly differentiated from the rest, a dorsal, a vontral, and two latcral, all of which run the entire length of the animal. Of these the dorsal receives slightly more emphasis than the others, since it continues forward to the base of the proboscis, where it divides into two diverging branches, which encircle it in the form of a ring. As for the notochord, this has been doubtfully identified with a small diverticulum, which arises from the dorsal wall of the pharynx, and extends some distance forward into the proboscis, and this supposition has been greatly strengthened through the recent discovery of an allied form belonging to a new genus (Harrimania) in which the diverticulum is much

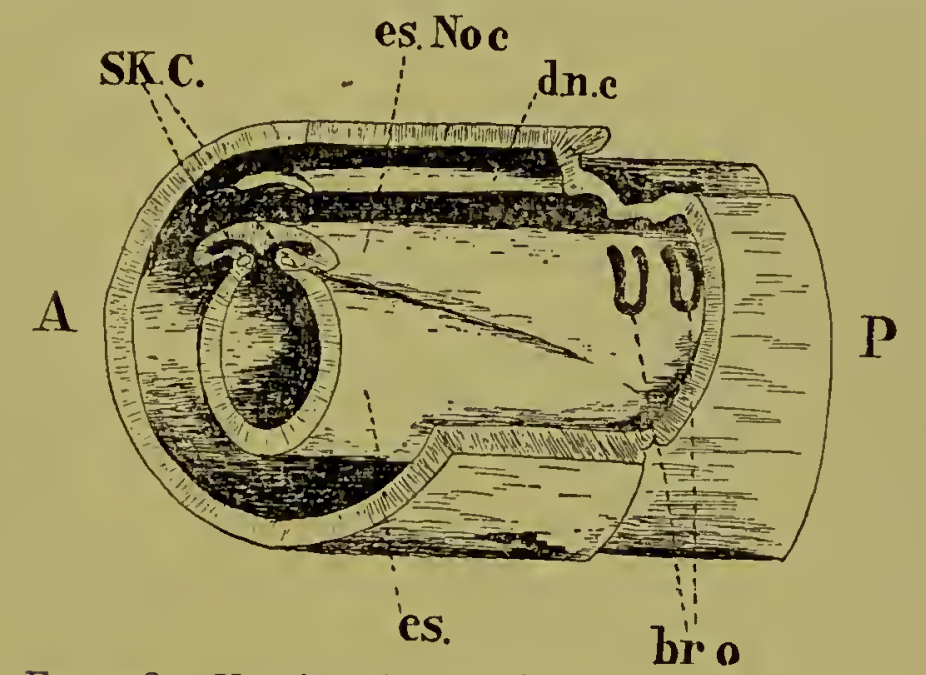

Fig. I48. Harrimania naculosa. [After RitTer.]

Schematic representation of dissection, including collar and small portion of the anterior pharyngeal region. The anterior and posterior aspects are designated as $\mathrm{A}$ and $\mathrm{P}$, respectively. cs, cesophagus; es. No-c, œsophageal notochord; $d$. n. c, dorsal nerve cord; $S K . C$, skeletal crura; $b r$. 0 , branchial orifices.

larger, and in its mode of origin is strikingly similar to that of the true vertebrate notochord, and is thus without much doubt homologous with this organ.

From the testimony afforded by the structure of Balanoglossus and its allied genera (the group Enteropneusta) it may be quite confidently asserted that thesc forms lie ncarly in the line of vertcbrate descent, and represcnt an carlier stage than that of the tunicates. But here the chain secms to end, for Balanoglossus is itself umusually isolated and shows no close affinity to any other invertcbrate types. There is, in such cases, but 
one possible way out, a single remaining clew, and that is, the embryology of the form in question, and even here the primary, historic features may be overlaid with secondary changes rendered necessary as an adaptation, and thus the value of a given feature is often hard to estimate. In the case of Balanoglossus, however, it seems probable that the early development is in great part an actual repetition of the racehistory, but if so, it leads us to surprising and not very satisfying results, for the animal begins life as a minute transparent floating larvà, the Tornaria, furnished with bands of cilia, by which it moves, a larva strikingly like that of star-fish, seaurchins, and other echinoderms, and one which an unprejudiced
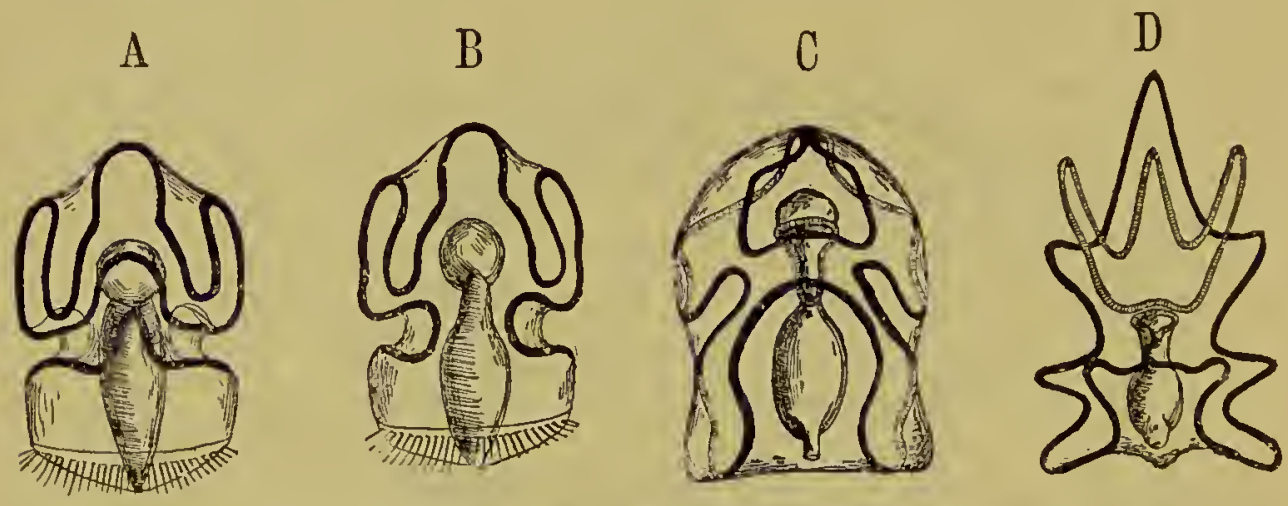

FIG. I49. Comparison of Tornaria larva with larval Echinoderms. [After O. HAMANN.] Main ciliated bands in black, lesser systems crosslined.

(A) Tornaria, ventral view. (B) Tornaria, dorsal view. (C) Auricularia, ventral view. (D) Bipinnaria, ventral view.

mind would not hesitate to classify with these latter. These larvæ are all of about the same size, all bilateral in structure, all transparent and equipped with bands of cilia, and there is even a close correspondence in the manner of disposal of these bands.

In the case of the echinoderms the universal occurrence of such larva is taken everywhere as a proof that they represent an early stage in the history of the Class, and that the ancestors of these radiate, crawling, or sessile, bottom forms were bilateral and pelagic. Now it would be highly improbable that 
an unrelated form should, as an adaptation, so modify its early stages as to resemble these echinoderm larvæ as closely as does the Tornaria, and the only alternative is to accept as a very ancient common ancestor of both echinoderms and vertebrates the form which all these larve may be said to copy; a form having the characteristics common to all, including bilaterality, minute size, transparency, locomotion by bands of cilia, and pelagic life. The lineal descendants of this hypothetical ancestor chose two paths, the one leading to the Echinodermata, the other to Balanoglossus, the Tunicata, Amphioxus, and eventually the Vertebrata.

This theory, although incomplete and unsatisfactory in

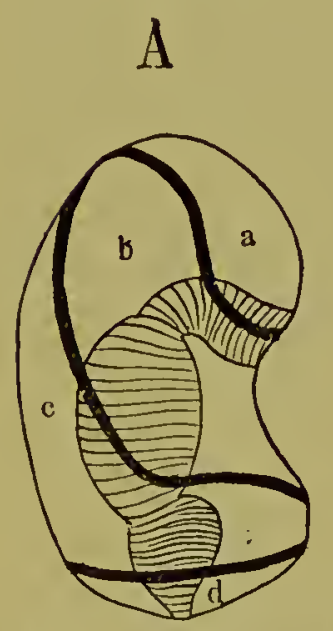

FIG. I50. Comparison of Tornaria and Echinoderm larvæ, lateral views. [After BALFour.]

(A) Tornaria. (B) Auricularia. (C) Bipinnaria.

$a$, apical area; $b$, oral area; $c$, post-oral area; $d$, anal area.

parts, is consistent with the most approved lines of biological thought; it rests upon development as well as adult structure, and bears the indorsement of the majority of investigators at the present time. The weakest part of the argument is that of the significance of the Tornaria larva; and while the acceptance of this gives us very little enlightenment, to abandon it would be to sacrifice but little, and would render the gulf between the adult Balanoglossus and other invertebrates only a little more profound. To summarize in the words of two recent 
authors, " The question of the descent of the Chordata is not solved by accepting their relationship to the Enteropneusta, since this latter group holds an uncommonly isolated position. Only from the structure of the Balanoglossus larva can there be concluded a distant connection with the echinoderms. We must resign ourselves to the thought that at the present time we are not in a condition to assert from what ancestral form the Chordata, and with them Balanoglossus, are to be derived. The origin of the vertebrates is lost in the obscurity of forms unknown to us."

* Korschelt u. Heider. Entwickelungsgeschichte. Jena, I893, p. I465. "Die Frage nach der Abstammung der Chordaten wird durch die Annahme von verwandtschaftlichen Beziehungen derselben zu den Enteropneusten nicht gelöst, da die letzere Gruppe selbst ungemein isolirt dasteht. Nur aus dem Bau der Balanoglossuslarve lässt sich eine entferntere Zusammenhang mit den Echinodermen erschliessen. Wir inüssen uns bei den Gedanken resigniren, dass wir vorläufig nicht im Stande sind, anzugeben, von welchen Urformen die Chordaten und mit ihnen Balanoglossus herzuleiten sind. Der Ursprung der Wirbelthiere verliert sich in das Dunkel uns unbekannter Formen." 


\section{APPENDIX}

\section{CLASSIFICATION OF THE VERTEBRATA.}

The following list of the larger subdivisions of the Vertebrata, arranged in synoptical form, may be of use in explaining the names of groups as used in the body of the work. Through the labors of palæontologists so many forms have been unearthed and so many new groups established that it seems best to include in the list these latter as well as modern animals, especially since many of the extinct groups consist of generalized forms from which several living groups have differentiated. The names of all groups, of whatever rank, that contain living representatives are printed in bold-faced type; those groups in which these latter are represented by but one or two isolated forms are farther designated with an asterisk. In this way the amount of damage wrought in the phylogenetic record, as well as the relative position of the groups, may be seen at a glance. The synopsis follows :-

\section{VERTEBRATA (or Chordata).}

\section{Division I. Cyclostomata.*}

\section{Class I. Marsipobranchii.*}

\section{Sub-Class I. Cyclostomi.*}

Order I. Myxinoidea* (Myxinc, the hag-fish).

Order 2. Petromyzontoidea* (Pctromyzon, the lamprey eel).

Sub-Class II. Ostracodermi.

Order I. Heterostraci (Ptcraspis).

Order 2. Osteostraci (Ccphalaspis).

Order 3. Antiarchi (Ptcrichthys). 
Division II. Gnathostomata.

Super-Class 1. Ichthyoidea.

Class I. Pisces.

Stıb-Class I. Elasmobranchii.

Order I. Pleuropterygii (Cladoselache).

Order 2. Ichthyotomi (Plcuracanthus).

Order 3. Acanthodii (Acanthodes, Diplacanthus).

Order 4. Selachii.

Sub-Order I. Squali (sharks, dog-fish).

Sub-Order 2. Raiæ (skates).

Sub-Class II. Holocephali.*

Order I. Chimæroidei * (Chimara).

Sub-Class III. Dipnoi.*

Order I. Sirenoidei * (Protopterus, Ceratodus).

Order 2. Arthrodira (Coccosteus, Dinichthys).

Sub-Class IV. Teleostomi.

Order I. Crossopterygii.*

Sub-Order I. Haplistia (Tarrasius).

Sub-Order 2. Rhipidistia (Holoptychius; Osteolcpis).

Sub-Order 3. Actinistia (Colacanthus; Undina).

Sub-Order 4. Polypteroidei* (Polypterus; Calamoichthys).

Order 2. Actinopterygii.

Sub-Order I. Chondrostei * (Palconiscus; Acipenser, sturgeoni).

Sub-Order 2. Protospondyli * (Lepidotus; Eugnathus; Amia, bow-fin).

Sub-Order 3. Etheospondyli * (Aspidorhynchus; Lepisostcus, gar-pike).

Sub-Order 4. Isospondyli (Lcptolcpis; herring; salmon; trout).

Sub-Order 5. Eventognathi (carp; minnow; sucker).

Sub-Order 6. Nematognathi (siluroids, e.g: bullheads, cat-fish, etc.).

Sub-Order 7. Haplomi (pike; killifish).

Sub-Order 8. Apodes (eels).

Sub-Order 9. Synentognathi (flying-fish). 
Sub-Order Io. Lophobranchii (sea-horse; pipefish).

Sub-Order I I. Hemibranchii (stickleback).

Sub-Order 12. Acanthopteri (mackerel; cod; perch; sculpin; flounder).

Sub-Order I3. Pediculati (angler-fish; frog-fish).

[In the Class of Pisces the process of extinction has left in our modern fauna five more or less isolated groups, usually treated as Orders. These are the Selachii, Holocephali, Dipnoi, Ganoidei and Teleostei. The first is the only remaining group of the elasmobranchs, of the second and third the only fossils known are much like those of the present day and their affinities have thus not been definitely traced; and the fourth and fifth are respectively the earlier and later types of teleostomes.

The living ganoids are very few in number and are for the most part unrelated to one another. There are but two crossoptergyians, and but one or two living genera of each of the three groups Chondrostei, Protospon$d y l i$, and Ethcospondyli. At this point the "ganoids" are considered to end, and the remaining Sub-orders, beginning with the Isospondyli, are included with the teleosts (i. e. Teleostei, to be carefully distinguished from Teleostomi, the larger group).

Sub-orders $\dot{4}-8$ are sometimes grouped as the Physostomi and the remaining sub-orders, 5-I3, as the Physoclysti. In the former of these the air-bladder retains its connection with the alimentary canal; in the latter this becomes lost during development and the air-bladder is a closed sac.]

Class II. Amphibia (Batrachia).

Order I. Urodela.

Sub-Order I. Perennibranchiata (Necturus; Siren).

Sub-Order 2. Derotremata (Cryptobranchus).

Sub-Order 3. Salamandrida (newts; salamanders).

Order 2. Gymnophiona (subterranean forms, with-

Order 3. Anura. out limbs or eyes).

Sub-Order r. Aglossa * (Surinam toad).

Sub-Orcler 2. Arcifera (toads; tree-toads).

Sub-Order 3. Firmisternia (frogs). 
Order 4. Stegocephali.

Sub-Order I. Branchiosauria (Branchiosaurus).

Sub-Order 2. Aistopoda (snake-like forms, without limbs).

Sub-Order 3. Microsauria (small forms, in shape like salamanders).

Sub-Order 4. Labyrinthodontia (Archagosaurus, Mastodonsaurus).

[Of the four Orders of Amphibia, one is entirely extinct and the other three essentially modern, and with few traces of older representatives. With regard to the extinct group, that of Stegocephali, it could be placed in the list either at the first or the last, since it shows strong affinities to both ganoids and reptiles and thus lies intermediate between the two. The modern Orders seem to have arisen directly from the Stegocephali, the Urodela being the least altered and hence the most important morphologically. This complex relationship between the groups mentioned, the Ganoidei, Stegocephali, Urodela, Reptilia, etc., cannot thus be represented in linear lines but may be partly expressed in the form of a tree, as in the diagram given in Chapter II. (p. 28). The three modern Orders of Urodela, Gymnophiona, and Anura, are quite distinct from one another, and, the first and third especially, are well represented in the living fauna.]

\section{Super-Class II. Sauropsida.}

Class III. Reptilia.

Order I. Theromorpha.

Sub-Order I. Pariasauria (Pariasaurus).

Sub-Order 2. Theriodontia (Cynognathus; Tritylodon).

Sub-Order 3. Dicynodontia (Dicynodon; Gordonia).

Order 2. Sauropterygia (Plcisiosaurus; Cryptocli$d u s)$.

\section{Order 3. Chelonia.}

Sub-Order I. Cryptodira (the majority of living turtles).

Sub-Order 2. Pleurodira (Miolania; Chclys).

Sub-Order 3. Trionychia (soft-shelled turtles).

Order 4. Ichthyopterygia (Ichthyosaurus). 
Order 5. Rhynchocephalia.*

Sub-Order I. Proterosauria (Proterosaurus; $\mathrm{Pa}$ laohatteria).

Sub-Order 2. Rhynchocephalia vera * (Sphenodon [Hatteria], the only living representative of the Order).

\section{Order 6. Squamata.}

Sub-Order I. Dolichosauria (Dolichosaurus).

Sub-Order 2. Pythonomorpha (Mosasaurus).

Sub-Order 3. Lacertilia (lizards).

Sub-Order 4. Ophidia (snakes).

Order 7. Dinosauria.

Sub-Order I. Theropoda (Anchisaurus).

Sub-Order 2. Sauropoda (Brontosaurus).

Sub-Order 3. Ornithopoda (Iguanodon; Stego-

\section{Order 8. Crocodilia.*}

$$
\text { saurus). }
$$

Sub-Order I. Parasuchia (Belodon).

Sub-Order 2. Mesosuchia (Pelagosaurus; Teleosaurus).

Sub-Order 3. Eusuchia * (Thoracosaurus; Crocodilus; Alligator).

Order 9. Pterosauria (Pterodactylus; Rhamporhynchus).

[As is indicated above by the difference in type the process of extinction in the group of Reptilia has gone very far, leaving but four isolated spots to be represented among the living forms; ( $\mathrm{I}$ ) the Chelonia, essentially a modern group, (2) the Rhynchocephalia, represented by a single living species, (3) the last two Sub-Orders of the Squamata, the lizards and snakes, and (4) a very few modern representatives or the Crocodilia. In arrangements in which living forms are alone taken into consideration, these are given as five Orders; the lizards and snakes count as two; in the earlier works Sphenodon was counted among the lizards, redicing the number to four. The Orders, as thus arranged, are as follows: Chelonia, Rhynchocephalia, Lacertilia, Ophidia, Crocodilia.]

Class IV. Aves.

Order I. Saurura (Archcopteryx; Laopteryx).

Order 2. Odontormæ (Ichthyornis; Apatomis). 
Order 3. Odontoholcx (Hesperornis; Lestornis).

Order 4. Eurhipiduræ.

Sub-Order I. Dromæognathi.

Section I. Struthiones (ostrich, casuary).

Section II. Aepiornithes (Appiomis).

Section III. Apteryges* (Apteryx).

Section IV. Crypturi (tinamoo, Crypturus).

Section V. Gastornithes (Gastornis).

Sub-Order 2. Impennes (penguins).

Sub-Order 3. Euornithes [a recent group, beginning in the Eocene].

Section I. Desmognathæ (ducks; herons; eagles; hawks; owls; cuckoos; kingfisher; trogon; parrot).

Section II. Schizognathæ (grebes; loons; gulls; snipes; grouse; quails; pigeons; humming-birds; woodpeckers).

Section III. Egithognathæ (Passeres, a group which includes over one-half of the species of living birds).

\section{Super-Class III. Mammalia.}

Class V. Mammalia.

Sub-Class I. Prototheria * [Ornithodelphia].

Order I. Pantotheria (Dromotherium; Amphilestes).

Order 2. Multituberculata (Ctenadon; Polymastodon).

Order 3. Monotremata * (Ornithorhynchus; Echidna).

Sub-Class II. Eutheria.

Super-Order I. Didelphia [Marsupialia].

Order I. Polyprotodontia (opossum; Thylacinus).

Order 2. Paucituberculata* (Cinolestes).

Order 3. Diprotodontia (Petaurus; wombat; kangaroo).

Super-Order 2. Monodelphia [Placentalia].

Order I. Insectivora (moles; shrews; hedgehog).

Order 2. Cheiroptera (bats).

Order 3. Galeopithecidæ* (flying lemur).

Order 4. Edentata. 
Sub-Order I. Tubulidentata * (Orycteropus, the earth-hog or " aard-vark.").

Sub-Order 2. Pholidota * (Manis, a scaled animal of Asia and Africa).

Sub-Order 3. Xenarthra (Glyptodon; Megatherium; Grypotherium; ant-eaters; armadilloes; sloths).

Order 5. Rodentia.

Sub-Order I. Tillod ontia (Tillotherium; Esthony'x).

Sub-Order 2. Duplicidentata (hares; rabbits).

Sub-Order 3. Simplicidentata (squirrels; beavers; mice).

Order 6. Primates.

Sub-Order I. Mesodonta (Adapis; Anaptomorphus).

Sub-Order 2. Lemuroidea (Lemurs; Chiromys; Tarsius).

Sub-Order 3. Anthropoidea.

Division I. Platyrrhini (Hapale; Midas; Cebus).

Division 2. Catarrhini (Cercopithecus; Scmnopithecus; Gorilla; Homo).

Order 7. Creodonta (Arctocyon; Hycenodon).

Order 8. Carnivora (cats; dogs; bear; weasel).

Order 9. Pinnipedia (seals; walrus; sea-lion).

Order IO. Cetacea (whales; porpoises).

Order II. Condylarthra (Phenacodus).

Order I2. Hyracoidea* (Procavia [Hyra.r]).

Order I3. Amblypoda (Coryphodon).

Order I4. Sirenia* (manatee; dugong).

Order I5. Proboscidea * (Mastodon; elephants).

Order 16. Ancylopoda (Homalodontotherium).

Order I7. Typotheria (Typotherium).

Order I8. Toxodontia (Toxodon).

Order 19. Litopterna (Proterotherium).

Order 20. Perissodactyla (Palcotherium; Titanotherium; rhinoceros; horse).

Order 21. Artiodactyla. 
Sub-Order I. Suina (Elotherium; pigs; peccaries; hippopotamus).

Sub-Order 2. Tylopoda (Oreodon; camel; llama). Sub-Order 3. Anthracotherioidea (Anthracotherium).

Sub-Order 4. Dichobunoidea (Dichobune; Anoplotherium).

Sub-Order 5. Traguloidea (Tragulus, several small species in E. Indies).

Sub-Order 6. Pecora (deer; sheep; cattle; giraffe).

[The arrangement of animal groups in the form of a list, in which they follow one anothcr in a single serics, is scldom more unsatisfactory than it is in the casc of mammals. The inadequacy of this method in expressing the true relationships is seen if the list be compared with the phylogenetic tree given in Chapter II. (p. 36). There are scveral distinct stems to be followed and the order in which they are taken in a list is largely a matter of preference. Here the attempt is made to proceed from the generalized to the more specialized ones, and thus the main stem of the Insectivora is taken first; then that of the Primates, and lastly the complex and highly specialized branch leading to the carnivore and ungulate Orders. This arrangement has the advantage of emphasizing the primitive and rather generalized structure of the Primates as comparcd with the groups just mentioned, a comparison entirely lost sight of by the usual arrangement, which places the apes and man at the top. If the arrangement be made solely on the basis of the development of the nervous system thcre can be no question of the rightfulness of this position; but if all the systems be taken into consideration, and especially the bones, muscles and teeth, which in other groups form the principal criteria for the purpose of classification, the Primates are found to have retained a larger number of primitive characters than any other placental group with the exception of the Insectivora, Rodentia, and Edentata, and thus to stand far lower in the scale of specialization than the manifold descendants of the Creodonta and Condylarthra.

The arrangement of the subdivisions of the Primates given above is a conscrvative onc, and will accord with the most of the literature on the subject. Ccrtain inportant modifications have, however, been recently pro- 
posed, based upon a more complete study of anatomical characters. Through these the extinct genus Anaptomorphus, which is probably very near the direct ancestral line leading to Man, has been placed in Sub-order 3, Anthropoidca, and with it has been placed the living genus, Tarsius, a closely related form.

For convenience in classification all the descendants of the Condylarthra, together with this latter, but excepting the aberrant Sirenia, are often grouped together under the single Order of Ungulata, or hoofed animals, connected both by descent and by the common peculiarities embodied in the name. This will include Orders II-2I in the above list. In the same way the Creodonta may be included in the Carnivora, although the Cetacea are treated as a separate, though allied group. The Galeopithecoidea are often included within the Insectivora. This reduces the Orders of placental mammals to nine, viz: Insectivora, Cheiroptera, Edentata, Rodentia, Primates, Ungulata, Sirenia, Cetacea, Carnivora. There are, of course, as in all groups of animals, many other possible arrangements, the differences being based on the relative value of the various groups, their relationships to one another, and the comparative degree of specialization of each; points upon which there is much room for difference of opinion.]

Owing to the extinction of so many of the groups, especially those forming the connection between two others, a classification that rests wholly upon living forms is far from complete and in some points differently arranged from one that includes all known forms. Thus among the fishes the selachians alone are left of all the elasmobranchs; a few remnants remain of the first few Orders of teleostomes, isolated from one another and from the others; and of the Holocephali and Dipnoi only a few species occur. Among the amphibians, the Stegocephali, the most inportant Order of all, have disappeared entirely, and among the reptiles a still greater destruction has left but four isolated spots in a once continuous history. This loss has affected also all the transition forms between reptiles and the modern type of birds. and completely isolated the Aves from all related forms. The mammals are still rich in Orders but the synthetic types that once united them have long since disappeared. Without going into the Sub-Orders this abbreviated classification, in some respects clifferent from the above synopsis, may be given here for convenience in comparison. Only the gnathostomes may be considered. 
SYNOPSIS OF VERTEBRATA (living forms alone).

Class I. Pisces.

Sub-Class I. Selachii.

Sub-Class II. Holocephali (often considered with the

Sub-Class III. Ganoidei. previous group).

Sub-Class IV. Teleostei.

Sub-Class V. Dipnoi.

Class II. Amphibia.

Order I. Urodela.

Order 2. Gymnophiona.

Order 3. Anura.

Class III. Reptilia.

Order I. Chelonia.

Order 2. Lacertilia (including Sphenodon).

Order 3. Ophidia.

Order 4. Crocodilia.

Class IV. Aves.

Sub-Class I. Ratitæ (running birds; with flat breastbone, e.g. Ostrich).

Sub-Class II. Carinatæ (flying birds; with keeled breast-bone).

Class V. Mammalia.

Sub-Class I. Prototheria.

Order I. Monotremata.

Sub-Class II. Eutheria.

Super-Order I. Didelphia (Marsupialia).

Super-Order 2. Monodelphia (Placentalia).

Order I. Edentata.

Order 2. Insectivora.

Order 3. Rodentia.

Order 4. Cetacea.

Order 5. Sirenia.

Order 6. Ungulata.

Order 6a. Proboscidea (occasionally separated from the Ungulata).

Order 6b. Hyracoidea (occasionally separated from the Ungulata). 
Order 7. Carnivora.

Order 8. Cheiroptera.

Order 9. Primates.

In this arrangement there will be noticed especially the separation of ganoids and teleosts, the small number of reptilian Orders, the complete isolation of the birds, and the arrangement of the mammalian Orders in such a way as to bring the Primates at the top. Certain of these faults, like the isolation of the birds, have been corrected for some time, the separation of ganoids and teleosts is a convenient one for purposes of comparative anatomy, and is employed for this purpose in the body of this work. The arrangement of the mammals to show the supremacy of Man is natural, and is based, of course, in part, on the high development of the brain, but much is due to natural human pride which recognizes man's mental supremacy, and feels that a supremacy in physical structure must also be granted. As a matter of fact, in all other aspects save that of the brain, the apes and man are rather primitive in their structure and show a far less bodily specialization than almost any of the other living Orders of mammals, the Insectivora and a few others being alone excepted. 



\section{INDEX}

(In using this index consult, for a given animal, both the scientific and common names.)

\section{A}

abdominal ribs, I4I

abomasus, 292

achselbogen, 25 I

acinous glands, 97, II 2

Acipenser, I7 I

acœlous vertebræ, I3I

acoustic hairs, 467

acoustic maculæ, 409

adenoid tissue, 362

afferent nerves, 408, 435

air-bladder, 270, 3IO, 3 I I

air-cells, 3 I 4

albatross, 478

alimentary canal, 258, 259, 260, $261,262,263,264,265,266,298$, 299,365

alisphenoids, I48

allantoic arteries, 70, 7I

allantoic veins, $70,7 \mathrm{I}$

allantois, $70,7 \mathrm{I}, 322,323,324,347$, 377,378

alveoli, of jaws, 273

alveoli, of lungs, 3 I4

Amblypoda, 38

Ammocœes, 477

Ammon's horn, 4I7

amnion, 70, $7 \mathrm{I}$

Amniota, I9, 70, 78, I 74, 373, 375, $378,383,384,385,393,427,457$ amniotic fluid, $70,7 \mathrm{I}$

amplibians, I I0, I48, I66, I70, I72,

I74, I78, 200, 202, 203, 220, 237,

$239,245,253,27 \mathrm{I}, 272,283,285$,

288, 29 I, 294, 296, 308, 310, 319,

$322,328,33 \mathrm{I}, 344,346,347,352$,

$353,356,358,369,375,383,385$,

$392,398,409,415,416,419,425$,

$427,439,444,456,464,465,469$,
amphibians-(Continued)

$472,479,484,488,489,49 \mathrm{I}, 504$, $5 \mathrm{I} 8$

amphicœlous vertebræ, 127

Amphioxus, 24, 26, 27, 28, 29, 44, $59,61,63,66,67,76,123,143$, I52, I54, I62, I63, 200, 260, 267, $289,290,303,305,306,353,365$, $4 \mathrm{II}, 432,436,443,445,460,46 \mathrm{I}$, $476,503,5 \mathrm{I} 5,5 \mathrm{I} 7,52 \mathrm{I}, 525,526$, $527,528,529,53 \mathrm{I}, 533,534,537$ amphirrhine condition, 476

Amphiuma, 305, 3I3

amplexation, 383, 397

ampullæ, of semicircular canals, 487 ampullæ, of slime canals, 47 I

anal fin, 164

anal sacs, II3

Anamnia, 70, 78, 384

Anarrhichas, 489

Ancylopoda, 38

angulare, 156

annelids, 368, 460, 5I2, 5I3, 5I4,

$515,517,5$ I $8,519,520,524$

annelid theory of vertebrate ances-

try, 513, 520

ansa hypoglossi, $45^{8}$

ant-eater, 3I6, 393

anterior girdle, I30

anthropoids, 38, 4I, 43, 45, 90, 2I 4, $226,230,25 \mathrm{I}, 256,298,409,447$,

$480,496,500,50.4$

antitropists, 244

antrum of Highmore, 482

Anura, 3I, 220, 3I3, 355, 486, 493, 495

anus, 258, 259, 296

aorta, aortx, $320,326,339,35 \mathrm{I}, 354$

apes, 45, 2I $4,223,229,253,297$, 390

apical pads, 9I 
appendages, 510

appendicular muscles, I90, I92, I93

appendicular skeleton, I22, 162

appendix, 297, 298

appendix testis, 392, 394

aqueductus ccrebri, 4I2

aqueductus Sylvii, 4I2

aqueductus vestibuli, 486

aqueous humor, of cyc, 501

arachnoids, 522,524

arachnoid theory of vertebrate ancestry, 522, 525

Archaopteryx, I9, 32

archencephalon, 4I I , 443, 527

archetype, 500, 509

archipterygium, I76, I85

archisternum, I38, I4I

area centralis, of retina, 499

armadillo, 85, 393

Artemia, 55

arterial arches, 320, 321, 328-339

artery, or arteries:- 318,328 ; allantoic, 322; anonyma, 332 ; aorta, aortæ, 320, 326, 339, 35I, 354; aortic arch, 33I, 356; afferent branchials, 325,329 ; arterial arches, 320, 32I, 328; branchials, afferent, 325, 329; branchials, efferent, 326; carotid, 321, 326, 332-339; carotis cerebralis, 333 , 338; caudal, 339 ; common carotid, 338; ductus arteriosus, 33I; ductus botalli, 33I ; efferent branchials, 326 ; external carotid, 338; hypoglossal, 335 ; iliac, 322, 326,339 ; innominata, 332 ; infra-orbital, 335 ; intercostal, 339 ; internal carotid, 338; ligamentum arteriosum, 330; ligamentum botalli, 330, 33I ; lingualis, 335; lumbar, 339; mandibularis, 335, 338; maxillaris, 335 ; mesenteric, 339, 340; posterior aorta, 320; pulmocutaneous, 330; pulmonary, 330; 354; sacralis media, 339, 340; segmental, 335; stapedialis, I59, 337,338 ; subclaviæ sccundariæ, 331 ; subclavian, $322,326,332$, 339 ; supra-orbital, 335 ; umbilical, 322 ; vertebralis cerebralis, 335 . articillare, I59, 495

articulates, 258, 259, 463

articulation of jaw, 450

Artiodactyla, 39

arytænoids, I6o, 3r 3

Ascalabotx, 487

Ascaris, 54

assimilation, 2

Ateles, 254

atlas, I33

atrioporc, of Amphioxus, 526

atrium, 324, 353, 357

auditory hairs, 488

auditory ossicles, I59

auditory tube, 494

auricula, 496, 497

Auricularia, 536, 537

axial muscles, 190, 192, 200, 217

axial skeleton, I22

axillary arch, 25I

axis, I33

axolotl, 440

\section{B}

babyroussa, 274

Balanoglossus, 26, 303, 533, 534, $535,536,537$

basihyal, I6o, 284

basioccipital, 150

basipterygium, I69, I75

basisphenoid, I50

bat, $183,184,292,394,433,496$

bats, insectivorous, $30 \mathrm{I}$

beak :- of birds, 84 , I05; of turtles, 84 .

beaker cells, i Io

belly, of a muscle, 197

biogenesis, law of, I5, 58

Bipinnaria, 536, 537

birds, III, I72, I74, I82, I83, I84, 201 , $202,203,220,249,253,263,268$, $272,290,296,315,352,353,354$, $355,356,360,375,377,385,386$, $398,414,415,416,419,426,437$, $439,465,475,483,488,489,491$, $495,500,504$

birds, toothed, 272

bisexual, 49

bladder, 377,378

blastocœle, 59

blastodernic vesicle, 73

blastula, $5^{8}$ 
blood, 3I8, 356

blood vessels, $65,79,318$

body axis, 6o, 6I

body, of a vertebra, 127

body wall, 365

bone complexes of skull, I5I

bone, or bones (see skeletal elements)

bony labyrinth, 492, 493

Bovidæ, 276

Bowman's capsule, 373,374

brachial plexus, 438, 439, 440, 44I

brain, communication with outside world, 409

brain, development of, 410, 427

branchial arches, I55

branchial system, 260

Branchiostoma (see Amphioxus)

broad ligament, of uterus, 387,390

bronchi, origin of, 270

bronchioli, 3I5

Bubo, 489

buccal glands, 285

bulbo-urethral glands, . 403

bull-heads, 475

bursa inguinalis, 395

bursa ovarica, 387

\section{C}

caducibranchiate amphibians, 307

cænogenetic characters, I6

canalis centralis, 62

canalis centralis, of spinal cord, 406

canals of Lorenzini, 469, 473, 502

Canidæ, 286

canines, 275

Canis, 87

capillaries, $3 \mathrm{I} 8$

carapace:-armadillo, 85; turtles, 105

Carchesium, 8

cardiac end of stomach, 29 I

Carnivora, 37, 38, I75, 239, 250, $29 \mathrm{I}, 394,402,417,480,484$

carotid gland, 288

carpus:-nomenclature of, $\quad 177$, I78, I79, I80; various forms of, I79; primitive condition of, I79; supernumerary clements, I8I.

cartilage, or cartilages (see skelctal clements) cartilage bones, I48

cartilago lateralis, I60

cassowary, 440

cat, 27I, 276, 287, 419, 49I

Catarrhini, 4I, 278

cauda equina, 43I

caudal fin, I64

caudal vertebræ, I30

cavernous tissue, 398, 399, 404

cell membrane, 3

centers of ossification, 83, I48

centrifugal nerves, 435

centripctal nerves, 408,435

centrum of a vertebra, I27, 508, 5 IO

Cephalophus, I I 2

cephalopod eye, 499

cephalopods, 499, 501

cerato-hyal, I6o, 284

Cercopithecidæ, 45

cerebellum, 4I2, 4I7, 425, 426

cerebral hemispheres, 4II, 4I3, 4I6

cerebrum, 4II, 4I5-4I8, 425

cervical fistula, 269

cervical intumescence, in cord, 433

cervical rib, I38

cervical vertebræ, I30

Cervus, 299

Cestracion, I86

Cetacea, 20, 38, 40, 98, II3, II6, I75, I $84,275,277,285,300,316$, 393

Chelonia, 33, 44

Chimæra, 489

chimpanzee, 45

chiridium, derivation from fin, I86, 220

Chironomus, 429

Chiroptera, 37

chiropterygium, I67, I84

chiropterygium, typical form of, I77

chiropterygium vs. ichthyopterygium, I84-I 88

Chlamydoselachus, I86

choanx, 269, 478

Cholœpus, II2, II3

chondrocranium, I45, I46, I53, I56

chordo gubernaculi, 395

Chordata, 538

chorioid plexuses, 4I3, 4I9, 420

chorion, $70,7 \mathrm{I}, 72$ 
chorionic villi, 70, $7 \mathrm{I}$

chorioid coat of eye, $498,50 \mathrm{I}$

chorioid fissure of eye, 500

chromatin, 54, 58

chromosomes 54,58

chromosomes:-number of, 54, 55, 57

Chrysochloris, II2, II3

ciliary ganglion, 447

ciliary glands, 505

circulation, in selachians, 324,325

circumvallate papillæ, 476

cisternæ chyli, 362

clavicle, I73, I74, I75

claws, I05, 106, 107

cleithrum, I73

clitoris, 398, 404

cloaca, 266, 296, 370, 375, 378, 38I, 383,403

cloacal cœca, 266

cloacal glands, i io

closed type of circulation, 317,318

Cobitis, 303

coccyx, I 35

cochlea, 488, 491, 492

cœca, of intestine, 296, 297, 298

Colenterata, 59, 60

Cœlogenys, 86, 49I

cœlom, 64, 66, 258, 365, 368, 369,

$37 \mathrm{I}, 373,374,375,376,378,38 \mathrm{I}$, 382

colic cœca, 266

colon, 298

colon labyrinths, 298

columella auris, 494

columns, of spinal cord, 434

commissures, of brain, 418, 427

concha, of ear, I6r

conchæ, of nose, $480-482$

concrescence theory, 280

Condylarthra, 38, 40

conjunctiva, 498, 504

contact sense, 468

continuity of germ plasm, 58

continuity of life, I2

conjugation, $6,49,50,5 \mathrm{I}$

conus arteriosus, 324, 353

conus inguinalis, 395

Cooper's fascia, 396

convolutions, of brain, 4I7

copulation, 49, 380, 38I, 398 coracoid, 174

coracoid process, I75

coral polyps, 60

corium, 76,78

cornea, 50I

corpora bigemina, 425

corpora cavernosa, 399, 404

corpora striata, 4I3, 4I6, 426

corpora quadrigemina, 425

corpus callosum, 4I8

corpus cavernosum urethræ, 404

corpus fibrosum, 398

corpus spongiosum, 404

cortex cerebri, 4I7

costal cartilages, I36

cotyledonal placenta, 72

cranial nerves, $427,442-458$

cremaster, 396

Creodonta, 37, 40

cricoid, 3 I4

cristæ acusticæ, 490

Crocidura, 92

crocodile, I64, 272, 360, 377, 398,

$399,483,491,495$

crop, 290

crura cerebri, 426,427

crustaceans, 259, 463

Cryptobranchus, 3I, 235, 305

crystalline lens, 423, 498, 50I, 502

ctenoid scales, 83

cuticula of invertebrates, 76

cutis, 76

Cuvier, theories of types, 506, 507 cyanosis, 357

cycloid scales, 83

Cyclops, 55

cyclostoma, 266

cyclostomes, 28, 29, 44, 49, 200, $267,286,287,289,305,347,365$, $369,379,380,4 I I, 420,433,437$, $444,46 \mathrm{I}, 487,488,489,490,5$ I 6

\section{$\mathrm{D}$}

Darwin, Charles:- theory of descent, $5 \mathrm{IO}, 5 \mathrm{I} 2$

Darwin's point, on ear, 497

Dasyurus, 86

decidua, 72

deciduate placenta, 73

deer, 299

Delphinus, 277 
dental formulæ, 276-277

dermal bones, $79,82,146,147,156$

dermal scutes, 79, 83, I47

dentary, 156

dentine, 79

dermal canal system, 448

descensus ovariorum, 392

descensus testiculorum, 203, 392, 393

Desmodus, 292

Desmognathus, 309, 343

developmental history, I5

development of hair, 96

devil-fish, 499

diaphragm, 3 I6

diapophyses, I38, 508, 5 10

Didelphia, 36

diencephalon, 4II, 4I8-425, 443

diffuse placenta, 72

digestive cavity, 258, 315

digestive glands, 262

digits:- names of, I77; number of, I82; reduction of, I82.

dinosaurs, 32

diphyodont dentition, $28 \mathrm{O}$

dipnoans, $30,176,449,453,454$, 457,478

discoidal placenta, 72

dissepiments, 367

diverticula of intestine, 266

dog, 27r, 286

dog-fish, 4I4, 4I5, 4I9, 446, 46I, 478

dorsal fin, I64

dorsal nerves, 436

dragon-fly, 428

drum of ear, $493-496$

Dryopithecus, 44

duck, 296

duck-billed platypus, 33

duct of Cuvier, 322

ductus arteriosus, 33I

ductus Botalli, 33I

ductus Cuvieri, 322

ductus deferens, $377,383,385,386$,

$392,393,394,4$ or

ductus endolymphaticus, 486

ductus venosus Arantii, $3+8$

duodenum, 266, 294

duplex placenta, 72

Duplicidentata, 37
$\mathrm{E}$

ear, 485-497

ear, of anthropoid, 496-497

ear, external, 16r, 496, 497

earth-worm, 460

ecdyses, 77 ; of epitrichium, 95

Echidna, mammary pocket of, II4

Echidna, 33, 288

echinoderms, 26, 258, 536-538

ectoderm, 60, 63, 257

ectoturbinalia, 480

Edentata, 37, 29r

eel, 432

efferent nerves, 435

eggs, 50, 5I, 52, 53, 56, 57, 69, 73

elephant, 274,484

enamel, 79

endochondral ossification, I48

endoderm, 60, 63, 257

endolymph, 488, 492

endolymphatic space, 357

endolymphatic cavity, 493

endolymphatic duct, 486

endoskeleton, I22

endoskeleton, parts of, I22

endostyle, 289, 528, $53 \mathrm{I}$

endoturbinalia, 480

Enteropneusta, 260, 535, 538

entocone, 279

epi-hyal, I60, 284

epidermic warts, $88,89,90$

epidermis, 76,408

epididymis, 385, 386, 394

epiglottis, I60, 3 I 3

epimere, 64,65

epimeres, I90, 245

epimeric muscles, I90, 192

epi-otics, 148

epiphysis, 420, 42I, 422

episternum, I4I

epithelial bodies, 363

epithelial corpuscles, 286

epitrichium, 95

eponychium, 95

epoöphoron, 39I, 394

Equus, 390

erectile tissue, 398, 399

Erinacel1s, 402, 49r

erythrocytes, 318,364

ethmoid, I48

ethmo-turbinal, 479,480 
Eurypterus, 524

Eustachian tube, 269, 494

Eutheria, 35

excretory tubules, 365,366

exoccipitals, I 48

exoskeleton, 79

cxoskeleton of reptiles and birds, 84

external ear, I6I, 496, 497

external genitals:-male, 398-40I ; female, 403; development of, 403-405.

\section{exuvia, 77}

external nose, 484

eye, $497-5.05$

eyeball, muscles of, 503

eyeball, skeletal elements of, I44,

I45, I5 I

eyeball, size of, 503

eyebrows, 505

eye-capsules, I45

eye, development of, 422, 423

eyelids, 498,503, 504

eye of cephalopod, 499

\section{$\mathrm{F}$}

Fallopian tube, 387

fascia cremasterica, 396

femoral glands, of lizard, IIo, III

fenestra cochleæ, 494

fenestra ovalis, of ear, 493

fertilization, 50, 379, 397, 398

fetal circulation, 320-324, 332-339, $342-352,357$

field-mouse, 91

fifth ventricle, 418

filum terminale, 43I

fins of fishes, I64, I67

fin, development of, I68, 218-219

fin-fold, 327

fin-fold theory, 163,238

fin rays vs. digits, 184,185

fin spines, 162

fin vs. hand, I84-I88

fishes, 29, 30, II0, I48, I63, I64, I65, I67, I75, I92, 200, 245, 247 , $262,263,268,27 \mathrm{I}, 272,285,29 \mathrm{I}$, 296, 304, 306, 307, 310, 311, 324, $330,339,342,347,352,353,355$, $358,375,409,425,436,437,452$, $460,465,468,469,47 \mathrm{I}, 484,485$, $488,490,49 \mathrm{I}, 503,517,519,527$. fission, 4,5

fissues, of brain, 4I7

fissue of Rolando, 4I 7

fissue of Sylvins, 4I7

flat-worms, 520

flexures, in brain, $4 \mathrm{I} 4$

fly, 428

fly, nervous system of, 428

foliate papillæ, 476

foramen epiploicum, 294

foramen interventriculare, $4 \mathrm{II}, 4 \mathrm{I} 6$, 420

foramen of Monro, 4II

foramen ovale (of heart), 357

foramina of Majendie, 4I3

forebrain, 4II, 443

fossa rhomboidalis, 427

fourth ventricle, of brain, 412

fovea centralis, of retina, 499

free-limbs:-development of, 218, 219; early history of, 175 , I76, I77; modifications of, I65, I82, I83, 184 ; nomenclature of, 178 ; origin of, I65; serial homology of, I78; typical skeleton of, I76. free nerve endings, 473

friction ridges, 90, I07; formation of, 88, 89, 90; ground plan of, 92 ; relation to pads, 90, 91.

friction ridge patterns, 92,93

frog, $198,263,264,285,290,307$,

3I 3, 359, 37I, 397, 420, 426, 428, $430,439,440,489,526$

frontal organ, 420

fundus of stomach, 292

furcula, I74

G

galea aponeurotica, 255

Galeopithecus, 37

gametes, 7, 49, 55

ganglion, or ganglia:- buccale, 456 ; ciliary, $447,452,464$; gasserian, 448, 456; geniculare, 454, 456; jugulare, 453, 456; laterale, 453,456 ; list of, in head, 456 ; mandibulare, 456 ; of articulates, $428,429,463$; of fly, 428, 429; of myriapod, 428; otic, 45I, 452, 455,464 ; ophthalmicum profundum, 456; ophthalmicum superficiale, 456 ; petrosum, 454,456 ; 
ganglion, or ganglia-(Continued)

semiluna re, 448,449 ; spinal, 436 ; spheno-palatine, $452,455,456$, 464; submaxillary, 452,464 ; sympathetic, $45 \mathrm{I}, 463$.

ganoids, 29, 44, I48, I49, I70, I7I,

I73, I76, I84, 230, 270, 3II, 369, 4 I6, 444

ganoid scales, 8I

Gärtner's duct, 39I

gastrocœle, 59, 258, 365

gastrula, 59, 61, 62, 257, 365

gall-bladder, 259

gecko, 487

geese, 296

genital cleft, 403

genital ridge, 403

genital tubercle, 403

germ cells, 7, 366, 368, 378, 379

germ glands, 49, 366, 367, 391, 394

germ layers, derivatives of, 74, 75

germ plasm, continuity of, 58

germinal epithelium, 378, 379

gibbon, 45

Gigantostraca, 524

gill arches, fifth pair, 312

gill-flap, 306

gill, innervation of, 453,454 ; of Amphioxus, 526, 527; of Balanoglossus, 533, 534; of tunicates, $530,531$.

gills, $302-307$

gill-slits, 267, 268, 303, 305

gill system, 260

gland, or glands:-acinous, 97,

II 2; anal sacs, II3; beaker-cells,

IIO, 262; buccal, 285 ; bulbourethral, 403; carotid, 288; ciliary, 505; cloacal, I Io; femoral, of lizard, IIo, I I ; germ, 49; glandulæ ductus deferentis, $40 \mathrm{O}$; glandulæ vesicales, 401 ; harderian, 504; integumental, 78, I07; intermaxillary, 285; labial, 285; lacrimal, 504; lingual, 285; mammary, 33, II4-II8; meibomian, II3, 505; mesenteric, 362; molar, 286; musk, IIo; necrobiotic, IIo; odoriferous, II4; of mouth cavity, 285, 286; of mucosa, 262; orbital, 286; parotid, 286 ; preputial, II3, 403; prostate, 403; gland, or glands-(Continued)

rectal, II4; retrolingual, 286; salivary, 286; sebaceous, II3; sublingual, 285; submandibular, 285 ; submaxillary, 285 ; sweat, II2, II3; tarsal, II3, 505; thymus, 286, 287, 290, 363; thyreoid, $286,289,290,363$; types of, I08, I09; Tyson's, II3; tubular, 97, I09, II2, 262; urethral, 40I ; uropygeal, III; vitally secretory, IIO.

glandular area, II4

glans penis, 404

Globicephalus, I83

glomeruli, 37I, 374

glottis, 270, 3IO, 3II, 3I2

Glyptodon, 37

gnathostoma, 267

gnathostomes, 29

Goethe, theories of the "Urbild", 506,507

gonads, 49, 63, 366

Gorilla, 45

gorilla rib, I38

Grandry's corpuscles, 474

grasshopper, 428

gray matter, 408,428

growth, 2, 3

greater curvature of stomach, 29I gubernaculum, 395

Gymnophiona, 3I, 84, 313, 373, 398, 442,483

gynæcomastism, I 8

gyri, of hemispheres, 4I7

H

hæmal arches, I26, 508

hæmal spine, 508, 510

hæmal system, 3 I8

Hæmamœba, 5

hæmapophyses, 508, 5 I0

hair, 469, 47I, 472; arrangement of,

86,87 ; development of, 96 ; direction of, IOI, I03, IO4, I05; distribution of, 99; of mammals, 95-105; structure of, 97, I00; varieties of, 97, 98 .

hair currents, IOI

hair groups, 87,88 
hair of Man:-direction of, I02, I03, I04; racial differences, IOO; shape of cross section, Ioo.

harderian glands, 504

hard palate, 270, 27I, 479

Harrimania, 535

Hatteria, 32

head cavities, $46 \mathrm{I}$

head, formation of, I24

head, relation to vertebral column, I 34

heart $318,320,324,352-357$; of amphibians, $354,355,356$; of Amphioxus, 353, 528; of fishes, 353,354 ; of mammals, 356,357 ; of reptiles, 356 .

hedgehog, 49I

Heptanchus, 305

heredity, material basis for, 53, 57, 58

hermaphrodites, 379

hermaphroditic, 49

hemispheres, of cerebrum, 4II, 4I3

heterodont dentition, 275

Hexanchus, 305

hibernation of Dipnoi, 478

hind brain, 4I I

hip-girdle, I28

hippocampus, 4I7

Hippopotamus, 274, 292, 505

Holocephali, 444

Homo, 87, 88

homodont dentition, 275

homology, of body somites, 439; of limbs, I77-I-So, 237-245; sexual, 394, 404, 405

Homo neanderthalensis, 42

Homo primigenius, 42, 43, 45

Homo sapiens, 42, 45

honeycomb stomach, 292

hoofs, I06, 107

horn, 78

horns of mammals, Io6

horny structures, 105, 106

horse, I $82,250,39 \mathrm{I}$

horse-shoe crab, 522

human phylogenesis, 44, 45

hydatid of Morgagni, 392

Hydra, 60

Hydromys, 277

hyobranchial apparatus, 283

hyobranchial complex, I6o, 283 hyoid apparatus, I60, 283

hyoid arch, I55, I57

hyoid bone, 314; body, I60; cornua, separate elements of, I60, I6I

hyomandibular, I55, I 57

hyperdactylism, $\mathrm{I} 82$

hypermastism, II7

Hyperoödon, 277

hyperphalangy, I83

hyperthelism, II7

hypertrichosis, 99

hypobranchial groove, 289

hypospadias, 399

hypothenar pads, 9 I

hypocone, 279

hypoglossus, 427

hypomeres, 64, 65, I90, 245

hypomeric muscles, 192

hypophysis, 4I4, 423, 424, 477

Hyracoidea, 38

Hyrax, 38

\section{I}

Ichthyopsida, 473

Ichthyopterygitum, I67, I84

Ichthyosaurus, 20, I83, I84, I85, I86

ilium, I28, I7 I

immortality of Protozoa, 5

incisors, 275

incus, I59, I50, I95

inferior turbinated bone (see maxillo-turbinal)

infundibulum, 424

ingluvies, 290

inguinal ligament, $386,387,391$, 395

inner ear, 469, 472, 473, 485-493

insect, insects, 259, 428, 460, 463, 506,512

Insectivora, insectivores, 37, 40, 44,45, II 3, I75, 239, 250, 277, 29I, 393, 394, 40I, 495, 500 insertion, of a muscle, 195, 197 integument:- of amplibians, 83 ,

84; of Amphioxus, 76 ; of fishes.

$80-83$; of invertebrates, 76 ; of manmals, 85 ; of reptiles, 84,85 ; pigmentation of, I IS-I2I.

integumental glands, 78, Io integumental muscles, I90, I92 
integumental respiration, 308

integumental sense-organs, 448

interclavicle, I4I, I74

interdigital pads, 9I

interdural space, 357

intermaxillary glands, 285

intermuscular lympl spaces, 358

interventricular foramina, 416,420

intestinal diverticula, 266

intestinal respiration, 303

intestine, 266, 294

intestine, length of, 299, 300, 301

intestinum crassum, 294

intestinum tenue, 294

introitus vagina, 404

intromittent organ, 398

intumescentiæ of spinal cord, 432 , 434

involuntary muscles, 189

iris, $50 \mathrm{I}$

ischium, I72

iter a tertio ad quartum ventriculum, 4I2

\section{$\mathrm{J}$}

Jacobson's cartilage, 483

Jacobson's nerve, 45I

Jacobson's organ, 483

jaws, origin of, I53, I54

jumping mice, 298

\section{K}

karyokinesis, 55

keratin, 84

kidneys, 323, 345, 369, 385, 386

kiwi-kiwi, I65

Krause's corpuscles, 474

L

labial cartilages, I53, I55

labial glands, 285

labia minora, 404

labia majora, 404

labyrinth, 452, 485-493; bony, 492;

development of, $485-490$; sensory areas of, $488-490$.

Lacerta, 287, 344

Lacertilia, 32

lacrimal apparatus, 504, 505

lacrimal apparatus, 504, 505

lacrimal bone, origin of, 83 lacrimal fluid, 504

lacrimal glands, 504

lacunæ, 3 I 8

lacunar circulation, 3 I7

Lxmargus, 470

lagena, 488, 49I, 492

language, development of, 256

lanugo, 98

large intestine, 294

larynx, I60, 270, 307, 3IO, 3II, 3I2 ;

of amphibians, 3I2, 3I3; of

mammals, 3I4, 3I5; of Saurop-

sida, 3I3, 3I 4

larynx dorsalis, 3IO, 3I I

larynx ventralis, 3 Io, 3 I I

lateral cartilages, of larynx, I60

lateral cerebral fissue, 4I7

lateral line, I92, 448

lateral line organs, 469

lateral ventricles, of brain, 4II, 4I6

lemmings, 298

Lemuroidea, $4 \mathrm{I}$

Lemurs, 38, 45, 89, 90, 284, 298, 3I I, 394

lesser curvature, of stomach, 29I

lesser omentum, 295

lesser peritoneal cavity, 294

leucocytes, 318, 362

life cycle, 9, Io

ligamentum :-arteriosum, 330, 33I ; Botalli, 330, 33I ; hepato-gastricum, 295; hepato-umbilicale, 347 ; inguinale, 386, 387, 391 ; nuchæ, I34; suspensorium hepatis, 295; teres hepatis, 347

limb muscles, I30

limb girdles, 128

limb plexuses, $438-442$

limbs, I64-I88; of primates, 238, 239 ; reduction of, 165 , I66; redundancy of, I66; serial homology of, 177-180, 237-245

Limulus, 522, 523, 524

linea alba, 65

Lingula, I9

lion, 282

Lithobius, 429

Litopterna, 38

liver, 266, 294, 323, 348

lizards, I In, III, I66, 287, 288, 342, $344,398,416,420,422,483$ 
lobes, of lungs, 315,316

lobi optici, 425,426

Loncheres, 86

love antics of salamanders, 397

lumbar intumescence, 433

lumbar vertebræ, I30

lumbo-sacral plexus, 438

lungless salamanders, 308

lungs, $307,310-316$

lungs, origin of, 270

lung system, 260

lymph, 3I9

lymph glands, 3 I8, 362

lymphatic nodes, 362

lymphatics, $65,3 \mathrm{I} 8,358$

lymphatic spaces, $318,357,358$

lymphatic system, 3I8, 319, 357364 ; development of, 360 ; origin of, 363

lymphatic vessels, $3 \mathrm{I} 8,35^{8}$

lymphocytes, 362

lymph hearts, $318,358,359$

lymphoid tissue, 362

lyssa, 385

\section{M}

Macacus, 91, 92, 93

macrogametes, $7,48,49,50,58$

maculæ acusticæ, 490

macula lutea, 500

malleus, I59, 450, 495

Malpighian corpuscles, 373

mammæ, II6, I17; inguinal, I16; pectoral, II7; unusual position of, II7.

Mammalia, mammals, 39, III, II3, I48, I72, I74, I75, I79, I98, 200, 20I, 203, 206, 2I2, 215, 216, 226, $227,229,236,247,248,249,250$, $263,264,268,269,27 \mathrm{I}, 275, \cdot 280$, $283,284,288,290,291,294,296$, $301,314,315,319,322,324,33$, $333,337,347,349,350,35 \mathrm{I}, 353$, $354,355,356,357,36 \mathrm{I}, 363,373$, $377,385,386,388,389,390,39 \mathrm{I}$, $395,398,399,400,401,402,409$, $4 \mathrm{I} 4,4 \mathrm{I} 5,4 \mathrm{I} 9,425,426,43 \mathrm{I}, 437$, 439, 444, 450, 45I, 452, 455, 465, $469,47 \mathrm{I}, 472,475,479,48 \mathrm{I}, 486$, $488,489,491,496,504,509$ mammary glands, 33 , I I4-I I8 mammary pockets, II4, II5 mammary ridge, I 6

mammuth, 42

mandibular articulation, I59

mandibular cartilages, I53, I56, 157

Manida, 85

marrow, 363

marsupials, 33, 44, 203, 223, 276, $284,292,297,357,388,389,399$, $400,40 \mathrm{I}, 402,4 \mathrm{I} 7$

marsupium, 34 , I I6

maxillo-turbinal, 479, 48o, 48I

Meckel's cartilage, I56, I59

median fins, I63, I64

medulla, of brain, 427

Medusa, 60

Megalonyx, 37

Megatherium, 37

meibomian glands, II3, 505

Meissner's corpuscles, 473

membraneous labyrinth, 485-492

Merostomata, 522, 523

mesencephalon, 4II, 425, 426

mesenchyme, 6o, 64

mesenteries, 63, 26I, 265, 367, 368

mesenteric glands, 362

mesodæum, 259

mesoderm, 60, 63, 66

mesodermic diverticula, $63-67$

mesodermic somites, 68, 69

Mesodonta, 38, 40, 4I, 45

mesomeres, 64, 65

meso-hypomeres, 64, 65, 68

mesogastrium, 293

mesonephridia, 373,374

mesonephros, 370, 373-375; 382, $385,386,390,39$ I, 394

mesonephros; remains in Amniota, 385

mesonephrotic duct, $374,375,376$, 38 I, $383,385,387$

mesonephrotic ligament, 386, 390, 391

mesonephrotic system, 373-375;

vestiges of in female, $39 \mathrm{I}$; vestiges of in male, 392

mesonephrotic tubules, 373,374

mesopterygium, $176, \quad 185-187$

mesorchium, 369-387

mesovarium, 369, 387, 39I

metacœle, $60,63,64,365,367,368$, $38 \mathrm{I}$

metacone, 278 
metacœlic sacs, 367,368

metamerism in vertebrate head, 460

metanephridia, 376

metanephros, $370,375-377,383$

metanephrotic system, 375, 377

metapleural folds, 527

metapterygium, I76, I85-I87

Metazoa, 257, 3I7

metencephalon, 4I I, 426, 443

microgametes, $7,48,49,50,58$

Midas, 86, 88

midbrain, 4II

middle ear, 493-496

milk glands, I I4-I I8

mitosis, 54, 55

molar glands, 286

molars, 275

mole, $277,287,284$

mole, star-nosed, 90

monkeys, 90, 9I, 92, II 2, 223, 254, 277

Monodelphia, 36

Monodon, 277

monophyodont dentition, 280

monorrhine condition, 476

monotremes, 33, 44, 236, 255, 337,

$357,388,393,399,400,402,49 \mathrm{I}$

motor nerves, 434

motor roots, of nerves, 435,436

mouse, 316, 402

mouth, 466, 467

mouth cavity, 466

mouth, origin of, 258, 259

mucosa, 260, 262

Müller's duct, 382, 387, 388, 39I, 392, 394

Multituberculata, 35

Mus, 402

muscle, or muscles:-abductor caudæ dorsalis, 2I2; abductor caudæ ventralis, 212 ; abductor coccygis, 2I4; abductores breves, 234; abductors, of pollex and minimus, 236; achselbogen, 25I ; acromiodeltoid, 226; adductores (femoris), 229; adductor laryngis, 3I3; adductor mandibulæ, 247 ; adductor mandibulæ, 495; anconcus, 222, 226; auricularis anterior, 255; auricularis posterior, 255; auricularis superior,
muscle-(Continued)

255 ; auriculo-labialis, 55 ; auriculo-occipitalis, 255; appendicular, I90, I92, I93, 217-245; axial, 190, I92, 200-2I7; axillary arch, 25I; biventer cervicis, 226, 227; biceps femoris, 229; brachialis, 226, 227; brachioradialis, 240; buccinator, 255; caudal, 212-214; caninus, 255 ; cervicalis ascendens, 208; coccygeus, 2I4; coraco-brachialis; 226 ; coraco-brachialis brevis, 222 ; coracobrachialis longus, 222 ; complexus, 210; crureus, 229; cremaster, 396; cucullaris, 223; curvatores coccygis, 2r4; deltoid, I95, 226; depressors of visceral arches, 245, 246, 247; diaphragm, 316; diaphragma pelvis, $2 \mathrm{I} 4$; digastricus, I96, 247, 248, 438; dilatator laryngis, 3I3; dorsalis antebrachii, 23I, 235; dorsalis scapulæ, 222; dorso-laryngeus, 247,248 ; extensor caudæ lateralis, 212; extensor caudæ medialis, 212; extensor carpi radialis, 237, 239; extensor carpi ulnaris, 237,239 ; extensor communis digitorum, 235; extensores breves, 23I; extensor radialis, 23I ; extensor ulnaris, 23I ; facial muscles, 254-256; femero-fibularis, 228; flexores breves profundi, 234; flexores breves superficiales, 234; flexor caudæ, 212; flexor carpi ulnaris, 237, 239; flexor carpi radialis, 237, 239; flexor digitorum profundus, 236 ; flexor digitorum sublimis, 236; flexor pollicis longus, 236; flexor ulnaris, 234; flexor radialis, 234; galea aponeurotica, 55 ; gastrochnemius, 239; genio-glossus, 248 ; glutæo-cruralis, 229; glutæi, 229 ; gracilis, 229; hyo-glossus, 248; hyo-laryngeus, 247; homology of, I94-I99; humero-antebrachialis, 222,227 ; ilio-coccygeus, 2I4; ilio-costalis, 208; ilio-femoralis, 228, 229; ilio-fibularis, 228, 229 ; ilio-extensorius, 228, 229; ilio- 
muscle-(Continucd)

psoas, 229; in fishes, 200, 20I ; infra-spinatus, 226; insertion, I95 ; integumental, I90, I92, 249256 ; intercostales, externi et interni, 204; intermetacarpales, 234; intermandibularis anterior, 247; intermandibularis posterior, 247 ; interossei, 236; interossei dorsales, 236; interossei palmares, 236; interspinales, 210; intertransversarii, 2IO, 2II ; intertransversarii caudæ, 213; intervertebral system, 2IO-2I2; involuntary, I89; ischio-cavernosus, 2I4, 2I5; laryngeus dorsalis, 247; laryngeus ventralis, 247 ; laryngei, 247, 248, 3I3; latissimus dorsi, I99, 206, 22I, 223, 226, 249, 250, $25 \mathrm{I}$; levator ani, $2 \mathrm{I} 4$; levator anguli oris, 255 ; levatores arcuum, 247; levatores costarum, 204; levator menti, 255; levator of visceral arches, $245,246,247$; levator scapulæ, 222, 223; lingualis, 249; longissimus, 208, 212, 215; longus colli, 202; longus capitis, 204 ; masseter, 248; mimetic muscles, 253-256, 45I ; multifidus, 210, 2I2; mylo-hyoideus, 248; nasal muscles, 255; obliqui capitis, I98, 2I5; obliqui oculi, 2I6, 447 ; obliquus capitis inferior, 2 I2; obliquus capitis superior, $2 \mathrm{I} 2$; obliquus externus abdominis, I98, 203, 204; obliquus inferior oculi, 447; obliquus internus abdominis, 203, 204; obliquus superior oculi, 447; obturator externus, 229; obturator internus, 229 ; occipitofrontalis, 255; of eyeball, 2I6, 447 ; of hip girdle and thigh, 227 , 230; of shoulder and upper arm, 217,227 ; of the free limbs, 230 , 237 ; opponens hallucis, 242 ; opponentes of pollex and minimus, 236 ; orbicularis oris, 255 ; orbicularis oculi (palpebrarum), 255; origin, I95; palmaris, 236 ; palmaris profundus, 233; palmaris longus, 236; palmaris superficialis, 233; panniculus carnosus, 250,
muscle-(Continued)

25I ; parietal, I90, I92; patagial muscles, 249; pectoralis abdominalis, 25I ; pectoralis, I99, 222, 226, 249, 250, 25I, 253; pectoralis major, 226; pectoralis minor, 226; peronæus brevis, 239 ; peronæus longus, 239; peronæus tertius, 24I; petro-hyoideus, I 88 ; pharyngeal constrictors, 249 ; piriformis, 230; platysma, I96, 253; prevertebral, 202; principles of muscle formation, ig8; procoraco-humeralis, 222, 226; pronator, 234, 237; pronator teres, 240; pronator quadratus, 240 ; propatagialis, 249; pterygoideus externus, 248 ; pterygoideus internus, 248; pubo-coccygeus, 2I4; pubo-ischio-femoralis externus, 22\%, 229; pubo-ischio-femoralis internus, 228, 229; pubo-ischiotibialis, 228, 229; pubo-tibialis, 228; pyramidalis, 203; quadratus labii inferioris, 255; quadratus lumborum, 203; quadriceps femoris, 229; recti oculi, 2I6, 447 ; rectus abdominis, I99, 203; rectus capitis anterior, 204; rectus capitis posterior major, 2I2; rectus capitis posterior minor, 2I2; rectus femoris, 229; rectus externus oculi, 447 ; rectus inferior oculi, 447; rectus internus oculi, 447; rectus lateralis capitis, 204; rectus superficialis, 222; rectus superior oculi, 447 ; retractor bulbi, 447 ; rhomboidei, 206, 226; rhomboideus capitis, 226; rhomboideus dorsi, 226; rhomboideus major, 206, 226; rotatores, 210; sacro-coccygei anteriores, 2I4; sacro-coccygei, posteriores, 2I4; sacro-lumbalis, 207 ; sacro-transverso-transversalis system, 207, 208, 2I5; sartorius, 229; scalenus anterior, 204; scalenus medius, 204; scalenus posterior, 204; semi-membranosus, 229; semi-spinalis, 210; semitendinosus, 229; serratus anterior (magnus), 223; serratus 
muscle-(Continued)

magnus, 222, 223; serrati posteriores, 203, 206; soleus-gastrochnemius, 239; sphincter ani, 2I4; sphincter colli, 253; sphincter cloacæ, 250; sphincter marsupii, 250 ; spinalis capitis, 209; spinalis cervicis, 208; spinalis dorsi, 208; spino-deltoid, 226; spino-spinalis system, 208, 215; spino-transversalis system, 207; splenius capitis, 207; splenius cervicis, 207, 2 I5; stapedius, I96, 248, 438, 495; sternalis, 253; sterno-cleido mastoideus, 223; sterno-cleido mastoideus, 457; striated, I89, I90; stylo-glossus, 248 ; stylo-hyoideus, 248; subclavius, 226 ; subcutaneus faciei, 255; supinator, 231, 237 ; supinator brevis, 240; supinator longus, 240; supracoracoideus, 222, 226; supra-spinatus, 226; temporalis, I90, 248; tenuissimus, 229; tensor tympani, 248, 495; teres major, 223, 226; teres minor, 226; tibialis anterior, 239; tibialis posterior, 239; trachelor-mastoid, 208; transversalis abdominis, 203 , 204; transversalis, colli, 208; transverso-spinalis system, 210 , 2I5; transversus thoracis, 204; trapezius, 206, 222, 223, 457; triangularis sterni, 204; triceps, 222, 226; unstriated, 189 ; vastus group, 229; visceral, I90, I92, 245249 ; voluntary, I89, I90 ; zygomaticus, 255

muscles of Necturus, I9I-I93, 220$222,227-229,230-235$

muscles, of alimentary canal, 290, $29 I$

muscle somites, I92

muscular homology, 194-I99

musculosa, 260

musk glands, I Io

muskrat, 297

myelencephalon, 4I2, 426, 42خ, 443

myocommata, 27, I92

myology, comparative, I95

myomeres, 27 (see also myotomes)

Myopotamus, 86

myotomes, I92, 46I myotomic buds, 2I7, 2I8, 219

myriapod, 428, 429, 460

myriapod, nervous system, 428

Myrmecophaga, 277

Myrmecophagidæ, 33

Myxine, 437, 476, 488, 489, 516

myxinoids, 49

nails, 105, 106, 107

nares, anterior, 478

nares, posterior, 478

narwhal, 277

nasal capsules, 145

nasal cavities, 269

Nasodon, 28I

naso-lacrimal duct, 504

naso-palatine canal, 479

Neanderthal man, 42

neck, I32, I33

neck, formation of, I30

Necturus, I28, I38, I71, I72, I83, I86, I9I, 220, 227, 228, 229, 230, $23 \mathrm{I}, 232,233,234,235,237,238$, 240, 246, 247, 3I2, 3I3, 430, 442 nemerteans, 520, $52 \mathrm{I}$

nemertean theory of vertebrate ancestry, 520, 521,522

nerve, or nerves:-abducens, 216 , $443,445,446,447,461,462$; accessorius, $443,452,455,457$; acusticus, $443,448,452,462$; auditorius (see acusticus); ansa hypoglossi, 458 ; brachial plexus, 438, 43944I; buccalis, 449; chorda tympani, 450, 45I ; communicans IX, 454,456 ; facialis, I96, 246,248 , $256,443,448-452 ; 453,454,46 \mathrm{I}$, 502; glosso-pharyngeus, 246, 247 , $443,45 \mathrm{I}, 452,457,46 \mathrm{I}$,502 ; gustatorius, 452; hyomandibularis, 449; hypoglossus, 443, 457-459, 462,463 ; intestinalis, 454, 457; Jacobson's, 45 T, 454, 456; lateralis $X, 453,457$; lingualis, 452 ; 454; lumbo-sacral plexus, 438; mandibularis externus VII, 449; mandibularis internus VII, 449; mandibularis $V, 449,45 I$; maxillaris, 449; motor, 434; motor oculi, 2I6, 443, 445, 446, 447, 462; occipital, 444; occipito-spinal, 444 ; olfactorius, 443,444 ; ophthalmicus, $45 \mathrm{I}$; ophthalmicus 
profundus, 449, 462, 463; ophthalmicus superficialis V, 448 ; ophthalmicus superficialis VII, 448; opticus, 443, 444; palatinus, 449; palatinus major, 450, 456; patheticus (v. trochlearis) peripheral, 434, 437; petrosus profundus major, 456; petrosus profundus minor, 456; petrosus superficialis major, 450, 456; petrosus superficialis minor, 456 ; plexus, 196, I97; plexus brachialis, 438, 439-44I ; plexus lumbosacralis, 438 ; plexuses, of sympathetic system, 464; pneumogastric (v. vagus); posttrematici, 454,462 , prætrematici, 454,462 ; sensory, 434; spinal accessory (v. accessorius); spino-occipital, 444; stapedialis, 45I ; sympathetic plexuses, 464; sympathetic system, $45 \mathrm{I}, 463,464$; terminalis, 445 ; trifacial (v. trigeminus); trigeminus, 247, 248, $443,447,448-452,46 \mathrm{I}, 495$; trochlearis, 216, 443, 445, 446, 447, 449, 462; tympanic, 45I, 454, 456 ; vagus, $247,443,452-457$, $46 \mathrm{I}$

nerves, motor, 434

nerves, peripheral, 434, 437

nerves, sensory, 434

nerve supply to muscles, 438

nervous system:-anlage of, 62 ; origin of, 406,407

neosternum, I38

neostoma, I54, 424

nephridia, 366, 368, 37o, 37 I, 38I, 382,383

nephrostomes, $366,373,374,382$, 383

neural arches, I26, 508

neural processes, I26

neural spine, 508, 5 I0

neural tube, 62, 406; development of, 407, 408; early history of, 406,407

neurapophyses, 508, 5 Io

neurenteric canal, 258

neurilemma, 434

newt, $3 \mathrm{I} 3$

nictitating membrane, 504 nipples, II5-II8; morphology of, II5; rudimentary, II7

noduli lymphatici aggregati, 362, 363

Nomarthra, 37

nose, cartilages of external, I5 I

nose, external, I5I, 484

notochord (see under skeletal elements)

notochord:-of Amphioxus, I23, 527; of Balanoglossus, 535; of tunicates, 53I

nucleus, 3

\section{$\mathrm{O}$}

obturator foramen, I72

occipital condyles, I33

odontoblasts, 273

odontoid process of axis, 33

œsophagus, 290

Oken, Lorenz, his theories, 506

olfactory buds, 485

olfactory lobes, 4I6, 445

olfactory nerve, 4 I 6

olfactory pit of Amphioxus, 445

olfactory surface, increase of, 479 , 482

omasus, 292

omentum, 293

ontogenesis, I5

ontogenesis, laws of, 20-25

oögonium, $55,56,57$

open type of circulation, 3I7

operculum, of ear, 493

operculum, of gills, I57, 306

Ophidia, 32

opossum, 33, 276

optic cup, 422,423

optic lobes, 4II, 425

optic stalks, 445

optic vesicle, 422,423

optici thalami, 425

opisthocœlous vertebræ, I3I

orang-uttan, 45

orbital glands, 286

organs of Corti, 492

organs of Giraldés, 392

organ of hearing, $485-497$

organ of Rosenmüller, $39 \mathrm{I}$

origin, of a muscle, 195

Ornithorhynchus, 33, 90, 432

Orthagoriscus, 430, 43 I 
ossicula auditus, I59

ostium tubre, 382

ostrich, I65, I72, 433

otic capsules, I 45

otic vesicle, 473,486

otter, 505

ova, $366,379,382,389$

ovaries, $49,367,379,383,387,389$,

$391,393,394$

oviduct, $382,383,385,387,388,389$,

39I, 394

ovum, $48,49,50,51,55,58$

Owen Sir Richard, his theories,

$507-512$

owl, 489

ox, I82, 49I

\section{$\mathrm{P}$}

Pacini's corpuscles, 474

pads, mammalian, 90, 9I

Palæostoma, 154, 424, 478

palatine cleft, 27 I

palingenetic characters, I6

pallium, $4 \mathrm{I} 6$

palm print of boy, 94

pancreas, 266, 294

Pantotheria, 35, 44

papilla acustica basilaris, 490, 49I

papilla acustica legenæ, 490

parachordal elements, I44, I45

parachordal region of head, I24,

I 44,460

paracone, 278

paradidymis, 392

Paramœecium, 4, 6

paraphysis, 420, 422

parapophyses, 508

pars olfactoria, of nose, 478

paraseptal cartilage, 483

parasternum, I4I

pars respiratoria, of nose, 478

parathyreoid bodies, 289

paired fins, 163, 164

parietal foramen, 420

parietal eye, 422

parietal mesoderm, 63, 66

parietal muscles, I90, 192

parietal organ, 420

paroöphoron, 39I, 394

parotid glands, 286

parrots, 296

patagium, 2.49 patella, 178

paunch, 292

pearl organs, 472

pectoral fins, I64

pectoral girdle, I30

pedunculi cerebri, 426

pelvic girdle, 169-172; phylogenesis

of, $170-172$

pelvis, of kidney, 376

penis, 398, 399, 400, 40 I

penis, position of, 399,400

perennibranchiate amphibians, 307,

330

perilymph, 492

perilymphatic cavity, 492-493

perilymphatic space, 357

perinæum, 389,401

perineurium, 434

Perissodactyla, 39

peristalsis, 265

peritoneal cavity, 367-369, 380

peritoneal cavity, lesser, 294

peritoneum, 66, 261, 293, 294, 316, 367,374

perivisceral fluid, 317

perspiration, II2

pes hippocampi, 4I7

petrel, 478

Petromyzon, 289, 420, 477, 488

Peyer's patches, 362

phallus, 398

pharyngeal pockets, 268

pharyngeal pouches, 265,266

pharyngo-œsophageal respiration, 308,309

pharynx, 265, 266

Phascoloarctus, 297

philthrum, 27 I

phylogenesis, I5

phylogenesis, laws of, 16-20

phylogenesis of man, 44, 45

phylogenetic tree, of mammals, 36 ,

39 ; of vertebrates, 28

pig, I82, 274, 361, 391, 437, 489

pigment, 78 , II8-I2I

pigment speck, of Amphioxus, 445

pineal gland, 42I

pineal organ, 420

pinna, of ear, I6I, 496, 497

Pinnipedia, 38

Pisces, 29, 30

Pithecanthropus, 43 
pituitary body, 424

placenta, 34, 70, 71, 72, 73, 377, 388

placoderms, 29, 83, 524, 525

placoid scales, 80, 8I, 267

placoid scales on teeth, I54

plasma, 318, 319

plastron of turtles, I05

platyhelminths, 520

Platyrrhini, 278

Pleisiosaurus, I85

pleura, 66, 316

pleurapophyses, I38, 508, 5 Io

pleuro-peritoneal cavity, 64,66

plexus, blood vessels in brain, $4 \mathrm{I} 3$, 419,420

plexus brachialis, 438, 439-14I

plexuses, chorioid, 4I3, 4I 6, 4I9, 420

plexuses, of nerves, 438

plexuses, sympathetic, 464

plexus formation, nerves, 438

plexus lumbo-sacralis, 438

plica fimbriata, 284

plica semilunaris, 504

pneumatic cyst, 3IO, 3II

poikilothermous animals, 356

poison fangs of serpents, 280

polar globules, 56, 57

Polypterus, I7 I, I86, I87, I88, 3II

Polypterus, ribs of, I37

polyspermy, 53

pons Varolii, 426, 427

Pontoporia, 30I

pori abdominalis, $369,380,38 \mathrm{I}$

porpoise, 478, 49I, 505

postbranchial bodies, 288, 289

posterior limb, I69

posterior nares, 269,478

post-minimus, I82, 242

præchordal elements, I44, I45

præchordal portion of head, 460

præchordal region of head, I24, I H4

pre-hallux, I82

prehistoric man, 42

premolars, 275

pre-pollex, I82, 242

preputial glands, II3,403

Prevertebrata, 26

primary body cavity, 365

Primates, 38, 40, 4I, 90, 9I, 92, 93, I65, I75, 2I6, 223, 238, 250, 277, 29I, 394, 402, 4I 7

Primates, limbs, 239 primitive dentition, 277

primordial brain, 4 I I

primordial skull, I45

Proboscidea, 38, 39

Procavia, 38

processus vaginalis, 395

procolous vertebrx, I3I

procoracoid, I74

proctodxum, 259

pronephridia, $37 \mathrm{I}$

pronephros, $370-373$

pronephrotic duct, 370

pronephrotic tubules, $37 \mathrm{i}, 374$

Propithecus, 300

propterygium, I76, I85-I87

prosencephalon, 4I I

prostate gland, 403

prostatic vesicle, 393, 394

Proteus, 23I, 247, 300, 3I2, 3I3

Protochordata, 26

protoccele, $63,64,365,367$

protocone, 278

protoplasm, characteristics of, I

protostome, 59, 6I

Prototheria, 35

Protozoa, 3, 257; immortality of, 5 ;

conjugation of, 6

pseudohypertrichosis, 99

Pterichthys, 524

Pterodactyl, 183, I84

pterygoid canal, 450

pubic bones, I72

pubo-ischiadic symphysis, I72

pulmonary system, 260, 270, 304, $308,310-316$

pulsating vessels, 317

pyloric cœca, 266

pyloric end of stomach, 291

pylorus, 265, $29 \mathrm{I}$

\section{$\mathrm{R}$}

rabbit, 297, 334, 345, 346, 49 I

race history, 15

Rana, 3I3, 489, 526

Ranodon, I86

rat, 336,338

ratio of surface to mass, $3,26 \mathrm{I}, 262$

ray, 453

receptacula chyli, 362

recessus utriculi, 487

rectum, 298, 389

red blood corpuscles, .3:8, 364 
reduction of limbs, I65, I66

reduction of teeth, 277

Reissner's membrane, 492

renal corpuscle, 373

reproduction by fission, 4

reproductive system, $378-405$

reptiles, 44, I72, 20I, 203, 2I2, 220, $229,248,253,255,268,272,280$, $296,328,330,33 \mathrm{I}, 352,353,355$, $356,358,375,377,385,386,399$, $409,4 I 5,4 I 6,4 I 9,425,426,465$, $472,487,488,504$

respiration, 30I-3I6; in amphibians, 306-308, 312-3I3; in Amphioxus, 304; in Balanoglossus, 304; in fishes, $304,305,306$; integumental, 308 ; intestinal, 303 ; pharyngo-œsophageal, 308; pulmonary, 307, 3 I0-3I6

reticulum, 292

retina, $409,422,497,498$

retrolingual glands, 286

rhinencephalon, 4I6, 445

Rhineura, 20

Rhinoceros, 394

rhombencephalon, 427

Rhynchocephalia, 32

ribs, I26, I35, I36, I37, I38; distri-

bution of, I37, I38; two types of,

I35, I37; variation in, I29, I30

rodents, 37, 45, 175, 203, 223, 239,

274, 277, 297, 298, 316, 394, 40I,

$4 \mathrm{I} 7,480,495,500$

roots, of nerves, 435

roots, of teeth, 273,274

rods and cones, of retina, 422,467 , 498

rods of Corti, 492

rostral plates of skull, I46

round ligament of uterus, 387,390

rumen, 292

ruminants, 292, 297, 484

\section{$\mathrm{S}$}

sacculus, 487,488

saccus endolymphaticus, 486

sacral vertebrx, I29, I30

sacrum, variation in, I28, I29

St. Hilaire, his theories, 506, 512

salamanders, I79, I85, I86, I92, 220 , $223,307,308,309,354,383,388$, $397,429,493$ saliva, 285

salivary glands, 285

Sauropsida, I I0, 200, 264, 283, 284, $285,294,296,313,3$ I 4, 319, 322, $323,324,347,360,373,375,377$, $39 \mathrm{I}, 444,457,464,479,49 \mathrm{I}, 495$. 497,499

scales:-ctenoid, 83 ; cycloid, 83 ; epidermic, 84 ; of fishes, 80 ; of ganoids, $8 \mathrm{I}$; of mammals, 85,86 , 90; of palmar and plantar surfaces, 95; of Stegocephali, 84; placoid, 8o; structure of, 80

Scaphyrhynchus, I7I

schizocole, 68

sclerotic coat, of eye, $423,498,501$ scorpion, 524

scrotal raphé, 404

scrotal sac, 395,396

scrotum, 394, 395

sculpin, 419

scutes, $79,83,146$

seal, 282,316

sebaceous glands, II3

segmentation of head, $458,460,46 \mathrm{r}$ selachians, 29, 44, I52, I53, I70, I72

I73, I84, I85, 2I 7, 218, 2 I9, 253 $272288,305,324,325,326,327$. $328,34034 \mathrm{I}, 369,380,38 \mathrm{I}, 382$ $392,4 I 6,4 I 9,420,436,460,486$ $488,489,490,5$ I 2,5 I 5

selachians, circulation in, 324, 325 selachians, skull of, I45, I46, I 53

sella turcica, 424

semicircular canals, 487

seminal fluid (see spermatic fluid)

seminal groove, 399

sensations, 467,468

sense of contact, 468

sense-organs, 408, 465-468

sense-organs, accessory parts, 467

sense-organs, cells of, 466

sense organs of head, I43

sensory cells, 408, 466, 467

sensory nerves, 408, 434

sensory roots of nerves, 435,436

scptum atriorum, 354

septum linguæ, 285

septum pellucidum, 4I8

scrial homology of limbs, I78, 237-

245

serosa, 260 
serous cavities, 3 I 8

serpents, 285

sesamoid bones, 178

sex determination, 403

sexes, definition of, 49

sexual homologies, 393, 394, 404, 405

sexual kidney, $382,383,385$

sharks, 388

sheep, 276

shoulder-girdle, I72-175; of Amniota, 174, 175; of amphibians, I74; of ganoids, I73; of teleosts, I74; of selachians, I73

shrew-mouse, 92

sigmoid flexure, 298

Simplicidentata, 37

sinuses, in bones of face, 482

sinus maxillaris, 482

sinusoids, $3 \mathrm{IS}$

sinus utriculi posterior, $4 \delta 7$

sinus utriculi superior, 487

sinus venosus, $324,326,340,34 \mathrm{I}$, 353-357

Siren, 165, 246, 442, 471

Sirenia, 38, 39, 98, II3, II7, 3I6, 393

skates, 453

skeletal elements:-abdominal ribs, I4I; acclous vertebræ, I3I ; alisphenoids, I48; alveoli of jaws, 273; amphicœlous vertebræ, 127; angulare, I56; appendicular skeleton, I22; appendicular skeleton, I62 ff.; a rchisternum, I38; articulare, I59, 495; a rytrenoids, I60, 313; atlas, I33; auditory ossicles, I59; a uricula, 496, 497 ; axial skeleton, I22; axis, I33; basihyal, I6o, 284; basioccipital, I50; basipterygium, I69, I75; basisphenoid, 150; bone complexes of skull, I5I; bony labyrinth, 492; branchial arches, 152 , 155 ; carpus, I77ISI; cartilage bones, I48; cartilago lateralis, I60; centers of ossification, I48; centrum of vertebra, I27; ceratohyal, I60, 284; cervical rib, I38; cervical vertebræ, r3o; cliondrocranium, 145 , 146, I53, I56; clavicle, I73-I75; cleithrum, I73; $\operatorname{coccyx,~I35;~col-~}$ skeletal elements-(Continued) umella auris, 494; conclix of nose, 480-482 ; coracoid, I74; costal cartilages, 136 ; cricnid, 3I4; dentary, I56; dentine, 79; dermal bones, 79, 82, I46, I47, I56; dermal bones of skull, 82, I46, 147, I56; diapophyses, I38; dorsal vertebræ (see thoracic vertebræ) ; ear, external, 496, 497 ; ectoturbinalia, 480; endochondral ossification, I48; endoturbinalia, 480 ; entoglossum, 283-285; epiglottis, I60, 3I3; epihyal, I60, $2 \mathrm{~S}_{4}$; epiotics, I48; episternum, I 4 I ; ethmoid, I 48 ; ethmo-turbinal, 479, 480; exoccipitals, I48; external ear, 496, 497; eyeball, skeleton, elements of, I44, I 45 , I5I ; eye capsules, I45; falciforme, I82; fangs of serpents, 280; fin spines, 162 ; free-limb, skeleton of, I76-I78; frontals, 82, 83, I47; furcula, I74; ganoid scales, $\delta_{I}$; gill arches, 3I2-3I4; hæmal arches, I26; hard palate, 270, 27I, 479; hipgirdle, I28; hyobranchial apparatus, 283 ; hyobranchial complex, 160, 283; hyoid, I60, I6r; hyoid apparatus, r6o, 283; hyoid arch, I55, I57; hyoid bone, 3I4; hyoid complex, 3I4; hyomandibular, I55, I57; ilia, I7I; ilium, I28; incus, I59, 450, 495; inferior turbinated bone (see maxillo-turbinal); interclavicle, I4I, I74; ischia, r72; Jacobson's cartilage, 483 ; jaws, origin of, I53, I54; labial cartilages, 153 , 155; labyrinth, bony, 492; lacrimal, 83 , I47; lateral cartilages of larynx, I60; limb-girdles, I28; lumbar vertebræ, I30; malleus, I59, 450, 495; mandibular cartilage, I53, I56, r57; maxillaries, 83, I56; maxillo-turbinal, 479-48I; Meckel's cartilage, I56, I59; mesopterygium, I76, I85-I87; metapterygium, I76, I85-I87; nasal capsules, I45; nasals, 82, I 47 ; naso-turbinal, 479, 480; neo- 
skcletal elements-(Continued)

stcrnum, I38; neural arches, I26; neural processes, I26; nose, cartilages of external, I57; notochord, 26, 27, 63, I22, I23; notochord, of Amphioxus, 527; notochord, of Balanoglossus, 535; notochord, of tunicates, 53I ; occipital condyles, I33; odontoid process of axis, I33; omosternum, I42; operculum, of ear, 493; operculum, of gills, I47, I57; opercular bones, I47; opisthocœlous vertebræ, I3I; opisthotics, I48; optic capsules, I45; orbitals, 83 , I47; orbitosphenoids, I48; os entoglossum, $283-285$; os falciforme, I82; ossicula auditus, I59; ossification, centers of, I48; otic capsules, I45; palate, 270; 27I; palatines, 83, I47, I56; patella, I78; parabasal, 83, r47; parachordal elements, I44, I45; paraseptal cartilage, 483; parasphenoid, I48; parasternum, I4I ; parietals, 82, 83, I47; pectoral girdle, I30, I69-I72; petrosal bone, 493; petrosals, I48; pinna, 496, 497; placoid scales, 80, 8I, 267; placoid scales, as teeth, I54; plastron, of turtle, I05; pleurapophyses, I38; post-frontals, 82, I47 ; post-temporals, I74; præchoidal elements, I44, I45; præfrontals, 82, I47; præmaxillary, I56; præsphenoid, I50; primordial skull, I45; procœlous vertebræ, I3I; procoracoid, I74; prootics, I48; propterygium, I76, I85-I87; pterygoids, I47, I56; pubic bones, I72; quadrate, I56, 450, 495; quadrato-jugal, 496 ; ribs, I26, I29, I30, I35I38; ribs, abdominal, I4I ; ribs, distribution of, I37, I38; rostral plates, I46; sacrum, I29; sacral vertebræ, I29, I30; scales, ganoid, 8I ; scales, placoid, 80, 8I, 267 ; scapula, I74; scapulocoracoid, I73; sesamoid bones, I78; shoulder girdle, I73-I75; sinuses, in bones of face, 482 ; skelctal clements-(Continucd) skull, amniote stagc, I50, I5I ; skull, amphibian stage, I50; sku1l, bone complexes of, I5I ; sku1l, development of, I42-I45; skull, ganoid stage, I46; sktull, of cyclostomes, I43; skull, of selachians, I44, I 45, I46, I53; sliull, primordial, I45; skull, selachian stage, I44-I46; spinous processes, I34; spiracular cartilage, I55; squamosals, 82, 83, I47; stapes, I59, 337, 495; sternebra, I40; sternum, I38, I39, I42; stylo-liyal, I6I, 284 ; styloid process, I6I, 284 ; supra-clavicles, I74; supra-cleithra, I74; supra-occipital, 82, I47; suspensorium, of jaws, I55; tarsal cartilages, 504; tarsus, I77I82; teeth, 267, 27I-283; teeth, evolution of shapes, 278-280; teeth of birds, 84 ; teeth of selachians, $8 \mathrm{I}$; teetl, origin of, $8 \mathrm{I}$, I53, I54; teeth, replacement of, 280-283; thecre of jaws, 273; thoracic vertebræ, I30, thyreohyal, 284, 3I4; thyreoid, 3I4; thyreoid cartilage, I6o; tonguebars, of Amphioxus, 290; tooth, structure of, 80 ; trabecula, I44; trachea, 270, 3I2; tracheal pieces, I6o, 3І3; tracheal rings, 3I3, 3I4; trunk vertebra, I30 ; turbinalia, 479-482; tympanic bone, 496; tympanic bulla, 496 ; tympano-hyal, I6I, 28+; typical vertebræ, 507-5II; uncinate processes, I36; urostyle, I36; vertebræ, I29, I30; vertebræ, development of, $12 \nmid-127$; vertebræ, typical, 507-5II; vertebral column, I30-I33; vertebral colımm, devclopment of, I24-I27; visceral arches, 267; visceral skelcton, I22, I52-I62; vomero-nasal cartilage, 483 ; vomers, 83 , I47, I 50 ; Weber's apparatus, 486; wishbone, I4I.

skeleton, rclation to soft parts, I23 skin, 77 skin color in Man, II9 skin, pigmentation of, IIS-r2I 
skull:-amniote stage, I50, I5I; amplibian stage, I50; development of, I42-I45; ganoid stage, I46; selachian stage, I44-I46.

slime canals, 469

sloths, II3, 316, 393

small intestine, 294

smell, 468, 476-485

smell-buds, $47 \mathrm{I}$

snakes, I Io, I66, 377, 398, 432, 433, $44 \mathrm{I}, 483,487,505$

soft palate, $266,27 \mathrm{I}$

soma, 7, II, 48, 58

somatic cells, 55

somites, 27

somites of head, $458-463$

sparrow, 4I9

spermatic cord, 396

spermatic fluid, $49,52,380,381,383$

spermatogonium, $55,56,57$

spermatophores, 383

spermatozoön, 48, 49, 50, 5I, 52, 55, $58,366,379,382,397,401$

Sphenodon, 32

spider, 259

spinal cord, 427, 428; caliber of,

432,433 ; columns of, 434 ; length,

428-43I; shape of cross-section, 433,434

spinal ganglia, 436,437

spinal nerves, $427-434$

spinous processes, excessive development of, 134

spiny ant-eater, 33

spiracular cartilage, I55

spiracular opening, 493, 494

spiraculum, I55, 269

spleen, 293, 363 .

Squalus, 388,446

squid, 499

squirrel, 3 I6

squamosals, 82,83 , I 47

stapedial artery, I59

stapes, I59, 337, 495

Stegocephali, 30, 3I, 32, 44, 84, 230

St egosaurus, 433

Stenson's canal, 479

sternebræ, I 40

sternum, I38

sternum, morphology of, I39

sternum of monotremes, $\mathrm{I}_{42}$

stoma, 266

stomach, 265, 290, 29I. 292 stomatodxum, 259, 476

stomato-pharyngeal cavity, 266

stratum corneum, 77

stratum germinativum, 77

stratum lucidum, 77

stratum mucosum, 77

striated muscle, 189, 190

sturgeon, 29

stylo-hyal, I6I, 284

stylo-hyoid ligament, I6I

styloid process, I6 1,284

sub-cutaneous lymph sâcs, 358

subdural space, 357

sub-lingua, 284

sublingual glands, 285,286

submandibular glands, 285, 286

submaxillary glands, 285, 286

submucosa, 260

subperitoneal space, 357

subvertebral space, 357,363

sulci, of brain, 417

sulcus centralis, 4I7

superciliary structures, 505

supra-clavicles, 174

supra-cleithra, I74

suprapericardial bodies, 288

Sus, 86, 489

suspensorium of jaw, I55

sweat-glands, II2, II3

swine, 484, 49 I

Sycandra, 50

sympathetic plexuses, 464

sympathetic system, 451, 463, 464 .

symphysis pubic, I72

syntropists, 244

syrinx, $3 \mathrm{I} 4$.

\section{$\mathrm{T}$}

tactile cells, 473

tactile corpuscles, 473

tactile sense, distribution of, 475

tactile spots, 473

tænia ventriculi quarti, 427

tæniæ chorioides, 4I9, 420

tail, I30, I32, I34, I35

tail, nerves of, $43 \mathrm{I}, 432$

talon, 279

Talpa, 277

tapetum nigrum, 423

tapir, 394

tarsal cartilages, 504

tarsal glands, II3, 505 
Tarsipes, 292

tarsus:-nomenclature of, I77-180; primitive condition of, I79; supernumerary elements, I8I, I82 taste, 475,476

taste-beakers, 475

taste buds, 469, 47I, 475

Taxeopoda, 38

teleosts, 30, 174, 287, 303, 305, 415, $4 \mathrm{I} 6,4 \mathrm{I} 9,430,486,489$

teeth, 267, 27I, 283. (V. also tooth):- -evolution of shapes, $278-280$; growth of, 273,274 ; kinds of, 275 ; of birds, 84 ; of selachians, $8 \mathrm{I}$; origin of, $8 \mathrm{I}$, I53, $154,271,272$; parts of, 273; reduction of, 277 ; replacement of, $280-283$

tela chorioidea, $4 \mathrm{I} 9$

telencephalon, 4II, 4I5-4I8, 443

temperature of animals, 356

tentorium, 4I7

testes, $49,367,379,383,394,396$

testes, position of, 393,395

thalami optici, 425

thecadont articulation, 273

thecæ of jaws, 273

thenar. pads, 9 I

theories of vertebrate ancestry:from annelids, 513-520; from arachnoids, 522-525; from archetype, 506-512; from articulates, 5I2, 513; from insect, 506, 512; from nemertean, 520-522; from protochordata, $525-538$

theromorphs, 32, 44

third ventricle of brain, 4II

thoracic duct, 357,360

thoracic vertebræ, I30

thymus gland, 286, 290, 363

thymus, origin of, $286-287$

thyreoid cartilage, I60, 3I4

thyreoid gland, 286, 289, 290, 363

thyreo-hyal, 284, 314

Tillodontia, 37

toad, $285,290,397,420,442$

tongue, $283-285$

tongue bars, of Amphioxus, 290

tonsils, 363

tooth. (V. also teeth)

tooth generations, 28I

tooth, primitive form of, 274

tooth, structure of, 80
Tornaria, 536, 537

tortoise-shell, 105

trabeculæ, I 44

trachea, 3I2

trachea, origin of, 270

tracheal pieces, I60, 3I 3

tracheal rings, 3I $3,3 \mathrm{I} 4$

triconodont dentition, 279

trigonodont dentition, 279

trilobites, I9

triradii, 9 I

Triton, I9, 67, 3I3, 483

tritubercular theory, 278,279

truncus arteriosus, 324

trunk vertebræ, I30

tuba auditiva, 269

tuberculum auriculi, 497

tubular glands, 97, I I2

tunica dartos, 396

Tunicata, 26, 260, 303, 529, 530, 53I, $532,533,535,537,538$

tunica vaginalis communis, 396

tunica vaginalis propria, 396

turtles, 33, 179, 20I, 253, 331, 360, 398, 399, 4I9, 431, 433, 483

Tylopoda, 39

tympanic bone, 496

tympanic bulla, 496

tympanic cavity, ossicles of, I59

tympanic membranes, 494

tympano-hyal, I6I, 284

tympanum, 493-496

typical vertebra (Owen's), 507, 508,

5 IO, 5 II

Tyson's glands, II3

\section{U}

ultimo-branchial bodies, 289

umbilical arteries, 70, 7I

umbilical cord, 377

umbilical veins, $70,7 \mathrm{I}$

umbilicus, 347

umbilicus, relation to bladder, 378

uncinate processes, I 36

ungulates, $394,417,480,496$

unstriated intrscle, 189

ureter, 376,383

urethra, 377, 393, 394, 401

urethral glands, $40 \mathrm{I}$

urinary bladder, 377,378

urinary organs, $369-378$

urinary papilla, 374 
urinary system, 369-378

urodeles, 3 I, 44, I74, I87, 20 I, 206, $227,229,238,247,287,288,346$, $354,355,440,442,47 \mathrm{I}, 493,503$

urogenital sinus, $378,387,388,393$, 394

urogenital system, origin of, 64,65 uropygeal gland, III

urostyle, I 35

Ursus, 87

uterine ligaments, 390

uterine mucous membrane, 72

uterus, 383, 387, 388, 389, 394;

bicornis, 390; bipartitus, 390 ;

duplex, 390; masculinus, 393;

simplex, 390

utriculo-saccular canal, 487

utriculus, 487 .

\section{V}

vagina, $387,389,390,394$

vasa aberrantia, 392

vasa efferentia, $38 \mathrm{I}, 383,385$

vascular system, 65,3 I7-319

vas deferens, 377. (See ductus deferens)

vein or veins:- 3 I8, 328 ; abdominal, 342 ; allantoic, $322,346,347$, 348; anterior cardinal, 32I, 326, 349, 350; azygos, 344, 350, 35I ; cardinal, 32I, 326, 34I; caudal, 327, 34I, 343; Cuvierian duct, 322, 326,346 ; duct of Cuvier, 322, 326, 346; ductus venosus Arantii, 348, 349 ; external jugular, 350 ; hemiazygos, 35I; hepatic, 323, 327; hepatic portal, 324, 327, 342, 344 ; iliac, $326,346,358$; internal jugular, 350 ; jugular, 350 , 358 ; lateral, 326,346 ; omphalomesenteric, $323,347,348$; ovarian, 346 ; portal, 323,327 ; post cava, 342, 344, 345, 349; posterior . cardinal, $32 \mathrm{I}, 326,343,344,345$, 349, 358; pulmocutaneous, 354; renal, 345 ; renales advehentes, 324,327 ; renales revehentes, 324 , 327 ; renal portal, 324, 327, 34I ; spermatic, 346; subclavian, 327 , 350, 358; subintestinal, 340,349 ; subintestinal of Amphioxus, 528;

\section{veins-(Continued)}

umbilical, $322,346,347,348$; vena anonyma, 350 , 352 ; vena cava anterior, 352 ; vena cava posterior, $342,344,345,349$; vitelline, 320, 340, 347, 353; yolk, 320, 340,347

velum palati, 27 I

ventral fins, $16_{4}$

ventral nerves, 436

ventricle, 324, 353-357

ventricles of brain, 62, 406, 4II, 4I2 Vermes, $25^{8}$

vertebræ, articulations of, I3I

vertebræ, development of, I24-I27

vertebral column, development of, I24-I 27

vertebral column of birds, I 32

vetebral column, regional differentiation of, 130

vertebral foramina, I3 8

vertebrate history, sketch of, I3-I5 vertebrates, phylogenetic tree of, 28 vesicles of brain, 4 II

vesicula prostatica, 393, 394

vernix caseosa, 95

vibrissæ, 472

vidian canal, 450

villi, chorionic, 70, 7I

visceral mesoderm, 63, 66

visceral arches, 267

visceral muscles, I90, I92, I93

visceral skeleton, I22, I52-I62

visceral skeleton, metamorphoses of, $\mathrm{I} 6 \mathrm{I}$

vitreous humor, 423,498 , 5 OI

vomero-nasal cartilage, 483

vomero-nasal organ, 483

voluntary muscle, 65, I89, I9o

\section{W}

Weber's apparatı1s, 486

whale, I65, 478, 49I, 505

white blood corpuscles, 3 I8, 362

white matter, 428

wish-bone, I4I

Wolffian body, 373, 386

Wolffian duct, $64,374,375,387,392$

woodchuck, 297

woodpecker, 283. 
X

Xenarthra, 37.

Y

yolk, 52, 53, 69, 73

yolk sac, 69, 70, 319 yolk stalk, 69,70

\section{Z}

zona radiata, 52

zonary placenta, 72

zygapophyses, 508

zygote, $7,48,58$. 



\section{Emerican 戸ctence ‡eries}

The two principal objects of the series are to supply author itative books whose principles are, so far as practicable, illustrated by American facts, and also to supply the lack that the advance of science perennially creates, of text-books which at least do not contradict the latest generalizations.

\section{Physics.}

By A. I. Kimbal, , Professor in Amherst College. (In preparation.)

\section{Physics.}

By GEORGE F. BARKER. $\mathrm{x}+902$ pp. $\$ 3.50$.

\section{Chemistry.}

By IRA Remsen, President of the Johns Hopkins University.

Advanced Course. $\quad x x i i+853$ pp. $\$ 3.00$.

College Chemistry. $\mathrm{xx}+689 \mathrm{pp} . \$ 2.25$.

Briefer Course. xxiv +5 I6 pp. \$I.25.

Elementary Course. $x+287$ pp. 80 cents.

\section{Astronomy.}

By Simon Newcomb and EDward S. Holden.

Advanced Course. $\quad x i i+512$ pp. $\$ 2.00$.

Briefer Course. $\mathrm{x}+366$ pp. $\$ \mathrm{I} .2 \mathrm{O}$.

Elementary Course. $\mathrm{xv}+446 \mathrm{pp}$. \$1.20.

\section{Geology.}

By Thomas C. Chamberlin and Rollin D. Salisbury, Professors in the University of Chicago. 3 vols. 8 vo.

Vol. I. Geological Processes and their Results. xix +654 pp. \$4.00.

Vols. II and III. Earth History. xxxvii + 1316 pp. (Not sold separately.) \$8.00.

\section{Physiography.}

By Rollin D. Salisbury, Professor in Chicago University.

Advanced Course. $\mathrm{xx}+770 \mathrm{pp} . \quad \$ 3.50$.

Briefer Course. viii + 53I pp. \$I.50. 


\section{General Biology.}

By William 'T. SEn GWICK, Professor in the Mass. Institute, and EDMUND B. Wilson, Professor in Columbia University. $x i i+231 \mathrm{pp} . \quad \$ 1.75$.

Botany.

By Charles E. Bessey, Professor in the University of Nebraska.

Advanced Course. $x+611$ pp. $\$ 2.20$.

Briefer Course. vii +356 pp. \$1.12.

\section{Zoology.}

By A. S. Packarn.

Advanced Course. viii +722 pp. $\$ \$ 2.50$.

Briefer Course. viii +338 pp. 4.12 .

Elementary Course. viii $+290 \mathrm{pp}$. 80 cents.

The Human Body.

By H. NEWELL MaRTIN.

Advanced Course. $x v i+685$ pp. $\$ 2.50$.

Briefer Course. $x i v+408 \mathrm{pp} . \$ 1.25$.

Elementary Course. vi +261 pp. 80 cents.

\section{Psychology.}

By William James, Professor in Harvard University.

Advanced Course. 2 volumes. $\$ 5.00$.

Briefer Course. $x i i i+478$ pp. $\$ 1.60$.

\section{Ethics.}

By JoHn DeWEy, Rrofessor in Columbia University, and JAMEs H. TuFts, Professor in the University of Chicago. (In press.)

\section{Political Economy.}

By Francis A. Walker.

Advanced Course. viii +537 pp. $\$$ \$2.00.

Briefer Course. viii +415 pp. $\$ 1.20$.

Elementary Course. $\mathrm{x}+323 \mathrm{pp} . \$ 1.00$.

\section{Finance.}

By HENRy C. ADAms, Professor in the University of Michigan. $x i v+573$ pp. $\$ 3.00$. 


\section{THE AMERICAN NATURE SERIES}

In the lope of doing something toward furnishing a series where the nature-lover can surely find a readable book of high authority, the publishers of the American Science Series have begun the publication of the American Nature Series. It is the intention that in its own way, the new series shall stand on a par with its famous predecessor.

The primary object of the new series is to answer questions which the contemplation of Nature is constantly arousing in the mind of the unscientific intelligent person. But a collateral object will be to give some intelligent notion of the "causes of things."

While the coöperation of foreign scholars will not be declined, the hooks will be undar the guarantee of American experts, and generally from the American point of view; and where material crowds space, preference will be given to American facts over others of not more than equal interest.

The series will be in six divisions :

\section{NATURAL HISTORY}

This division will consist of two sections.

Section A. A large popular Natural History in several volumes, with the topics treated in due proportion, by authors of unquestioned authority. 8vo. $i \frac{1}{2} \times 10 \frac{1}{4}$ in.

The books so far publisht in this section are:

FISHES, by Davin Starr Jorman, President of the Leland Stanford Junior University. \$6.00 net; carriage extra.

AMERICAN INSECTS, by Vernor L. Ketrogg, Professor in the Leland Stanford Junior University. $\$ 5.00$ net; carriage extra.

BIRDS OF THE WORLD. A popular account by FraNK $\mathrm{H}$. Knowlton, M.S., Ph.D., Member American Ornithologists Union, President Biological Society of Washington, etc., etc., with Chapter on Anatomy of Birds by FreDERIC A. LuCAs, Chief Curator Brooklyn Museum of Arts and Sciences, and edited by Roben' Ringway, Curator of Birds, U. S. National Museum. $\$ 7.00$ net; carriage extra.

\section{Arranged for are:}

SEEDLESS PLANTS, by Grorgr: T. Moore, Head of Department of Botany, Marine Biological Laboratory, assisted by other specialists.

WILD MAMMALS OF NORTH AMERICA, by C. HART MERrian, Chicf of the United States Biological Survey. 
REPTILES AND BATIRACHIANS, by Lronham STEJ Curator of Reptiles, U. S. National Museum.

Section B. A Shorter Natural History, mainly by the Authors of Section A, preserving its popular character, its proportional treatment, and its authority so far as that can be preserved without its fullness. Size not yet determined.

\section{CLASSIFICATION OF NATURE}

1. Library Series, very full descriptions. 8 vo. $7 \frac{1}{2} \times 10 \frac{1}{1}$ in.

\section{Already publisht:}

NORTH AMERICAN TREES, by N. L. BRITTON, Director of the New York Botanical Garden. \$7.00 net; carriage extra.

FERNS, by Camphelt. E. Waters, of Johns Hopkins University, 8 vo, pp. xi+362. \$3.00 net; by mail, \$3.30.

2. Poc'set Series, Identification Books - "How to Know," brieŕ and in portable shape.

\section{FUNCTIONS OF NATURE}

These books will treat of the relation of facts to causes and effects - of heredity and the relations of organism to environment. 8 vo. $6 \frac{5}{8} \times 8 \frac{7}{8}$ in.

\section{Already publisht:}

THE BIRD : ITS FORM AND FUNCTION, by C. W. BEEBE, Curator of Birds in the New York Zoological Park. 8vo, 496 pp. $\$ 3.50$ net; by mail, $\$ 3.80$.

\section{Arranged for:}

THE INSECT: ITS FORM AND FUNCTION, by VERNoN L. KELLOG , Professor in the Leland Stanford Junior University.

THE FISH : ITS FORM AND FUNCTION, by H. M. SмITH, of the U. S. Bureau of Fisheries.

\section{WORKING WITH NATURE}

How to propagate, develop, care for and depict the plants and animals. The volumes in this group cover such a range of subjects that it is impracticable to make them of uniform size.

\section{Already publisht:}

NATURE AND HEALTh, by Edward Curtis, Professor Emeritus in the College of Physicians and Surgeons. 12mo. \$1.25 net; by mail, $\$ 1.37$. 


\section{A M E R I C A N A T U R E E R I ES (Continued)}

THE FRESHWATER AQUARIUN AND ITS INHABITANTS.

A Guide for the Amateur Aquarist, by OTTo Eggeing and

Fredehick Ehuenberg. Large $12 \mathrm{mo}$. \$2.00 net; by mail, \$2.19.

THE LifE OF a FOSSIL HUNTER, by Charles H. Sternibig. Large $12 \mathrm{mo}$. $\$ 1.60$ net; by mail, $\$ 1.72$.

\section{Arranged for:}

PhOTOGRAPHING NATURE, by E. R. Sanborn, Photographer of the New York Zoological Park.

THE SHELLFISH INDUSTRIES, by James L. Kellogg, Professor in Williams College.

Chemistry of DAILY Life, by Henry P. Talbot, Professor of Chemistry in the Massachusetts Institute of Technology.

DOMESTIC ANIMALS, by WiritaM H. Brewer, Professor Emeritus in Yale University.

THE CARE OF TREES IN LAWN, STREET AND PARK, by B. E. Fernow, Professor of Forestry, University of Toronto.

\section{DIVERSIONS FROM NATURE}

This division will include a wide range of writings not rigidly evstematic or formal, but written only by authorities of standing. Large $12 \mathrm{mo} .5 \frac{7}{4} \times 8 \frac{1}{8}$ in.

Already publisht:

INSECT STORIES, by Vernon L. Kellogg. \$1 50 net; by mail, $\$ 1.62$.

FISH STORIES, by Charles F. Holder and David Starr Jozdax. Larye $12 \mathrm{mo}$. $\$ 1.75$ net; by mail, $\$ 1.87$.

Arranged for:

HORSE TALK, by WILIIAM H. BrEWER.

BIRD NOTES, by C. W. BEERE.

\section{THE PHILOSOPHY OF NATURE}

A Series of volumes by President Jondan, of Stanford University, and Professors Brooks of Johns Hopkins, Lull of Yale, Tromson of Aberdeen, Przibran of Austria, zur Strassen of Germany, and others. Edited by Professor Kellogg of Leland Stanford. 12mo. $5 \frac{1}{8} \times 7 \frac{1}{2} \mathrm{in}$.

JUNE, '09.

HENRY HOLT AND COMPANY, NEW YORK 


\section{Fitz's Physiology and Hygiene}

By GRORGe W. FrTz, M.D., former Assistant Professor of Pliysiology and Hygiene in Harvard University, and sometime 'Teacher of Science in Cook County Normal School, Ill. xiii +357 pp. I2mo. \$r.I2.

The book is suitable for use in the first year of the high school. Special emphasis is laid upon hygiene and sanitation, and experimental work is fully outlined in connection with each chapter.

- Dr. G. L. Meylan of Columbia University in the "Edu. cational Review":- This volume marks a distinct advance in the effort to produce a satisfactory text-book of physiology and hygiene for high schools. . . . It includes chapters on physiology, personal hygiene, community hygiene, and first aid to the injured. . . . An abundance of exceptionally good illustrations and the addition at the end of each chapter of simple experiments constitute valuable aids in understanding the structure and functions of the various organs.

\section{Martin's The Human Body Briefer Course.}

By H. Newell Martin, F.R.S., sometime Professor in the Johns Hopkins University. Fifth Edition, Revised, with Practical Exercises, by GEORGE W. FITz, former Assistant Professor in Harvard University. xiv $+408 \mathrm{pp}$. I2mo. (American Science Series.) \$1.25.

\section{Peabody's Laboratory Exercises in Anatomy and Physiology}

By JAmEs E PEABody, Instructor in the Morris High School, New York. $x+79$ pp. I2mo. Interleaved. 60 cents.

Martin's The Human Body Advanced Course.

By H. Newell Martr, sometime Professor in the Johns Hopkins University. New Edition. xvi 685 pp. $8 v o$. (American Science Series.) \$2.50. Copies without the Chapter on Reproduction will be supplied when specially ordered.

\section{HENRY HOLT AND COMPANY NEW YORK







